\title{
HIGH-TEMPERATURE PHOTOCHEMICAL DESTRUCTION OF TOXIC ORGANIC WASTES USING CONCENTRATED SOLAR RADIATION
}

B. Dellinger

J.L. Graham

J.M. Berman

P.H. Taylor

Environmental Sciences and Engineering University of Dayton 300 College Park Avenue Dayton, OH 45469-0132

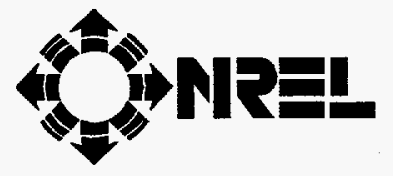

National Renewable Energy Laboratory 1617 Cole Boulevard Golden, Colorado 80401-3393

A national laboratory of the U.S. Department of Energy Operated by Midwest Research Institute for the U.S. Department of Energy Under Contract No. DE-AC02-83CH10093

Under Subcontract No. TAE-2-12246-01 MASTER May 1994 OBMEBUTION OF THIS QDCUMENT IS UNLLMTTED 


\section{NOTICE}

NOTICE: This report was prepared as an account of work sponsored by an agency of the United States government. Neither the United States government nor any agency thereof, nor any of their employees, makes any warranty, express or implied, or assumes any legal liability or responsibility for the accuracy, completeness, or usefulness of any information, apparatus, product, or process disclosed, or represents that its use would not infringe privately owned rights. Reference herein to any specific commercial product, process, or service by trade name, trademark, manufacturer, or otherwise does not necessarily constitute or imply its endorsement, recommendation, or favoring by the United States government or any agency thereof. The views and opinions of authors expressed herein do not necessarily state or reflect those of the United States government or any agency thereof.

Printed in the United States of America

Available from:

National Technical Information Service

U.S. Department of Commerce

5285 Port Royal Road

Springfield, VA 22161

Price: Microfiche A01

Printed Copy A13

Codes are used for pricing all publications. The code is determined by the number of pages in the publication. Information pertaining to the pricing codes can be found in the current issue of the following publications which are generally available in most libraries: Energy Research Abstracts (ERA); Government Reports Announcements and Index (GRA and I); Scientific and Technical Abstract Reports (STAR); and publication NTIS-PR-360 available from NTIS at the above address. 


\section{DISCLAIMER}

Portions of this document may be illegible in electronic image products. Images are produced from the best available original document. 


\section{PREFACE}

Continuing concern from environmental and citizens groups over the potential dangers resulting from incineration of hazardous waste, has resulted in considerable interest in developing alternative treatment methods. Solar energy is one of our greatest and undoubtedly most underutilized natural resources. In response to a 1984 request from the then Department of Energy's Solar Energy Research Institute for innovative uses of solar energy, we propose to use concentrated solar radiation to destroy hazardous waste. As opposed to the use of just the thermal energy in solar radiation to "burn" the waste, our concept focused on the synergistic combination of thermal energy (viz. infrared and visible solar radiation) and photolytic energy (viz. ultraviolet solar radiation) to induce a thermally enhanced photochemical destruction of the waste.

Our own research which began in 1985 as well as that conducted by NREL researchers and investigators at other institutions has shown that this approach may be practical but also involves scientific concepts that have not previously been explored. The progress made to date, although not always as rapid as one would like, has been gratifying from a pure science and environmental perspective. The currently planned, large-scale demonstration that is co-sponsored by the Department of Energy, the USEnvironmental Protection Agency and the U.S. Army Toxic and Hazardous Materials Agency represents a culmination of 8 years of research by many researchers. We hope that our contributions will result in the implementation of this technology and help those who will continue in its development and improvement in the future. 
TABLE OF CONTENTS

Section

Preface

Table of Contents

List of Figures

List of Tables

Acknowledgements

Project Summary

1.0 Introduction

2.0 Theory of High-Temperature Photochemistry 3

2.1 Basic Physical, Thermal/Photolytic Reaction Model 3

2.2 Basic Kinetic Formulation

2.3 Temperature Dependence of Elementary

Photochemical Processes

7

2.4 References

3.1 Thermal/Photolytic Reactor System

3.2 Advanced Thermal/Photolytic Reactor System

3.3 High Temperature Absorption Spectrometer

3.4 Experimental Approach

4.0 Enhanced Destruction of Pure Compounds 33

4.1 Effect of Temperature 33

4.2 Effect of Time $\quad 39$

4.3 Effect of Light intensity 41

4.4 Effect of Fuel/Air Stoichiometry 45

4.5 Destruction of 2,6-Dinitrotoluene $\quad 47$

4.5.1 Experimental $\quad 48$

4.5.2 Results and Discussion $\quad 48$

4.6 References $\quad 54$

5.0 Mineralization of By-Products 56

5.1 PICs from the Pyrolysis of 1,2,3,4-Tetrachlorodibenzo-
dioxin.

5.2 Thermal/Photolytic Oxidation of Monochlorobenzene 58

5.2.1 Experimental $\quad 58$

$\begin{array}{ll}5.2 .2 \text { Results } & 60\end{array}$

5.2.3 Discussion 64

5.3 Thermal/Photolytic Oxidation of $3,3^{\prime}, 4,4^{\prime}$-Tetrachlorobiphenyl

5.3.1 Experimental $\quad 72$ 
TABLE OF CONTENTS (continued)

5.3.2 Results

5.3.3 References

6.0 Effects on Mixtures 82

6.1 Oxidation of a Spiked, Fuel Oil Mixture-I

6.2 Oxidation of a Spiked, Fuel Oil Mixture-ll 83

6.3 Oxidation of a Synthetic 8-Component Mixture 86

6.4 References $\quad 89$

7.0 High-Temperature Spectroscopy 94

7.1 The Ground Electronic State 94

7.1.1 Experimental 94

7.1.2 Results 95

7.1.3 The Ground Electronic State $\quad 95$

7.2 Excited Electronic States 106

7.3 References 111

8.0 Engineering Scale-Up 113

8.1 Field Demonstration of Destruction of TCDD 113

8.1.1 Experimental 113

8.1.2 Results 116

8.1.3 Discussion 119

8.2 Droplets in an Intense Radiative Field 123

8.2.1 Radiative Heating 124

8.2.2 Photo-Induced Ignition $\quad 128$

8.2.3 Droplet Lensing 128

8.3 References 130

9.0 Destruction of Non-Absorbing Compounds $\quad 131$

9.1 Homogeneous Initiation 131

9.1.1 Energy Transfer Sensitization 132

9.1.2 Photo-Generated Radical Initiation 136

9.2 Heterogeneous Initiation 141

9.2.1 Generation of Reactive Species 142

9.2.2 Effectiveness of Reactive Species 151

9.2.3 Mixing the Reactive Species and Bulk Waste 156

9.2.4 Mechanistic Aspects of Photo-Catalytic

Generation of Reactive Species $\quad 157$

$\begin{array}{lll}9.3 & \text { References } & 164\end{array}$

$\begin{array}{ll}10.0 \text { Conclusions } & 165\end{array}$

$\begin{array}{lll}11.0 & \text { Recommendations } & 168\end{array}$ 
12.0 Bibliography

Appendices

Appendix 1

Appendix 2

202

Appendix 3

Appendix 4 244

Appendix 5

253

Appendix 6

261

Appendix 7 


\section{LIST OF FIGURES}

Figure 2.1. An air mass 1.5 (AM 1.5) solar irradiance spectrum at the earth's surface through typical clean-air atmospheric conditions.

Figure 2.2. Energy diagram illustrating the central principals of the solar detoxification process.

Figure 2.3. Three state model for thermal-photolytic dissociation. $k_{a b}$ is the rate of energy absorption. $\mathrm{kF}_{\mathrm{F}}$ is the rate of excited state deactivation. $k_{1}[M]$ and $k_{-1}[M]$ are the pressure dependent rates of thermal activation and deactivation, respectively. State 1 is the reactive state corresponding to the intrinsic energy barrier to reaction with the rate of passage over the barrier given by $k_{p}$.

Figure 3.1. Schematic of the Thermal/Photolytic Reactor System (TPRS) showing the instrument's general layout and major features.

Figure 3.2. Schematic of the Advanced Thermal/Photolytic Reactor System (ATPRS) showing the instrument's general layout and major features.

Figure 3.3. Schematic of the High-Temperature Absorption Spectrophotometer (HTAS) showing the instrument's general layout and major features.

Figure 3.4. Relative output of the TPRS's solar simulator fitted with a dichroic mirror and air mass filters and a AM 1.5 solar spectrum.

Figure 3.5. Relative filtered solar simulator emission and chlorine absorption spectra.

Figure 3.6. Test results actinometer destruction efficiencies are very different under deuterium lamp and Argon ion $\left(\mathrm{Ar}^{+}\right)$laser irradiation.

Figure 4.1. TPRS data for 1-nitronaphthalene $(\sim 10.5 \mathrm{M})$ exposed to 0 and 307 AM 1.0 suns simulated for 5 seconds in air.

Figure 4.2. Examples of the thermal/photolytic enhancement ratio (oxidation) as a function of temperature for which a maximum enhancement was bserved. These include 1-chloronaphthalene ( 307 AM 1.0 suns for 5 s $n$ air), 3,3', 4,4'-tetrachlorobiphenyl (273 AM 1.5 suns for $10 \mathrm{~s}$ in air), nd 1,2,3,4-tetrachlorodibenzo-p-dioxin (307 AM 1.0 suns for $10 \mathrm{~s}$ in air). 
Figure 4.3. Examples of the thermal/photolytic enhancement ratio (oxidation) as a unction of temperature in which a maximum enhancement was not bserved. These include 1-nitro-naphthalene ( 307 AM 1.0 suns for $5 \mathrm{~s}$ in air), 3,3',4,4'-tetrachlorobiphenyl (307 AM 1.0 suns for $10 \mathrm{~s}$ in helium), and xanthone ( 307 AM 1.0 suns for $5 \mathrm{~s}$ in air).

Figure 4.4. The molar extinction spectra for methylene chloride at 200, 400, 600 , and $700^{\circ} \mathrm{C}$.

Figure 4.5. The rate of photon absorption for 3,3',4,4'-tetrachlorobiphenyl exposed to $273 \mathrm{AM} 1.5$ suns illustrating that $k_{a b}$ increases exponentially with temperature.

Figure 4.6. The photochemical quantum yield as a function of temperature for 3,3', 4,4'-tetrachloro-biphenyl $\left(3.78 \times 10^{-6} \mathrm{M}\right)$ exposed for 10 seconds in air to 0 and 273 AM 1.5 suns.

Figure 4.7. The photochemical quantum yield as a function of temperature for monochlorobenzene $\left(2.95 \times 10^{-5} \mathrm{M}\right)$ exposed for 10 seconds in air to pulsed laser illumination at $280 \mathrm{~nm}\left(862 \mathrm{~mW} / \mathrm{cm}^{2}\right)$.

Figure 4.8. TPRS data for 3,3',4,4'-tetrachlorobiphenyl $\left(\sim 10^{-5} \mathrm{M}\right)$ exposed for 5 and 10 seconds in air to 307 AM 1.0 suns (simulated).

Figure 4.9. The enhancement ratio for xanthone $\left(\sim 10^{-5} \mathrm{M}\right)$ exposed at $650^{\circ} \mathrm{C}$ for 5 seconds in air with simulated solar radiation ranging from 0 to 307 AM 1.0 suns.

Figure 4.10. The enhancement ratio for monochlorobenzene $\left(2.95 \times 10^{-5} \mathrm{M}\right)$ exposed to $600^{\circ} \mathrm{C}$ for 10 seconds in air and with laser illumination at $280 \mathrm{~nm}$ from 0 to $862 \mathrm{~mW} / \mathrm{cm}^{2}$.

Figure 4.11. TPRS data for 1,2,3,4-tetrachlorodibenzo-p-dioxin $\left(\sim 10^{-5} \mathrm{M}\right)$ exposed in air (oxidation) and helium (pyrolysis) for 10 seconds to 307 AM 1.0 suns simulated.

Figure 4.12. The ratio of fraction remaining from pyrolysis versus oxidation for 1,2,3,4-tetrachlorodibenzo-p-dioxin $\left(\sim 10^{-5} \mathrm{M}\right)$ exposed to $307 \mathrm{AM}$ 1.0 suns simulated for 10 seconds in air (oxidation) and helium (pyrolysis). 
Figure 4.13. The enhancement ratio as a function of temperature for 1,2,3,4tetrachlorodibenzo-p-dioxin $\left(\sim 10^{-5} \mathrm{M}\right)$ exposed to 0 and $307 \mathrm{AM}$ 1.0 suns simulated for 10 seconds in air (oxidation) and helium (pyrolysis).

Figure 4.14. TPRS chromatograms from the thermal and thermal/photolytic decomposition of 2,6 -dinitrotoluene $\left(5 \times 10^{-8} \mathrm{M}\right)$ exposed to $350 \mathrm{AM}$ 1.5 suns (simulated) for 10 seconds in helium

Figure 4.15. TPRS chromatograms from the thermal and thermal/photolytic decomposition of 2,6 -dinitrotoluene $\left(5 \times 10^{-8} \mathrm{M}\right)$ exposed to 350 AM 1.5 suns (simulated) for 10 seconds in air.

Figure 4.16. TPRS data for 2,6-dinitrotoluene $\left(5 \times 10^{-8} \mathrm{M}\right)$ exposed to $350 \mathrm{AM}$ 1.5 suns (simulated) for 10 seconds in helium.

Figure 4.17. TPRS data for 2,6 -dinitrotoluene $\left(5 \times 10^{-8} \mathrm{M}\right)$ exposed to $350 \mathrm{AM}$ 1.5 suns (simulated) for 10 seconds in air.

Figure 5.1. TPRS data for 1,2,3,4-tetrachlorodibenzo-p-dioxin $\left(\sim 10^{-5} \mathrm{M}\right)$ exposed to 0 and 307 AM 1.0 suns (simulated) for $10 \mathrm{~s}$ in helium.

Figure 5.2. TPRS chromatograms for 1,2,3,4-tetrachlorodibenzo-p-dioxin $\left(\sim 10^{-5} \mathrm{M}\right)$ exposed in helium to $307 \mathrm{AM} 1.0$ suns (simulated) for $10 \mathrm{~s}$ at $500^{\circ} \mathrm{C}$ (top) and under purely thermal conditions at $700^{\circ} \mathrm{C}$ (bottom).

Figure 5.3. TPRS data for monochlorobenzene $\left(2.95 \times 10^{-5} \mathrm{M}\right)$ exposed under purely thermal conditions for $10 \mathrm{~s}$ in air.

Figure 5.4. TPRS data for monochlorobenzene $\left(2.95 \times 10^{-5} \mathrm{M}\right)$ exposed to $862 \mathrm{~mW} / \mathrm{cm}^{2}$ of $280 \mathrm{~nm}$ radiation for $10 \mathrm{~s}$ in air.

Figure 5.5. Temperature dependent $S_{1} \leftarrow S_{0}$ absorption spectra for monochlorobenzene at 150,400 , and $700^{\circ} \mathrm{C}$.

Figure 5.6. State/process diagram for monochlorobenzene.

Figure 5.7. Proposed pathway to the organic products observed from the thermal/photolytic oxidation of monochlorobenzene.

Figure 5.8. Two example TPRS chromatograms from 3,3',4,4'-tetrachloro-

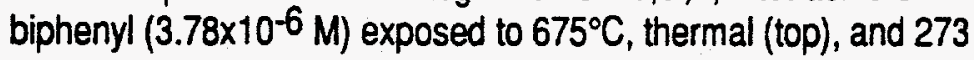
AM 1.5 suns (simulated) at $550^{\circ} \mathrm{C}$ for $10 \mathrm{~s}$ in air (bottom). 
Figure 5.9. The relative molar yield as a function of temperature for the product, 1,2-dichlorobenzene from 3,3', 4, 4'-tetrachlorobiphenyl (3.78 x $10^{-6} \mathrm{M}$ ) exposed to 0 and $273 \mathrm{AM} 1.5$ suns (simulated) for $10 \mathrm{~s}$ in air.

Figure 5.10. The relative molar yield as a function of temperature for the product, 3,4,4'-trichlorobiphenyl from 3,3',4,4'-tetrachiorobiphenyl (3.78x $10^{-6} \mathrm{M}$ ) exposed to 0 and 273 AM 1.5 suns (simulated) for $10 \mathrm{~s}$ in air.

Figure 5.11. The relative molar yield as a function of temperature for the product, tetrachlorodibenzofuran from 3,3',4,4'-tetrachlorobiphenyl (3.78x $10^{-6} \mathrm{M}$ ) exposed to 0 and $273 \mathrm{AM} 1.5$ suns (simulated) for $10 \mathrm{~s}$ in air.

Figure 6.1. TPRS data for the oxidation of a fuel oil mixture spiked with chloronitrobenzene, dichlorobenzene, trichloroethylene, and carbon tetrachloride exposed to 0 and $307 \mathrm{AM} 1.0$ suns (simulated) for $5 \mathrm{~s}$ in air. These curves are for total HFID response that includes feed components and PICs.

Figure 6.2. TPRS data for the combined carbon tetrachloride and trichloroethylene components of a spiked fuel oil exposed to 0 and 307 AM 1.0 suns (simulated) for $5 \mathrm{~s}$ in air.

Figure 6.3. TPRS data for the dichlorobenzene component of a spiked fuel oil exposed to 0 and 307 AM 1.0 suns (simulated) for $5 \mathrm{~s}$ in air.

Figure 6.4. TPRS data for the chloronitrobenzene component of a spiked fuel oil exposed to 0 and $307 \mathrm{AM}$ suns (simulated) for $5 \mathrm{~s}$ in air.

Figure 6.5. TPRS data for the oxidation of a fuel oil mixture spiked with dichlorobenzene, carbon tetrachloride, toluene, and naphthalene exposed to 0 and 273 AM 1.5 suns (simulated) for 10 s in air. These curves are for total HFID response and include both feed components and PICs.

Figure 6.6. TPRS data for the toluene component of a spiked fuel oil exposed to 0 and 273 AM 1.5 suns (simulated) for 10 s in air.

Figure 6.7. TPRS data for the naphthalene component of a spiked fuel oil exposed to 0 and 273 AM 1.5 suns (simulated) for $10 \mathrm{~s}$ in air.

Figure 6.8. TPRS data for the dichlorobenzene component of a spiked fuel oil exposed to 0 and 273 AM 1.5 suns (simulated) for 10 s in air. 
Figure 6.9. TPRS data for the methylene chloride component of an 8 component mixture exposed to 0 and 192 AM 1.0 suns (simulated) for $10 \mathrm{~s}$ in air.

Figure 6.10. TPRS data for the trichloroethylene component of an 8 component mixture exposed to 0 and 192 AM 1.0 suns (simulated) for $10 \mathrm{~s}$ in air.

90

Figure 6.11. TPRS data for the aniline component of an 8 component mixture exposed to 0 and 192 AM 1.0 suns (simulated) for 10 s in air.

Figure 6.12. TPRS data for the pyridine component of an 8 component mixture exposed to 0 and 192 AM 1.0 suns (simulated) for 10 s in air.

Figure 6.13. TPRS data for the nitrobenzene component of an 8 component mixture exposed to 0 and $192 \mathrm{AM} 1.0$ suns (simulated) for $10 \mathrm{~s}$ in air.

Figure 6.14. TPRS data for the naphthalene component for an 8 component mixture exposed to 0 and 192 AM 1.0 suns (simulated) for 10 s in air.

Figure 6.15. TPRS data for the 1-nitronaphthalene component of an 8 component mixture exposed to 0 and $192 \mathrm{AM} 1.0$ suns (simulated) for $10 \mathrm{~s}$ in air.

Figure 7.1. The $S_{1} \leftarrow S_{0}$ absorption spectra of benzene from 22 to $600^{\circ} \mathrm{C}$ showing the loss of fine spectra features, and an overall increase in absorption intensity and shift towards longer wavelengths.

Figure 7.2. Temperature dependent $S_{1} \leftarrow S_{0}$ absorption spectra of chlorobenzene.

Figure 7.3. Temperature dependent $S_{1} \leftarrow S_{0}$ absorption spectra of tetrachlorobiphenyl.

Figure 7.4. High temperature absorption spectroscopy. Ground state thermal excitation allows for a reduction of energy required to access electronically excited states.

Figure 7.5. Thermally induced red shifts of the electronically excited singlet states of benzene, chlorobenzene, and tetrachlorobiphenyl.

Figure 7.6. Thermal and (xenon lamp simulated) concentrated solar-assisted pyrolysis of chlorobenzene. $[\phi \mathrm{C}]=\left(10^{-5}\right) \mathrm{M}$, retention time $=10.0 \mathrm{~s}$, in a helium carrier. 
Figure 7.7. State $\mid i>$ lends character to states $\mid f>$ and $\mid g>$ via a vibronic perturbation, $H_{\text {V.C. }}, f^{\prime}=f+\alpha i$, and $g^{\prime}=g+\beta i$.

Figure 7.8. Ambient temperature $\left(\sim 20^{\circ} \mathrm{C}\right)$ and $300^{\circ} \mathrm{C}$ absorption spectra of molecular chlorine. Note that the integrated intensity remains constant.

Figure 7.9. Diagram of the High Temperature Emission Spectrometer (HTES).

Figure 7.10. The $S_{1} \rightarrow S_{0}$ dispersed fluorescence spectrum of toluene at $150^{\circ} \mathrm{C}$.

Figure 8.1. General schematic of the White Sands Solar Furnace which was used for the field tests reported here

Figure 8.2. Detailed schematic of the bench-scale reactor which was designed by NREL and installed in the White Sands Solar Furnace.

Figure 8.3. Summary of the field data for 1,2,3,4-TCDD including thermal (nearUV below $400 \mathrm{~nm}$ attenuated), solar (full solar spectrum).

Figure 8.4. Summary of the laboratory and field data for 1,2,3,4-TCDD with a mean exposure time of $5 \mathrm{~s}$ in air.

Figure 8.5. Thermal performance of the bench scale reactor for the oxidation of $1,2,3,4-T C D D$ with a mean exposure time of $5 s$ as predicted by a compartment model of the reactor, and measured at the WSSF with wavelengths $<400 \mathrm{~nm}$ removed from the solar spectrum.

Figure 8.6. Solar performance of the bench scale reactor for the oxidation of $1,2,3,4-T C D D$ with a mean exposure time of $5 \mathrm{~s}$ as predicted by a compartment model of the reactor, and measured at the WSSF using the entire ambient solar spectrum.

Figure 8.7. Absorption spectrum of 1,2,3,4-TCDD @ $23^{\circ} \mathrm{C}$ in benzene and an AM 1.5 solar irradiance spectrum.

Figure 8.8. A typical gasification profile for a droplet.

Figure 8.9. Calculated ray traces for a $500 \mu \mathrm{m}$ diameter droplets with indices of refraction, nD of 1.4 and 1.9. Note, an nD of 1.4 is typical for that of No. 5 fuel oil. 
Figure 9.1. Electronic state energy diagram for acetone and benzene illustrating to slightly endothermic triplet-triplet energy transfer.

Figure 9.2. Electronic state energy diagram for benzophenone and naphthalene illustrating exothermic triplet-triplet energy transfer.

Figure 9.3. TPRS data for benzene $\left(\sim 10^{-5} \mathrm{M}\right)$ in the presence of acetone $\left(\sim 10^{-5} \mathrm{M}\right)$ exposed to 0 and 307 AM 1.0 suns (simulated) for $5 \mathrm{~s}$ in helium.

Figure 9.4. TPRS data for naphthalene $\left(\sim 10^{-5} \mathrm{M}\right)$ in the presence of benzophenone $\left(\sim 10^{-5} \mathrm{M}\right)$ exposed to 0 and 307 AM 1.0 suns (simulated) for $5 s$ in helium.

Figure 9.5. TPRS data for benzophenone $\left(\sim 10^{-5} \mathrm{M}\right)$ exposed to 0 and $307 \mathrm{AM}$ 1.0 suns (simulated) for $5 s$ in air.

Figure 9.6. TPRS data for the methylene chloride $\left(\sim 10^{-5} \mathrm{M}\right)$ component of a chlorine/methylene chloride mixture $(3: 1, v: v)$ exposed to 0 and 307 AM 1.0 suns (simulated) for 10 s in helium.

Figure 9.7. TPRS data for the methylene chloride component of a chlorine/ methylene chloride mixture $(3: 1, \mathrm{v}: \mathrm{v})$ exposed to 0 and 307 AM 1.0 suns (simulated) for $10 \mathrm{~s}$ in air. Note that the fraction remaining under the simulated solar conditions is below the detection limit of $0.01 \%$ at all temperatures.

Figure 9.8. TPRS data for the methylene chloride component of a chlorine/ methylene chloride mixture with the initial molar ratio of molecular chlorine to methylene chloride $\left(\sim 10^{-5} \mathrm{M}\right)$ varying from 0 to 1.25 exposed to $307 \mathrm{AM} 1.0$ suns (simulated) and $400^{\circ} \mathrm{C}$ for $10 \mathrm{~s}$ exposure in air.

Figure 9.9. TPRS data for trichloroethylene $\left(\sim 10^{-5} \mathrm{M}\right)$ exposed to 0 and 307 AM 1.0 suns (simulated) for 10 s in air.

Figure 9.10. TPRS data for the trichloroethylene $\left(\sim 10^{-5} \mathrm{M}\right)$ component of a hydrogen peroxide/trichloroethylene mixture $(10: 1, \mathrm{v}: \mathrm{v})$ exposed to 0 and 307 AM 1.0 suns (simulated) for 10 s in air.

Figure 9.11. Generalized diagram of a reactor using the Solar Catalytically Activated Destruction (Sol-CAD) process. 
Figure 9.12. A diagram of a pump/probe experimental apparatus modified to study the Sol-CAD process.

Figure 9.13. A detailed sketch of the Sol-CAD test cell assembly.

Figure 9.14. a) Typical baseline $\mathrm{OH}$ signal at $300^{\circ} \mathrm{K}$. Baseline tests consisted of no precursor, no pump laser, no probe laser, or probe laser turned off-resonance. b) Time dependent gas-phase $\mathrm{OH}$ signal from $\mathrm{H}_{2} \mathrm{O}_{2}$ over a $\mathrm{TiO}_{2}$ catalyst at $300^{\circ} \mathrm{K}$ irradiated with $351 \mathrm{~nm}$ excimer laser radiation.

Figure 9.15. $\mathrm{OH}$ signal decays as a function of cell temperature for $\mathrm{H}_{2} \mathrm{O}$ over a $\mathrm{TiO}_{2}$ catalyst irradiated with $351 \mathrm{~nm}$ excimer radiation.

Figure 9.16. Monochlorobenzene destruction as a function of water vapor splitting catalyst efficiency. mole fraction of monochlorobenzene $=0.1028$, $\phi_{f / a}=1.0$, mole fraction of water $=0.089$.

Figure 9.17. $\mathrm{H}$ and $\mathrm{OH}$ temporal profiles calculated from the rapid, irreversible, solar-catalyzed dissociation of $\mathrm{H}_{2} \mathrm{O}$ in the presence of chlorobenzene and air in $973^{\circ} \mathrm{K}$.

Figure 9.18. Catalytically stabilized combustion generation of gas phase $\mathrm{H}$ and $\mathrm{OH}$.

Figure 9.19. Energy required for excitation of a molecule more strongly bound in the excited electronic state, $E$, than the ground state, $G$. Note that hu (molec) > hu (complex).

Figure 9.20. Electron (hole) and overall energetics of the spliting of $\mathrm{H}_{2} \mathrm{O}$ to form $\mathrm{H}_{2}$ and $\mathrm{O}_{2}$ and the steam reforming of $\mathrm{CH}_{3} \mathrm{Cl}$. 


\section{LIST OF TABLES}

Page

Table $3.1 \quad$ TPRS Destruction of $\mathrm{C}_{6} \mathrm{H}_{12}$

Table 3.2 Argon Laser Destruction of $\mathrm{C}_{6} \mathrm{H}_{12}$ in a Static Cell 30

Table 3.3 Comparison of Resultant TPRS and Argon Laser Absorbance

Rate Constants, $\mathrm{kab}_{\mathrm{ab}}\left(\mathrm{s}^{-1}\right)$

Table 4.3 Maximum Observed Enhancement Ratios for 3,3',4,4', Tetrachlorobiphenyl and 1,2,3,4-Tetrachlorodibenzodioxin Exposed to 0 and 307 AM 1.0 Suns (Simulated) for 5 and $10 \mathrm{~s}$

Table 4.2 Summary of Maximum Observed Photo enhancements and Values Corrected to 1,000 Suns

Table 4.3 Enhancement Ratios $(R=(350))$ for the Thermal/Photolytic Decomposition of 2,6-DNT for 57 AM 1.5 Suns and a Residence Time of $10.0 \mathrm{~s}$

Table 5.1 Summary of the Thermal Oxidation Data for Chlorobenzene and its Major Products Given as Mole \% Referenced to the Original Amount of Chlorobenzene

Table 5.2 Summary of the Thermal/Photolytic Oxidation Data for Chiorobenzene and its Major Products Given as Mole \% Referenced to the Original Amount of Chlorobenzene

Table 5.3 Product Yields from the Thermal Oxidation of 3,3'4,-4,4'-Tetrachloro biphenyl for $10.0 \mathrm{~s}$ in Air

Table 5.4 Product Yields from the Thermal/Photolytic Oxidation of 3,3',4,4'.

Tetrachlorobiphenyl Exposed to $273 \mathrm{AM}-1.5$ Suns for $10.0 \mathrm{~s}$ in Air

Table 5.5 Summary of TPRS Data for 3,3,4,4'-Tetrachlorobiphenyl Exposed to 0 and 273 AM 1.5 Suns (Simulated) for $10 \mathrm{~s}$ in Air

Table 7.1 Benzene $S_{1} \leftarrow S_{0}$ and 3,3',4,4'-Tetrachlorobiphenyl $S_{2} / S_{1} \leftarrow S_{0}$

Temperature Dependent Parameters

Table 7.2 Explicit Expectation Values for a Diatomic Harmonic Oscillator

Table 8.1 Summary of the WSSF Field Data for 1,2,3,4-Tetrachlorodibenzo-pdioxin Exposed for 5 Seconds in Air 
LIST OF TABLES (CONTINUED)

Table 8.2 Droplet Vaporization Times for Fuel Oil in Air @ 300 Suns

Table 8.3 Droplet Vaporization Times for Methylene Chloride in Air @ 300 Suns

Table 9.1 Monochlorobenzene Sensitivity Analysis Calculations 


\section{ACKNOWLEDGEMENTS}

The opportunity that NREL and DoE have provided us to be involved in research that is so scientifically enticing while also being of immense practical value is recognized and enormously appreciated. The support and advice of our current Project Monitor, Dr. Tom Milne and our previous Project Monitor, Dr. Greg Glatzmaier have helped us to be responsive to DoE's desires and NREL's research program goals. The collaborative efforts have been truly rewarding and we only wish there had been more opportunities to do so.

Extra special recognition must be reserved for Mr. Frank Wilkins, the DoE Solar Program Manager and Dr. Gerry Nix, our original NREL Project Manager. Both individuals showed tremendous foresight, exceptional organizational abilities, and a special penchant for providing guidance at the right time and place. Mr. Wilkins' continued interest and involvement has undoubtedly been key to any success the program has experienced.

We truly owe a special debt of gratitude to the entire DoE and NREL program staff and managers. 


\section{PROJECT SUMMARY}

This report summarizes eight years of research by the University of Dayton researchers on the use of concentrated solar energy to destroy toxic organic wastes. This work was conducted under the sponsorship and guidance of the US-Department of energy (DoE) and the National Renewable Energy Laboratory (NREL) under NREL Sub-contract XX-6-06082 and DoE Contract DE-AC03-84SF15354.

As originally envisioned and proposed, concentrated solar radiation would be used to destroy hazardous waste in a thermally activated or assisted, photochemical process. At the outset of the program, it was not clear if elevated temperatures would increase the rates of photochemical reactions or if they might actually decrease. However, the concept of a process that synergistically uses thermal energy (infrared radiation and most of the visible electromagnetic spectrum) and photolytic energy (ultraviolet radiation) was particularly attractive as an application of solar energy since solar energy is unique as an energy source in that it delivers both types of energy in one package. In contrast, electricity generation schemes use only a small portion of the ultraviolet and visible spectrum to induce photo electrochemical processes and most other applications use only thermal energy.

Within the first year of research, we demonstrated that there was indeed a significant increase in the rate of photochemical decomposition of many hazardous organic compounds at moderately elevated temperatures (i.e., $300-600^{\circ} \mathrm{C}$ ) and modest level of simulated solar flux (i.e., approximately $10 \mathrm{watts} / \mathrm{cm}^{2}$ or 100 Air mass 1.5 (AM-1.5) suns). For the next two years, we concentrated on improving our experimental apparatus and techniques as well as developing a basic model for "thermal/photolytic processes." By careful instrument and experimental design, we succeeded in conclusively demonstrating that the experimentally observed decompositions were indeed photochemical and not induced by radiative heating of the sample, a problem that continuously plagued attempts of other research groups to demonstrate a "non-thermal", solar-unique, or solar-beneficial process.

Examination of the experimental data and consideration of the physio-chemical processes involved suggested that the rate of absorption of light and quantum yield of photodissociation might increase with temperature. Accordingly, a high-temperature absorption spectrometer was designed and constructed and 
spectroscopic studies were undertaken. Temperature dependent absorption spectra revealed that not only was the expected red-shift of absorption observed but there was also a 2-4 fold increase in the oscillator strength. This often resulted in a 10 to 100 fold increase in the rate of absorption of solar photons.

Attempts were also made to obtain accurate photochemical quantum yields. For almost all samples tested, there was an increase in quantum yield with increasing temperature. Quantum yields of greater than 1.0 were sometimes observed suggesting that chain reactions were occurring. This also suggested that thermal/photolytic decomposition of one absorbing component of a waste might result in the destruction of other non-absorbing components by a radical-molecule reaction mechanism.

We also began a detailed studies of the efficiency of the solar thermal/photolytic process on preventing the formation and/or destroying organic reaction intermediates, or in incineration terminology products of incomplete combustion (PICS). Since solar detoxification of hazardous waste is not combustion, the term PIC might more properly be used to stand for products of incomplete conversion.

These experimental studies consistently showed the higher molecular weight products were either formed in lower yields or destroyed at lower temperatures than by purely thermal destruction. Lower molecular weight products were either unaffected or slightly reduced in yield. Particularly notable results were the near elimination of polychlorinated dibenzofurans (PCDFs) as products from the degradation of PCBs and the striking reduction in formation of partially dichlorinated dioxin isomers from the degradation of tetrachloro-dibenzo-p-dioxin (TCDD).

At this point, we had largely determined the breadth of applicability of the process and completed the most basic parametric studies of its effectiveness as a function of reaction temperature, time, stoichiometry, and to some extent structure and composition of the waste. However, we did still not have a very complete understanding of the fundamental processes involved. Consequently, our research took on a more fundamental focus for about a year and a half between 1990 and 1992.

We conducted detailed mechanistic studies of the oxidation of chlorobenzene and 3,3',4,4'tetrachlorobiphenyl. Significant mechanistic changes were observed for monochlorobenzene. 
We also thoroughly examined the temperature dependence of the absorption oscillator strength. After lengthy examination of theory and data it was determined that the phenomenon could in all likelihood be attributed to an increase in vibronic coupling which made partially fortidden states more allowed. The formulation for this theory was largely worked out, although many detailed calculations remain to be completed.

We also developed the basic elements of a chemical dynamic model of the temperature dependence that complemented our previously developed photochemical model. This model, although very simple, clearly demonstrated the need to know the temperature dependence of radiative and radiationless excited state decay processes versus the temperature dependence of excited state reactions. Excited singlet state lifetimes may not be long enough for thermal activities. Triplet lifetimes are long enough for thermal activation unless radiative and/or radiationless decay rates also increase significantly with temperature. In order to study these processes, we constructed a high temperature emission spectrometers. However, increasing emphasis on the scale-up and demonstration of the technology precluded further study. The fundamental research plan has been developed but is awaiting execution.

Although many fundamental scientific issues remained to be resolved, the potential utility of the process had been made apparent. Thus, demonstration of the technology at pilot- and near, full-scale became feasible and desirable.

In 1988 and early 1989, we participated in a demonstration of solar detoxification of 1,2,3,4tetrachlorodibenzo-p-dioxin (1,2,3,4-TCDD) was conducted by NREL researchers at the White Sands Solar Simulation Facility (WSSF). In this study, a solar reactor, designed and constructed by NREL with our assistance, was irradiated with 540-1,1200 suns of true solar radiation. The effluent samples obtained by NREL and analyzed by us conclusively demonstrated that 1,2,3,4-TCDD was destroyed with an efficiency of $>99.9999 \%$ at all temperatures above $750^{\circ} \mathrm{C}$. Furthermore, filtering of the wavelengths $<400$ um clearly demonstrated that the ratio of purely thermal to thermal/photolytic destruction was on the order of 0.001 . We subsequently developed a reactor and kinetic model that demonstrated the consistency of the field and previously obtained laboratory results. 
With the success of this study, NREL began solar, flame reactor studies in 1992 and a near fullscale demonstration project was begun under the sponsorship of the US-Department of Energy, USEnvironmental Protection Agency and the US-Army Toxic and Hazardous Materials Agency. This socalled Tri-Agency project and NREL study required that our laboratory studies take on more of a support role.

As a result, we performed three tasks. In the first, the behavior of droplets in an intense radiant field were evaluated with respect to radiative heating, photoinduced ignition, and lensing of the radiation. The most significant results of these studies was that focusing of the radiation by high index of refraction, spherical droplets could result in significant internal heating and secondary droplet atomization.

In a project co-funded by the US-EPA we also studied the thermal and thermal/photolytic destruction of organic compounds volatilized from virgin and contaminated soils. This study generated treatability type data to be used in the design of the demonstration scale facility for the Tri-Agency project which will address remediation of contaminated soils.

In addition, we conducted the calculations necessary to design a soil decontamination/off-gas solar detoxification system that utilized activated carbon as an intermediate trap for the soil off-zones. With this approach, soil could be thermally treated day and night, the off-gas contaminants stored and concentrated on carbon, and treated efficiently during periods of peak solar flux.

As a final, recurring effort throughout the program, we have attempted to address how to treat contaminants that do not absorb solar radiation. Elevated temperature results in significantly increased absorption by many species, but alkanes and non-conjugated olefins and simply do not absorb solar radiation. Fortunately, our mixture results indicate that many mixtures that contain a component that does absorb solar radiation and decomposes can initiate decomposition of all the mixture components. However, this approach is not always, completely reliable.

Energy transfer sensitizes were found to be ineffective very early in the program. Theoretical considerations suggest electronics energy transfer to be inefficient at high temperature. Furthermore, most 
sensitizers are thermally or photochemically fragile. Addition of photoactive radical generators holds some promise, but may be expensive. Gas-surface photocatalysis, that is being investigated by NREL, also shows some promise but may suffer from contamination of the catalyst by components of the waste material.

Consequently, we have begun research on a technique called Solar Catalytically Activated Destruction (Sol-CAD). In this approach, we pass radical precursors through a photo-catalyst with the intent of decomposing the precursor and mixing the resulting radicals with the waste outside the catalyst to initiate decomposition. This approach has the advantage that it does not poison the catalyst.

Modeling suggests that the approach is feasible. We also succeeded in observing a gas phase $\mathrm{OH}$ signal above the surface of a $\mathrm{TiO}_{2}$ catalyst bed which was continuously treated with water vapor and irradiated at $351 \mathrm{~nm}$. Obviously, the apparent splitting of water is an important result. If $\mathrm{OH}$ can be generated in sufficient quantities our modeling, the combustion literature, and experience suggests that waste destruction can be initiated at low temperatures and be very efficient. This vein of research is our only currently on-going activity concerning solar detoxification.

Implementation of solar detoxification currently stands at a threshold. On one hand, the homogeneous, gas-phase thermal/photolytic approach is being tested at a large scale. Hopefully the many technological questions will be overcome and the demonstration will be successful.

On the other hand, the fundamental chemistry and physical principles involved are only moderately understood. Consequently, many basic improvements in process efficiency are possible with a better fundamental understanding. The photo-catalyst and Sol-CAD approaches are valuable for this application and as a contribution to fundamental science and even energy generation. The track that the future research in this area will take will undoubtedly be controlled by the perceived national needs and the organizational strength and technical capabilities of the institutions interested in developing this technology. 


\section{SECTION 1.0 \\ INTRODUCTION}

Disposal of hazardous wastes is among our most pressing environmental dilemmas. As restrictions on land disposal have increased, there has been ever-increasing emphasis on development of disposal techniques resulting in complete destruction of the waste. Controlled, high-temperature incineration has emerged as the most extensively used waste disposal technique and its use has been widely accepted by the US-EPA. However, siting of new facilities has become increasingly difficult and skepticism has become the rule, rather than the exception concerning incineration. Although much of the public opposition is socio-economic, there is a general lack of understanding of the combustion properties of hazardous organic wastes. This has prevented scientists and engineers from providing firm answers on key health and ecology issues. Consequently, we are currently dealing with a society that has all but completely banned land disposal and firmly resists incineration. As a result, there is intense interest in developing alternative disposal techniques. Regularly mandated clean-up of abandoned dump sites, in which billions of tons of soil are contaminated with hazardous wastes, has further accelerated the need for efficient, cost-effective methods of destroying toxic materials.

Application of concentrated solar energy has been proposed to be a viable waste disposal option. As currently conceived, the process will be homogeneous, high-temperature, photolysis of the waste. This is a new concept and is distinctly different from a purely solar-thermal destruction and ambient temperature photolysis. Specifically, this concept of solar induced high-temperature photochemistry is based on the synergistic contribution of concentrated infrared (IR) radiation, which acts as an intense heating source, and near ultraviolet and visible (UV-VIS) radiation, which can induce destructive photochemical processes. In fact, currently completed studies indicate that heating increases the rates of photochemical reactions and photochemistry increases the rates of thermal reactions.

Research completed to date must still be classified as exploratory in nature. Still some significant advances have been made in the theoretical framework of high-temperature photochemical processes (Section 2) and development of experimental techniques for their study (Section 3). Basic "thermal/photolytic" studies have addressed the effect of temperature on the photochemical destruction of pure compounds (Section 4 Appendix VI and VII). Detailed studies of the destruction of reaction byproducts have been conducted on selected waste molecules (Section 5 ). Some very limited results are available on the destruction of mixtures (Section 6 and Appendix VI). Although these studies have been useful in defining the generality and range of the concept of high-temperature photochemical destruction, they only indirectly address the fundamental underlying chemistry and physics. Fundamental spectroscopic studies have been recently initiated (Section 7 ). The results to date have been used to 
conduct some relatively simple scale-up studies of the solar detoxification process. These include NREL conducted tests in a "flaming reactor", pilot scale testing at the White Sands Solar Furnace (WSSF), simulation of effects on droplets in an intense radiative field (Section 8). More recent work has focused on destruction of compounds that do not directly absorb solar radiation. Research efforts have focused on homogeneous as well as heterogeneous methods of initiating destructive reaction pathways (Section 9).

Although many conclusions at this point must be considered tentative due to lack of basic research, a clearer picture of the overall process is emerging (Section 10). However, much research remains to be performed and must follow several veins, including photochemical, spectroscopic, combustion kinetic, and engineering scale-up (Section 11). 


\section{SECTION 2.0}

\section{THEORY OF HIGH-TEMPERATURE PHOTOCHEMISTRY}

High-temperature photochemistry is a general concept based on the notion that elevated temperatures can increase the rate of photochemical reaction of a given chemical system[1]. The term is intended to apply to any excitation wavelengths that can promote molecules to their excited electronic states. The term solar detoxification of hazardous wastes, as used in the report, refers to the use of concentrated solar radiation to induce thermal/photolytic reactions that decompose compounds and their reaction intermediates to thermodynamic end-products (e.g., $\mathrm{CO}_{2}, \mathrm{H}_{2} \mathrm{O}$, and $\mathrm{HCl}$ ). Because the solar spectrum at the Earth's surface has a short wavelength cut-off of $280-300 \mathrm{~nm}$ (cf. Figure 2.1), only the first excited singlet states of most molecules can be populated [2].

\subsection{BASIC PHYSICAL, THERMAL/PHOTOLYTIC REACTION MODEL}

The energy reaction coordinate diagram shown in Figure 2.2. depicts the basic concepts involved in high-temperature photochemistry. Purely thermal reactions occur in the singlet, ground electronic state, $S_{0}$. Unimolecular decomposition of most molecules requires energies on the order of $50-100 \mathrm{kcal} / \mathrm{mole}$ (e.g. carbon-chlorine bond rupture in chlorobenzene has a bond dissociation energy of about $95 \mathrm{kcal} / \mathrm{mole}$ ) [3]. To overcome the ground state activation energy barrier, $\mathrm{E}_{\mathrm{a}}\left(\mathrm{S}_{0}\right)$, temperatures of $600-1000^{\circ} \mathrm{C}$ are required for reaction times of $1.0-10.0 \mathrm{~s}[4]$.

When a typical organic molecule absorbs visible or ultraviolet radiation it is promoted to an electronically excited state[5]. For solar excitation this is almost always the first excited singlet state, $\mathrm{s}_{1}$. There may be sufficient electronic energy in $S_{1}$ to overcome the barrier to decomposition, or a small amount of additional energy may be required. This may be because the zero-point electronic energy of $S_{1}$, $E\left(S_{1}\right)$, is still slightly less than $E_{a}\left(S_{0}\right)$ or there is an additional intrinsic activation energy from $S_{1}, E_{2}\left(S_{1}\right)$. The latter case is depicted in Figure 2.2. In addition, the molecule initially prepared in $S_{1}$ may convert to the lowest triplet state, $T_{1}$, in a process referred to as inter-system crossing [7]. As shown in Figure 2.2, the electronic zero-point energy of $T_{1}$ is almost always less than $E_{a}\left(S_{0}\right)$ and thus additional energy is required for reaction. However, the lifetimes of lowest excited triplet states typically are much longer than lowest excited singlet states (e.g., $\sim 1 \mu \mathrm{s}-\sim 1 \mathrm{~s}$ versus $\sim 1 \mu \mathrm{s}-100 \mu \mathrm{s}$, respectively) and more time is available for reactions to occur before the molecule returns to the ground state $[7]$. For this reason the lowest triplet state is generally more photo-reactive than the lowest singlet[7]. 


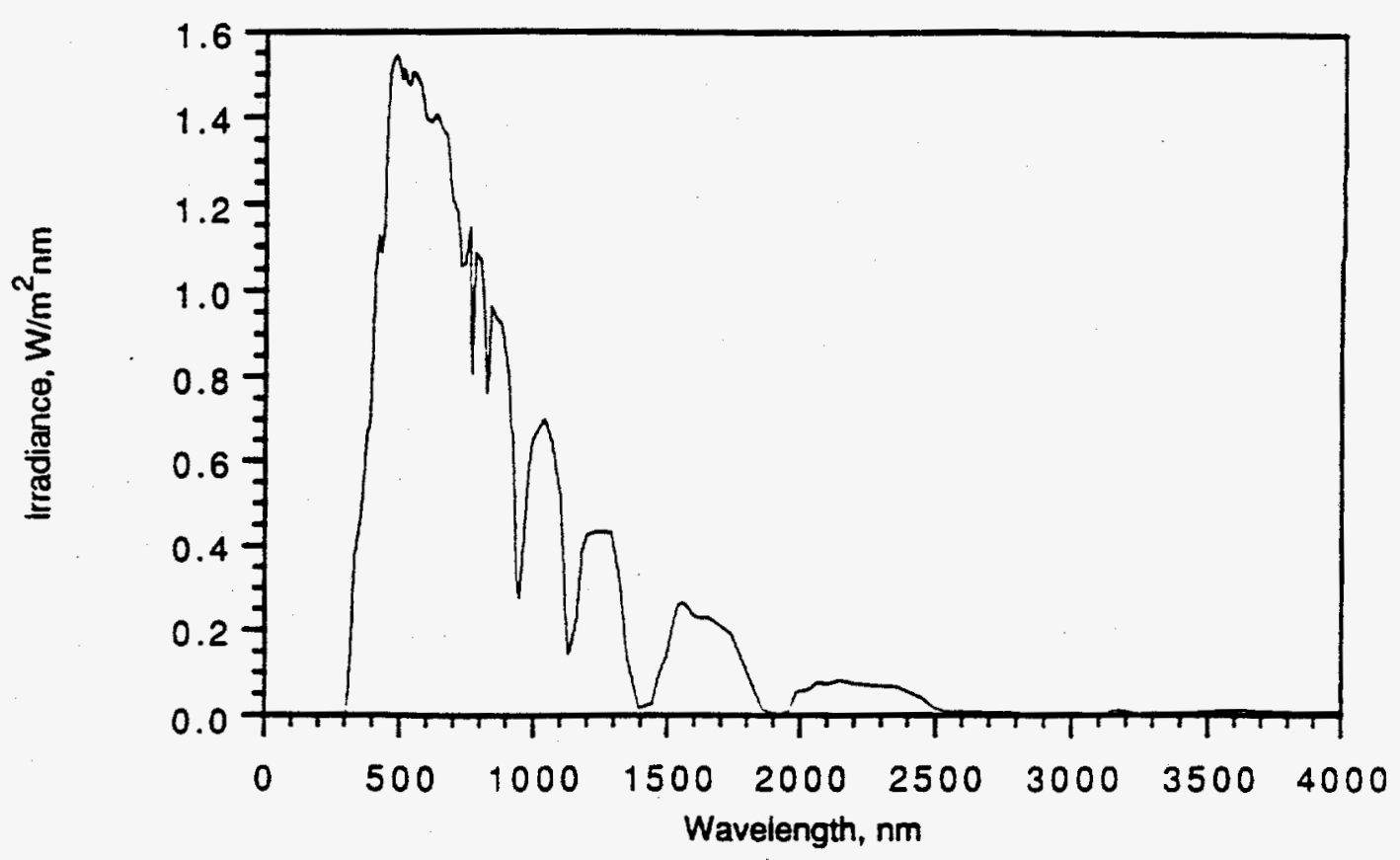

Figure 2.1. An air mass 1.5 (AM 1.5) solar irradiance spectrum at the Earth's surface through typical clean-air atmospheric conditions.

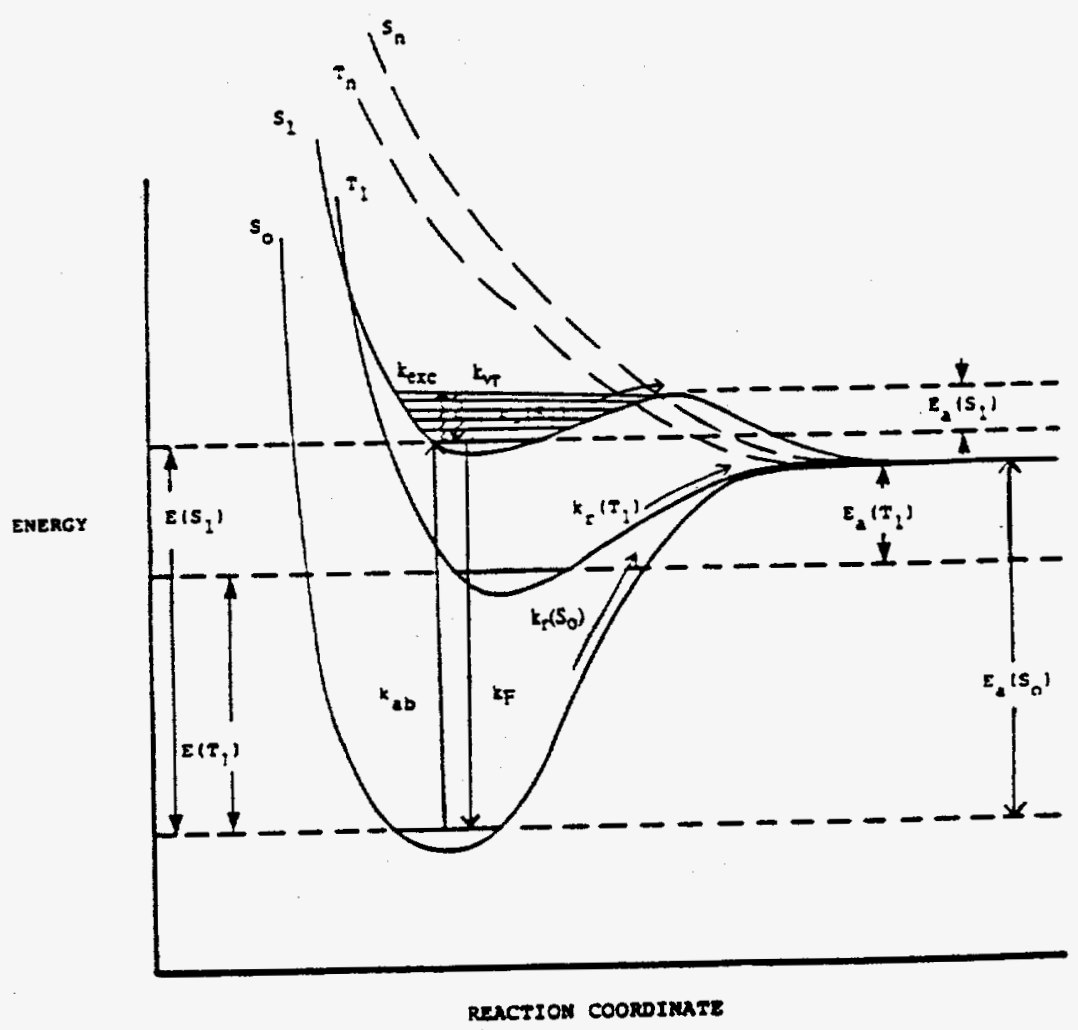

Figure 2.2. Energy diagram illustrating the central principals of the solar detoxification process.

4 


\subsection{BASIC KINETIC FORMULATION}

A relatively simple pseudo-first order kinetic model has been formulated that fits the bulk of the experimental data obtained thus far[1]. This model assumes that the rate of the ground state reaction may be described by a first-order kinetic expression;

$$
-\mathrm{d}[\mathrm{Hz}] / \mathrm{dt}=\mathrm{k}_{\mathrm{gnd}}[\mathrm{Hz}]
$$

where $\mathrm{k}_{\mathrm{gnd}}$ (also given below as $\mathrm{k}\left(\mathrm{S}_{0}\right)$ ) is first order with respect to the hazardous compound, $\mathrm{Hz}$. It implicitly assumes that any oxygen dependence or other non-unimolecular character of the ground state, thermal reaction can be empirically included in $\mathrm{k}_{\mathrm{gnd}}$. It also assumes that the rate of the excited state reaction may be described as;

$$
-\mathrm{d}[\mathrm{Hz}] / \mathrm{dt}=\mathrm{I}_{\mathrm{a}} \phi_{\mathrm{r}}
$$

where $I_{a}$ is the energy absorbed per unit time per unit volume and $\phi_{r}$ is the photochemical quantum yield. If one now assumes that the system weakly absorbs collimated radiation, the total rate of ground and excited state reaction can be shown to be[8]:

$$
d[H z] / d t=k_{e x}[H z]+k_{g n d}[H z]=\left(k_{a b} \phi_{r}+k_{g n d}\right)[H z]
$$

where $[\mathrm{Hz}]$ is the concentration of the hazardous compound $\left(\mathrm{mol}^{-1}\right), \mathrm{kex}_{\mathrm{ex}}$ is the total rate of the excited state reactions $\left(s^{-1}\right), k_{\text {gnd }}$ is the pseudo-first order rate constant of the ground state reaction $\left(s^{-1}\right)$, and $\phi_{r}$ is the total photochemical quantum yield of all excited state reactions (i.e., the fraction of molecules absorbing radiation that actually react while in an excited state). $k_{a b}\left(s^{-1}\right)$, rate of broad-band photon absorption can be expressed as;

$$
k_{a b}=1.92 \times 10^{-5} \int \varepsilon_{\lambda} I_{\lambda} d \lambda
$$

where $\varepsilon_{\lambda}$ is the molar absorption coefficient (mole $\mathrm{L}^{-1} \mathrm{~cm}^{-2}$ ) as a function of wavelength $\lambda(\mathrm{nm}), \mathrm{I}_{\lambda}$ is the intensity distribution of the radiation source $\left(\mathrm{W} \mathrm{cm}^{-2}\right)$, and the integral is evaluated over the wavelength interval over which the emission spectrum of the source and the absorption spectrum of the compound coincide. Equation 2.3 is readily integrated to give the familiar first-order, exponential decay of the concentration of $\mathrm{Hz}$. 


$$
f_{r}=[H z] /[H z] 0=\exp -\left(k_{a b} \phi_{r}+k_{g n d}\right) t
$$

where $f_{r}$ is the fraction of $\mathrm{Hz}$ remaining undestroyed and $[\mathrm{Hz}] \mathrm{O}$ is the initial concentration of $\mathrm{Hz}$.

Since we desire to determine the overall improvement of the high-temperature photochemical process over a purely thermal process, a useful quantity referred to as the thermal/photolytic enhancement ratio may be defined as,

$$
R=f_{\mathrm{r}}(\text { dark }) / f_{\mathrm{r}} \text { (ill) }
$$

where $R$ is the enhancement ratio, $f_{r}(d a r k)$ is the fraction of $\mathrm{Hz}$ remaining undestroyed following purely thermal (dark) exposure, and $\mathrm{f}_{(}(\mathrm{ill})$ is the fraction of $\mathrm{Hz}$ remaining undestroyed following thermal/photolytic (illuminated) exposure.

The thermal/photolytic enhancement ratio $(R)$, the ratio of excited versus ground state reactions $\left(k_{e x} / k_{g n d}\right)$, and the photochemical quantum yield $\left(\phi_{r}\right)$ may all be considered measures of the relative effectiveness of the thermal/photolytic process compared to a purely thermal process. Specifically, $\mathrm{k}_{\mathrm{ex}} \mathrm{K}$ gnd might be most meaningful to the kineticist, determination of $f \mathrm{r}$ is the domain of the photochemist, while $R$ would be the most significant quantity to the environmental engineer since it is essentially a measure of the overall relative destruction (or emissions) of $\mathrm{Hz}$. These quantities are all interrelated by the following expressions;

$$
\begin{aligned}
& R=f_{r}(\text { dark })_{r} f_{r}(\text { ill) })=\exp \left(\phi_{r} k_{a b} t\right) \\
& \left.\phi_{r}=\left\{\ln \left(f_{r}(\text { dark })\right)_{r}(\text { (ill) })\right)\right\} k_{a b} t=\ln (R) /\left(k_{a b} t\right) \\
& k_{e x} / k_{\text {gnd }}=-\phi_{r} k_{a b} t \ln \left(f_{r}(\text { dark })\right)=-\ln R / n f_{r} \text { (dark) }
\end{aligned}
$$

The exponential dependencies of $R$ on $\phi_{r}, k_{a b}$, and $t$ predicted by these equations are of particular interest.

Although the derivations of equations 2.5-2.7 require many assumptions and are based on an admittedly simple model, they have proven most useful. Still, other more specialized models have been developed. For example, we consider the case where the radiation is adsorbed by a "sensitizer", S, which transfers the excitation energy through triplet-triplet energy transfer to the acceptor, $\mathrm{Hz}$. 
For the case where the ground state thermal reaction of $\mathrm{Hz}$ is slow but the efficiency of energy transier is high, it can be shown that $[1]$;

$$
f_{r}(H z) \approx 1-t k_{a b}(S)[S]_{0} \phi_{i s c}(S) \phi_{e t} \phi_{r}, T(H z)
$$

where $k_{a b}(S)$ is the rate of absorption for the sensitizer, [S]0 in the initial concentration of the sensitizer, $\phi_{\text {isc }}(S)$ is the $S_{1} \rightarrow T_{1}$ intersystem crossing efficiency of the sensitizer, $\phi$ et is the triplet-triplet energy transfer efficiency for the sensitizer and acceptor, and $\phi_{r}, \mathrm{~T}(\mathrm{~Hz})$ is the quantum efficiency of the reaction of $\mathrm{Hz}$ from its lowest triplet state. It is interesting to note that, for this case, the destruction of $\mathrm{Hz}$ is now only linearly dependent on $f_{r}, k_{a b}(S)$, and $t$.

In contrast, when the efficiency of energy transfer is low, the exponential decay of $\mathrm{Hz}$ again results;

$$
f_{f}(H z)=\exp -\left(k_{a b}(S)[S] O \phi_{i s c}(S)\left(k_{e t} / k q\right) \phi_{r}, T(H z)\right)
$$

where $k_{\text {et }}$ is the rate constant for energy transfer from the triplet of the sensitizer to the triplet of the acceptor, $\mathrm{k}_{\mathrm{q}}$ is the rate constant for quenching of $T_{1}$ of the sensitizer by internal processes and other external quencher species, and the other variables are as previously defined.

\subsection{TEMPERATURE DEPENDENCE OF ELEMENTARY PHOTOCHEMICAL PROCESSES}

Consideration of the kinetic model expressed in Equation 2.3, suggests that the photolytic, kinetic parameters, $k_{a b}$ and $f_{r}$, may be temperature dependent. In fact, we have identified three elementary "photolytic" processes that appear to be operative in many of the systems thus far studied[?]. These are:

1. An increase in the rate of absorption of radiation as the temperature is increased;

2. An increase in the rate constant of the primary step of the excited state reaction as the temperature is increased; and

3. Thermally enhanced, photolytic initiation of complex radical chain reaction pathways that propagate at high temperature but not at low temperature.

Processes 1 and 3 have been directly observed experimentally $[9,10]$. Experimental observation of process 2 is not readily verifiable via conventional photochemical or kinetic techniques due to possible interference by concurrent occurrence of process 3 . However, process 2 , the temperature dependence of the rate of the primary photochemical event is of extreme theoretical interest as well as of practical importance[11]. 
Consequently, we have developed a simple, 3 state, excited state dynamics model that defines some of the issues involved in thermally activated photochemistry.

The process diagram for the model is displayed in Figure 2.3. The process described by this Figure is based on photoexcitation from the ground state of the molecule, $G$, to an excited but still nonreactive state, 0 , followed by thermal activation to a reactive state, 1 . Although not formally necessary in this model, state $G$ may be considered the ground electronic, $S_{0}$, while state 0 may be considered the $V=0$ purely electronic state of the excited state $S_{1}$. State 0 may be many states, e.g. the $V=1$ or $V=n$ vibronic state of $S_{1}$, or a thermally accessible triplet state. The rate of $0 \leftarrow G$ excitation is $k_{a b}$ and the rate of $0 \rightarrow$ $\mathrm{G}$ decay is denoted $\mathrm{k}_{\mathrm{f}}$. Once in state 0 , the remainder of the model is identical to the simple Lindeman thermal activation model in which the rates of activation to state $1, k,[n]$, and deactivation of state $1, k_{-1},[n]$, are pressure and temperature dependent. The rate of conversion from state 1 to products, $k_{p}$, is temperature but not pressure dependent.

Steady state treatment of the various processes involved results in the expression for the overall rate constant for the thermally activated photo-reaction.

$$
\mathrm{k}_{\text {diss }}=\frac{\left(\mathrm{k}_{\mathrm{ab}} / \mathrm{k}_{\mathrm{F}}\right) \mathrm{k}_{1} \mathrm{k}_{\mathrm{p}}[\mathrm{M}]}{\mathrm{k}_{-1}\left(\varepsilon_{0} \varepsilon_{1}+1\right)[\mathrm{M}]+\mathrm{k}_{\mathrm{p}}}
$$

where the efficiencies of the forward steps in the from state 0 has been defined as $\varepsilon_{0}=k_{1} / k_{f}$ and the forward efficiency from state 1 has been defined as $\varepsilon_{1}=k_{p} / k_{-1}$. Physical interpretation of Equation 2.10 is facilitated by considering two cases: (1) the thermal/photolytic reaction is inefficient, i.e. $\varepsilon_{0} \varepsilon_{1} \ll 1$, and (2) the thermal/photolytic reaction is efficient $\varepsilon_{0} \varepsilon_{1} \gg 1$. Since we are only interested in cases where the thermal/photolytic reaction is efficient, we only consider case 2. For case 2, Equation 2.10 reduces to;

$$
k_{\text {diss }}=\frac{\left(k_{\mathrm{ab}} / \mathrm{k}_{\mathrm{F}}\right) \mathrm{k}_{\mathrm{l}} \mathrm{k}_{\mathrm{p}}[\mathrm{M}]}{\mathrm{k}_{\mathrm{p}}\left(\mathrm{k}_{1} / \mathrm{k}_{\mathrm{F}}\right)[\mathrm{M}]+1} \quad \varepsilon_{0} \varepsilon_{1} \gg 1
$$

at low pressures $([\mathrm{M}] \rightarrow 0)$, Equation 2.11 approaches the pressure dependent rate constant, $\mathrm{k}_{\text {diss }}(\mathrm{M} \rightarrow$ $0)$ is equal to $\left(k_{a} / k_{f}\right) k_{1}[M]$. In the high pressure limit, the rate constant is independent of pressure and is limited by the rate of absorption of radiation, i.e. $k_{\text {diss }}(M \rightarrow \infty)$ is equal to $k_{a b}$. Since $\phi_{r}$ is equal to the ratio of $k_{\text {diss }}$ to $k_{a b}, \phi_{r}$ behaves in the following manner;

$$
\phi_{r}(M \rightarrow 0)=k_{1}[M] / k_{f}
$$




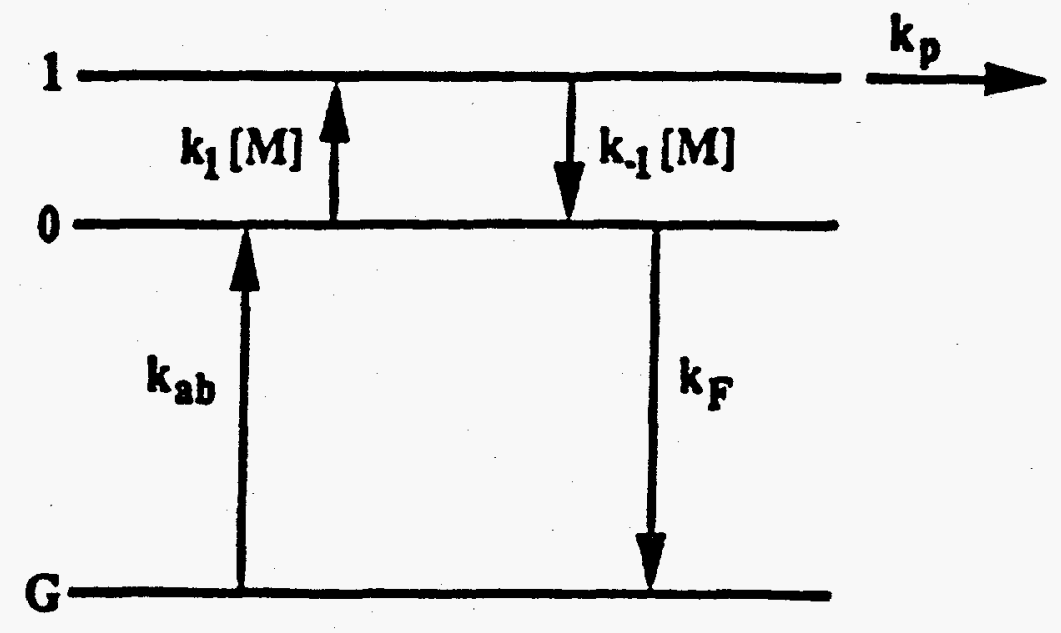

Figure 2.3. Three state model for thermal-photolytic dissociation. $k_{a b}$ is the rate of energy absorption. $k_{F}$ is the rate of excited state deactivation. $k_{1}[M]$ and $k_{1}[M]$ are the pressure dependent rates of thermal activation and deactivation, respectively. State 1 is the reactive state corresponding to the intrinsic energy barrier to reaction with the rate of passage over the barrier given by $k_{p}$. 


$$
\phi_{r}(M \rightarrow \text { in } f)=1.0
$$

Thus, $\phi_{r}$ is pressure (and temperature) dependent at "low" pressures. If $k_{f}$ increases faster with temperature than $k_{1}$, then $\phi_{r}$ becomes more pressure dependent. At sufficiently high pressures and temperatures, the process is fully "thermally activated" and the low temperature value of $\phi_{r}$ is increased to its maximum value of 1.0. However, from Equation 2.12, the required temperature and pressures are not clear. To assess the requirement for thermal activation, we turn to RRKM theory. Under the formulation of this theory;

$$
k_{\text {diss }}(M \rightarrow 0)=\beta_{c} Z[M] F^{*}
$$

where $\beta_{c}$ is the modified, strong collision efficiency of energy transfer from the bath molecules to the reactant, $Z$ is the gas kinetic collision efficiency at unit concentration, and $F^{*}$ may be expressed as;

$$
F^{*}=\int F(E) d E
$$

where $F(E)$ represents the Bolzman distribution of states with energy, $E$, above that of state 1 , and the integral is evaluated from $E_{1}$ to infinity [12]. We have not yet made the complex evaluation of $F^{*}$ under RRKM formalism. However, we can readily evaluate the remaining terms that together yield effective collision times for the system $\tau_{c o l l}=\beta_{c} Z[M]$. For a bath gas of helium at atmospheric pressure at $1000^{\circ} \mathrm{C}$, $\tau_{\mathrm{coll}}=10^{-9} \mathrm{~s}$. In comparison the lifetimes of the zero point level of $S_{1}$ states, $\tau_{S 1}$, are $10^{-9} \cdot 10^{-6} \mathrm{~s}$ at room temperature or below $[6,7,8]$. Triplet lifetimes, $\tau_{T 1}$, are $10^{-6}-10^{-9} \mathrm{~s}$ at room temperature or below $[6,7,8]$. These calculations suggest that if the lifetimes of the states are not reduced with increasing temperature, then long lived (e.g., $\left.n \pi^{*}\right)$ states will be thermally activated by collision while shor lived (e.g., allowed $\pi \pi^{*}$ ) states will be only partially activated. In contrast, triplet states appear to be sufficiently long lived, but again, only if their rate of deactivation is not significantly increased with temperature.

Unfortunately, an extensive review suggests that nothing is available in the literature concerning the temperature dependence of the lifetime of excited electronic states. Thus, a theoretical understanding of the effects of temperature and pressure on the quantum yields of the primary step of photochemical reactions will have to wait for direct photochemical measurements of $\phi_{r}$ as a function of temperature, spectroscopic measures of the temperature dependence of $\tau_{S 1}$ and $\tau_{T 1}$, or a theoretical formulation of the effects of temperature on radiative and radiationless processes. 


\subsection{REFERENCES}

1. J. L. Graham and B. Dellinger, Energy, 12, No. 3/4, 303, 1987

2. J. L. Graham, et al., Emerging Technologies in Hazardous Waste Management II, 93, ACS, Washington, DC, 1990.

3. R. Hulstrum, R. Bird, and C. Riordon, Solar Cells, 15, 365, 1985.

4. D. F. McMillin and D. M. Golden, Amer. Res. Phys. Chem., 33, 493, 1982.

5. B. Dellinger, et al., Hazardous Waste, 1, No. 2, 187, 1984.

6. J. A. Bartirop and J. P. Coyle, Principles of Photochemistry, New York, NY, John Wiley and Sons, Inc., 1978.

7. S. L. Murov, Handbook of Photochemistry, Marcel Dekker, New York, NY, 1973.

8. S. P. McGlynn, T. Azumi, and M. Kinadhita, Molecular Spectroscopy of the Triplet State, Prentice Hall, Inc., Englewood Clitts, NJ, 1969.

9. A. A. Lamola, Creation and Detection of the Excited State, Vol. 1, Part A, Marcel Dekker, New York, NY, 1971.

10. J. L. Graham, J. M. Berman, and B. Dellinger, Accepted, ل. Photochem. Photobiol.

11. J. M. Berman, J. L. Graham, and B. Dellinger, L. Photochem. A: Chem., 68, 353, 1992.

12. Potential Applications of Concentrated Solar Energy, National Academy Press, Washington, DC, 1991.

13. W. C. Gardiner, Jr. and Jürgen Troe, "Rate Coefficients of Thermal Dissociation, Characterization, and Recombination Reactions," Combustion Chemistry, p. 173, W.C. Gardiner, Jr., ed., SpringerVerlag, NY, 1984. 


\section{SECTION 3.0 \\ DEVELOPMENT OF EXPERIMENTAL TECHNIQUES}

The experimental investigation of the solar thermal/photolytic destruction of toxic organic wastes required the development of laboratory instrumentation and experimental techniques that were not previously available. Specifically, studying of the mechanistic and kinetic characteristics of hightemperature solar detoxification required the development of an experimental system in which the process variables of exposure time, atmosphere, temperature, and the intensity of the incident radiant flux could be controlled. Furthermore, since radiative heating of the sample and/or measurement devices could interfere with discerning whether an observed effect was thermal or photolytic in nature, separate control of temperature and radiant flux was considered a critical design requirement. These goals were successfully implemented with the development of the Thermal/Photolytic Reactor System (TPRS) and the Advanced TPRS (ATPRS).

The TPRS is a high-temperature, gas-phase, flow reactor system which uses a filtered xenon arc lamp as the radiation source and an in-line gas chromatograph-flame ionization detector (GC-FID) for analysis of the reactor effluent. The ATPRS, which employs a pulsed laser system as the excitation source and a GC-mass spectrometer (GC-MS) system for effluent analysis, was developed to address the need for more accurate photochemical quantum yield and kinetic measurement than could be achieved with the TPRS. A high-temperature photochemical actinometry approach has been developed to improve the ability to measure quantum yields on the TPRS.

As research progressed, it was recognized that there was a need to study the high-temperature spectroscopy of the test materials. The High-Temperature Absorption Spectrophotometer (HTAS) was developed to obtain gas-phase absorption spectra at temperatures as high as $1000^{\circ} \mathrm{C}$. The HighTemperature Emission Spectrophotometer (HTES), which is currently under development, will have the capabilities to study the temperature dependence of excited state lifetimes and emission quantum yields.

The following paragraphs discuss, in some detail, the design and operation of the TPRS, ATPRS, and HTAS as well as development work on the high-temperature, photochemical actinometer. The initial work on the HTES is described in Section 7.2.

\subsection{THERMAL/PHOTOLYTIC REACTOR SYSTEM}

The TPRS was built to satisfy the original need for a flow reactor system capable of exposing a gas phase process stream simultaneously to high temperature and intense, simulated, solar radiation. 
Furthermore, the system was designed to permit independent control of temperature, mean residence time, excess air, and the intensity of the radiant exposure.

Since its completion in 1985, the TPRS has undergone several revisions. However, its general layout has remained the same. The schematic shown in Figure 3.1 shows the instrument's overall design features and its current configuration. As this Figure illustrates, the TPRS consists of a sample inlet chamber, reactor, and cryogenic trap, all housed within a thermally insulated aluminum enclosure. The entire reactor was fabricated from fused quartz to give a seamless, chemically inert flow path that was transparent to near UV radiation and able to tolerate temperatures in excess of $1000^{\circ} \mathrm{C}$.

The sample inlet chamber utilizes a unique tube-in-shell design surrounded by a small tube furnace (1-1/2 $\times 6$ in) for versatile temperature control. Specifically, the inlet may be operated isothermally, or the temperature may be altered in a variety of fashions using a combination of heat supplied by the tube furnace, and purging cooling gas through the shell.

To accommodate a wide range of sample types and phases, the inlet chamber may be fitted with a variety of sample inlet probes. These include probes for fluid (gas or liquid) injection and solid insertion. Fluids may be batch charged (pulsed injection) or introduced at a controlled rate using a syringe pump. Similarly, solids may be batch charged using a special probe onto which the sample is solvent deposited, or introduced at a controlled rate using a miniature ram feed probe in conjunction with a modified syringe pump. Finally, bulk solids may be batch charged using another probe assembly which consists of a miniature sample tube and a carrier probe.

To enhance the ability to control the rate at which the sample leaves the insertion region and flows into the reactor, carrier gas is admitted to the TPRS at two points. Specifically, the first carrier gas line enters the system upstream from the inlet chamber and sweeps the sample insertion region. The second inlet line enters just down stream of the insertion chamber. By varying the ratio of the flow through these two lines, the sample concentration as a function of time from batch charging of sample can be varied over a considerable range. Furthermore, the sum of the flows entering the system determines the mean residence time (i.e., mean exposure time) the sample will experience in the reactor.

The reactor vessel itself is of a fairly simple design. Specifically, it is in the form of a small cylinder measuring $1.2 \times 8.2 \mathrm{~cm}$. The entrance and exit flow lines are fused to the reactor's side wall at the very ends of the vessel so that the process stream enters and exits the reactor smoothly, without the possibility of stagnant or by-passed regions within the reactor volume. The ends of the reactor are sealed with flat 


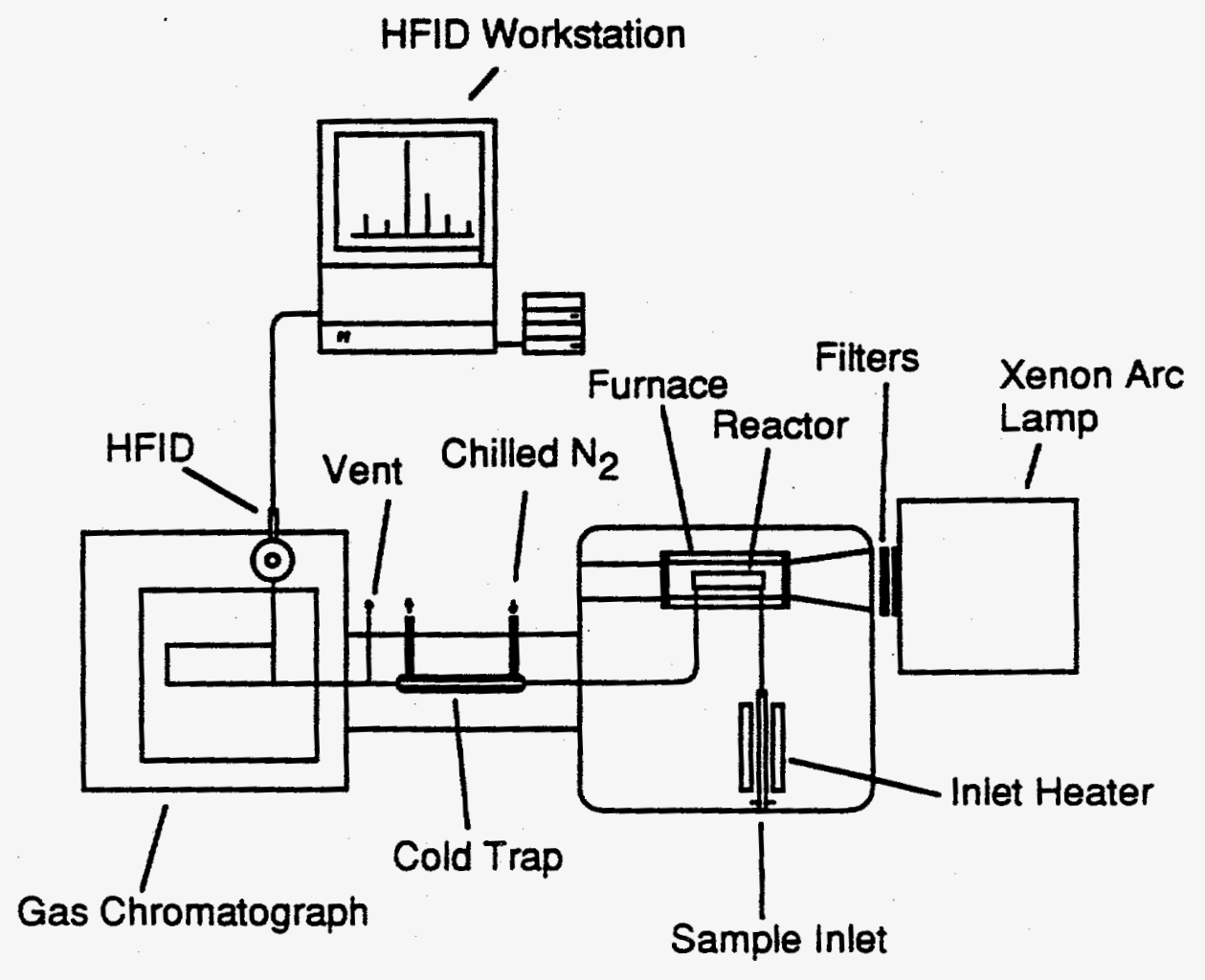

Figure 3.1. Schematic of the Thermal/Photolytic Reactor System (TPRS) showing the instrument's general layout and major features. 
quart windows so that the interior can be evenly illuminated by the solar simulator. Similarly, the reactor's tube furnace is also sealed with flat quart windows to provide a thermally stable enclosure.

The transfer lines between the sample inlet chamber and the reactor are heated by the waste heat produced by these two elements, while the longer run between the reactor exit and the cryogenic trap is heat traced to maintain a temperature of at least $300^{\circ} \mathrm{C}$. This arrangement ensures that the organic content of the process stream is quantitatively transported through the system from the inlet to the trap.

The cryogenic trap is of a simple shell and tube design. The inside diameter of the tube within the trap widens from 1 to $4 \mathrm{~mm}$ to slow the linear velocity of the gas, and it is lightly packed with quartz wool to improve heat transport and to provide a surface for condensation. The shell is cooled with dry nitrogen which has passed through a copper coil immersed in liquid nitrogen. This system allows the trap to be cooled to approximately $-150^{\circ} \mathrm{C}$. The environment surrounding the trap is maintained at $350^{\circ} \mathrm{C}$, so the trap may be rapidly heated by simply shutting off the flow of coolant to the shell.

The gas chromatograph (GC) is a Varian Vista 3700 usually fitted with a $320 \mathrm{~mm} \times 15 \mathrm{~m}$ nonpolar column (J\&W Scientific DB-1). A hydrogen flame ionization detector (FID) is used for solute detection.

The solar simulator is a Spectral Energy, Model SS-1000X system fitted with a $1000 \mathrm{~W}$, high pressure, short arc xenon lamp, dichroic mirror, and air mass filters. This system was modified with custom filter holders and with a slightly altered arc location to extend the focal zone from 5 to $20 \mathrm{~cm}$ outside the lamp housing. The combination of the dichroic mirror, which is reflective from 300 to $550 \mathrm{~nm}$, and air mass filters gives a relative spectral distribution very similar to an air mass (AM) 1.5 (42 degree sun elevation at sea level) solar distribution. When an aluminized mirror is used in place of the dichroic mirror, the system delivers a spectral distribution similar to an AM 1.0 (90 degree sun elevation at sea level) solar distribution. The beam from the simulator is softly focused by an ellipsoidal reflector in the lamp housing. The focused region provides a somewhat evenly illuminated volume measuring about $1.0 \times 10 \mathrm{~cm}$ centered on the reactor vessel. The maximum radiant intensity which can be generated in this volume is about 30 W/ $\mathrm{cm}^{2}$, or about 300 times terrestrial sunlight.

\subsection{ADVANCED THERMAL/PHOTOLYTIC REACTOR SYSTEM}

The TPRS has proven to be a valuable tool for simulating the conditions in a small solar furnace. However, its rather simple design limits its usefulness in providing detailed data required for fundamental research. For this purpose the ATPRS was constructed. 
As the schematic shown in Figure 3.2 illustrates, the design of the ATPRS follows the same general pattern of the TPRS (e.g., one-piece quartz construction, thermally insulated enclosures, small tube furnaces, etc.). However, in contrast to the single, multipurpose sample inlet of the TPRS, the ATPRS includes two inlet channels. The first inlet is designed for the controlled introduction of fluids. Specifically, it is constructed from the same narrow tube stock $(1 \mathrm{~mm} \mathrm{I.D.)} \mathrm{as} \mathrm{the} \mathrm{transport} \mathrm{lines.} \mathrm{This} \mathrm{preserves} \mathrm{the}$ sample input profile from the injection process (e.g., pulsed injection or continuous syringe pump). The second inlet is similar to that used on the TPRS in that it consists of a chamber whose volume is large enough to admit a variety of special purpose probes. Though it was designed primarily for the introduction of solid samples, it may be fitted with probes for injecting fluids as well. This inlet differs from the TPRS in that it does not have the purgable shell surrounding it.

The two inlet chambers are each purged with their own carrier gas supply lines. The gas leaving the chambers are joined together into a single transfer line before flowing into the reactor. The flow of gas through the two inlets may be manipulated in a manner similar to the flow through the two carrier gas supply lines for the TPRS in order to provide control of the sample inlet profile and the total flow of gas to the reactor.

The reactor vessel is similar in form to the TPRS reactor in that it is cylindrical in shape with flat windows at either end and has carrier gas lines fused to the side walls immediately adjacent to the windows. However, to take advantage of the ATPRS's highly collimated illumination source (i.e., laser illumination), the reactor used here is relatively long and slender $(0.4 \times 25 \mathrm{~cm})$. This geometry, in conjunction with the laser illumination source, gives a very well defined, plug flow exposure.

The cold trap arrangement on the ATPRS is similar to the TPRS, but features an improved interface with the GC, and an approximately $25 \%$ greater internal volume. The insulation and nitrogen cooler have also been improved so that temperatures as low as $-180^{\circ} \mathrm{C}$ can be achieved.

The analytical system on the ATPRS consists of a Hewlett-Packard $5890 \mathrm{GC}$ fitted with a 5970A scanning mass selective detector (MSD) and an FID. The GC is equipped with a dual column assembly which permits data to be acquired from both detectors simultaneously. Specifically, the MSD channel was fitted with a $200 \mathrm{~mm} \times 20 \mathrm{~m}$ column, while the HFID was fitted with a $320 \mathrm{~mm} \times 15 \mathrm{~m}$ column. Both columns were fused silica lined with a nonpolar liquid phase (J\&W Scientific, DB-1).

The illumination system for the ATPRS is a frequency doubled Nd:YAG (Continuum Model 682-20) pumped dye laser (Continuum Model TDL-51). The output of the dye laser is also frequency doubled. The system is currently operated using Rhodamine 590 dye and is tuned to deliver up to $880 \mathrm{~mW}$ of average 


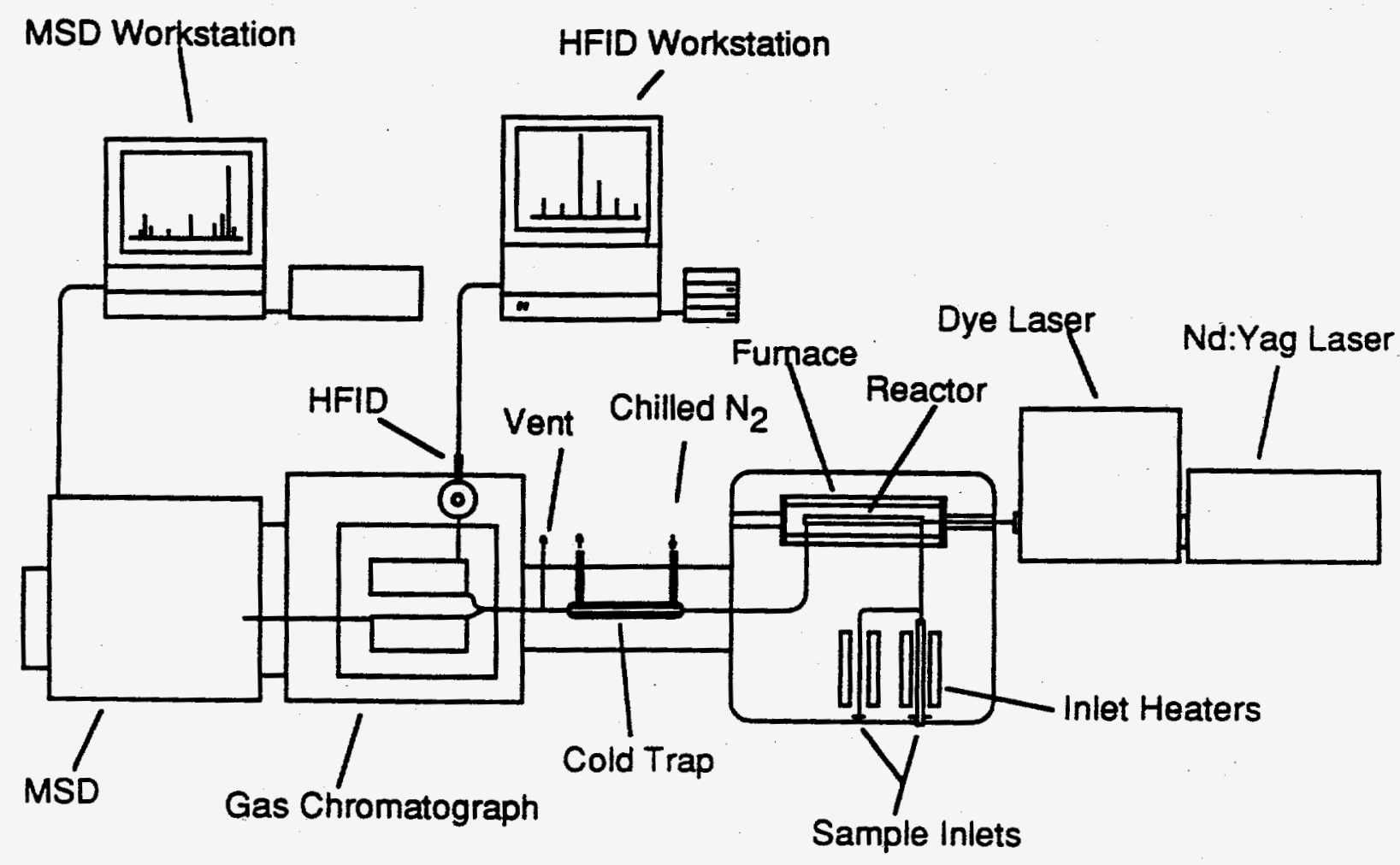

Figure 3.2. Schematic of the Advanced Thermal/Photolytic Reactor System (ATPRS) showing the instrument's general layout and major features. 
power at $280 \mathrm{~nm}$ in a beam measuring approximately $6 \mathrm{~mm}$ in diameter. The radiation is delivered in pulses with a mean time width of $6 \mathrm{~ns}$, and a repetition rate of 20 pulses per second.

\subsection{HIGH TEMPERATURE ABSORPTION SPECTROMETER}

Consideration of the spectroscopic theories of molecular absorption of radiation, suggested that absorption spectra would red-shift as the temperature of the sample is increased. It was also recognized that thermal effects could affect absorption properties in other ways. Since most organic molecules are weak absorbers of solar radiation, small shifts or changes in the intensity of absorption could significantly affect $k_{a b}$ and the overall thermal/photolytic destruction rates. Consequently, considerable effort was expended in the development of the HTAS which could be used to accurately determine gas-phase absorption spectra up to relatively high temperatures (i.e., $1000^{\circ} \mathrm{C}$ ).

As shown in the schematic given in Figure 3.3, the HTAS is built on a pattern almost identical to the ATPRS. Specifically, the HTAS features seamless, all-quartz construction, two sample inlets (one for fluids, the other for solids), a long $(1.2 \times 20 \mathrm{~cm})$ cylindrical absorption cell, and a heat traced exhaust line. However, unlike the TPRS and ATPRS, the HTAS is specifically designed to avoid the decomposition of samples in its flow cell. To achieve this, the system is designed to accommodate inert carrier gasses (e.g., nitrogen and helium), and the space velocities through the absorption cell are relatively high (greater than 1 $\mathrm{s}^{-1}$ as compared to $0.1-1 \mathrm{~s}^{-1}$ for the TPRS and ATPRS) in order to reduce the possibility of thermal reactions. Furthermore, since the HTAS is not designed as a chemical reactor, it has no provision for exhaust gas analysis. Instead, the exhaust is processed with a particulate filter to trap solid condensates and aerosols, an activated charcoal filter to attenuate volatile components, and is then vented to a fume hood.

The HTAS operates as a single beam spectrophotometer. Specifically, to measure the absorption spectra of a sample, the absorption cell is illuminated with collimated radiation from a deuterium lamp (Oriel Model 6316). After passing through the cell, the intensity of the light as a function of wavelength is measured by focusing the beam through a $0.25 \mathrm{~m}$ Ebert Monochrometer (Jarrel-Ash Model 82-410) which disperses it onto a 512 channel ( $12 \mathrm{~mm}$ wide) photodiode array (Tracor Northern Model 6100). An absorption spectrum is obtained by comparing the spectra of the radiation passing through the cell with sample flowing through the instrument with an identical spectra taken without sample. 


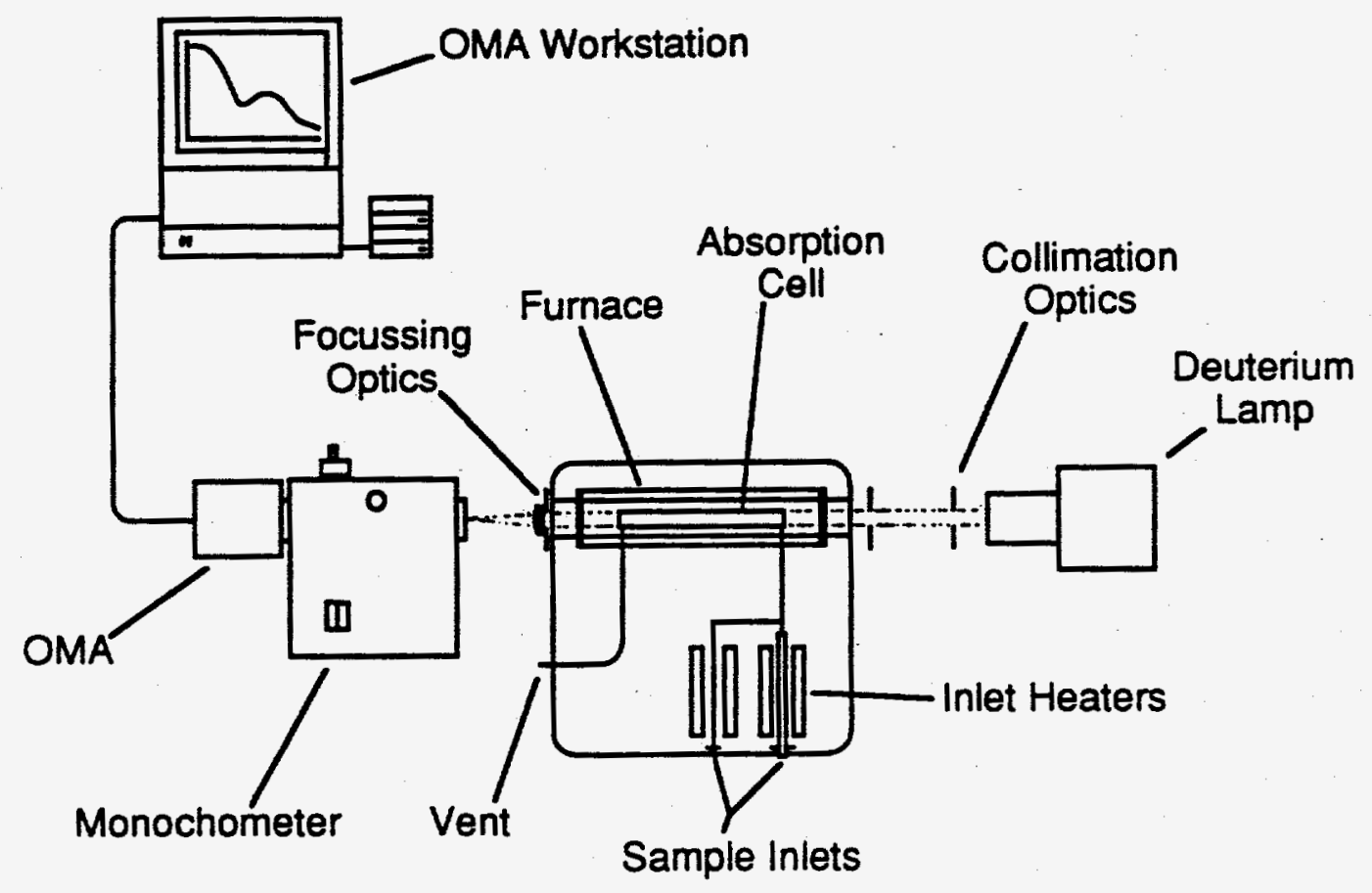

Figure 3.3. Schematic of the High-Temperature Absorption Spectrophotometer (HTAS) showing the instrument's general layout and major features. 


\subsection{EXPERIMENTAL APPROACH}

The overall operation of the systems described above may be categorized as: sample introduction, exposure, analysis, and data reduction. In the paragraphs below, each of these will be discussed in tum.

In broadest terms, sample introduction may be categorized as either pulsed or fixed rate. Pulsed sample introduction includes rapid syringe injection of fluids (e.g., gases or liquids), or batch charging of small quantities of either solids or mixed phase (liquid/solid) samples. Fixed rate injection includes controlled rate syringe injection of fluids, or controlled rate ram feeding of either solids or mixed phase (liquid/solid) samples. Fixed rate injection includes controlled rate syringe injection of fluids, or controlled rate ram feeding of either solids or mixed phase samples.

Since the analytical systems used on the TPRS and ATPRS have limited sample capacities (typically on the order of a few micrograms), the amount of sample admitted to these instruments is likewise limited. For this reason, pulsed injection is often employed as it is well suited for delivering minute amounts of material. Pulsed injection may be used with neat or dilute gases (samples in which the gaseous sample is diluted with an inert bath gas in a glass sample bulb), and solid phase samples solvent deposited on a special probe insert. Furthermore, on the two reactor systems, pulsed injection is always used for liquids, as fixed rate feed of microgram quantities of liquids is impractical.

Since microgram quantities of gasses, and dilute solid phase materials in solution, are relatively easy to handle, these types of samples are well suited for controlled rate insertion. Specifically, gases may be injected at a controlled rate using a syringe pump (a device that depresses a syringe plunger at a preset rate). Solid materials are first dissolved in a volatile solvent, then a small quantity is placed in a $0.4 \times 4 \mathrm{~cm}$ trough which is on the end of a special probe. After the solvent evaporates, a thin film of the sample remains along the bottom of the trough. This probe is then inserted into the inlet (which is typically preheated to $300^{\circ} \mathrm{C}$ ) at a controlled rate using a modified syringe pump.

Mixed phase samples, such as a liquid solvent mixed in a solid matrix, present a special challenge. Specifically, since these samples often contain a wide variety of materials with a broad range of vapor pressures, it is desirable to uniformily heat them as quickly as possible to minimize partitioning of the sample. This is done by placing the sample in miniature $(0.1 \times 3 \mathrm{~cm})$ glass test tubes which are then placed in the preheated inlet using a special carrier probe. 
The HTAS has a unique set of sample inlet requirements that are very different from the TPRS and ATPRS. Specifically, in order to get an adequate signal strength, a high concentration of sample is often required. Consequently, pulse injection of large quantities of neat gases (typically several $\mathrm{ml}$ ) or solids (tenths of $\mathrm{mg}$ ) is often used. Liquids may be introduced at a fixed rate either neat, or in a UV transparent solvent. Solids may also be injected in solution if the background (i.e., solvent only) is carefully accounted for.

In the TPRS and ATPRS, analyses are conducted in pairs with one exposure conducted with the reactor in darkness (thermal) and the other with the reactor illuminated with the respective radiation source (e.g., simulated solar radiation in the TPRS, and laser irradiation in the ATPRS). The radiant intensity from the solar simulator is set using the lamp current and lamp voltage settings on the unit's power supply. These values are periodically calibrated against the simulator's total power output measured with a thermopile power meter (Scientech Model 362). The response of this power meter has been checked against that of a high-temperature, photochemical actinometer developed in our laboratories expressly for these studies (cf. Section 3.5). The simulator's output as a function of wavelength is determined from a relative energy versus wavelength calibration curve which was carefully established for the system (cf. Figure 3.4). The radiant intensity from the laser system is measured before and after each run using the same power meter just described. The mean duration of each exposure is determined from the measured volumetric flow rate of gas through the system, temperature, and volume of the reactor vessel. The temperature of the exposure is measured with a radiation shielded thermocouple which is placed adjacent to the reactor side wall. This thermocouple also serves as the feedback element to the furnace temperature controller. The pressure of the vessel is taken as ambient (typically $745 \mathrm{~mm} \mathrm{Hg}$ ).

Measuring the extent of conversion (destruction) using the TPRS and ATPRS begins with obtaining reference GC/FID chromatograms under conditions where reactions are not expected to occur. This usually means thermal exposures (the solar simulator or laser is not used) at $300^{\circ} \mathrm{C}$. These chromatograms provide a record of the retention time(s) of the parent compound(s), and the integrated peak area(s) for the unconverted sample. The extent of conversion from subsequent tests is then calculated from (recall that the FID responses linearly to mass);

$$
f_{r}=A_{T, I 0} / A_{0} \times 100 \%
$$

where $f_{r}$ is the fraction remaining (weight or mole \%), ATIo is the integrated chromatographic peak area (area counts) at temperature $\mathrm{T}\left({ }^{\circ} \mathrm{C}\right)$ and with radiant flux $\mathrm{I}_{0}\left(\mathrm{~W} / \mathrm{cm}^{2}\right.$ or the equivalent in solar constants), and $A_{0}$ is the reference area (area counts) measured under non-destructive conditions (i.e., $A_{300,0}$ ). On 


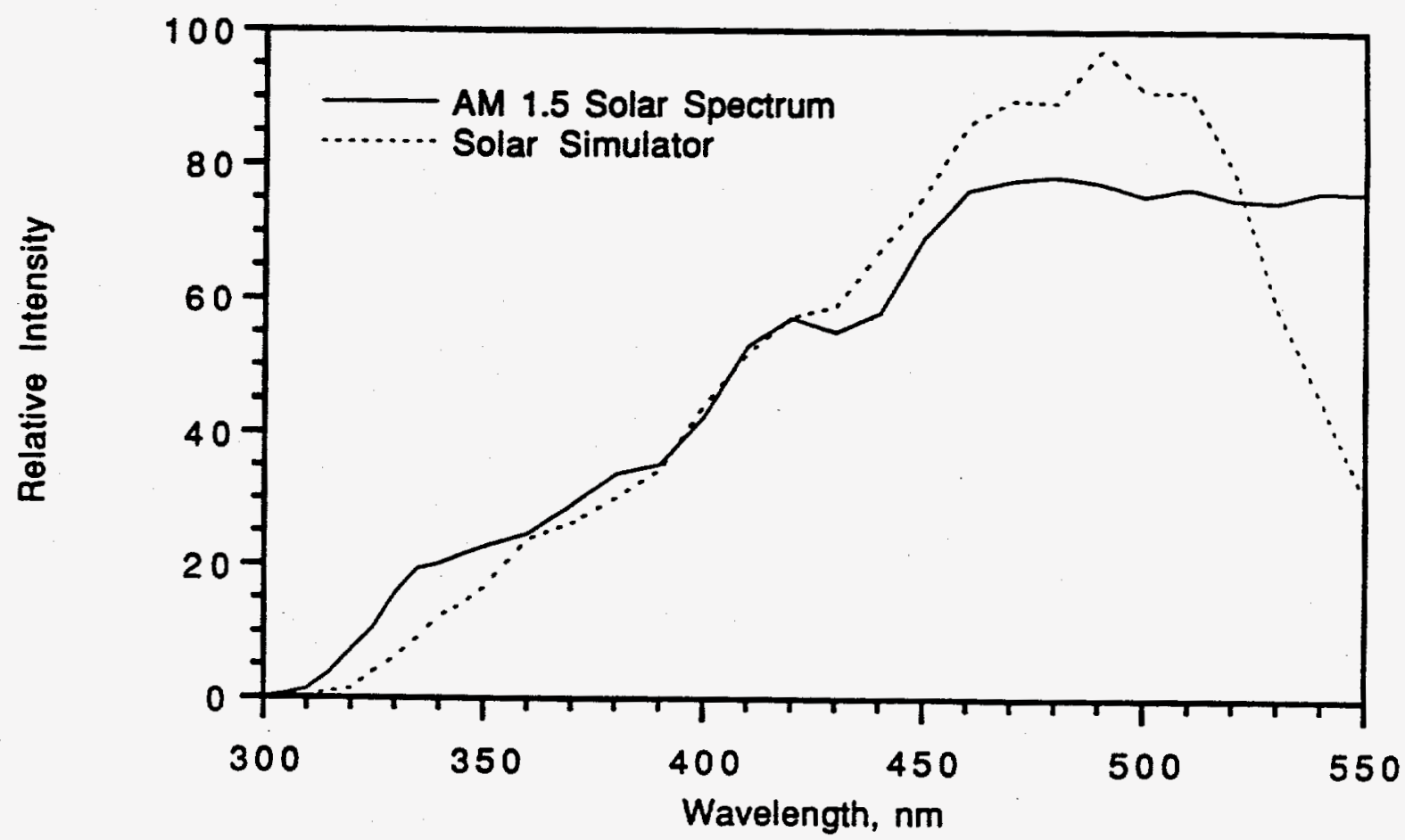

Figure 3.4. Relative output of the TPRS's solar simulator fitted with a dichroic miror and air mass filters and a AM 1.5 solar spectrum. 
the TPRS parent species are indentified by their chromatographic retention times, and on the ATPRS by there retention times and mass spectra.

In a similar manner, organic reaction products (recall that the FID does not respond to inorganic products) are identified as peaks not observed in the reference chromatograms. By itself, the TPRS cannot be used to conclusively identify products. However, since nonpolar liquid phases are used in the analysis process, general statements concerning the molecular weight can be made. Specifically, compounds with molecular weight higher than the parent will have longer retention times, and vice versa. Also, a crude approximation of product yields can be made using the integrated chromatographic peak area as;

$$
f_{p}=A^{\prime} T, I 0 / A_{0} \times 100 \%
$$

where $f_{p}$ is the fraction produced (weight or mole \% taken as the reference compound), and $A^{\prime}$, Io is the integrated chromatographic peak area (area counts) for the product.

If product identification information is available (see below), products on the TPRS can be quantified as;

$$
\text { Moles product }=A^{\prime} T, I 0 \times k_{\text {resp }}
$$

where $k_{\text {resp }}$ is the calibrated response factor (moles per area count) obtained by analyzing known quantities of reference standards with the TPRS under nondestructive conditions.

Quantitative data from the ATPRS is obtained in a similar fashion as described above for the TPRS, except here the GC/MSD channel provides identification information for both parent species and products. Parent compounds are defined as those peaks observed with the ATPRS operating in a nondestructive mode (i.e., $300^{\circ} \mathrm{C}$ without laser illumination). Products are taken as any peak observed in subsequent runs which were not seen in the reference chromatograms. Initial identifications are made with the mass spectral information provided by the GC/MSD channel. These assignments are then confirmed using analytical standards when they are available. Once identifications are confirmed by GC/MSD retention times and mass spectra, the peaks are quantified using the GC/FID channel as described by Equation 3.3. In cases where analytical standards are not available, structurally similar compounds are used. 
In addition to serving in it's own role, the ATPRS can be used to provide identifications of major products from the TPRS. This is accomplished by conducting tests on the ATPRS under the same conditions of exposure time and temperature as the TPRS, then using the GC/MSD channel of the ATPRS to make identification assignments, and the GC/FID to provide a record of relative retention times which may be transferred to the TPRS chromatograms. Product identifications made in this manner are subject to the same confirmation by analysis of reference standards as described above. Note that this type of cross-referencing is considered valid only for thermal exposures and, in the cases where it has been employed the distribution of products from the thermal and simulated solar exposures were very similar.

In HTAS data is also taken in pairs with one data set serving as a reference, and the other containing the information of interest. Specifically, the first set of data is taken with clean carrier gas (normally dry nitrogen) flowing through the cell. This data set may be from a single reading of the detector array, or from the average of 4, 9 (normal), or 16 runs. The latter improves the signal-to-noise ratio by 2, 3, or 4 times respectively. Knowing the centerline wavelength set on the monochrometer (read from the monochrometer's indicator which has been calibrated against the prominant lines of $\mathrm{Hg}$ emission), the spacing of the detector elements $(0.025 \mu \mathrm{m})$, and the dispersion of the grating (either 1.65 or $3.30 \mathrm{~nm} / \mathrm{mm}$ ) record of transmitted light intensity as a function of wavelength for the system is provided. Next, similar data is taken with sample flowing through the cell. Exactly how this data is taken depends on the method of sample introduction, i.e., continuous or single pulse.

The case where sample is continuously fed to the absorption cell is relatively straight forward. Specifically, as the sample is being admitted to the HTAS, the output from a single detector element (any one of the 512 elements may be used) is monitored to ensure that the system is at steady state. Once a steady signal is observed the operator can direct the HTAS to obtain a single set of data from the detector array, or the average of 4,9 , or 16 sets as described above.

Obtaining data in cases where the sample is introduced as a single pulse requires more care. In this case the HTAS's data system displays and records a continuous readout of the signal from a single detector element (any one of the 512 elements may be used) as compared against the same element from the reference data set. As the sample pulse passes through the cell, the relative intensity of the monitor element is observed to deviate from a baseline value representing $100 \%$ transmission and approach a relatively steady value showing that the sample is absorbing the radiation passing through the cell. As the signal approaches this value, the data system is directed to store 4 complete data sets from the detector array, after which the recording of data from the single element continues until the signal is seen to have returned to baseline. From the 4 complete spectra collected, the spectra with the strongest signal is selected for final analysis. 
From either sample injection technique the data may be reduced to transmission as (recall that photodiodes respond linearly to light intensity);

$$
T_{\lambda}=I_{\lambda} I_{0 \lambda}
$$

where $T_{\lambda}$ is the transmission at wavelength $\lambda(\mathrm{nm}), \mathrm{I}_{\lambda}$ is the light intensity measured by the detector element corresponding to I with the sample flowing through the cell, and $\mathrm{I}_{0 \lambda}$ is the reference light intensity measured by the detector element corresponding to $\lambda$. The data may also be expressed as absorbance;

$$
A_{\lambda}=\log _{10}\left(1 / T_{\lambda}\right)
$$

where $A_{\lambda}$ is the absorbance at wavelength $\lambda$. Finally, knowing the path length of the cell $(20 \mathrm{~nm})$ and mean molar concentration of the sample in the cell, the absorption of the sample may be calculated as;

$$
\varepsilon_{\lambda}=A_{\lambda} /(b c)
$$

where $\varepsilon_{\lambda}$ is the molar extinction coefficient $\left(\mathrm{L} \mathrm{mol}^{-1} \mathrm{~cm}^{-1}\right)$ at wavelength $\lambda, b$ is the path length $(\mathrm{cm})$, and $c$ is the molar concentration (mol $\mathrm{L}^{-1}$ ). in the case where sample is admitted to the HTAS at a constant rate, Equation 3.6 can be readily applied as the mean molar concentration can be easily calculated from the molar feed rate of sample, total volumetric flow rate through the cell, and the cell temperature and pressure. However, in the case of the pulse injection, calculating the concentration is a little more involved.

At the conclusion of a test run with the pulse injection technique, a record of the sample transmission at a single wavelength as a function of time is available as well as 4 complete spectra taken as the signal strength passes through a maximum. The transmission versus time data can be converted to an absorbance versus time record using Equation 3.5 . Since absorbance varies linearly with concentration (recall that absorbance is the product of the molar extinction coefficient, path length, and molar concentration), this record can be converted to concentration versus time data by applying an appropriate constant of proportionality. Specifically;

$$
c=A_{\lambda, t}\left[M /\left(v \int A_{\lambda, t} d t\right)\right]
$$

where $A \lambda, t$ is the absorbance at wavelength $\lambda(\mathrm{nm})$ and time $t(\mathrm{~s}), M$ is the total number of moles of sample injected (mol), $v$ is the volumetric flow rate through the cell $(\mathrm{L} / \mathrm{s})$, and the integration is carried out over the 
time of the run. In these tests the integration was conducted numerically using a simple trapezoidal approximation. Once $\mathrm{c}$ is known for the selected spectra, the molar extinction spectra can be constructed as shown in Equation 3.6.

In the TPRS and ATPRS knowledge of the sample concentration was gained using relatively simple methods. Specifically, for continuously fed samples, the mean concentration was calculated as described above (i.e., from knowledge of the molar feed rate of sample, total volumetric feed rate of sample and carrier gas, the reactor temperature and pressure, and assuming ideal behavior). In the case of pulsed injection into these systems, the mean concentration was estimated assuming the sample is evenly distributed to a $10 \mathrm{ml}$ volume (the approximate volume of the inlet chambers) prior to entering the reactor.

In the two reactor systems, the condensable content of the exhaust stream is captured cryogenically at either approximately $-130^{\circ} \mathrm{C}$ (TPRS) or $-160^{\circ} \mathrm{C}$ (ATPRS). The HTAS exhaust passes through a particulate/aerosol trap (which is at room temperature) to remove nonvolatile components, then through an activated carbon filter to remove semivolative components, then to a fume hood for disposal of the dilute or inert volatile components.

In the two reactor systems, the cryogenic trap is first purged with the carrier gas which will be used for the chromatographic analysis (typically helium). The exhaust vent is sealed, directing the carrier gas to the $\mathrm{GC}$, and the trap is then rapidly heated to $350^{\circ} \mathrm{C}$ releasing the captured components on to the GC column(s).

The GC column(s) are initially held at a sub-ambient temperature (typically $-80^{\circ} \mathrm{C}$ ) to condense the analytes on the column inlet. The temperature is then raised at $10^{\circ} \mathrm{C} / \mathrm{min}$ to $0^{\circ} \mathrm{C}$, then at $20^{\circ} \mathrm{C} / \mathrm{min}$ to $260^{\circ} \mathrm{C}$, where the temperature is held until solute peaks are no longer observed. Data for parent species (those observed under nondestructive conditions) are quantified by comparing the integrated chromatographic peak area at low (nondestructive) temperature, with areas measured at higher temperatures. For GC/FID analytes, identifications are made by comparing relative retention times of unknowns with analytical reference standards. For GC/MS (and GC/HFID/MS) data, identifications are made using both mass spectra, quantitative standards (when available), and comparing relative retention times of unknowns with retention time of reference standards.

Analyses of noncondensable compounds (e.g., methane, carbon monoxide, carbon dioxide, etc.) are conducted by collecting the reactor exhaust in Tediar bags. The content of these bags are then quantitatively analyzed for specific compounds using a packed column GC (Varian 3700) fitted with a 
nonpolar column ( $2 \mathrm{~mm} \times 2 \mathrm{~m}, 80 / 120$ mesh Porapak Q) and a methanizer/FID (Baseline Industries, Model 2400A) for solute detection.

\subsection{HIGH-TEMPERATURE PHOTOCHEMICAL ACTINOMETRY}

The TPRS geometry, xenon lamp characteristics, and optical imaging problems have made it extremely difficult to obtain in-situ measurements of the amount of light that passes through the reactor vessel. It would be desirable to develop a system capable of providing a true in-situ measurements of the near UV photon flux. We have therefore attempted development of a chemical actinometer in hopes of obtaining in-situ measurements of TPRS photon flux.

It was determined early on in our research that most conventional chemical actinometers would not be acceptable because they were not calibrated at high temperature or even the minimum temperature at which the TPRS could operate. An additional restriction that eliminated many chemical actinometers from consideration was the requirement that the extent of reaction be analyzable using the capillary column GC techniques employed in the TPRS. As a result it became immediately evident that conventional gas-phase actinometers such as acetone and methyl ethyl ketane would not work on the TPRS. A great deal of enabling work yielded an actinometer system consisting of cyclohexane $\left(\mathrm{C}_{6} \mathrm{H}_{12}\right)$ and molecular chlorine $\left(\mathrm{Cl}_{2}\right)$ in a 1:3 molar ratio. This system was based on the belief obtained from the literature that the quantum yield of chlorine atom production from photolysis of $\mathrm{Cl}_{2}$ was known and independent of excitation wavelength over the wavelength range of $300-550 \mathrm{~nm}$ (i.e., $\phi_{r}=2.0$ ). Thus, it was suspected that $\mathrm{Cl}_{2}$ dissociation and subsequent rapid reaction of $\mathrm{Cl}$ with cyclohexane would result in $\mathrm{GC}$ measurable disappearance of the cyclohexane. By measuring the extent of reaction using a well characterized illumination source (e.g., a laser), the reaction quantum yield could be accurately determined. With knowledge of $\phi_{r}$ and necessary spectroscopic parameters in hand, the actinometer system could then be used to determine the photon flux of the TPRS. However, it was determined after many painstaking experiments that the reaction rate was very sensitive to reaction conditions. Many trial and error experiments were required to identify any set of experimental conditions that yielded reproducible results. Finally, such a set of conditions was identified.

Specifically, a premixed feed consisting of $1.32 \times 10^{-8} \mathrm{~mol} / \mathrm{L}$ cyclohexane and $4.02 \times 10^{-8} \mathrm{~mol} / \mathrm{L}$ chlorine gas was exposed for $10 \mathrm{~s}$ in dry helium at $200^{\circ} \mathrm{C}$. The solar simulator was fitted with a dichroic mirror (reflecting from 300 to $550 \mathrm{~nm}$ ), one AM-1 glass filter (Spectral Energy LHA 175/3) and one neutral density (ND-1) 10\% transmission filter. These conditions were maintained for all experiments. In Figure 3.5, we show the AM-1 filtered lamp output, the AM-1 + ND-1 filtered lamp output, and the amount 


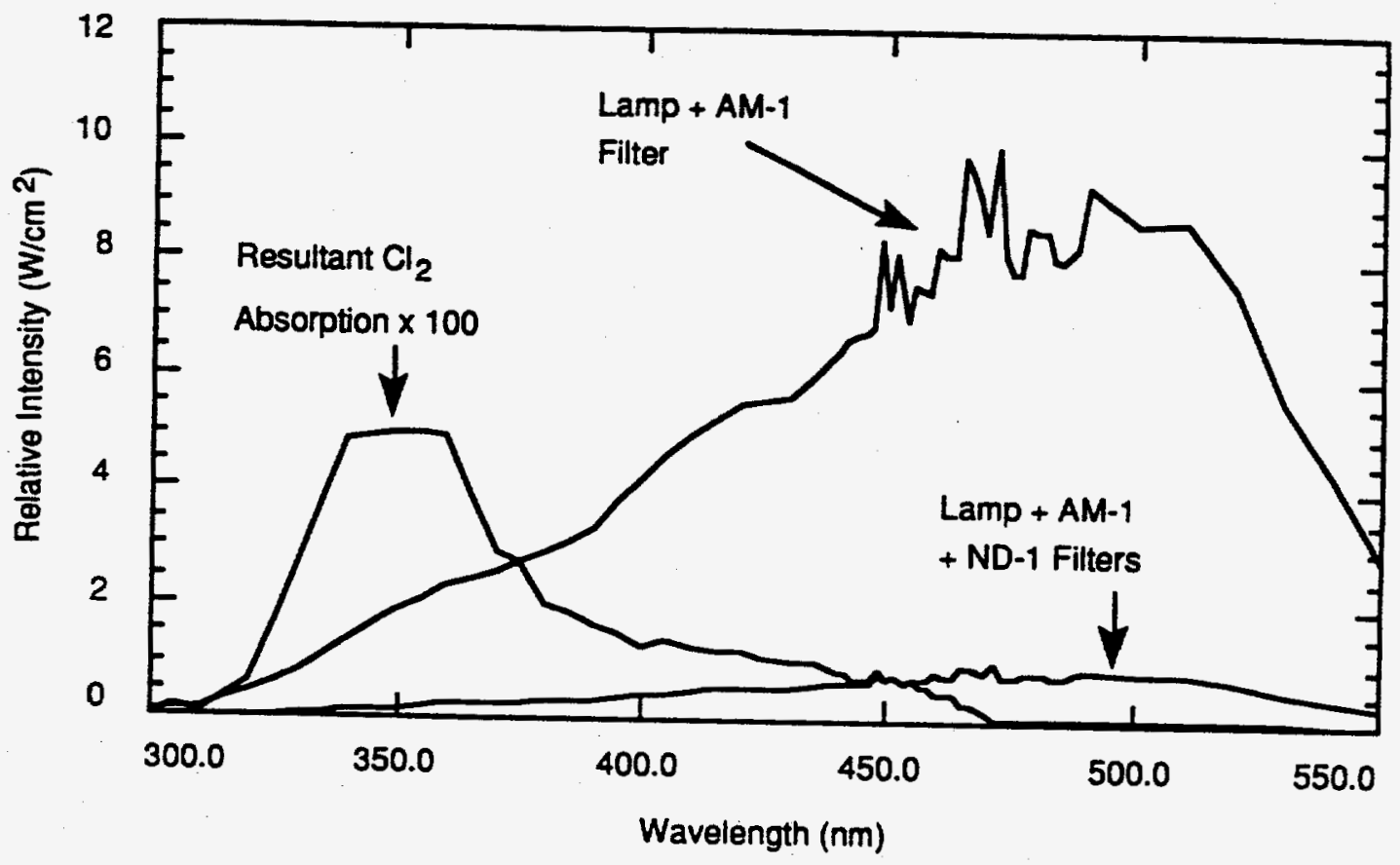

Figure 3.5. Relative filtered solar simulator emission and chlorine absorption spectra. 
(multiplied by 100) which is absorbed by chlorine under these conditions. The need for extreme attenuation of the lamp output in order to control the rate of reactions was met with the neutral density filter.

Upon absorption of near UV radiation, molecular chlorine dissociates into atomic chlorine, $\mathrm{Cl}$, which react with cyclohexane. The extent of cyclohexane destruction was measured with the TPRS. Results are given in Table 3.1.

TABLE 3.1

TPRS DESTRUCTION OF $\mathrm{C}_{6} \mathrm{H}_{12}$

Power Supply Reading (Watts)

Avg. Fraction

$\mathrm{C}_{6} \mathrm{H}_{12}$ Remaining

\begin{tabular}{cc}
\hline 600 & 0.39 \\
800 & 0.33 \\
1000 & 0.27 \\
\hline
\end{tabular}

The photochemical quantum yield of chlorine dissociation is known to be unity, however, the efficiency of reaction with cyclohexane was unknown. Therefore to calibrate the cyclohexane/chlorine as an actinometer we needed to determine the photochemical quantum yield of the system. We accomplished this by illuminating a static cell with an Argon ion laser maintaining the same concentrations, exposure time, and temperature conditions as with the TPRS flowing cell. After laser irradiation, samples (kept isolated from room lights) were next analyzed using the TPRS to determine the fraction of cyclohexane remaining unreacted. The quantum yield was then found from the following relation:

$$
\phi_{\mathrm{r}}=-\ln \left(\mathrm{f}_{\mathrm{r}}\right) /\left(1.92 \times 10^{-5} \mathrm{t}_{\mathrm{r}} \Sigma \lambda_{\varepsilon_{\lambda}} \Delta \mathrm{I} \lambda\right)
$$

where $\phi_{r}$ is the photochemical quantum yield, $f_{r}$ is the fraction remaining, $t_{r}$ is the mean residence (exposure) time (s), $\lambda$ is wavelength $(\mathrm{nm}), \varepsilon_{\lambda}$ is the molar extinction coefficient $\left(\mathrm{L} \mathrm{mol}^{-1} \mathrm{~cm}^{-1}\right)$ at $\lambda, \Delta \mathrm{I} \lambda$ is the light intensity $\left(W / \mathrm{cm}^{2}\right)$ over the step interval in $\lambda$, and the summation is carried out over the wavelength region of overlap between the absorption spectrum of the actinometer and the emission spectrum of the source. In the calibration tests, an argon ion laser was used which had emission lines at 349.5 and 362.2 nm with relative intensities of $46.6 \%$ and $53.4 \%$, respectively. The results of these tests are given in Table 3.2.

Calibration may be achieved via the set of numerics, $C_{0}$, found in the following expression for quantum yield: 


$$
\phi_{r}=-\ln \left(f_{r}\right) /\left(t_{r} C_{0} \int \lambda \varepsilon \lambda \Delta I_{\lambda}\right)
$$

where the integration is carried out over the wavelength region of overlap between the absorption spectrum of the actinometer and the emission spectrum of the source. The results, shown in Table 3.2, suggest that the low energy trials were made in an "onset" regime, while the high energy measurements were taken in a "saturation" regime.

TABLE 3.2

ARGON ION LASER DESTRUCTION OF $\mathrm{C}_{6} \mathrm{H}_{12}$ in a STATIC CELL

\begin{tabular}{cccc} 
Power $(W)$ & Intensity $\left(\mathrm{W} / \mathrm{cm}^{2}\right)$ & $\begin{array}{c}\text { Avg. Fraction } \\
\mathrm{C}_{6} \mathrm{H}_{12} \text { Remaining }\end{array}$ & $\phi_{\mathrm{r}}$ \\
\hline 0.025 & 0.009 & 1.0 & \\
0.050 & 0.018 & 1.0 & \\
0.075 & 0.026 & 1.0 & 1.5 \\
0.088 & 0.031 & 1.0 & 2.6 \\
0.184 & 0.065 & 0.89 & 2.3 \\
0.368 & 0.053 & 0.69 & 1.9 \\
0.553 & 0.053 & 0.60 & 1.8 \\
0.737 & 0.053 & 0.58 & 1.4 \\
0.745 & 0.262 & 0.59 & 1.0 \\
0.922 & 0.325 & 0.60 & 0.63 \\
1.150 & 0.404 & 0.0 & \\
\hline
\end{tabular}

We tested the compatibility of the TPRS and laser results by calculating the absorbance rate constants. For the laser, the rate of absorbance was calculated as;

$$
k_{a b}=1.92 \times 10^{-5} \Sigma \lambda_{\varepsilon_{\lambda}} \Delta \mathrm{I}_{\lambda}
$$

where $k_{a b}$ is the absorption rate constant $\left(\mathrm{s}^{-1}\right)$ and the summation was carried out for the 349.5 and 362.2 $\mathrm{nm}$ argon ion emission lines. Similarly, for the broad-band solar simulator, $k_{a b}$ was found from;

$$
k_{a b}=1.92 \times 10^{-5} \int \lambda \varepsilon_{\lambda} d I_{\lambda}
$$

where the integration was carried out numerically from 300 to $550 \mathrm{~nm}$.. Results from these tests are given in Table 3.3 and Figure 3.6. These data show divergent destruction efficiencies exhibited by TPRS and argon ion irradiation of our actinometer. Despite lower overall energies, the TPRS lamp shows higher 
destruction efficiencies. Since the laser is nearly monochromatic and the lamp is polychromatic, this implies a possibility that the quantum yield associated with molecular chlorine dissociation is not constant with wavelength. We tentatively attribute the disparity in results to absorption by a weak $\mathrm{Cl}_{2}$ triplet Rydberg state, the $A\left({ }^{3} \Pi^{+} u \leftarrow X\left({ }^{1} \Sigma^{+} g\right)\right.$, at $546 \mathrm{~nm}$ which is a transition accessible only to the solar simulator.

We have demonstrated that the $\mathrm{C}_{6} \mathrm{H}_{12}: \mathrm{Cl}_{2}$ system has some potential as an actinometer. Unlike conventional actinometers which we also attempted to use, this system actually worked under our unusual experimental conditions. There are, however, several problems. The system is extremely sensitive over a narrow range. In addition, it appears that $\mathrm{Cl}_{2}$ quantum yield may not be strictly constant with wavelength. If this system is to be used as a proper chemical actinometer new data will have to be generated using a green-filtered TPRS with the argon ion laser in order to eliminate any possible contributions from the $\mathrm{Cl}_{2}$ $546 \mathrm{~nm}$ triplet Rydberg state.

It may be that the main value of the $\mathrm{C}_{6} \mathrm{H}_{12}: \mathrm{Cl}_{2}$ system will emerge as a check of the power delivered by the TPRS. As such,. we would measure the power distribution irradiating a cell mock-up with a well-calibrated power meter. Next, $\mathrm{C}_{6} \mathrm{H}_{12}$ destruction measurements would be taken in the same mockup using the actinometer. Lastly, the TPRS cell is aligned until the same $\mathrm{C}_{6} \mathrm{H}_{12}$ destruction is observed.

TABLE 3.3

COMPARISON OF RESULTANT TPRS AND ARGON ION LASER ABSORBANCE RATE CONSTANTS, $\mathrm{k}_{\mathrm{ab}}\left(\mathrm{s}^{-1}\right)$

\begin{tabular}{ccc} 
Power $(W)$ & $100 \mathrm{ka}^{-1}\left(\mathrm{~s}^{-1}\right)$ & $-\ln (\mathrm{fr})$ \\
\hline 0.106 & $\frac{\text { IPRS }}{0.722}$ & 0.94 \\
0.106 & 0.722 & 1.03 \\
0.106 & 0.722 & 0.85 \\
0.146 & 0.994 & 1.11 \\
0.146 & 0.994 & 1.27 \\
0.146 & 0.994 & 0.95 \\
0.201 & 1.36 & 1.31 \\
0.201 & 1.36 & 1.23 \\
0.201 & 1.36 & 1.36 \\
& Argon ion laser & \\
0.184 & 0.723 & 0.11 \\
0.368 & 1.44 & 0.38 \\
0.553 & 2.17 & 0.50 \\
0.737 & 2.90 & 0.55 \\
0.745 & 2.93 & 0.53 \\
0.922 & 3.90 & 0.52 \\
1.150 & 4.52 & 0.46 \\
\hline \hline
\end{tabular}




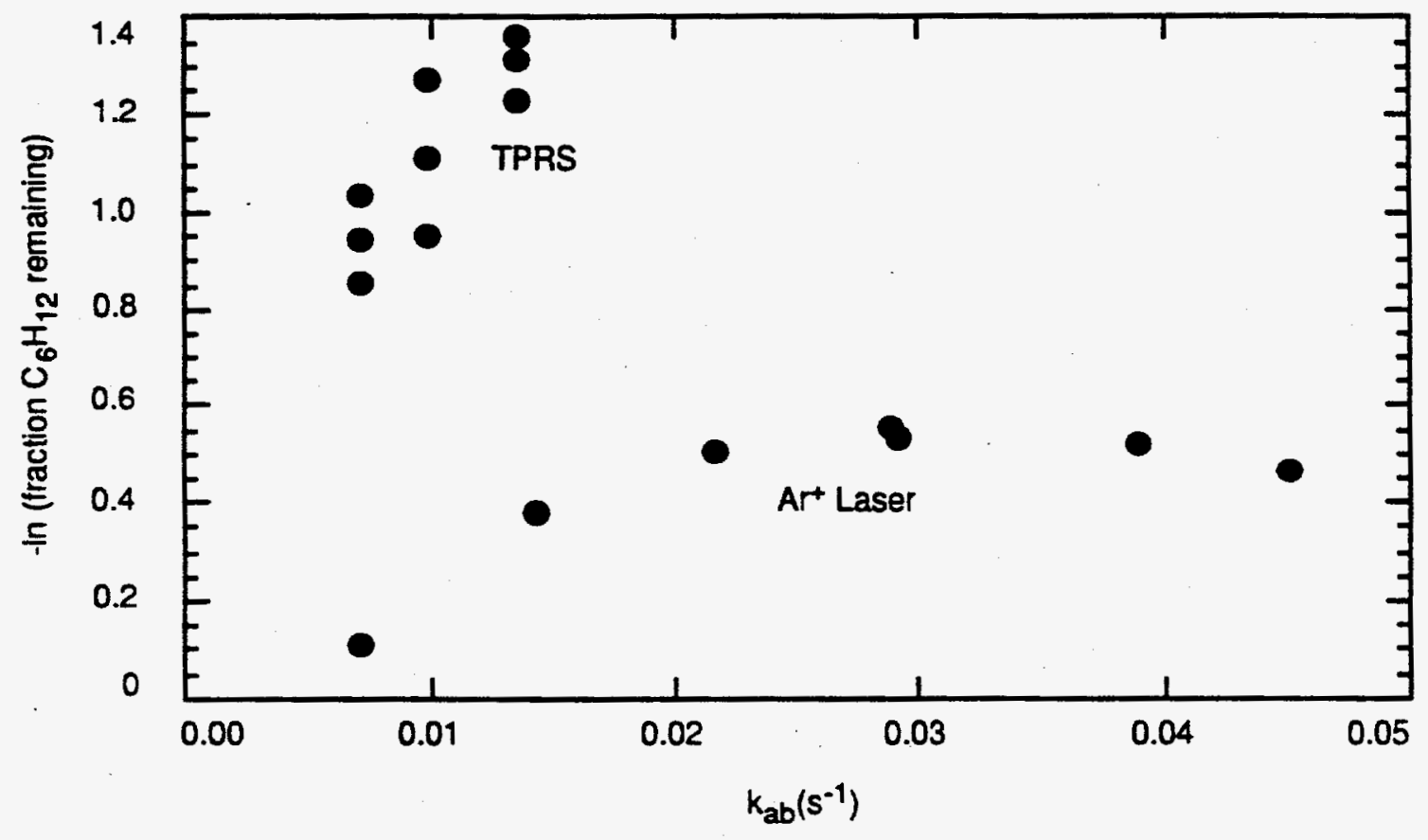

Figure 3.6. Test results actinometer destruction efficiencies are very different under deuterium lamp and Argon ion $\left(\mathrm{Ar}^{+}\right)$laser irradiation. 


\section{SECTION 4.0 \\ ENHANCED DESTRUCTION OF PURE COMPOUNDS}

The simple, pseudo-first-order kinetic model described in Section 2 suggests that the thermal/photolytic enhancement, as quantified by the enhancement ratio $R$, is exponentially dependent on $\phi_{r}, I_{0}, \varepsilon$, and $t$ (ct. Equation 2.7a). We have further argued that $\phi_{r}$ and $\varepsilon$ increase with increasing temperature in a complex manner while $k_{g n d}$ obeys simple Arrhenius kinetics. Although not explicitly included in the theoretical development, $R$ may vary with reaction atmosphere, i.e., oxidation versus pyrolysis. In the following Section we present and discuss selected data which illustrate the dependence of $R$ on temperature, time, light intensity, and excess air.

\subsection{EFFECT OF TEMPERATURE}

The dominant variable of interest throughout most of the solar detoxification tests is the mean exposure temperature. In this section the specific impact of temperature on the overall process performance will be discussed along with it's effect on the extent of solar enhancement, the rate of photon absorption, and quantum efficiency.

Examining the data presented in Appendix I for all pure compounds studied during the course of this research program it can be seen that the overall process efficiency of the solar detoxification process always increases with temperature. As a typical example, consider the data for 1-nitronaphthalene (cf. Figure 4.1) exposed to 0 and $307 \mathrm{AM} 1.0$ suns for $5 \mathrm{~s}$ in air. Note that at $300^{\circ} \mathrm{C}$, the lowest temperature for which data is available, $66.6 \%$ of the parent compound was destroyed from the simulated solar process ( $R$ $=2.99$ ). The thermal process does not show a measurable level of conversion (onset detection limit is $\sim 98$ mole \% remaining) until $500^{\circ} \mathrm{C}$ where $44.6 \%$ is destroyed thermally while $97.11 \%$ has been destroyed by the simulated solar process $(R=19.2)$. Even at high levels of conversion the simulated solar process remains very efficient with $99.9754 \%$ destroyed at $625^{\circ} \mathrm{C}$ as compared to only $98.58 \%$ by the comparable thermal process $(R=58.4)$.

An important observation in the thermal/photolytic destruction of pure compounds is the appearance in many cases of a temperature range in which the photolytic effects pass through a maximum (i.e., a maximum in R). Reviewing the data summarized in Appendix I, this is seen as a common behavior and several specific examples are summarized in Figure 4.2. However, there are also examples, some of which are shown in Figure 4.3 , in which a maximum in $R$ is not observed over the temperature range studied. As a simple summary of the data on pure compounds which were irradiated with simulated sunlight, there are a total of 22 data sets, in which $14(64 \%)$ showed a maximum in $R$. There is no clear 


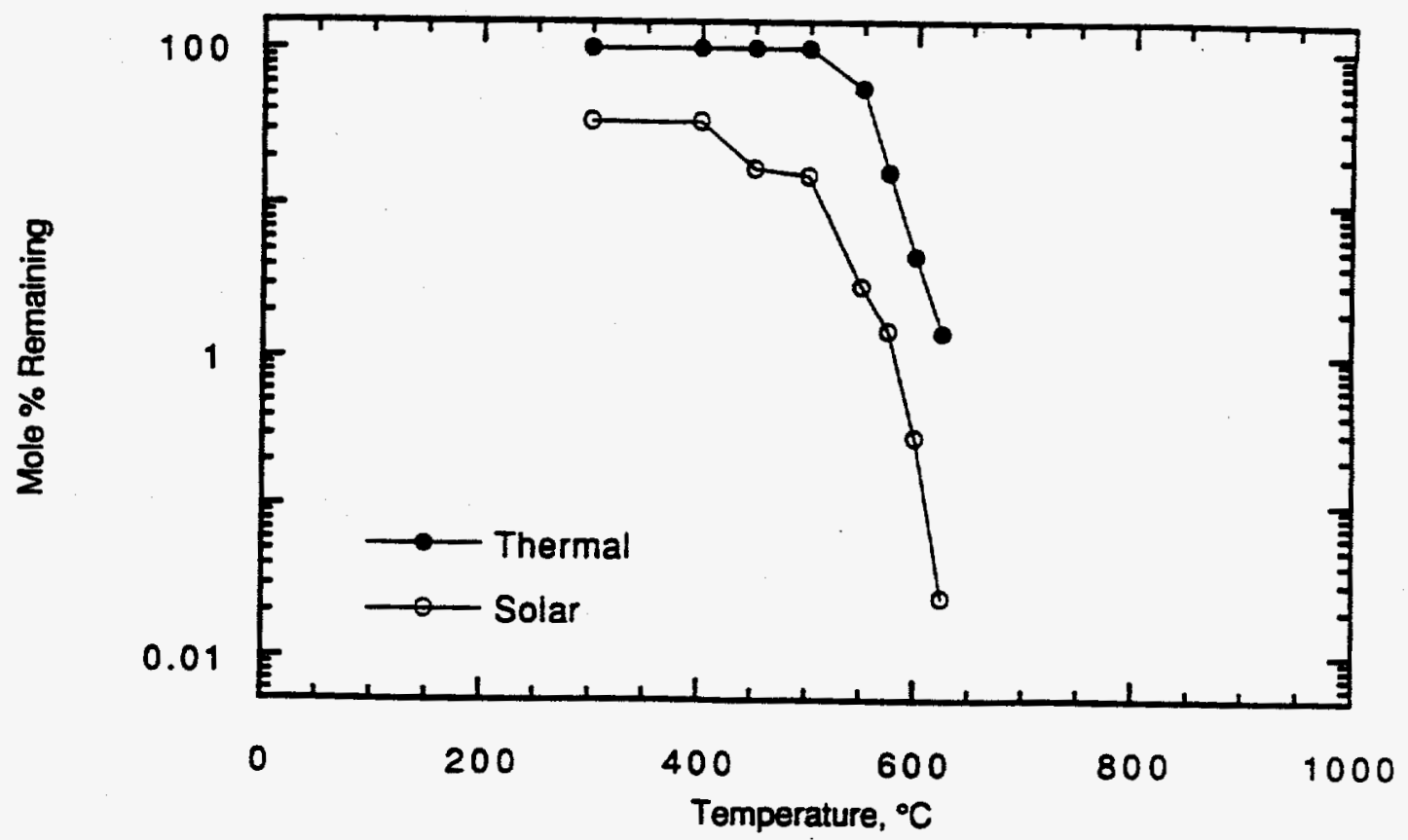

Figure 4.1. TPRS data for 1-nitonaphthalene $\left(-10^{-5} \mathrm{M}\right)$ exposed to 0 and 307 AM 1.0 suns (simulated) for 5 seconds in air.

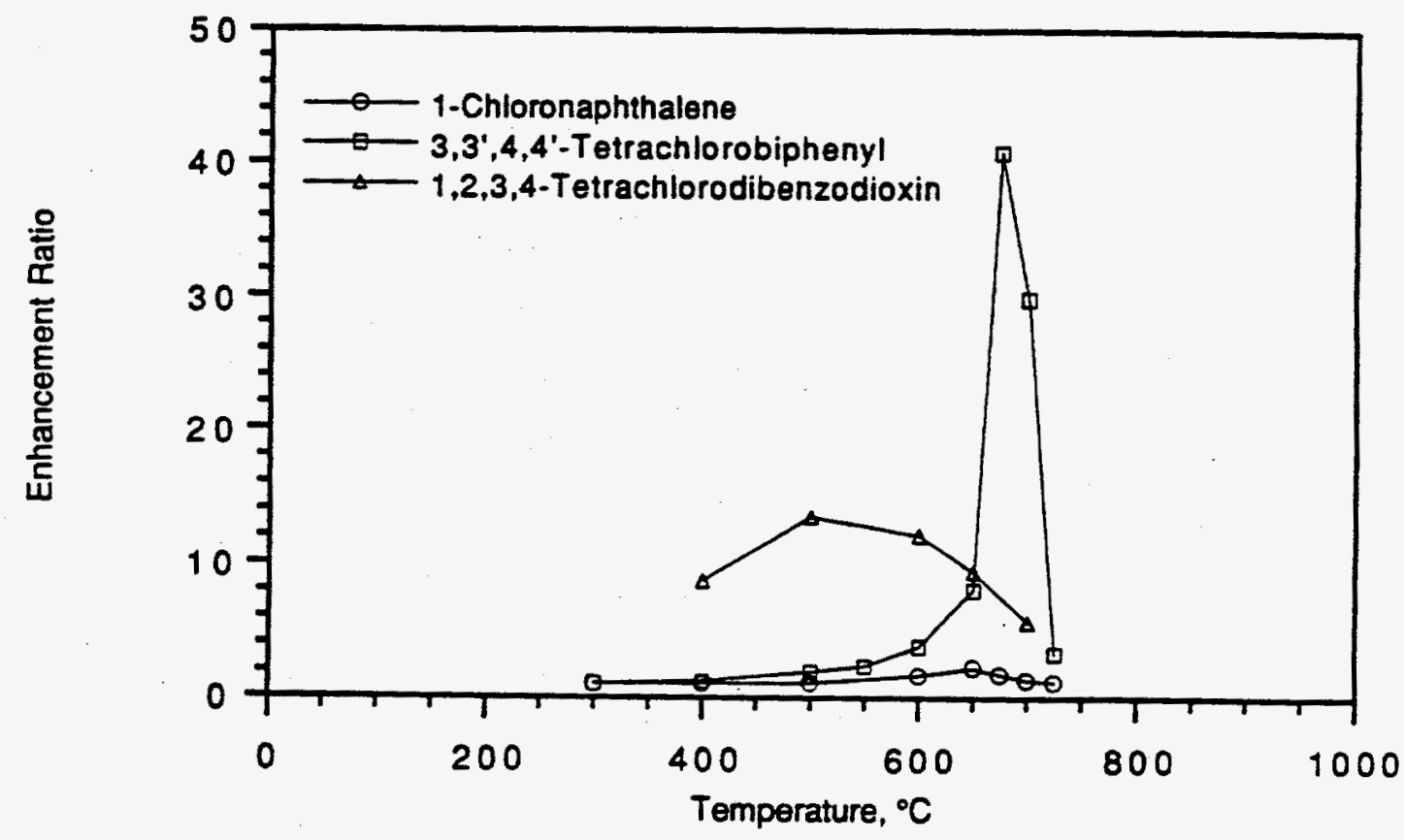

Figure 4.2. Examples of the thermal/photolytic enhancement ratio (oxidation) as a function of temperature for which a maximum enhancement was observed. These include 1-chioronaphthalene (307 AM 1.0 suns for 5 s in air), 3,3', 4,4'tetrachiorobiphenyl (273 AM 1.5 suns for $10 \mathrm{~s}$ in air), and 1,2,3,4-tetrachiorodibenzo-p-dioxin (307 AM 1.0 suns for 10 s in air). 


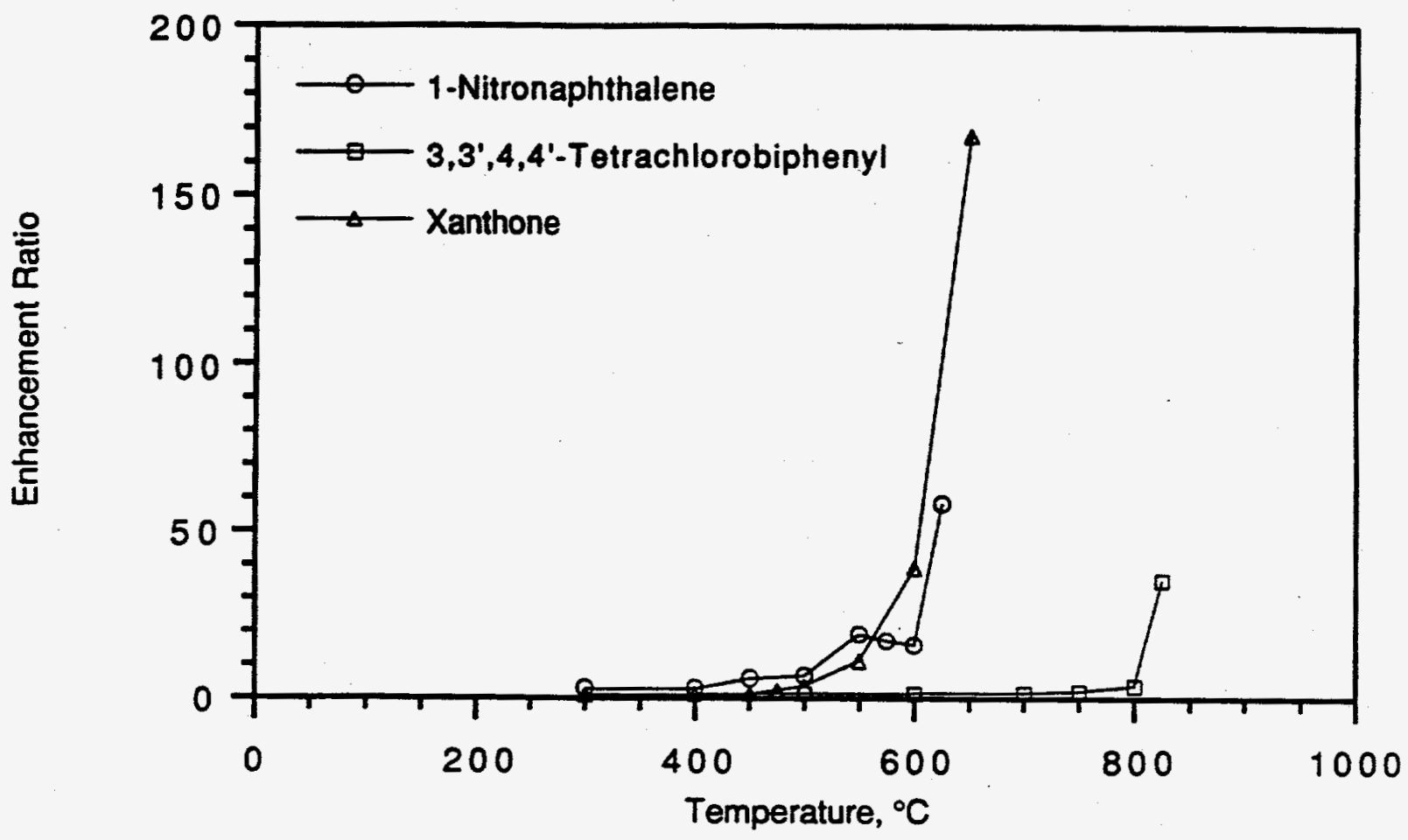

Figure 4.3. Examples of the thermal/photolytic enhancement ratio (oxidation) as a function of temperature in which a maximum enhancement was not observed. These include 1-nitronaphthalene (307 AM 1.0 suns for $5 s$ in air), 3,3',4,4'-tetrachlorobiphenyl (307 AM 1.0 suns for $10 s$ in helium), and xanthone (307 AM 1.0 suns for $5 s$ in air). 
relationship between the various data sets which suggests which class of compounds or exposure condition (e.g., exposure time or atmosphere) are likely to exhibit this behavior. However, this observation indicates that, unlike thermal destruction where higher temperatures are considered to always yield higher destruction, this may not be the case in a solar process if the goal is to maximize the solar benefit. Clearly, a sound understanding of this phenomena is important if the operating conditions for a full-scale facility are to be unambiguously established.

As will be more thoroughly discussed in Section 7.1, we have reason to believe that for many molecules, the extent of photoenhancement will be controlled by the temperature dependence of $k_{a b}$. This rate is controlled by the extent of overlap and relative intensities of the emission spectrum of the radiation source and the absorption spectrum of the waste. As summarized in Appendix III, the absorption spectra of several compounds have been measured at temperatures as high as $700^{\circ} \mathrm{C}$ and all show a red-shift and an increase in intensity with increasing temperature. As a typical example, consider the molar extinction spectra for methylene chloride from 200 to $700^{\circ} \mathrm{C}$ (cf. Figure 4.4). As this figure shows, the intensity of the absorption steadily increases, and shifts towards longer wavelengths (lower energy) as the temperature increases. This behavior demonstrates that as the temperature is increased, the target molecule absorbs solar energy with increasing effectiveness. Combining the measured absorption spectra for $3,3^{\prime}, 4,4^{\prime}$ tetrachlorobiphenyl (cf. Figure 7.3 and Appendix V) with the published emission spectra for AM 1.5 solar energy, the rate of photon absorption can be calculated. As shown in Figure 4.5, the rate of photon absorption increases exponentially with temperature.

Using the experimentally determined thermal/photolytic reaction and photon absorption rates, the quantum yield may be calculated using Equation $2.7 \mathrm{~b}$. Quantum yields as a function of temperature were calculated for 3,3',4,4'-tetrachlorobiphenyl thermal/photolytic oxidation with 273 simulated AM 1.5 suns and for monochlorobenzene oxidation using pulsed laser illumination at $280 \mathrm{~nm}$ (cf. Figures 4.6 and 4.7 ). In the case of monochlorobenzene, ff gently declines from 0.4 at $300^{\circ} \mathrm{C}$ to 0.35 at $500^{\circ} \mathrm{C}$, then to rises to a value of 0.58 by $700^{\circ} \mathrm{C}$. In contrast, $\phi_{r}$ for $3,3^{\prime}, 4,4^{\prime}$-tetrachlorobiphenyl increases from 0.6 at $300^{\circ} \mathrm{C}$ to approximately 7 at $675^{\circ} \mathrm{C}$, before declining to approximately 2 at $725^{\circ} \mathrm{C}$.

The calculated quantum yields clearly display a complex temperature dependence. We rationalize the relative insensitivity of monochlorobenzene by recognizing that carbon-chlorine bond rupture is exothermic from $S_{1}$. If the reaction occurs from $S_{1}$, then little or no temperature dependence is expected as only a small barrier to dissociation is expected from this state. This point is discussed in more detail in Section 7. 


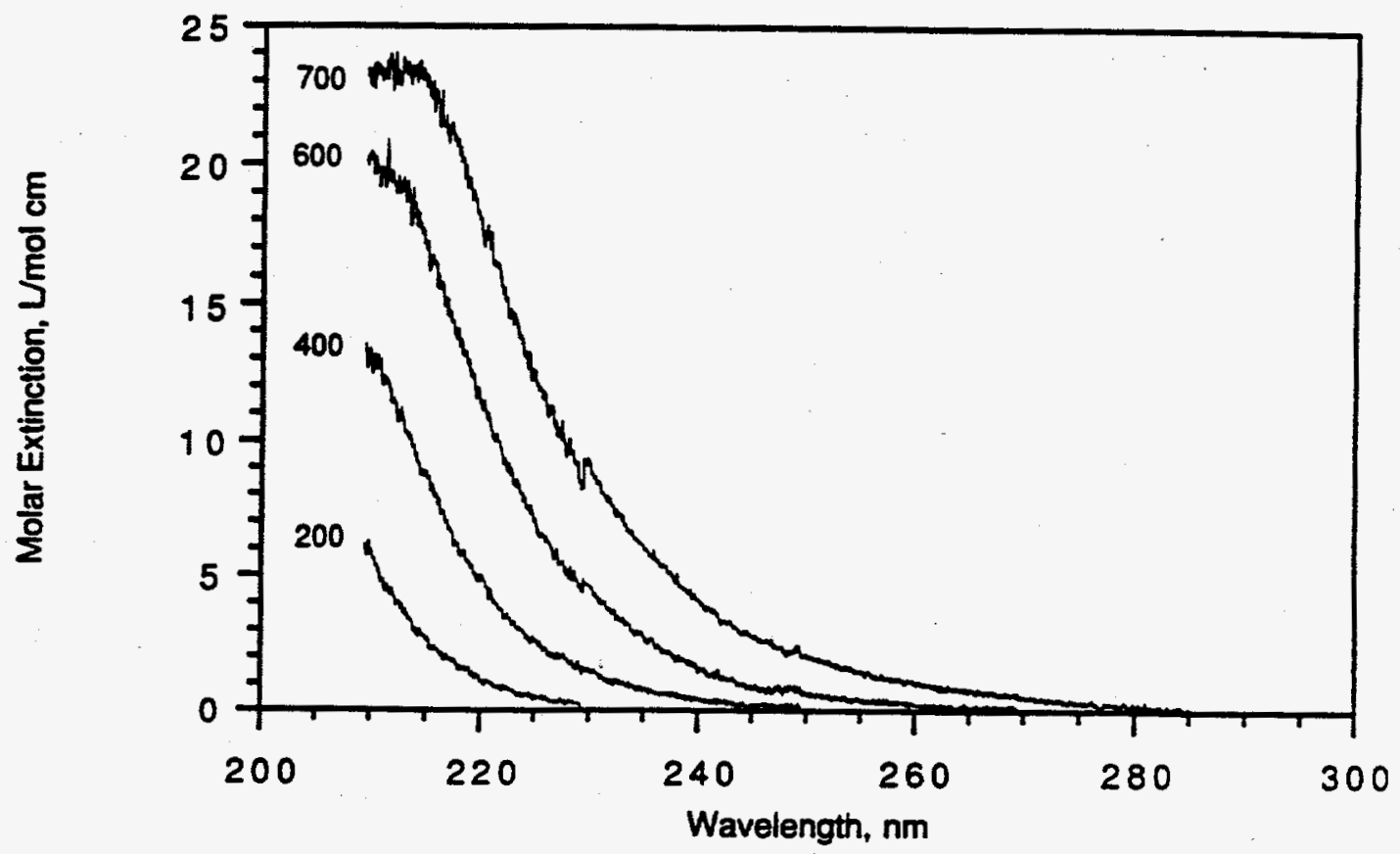

Figure 4.4. The molar extinction spectra for methylene chloride at $200,400,600$, and $700^{\circ} \mathrm{C}$.

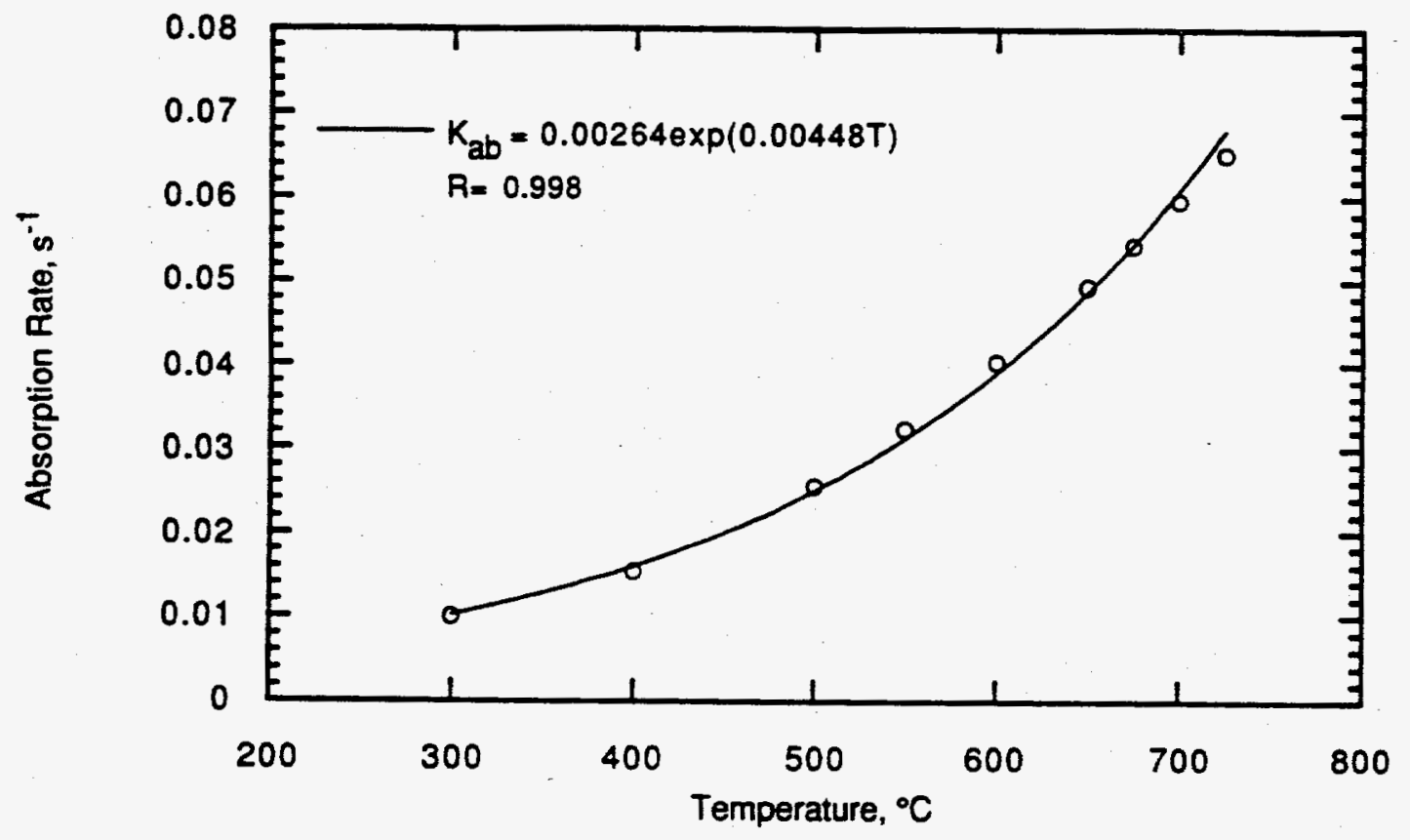

Figure 4.5. The rate of photon absorption for 3,3',4,4'-tetrachlorobiphenyl exposed to 273 AM 1.5 suns illustrating that $k_{a b}$ increases exponentially with temperature. 


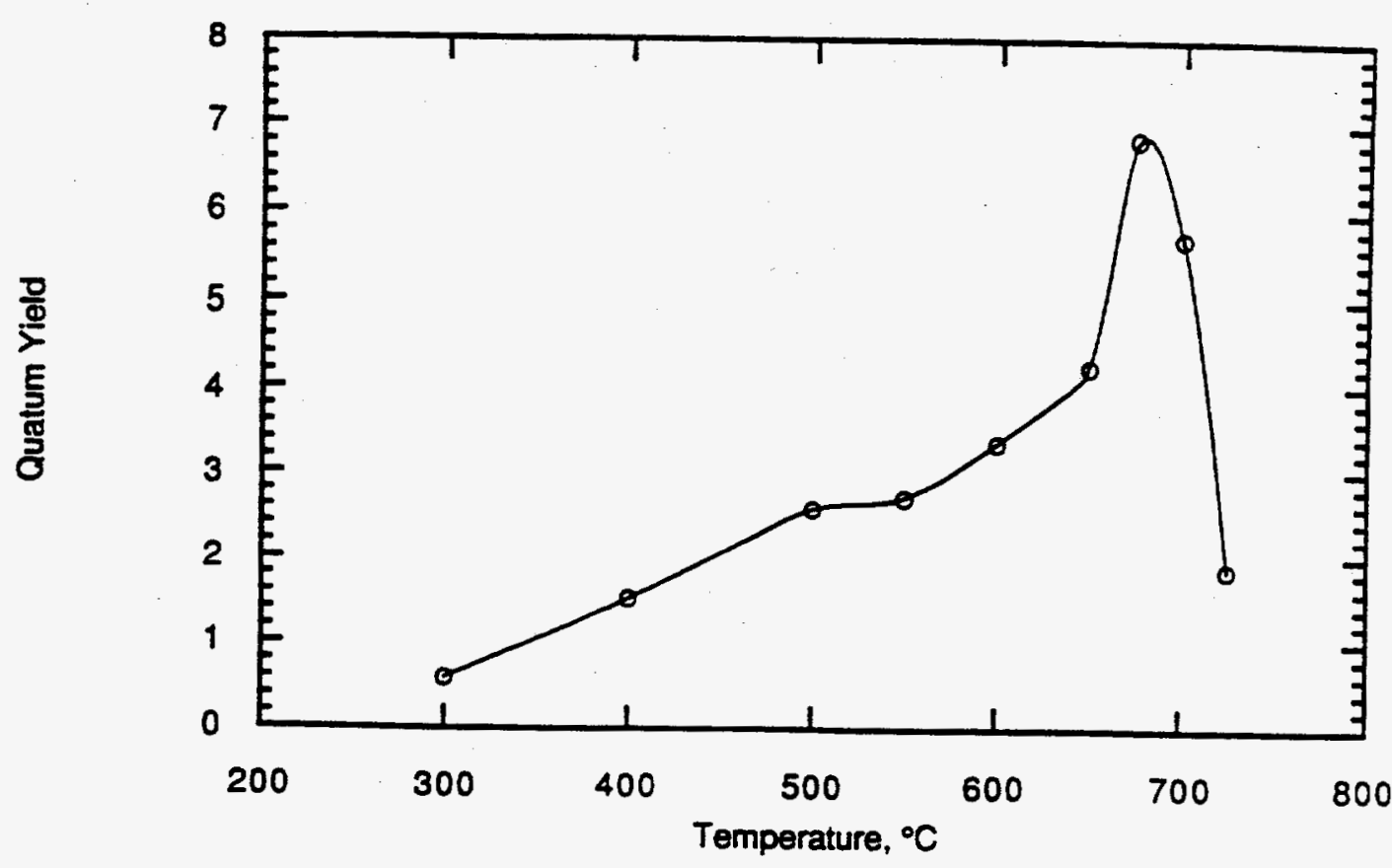

Figure 4.6. The photochemical quantum yield as a iunction of temperature for $3,3^{\prime}, 4,4^{\prime}$-tetrachiorobiphenyl $\left(3.78 \times 10^{-6} \mathrm{M}\right.$ ) exposed for 10 seconds in air to 0 and $273 \mathrm{AM} 1.5$ suns.

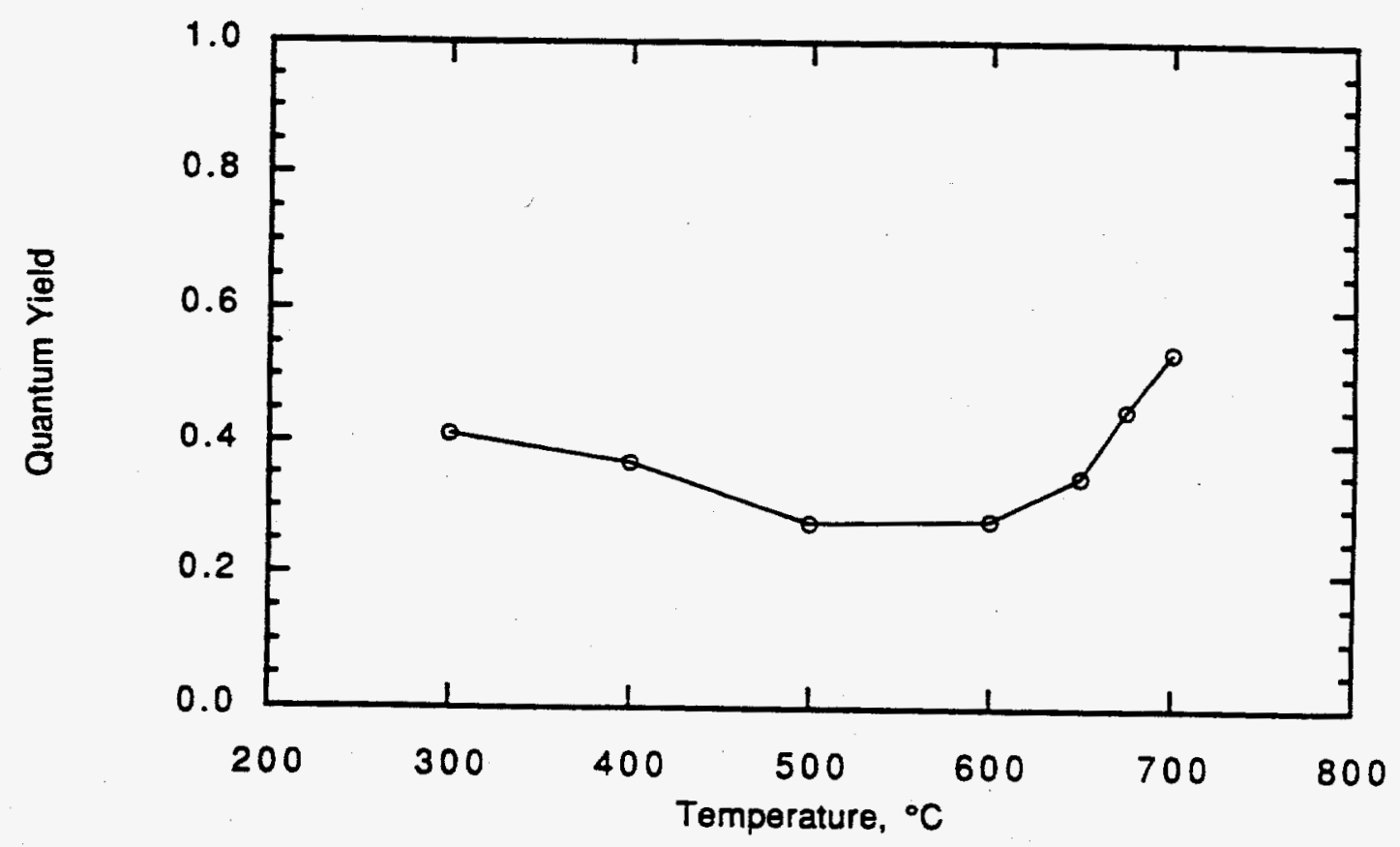

Figure 4.7. The photochemical quantum yield as a function of temperature for monochiorobenzene $\left(2.95 \times 10^{-5} \mathrm{M}\right)$ exposed for 10 seconds in air to pulsed laser illumination at $280 \mathrm{~nm}(862$ $\mathrm{mW} / \mathrm{cm}^{2}$ ). 
In contrast, the quantum yield of 7 for $3,3^{\prime}, 4,4^{\prime}$-tetrachlorobiphenyl requires that secondary chain reactions be occurring. These secondary reactions obscure any possible thermal enhancement effects on the primary photochemical event. Determination of the mechanism of this photo-initiated chain reaction has not yet been explained, but it is clearly of significant importance.

\subsection{EFFECT OF TIME}

The plug flow reactor performance model (Equation 2.7a) predicts that the thermal/photolytic enhancement will increase with exposure (mean residence) time. Although not immediately intuitive, it is simply a result of the overall thermal/photolytic rate coefficient being greater than the purely thermal rate coefficient.

As an example, consider the ratio of the fraction remaining following two thermal exposures (assuming first order processes);

$$
f_{r}(0) / f_{r}(0)=\exp \left(-k_{g n d}\left(t^{\prime}-t\right)\right)
$$

where $f_{r}(0)$ and $f_{r}^{\prime}(0)$ are the fractions remaining after exposures for times $t$ and $t^{\prime}$ respectively. For thermal/photolytic exposures to $n$ suns, Equation 4.1 becomes;

$$
f_{r}(n) / f_{r}^{\prime}(n)=\exp \left(-\left(k_{g n d}+k_{e x}\right)\left(t^{+}-t\right)\right)
$$

since clearly the sum of the ground and excited state rate terms ( $\mathrm{kgnd}_{\mathrm{gn}}$ and $\mathrm{k}_{\mathrm{ex}}$ ) is greater than the ground state term alone, it follows that;

$$
f_{r}(n) / f_{r}^{\prime}(n)>f_{r}(0) \mu_{r}^{\prime}(0)
$$

so the extent of thermal/photolytic conversion should increase more rapidly than purely thermal conversion, and the former is a more sensitive function of time.

The relative effect of time on the thermal/photolytic reactor performance is clearly shown in Figure 4.8 which summarize the degradation of $3,3^{\prime}, 4,4^{\prime}$-tetrachlorobiphenyl from simulated solar exposures to 5 and 10 seconds. Clearly, the exposure time had a significant impact on the simulated solar reactor performance. With a $5 \mathrm{~s}$ exposure only $6.80 \%$ of the tetrachlorobiphenyl was destroyed at $400^{\circ} \mathrm{C}$ as compared to $67.2 \%$ following a 10 s exposure. Similarly, while $97.62 \%$ of the tetrachlorobiphenyl was destroyed at $725^{\circ} \mathrm{C}$ following a $5 \mathrm{~s}$ exposure, $99.877 \%$ was destroyed following a $10 \mathrm{~s}$ exposure. 


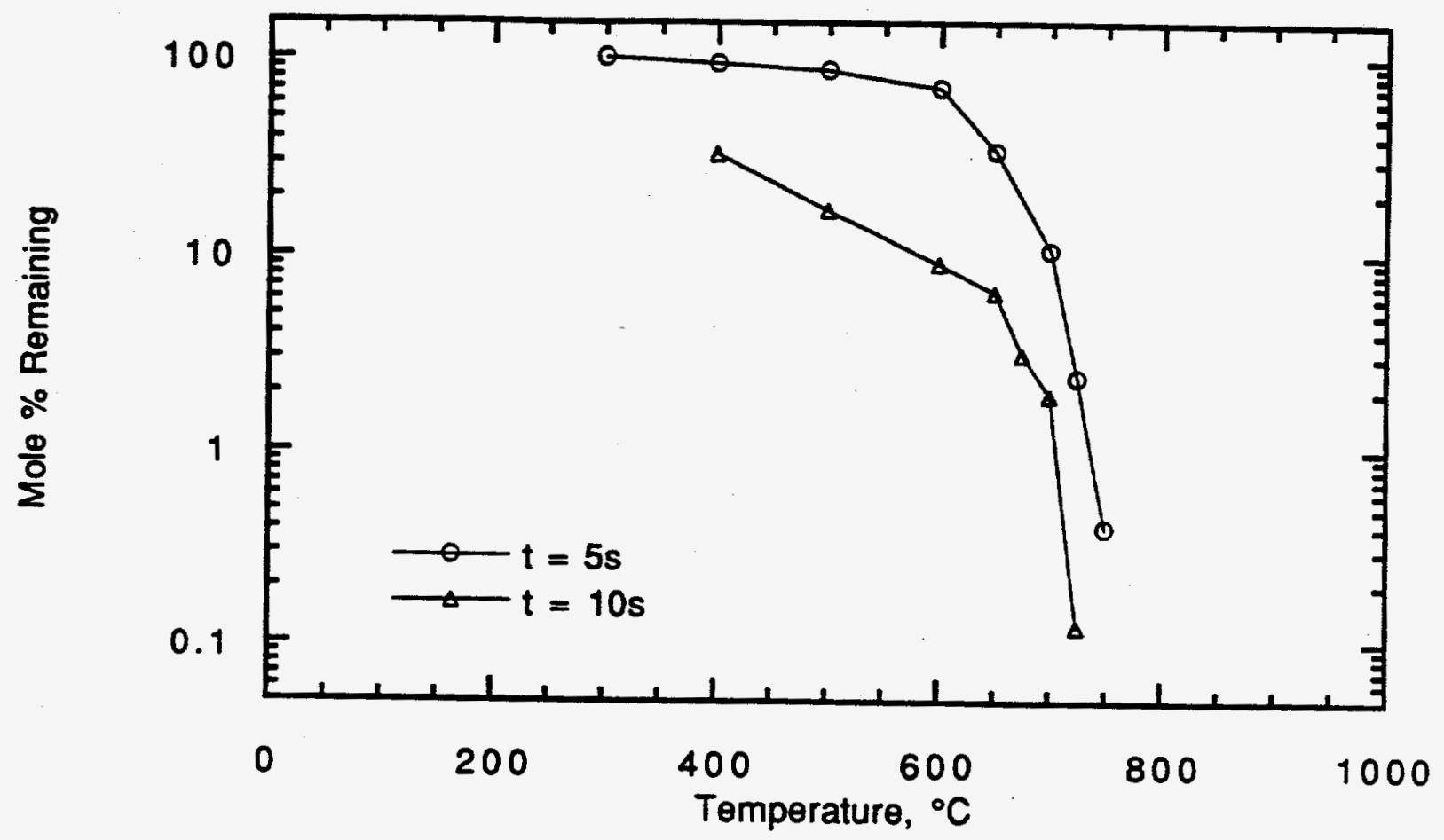

Figure 4.8. TPRS data for $3,3^{\prime}, 4,4^{\prime}$-tetrachlorobiphenyl $\left(-10^{-5} \mathrm{M}\right)$ exposed for 5 and 10 seconds in air to 307 AM 1.0 suns (simulated). 
The effect of exposure time also had a significant impact on the maximum observed enhancement ratios. These data for three sets of comparable data are summarized in Table 4.3. As these data illustrate that doubling the mean exposure time more than doubled the maximum enhancement ratio.

TABLE 4.3

\section{MAXIMUM OBSERVED ENHANCEMENT RATIOS FOR \\ 3,3',4,4'-TETRACHLOROBIPHENYL AND 1,2,3,4-TETRACHLORODIBENZODIOXIN EXPOSED TO 0 AND 307 AM 1.0 SUNS (SIMULATED) FOR 5 AND $10 \mathrm{~s}$}

\begin{tabular}{|c|c|c|c|c|c|}
\hline $\begin{array}{l}\frac{\text { Name }}{\text { Tetrachlorobiphenyl| }} \\
\text { Tetrachlorobiphenyl }\end{array}$ & $\begin{array}{l}\frac{\pi}{5} \\
10\end{array}$ & $\begin{array}{l}\frac{\text { atm }}{\text { air }} \\
\text { air }\end{array}$ & $\begin{array}{l}\frac{T(C)}{700} \\
600\end{array}$ & $\frac{B(307)}{2.68}$ & $\frac{B(10) / R(5)}{2.93}$ \\
\hline $\begin{array}{l}\text { Tetrachlorodibenzodioxin } \\
\text { Tetrachlorodibenzodioxin }\end{array}$ & $\begin{array}{c}5 \\
10\end{array}$ & $\begin{array}{l}\text { air } \\
\text { air }\end{array}$ & $\begin{array}{l}700 \\
500\end{array}$ & $\begin{array}{l}5.91 \\
13.5\end{array}$ & 2.28 \\
\hline $\begin{array}{l}\text { Tetrachlorodibenzodioxin } \\
\text { Tetrachlorodibenzodioxin }\end{array}$ & $\begin{array}{c}5 \\
10\end{array}$ & $\begin{array}{l}\text { Helium } \\
\text { Helium }\end{array}$ & $\begin{array}{l}700 \\
750\end{array}$ & $\begin{array}{l}10.2 \\
83.3\end{array}$ & 8.17 \\
\hline
\end{tabular}

'The enhancement ratio was still increasing at the highest temperature for which data are available.

\subsection{EFFECT OF LIGHT INTENSITY}

As shown by Equation 2.4, the rate of photon absorption is a linear function of the intensity of the radiant intensity (assuming the emission spectra of the source is not of a function of intensity). Therefore, as shown by Equation 2.5 , the reactor performance should increase exponentially with radiant flux. The best measure of the improvement in overall reactor performance is the enhancement ratio (cf. Equation 2.7a), and considering the theoretical functionality of the thermal/photolytic reactor performance, $R(n)$ should also increase exponentially with increasing radiant flux.

Two examples of the radiant intensity dependence of the thermal/photolytic process are available from the data summarized in the Appendices. Specifically, data has been obtained for the oxidation of xanthone exposed at $650^{\circ} \mathrm{C}$ for 5 seconds in air with simulated solar radiation ranging from 0 to $307 \mathrm{AM} 1.0$ suns, and for the oxidation of monochlorobenzene exposed to $600^{\circ} \mathrm{C}$ for 10 seconds in air and with laser illumination at $280 \mathrm{~nm}$ from 0 to $862 \mathrm{~mW} / \mathrm{cm}^{2}$. The enhancement ratio for these two compounds are shown in Figures 4.9 and 4.10 , respectively, as the log of the enhancement versus the radiant intensity. As these Figures demonstrate, the enhancement ratio varies exponentially with increasing radiant flux, even 


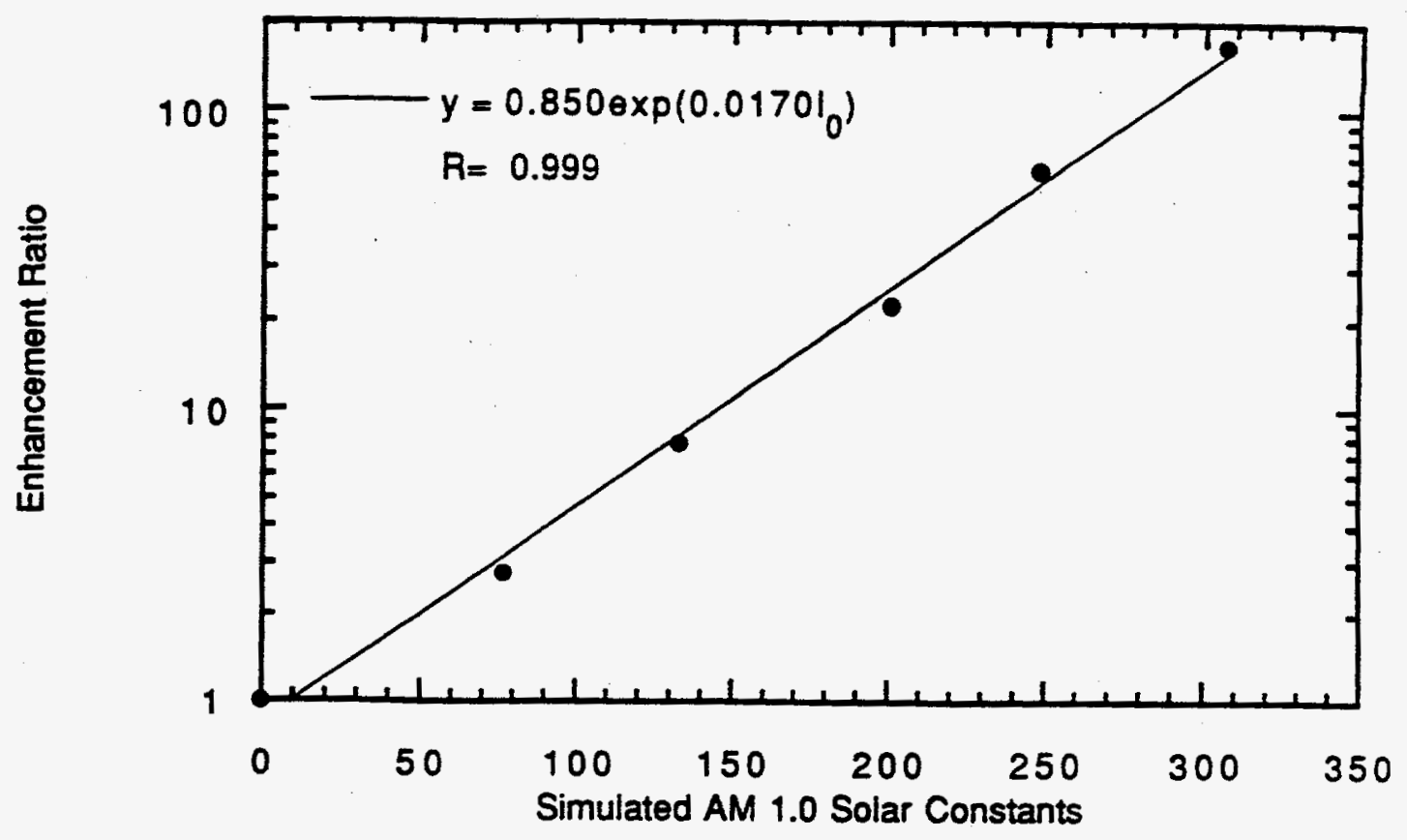

Figure 4.9. The enhancement ratio for xanthone $\left(-10^{-5} \mathrm{M}\right)$ exposed at $650^{\circ} \mathrm{C}$ for 5 seconds in air with simulated solar radiation ranging from $O$ to 307 AM 1.0 suns.

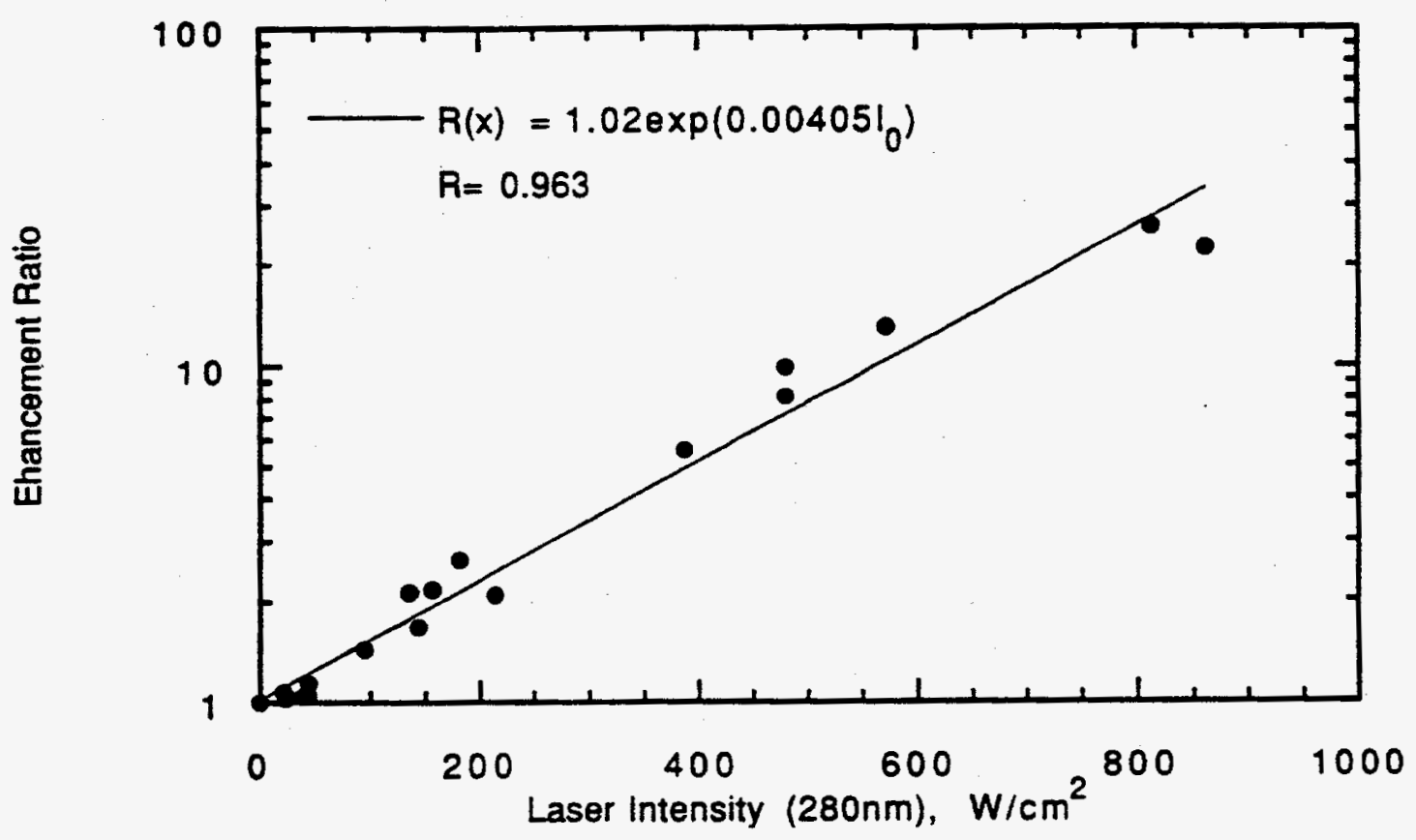

Figure 4.10. The enhancement ratio for monochiorobenzene $\left(2.95 \times 10^{-5} \mathrm{M}\right)$ exposed to $600^{\circ} \mathrm{C}$ for 10 seconds in air and with laser illumination at $280 \mathrm{~nm}$ from 0 to $862 \mathrm{~mW} / \mathrm{cm}^{2}$. 


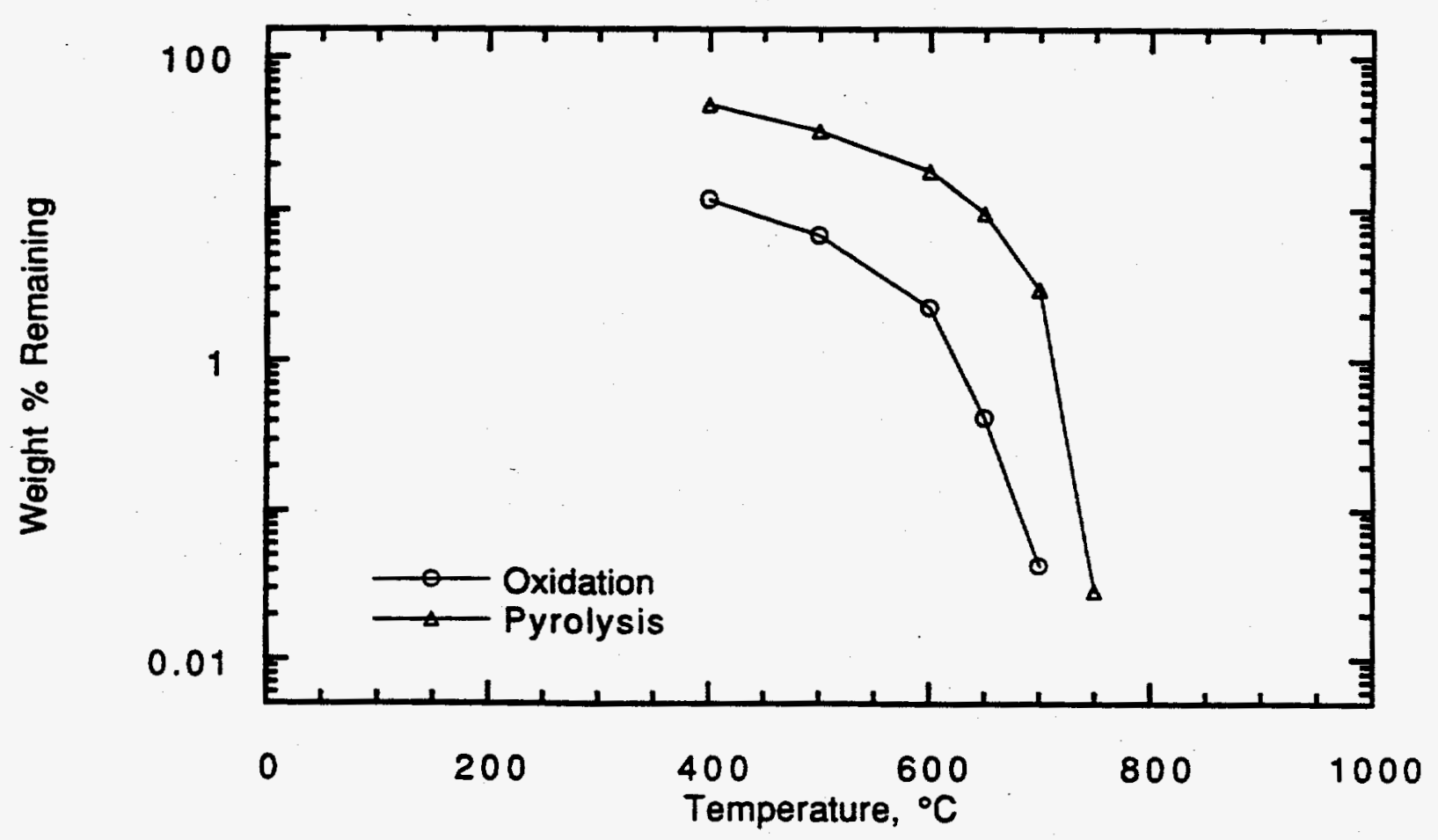

Figure 4.11. TPRS data for 1,2,3,4-tetrachlorodibenzo-p-dioxin $\left(\sim 10^{-5} \mathrm{M}\right)$ exposed in air (oxidation) and helium (pyrolysis) for 10 seconds to 307 AM 1.0 suns simulated. 
over a considerable range of radiant intensities. Therefore, the thermal/photolytic process benefits greatly from increasing UV solar flux.

One benefit of knowing how the efficiency of solar detoxification process varies with the intensity of radiant flux is that the laboratory data which has been taken under relatively mild solar concentrations as compared to what is achievable in large solar concentrators may be extrapolated to much higher flux levels. This also offers the opportunity to summarize the laboratory data on a common radiant intensity scale. A summary of this sort is presented in Table 4.2 which lists the maximum observed enhancement ratios for all of the compounds involved in this study extrapolated to 1,000 suns. As this Table illustrates, at radiant intensities easily achievable with existing concentrator technology, very high levels of solar enhancement may be expected.

TABLE 4.2

Summary of Maximum Observed Photoenhancements and Values Corrected to 1,000 Suns

\begin{tabular}{|c|c|c|c|c|c|c|}
\hline Name & $I_{n}$ & $t_{p}$ & Atm. & $T\left({ }^{\circ} \mathrm{C}\right)$ & $R(1)$ & $R(1000)$ \\
\hline Azulene ${ }^{*}$ & 307 AM 1.0 & 5 & air & 650 & 3.31 & 49.3 \\
\hline Benzene* & 307 AM 1.0 & 5 & air & 725 & 1.31 & 2.41 \\
\hline Bromonaphthalene & 307 AM 1.0 & 5 & air & 700 & 3.67 & 69.1 \\
\hline Benzolelpyrene & 307 AM 1.0 & 5 & air & 650 & 2.09 & 11.0 \\
\hline Chloronaphthaiene & 307 AM 1.0 & 5 & air & 650 & 2.28 & 14.7 \\
\hline 2,6-Dinitrotoluene & 180 AM 1.5 & 10 & air & 450 & 18.0 & $9,410,000$ \\
\hline 2,6-Dinitrotoluene & 356 AM 1.5 & 10 . & air & 500 & 17.8 & 3,250 \\
\hline 2,6-Dinitrotoluene & 356 AM 1.5 & 10 & helium & 450 & 7.34 & 270 \\
\hline Hexachlorobenzene & 307 AM 1.0 & 5 & air & 750 & 2.66 & 24.2 \\
\hline Iodonaphthalene & 307 AM 1.0 & 5 & air & 550 & 3.28 & 47.9 \\
\hline Naphthalene & 307 AM 1.0 & 5 & air & 675 & 1.72 & 5.85 \\
\hline Nitronaphthalene* & 307 AM 1.0 & 5 & air & 625 & 58.4 & 567,000 \\
\hline Nitronaphthalene" & 307 AM 1.0 & 5 & helium & 625 & 4.60 & 144 \\
\hline Tetrachlorobiphenyl" & 307 AM 1.0 & 5 & air & 700 & 2.68 & 24.8 \\
\hline Tetrachlorobiphenyl & 273 AM 1.5 & 10 & air & 675 & 40.9 & 801,000 \\
\hline Tetrachiorobiphenyl" & 307 AM 1.0 & 10 & helium & 825 & 35.5 & 112,000 \\
\hline Tetrachlorodibenzodioxin & 307 AM 1.0 & 5 & air & 700 & 5.91 & 326 \\
\hline Tetrachlorodibenzodioxin & 307 AM 1.0 & 5 & helium & 700 & 10.2 & 1,930 \\
\hline Tetrachlorodibenzodioxin & 307 AM 1.0 & 10 & air & 500 & 13.5 & 4,810 \\
\hline Tetrachlorodibenzodioxin" & 307 AM 1.0 & 10 & helium & 750 & 83.3 & $1,800,000$ \\
\hline Xanthone* & 307 AM 1.0 & 5 & air & 650 & 167.9 & $17,700,000$ \\
\hline Xanthone* & 307 AM 1.0 & 5 & helium & 850 & 65.8 & 837,000 \\
\hline
\end{tabular}

"The value of $R$ was still increasing at the last temperature studied. 


\subsection{EFFECT OF FUEL/AIR STOICHIOMETRY}

Although the global kinetic models constructed to describe the thermal and thermal/photolytic detoxification processes do not explicitly include a description of the oxygen dependence of the respective decomposition processes, it is important to explore how the reactor performance is effected by varying levels of excess air. Specifically, in a full scaie detoxification system, the level of theoretical air will fluctuate as feed rate and the uniformity of the feed vary with time. It is also important to understand how a reactor will perform under extreme "failure-mode" conditions (e.g., unintentional overloading of a reactor, poor mixing of waste and oxidizer, atomizer failure, etc.) which could result in the partial destruction of feed through pyrolytic processes.

As an example of a compound for which comparable oxidation and pyrolysis data are available, consider the data shown in Figure 4.11 for 1,2,3,4-tetrachlorodibenzo-p-dioxin exposed to 307 AM 1.0 suns for 10 seconds in air and helium. It is clear that the absence of oxygen has a significant impact on reactor performance. This is illustrated in Figure 4.12 which shows that the ratio of fraction remaining from pyrolysis versus oxidation steadily increases with temperature. This difference in the rate of change in the reactor performance with oxygen concentration is also reflected in the enhancement ratio. Specifically, as Figure 4.13 demonstrates, under oxidative conditions, the enhancement ratio passes through a maximum, while the ratio steadily increases with temperature under pyrolysis.

The reason (or reasons) for this varied behavior have not been experimentally explored. However, detailed, elementary reactor kinetic modeling on monochlorobenzene thermal/photolytic oxidation has suggested an interesting possible explanation. Specifically, photo-dissociation of monochlorobenzene into phenyl radicals and chlorine atoms seems to result in inhibition of oxidation by chlorine catalyzed scavenging of hydrogen atoms, thus preventing the chain branching reaction; $\bullet \mathrm{H}+\mathrm{O}_{2} \rightarrow \bullet \mathrm{OH}+\bullet \mathrm{O}$. The model suggests this inhibition increases with temperature. Thus, it may be possible that the initial acceleration of decomposition by photo-induced $\phi-\mathrm{Cl}$ bond rupture may be overcome by $\cdot \mathrm{Cl}$ induced inhibition of oxidation at higher temperatures. This would result in an optimum temperature for oxidation. However, this inhibition would not occur under pyrolysis, and $R$ would continue to increase with temperature.

Although the explanation for the observed behavior may be theoretically sound, it is not the only possibility, and other theories, such as oxygen quenching of the excited triplet state, may serve as well. Therefore, a carefully planned and executed series of tests designed specifically to address this issue is necessary to thoroughly explain the global effects of oxygen, i.e., excess air, on the thermal/photolytic reactor performance. 


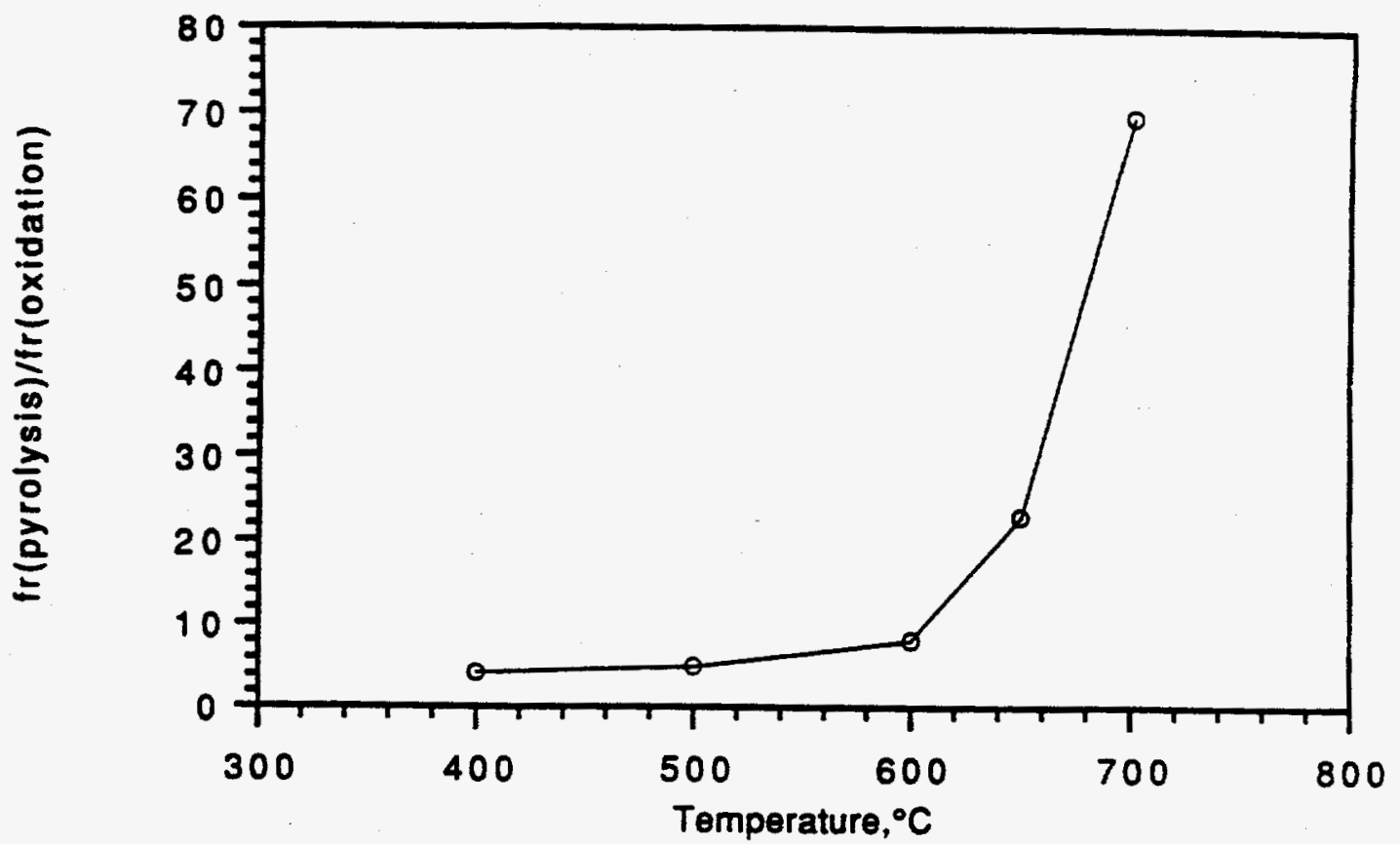

Figure 4.12. The ratio of fraction remaining from pyrolysis versus oxidation for 1,2,3,4-tetrachiorodibenzo-p-dioxin $\left(-10^{-5} \mathrm{M}\right)$ exposed to $307 \mathrm{AM} 1.0$ suns simulated for 10 seconds in air (oxidation) and helium (pyrolysis).

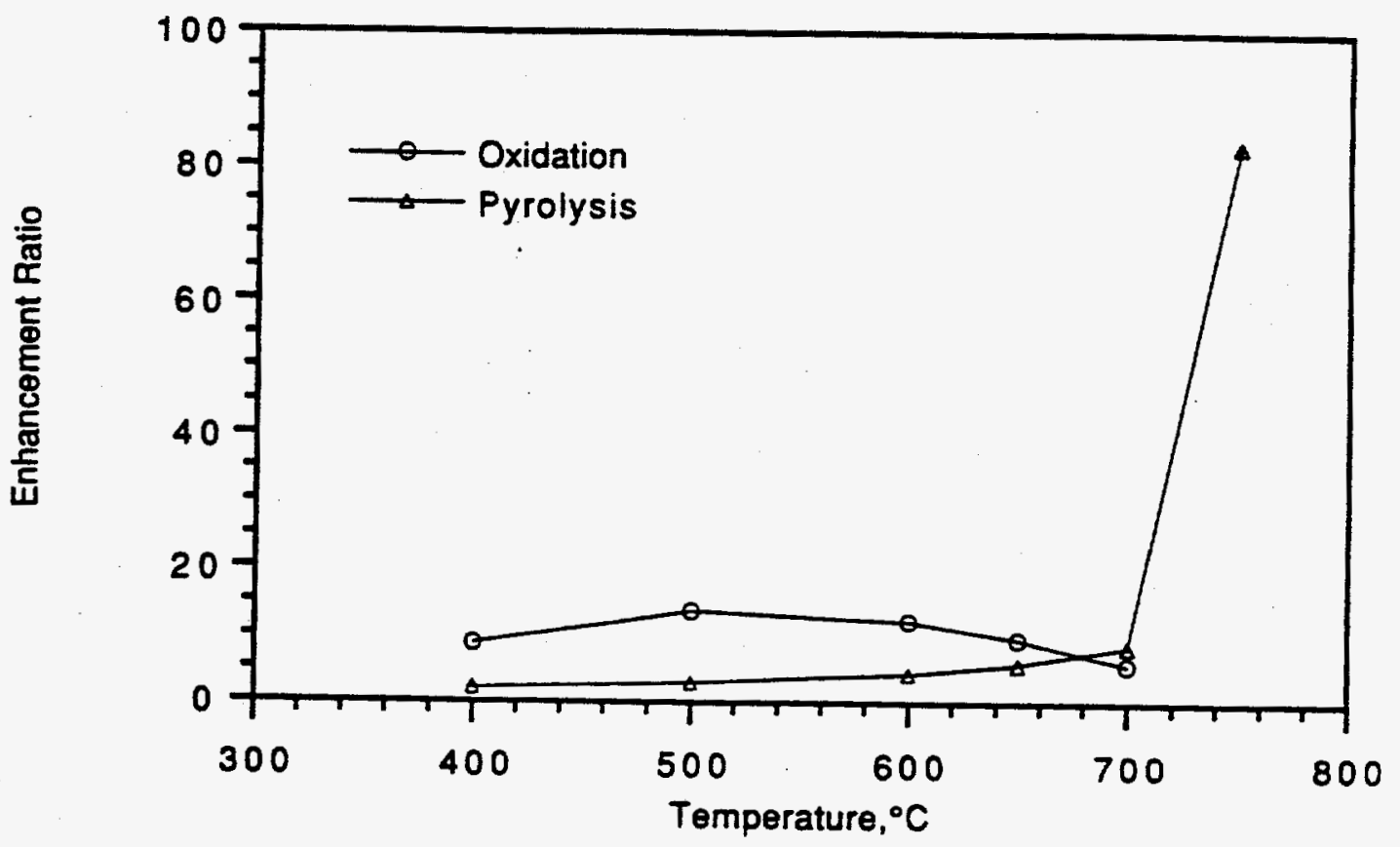

Figure 4.13. The enhancement ratio as a function of temperature for 1,2,3,4-tetrachiorodibenzo-pdioxin $\left(-10^{-5} \mathrm{M}\right)$ exposed to 0 and 307 AM 1.0 suns simulated for 10 seconds in air (oxidation) and helium (pyrolysis). 


\subsection{DESTRUCTION OF 2,6-DINITROTOLUENE}

Environmentally safe disposal of acutely toxic chemical armaments and energetic materials has become and area of increasing concern. Many of these materials are not amenable to conventional incineration, while public opposition hinders the thermal destruction of those that are. Thus there has been considerable interest in developing new technologies which are capable of efficiently destroying these types of wastes.

Our research on the use of concentrated solar energy to destroy hazardous organic wastes has shown the process to be particularly effective on substituted aromatic compounds. A review of the literature indicates that by-products from the manufacture of 2,4,6-trinitrotoluene (i.e., TNT) contain large quantities of materials that may be susceptible to disposal by this new technology.

During the manufacturing process, unsymmetrical trinitrotoluene isomers and various oxidation products are removed from the process stream and made water soluble. These impurities, considered to be the most refractory components in the waste stream, absorb in the blue end of the visible spectrum which give the wastewater a characteristic red color, and hence it is often referred to as "pink water" or "red water." In addition, high pH and/or exposure to sunlight produce extensive trinitrotoluene decomposition products. The recent construction and operation of a number of trinitrotoluene manufacturing plants has introduced the problem of disposal of red water. Moreover, various sites near army ammunition plants are contaminated where these wastes have been dumped for approximately thirty years following World War II[1]. Much interest has been expressed in finding the most cost-effective and technologically-feasible permanent disposal method for this type of waste.

We have examined the gas-phase, thermal/photolytic decomposition of 2,6-dinitrotoluene (this isomer was selected as a model compound for red water) using simulated solar energy. This compound was subjected to a simultaneous exposure of high temperature and concentrated solar radiation (simulated) using the TPRS. These data were then compared to purely thermal data obtained under identical conditions of exposure time, temperature, and atmosphere to determine the extent of the potential solar benefit.

No studies were found in the literature which investigated the thermal or photolytic decomposition of 2,6-dinitrotoluene. Much of the available literature in this area pertains to the decomposition of molten or solution phase 2,4,6-trinitrotoluene[2-18], Although studied for over 60 years, the thermochemical decomposition mechanisms of trinitrotoluene and other nitroaromatic compounds are not well understood. 
Two studies were found in the literature which dealt with the thermal decomposition of gas-phase 2,4,6-trinitrotoluene. In 1956, Cook and Abegg [19] followed the trinitrotoluene decomposition reaction in both the vapor and liquid phases and found no measurable decomposition of trinitrotoluene in the vapor phase over the temperature range $250-310^{\circ} \mathrm{C}$. Specifically, the concentration of trinitrotoluene ranged from $3.37 \times 10^{-4}$ to $6.61 \times 10^{-4}$ mol/ in air, and the total pressure varied from 35 to $540 \mathrm{~mm} \mathrm{Hg}$. Rauch et al.[20] concluded in 1969 that, whereas decomposition is negligible for trinitrotoluene present only in the gas phase at $265^{\circ} \mathrm{C}$, further decomposition of the trinitrotoluene vapor occurred at this temperature if liquid trinitrotoluene was present.

No studies were found which investigated the photolytic decomposition of gas-phase dinitrotoluene or trinitrotoluene. However, some researchers have dealt with the photolytic decomposition of trinitrotoluene in solution[21-28].

\subsubsection{EXPERIMENTAL}

The experiments performed for this study were conducted with the TPRS. The sample used in this study (2,6-dinitrotoluene, 98\%) was obtained from the Aldrich Chemical Company, Inc. (\#D20,060-3). A stock solution of with a concentration $0.18 \mathrm{mg} / \mathrm{L}$ was prepared.

For this set of experiments, the reactor temperature was varied from 175 to $575^{\circ} \mathrm{C}$. The total carrier gas flow was set so that the sample experienced a mean residence time in the reactor of 10 seconds at each temperature. For each analysis the sample was prepared by depositing $3.2 \mathrm{~mL}$ of the stock solution ( $0.52 \mathrm{mg}$ of dinitrotoluene) onto a special probe insert. After the solvent evaporated, leaving a thin deposit of sample on the probe, it was sealed within the TPRS's inlet chamber. The reactor concentration was approximately $5 \times 10^{-8} \mathrm{~mol} / \mathrm{L}$. Data was obtained in pairs at various temperatures, first with the lamp off giving a purely thermal exposure, then with the lamp on, giving a simulated solar exposure (thermal/photolytic) equivalent to 350 AM 1.5 suns.

\subsubsection{RESULTS AND DISCUSSION}

The actual chromatographic data from the thermal and thermal/photolytic decomposition of dinitrotoluene under pyrolytic conditions is shown in Figure 4.14, while similar data for oxidative conditions are shown in Figure 4.15. In these Figures, the range of scale of the vertical axis is indicated in the upper right corner of each chromatogram along with the exposure temperature. The peak representing the parent 
compound is indicated by an arrow on each chromatogram. All other peaks correspond to either organic reaction products or impurities.

Under pyrolytic conditions, thermal decomposition was not observed at temperatures below $450^{\circ} \mathrm{C}$. Similarly, for oxidative conditions, thermal decomposition was not observed under any tests with exposure temperatures of less than $400^{\circ} \mathrm{C}$. Note that at for all conditions shown the conversion of dinitrotoluene is significantly higher for the simulated solar exposures than the similar thermal tests. Given that a complex mixture of products often accompanies the destruction of aromatic compounds, particularly under pyrolysis, the relative absence of products from the destruction of dinitrotoluene is an interesting result. Specifically, the sum of all the product yields (all product HFID response factors were taken as dinitrotoluene) never exceed $5 \%$ of the original amount of dinitrotoluene. Similarly, the lack of relatively heavy products (i.e., product higher in molecular weight that the parent dinitrotoluene as would be indicated by a longer chromatographic retention time) is also an unexpected result. These two observations illustrate that dinitrotoluene decomposes relatively efficiently to products of complete conversion.

The fraction of dinitrotoluene surviving each exposure as a function of temperature is summarized in Figures 4.16 and 4.17 for pyrolysis and oxidation, respectively. Also shown on these figure is the relative amount of all products formed (i.e., the sum of all peaks other than dinitrotoluene with their chromatographic response calibrated as dinitrotoluene. The detection limit shown in these figures was taken to be the point where the signal-to-noise ratio is equal to 2 . Solar enhancement ratios have been calculated for both the parent dinitrotoluene and the total product yields. These results are summarized in Table 4.3 for pyrolysis and oxidation, respectively.

TABLE 4.3

Enhancement Ratios $(R=(350))$ for the Thermal/Photolytic Decomposition of 2,6-DNT for 57 AM 1.5 Suns and a Residence Time of $10.0 \mathrm{~s}$

Parent (2,6-DNT) Total Products

\begin{tabular}{ccccc} 
Temp. $\left({ }^{\circ} \mathrm{C}\right)$ & Pyrolysis & Oxidation & Pyrolysis & Oxidation \\
\hline 175 & 3.2 & 2.6 & - & - \\
250 & 3.8 & 3.7 & - & - \\
325 & 4.5 & 5.9 & - & - \\
400 & 6.6 & 9.1 & - & 0.836 \\
450 & 7.1 & 12.5 & 0.142 & 1.38 \\
500 & 5.3 & 17.8 & 0.518 & 1.28 \\
525 & 6.1 & 15.0 & 0.957 & 1.79 \\
550 & 3.9 & - & 1.06 & - \\
575 & - & - & 1.90 & - \\
\hline
\end{tabular}


A significant solar enhancement in the destruction of dinitrotoluene was observed under our experimental conditions. For the pyrolytic exposures, the simulated solar process was 3 to 7 times more efficient than the purely thermal process. Under oxidative conditions, the enhancement reached values as high as 18. However, dinitrotoluene proved to be very thermally fragile. Thus, equivalent levels of thermal conversion were achieved at only slightly higher temperatures than for the simulated solar process. Although this rapid increase in the purely thermal destruction rate resulted in high overall destruction efficiencies, it made the calculation of the thermal/photolytic enhancement ratio very inprecise above 450 to $500^{\circ} \mathrm{C}$ as there were rather large differences in very small values of $f_{f}$. This lack of precision may be responsible for the exponential dependence of $R$ versus $T$ only being observable up to $400^{\circ} \mathrm{C}$ and $500^{\circ} \mathrm{C}$ under oxidative and pyrolytic conditions, respectively. Assuming that the extent of enhancement does indeed increase exponentially with the intensity of the radiant exposure, a solar reactor operating at 1,000 suns, as compared to the 350 suns used here, can expect enhancement ratios equivalent to the values reported for the TPRS exposures raised to the power of 2.9. Specifically, at 1,000 suns, enhancement ratios as high as 280 can be expected under pyrolytic conditions, and as high as 4,400 under oxidation.

Studying the decomposition of organic compounds under pyrolytic conditions is important for two reasons. First, pyrolysis is a simpler system to study compared to oxidation as the number of possible initiation reactions is limited. Second, it is thought that uncontrollable oxygen deficient regions within a conventional incinerator play an important role in limiting the performance of these units. The pyrolysis data for dinitrotoluene shows that even if these oxygen starved regions would occur in a solar incinerator, the solar process should remain efficient, even under this difficult operating condition.

For the few products detected from the decomposition of dinitrotoluene, the enhancement ratio increased with temperature (cf. Table 4.3 and Figures 4.17 and 4.18). At low temperatures the solar process appears to create products at a higher yield than the purely thermal process. However, this is largely an experimental artifact in that at low temperatures the thermal process simply hasn't begun to decompose dinitrotoluene. For example, note that under pyrolytic conditions there were comparable levels of conversion at $525^{\circ} \mathrm{C}$, thermal $\left(12.7 \%\right.$ remaining), and at $450^{\circ} \mathrm{C}$, solar $(12.2 \%$ remaining). In comparison, the products produced from the solar exposure $(2.23 \%)$ were significantly less than the thermal (3.49\%). This suggests that at equivalent levels of reactor performance with respect to the destruction of the waste feed, the solar reactor should be operating more cleanly (lower product yields) than a thermal reactor. This does not seem to hold for the case of oxidation where the levels of product formation are approximately equivalent. 

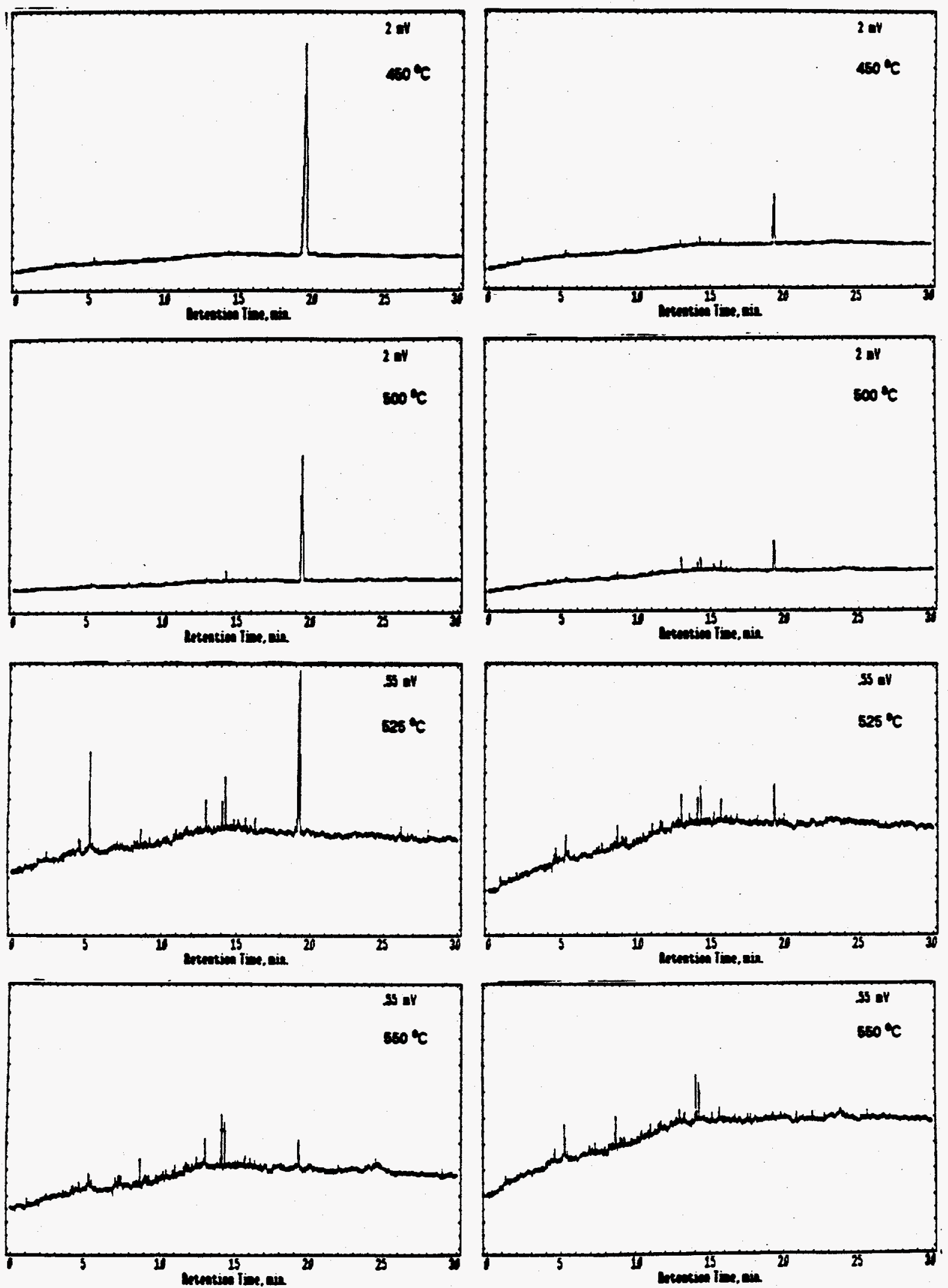

Figure 4.14. TPRS chromatograms from the thermal and thermal/photolytic decomposition of 2,6dinitrotoluene $\left(5 \times 10^{-8} \mathrm{M}\right)$ exposed to $350 \mathrm{AM} 1.5$ suns (simulated) for 10 seconds in helium. 

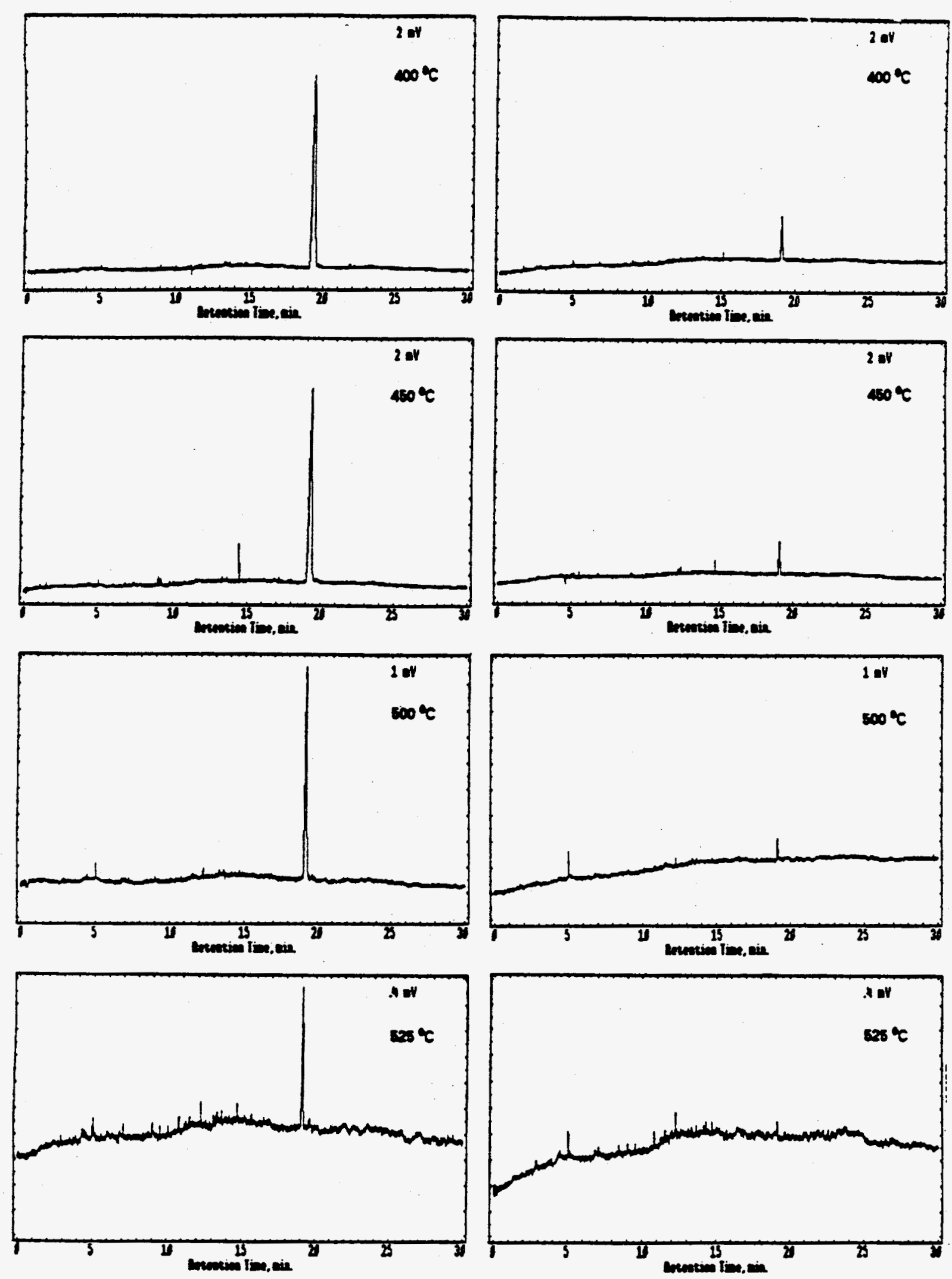

Figure 4.15. TPRS chromatograms from the thermal and thermalphotolytic decomposition of 2,6dinitrotoluene $\left(5 \times 10^{-8} \mathrm{M}\right.$ ) exposed to 350 AM 1.5 suns (simulated) for 10 seconds in air. 


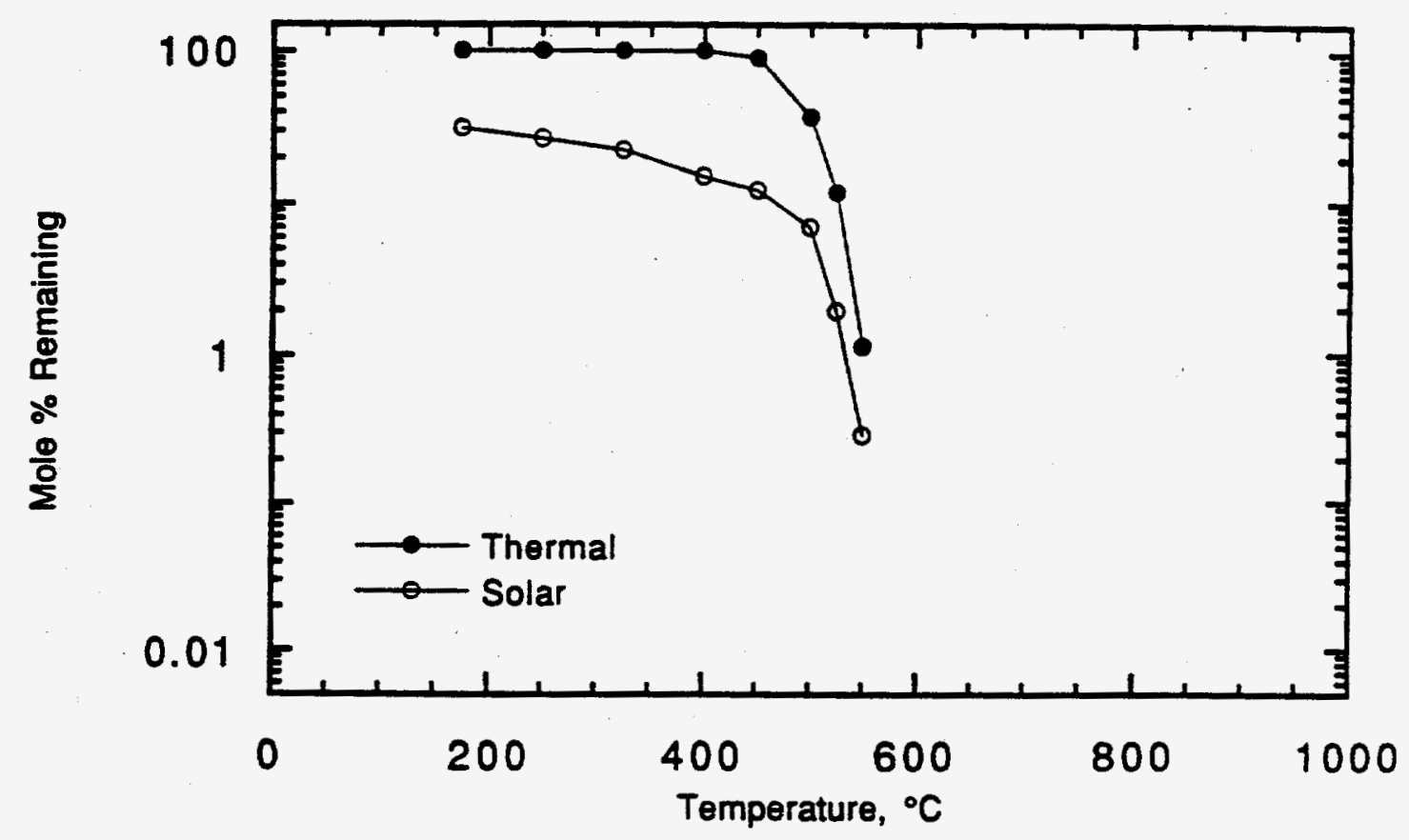

Figure 4.16. TPRS data for 2,6-dinitotoluene $\left(5 \times 10^{-8} \mathrm{M}\right)$ exposed to $350 \mathrm{AM} 1.5$ suns (simulated) for 10 seconds in helium.

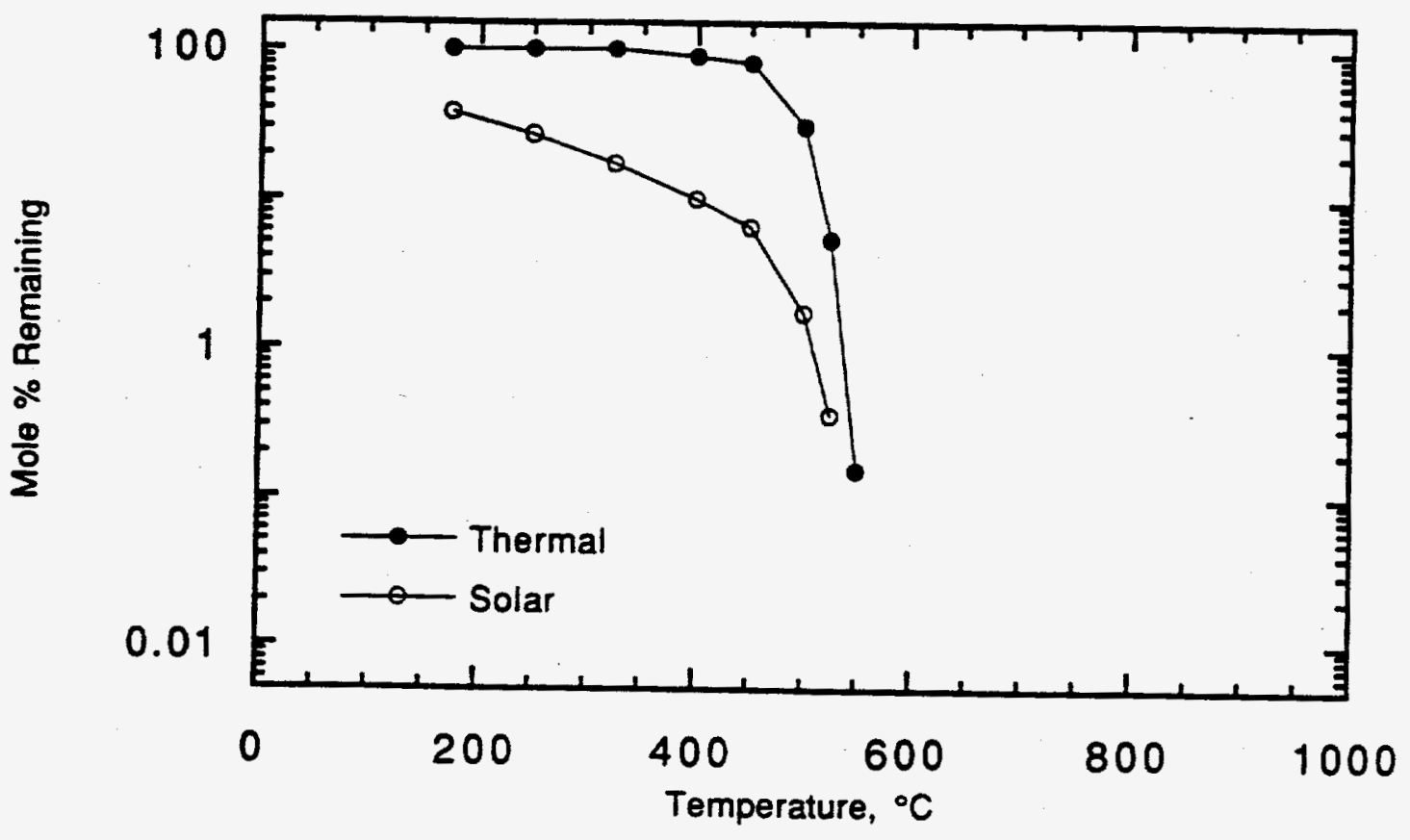

Figure 4.17. TPRS data for 2,6 -dinitrotoluene $\left(5 \times 10^{-8} \mathrm{M}\right)$ exposed to $350 \mathrm{AM} 1.5$ suns (simulated) for
10 seconds in air. 


\subsection{REFERENCES}

1. Nay, M. W., et al., "Biological Treatability of Trinitrotoluene Manufacturing Wastewater," Journal WPCE, Vol. 48, No. 3, March 1974, p. 485.

2. Guidry, R. M. and Davis L. P., Thermochemical Decomposition of Explosives. I. TNT Kinetic Parameters Determined from ESR Investigations," Thermochim. Acta, 32(1-2), 1979, pp. 1-18 and references therein.

3. Robertson, R., J. Chem. Soc., 119, 1921, p. 1.

4. Robertson, A. J. B., Irans, Faraday Soc., 44, 1948, p. 977.

5. Roginsky, S., Phys. Z. Soviet Union, 1, 1932, p. 640.

6. Urbanski, T. and Rychter, S., Acad.Sci., 208, 1939, p. 900.

7. Maksimov, Y. Y. and Pavlik, L. T., Russ. J.Phys. Chem., 49, 1975, p. 360.

8. Beckman, J. W., et al., "2,4,6-Trinitrotoluene Thermal Decomposition: Kinetic Parameters Determined by the Isothermal Differential Scanning Calorimetry Technique," Thermochim. Acta, 19, 1977, pp. 111. 118.

9. Adams, G. K., et al. Ministry of Supply Report A, C, 3982, Great Britain, 1943.

10. Rogers, R. N., Anal. Chem, 39, 1967, p. 730.

11. Dacons, J. C., et al., "Some Novel Observations Concerning the Thermal Decomposition of 2,4,6Trinitrotoluene," J.Phys. Chem., Vol. 74, No. 16, 1970.

12. Colman, W. P. and Rauch, F. C., Picatinny Arsenal Report A.D. No. 881190.

13. Janzen, E. G., J.Am. Chem. Soc., 87, 1965, p. 3531.

14. Turner, A. G. and Davis, L. P., "Thermal Decomposition of TNT: Use of 1-Nitropropene to Model the Initial Stages of Decomposition," \ـ Am. Chem. Soc., 106, 1984, pp. 5447-5451.

15. McKinney, T. M. et al., "EPR Observation of Nitroxide Free Radicals During Thermal Decomposition of Some Nitro Aromatic Compounds," J. Phys. Chem., 90, 1986, pp. 5968-5973 and references therein.

16. Tsang, W., et al., "Single-Pulse Shock-Tube Studies on C-No2 Bond Cleavage During the Decomposition of Some Nitro Aromatic Compounds," J. Phys. Chem., 90, 1986, pp. 5968-5973 and references therein.

17. Fields, E. K. and Meyerson, S., Adv. Free Radical Chem., 1965, 5, p. 101. 
18. Pasman, H. J., et al., "Thermal Decomposition of TNT," Explosivstoffe, 17(7), 1969, pp. 151-6.

19. Cook, M. A. and Abegg, M. T., ind. Eng Chem., 48, 1956, p. 1090.

20. Rauch, F. C. and Colman, W. P., Picatinny Arsenal Report A.D. No. 869226, 1970.

21. Kaplin, L. A. et al., "Photochemistry of TNT: Investigation of the 'Pink Water' Problem, Part II," NSWC Technical Report 75-152, Nov. 1975.

22. Suryanarayanan, K. and Capellos, C., Int. J. of Chem. Kinetics, 6, 1974, p. 89.

23. Sachs, F. and Hilpert, S., Chem. Ber., 37, 1904, p. 3425.

24. Capellos, Ch. and Iver S., "Energetic Nitroaromatic Transients and Their Role in Modifying Initiation Thresholds," U. S. Army Armanent Res. Dev. Command, Dover, N. J., 1979, p. 628.

25. Craig, B. B. and Atherton, S. J., "Laser Photolysis of Nitroaromatics," Proc. SPIE-Int. Soc. Opt. Eng., 482(Appl. Laser Chem. Diagn.), 1984, pp. 96-103.

26. Chattopadhyay, S. K. and Craig, B. B., "Picosecond Transient Absorption Studies of Dinitrotoluene in Solution," J. Phys. Chem., 1987, 91, pp. 323-326.

27. Mabey, W. R. et al., "Photolysis of Nitroaromatics in Aquatic Systems. 1. 2,4,6-Trinitrotoluene," Chemosphere, Vol. 12, No. 1, pp. 3-16, 1983.

28. Sandus, O. and Slagg, N., "Reactions of Aromatic Nitro Compounds I. Photochemistry," U.S. NTIS, AD-753923, p. 48, 1972. 


\section{SECTION 5.0 MINERALIZATION OF BY.PRODUCTS}

While the primary function of any detoxification process is the destruction of the waste feed, it is of little value if it produces an effluent which itself hazardous to the environment. Therefore, an essential secondary function of a detoxification process is the destruction, or mineralization (reduction to inorganic materials), of hazardous products. In the incineration industry, these materials are known as products of incomplete combustion (PICs). Although the solar detoxification process does not explicitly include combustion, the essence of the concern is the same. Therefore, to follow the naming convention established in the incineration literature, these products will also be referred to as PICs, whereas in this case the term stands for products of incomplete conversion.

In the course of the solar detoxification program, two thorough studies on the production and fate of products from the thermal/photolytic destruction of organic compounds have been conducted. Specifically, the thermal and thermal/photolytic oxidation of tetrachlorobiphenyl from exposure to 0 and 273 AM 1.5 suns (simulated), and the oxidation of monochlorobenzene to 0 and $862 \mathrm{~mW} / \mathrm{cm}^{2}, 280 \mathrm{~nm}$ laser radiation. These two data sets, along with a smaller data set for the pyrolysis of tetrachlorodibenzodioxin, will be discussed in detail below. Note that all of the available product data is summarized in the Appendices.

\subsection{PICS FROM THE PYROLYSIS OF 1,2,3,4-TETRACHLORODIBENZODIOXIN}

Although only qualitative information is available on the distribution of PICs from the pyrolysis of tetrachlorodibenzodioxin, the solar detoxification of this compound illustrates some intriguing results. In these tests approximately $2 \mathrm{mg}$ of tetrachlorodibenzodioxin was batch charged into the TPRS's sample inlet chamber which was preheated to $300^{\circ} \mathrm{C}$. This insured rapid evaporation of the sample, and resulted in a mean concentration in the reactor on the order of $10^{-5} \mathrm{~mol} / \mathrm{L}$. This sample was exposed to 0 and 307 AM 1.0 suns for 10 s in dry helium.

The data for the thermal and thermal/photolytic decomposition of tetrachlorodibenzodioxin is summarized in Figure 5.1. As this Figure shows, at $500^{\circ} \mathrm{C}$ there is a significant level of tetrachlorodibenzodioxin destruction from the simulated solar process, and there is no significant conversion from the purely thermal exposures. Therefore, the products observed from the thermal/photolytic exposure at this temperature result solely from the solar induced reactions and would represent the product distribution from a reactor operating in a dominantly solar (thermal/photolytic) mode. In comparison, there is data available from the purely thermal data at $700^{\circ} \mathrm{C}$ which is of a similar level of 


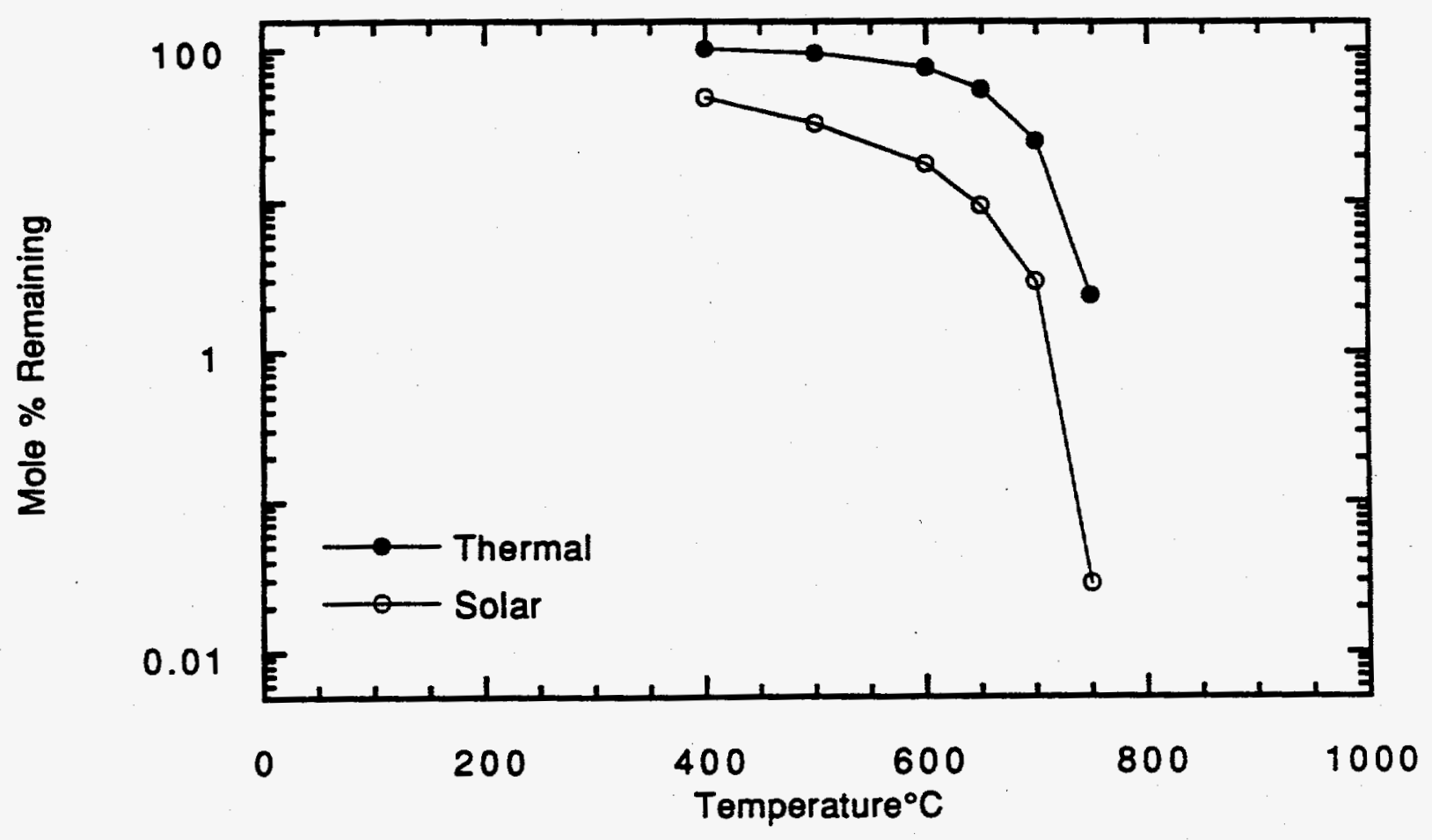

Figure 5.1. TPRS data for 1,2,3,4-tetrachlorodibenzo-p-dioxin $\left(-10^{-5} \mathrm{M}\right)$ exposed to 0 and 307 AM 1.0 suns (simulated) for $10 \mathrm{~s}$ in helium. 
conversion (75.1\% thermal versus $67.6 \%$ solar), from which the product distribution would be representative of a reactor operating in a purely thermal mode. The TPRS chromatograms from these two exposures are shown in Figure 5.2. Note the appearance of numerous PICs in the thermal data, which we would speculate include partially dechlorinated dioxins and chlorinated benzenes. In contrast, the chromatogram from the simulated solar exposure show an almost complete absence of these, or any, products. This data suggests that a solar detoxifier may be less susceptible to upsets due to fluctuating levels of excess air, and it may even be practical to operate a solar detoxifier with theoretical air levels at, or below, unity.

\subsection{THERMAL/PHOTOLYTIC OXIDATION OF MONOCHLOROBENZENE}

In this Section we present the results of a detailed study of the high-temperature thermal/photolytic reactions of monochlorobenzene (chlorobenzene) in a gas-phase, oxidative environment with a hightemperature flow reactor system that utilizes a puised, tunable laser system as a source of near UV radiation. Chlorobenzene is considered a prototypical, refractory chlorinated hydrocarbon whose purely thermal and photochemistry is relatively well characterized $[1-11]$. The laser photolysis study was selected because of the well-defined nature of the source (as compared to other sources such as xenon arc emission) and compatibility with planned spectroscopic studies.

\subsubsection{EXPERIMENTAL}

The thermal and thermal/photolytic kinetic data for this Section were obtained using the ATPRS. Specifically, the GC/MSD channel was fitted with a $180 \mu \mathrm{m} \times 20 \mathrm{~m}$ fused silica column with a $0.40 \mathrm{~mm}$ thick methylsilicone (nonpolar) liquid phase (J\&W Scientific, No. 123-1013). The MSD was operated in a scanning mode detecting ion masses from 10 to 200 AMU. The GC/FID channel was fitted with a $320 \mathrm{~mm}$ $\times 15 \mathrm{~m}$ fused silica column with a $0.25 \mathrm{~mm}$ thick methylsilicone (nonpolar) liquid phase (J\&W Scientific, No. 123-1012). The columns were simultaneously temperature programmed from $-80^{\circ} \mathrm{C}$ (initial hold of $5 \mathrm{~min}$ ) to $0^{\circ} \mathrm{C}$ at $12^{\circ} \mathrm{C} / \mathrm{min}$, then to $260^{\circ} \mathrm{C}$ at $20^{\circ} \mathrm{C} / \mathrm{min}$, where the temperature was held until the emergence of solute peaks was observed to be complete. Solute peak identifications were accomplished using the mass spectral data and subsequently confirmed using analytical standards when they were available. The standards were also used to quantify each product using comparative GC/FID peak areas. In the cases where standards were not available, structurally similar materials were used.

Volatile products (e.g., methane and oxides of carbon) were analyzed using samples collected from the ATPRS's exhaust port. Specifically, 1 liter Tedlar sample bags (SKC, No. 231-01) were attached to the port during a series of runs designed exclusively for the analysis of volatile species. These runs 

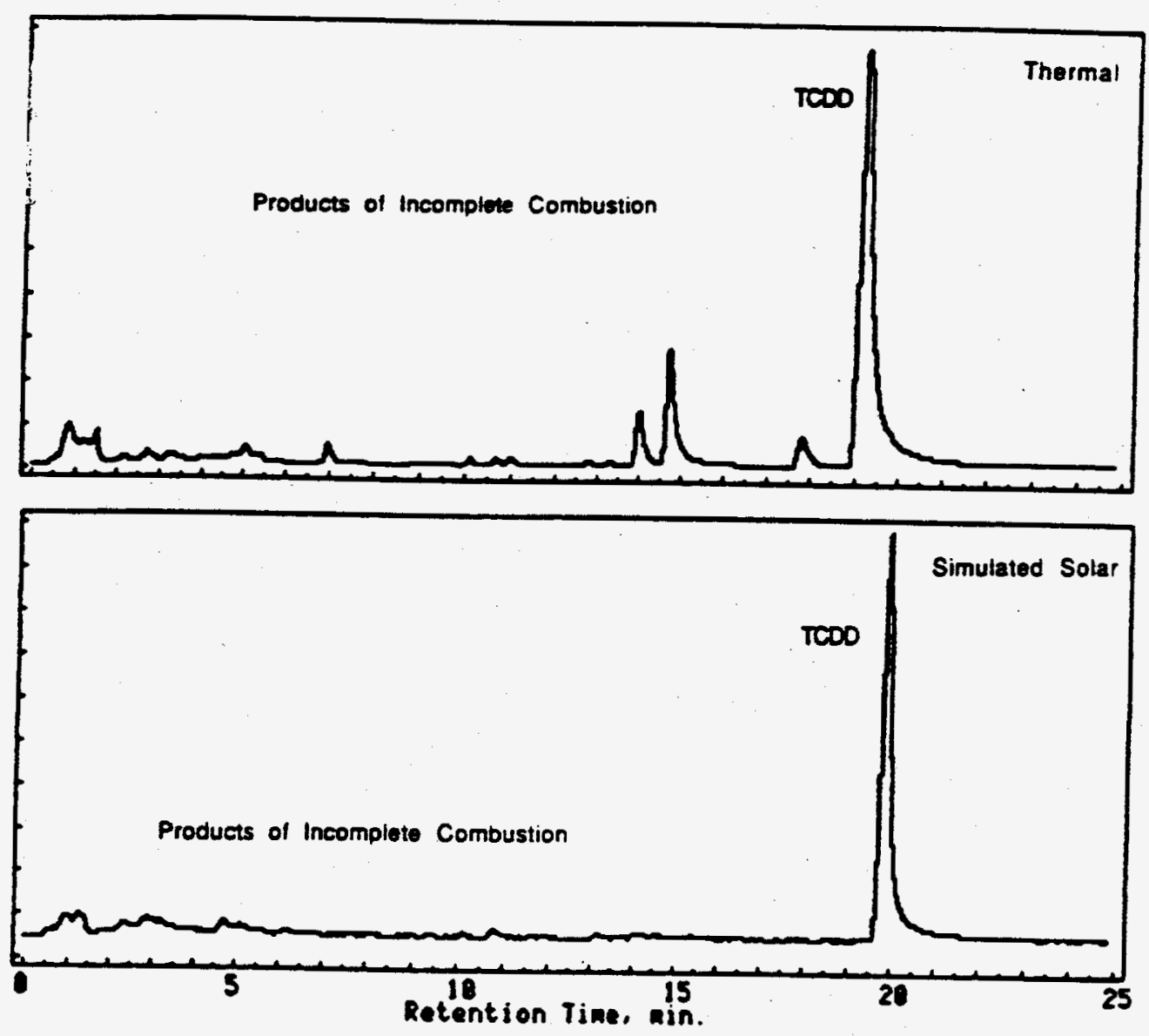

Figure 5.2. TPRS chromatograms for 1,2,3,4-tetrachlorodibenzo-p-dioxin ( $\left.10^{-5} \mathrm{M}\right)$ exposed in helium to purely thermal conditions at $700^{\circ} \mathrm{C}$ (top) and $307 \mathrm{AM} 1.0$ suns (simulated) for $10 \mathrm{~s}$ at $500^{\circ} \mathrm{C}$ (bottom). 
were conducted with the temperature of the cryogenic trap maintained at $350^{\circ} \mathrm{C}$ so that the transport of products to the collection bag would not be affected. At the conclusion of each run, a 2,000 $\mathrm{mL}$ sample from the bag was injected into a GC/methanizer/FID system fitted with a $2 \mathrm{~mm} \times 2 \mathrm{~m}$ TFE column packed with $80 / 100$ mesh Porapak $Q$. The column was temperature programmed from $-80^{\circ} \mathrm{C}$ (initial hold of $2 \mathrm{~min}$ ) to $150^{\circ} \mathrm{C}$ at $12^{\circ} \mathrm{C} / \mathrm{min}$ where it was held for 20 minutes. The observed products were identified and quantified using analytical standards.

Stock samples of chlorobenzene were prepared by injecting $45.3 \mathrm{~mL}$ of liquid chlorobenzene (Aldrich, 99.99\%, HPLC Grade) into a 1 liter glass sample bulb which had been previously cleaned and filled with dry helium. For each run, a 2,000 $\mathrm{ml}$ aliquote ( $100 \mathrm{mg}$ chlorobenzene) of the stock sample was injected at a rate of $20.3 \mathrm{~m} / \mathrm{s}$ for a period of approximately $100 \mathrm{~s}$. These conditions gave a mean concentration in the reactor of $29.5 \times 10^{-6} \mathrm{mo} / \mathrm{L}$. Since the volumetric flow rate of carrier gas was adjusted at each temperature to give a constant mean residence time, the concentration of oxygen in the reactor varied from $2.08 \times 10^{-3}$ at $300^{\circ} \mathrm{C}$ to $1.23 \times 10^{-3} \mathrm{~mol} / \mathrm{L}$ at $700^{\circ} \mathrm{C}$. Therefore, the level of theoretical air varies from $1,000 \%$ at $300^{\circ} \mathrm{C}$ to $600 \%$ at $700^{\circ} \mathrm{C}$.

\subsubsection{RESULTS}

The thermal and thermal/photolytic decomposition of chlorobenzene and associated carbon containing products (i.e., $\mathrm{HCl}$ and water were not measured) are summarized in Tables 5.1 and 5.2. The thermal and thermal/photolytic data are also summarized as mole percent (on a logarithmic scale, normalized by the original amount of chlorobenzene) versus temperature in Figures 5.3 and 5.4, respectively.

Our data clearly demonstrate an increase in the rate of decomposition of chlorobenzene via the thermal/photolytic pathways versus those induced by purely thermal exposure. For example, consider the data at $500^{\circ} \mathrm{C}$ where only $5.1 \%$ of the chlorobenzene was destroyed following thermal exposure, as compared to $61.5 \%$ following a thermal/photolytic exposure. The effect on product formation was even more starting as both the identity and yield of products were significantly altered. Phenol was a major organic product under both conditions. However, under purely thermal exposure, there was clear evidence of molecular growth pathways with formation of substituted benzenes and naphthalenes, while oxidation was enhanced under thermal/photolytic exposure with observation of partially oxidized organic species such as maleic anhydride and $2(3 \mathrm{H})$-furanone. Thermal/photolytic exposure resulted in a fewer number of by-products, of lower yield, of lower molecular weight, that decomposed at lower temperatures. This clearly has favorable implications for efficiency of destruction as a waste disposal method. 


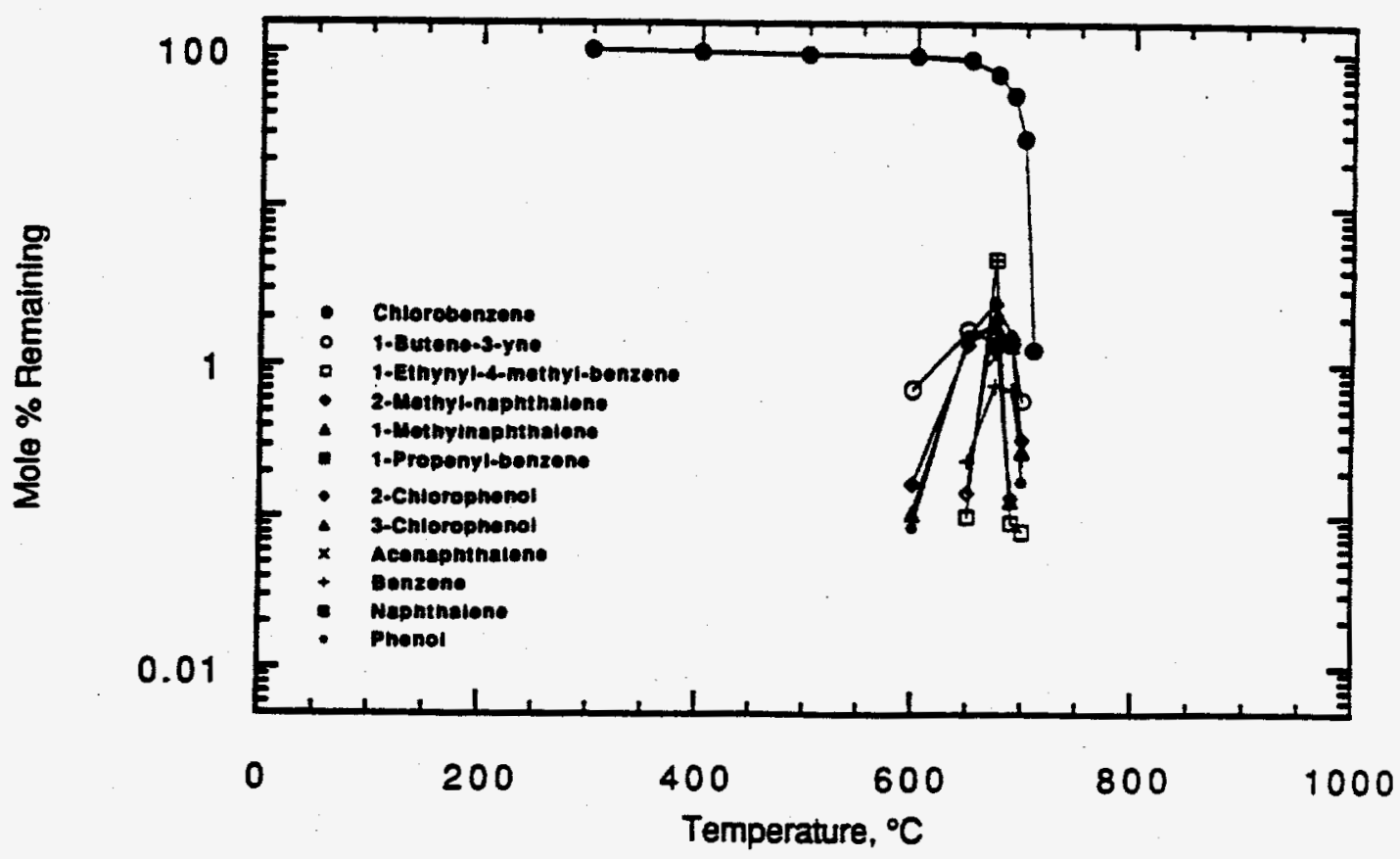

Figure 5.3. TPRS data for monochlorobenzene $\left(2.95 \times 10^{-5} \mathrm{M}\right)$ exposed under purely thermal conditions for 105 in air.

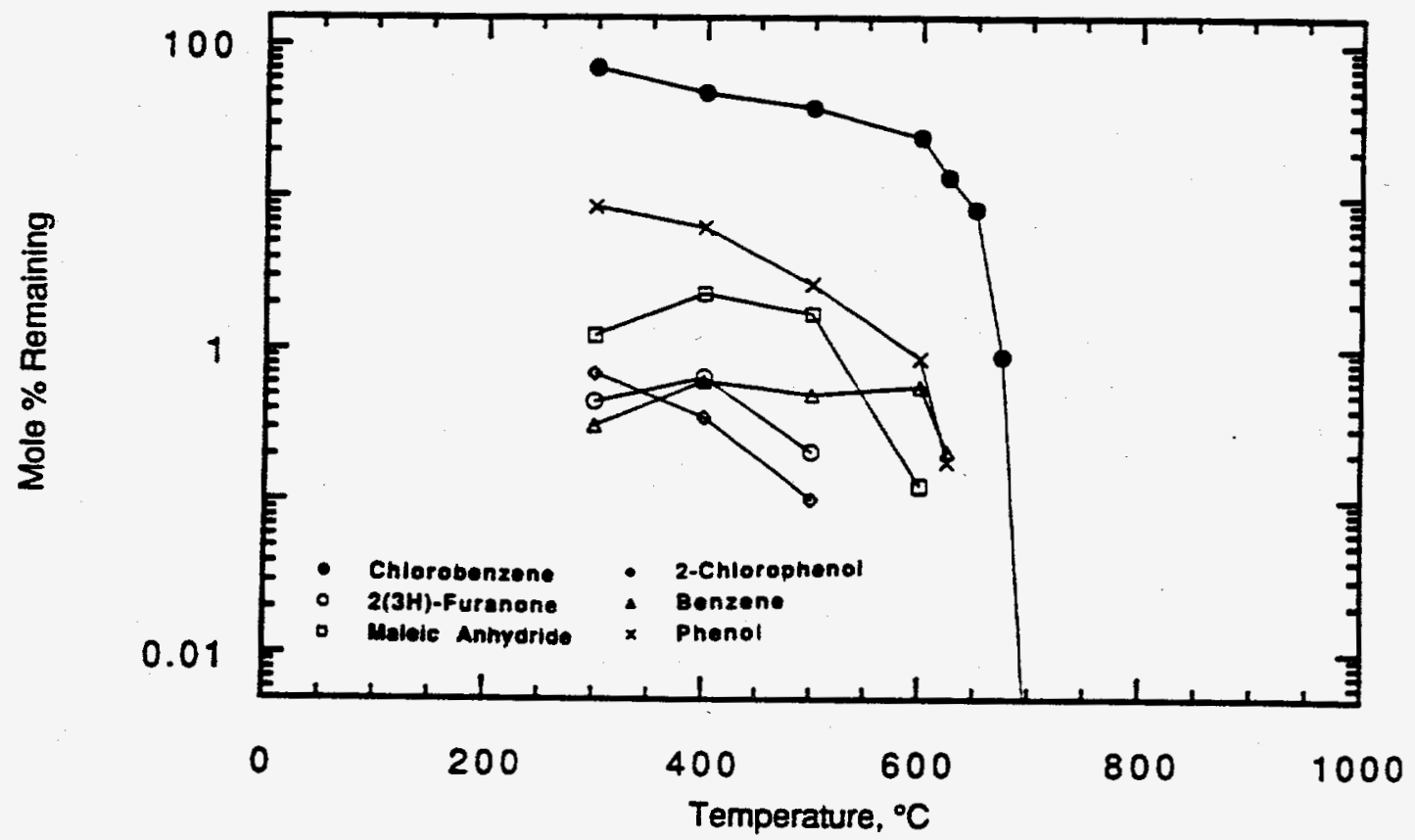

Figure 5.4. TPRS data for monochiorobenzene $\left(2.95 \times 10^{-5} \mathrm{M}\right)$ exposed to $862 \mathrm{~mW} / \mathrm{cm}^{2}$ of $280 \mathrm{~nm}$ radiation for 10 s in air. 
TABLE 5.1

SUMMARY OF THE THERMAL OXIDATION DATA FOR

CHLOROBENZENE AND ITS MAJOR PRODUCTS GIVEN

AS MOLE \% REFERENCED TO THE ORIGINAL

AMOUNT OF CHLOROBENZENE ${ }^{1}$

Temperature, ${ }^{\circ} \mathrm{C}$

Name

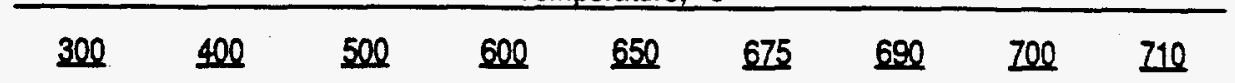

\begin{tabular}{|c|c|c|c|c|c|c|c|c|c|}
\hline Chlorobenzene (Parent) ${ }^{2}$ & 100 & 96.6 & 94.9 & 92.9 & 88.6 & 71.6 & 52.7 & 27.6 & 1.23 \\
\hline Carbon monoxide & & & & & 45.9 & 138 & 388 & 488 & 573 \\
\hline Carbon dioxide & & & & & 5.30 & 11.2 & 41.4 & 68.7 & 94.6 \\
\hline 1-Butene-3-yne ${ }^{3}$ & & & & 0.680 & 1.64 & 1.43 & 1.40 & 0.586 & \\
\hline $\begin{array}{l}\text { 1-Ethynyl-4-methyl } \\
\text { benzene }\end{array}$ & & & & & 0.102 & 4.67 & 0.093 & 0.081 & \\
\hline 2-Methyl-naphthalene ${ }^{2}$ & & & & & 0.146 & 2.45 & 0.136 & & \\
\hline 1-Methyl-naphthalene ${ }^{2}$ & & & & & & 1.65 & 0.130 & & \\
\hline 1.-Propenyl-benzene 4 & & & & & & 1.34 & & & \\
\hline 2-Chlorophenol ${ }^{2}$ & & & & 0.165 & 1.33 & 1.90 & 1.36 & 0.327 & \\
\hline 3-Chlorophenol ${ }^{2}$ & & & & 0.106 & 1.49 & 1.84 & 1.33 & 0.284 & \\
\hline Acenapthalene ${ }^{2}$ & & & & & & 1.11 & & & \\
\hline Benzene 2 & & & & & 0.239 & 0.735 & 0.689 & 0.248 & 0.141 \\
\hline Naphthalene ${ }^{2}$ & & & & & & 4.67 & & & \\
\hline Phenol $^{2}$ & & & & 0.085 & 1.54 & 2.48 & 1.55 & 0.172 & \\
\hline Total Carbon Recovered 5 & $100 \%$ & $96.6 \%$ & $94.9 \%$ & $93.3 \%$ & $103 \%$ & $131 \%$ & $131 \%$ & $122 \%$ & $113 \%$ \\
\hline
\end{tabular}

${ }_{1}^{1}$ Mole \% remaining of species i at temperature $T=$ Moles $T(i) /$ Moles $_{300}\left(\mathrm{ClBz}_{2}\right) \times 100 \%$.

${ }^{2}$ Quantified by analytical standard.

3 Quantified as butadiene.

${ }^{4}$ Quantified as ethynylbenzene.

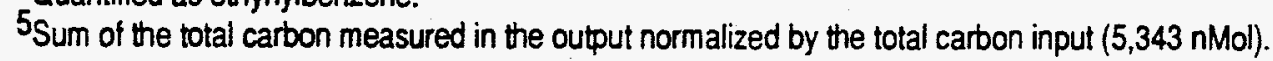


TABLE 5.2

\section{SUMMARY OF THE THERMALPHOTOLYTIC OXIDATION DATA FOR CHLOROBENZENE AND ITS MANOR PRODUCTS GIVEN \\ AS MOLE \% REFERENCED TO THE ORIGINAL AMOUNT OF CHLOROBENZENE ${ }^{1}$}

Temperature, ${ }^{\circ} \mathrm{C}$

Name

\begin{tabular}{lccccccc}
300 & 400 & 500 & 600 & 625 & 650 & $\underline{675}$ & $\underline{700}$ \\
69.8 & 47.8 & 38.5 & 24.7 & 13.5 & 8.36 & 0.895 & 0.00201 \\
203 & 356 & 465 & 516 & 564 & 612 & 565 & 518 \\
58.4 & 52.4 & 73.8 & 79.7 & 79.7 & 79.6 & 91.2 & 103 \\
0.447 & 0.647 & 0.207 & & & & & \\
1.22 & 2.31 & 1.71 & 0.125 & & & & \\
0.685 & 0.354 & 0.102 & & & & & \\
0.312 & 0.612 & 0.500 & 0.564 & 0.211 & & & \\
8.60 & 6.28 & 2.68 & 0.869 & 0.178 & & & \\
\hline $124 \%$ & $126 \%$ & $133 \%$ & $126 \%$ & $121 \%$ & $124 \%$ & $110 \%$ & $104 \%$
\end{tabular}

${ }_{1}^{1}$ Mole $\%$ remaining of species i at temperature $T=$ Moles $T(i) /$ Moles $_{300}(\mathrm{ClBz}) \times 100 \%$.

${ }^{2}$ Quantified by analytical standard. Two GC peaks were observed that were identified as maleic anhydride.

3 Quantified as 2H-Pyranone.

${ }^{4}$ Sum of the total carbon measured in the output normalized by the total carbon input $(5,343 \mathrm{nMol})$. 
Attempts at carbon balances were fairly successful with carbon recovery ranging from $94.9 \%$ to $133 \%$. The spread in recoveries is primarily attributed to the uncertainty in the collection and calibration procedures for carbon monoxide and carbon dioxide.

Temperature dependent $S_{1} \leftarrow S_{0}$ absorption spectra are presented in Figure 5.5 for the temperature range $150-700^{\circ} \mathrm{C}$. By $450^{\circ} \mathrm{C}$, vibronic resolution (under our experimental conditions) is completely lost due to spectral congestion. Hotbands (lower energy transitions originating from thermally populated ground state levels) result in a red-shift of both the $S_{1} \leftarrow S_{0}$ and $S_{2} \leftarrow S_{0}$ onsets. The intrinsic intensity of the spectrum was also observed to increase with increasing temperature with oscillator strength increasing from 0.0038 at $150^{\circ} \mathrm{C}$ to 0.119 at $700^{\circ} \mathrm{C}$. These two phenomena resulted in an increase in the rate of absorption of $280 \mathrm{~nm}$ radiation by a factor of 51 .

The photochemical quantum yield was found to be 0.19 at $300^{\circ} \mathrm{C}$, dropping to a minimum of 0.13 at $500-600^{\circ} \mathrm{C}$, then increasing again to 0.61 at $700^{\circ} \mathrm{C}$. In contrast, the enhancement ratio increases from 1.4 at $300^{\circ} \mathrm{C}$ to 13,700 at $700^{\circ} \mathrm{C}$ due to the increased rate of photon absorption as the temperature is increased. The ratio, $k_{e x} / k_{\text {gnd }}$, can only be estimated below $400^{\circ} \mathrm{C}$ due to the absence of any measurable thermal reaction below this temperature. A minimum value of 16.8 at $300^{\circ} \mathrm{C}$ is calculated based on a minimum detectable thermal conversion of $2.0 \%$. The general observation is that $k_{\text {ex }} / \mathrm{k}_{\text {gnd }}$ remains relatively constant at temperatures up to $650^{\circ} \mathrm{C}$. At $650-675^{\circ} \mathrm{C}$, the purely thermal reaction is initiated and $\mathrm{k}_{\mathrm{ex}} / \mathrm{k}_{\mathrm{gnd}}$ decreases as the rate of the thermal reaction increases more rapidly with temperature than the photochemical reaction. This is generally the case for catalyzed (photochemical, heterogeneous, etc.) versus thermal reactions, since the effect of the catalyst is to lower both the activation energy and the frequency factor of the reaction. This results in the catalytic reaction dominating at low temperature and the thermal reaction becoming increasingly significant at higher temperatures and finally dominating at a sufficiently elevated temperature.

We also investigated the possibility of multiphoton processes by varying the laser intensity from 1.0 to $43 \mathrm{~mW} / \mathrm{cm}^{2}$ per pulse. No evidence for multiphoton reactions was found, as the plot of log kex versus 10 yielded a straight line with a slope of $1.28 \pm 0.3$.

\subsubsection{DISCUSSION}

\subsubsection{ABSORPTION OF RADIATION}

The increased rate of solar radiation absorption is attributed to two temperature-dependent phenomena: 1) a red-shift in the onset of molecular absorption, and 2) an increase in overall molecular 


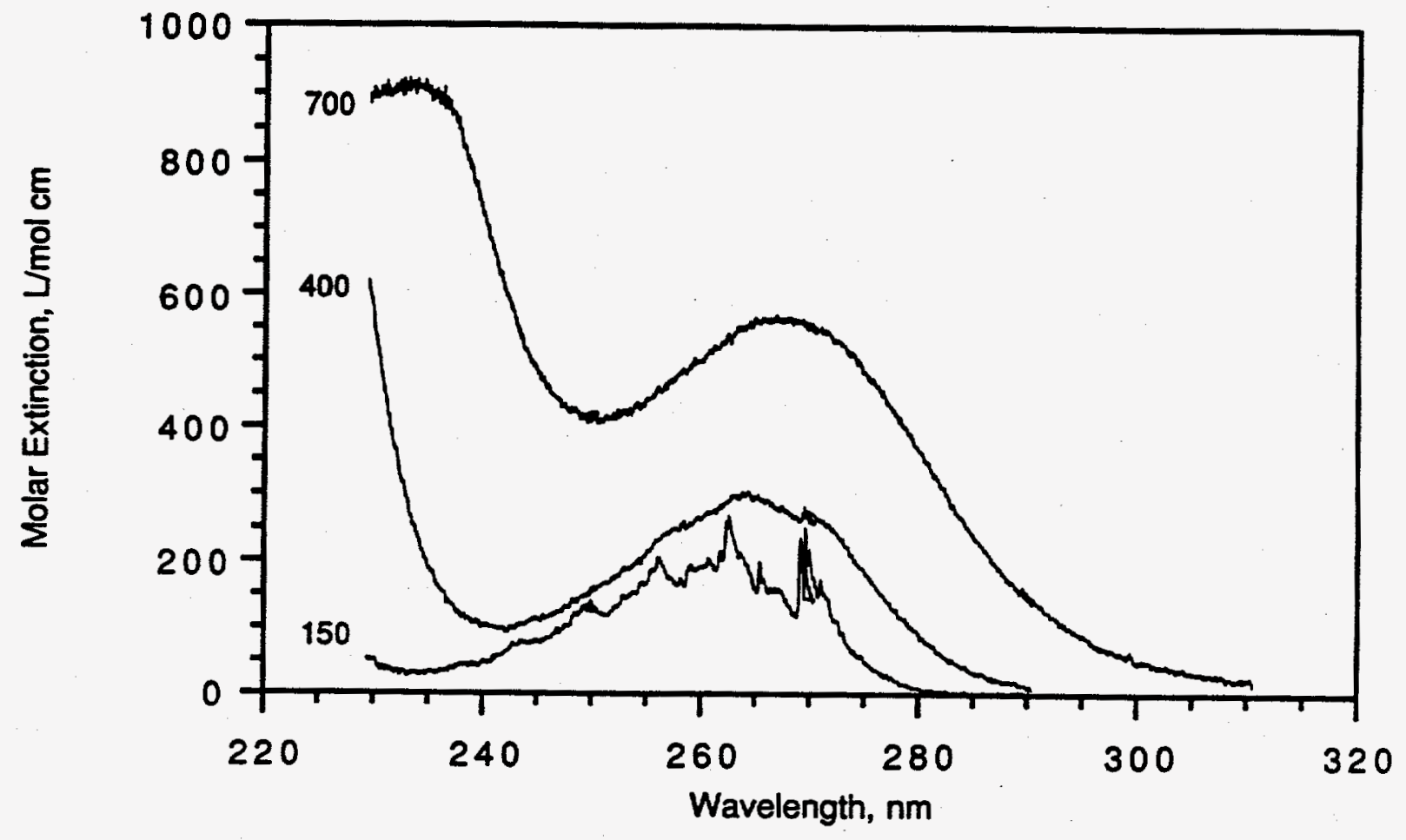

Figure 5.5. Temperature dependent $S_{1} \leftarrow S_{0}$ absorption spectra for monochlorobenzene at 150,400 , and $700^{\circ} \mathrm{C}$. 
absorption intensity. As temperatures increase, low-lying ground state vibrational modes become thermally populated as described by Boltzman statistics. This facilitates lower energy "hot" transitions. At the temperatures of this study, sufficient thermal energy is available to appreciably populate $n^{n}=1$ for lowlying vibration modes. Chlorobenzene has a number of modes that fit this description, particularly: $v_{6 a}$ $\left(417 \mathrm{~cm}^{-1}\right), v_{12}\left(707 \mathrm{~cm}^{-1}\right), v_{18 b}\left(295 \mathrm{~cm}^{-1}\right), v_{6 b}\left(615 \mathrm{~cm}^{-1}\right), v_{16 a}\left(403 \mathrm{~cm}^{-1}\right), v_{16 b}\left(467 \mathrm{~cm}^{-1}\right), v_{4}(684$ $\left.\mathrm{cm}^{-1}\right)$, and $v_{11}\left(197 \mathrm{~cm}^{-1}\right)[1,2]$. As the temperature is raised many modes are populated giving rise to a pseudo-continuum of rotationally-broadened vibronic transitions. Thus, the magnitude of the red-shitt in the onset of absorption may be predicted by a straightforward application of Boltzman statistics. Although the vibrationless $S_{1}\left(B_{1}\right) \leftarrow S_{0}\left(A_{1}\right)(0,0)$ transition is strongly allowed. Chlorobenzene derives much intensity from vibronic coupling $[1,2]$. According to the theoretical formulation of this mechanism, an electronic transition may gain in intensity when either state becomes distorted by a suitable vibration facilitating an electronic coupling with a third electronic state[12]. It is the intense $S_{3}\left(-55000 \mathrm{~cm}^{-1}\right)$ through vibrational mode $6 b, v_{6 b}$, which is believed to be the principle vibronic coupling state in the chlorobenzene $s_{i} \leftarrow S_{0}$ transition[3].

The observed intensity increase is attributed to a thermal enhancement of this phenomenon. Included in the formal treatment are the vibrational integrals, $\Sigma \int(n g) Q_{r}\left(m^{f}\right) d \tau$. $Q_{r}$ is the distorting vibration, $\mathrm{d} \tau$ indicates integration over all space, and $\mathrm{n} g$ and $\mathrm{m}^{\mathrm{f}}$ are the respective number of quanta of vibrational mode $r$ in the ground and final electronic states. In the nondisplaced harmonic oscillator approximation, as $n g$ increases (i.e., for hotbands) these integrals become more favorable. Integration for a Boltzman distributed set of levels, $n g$, results in a temperature dependence of the oscillator strength that increases as coth $(1 / 7)^{[12]}$. The observed increase in oscillator strength is expected to be even greater for species involving symmetry forbidden transitions.

\subsubsection{PRIMARY PHOTOCHEMICAL REACTION}

The direct impact of temperature on the rate constant of the initial step of the excited state photoreaction is difficult to discern in a system where secondary reactions are occurring. The observed dependence of the photoreaction quantum yield on temperature was complex and yielded no definitive information. A state/process diagram for chlorobenzene that summarizes the relevant literature data is depicted in Figure 5.6. The homolytic dissociation of the carbon-chlorine bond is $95.7 \mathrm{kcal} / \mathrm{mole}$ endothermic from $S_{0}$. However, from $T_{1}$ it is endothermic by only $16.3 \mathrm{kcal} / \mathrm{mole}$ and is actually exothermic from $S_{1}$. Thus, if there is no intrinsic barrier to dissociate from $S_{1}$, then no temperature dependence of the quantum yield of dissociation is to be expected. Conversely, the reaction yield from $T_{1}$ 


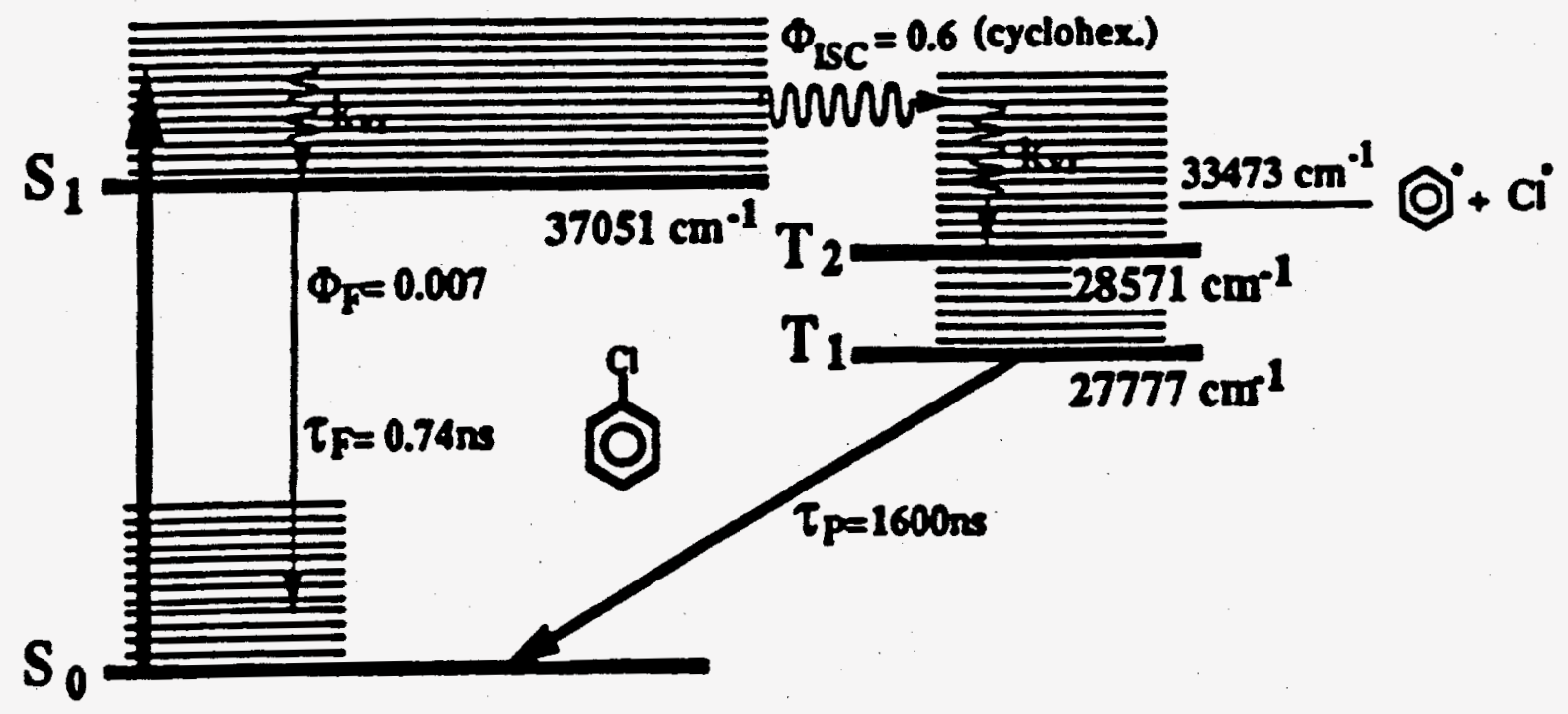

Figure 5.6. State/process diagram for monochlorobenzene. 
may be temperature dependent if the state is long-lived enough for the thermal energy of the bath gas to be transferred to the reactant state.

In liquid cyclohexane at room temperature, the $S_{1} \rightarrow S_{0}$ chlorobenzene fluorescence lifetime is only $0.74 \mathrm{~ns}[6]$. Thus, it is unlikely that any significant vibrational population of $S_{1}$ will be achieved at atmospheric pressure, although higher pressures may be effective if the excited state lifetime is not significantly shortened as the temperature is increased. If the gas-phase photodecomposition occurs from the $S_{1}$ state, this predicted lack of thermal activation and exothermicity of the photodissociation from $S_{1}$ may be responsible for the weak effect of temperature on $\phi_{r}$.

In contrast, the gas-phase phosphorescence lifetime of chlorobenzene has been reported to be $1,600 \mathrm{~ns}$, suggesting that thermal population of vibrational levels of $T_{1}$ may be possible[5,6,13]. Since the reaction is endothermic from $T_{1}$, a temperature dependence of $\phi_{r}$ could be observed. However, the rates of $S_{1} \rightarrow S_{0}$ fluorescence and internal conversion, $S_{1} \rightarrow T_{1}$ intersystem crossing, and $T_{1} \rightarrow S_{0}$ intersystem crossing may also be temperature dependent. Thus, the behavior of $\phi_{r}$ as a function of temperature may be quite complex and difficult to predict.

It is difficult to even speculate on the temperature dependence of $\phi_{r}$ since so little is known about the dynamics of the gas-phase photochemistry. Based on gas-phase photolysis studies in the presence of ethane at $15-85^{\circ} \mathrm{C}$, carbon-chlorine bond rupture has been proposed to occur via a predissociative $3 \sigma \sigma^{*}$ state which accounts for the observed competitiveness of the photoreaction with the fast internal conversion following excitation into the vibrational manifold of $S_{1}[13]$. Vibronic coupling from $S_{1}\left(\pi \pi^{*}\right)$ through a $\pi^{*} \sigma$ or $\sigma^{*} \pi$ singlet followed by spin orbit coupling to a $\pi \sigma$ or $\sigma^{*} \pi$ triplet state were suggested to account for the calculated intersystem crossing rate of $10^{9} \mathrm{~s}^{-1}$.

Hartree-Frock Cl-SCF calculations support the presence of these states[7]. Dual phosphorescences in a rigid matrix have been assigned as from $3 \pi \sigma^{*}\left(B_{1}\right)$ and $3 \pi \pi^{*}\left(A_{1}\right)$ states[5]. All of these states may play a role in the efficiency of intersystem crossing $\phi_{s_{1} \rightarrow T_{1}}=0.64$ ) and the weak fluorescence yield $(\phi F=0.007$ ). However, solution studies have observed $\mathrm{Cl} \bullet$ generation on the order of nanoseconds suggesting a stronger role for $S_{\mathcal{1}}$ in the photodissociation[8].

Direct spectroscopic measurement of the temperature dependence of the various excited state processes will be necessary to determine the nature of the effect of temperature on the efficiency of the primary step in this or other photochemical reactions. 


\subsubsection{PHOTO-INDUCED CHAIN REACTION}

There is clear evidence of changes in the secondary reaction pathways as we proceed from purely thermal to thermalphotolytic exposure. The purely thermal reaction products are readily explainable using well documented elementary reactions for oxidation and molecular growth of aromatic species $[14,15]$. (We have developed both pyrolytic oxidative kinetic models for chlorobenzene. The reader is referred to Appendix VI for a discussion of the predictive capability of these models.) However, the thermal/photolytic products introduce the prospect of new chemical pathways. Phenol and chlorophenol formation is readily explained via a photo-induced initiation step involving carbon-chiorine bond rupture. Figure 5.7 also outlines a pathway to the observed organic products initiated by a photo-activated bimolecular addition of $\mathrm{O}_{2}\left({ }^{3} \mathrm{\Sigma g}\right.$ or $\left.{ }^{1} \Delta \mathrm{g}\right)$ across the aromatic ring. Such additions have been previously proposed for reactions of singlet oxygen with unsaturated organic species[16]. A similar addition pathway may also be envisioned involving addition of $\mathrm{O}_{2}$ to phenyl radical formed from photo-induced carbon-chlorine bond rupture. Mechanisms that do not involve bicyclic addition of $\mathrm{O}_{2}$ can also be devised; however, rather improbable steps involving multiple hydrogen atom migrations must be invoked.

Although this proposed mechanism is entirely speculative, the individual steps seem thermodynamically and kinetically plausible. Efforts are currently underway to estimate the thermochemical properties of the individual species and develop a detailed reaction kinetic model. The modeling is complicated by the need to model a series of high intensity light reaction periods of approximately $10 \mathrm{~ns}$, corresponding to the laser pulse, followed by a relatively long period of $50 \mathrm{~ms}$ of dark reaction in which radical chains may propagate. Preliminary results for this and other systems indicate that thermal/photolytically generated radicals are produced in quantities sufficient to generate long chains at temperatures $>600^{\circ} \mathrm{C}$. This suggests that the chemistry of even non-absorbing compounds in a complex mixture may be significantly affected by thermal/photolytically induced initiation reactions.

\subsection{THERMAL/PHOTOLYTIC OXIDATION OF 3,3'4,4'-TETRACHLOROBIPHENYL}

The reported toxicity of chlorinated biphenyls (PCBS) and their combustion by-products has caused serious concern in the environmental and scientific community $[17,18]$. Consequently, they have been banned from further use in the United States, and safe methods of disposal have been of key interest since the late 1970 's $[19,20]$. Although controlled incineration has become an increasingly accepted method for disposal of most toxic organic wastes, the refractory nature of PCBs and their propensity for formation of toxic combustion by-products such as polychlorinated dibenzofurans (PCDFs) have severely hindered their disposal via incineration $[18,21,22]$. In fact, the U. S. EPA has required that it be demonstrated that an incinerator can achieve a destruction and removal efficiency (DRE) of $99.9999 \%$ for 


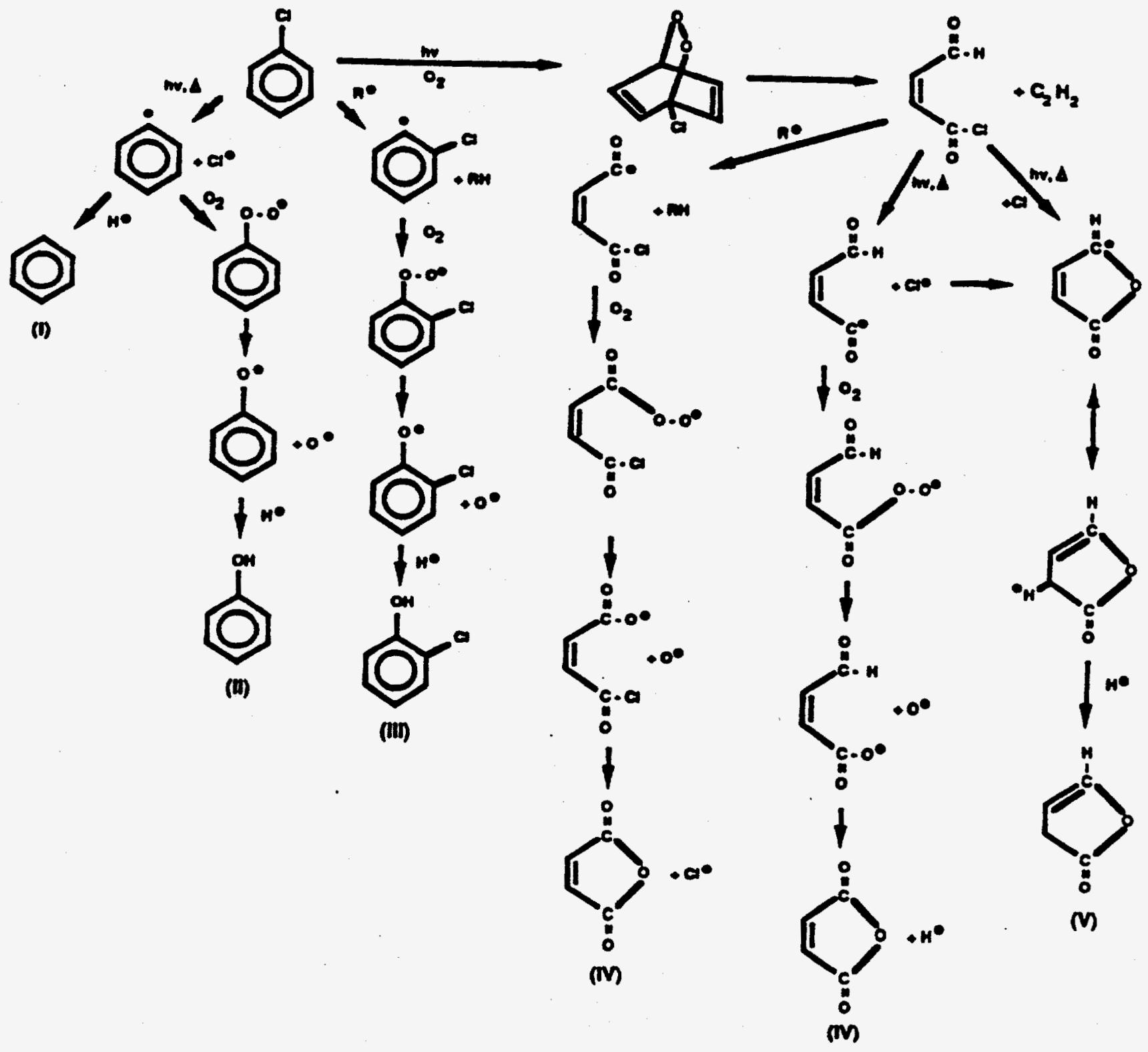

Figure 5.7. Proposed pathway to the organic products observed from the thermal/photolytic oxidation of monochlorobenzene. 
PCBs before it can receive an operating permit 19$]$. As a result there has been considerable research concerning the degradability and destruction of PCBs.

Early studies of PCB thermal degradation were conducted in sealed tubes at temperatures of less than $650^{\circ} \mathrm{C}$ with exposure times on the order of minutes with generally poorly defined reaction conditions[23-26]. More recent studies have been conducted in gas-phase flow reactors with precise control of temperature, residence time, pressure, and reaction atmosphere $[21,27,28]$. Such studies have clearly demonstrated that PCBs are exceedingly stable compounds and form PCDFs, chlorinated benzenes (PCBs), and lower chlorinated PCBs with yields on the order of $1-5 \%$ at $750^{\circ} \mathrm{C}$ and a 2.0 second gas phase residence timel[21].

Photochemical degradation and destruction studies of PCBs in solution have been even more numerous and the subject of a number of relatively recent reviews [29.32]. The utility of direct photolytic degradation of PCBs appears to be limited by the low disappearance quantum yield for most congeners $[29,33]$. The degradation of PCBs by solar radiation is also limited by their low absorption cross section with the solar spectrum. Furthermore, the parent $P C B$ is only partially dechlorinated under most reaction conditions, although the primary mode of destruction is cleavage of a carbon-chlorine bond[33]. Unwanted, potentially toxic reaction by-products, such as PCDFs and hydroxyl chlorobiphenyls, have been observed [33,34]. As a result, increasing emphasis has been placed on photochemical destruction using charge or electron transfer reagents $[31,33,35-42]$. Sodium borohydride has received the most attention and apparent success, although exciplex forming additives such as amines, cyanobenzenes, and thiols have also resulted in somewhat enhanced disappearance quantum yields.

Our research has shown that some of the limitations of high temperature thermal degradation and room-temperature photolysis can be overcome by combining intense heating with IR radiation and photolysis with near UV radiation in a process referred to as thermal/photolytic destruction. In fact, we have previously shown that PCBs, as well as many other toxic organic compounds are amenable to gasphase destruction using concentrated solar radiation[43]. For experiments in air and reaction times of $5.0 \mathrm{~s}$ where the temperature was held constant between $400-850^{\circ} \mathrm{C}$ using thermoelectric heaters, it was shown that irradiation with UV light with wavelengths $>290 \mathrm{~nm}$ resulted in photochemical destruction of the test material. For simulated solar irradiation intensities of 57 times that of natural sunlight ( 57 "suns"), the photochemical destruction efficiency enhancement over purely thermal destruction exhibited a maximum of $2.17(117 \%$ increase in $\mathrm{DE})$ at $850^{\circ} \mathrm{C}$. Photochemical destruction was observed at temperatures as low as $600^{\circ} \mathrm{C}(7 \%$ destruction). This suggests that thermally enhanced photochemical destruction is occurring. UV radiation effects were confirmed by measuring the destruction efficiency as a function of the UV cutoff of the radiation source. 
In the following paragraphs, we report the results of a detailed study of the thermal/photolytic destruction of 3,3,4,4'-tetrachlorobiphenyl (tetrachlorobiphenyl) using 273 simulated AM 1.5 suns (27.3 W/ $\mathrm{cm}^{2}$ with xenon arc emission limited to wavelengths $>300 \mathrm{~nm}$ ). Detailed analysis of reaction products were conducted in order to determine the overall effectiveness of the thermal/photolytic process.

Considerable effort was made to obtain mass balances and to determine the temperature dependence of the rate of photon absorption, the rate of thermal reaction, and the photochemical quantum yield.

\subsubsection{EXPERIMENTAL}

To examine the effectiveness of the thermal/photolytic process on the oxidation of tetrachlorobiphenyl, data was obtained with three dedicated laboratory systems; the HTAS, TPRS, and ATPRS. Since these systems have been described in considerable detail in Section 3, only the pertinent experimental procedures will be described here.

The gas-phase molar extinction spectra ( 280 to $320 \mathrm{~nm}$ ) for tetrachlorobiphenyl were measured with the HTAS from 200 to $700^{\circ} \mathrm{C}$. For each analysis, $250 \mathrm{mg}$ samples were placed into the HTAS's sample insertion chamber which was heated to $250^{\circ} \mathrm{C}$. Spectra were continuously recorded with the linear diode array every 2.4 seconds while the sample was passing through the system. From these data, along with reference spectra taken of the empty absorption cell, the instantaneous mean concentration in the cell, and hence the molar extinction coefficient, could be calculated.

The thermal and thermal/photolytic oxidation of tetrachlorobiphenyl was quantitatively examined with the TPRS. Specifically, $100 \mathrm{mg}$ samples of tetrachiorobiphenyl were fed to the reactor at $1.02 \mu \mathrm{g} / \mathrm{sec}$ using a special probe assembly. This gave a mean concentration of $3.78 \times 10^{-6} \mathrm{~mol} / \mathrm{L}$ in the reactor. Since the flow rate was adjusted at each temperature to give a fixed mean exposure tome of 10 seconds, the theoretical air varied from $43,500 \%$ at $300^{\circ} \mathrm{C}$, to $26,300 \%$ at $700^{\circ} \mathrm{C}$. Exposures where made in pairs with radiant intensities of 0 (thermal) and 273 air mass 1.5 (AM 1.5) suns. The TPRS reactor effluent was analyzed with a nonpolar column ( $320 \mathrm{~mm} \times 15 \mathrm{~m}$, J\&W Scientific DB-1) that was temperature programmed from -60 to $260^{\circ} \mathrm{C}$ at $15^{\circ} \mathrm{C} / \mathrm{min}$.

Products from the oxidation of tetrachlorobiphenyl were identified from selected thermal exposures using the ATPRS. Specifically, data was obtained at $675^{\circ} \mathrm{C}$ (the temperature of maximum thermal PIC production) with a mean exposure time of 10 seconds in air. The reactor effluent was simultaneously analyzed by GC/HFID and GC/MS using $320 \mathrm{~mm} \times 15 \mathrm{~m}$ and $200 \mathrm{~mm} \times 20 \mathrm{~m}$ columns, respectively. Both columns used nonpolar liquid phases (J\&W Scientific DB-1). The mass spectral data (35-350 AMU) was 
used to assign structural identifications to all major products. Relative retention times between the GCMS and GC/HFID traces were then used to assign identifications to the major effluent peaks observed in the TPRS chromatograms. Finally, analytical standards were used, when available, to confirm the identifications and provide detector response factors for quantification. Structurally similar analogs were used in the cases where analytical standards were not available. Quantification standards are noted in Tables 5.3 and 5.4 .

Carbon balances were conducted at $675^{\circ} \mathrm{C}$ for thermal, and $600^{\circ} \mathrm{C}$ for the simulated solar exposures. These temperatures represent the points at which maximum PIC yields were observed from the respective exposure conditions. During the sample introduction phase of each run, $1 \mathrm{~L}$ Tedlar sample bags were attached to the TPRS's cold trap exhaust port. Furthermore, during these runs the cryogenic trap was maintained at $350^{\circ} \mathrm{C}$ so that the transport of material through the trap would not be impeded. The contents of the sample bags were then quantitatively analyzed for carbon monoxide and carbon dioxide. For each analysis, 2,000 $\mu$ lof the bag sample was injected onto a $2 \mathrm{~mm} \times 2 \mathrm{~m}$ Teflon column packed with $80 / 100$ mesh Porapak $Q$ that was initially held at $-60^{\circ} \mathrm{C}$ for 2 minutes, then temperature programmed to $100^{\circ} \mathrm{C}$ at $15^{\circ} \mathrm{C} / \mathrm{min}$. The column was supplied with $25 \mathrm{~m} / / \mathrm{min}$ of dry helium, and a methanizer/HFID was used for effluent peak detection. The system was calibrated by collecting bag samples from runs in which known amounts of carbon monoxide and carbon dioxide had been injected into the instrument.

\subsubsection{RESULTS}

The temperature dependent absorption spectra of tetrachlorobiphenyl are presented in Figure 4.4. In accord with our previously explained understanding of the temperature dependence, the spectrum redshift and increase in intensity as the temperature is increased. The $200^{\circ} \mathrm{C}$ spectrum is in generally good agreement with that of room temperature, nonpolar solution spectra reported in the literature.

We have calculated the rate of photon absorption $\left(k_{a b}\right)$ under the conditions of these tests as shown in Figure 4.6. As these data show, $\mathrm{k}_{\mathrm{ab}}$ increases in a smooth, exponential manner from $0.01 \mathrm{~s}^{-1}$ at $200^{\circ} \mathrm{C}$ to $0.07 \mathrm{~s}^{-1}$ at $700^{\circ} \mathrm{C}$. Therefore, based on the rate of absorption of solar radiation alone, the overall rate of the photochemical process would be expected to increase by a factor of 7 based on theory.

The thermal and thermal/photolytic destruction data for tetrachlorobiphenyl oxidation are given in Table 5.3 through 5.5. Destruction data and profiles for the parent tetrachlorobiphenyl alone are presented in Table 5.5 and Figure 4.1. As these data illustrate, there is a thermal enhancement of the photolytic process over the entire temperature range studied. The thermal/photolytic enhancement ratio varied from 1.1 at $300^{\circ} \mathrm{C}$ to a maximum of 41 at $675^{\circ} \mathrm{C}$ before decreasing to 3.35 at $725^{\circ} \mathrm{C}$. The occurrence of a 
TABLE 5.3

PRODUCT YIELDS FROM THE THERMAL OXIDATION OF 3,3',4,4'-TETRACHLOROBIPHENYL FOR 10.0s IN AIR

\begin{tabular}{|c|c|c|c|c|c|c|c|}
\hline \multirow[b]{2}{*}{ Name } & \multicolumn{7}{|c|}{ Temperature $\left({ }^{\circ} \mathrm{C}\right)$} \\
\hline & 500 & 550 & 600 & 650 & 675 & 700 & 725 \\
\hline$T C B^{2}$ & $100^{1}$ & 100 & 98.1 & 66.9 & 42.1 & 5.11 & 0.0847 \\
\hline 1,2-Dichlorobenzene? & -8 & 0.00490 & 0.0172 & 0.120 & 0.153 & 0.0575 & 0.00910 \\
\hline 1,2,4-Trichiorobenzene ${ }^{2}$ & - & - & - & 0.0210 & 0.0410 & 0.0931 & - \\
\hline Dichlorostyrene ${ }^{3}$ & 0.0179 & 0.0331 & 0.0906 & 0.348 & 0.355 & 0.140 & - \\
\hline Dichlorobenzofuran 4 & - & 0.00711 & 0.0214 & 0.144 & 0.256 & 0.0149 & - \\
\hline Dichlorobenzofuran 4 & - & 0.00565 & 0.0087 & 0.0399 & 0.0603 & 0.301 & - \\
\hline 1,3-Butadienyl-dichloro-benzene ${ }^{4}$ & - & 0.0125 & 0.0501 & 0.1542 & 0.163 & 0.0614 & 0.0294 \\
\hline 1,3-Butadienyl-trichloro-benzene ${ }^{5}$ & - & 0.0116 & 0.0345 & 0.120 & 0.133 & 0.171 & - \\
\hline 1,3-Butadienyl-tetrachioro-benzene ${ }^{5}$ & - & 0.00799 & 0.0397 & 0.156 & 0.122 & 0.0748 & 0.0571 \\
\hline Trichloronaphthalene 6 & - & 0.0312 & 0.0646 & 0.354 & 0.453 & 0.220 & - \\
\hline Trichlorobipheny 6 & 0.0497 & 0.0723 & 0.0762 & 0.157 & 0.139 & 0.0536 & - \\
\hline Trichlorobiphenyl6 & 0.0321 & 0.0420 & 0.0490 & 0.106 & 0.0880 & 0.0204 & - \\
\hline Methyl-trichlorobipheny ${ }^{6}$ & 0.00227 & 0.00765 & 0.0298 & 0.142 & 0.171 & 0.0836 & - \\
\hline Methyl-trichlorobipheny 6 & - & 0.0125 & 0.0374 & 0.154 & 0.181 & 0.0709 & -. \\
\hline Tetachlorodibenzofuran? & 0.0221 & 0.0354 & 0.0860 & 0.415 & 0.602 & 0.404 & - \\
\hline Tetrachlorodibenzofuran 7 & 0.0169 & 0.0529 & 0.129 & 0.425 & 0.573 & 0.528 & - \\
\hline Tetrachlorodibenzofuran 7 & 0.0275 & 0.0606 & 0.0909 & 0.176 & 0.00658 & 0.00496 & - \\
\hline $\begin{array}{l}\text { Carbon Monoxide }{ }^{2} \\
\text { Carbon Dioxide } \\
\text { Total Carbon Recovered }\end{array}$ & & & & & $\begin{array}{l}475 \\
104 \\
136\end{array}$ & & \\
\hline
\end{tabular}

${ }^{1} \mathrm{All}$ data are mole $\%$ remaining referenced to initial number of moles of $T C B$ at $300^{\circ} \mathrm{C}$ and 0 suns (thermal).

${ }^{2}$ Quantified by analytical standard.

3 Quantified as 2,6-Dichlorostyrene.

4 Quantified as 2,6-Dichlorobenzene.

5 Quantified as 1,2,4-Trichlorobenzene.

6 Quantified as 3,4,4-Trichlorobiphenyl.

7 Quantified as 2,3,7,8-Tetrachlorodibenzofuran.

8 Below detection limit. 
TABLE 5.4

PRODUCT YIELDS FROM THE THERMALIPHOTOLITIC OXIDATION OF $3,3,4,4^{\prime}$-TETRACHLOROBIPHENYL EXPOSED TO 273 AM-1.5 SUNS FOR 10.0s IN AIR

\begin{tabular}{|c|c|c|c|c|c|c|c|c|c|}
\hline \multirow[b]{2}{*}{ Name } & \multicolumn{9}{|c|}{ Temperature $\left({ }^{\circ} \mathrm{C}\right)$} \\
\hline & 300 & 400 & 500 & 550 & 600 & 650 & 675 & 700 & 725 \\
\hline $\mathrm{TCB}^{2}$ & $94.3^{1}$ & 79.5 & 52.3 & 42.1 & 25.6 & 8.28 & 1.03 & 0.171 & 0.0253 \\
\hline 1,2-Dichlorobenzene 2 & - & 0.00426 & 0.0371 & 0.0841 & 0.124 & 0.110 & 0.0265 & 0.00432 & - \\
\hline 1,2,4-Trichlorobenzene 2 & - & 0.0180 & 0.401 & 0.557 & 0.0823 & 0.094 & 0.0708 & - & - \\
\hline Dichlorostyrene ${ }^{3}$ & 0.0121 & 0.0534 & 0.233 & 0.344 & 0.429 & 0.199 & 0.0294 & - & - \\
\hline Dichlorobenzofuran ${ }^{4}$ & 0.0170 & 0.0930 & 0.188 & 0.214 & 0.278 & 0.296 & 0.150 & - & - \\
\hline Dichlorobenzoturan 4 & - & - & - & - & - & - & - & - & - \\
\hline 1,3-Butadienyl-dichloro-benzene 4 & 0.0791 & 0.163 & 0.288 & 0.191 & 0.229 & 0.123 & 0.0301 & - & - \\
\hline 1,3-Butadienyl-trichloro-benzene 5 & 0.00564 & 0.124 & 0.447 & 0.464 & 0.402 & 0.217 & 0.0983 & - & -- \\
\hline 1,3-Butadienyl-tetrachloro-benzene ${ }^{5}$ & - & 0.0389 & 0.0872 & 0.0932 & 0.174 & 0.132 & 0.0328 & - & $\ldots$ \\
\hline Trichloronaphthalene 6 & - & - & - & 0.282 & 0.227 & 0.136 & 0.0693 & - & -. \\
\hline Trichlorobipheny 6 & 0.0483 & 0.0520 & 0.0714 & 0.0933 & 0.0887 & 0.0557 & 0.0140 & - & - \\
\hline Trichlorobipheny ${ }^{6}$ & 0.0418 & 0.0326 & 0.0407 & 0.0568 & 0.0527 & 0.0307 & 0.00605 & - & - \\
\hline Methyl-trichlorobiphenyl 6 & - & 0.0120 & 0.0275 & 0.0441 & 0.0610 & 0.0344 & 0.00746 & - & - \\
\hline Methyi-trichlorobipheny16 & 0.150 & 0.236 & 0.254 & 0.206 & 0.122 & 0.00647 & 0.00323 & -- & - \\
\hline Tetrachlorodibenzoturan 7 & 0.00374 & 0.0104 & 0.0389 & 0.0365 & 0.0156 & 0.0107 & 0.00666 & - & $\ldots$ \\
\hline Tetrachlorodibenzoturan ${ }^{7}$ & 0.00816 & 0.0199 & 0.0116 & 0.00876 & 0.00384 & 0.0139 & 0.00750 & - & $\ldots$ \\
\hline Tetrachlorodibenzohuran 7 & 0.00750 & 0.0232 & 0.0175 & 0.00350 & 0.00580 & - & - & - & ... \\
\hline $\begin{array}{l}\text { Carbon Monoxide } \\
\text { Carbon Dioxide }^{2} \\
\text { Total Carbon Recovered }\end{array}$ & & & & & & & $\begin{array}{l}515 \\
91.7 \\
127 .\end{array}$ & & \\
\hline
\end{tabular}

1All data are mole \% remaining referenced to initial number of moles of TCB at $300^{\circ} \mathrm{C}$ and 0 suns (thermal).

2 Quantified by analytical standard.

3 Quantified as 2,6-Dichlorostyrene.

${ }^{4}$ Quantified as 2,6-Dichlorobenzene.

5 Quantified as 1,2,4-Trichlorobenzene.

6 Quantified as 3,4, 4-Trichlorobiphenyl.

${ }^{7}$ Quantified as 2,3,7,8-Tetrachlorodibenzoturan

8 Below detection limit. 
TABLE 5.5

Summary of TPRS Data for 3,3,4,4'-Tetrachlorobiphenyl

Exposed to 0 and 273 AM 1.5 Suns (Simulated) for $10 \mathrm{~s}$ in Air

\begin{tabular}{cccc} 
Temp. & $f_{r}(0)$ & $f_{r}(0)$ & $R(10)$ \\
\hline 300 & 100 & 94.3 & 1.06 \\
400 & 100 & 79.5 & 1.26 \\
500 & 100 & 52.3 & 1.91 \\
550 & 100 & 42.1 & 2.38 \\
600 & 98.1 & 25.6 & 3.83 \\
650 & 66.9 & 8.28 & 8.08 \\
675 & 42.1 & 1.03 & 40.87 \\
700 & 5.11 & 0.171 & 29.88 \\
725 & 0.0847 & 0.0253 & 3.35
\end{tabular}


temperature range in which the photolytic enhancement is optimized has been observed in most of the previously presented thermal/photolytic oxidation studies.

From the temperature dependence of $k_{a b}$, summarized in Figure 4.5 , and the tetrachlorobiphenyl destruction data summarized in Table 5.3 and 5.4, the temperature dependence of the photochemical quantum yield may be found per Equation 2.7b. These results are summarized in Figure 4.6, which show the quantum yield varied from 0.58 at $300^{\circ} \mathrm{C}$ to 6.8 at $675^{\circ} \mathrm{C}$, then declined to 1.9 at $725^{\circ} \mathrm{C}$. Unfortunately, we feel that there is yet insufficient information on the detailed nature of the photochemistry and photophysics to meaningfully speculate on the reasons for observing quantum yields significantly higher than unity, though it certainly requires a complex reaction scheme.

In addition to the efficient destruction of the parent components in a waste, an environmentally acceptable disposal process must also destroy any potentially hazardous products that may be formed. Specifically, it is known that the decomposition of even relatively simple wastes involve complex reaction pathways that often result in the production of numerous stable products of incomplete combustion (PICs). Furthermore, it is even possible that organic products may be formed that are higher in molecular weight, structurally more complex, and more hazardous than the parent species in the waste stream.

Consider the two example TPRS chromatograms from the oxidation of tetrachlorobiphenyl shown in Figure 5.8. These chromatograms depict "snap shots" of the product distributions from the tetrachlorobiphenyl exposed to purely thermal conditions at $675^{\circ} \mathrm{C}$ and simulated solar at $550^{\circ} \mathrm{C}$. These temperatures were selected because the extent of conversion under the two conditions were essentially identical (i.e., 42.1\% remaining). Furthermore, the simulated solar data was obtained at a temperature where there are no significant purely thermal reactions occurring. Thus, these data sets illustrate the products that result from the purely thermal and purely solar oxidation of $57.9 \%$ of the tetrachlorobiphenyl.

The complexity of the chromatograms and the variety of products identified in Figure 5.8 demonstrate that the oxidation of tetrachlorobiphenyl proceeds through a complex series of radicalmolecule and radical-radical reactions. Furthermore, although nearly identical products were observed in both the thermal and simulated solar tests, the relative yields varied significantly.

Tables 5.3 and 5.4 summarize the relative molar yields (normalized to the original amount of tetrachlorobiphenyl) of the major identified products. These tables also give the results of a carbon balance conducted at the temperature of maximum PIC yields under the two conditions. Specifically, $127 \%$ of the carbon was recovered from the simulated solar exposure at $600^{\circ} \mathrm{C}$, and $139 \%$ from the thermal exposure at $675^{\circ} \mathrm{C}$. These results are similar to those conducted on other compounds and demonstrate 

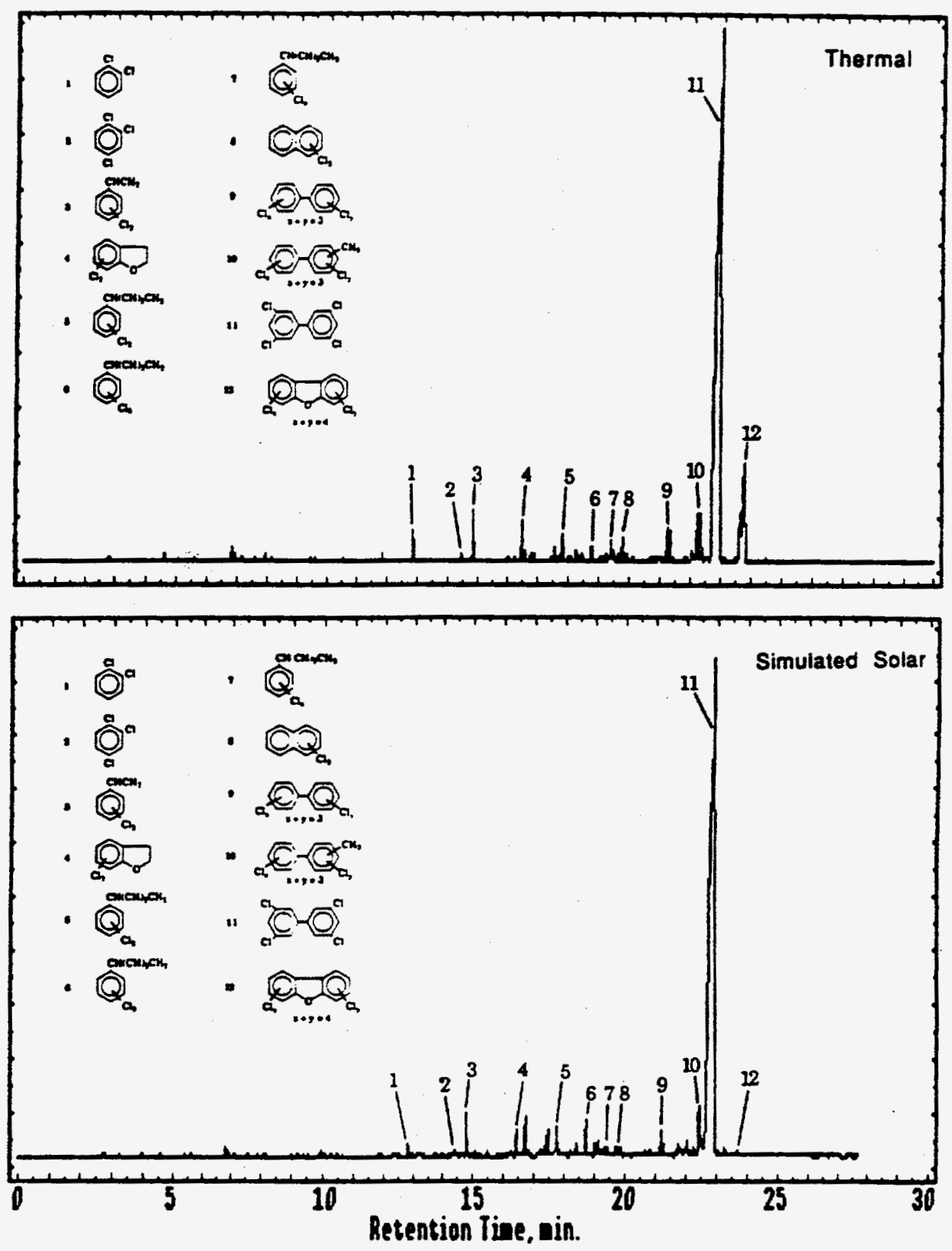

Figure 5.8. Two example TPRS chromatograms from $3,3^{\prime}, 4,4^{\prime}$-tetrachlorobiphenyl $\left(3.78 \times 10^{-6} \mathrm{M}\right)$ exposed to $675^{\circ} \mathrm{C}$, thermal (top), and $273 \mathrm{AM} 1.5$ suns (simulated) at $550^{\circ} \mathrm{C}$ for $10 \mathrm{~s}$ in air (bottom). 
that all major organic products have been accounted for. Also note that for some PICs, several isomers were observed, but isomer specific identification was not possible as in many cases analytical standards were not available.

The relative molar yield as a function of temperature for the lightest identified product, 1,2dichlorobenzene, is shown in Figure 5.9. This Figure shows that this product was formed from the solar exposure with a slightly lower maximum yield, and that it decomposed at a lower temperature than for the thermal tests. Since dichlorobenzene should not absorb the simulated sunlight to any meaningful extent direct photolysis of this product would not be expected. Therefore, the early disappearance of this product suggests a limited production rather than the thermal/photolytic destruction.

The data for 3,4,4'-trichlorobiphenyl, depicted in Figure 5.10, was selected as representative of the moderate molecular weight PICs formed. Note that since this product was observed in all of the low temperature exposures, it was apparently present as a contaminant in the original tetrachlorobiphenyl sample. However, it's increase in concentration with temperature suggests that it was also produced as a PIC. As with dichlorobenzene, the solar product reached a lower maximum yield and was destroyed at a lower temperature than it's thermal counterpart. However, unlike the light molecular weight products, the heavier products are likely to absorb the simulated sunlight. Thus thermal/photolytic decomposition may play an important role in limiting their ultimate yield.

The last example, shown in Figure 5.11, is for the first of three tetrachlorodibenzofuran isomers detected. These products are of interest not only because they are the highest molecular weight PICs observed, but also because they are known to be more toxic than the parent PCB. Furthermore, although it is quite difficult to conduct isomer specific identification for TCDFs on the column used in this analysis, comparisons with analytical standards suggests that this may the extremely hazardous $2,3,7,8$ tetrachlorodibenzofuran isomer. As with the trichlorobiphenyls, these products may have also been present as extremely low level contaminants in the original sample. As the data in Figure 5.11 clearly shows, the yield for the solar destruction of tetrachlorodibenzofuran was much lower than the thermal destruction, and that it was destroyed at a much lower temperature than the thermal destruction product. Furthermore, since tetrachlorodibenzofuran is very likely to be a good absorber of the simulated sunlight, significant thermal/photolytic destruction was likely.

\subsubsection{REFERENCES}

1. H. D. Bist, V. N. Sarin, A. Ojha and Y. S. Jain, Apolied Spec., 24, p. 292, 1970.

2. J. L. Murakami, K. Kaya and M. Ito, J. Chem. Phys., 72, p. 3263, 1980. 


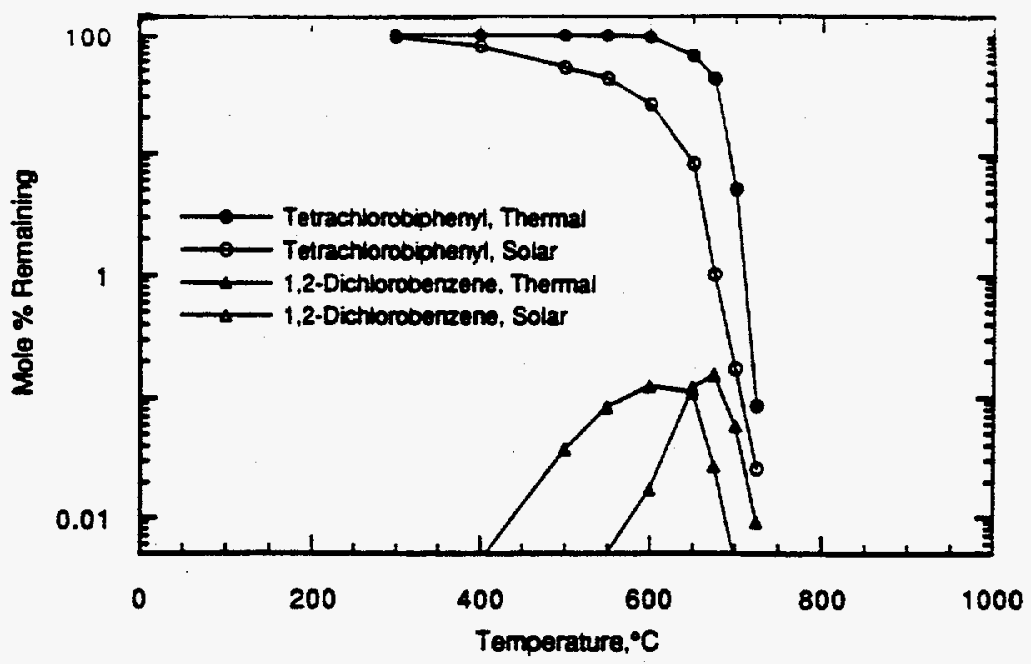

Figure 5.9. The relative molar yield as a function of temperature tor the product, 1.2-dichtoro-benzene

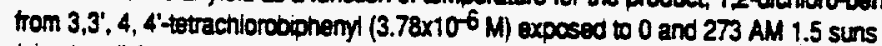
(simulated) for 105 in air.

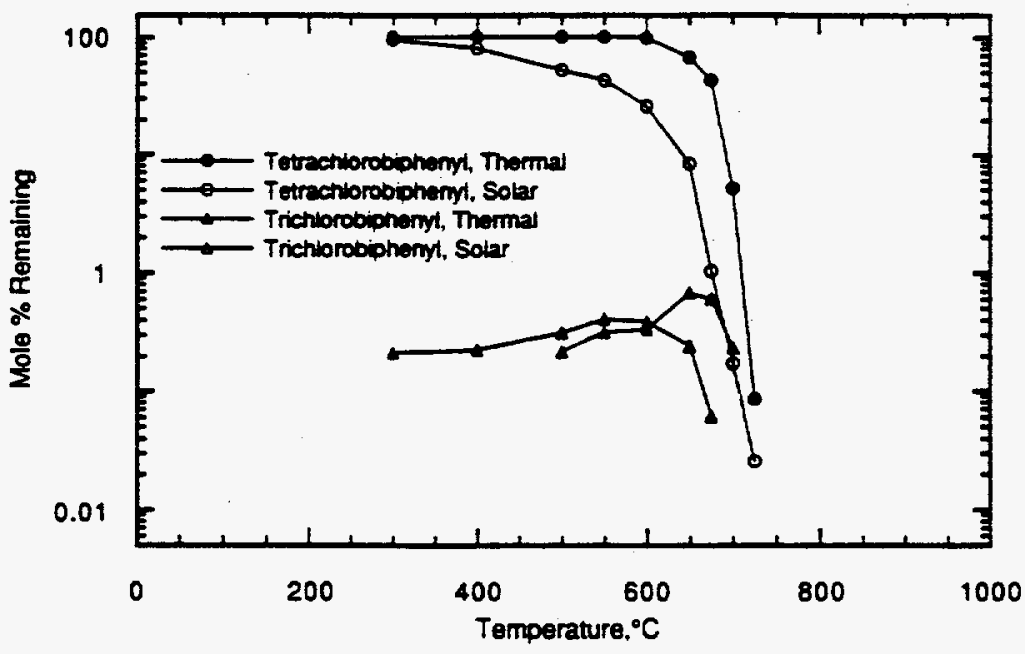

Figure 5.10. The relative molar yeid as a tunction of temperature for the product. 3.4,4-trichiorobiphenyl trom $3.3^{3}, 4,4^{\prime} \cdot$ tetractiorociphenyl $\left(3.78 \times 10^{-6} \mathrm{M}\right)$ exposed to 0 and $273 \mathrm{AM} 1.5$ suns (simuiated) tor $10 \mathrm{~s}$ in air.

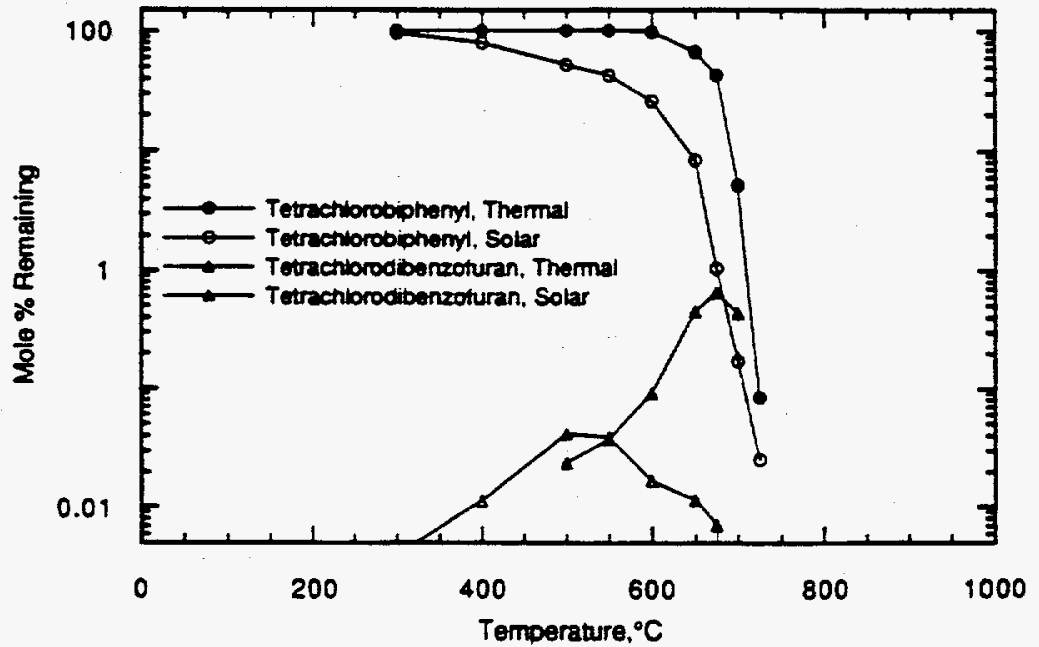

Figure 5.11. The relative molar yield as a tuneton of temperature for the product, tetrachiorodibenzofuran trom 3.3',4.4'-tetractiorocionenyl $\left(3.78 \times 10^{-6} \mathrm{M}\right)$ exposed to 0 and $273 \mathrm{AM} 1.5$ suns (simulated) for $10 \mathrm{~s}$ in air. 
3. Y. S. Jain and H. D. Bist, L.Mol. Spec., 47, p. 126, 1973.

4. R. P. Rava and L. Goodman, L.Am. Chem. Soc., 104, p. 3815, 1982.

5. T. Takeura, M. Fujita and S-i Nagaoka, Chem. Phvs. Lett., 130, p. 39, 1986.

6. C. M. Previtali and T. W. Ebbesen, J. of Photochem., 27, p. 9, 1984.

7. S-i Nagaoka, T. Takemura, H. Baba, N. Koya and K. Morokuma, J. Phys. Chem., 90, p. 759, 1986.

8. C. M. Previtali and T. W. Ebbesen, J.Photochem, 30, p. 259, 1985.

9. S. Nagaoka, T. Takemura and H. Baba, Bull. Chem. Soc. Jpn., 58, p. 2082.

10. A. Tissot, P. Boule and J. Lemaire, Chemosphere, 12, p. 381, 1984.

11. D. R. Arnold and P. C. Wong, J.Am. Chem. Soc., 99, p. 3361, 1976.

12. A. C. Albrecht, L. Chem. Phys., 33, No. 1, pp. 156-169, 1960.

13. T. Ichimura and Y. Mori, L. Chem. Phys., 58, p. 288, 1973.

14. E. R. Ritter, J. Bozzelli and A. M. Dean, Journal Phys. Chem., 94, pp. 2493-2504, 1990.

15. M. Frencklach, M. Ramachandra and A. Matula, Proceedings of the Symposium (Int.) Combustion, 20, 871, 1984.

16. D. R. Kearns, Chemical Review, 17, 395, 1971. 


\section{SECTION 6.0 EFFECTS ON MIXTURES}

Most of the solar detoxification research thus far completed has been conducted using pure analytical standards, and for good reasons. Only through the study of relatively simple reaction schemes can fundamental processes be identified and quantified. However, by their nature as waste products, actual wastes streams are rarely, if ever, available as clean, neat, process streams. Rather, they are typically extremely complex mixtures. Furthermore, it is common practice to blend wastes from a variety of sources to homogenize the waste feed to a detoxification device as much as possible. The problem these mixtures pose from a research standpoint is that the interactions between the numerous components is likely to be so complex, that obtaining any useful, fundamental information about the global process of solar detoxification is unlikely. However, it is useful to evaluate the effectiveness of the process on complex wastes in order to simulate actual waste streams, and to investigate the effect of the process on those components of the waste feed that do not directly absorb solar radiation.

At least two fundamental processes can occur in mixtures that can alter the implications of the results of studies of pure compounds. One is the transfer of excitation energy (or an electron) from one molecular species to another. This can be beneficial to the process of solar detoxification when a strong absorber (i.e., sensitizer) transfers energy (or an electron) to a weakly absorbing toxic component. However, energy transfer can also be detrimental when a toxic component is a strong absorber, but efficiently transfers the absorbed energy to a non-toxic component (i.e., a quencher). In contrast, electron transfer is still expected to be beneficial in this case.

A second process is photo-initiated radical chain reactions. This process would be expected to be largely beneficial. One strongly absorbing species may undergo decomposition to radical products which may then attack non-absorbing species. Once the molecule is converted into a radical by radical attack, then the elevated temperature of the solar detoxification process would be expected to cause the radical to continue to decompose. In contrast, these radicals may not decompose in low temperature photolytic processes and high yields of radical recombination products may result. In some limited instances, photoradical generation may be detrimental. For example, if only $\mathrm{Cl} \bullet$ is generated by photo-initiation, it may act as an oxidation inhibitor.

In the course of this study, three series of experiments were conducted to specifically determine the effect of a complex mixture on the solar detoxification process. Specifically, two studies were conducted in which several surrogate compounds were spiked into a fuel oil stock, and a third in which a 
mixture was composed of various selected analytical standards. Multi-component mixture data are summarized in Appendix III.

\subsection{OXIDATION OF A SPIKED, FUEL OIL MIXTURE-I}

The first set of experiments were conducted with a sample composed of $47 \%$ (w/w) fuel oil, $30 \%$ chloronitrobenzene, $8 \%$ dichlorobenzene, $8 \%$ trichloroethylene, and $7 \%$ carbon tetrachloride. This sample was obtained from a commercial hazardous waste incinerator and was taken from the actual feed stock used in a trial burn demonstration. The mixture was exposed in the TPRS to 0 and 307 AM 1.0 suns for 5 seconds in air. Of the specific contaminants, the carbon tetrachloride, trichloroethylene, and fuel oil do not absorb the simulated solar radiation, the dichlorobenzene may weakly absorb the simulated sunlight at high temperatures because of the spectral shift with temperature, while the chloronitrobenzene is expected to have a modest absorption in the solar regime.

The data shown in Figure 6.1 shows that the simulated solar exposure had a beneficial effect on the destruction of the mixture taken as a whole in only a small temperature range (approximately $\pm 50^{\circ} \mathrm{C}$ ) centered at $500^{\circ} \mathrm{C}$. Interestingly, the data suggest the presence, or production, of a low level ( 1\%) of extremely stable components at temperatures above $700^{\circ} \mathrm{C}$. In contrast, the data for the specific contaminants show varying degrees of solar enhancement. Specifically, the data for carbon tetrachloride and trichloroethylene, which were not chromatographically resolved (cf. Figure 6.2) again show enhanced destruction at $500^{\circ} \mathrm{C}$, comparable conversions at 500 and $600^{\circ} \mathrm{C}$, after which the solar process destroys these compounds more efficiently than the thermal process. This is a very interesting result as these compounds do not directly absorb any of the solar radiation, so their enhanced destruction has to be the result of an indirect solar process. Similar results are seen in Figure 6.3 for dichlorobenzene where there is comparable conversion up to $600^{\circ} \mathrm{C}$, after which an increasing level of solar enhancement is observed. In this case, the dichlorobenzene may weakly absorb solar energy at high temperatures, whereas it is unlikely to do so at low temperatures. Finally, the data for chloronitrobenzene in Figure 6.4 shows the solar process completely destroys this component at temperatures above $500^{\circ} \mathrm{C}$. Unlike the other contaminants, chioronitrobenzene is expected to absorb solar energy at all temperatures, so the enhancement of its destruction is likely the result of a direct solar process.

\subsection{OXIDATION OF A SPIKED, FUEL OIL MIXTURE-II}

The second series of tests was conducted with a contaminated fuel oil stock consisting of $40 \%$ fuel oil (v/v), $40 \%$ dichlorobenzene, $10 \%$ carbon tetrachloride, $5 \%$ toluene, and $5 \%$ naphthalene. This sample was prepared by Midwest Research Institute (MRI) for use in pilot-scale testing of the solar detoxification 


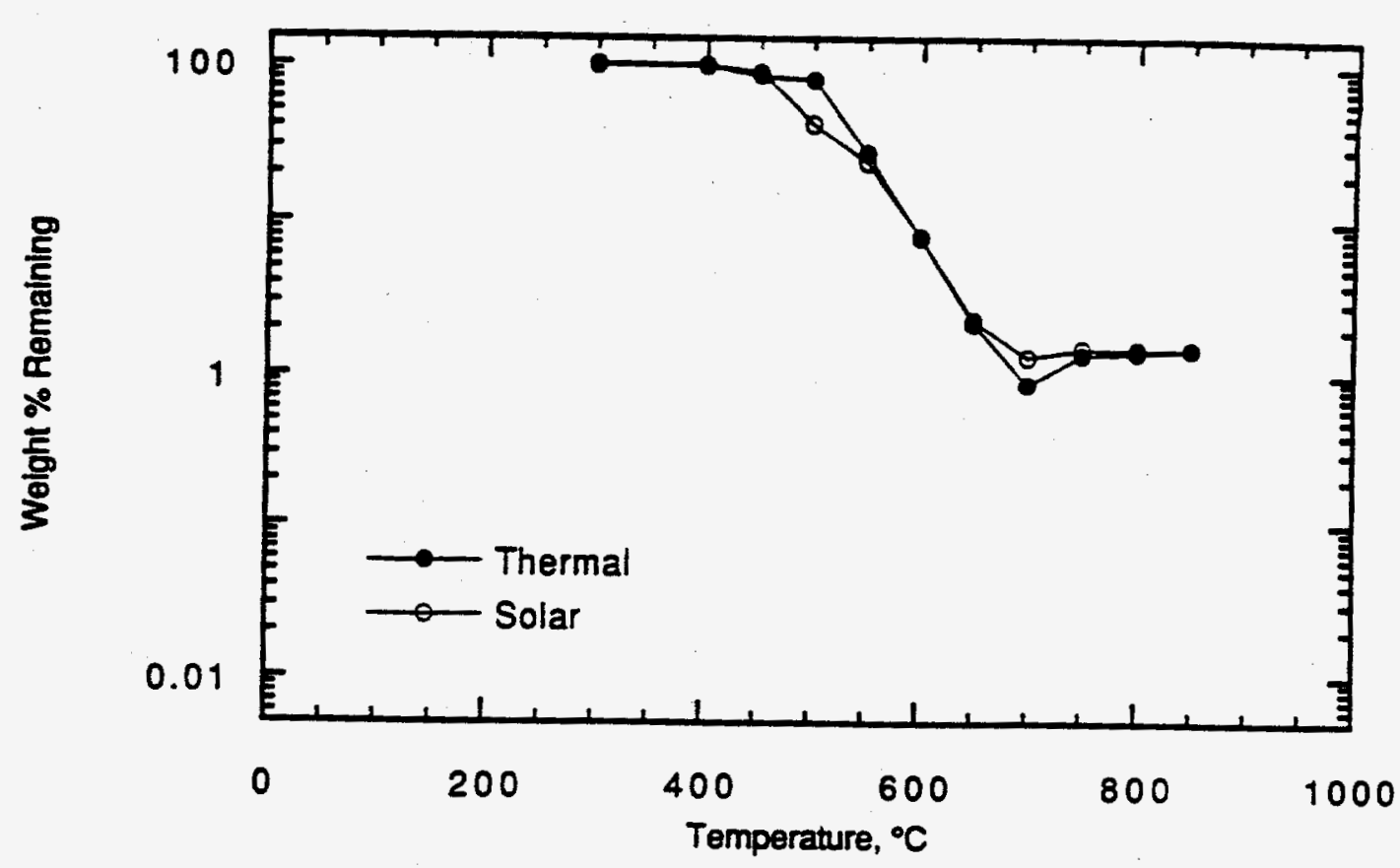

Figure 6.1. TPRS data for the oxidation of a fuel oil mixture spiked with chloronitrobenzene, dichlorobenzene, trichloroethylene, and carbon tetrachloride exposed to 0 and 307 AM 1.0 suns (simulated) for $5 s$ in air. These curves are for total HFID response that includes feed components and PICs.

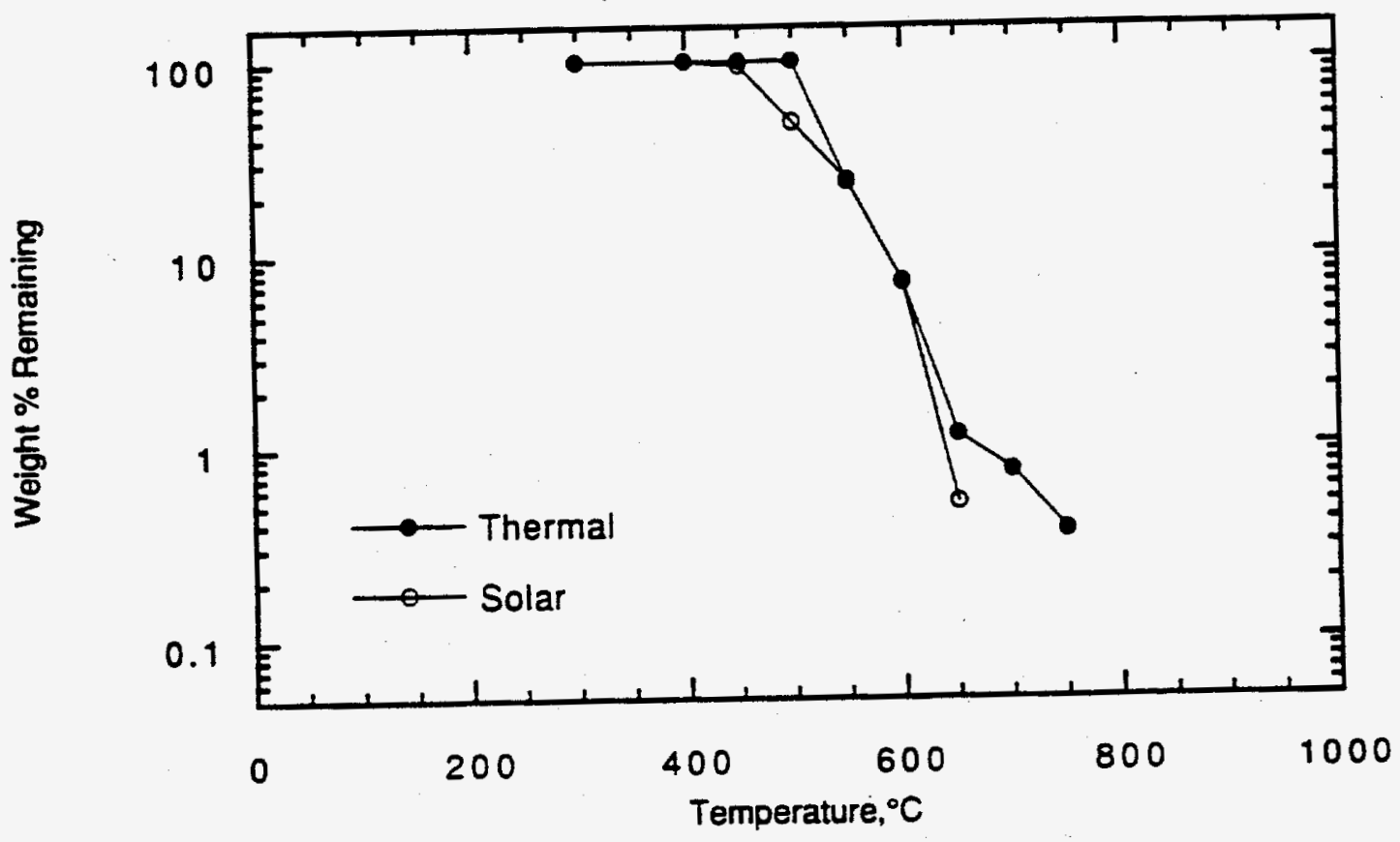

Figure 6.2. TPRS data for the combined carbon tetrachloride and tichloroethylene components of a spiked tuel oil exposed to 0 and 307 AM 1.0 suns (simulated) for 5 s in air. 


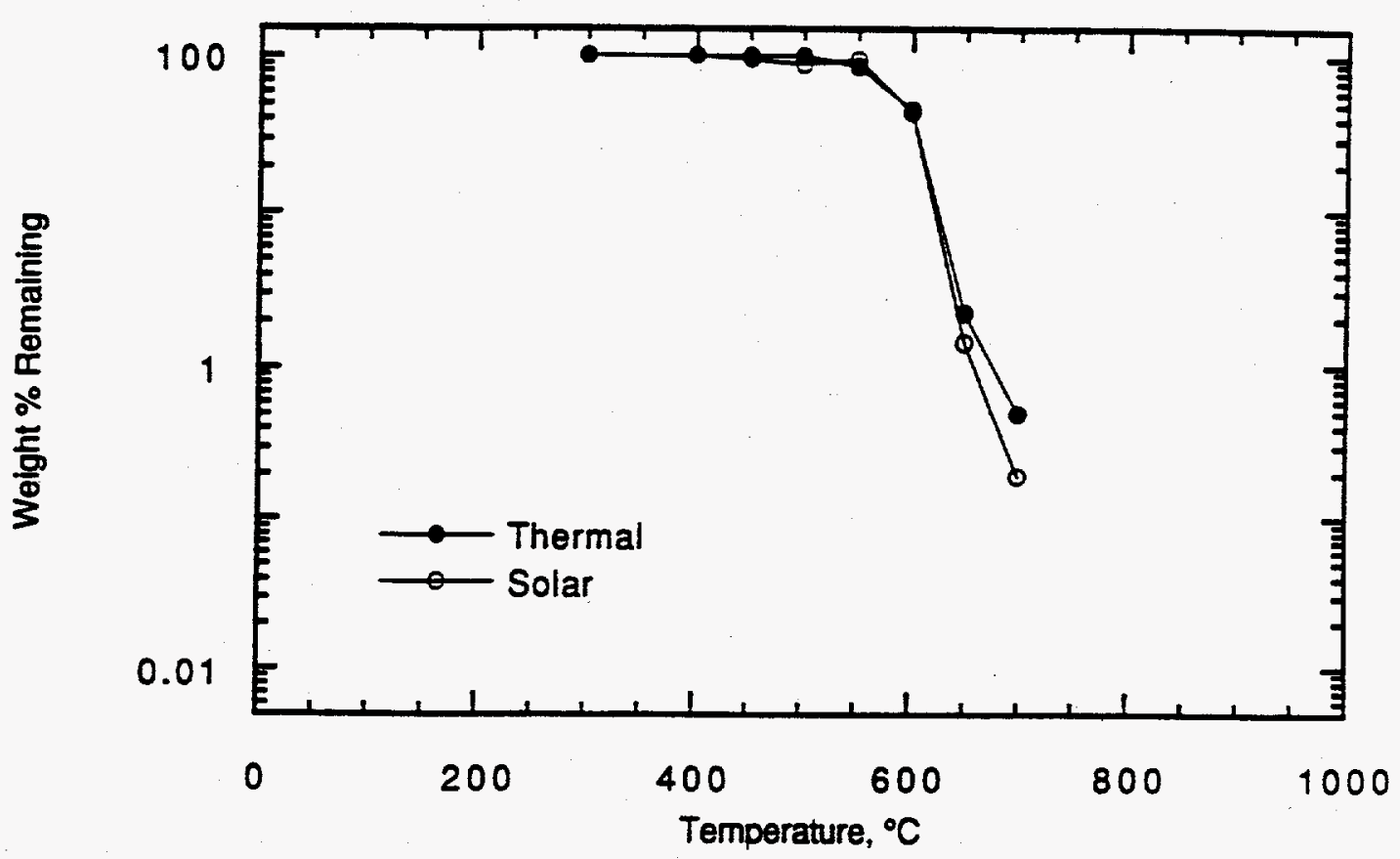

Figure 6.3. TPRS data for the dichlorobenzene component of a spiked tuel oil exposed to 0 and 307 AM 1.0 suns (simulated) for $5 \mathrm{~s}$ in air.

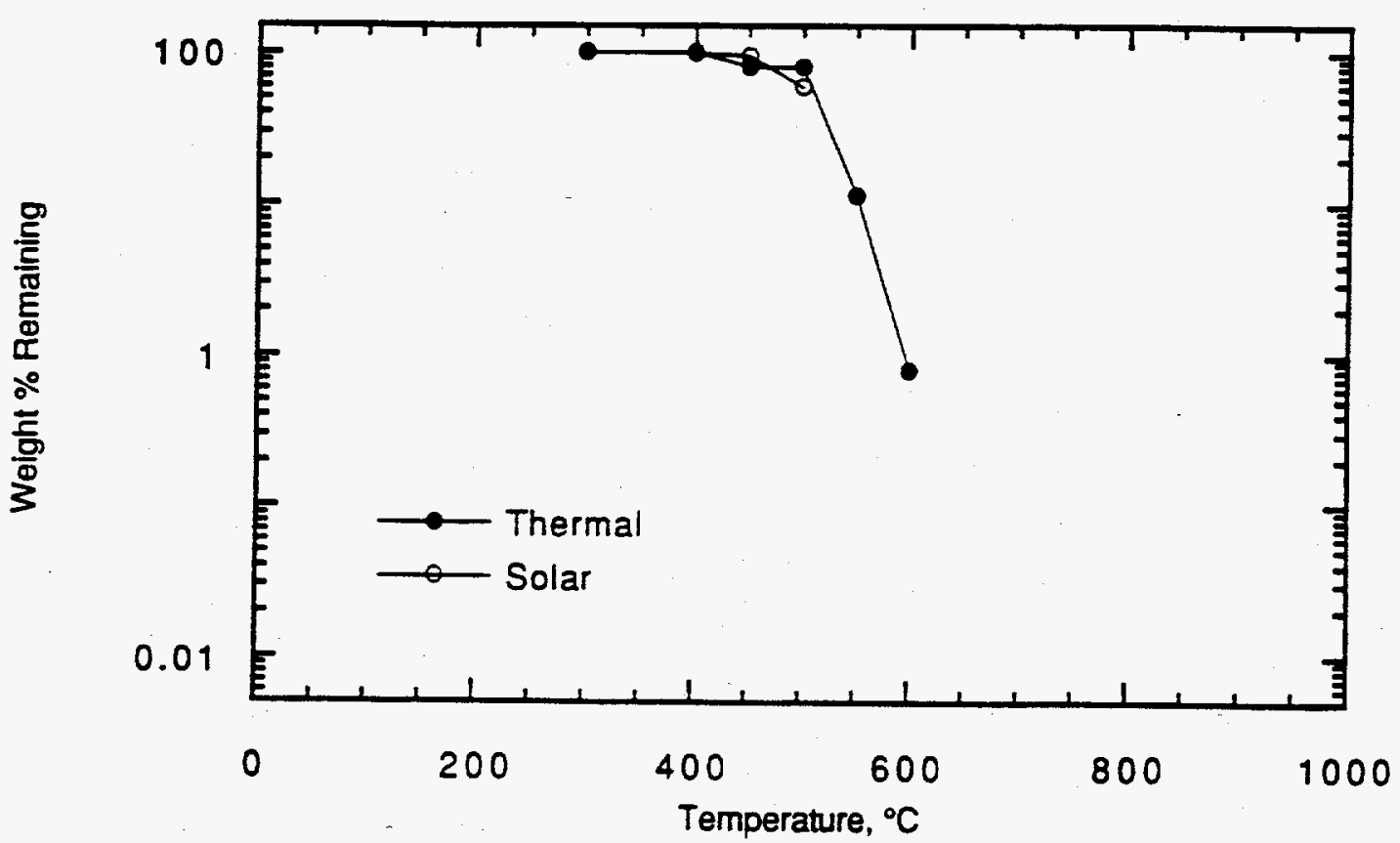

Figure 6.4. TPRS data for the chloronitrobenzene component of a spiked fuel oil exposed to 0 and 307 AM suns (simulated) for $5 s$ in air. 
process as part of the Tri-Agency (DOE, U.S.-EPA, USATHAMA) Initiative. Of the materials present in this mixture, only the dichlorobenzene and naphthalene components are likely to absorb solar energy, and then only very weakly. In these tests, the sample was exposed to 0 and 273 AM 1.5 suns for 10 seconds in air.

The TPRS data for the waste mixture taken as a whole is shown in Figure 6.5 , and demonstrates that the simulated solar radiation resulted in a small improvement in reactor performance between 400 and $700^{\circ} \mathrm{C}$. Above $800^{\circ} \mathrm{C}$ the data show an unexpected result of the solar exposure actually reducing the reactor performance. Similar results were found for the toluene (cf. Figure 6.6) and naphthalene (cf. Figure 6.7), where a small solar enhancement is seen at low temperatures, but the solar exposure apparently retarding the reactor performance at higher temperatures. In contrast, the reactor performance for the destruction of dichlorobenzene was essentially unaffected by the simulated solar radiation (cf. Figure 6.8).

The apparent reduction in reactor periormance upon irradiation for most of the components in this waste mixture is certainly an unexpected result. Quenching of photo-excited species by non-toxic or nonreacting components is a possible explanation. Alternatively, it is possible that chlorine produced from dichlorobenzene decomposition is acting as an oxidation inhibitor. Preliminary theoretical kinetic modeling studies (on a different mixture composition) suggest that could occur (see Appendix Vi for complete details of this study). It is also possible that some of these components are produced as PICs as the result of the enhanced solar destruction of some other species which were not quantified in this study. One mechanism to explore this issue further would be to compare the rates of destruction in that waste with the rate of destruction of the pure compounds to determine if the rates of destruction in the waste were significantly different than for the pure components.

\subsection{OXIDATION OF A SYNTHETIC 8 COMPONENT MIXTURE}

A third series of tests on a complex mixture was conducted on a sample composed of eight selected analytical standards. Specifically, the mixture consisted of $21 \%$ methylene chloride (mole/mole), $21 \%$ trichloroethylene, $22 \%$ pyridine, $17 \%$ aniline, $14 \%$ nitrobenzene, $2 \%$ naphthalene, $1.5 \%$ nitronaphthalene, and $1.5 \%$ xanthone. Of these components, methylene chloride, trichloroethylene, and pyridine are non absorbers of solar radiation (onset of absorption below $300 \mathrm{~nm}$ ), aniline and nitrobenzene are weak absorbers (onset below $305 \mathrm{~nm}$ ), naphthalene moderately absorbs (onset below $320 \mathrm{~nm}$ ), while nitronaphthalene and xanthone are strong absorbers (onset well above $360 \mathrm{~nm}$ ). Therefore, the nonabsorbing components comprise $64 \%$ of the mixture, compounds that have been shown to be photoactive within the solar spectrum make up $17 \%$ of the mixture, and the photosensitivity of the remaining $19 \%$ is questionable. During the testing the sample was exposed to 0 and $192 \mathrm{AM} 1.0$ suns for 10 seconds in air. 


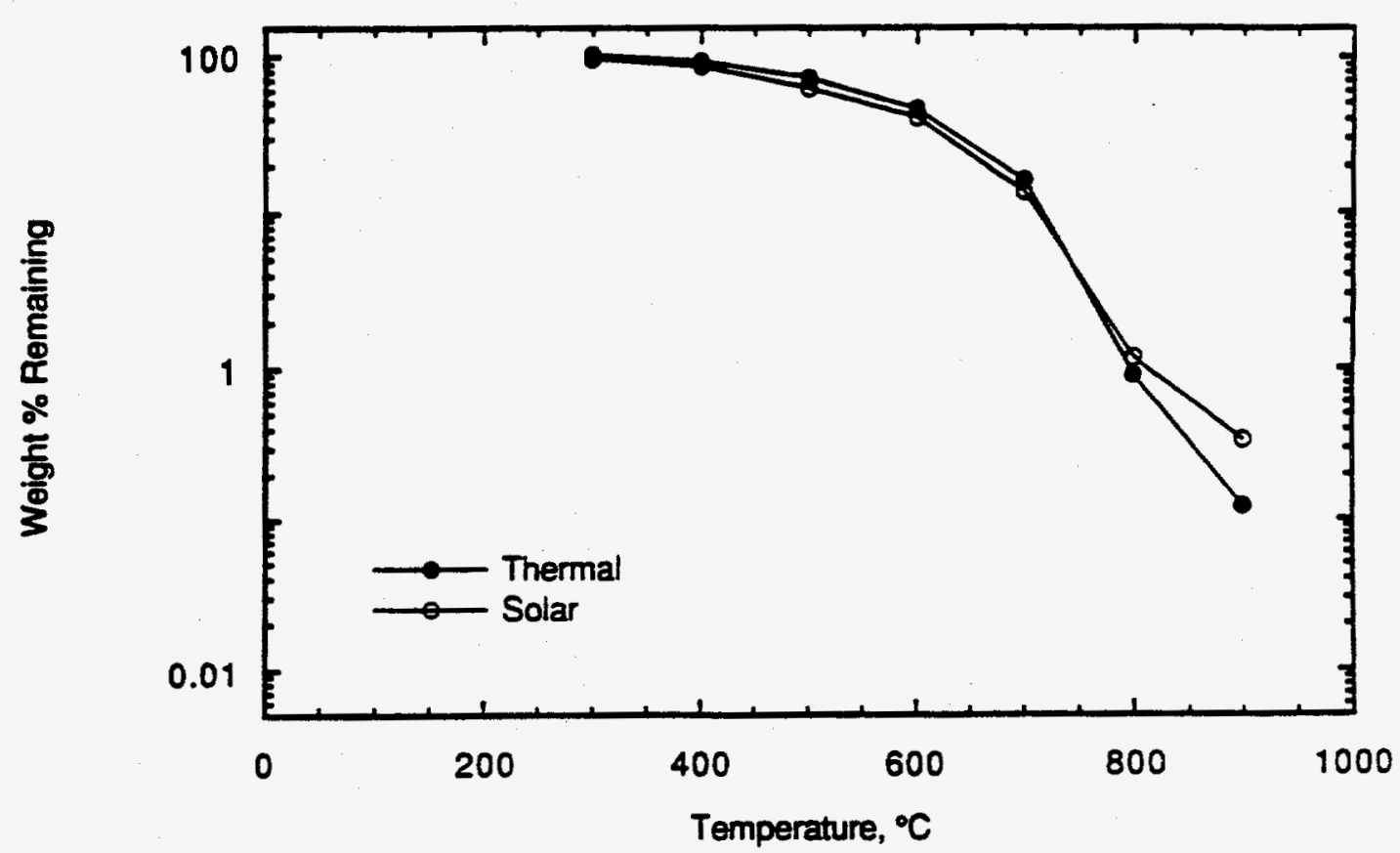

Figure 6.5. TPRS data for the oxidation of a fuel oil mixture spiked with dichlorobenzene, carbon tetrachloride, toluene, and naphthalene exposed to 0 and 273 AM 1.5 suns (simulated) for $10 s$ in air. These curves are for total HFID response and include both feed components and PICs.

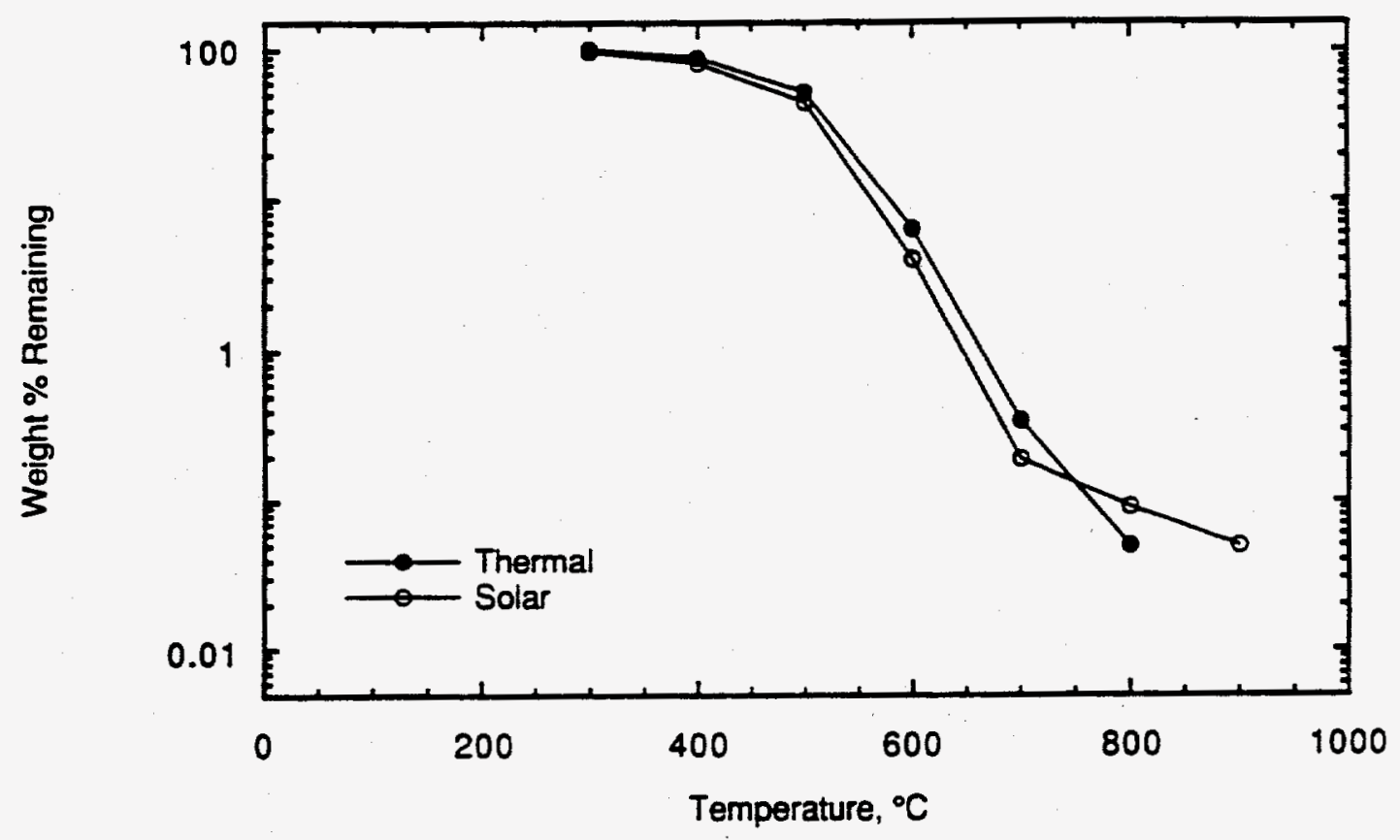

Figure 6.6. TPRS data for the toluene component of a spiked fuel oil exposed to 0 and 273 AM 1.5 suns (simulated) for 10 s in air. 


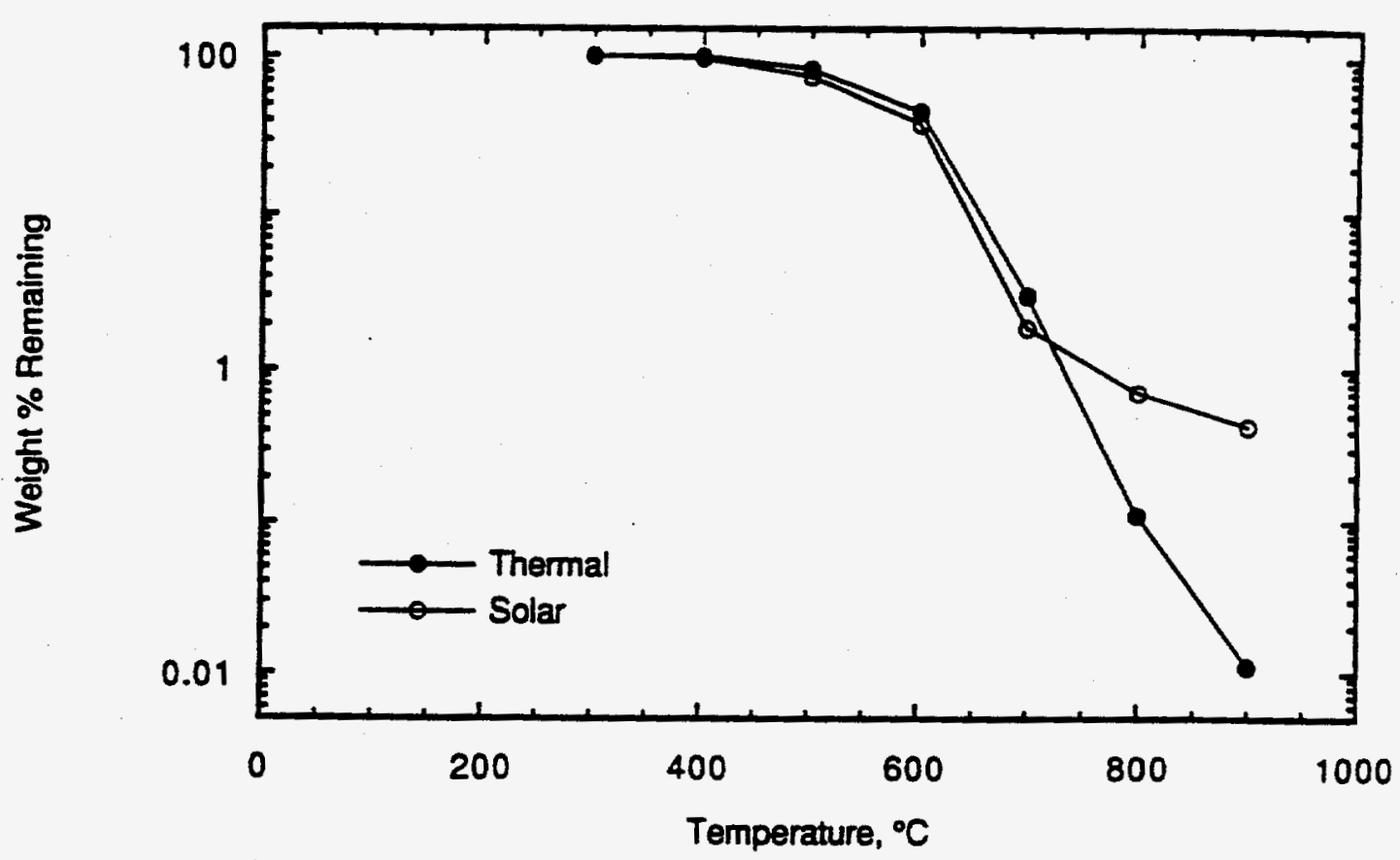

Figure 6.7. TPRS data for the naphthalene component of a spiked fuel oil exposed to 0 and 273 AM 1.5 suns (simulatod) for 10 s in air.

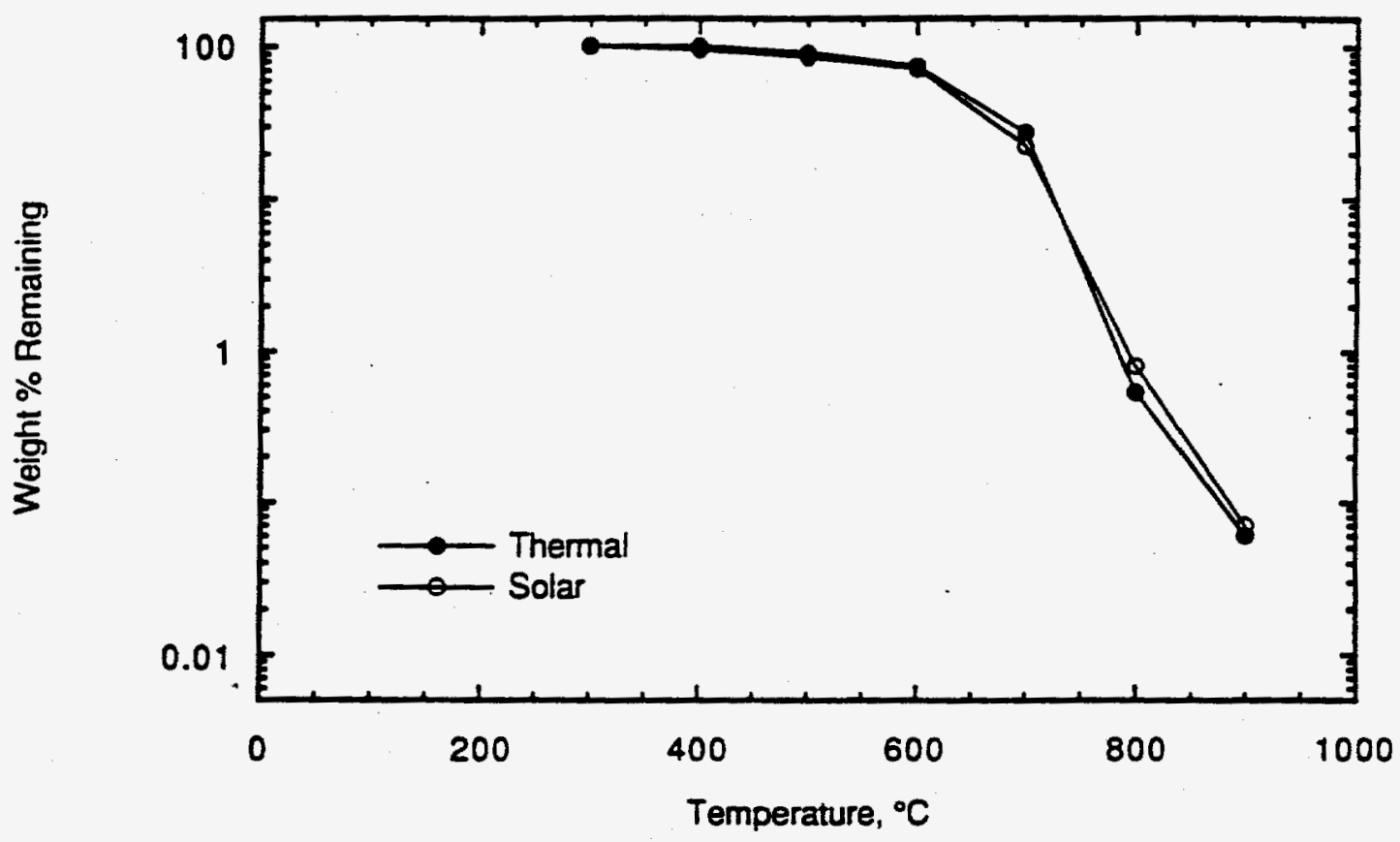

Figure 6.8. TPRS data for the dichlorobenzene component of a spiked fuel oil exposed to 0 and 273 AM 1.5 suns (simulated) for $10 \mathrm{~s}$ in air. 
The data for each of the components, with the exception of xanthone, are summarized in Figures 6.9 through 6.15. Unfortunately, the chromatography of xanthone was of insufficient quality to permit quantification of this component. In contrast to the contaminated fuel oils, most of the components of this mixture showed significant solar enhancement. Specifically, of the three compounds identified as nonabsorbers, methylene chloride (cf. Figure 6.9) showed a large enhancement at high temperature with a value of $R$ reaching a value of 48 , trichloroethylene (cf. Figure 6.10 ) showed a small level of enhancement with a mean $R$ of about 2 , and pyridine (cf. Figure 6.11) showing a large enhancement with a value of $R$ reaching 130. Of the weak absorbers, aniline (cf. Figure 6.12) showed a large enhancement ( $R$ of 54 ), and nitrobenzene (cf. Figure 6.13) showed a moderate level of improvement ( $R$ of approximately 5 ). Naphthalene (cf. Figure 6.14) which is a moderate absorber and has previously been shown to be relatively photochemically inactive under simulated solar radiation, and showed only a moderate level of enhancement ( $R$ of about 3 ). In contrast, nitrobenzene (Figure 6.15), which is considered a very strong absorber of the simulated solar radiation, showed a significant enhancement of it's destruction throughout the temperature range studied with a enhancement of up to 110 .

From the body of data here it is clear that complex mixtures have a variety of effects on the performance of a solar detoxifier. It is particularly interesting, and encouraging, that the destruction of non absorbing materials like methylene chloride can still benefit from the solar detoxification process, though the extent of improvement seems to be significantly effected by the composition of the mixture in which it is placed. Specifically, individual components of the two fuel oil wastes showed only moderate, or even detrimental, response to the solar detoxification process. In contrast, the simpler, yet still complex, waste showed an overall beneficial effect that was quite significant. In related, U.S.-EPA sponsored research, we studied the decomposition of the fuel oil mixture discussed in Section 6.2 spiked onto a humus rich soil (cf. Appendix VII). The desorbed materials from the soil resulted in improved destruction of the toxic components.

Unfortunately, the study of these complex waste mixtures raise more questions than answers. Additional, more fundamental studies on relatively simple mixtures (e.g., two or three components) designed specifically to investigate mixture effects are clearly needed. 


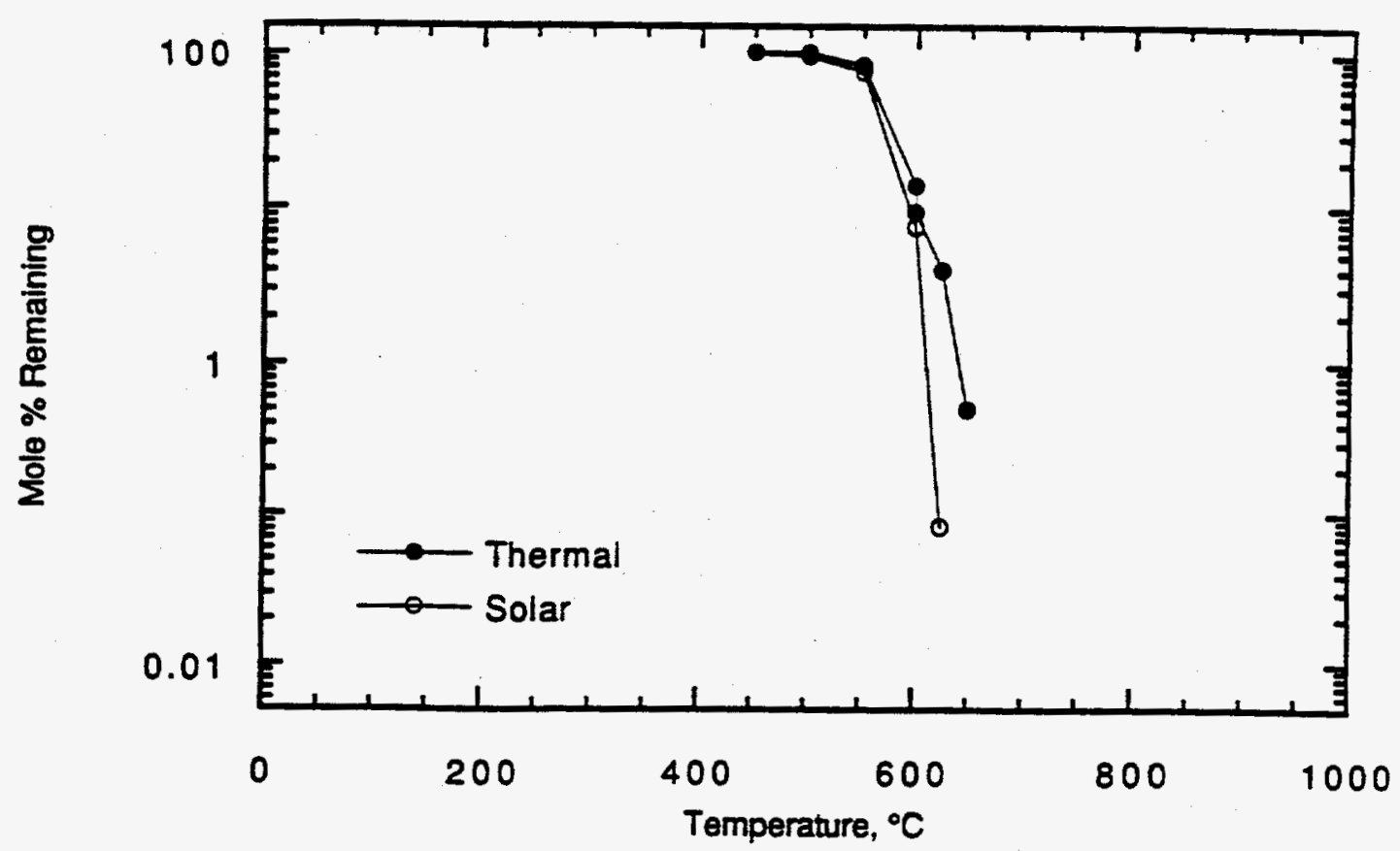

Figure 6.9. TPRS data for the methylene chioride component of an 8 component mixture exposed to 0 and 192 AM 1.0 suns (simulated) for 10 s in air.

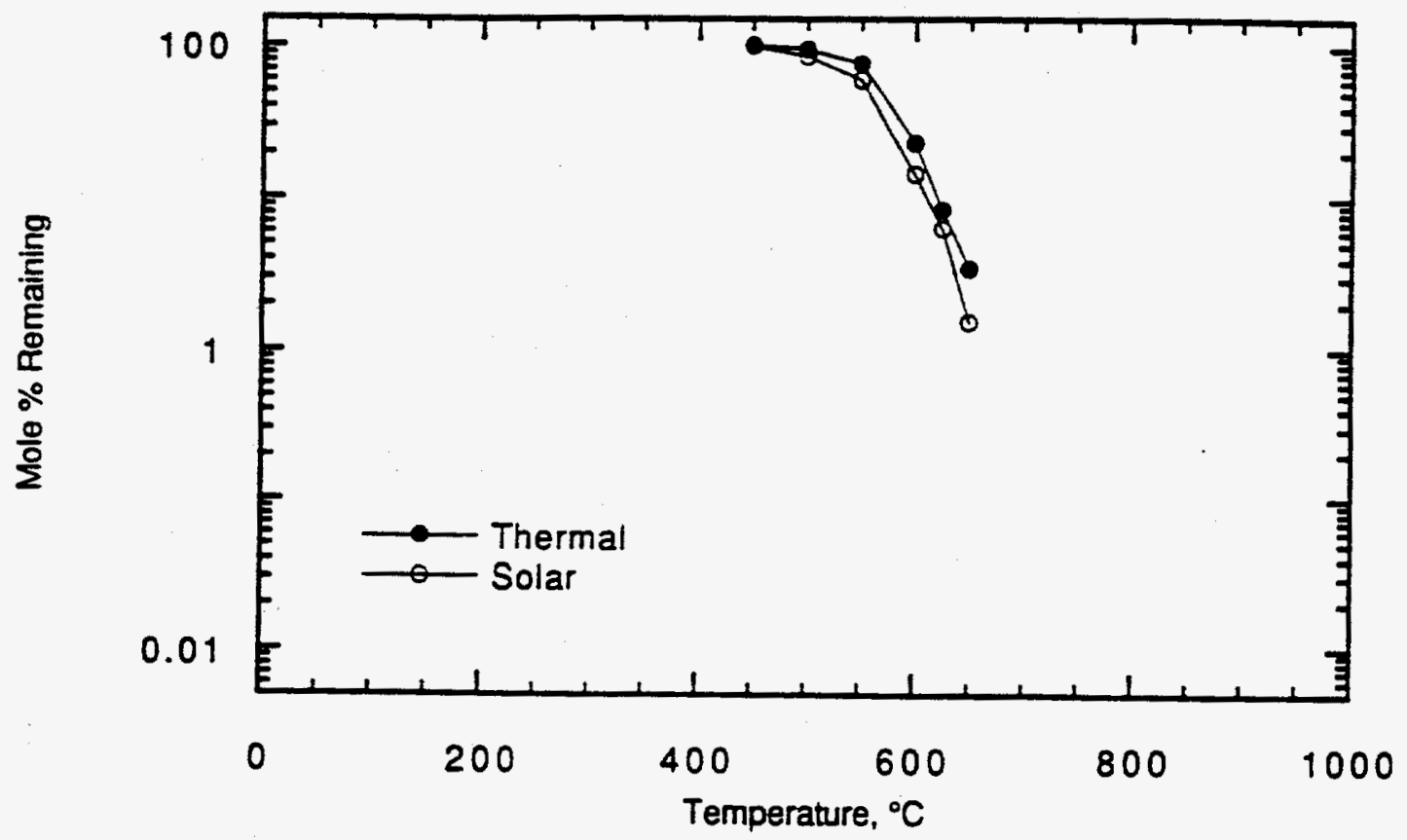

Figure 6.10. TPRS data for the trichloroethylene component of an 8 component mixture exposed to 0 and 192 AM 1.0 suns (simulated) for $10 \mathrm{~s}$ in air. 


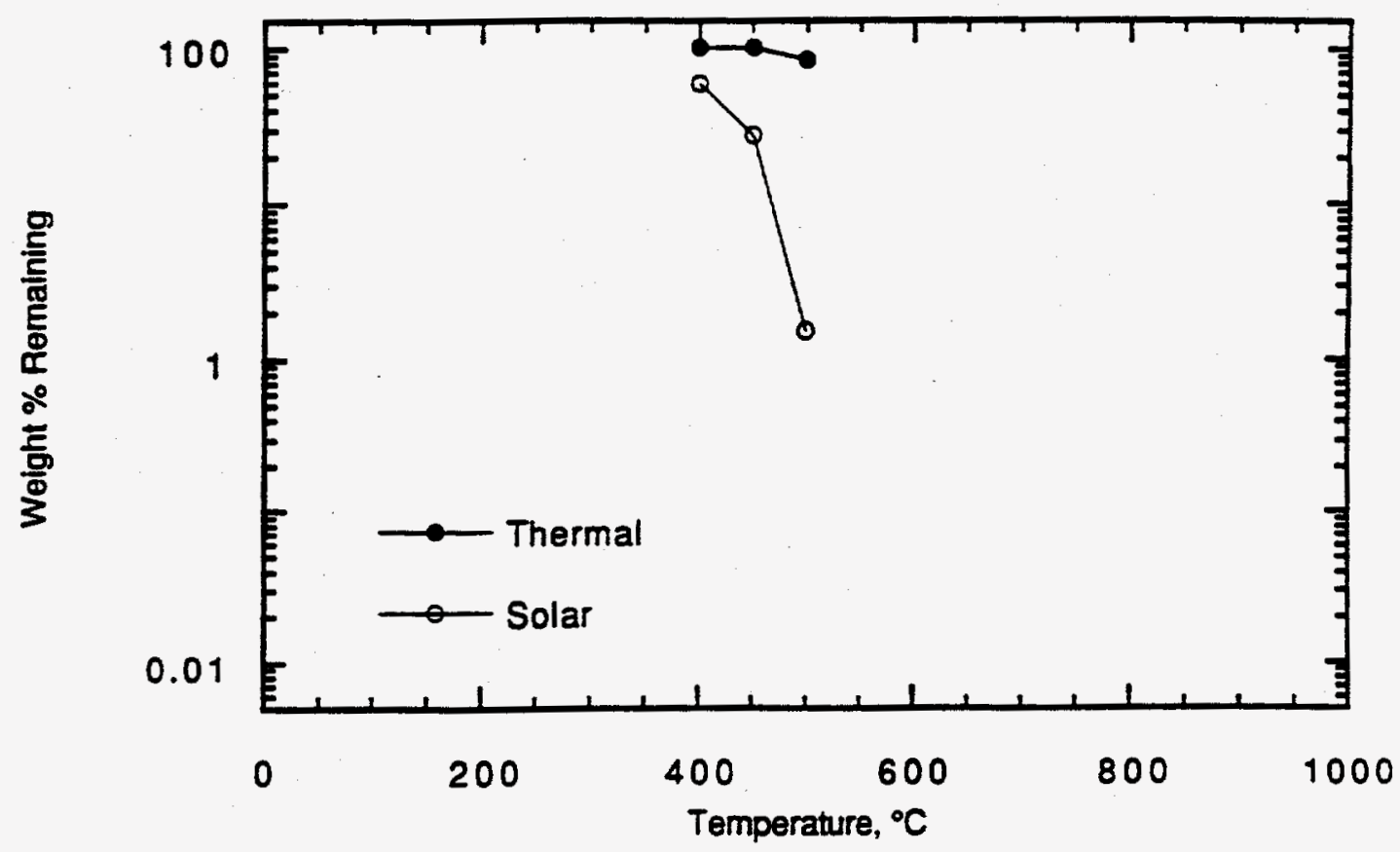

Figure 6.11. TPRS data for the aniline component of an 8 component mixture exposed to 0 and $192 \mathrm{AM}$ 1.0 suns (simulated) for $10 \mathrm{~s}$ in air.

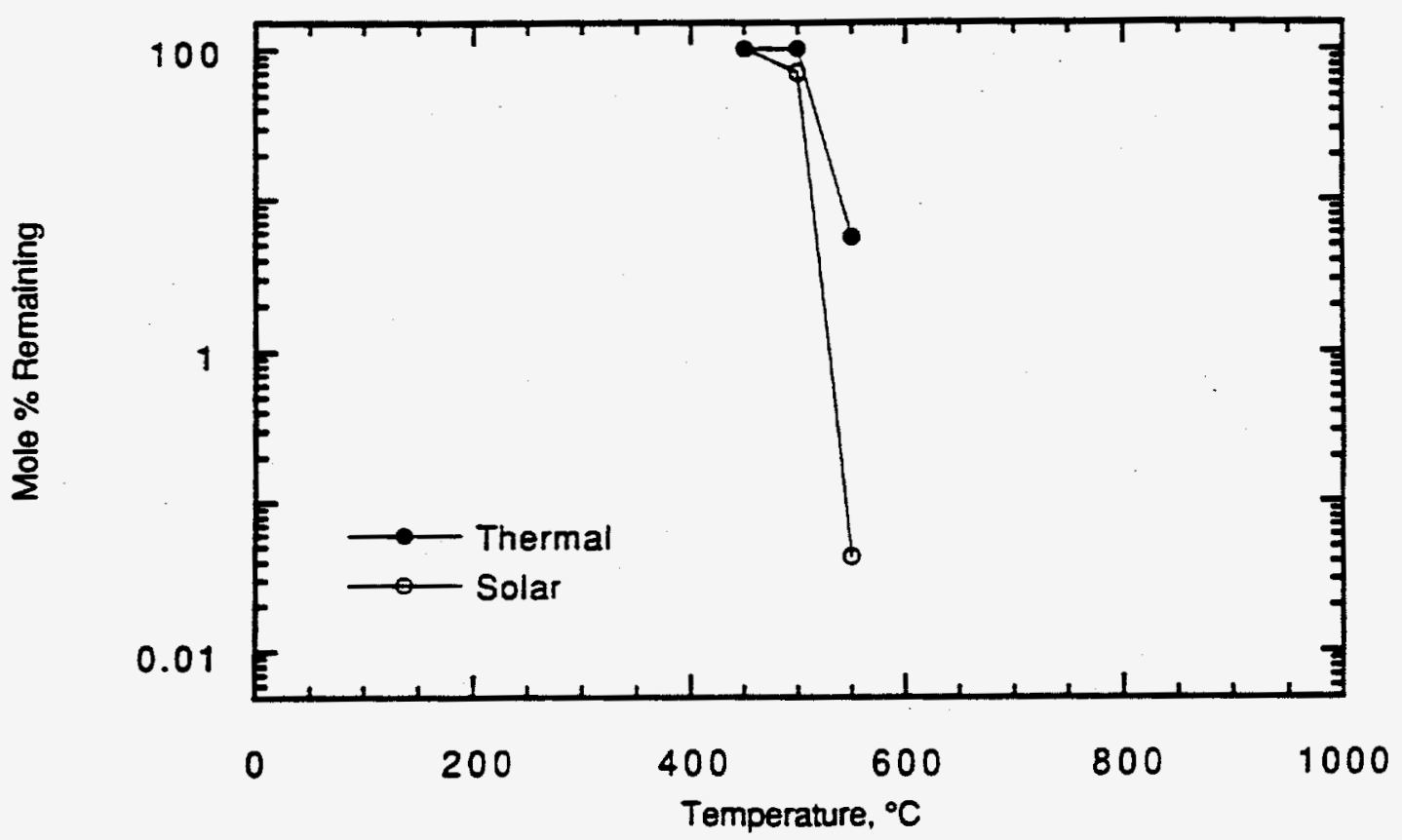

Figure 6.12. TPAS data for the pyridine component of an 8 component mixture exposed to 0 and 192 AM 1.0 suns (simulated) for $10 \mathrm{~s}$ in air. 


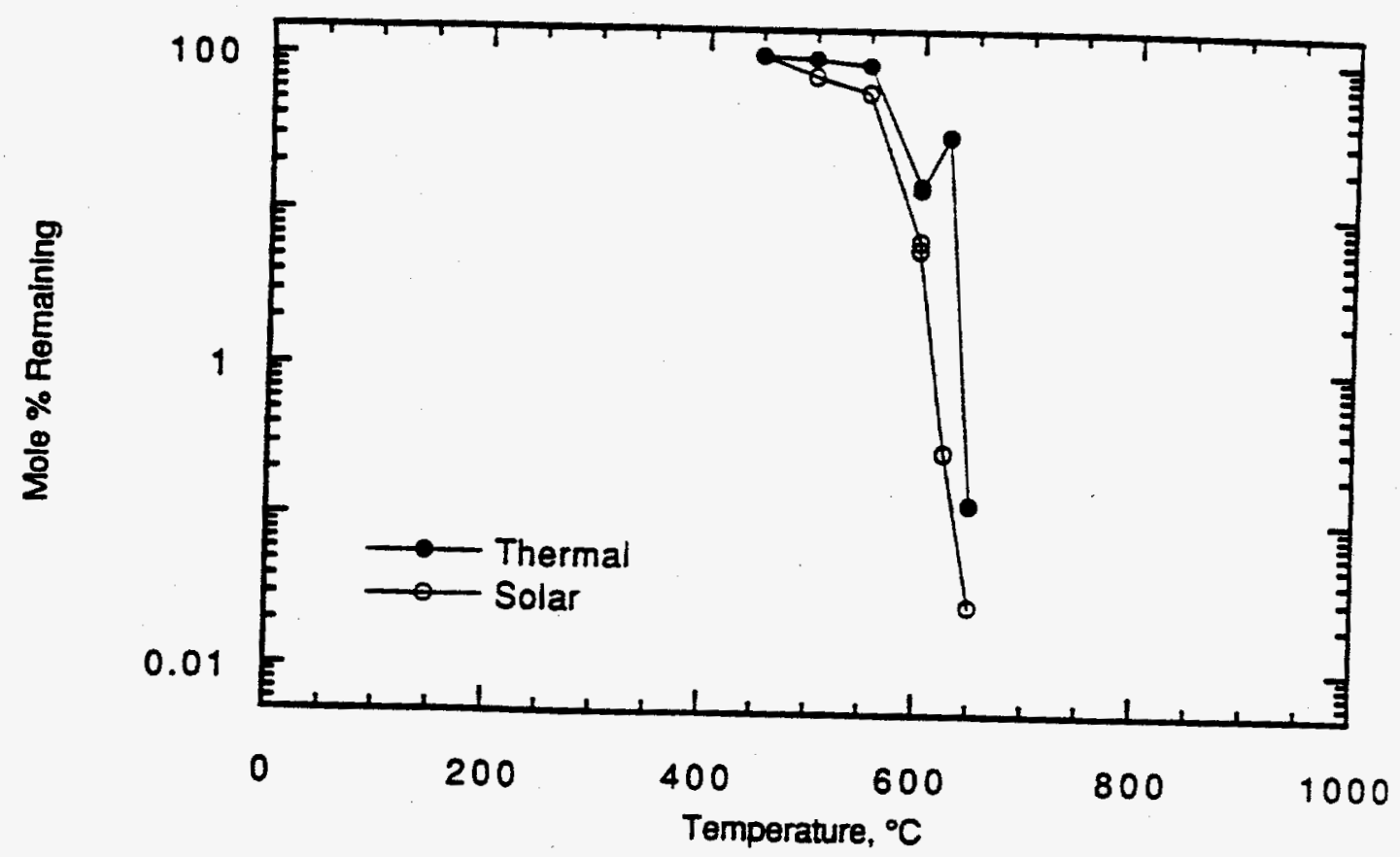

Figure 6.13. TPRS data for the nitrobenzene component of an 8 component mixture exposed to 0 and 192 AM 1.0 suns (simulated) for 10 s in air.

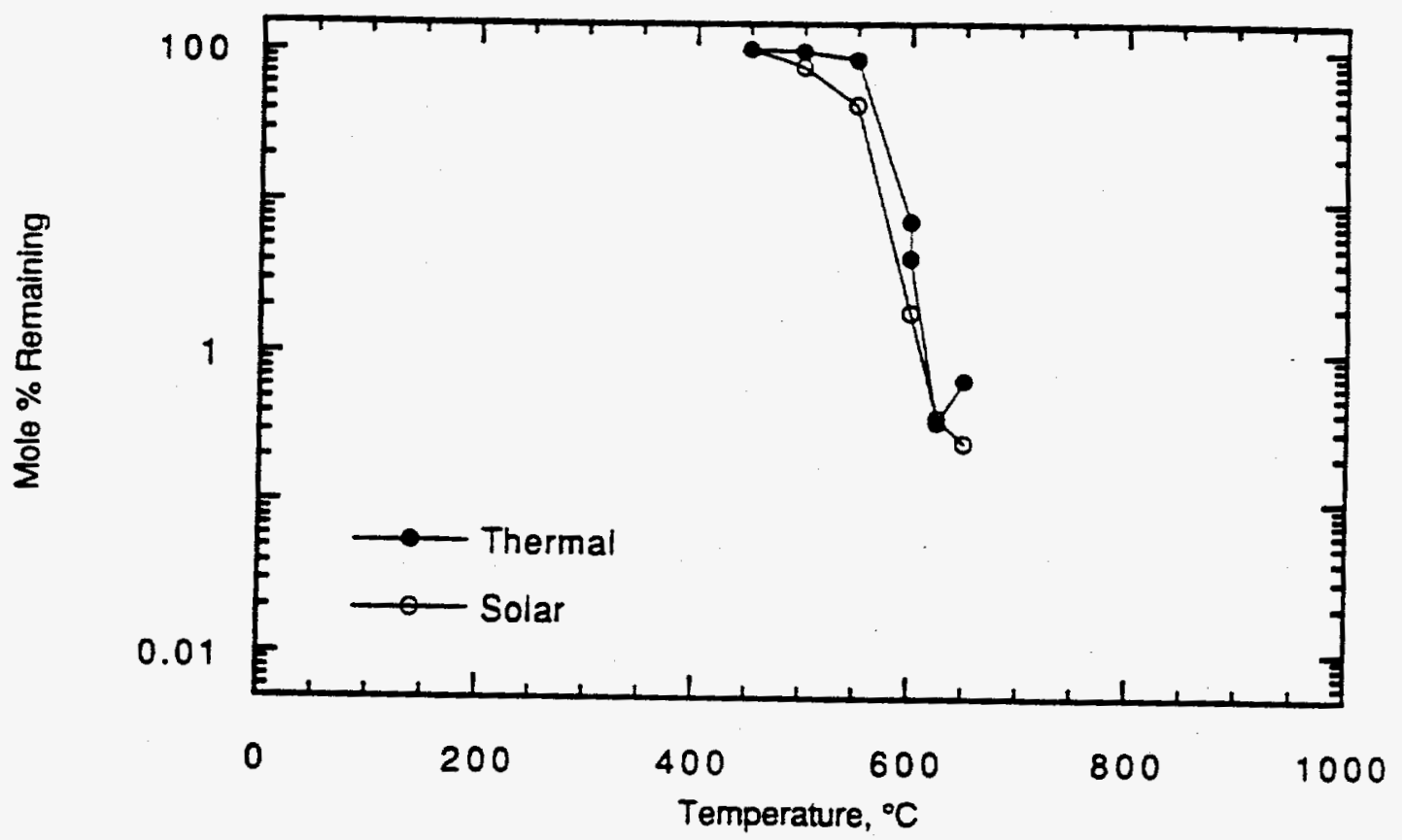

Figure 6.14. TPRS data tor the naphthalene component for an 8 component mixture exposed to 0 and 192 AM 1.0 suns (simulated) for 10 s in air. 


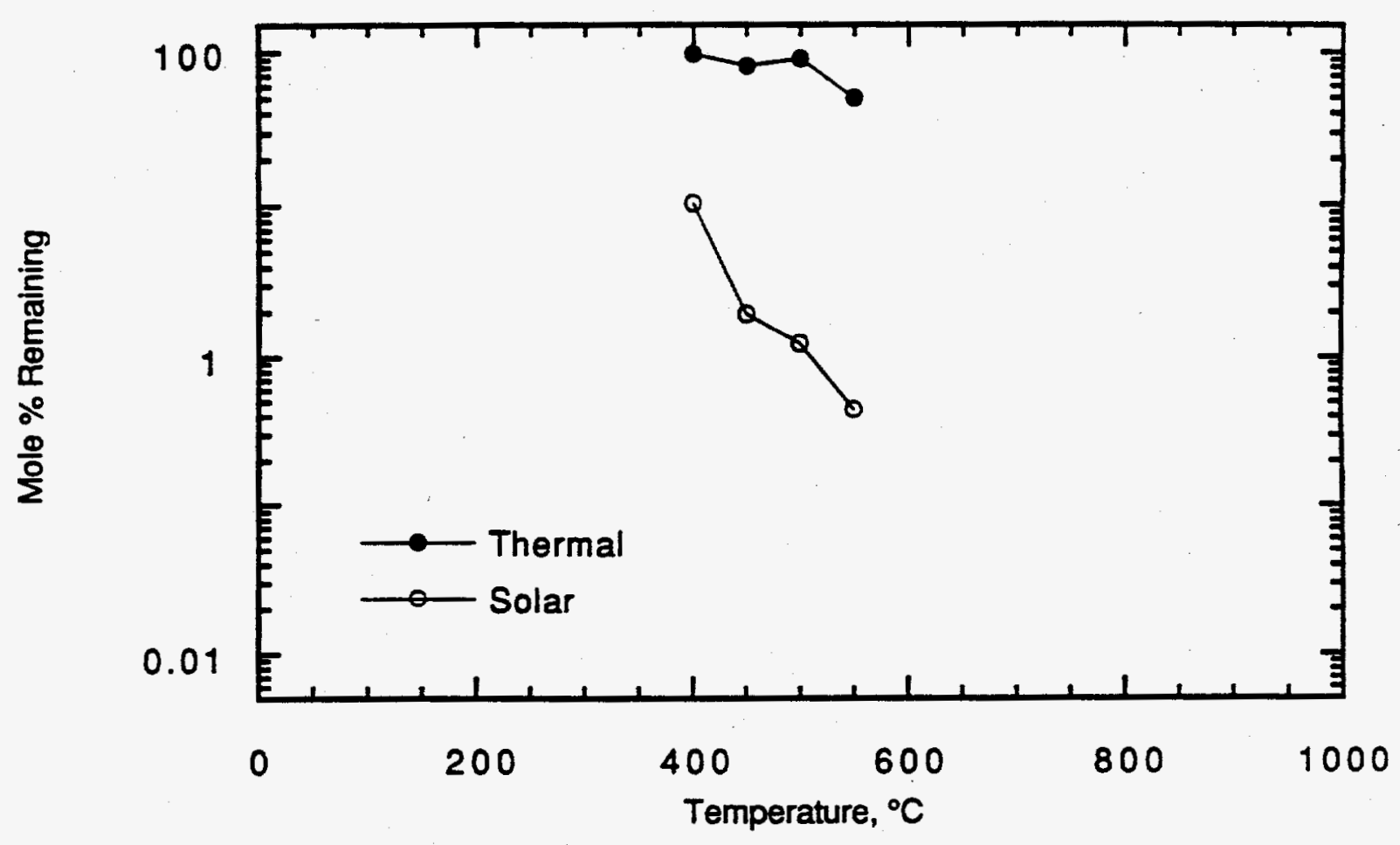

Figure 6.15. TPRS data for the 1-nitronaphthalene component of an 8 component mixture exposed to 0 and 192 AM 1.0 suns (simulated) for $10 \mathrm{~s}$ in air. 


\section{SECTION 7.0 \\ HIGH-TEMPERATURE SPECTROSCOPY}

Solar-enhanced destruction of hazardous waste is predicated on the general process that we have referred to as high-temperature photochemistry. Our solar photochemical studies have suggested that temperature can alter photochemical reaction rates by altering both the rate of absorption of radiation and the rate absorption of radiation and the rate of excited state reactions. The effects of temperature on the rates of photochemical reactions are essentially unresearched outside of our previous NREL sponsored studies.

To better understand the underlying physical and photochemical processes, we have begun a study of the high-temperature spectroscopy and molecular dynamics of benzene, chlorobenzene, and $3,3^{\prime}, 4,4^{\prime}$-tetrachlorobiphenyl. This research may be broken down into studies concerning the effect of temperature on UV absorption, and the effect of temperature on the rates of competing non-photochemical processes in the excited state via emission experiments.

\subsection{THE GROUND ELECTRONIC STATE}

As the temperature of a molecular system is increased, higher lying levels of the ground electronic state, $S_{0}$, will be increasingly populated. It is well known that this thermal energy decreases the frequency of electromagnetic radiation required to promote the molecule to an electronically excited state. However, population of ground state vibrational levels can also have more subtle effects that can significantly effect the solar detoxification process. To further explore these phenomena we constructed the HTAS and have thus far conducted a small series of tests on a limited number of compounds. The results of these tests are given in the following paragraphs.

\subsubsection{EXPERIMENTAL}

Benzene (Aldrich, 99.99\%, HPLC Grade) was syringe pump injected into the HTAS as a gas at its room temperature vapor pressure ( 81 Torr) producing a constant concentration of $7.0 \times 10^{-5} \mathrm{~mol} / \mathrm{L}$. Chlorobenzene (Aldrich, 99.99\%, HPLC Grade) was injected liquid phase expanding to a constant $9.1 \times$ $10^{-5}$ mol/L gas phase concentration. Tetrachlorobiphenyl (ULTRA Scientific, $99 \%+$ ) was introduced as a solid deposited on a probe and carried into the absorption celt. The mean tetrachlorobiphenyl concentration of $3.3 \times 10^{-6} \mathrm{~mol} / \mathrm{L}$ was determined as described in Section 3. Chlorine (Aldrich, $99 \%$ ) was injected as a gas at a concentration of $1.2 \times 10^{-3} \mathrm{~mol} / \mathrm{L}$. Sample mean residence times in the absorption cell were $1.0 \mathrm{~s}$ for both benzene and chlorobenzene, and 5.0 sec for both tetrachlorobiphenyl and chlorine. 


\subsubsection{RESULTS}

Figure 7.1 shows the $S_{1} \leftarrow S_{0}$ absorbance spectrum of benzene as it evolves from 22 to $600^{\circ} \mathrm{C}$. Prominent in the $220^{\circ} \mathrm{C}$ spectrum are the cold bands $6_{0}^{1}, 6_{0}^{1} 1_{0}^{1}, 6_{0}^{1} 1_{0}^{2}, 6_{0}^{1} 1_{0}^{3}, 6_{0}^{1} 1_{0}^{4}$, and $6_{0}^{1} 1_{0}^{5}$ (where $X_{0}^{1}$ denotes a transition originating at the zero point of the ground electronic state and ending in the excitation of one quantum of vibrational mode $X$ in the excited electronic state, and $X_{0}^{1} Y_{0}^{n}$ denotes a combination band excitation) $[1,2]$ Approximately $8 \mathrm{~nm}$ to the red of $6_{0}^{1}$ is its ground state hotband counterpart $6_{1}^{0}$ Built on each of these transitions is a train of hotbands to the red. As temperatures increase, hotband intensities increase and coldband activity decreases. By $600^{\circ} \mathrm{C}$ vibronic resolution (under our experimental conditions) is completely lost. Note both the $S_{1}$ and $S_{2}$ spectral regimes are red-shifted (exhibit onsets occurring at longer wavelengths). Note also that the intrinsic intensity of the spectrum increases with increasing temperature.

Figures 7.2 and 7.3 show results for the $S_{1} \leftarrow S_{0}$ absorbance of chlorobenzene and the $S_{2} / S_{1} \leftarrow S_{0}$ absorbance of tetrachlorobiphenyl, respectively. Although vibronic resolution is poor in the former and nonexistent in the latter, the trends, especially in chlorobenzene, are similar to those of benzene. We again note that observed excited state UV absorption red-shifts with increasing temperature, and that these transitions gain in integrated intensity as temperature is increased.

\subsubsection{THE GROUND ELECTRONIC STATE}

Our primary purpose has been to relate high-temperature absorption characteristics to solarinduced, high-temperature photochemistry. As temperatures rise, increasingly higher ground state vibrational levels become statistically populated. This, in turn, decreases the optical energy required to access electronic states and significantly affects the solar detoxification process in a positive way. The HTAS has allowed us to explore this and related phenomena for a number of molecules.

According to simple Boltzman statistics, in a polyatomic molecule the temperature dependent probability, $\mathrm{P}_{\mathrm{nr}}(\mathrm{T})$, of populating the $n$th level of a vibrational mode $r$, is independent of other ground state vibrational levels and is given by:

$$
P_{n r}(T)=\left\{\exp \left(-n_{F} h \omega_{r} /(k T)\right)\right\} /\left\{1-\exp \left(-h \omega_{r} /(k T)\right)\right\}
$$

where $\omega_{r}$ is the frequency of vibrational mode $r, k$ is the Bolzman constant, $h$ is Planck's constant, and $T$ is absolute temperature. Ground to excited state vibronic optical transitions may be divided into two sets 


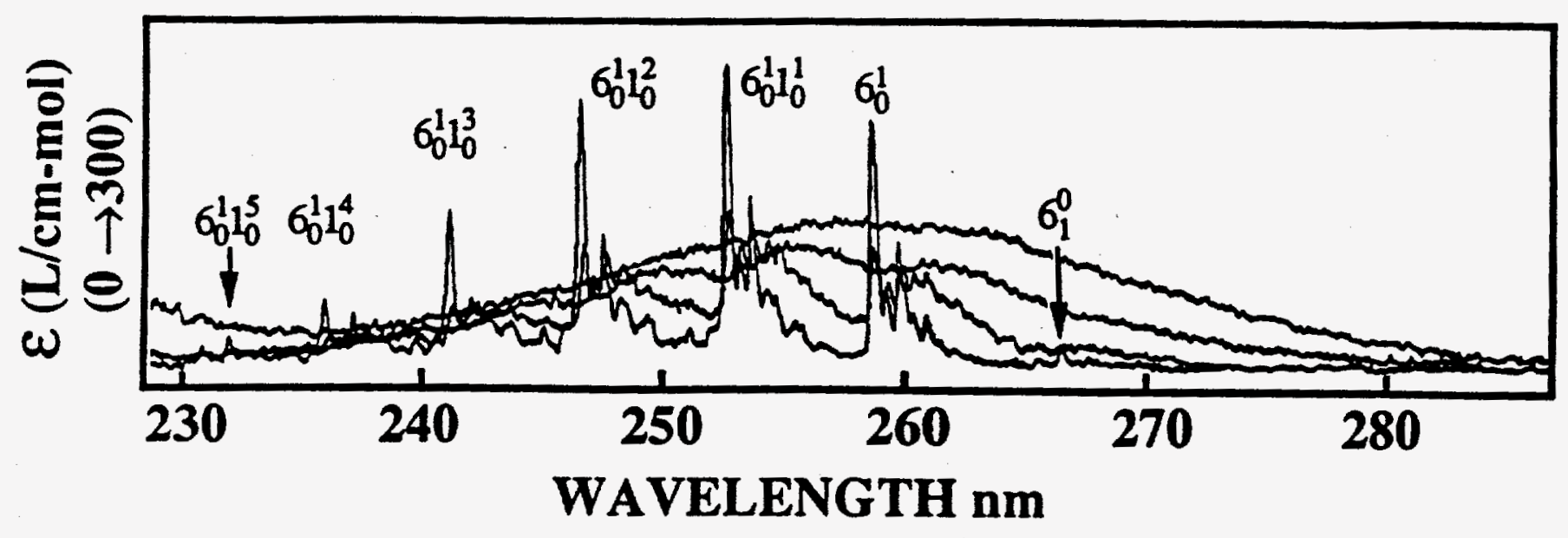

Figure 7.1. The $S_{1} \leftarrow S_{0}$ absorption spectra of benzene from 22 to $600^{\circ} \mathrm{C}$ showing the loss of fine spectra features, and an overall increase in absorption intensity and shift towards longer wavelengths. 

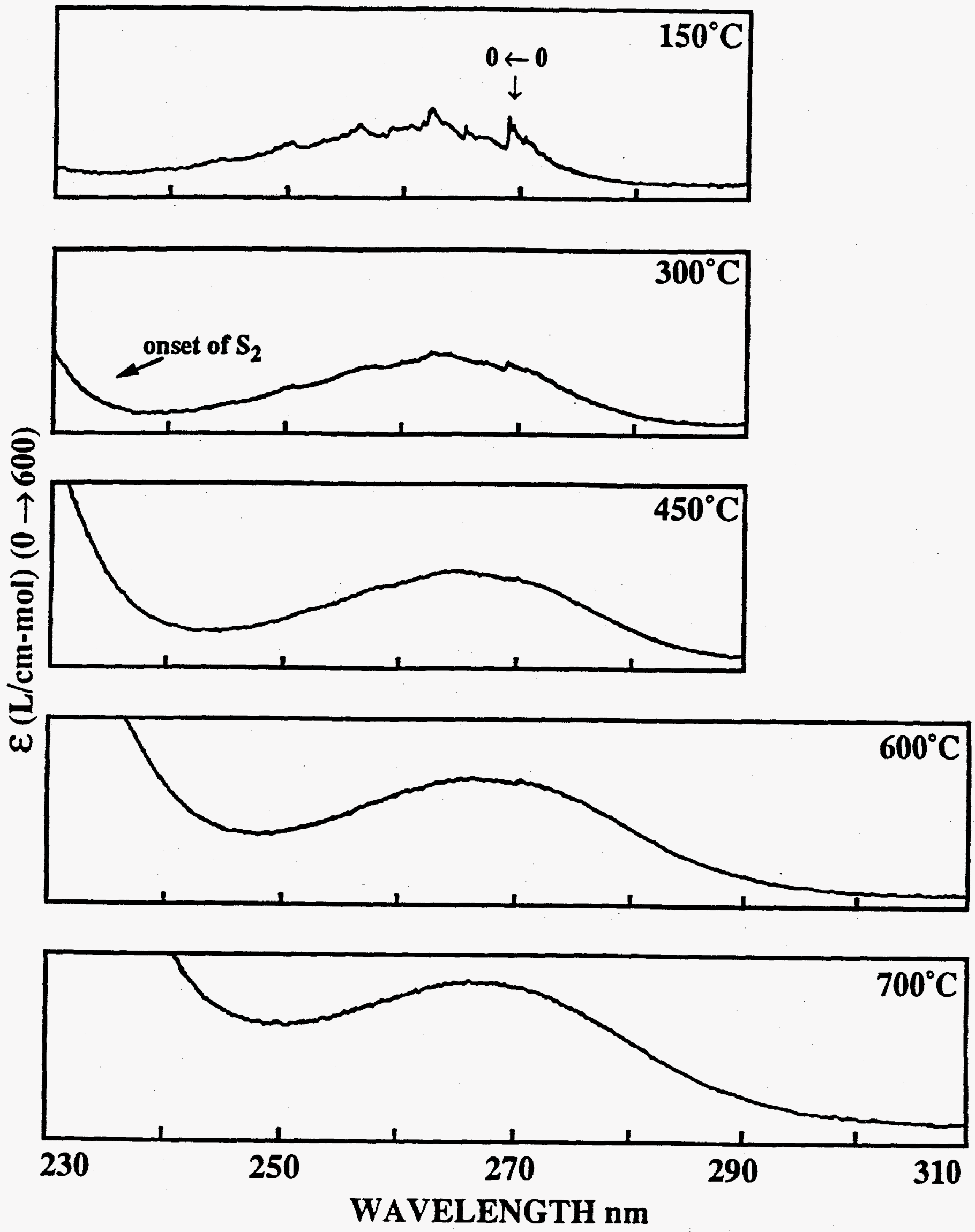

Figure 7.2. Temperature dependent $S_{1} \leftarrow S_{0}$ absorption spectra of chlorobenzene. 


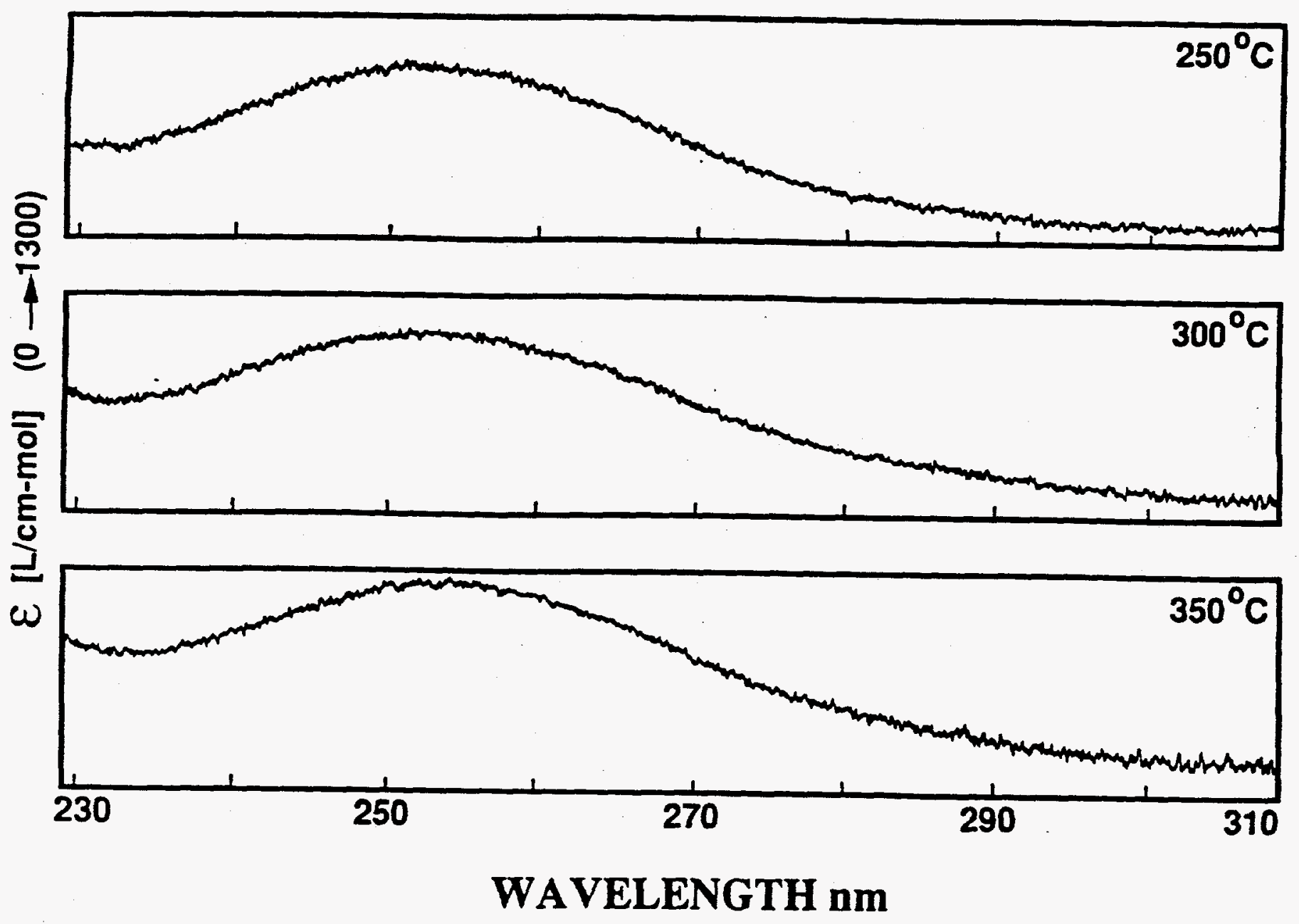

Figure 7.3. Temperature dependent $S_{1} \leftarrow S_{0}$ absorption spectra of tetrachlorobiphenyl. 
according to their Bolzman activity. Transitions originating from thermally populated ground state levels ("hotbands", where $n>0$ ) will (initially) gain in intensity with increasing thermal energy. Those transitions originating from the zero point of the ground state ("coldbands", where $n=0$ ) will lose intensity as thermal energy increases since populating the ground state vibrational levels depicts the zero point population.

The shitting of optical transitions toward longer wavelengths is depicted in Figure 7.4. As higherIying optically active vibrational ground states levels become thermally populated, less energy is required to access excited electronic states. As temperatures increase and observed absorption onsets migrate to the red (see Table 7.1), the excited state absorption spectrum and solar emission spectrum may overlap allowing UV absorption to assist in thermal destruction. Despite a $20 \mathrm{~nm}$ red-shift at $600^{\circ} \mathrm{C}$, benzene (Figures 7.1 and 7.5), does not access the solar spectrum. This is confirmed by the fact that concentrated, simulated solar energy produces no enhancement of the thermal destruction. Chlorobenzene (Figures 7.2 and 7.5 ) is an intermediate case. At elevated temperatures, the (red-shifted) $S_{1}$ onset slightly accesses the blue end of the solar spectrum resulting in a moderate enhancement of thermal destruction exhibited in Figure 7.6. Simple extrapolation indicates that benzene requires approximately $1345^{\circ} \mathrm{C}$ of thermal energy in order to access the solar emission spectrum. At $350^{\circ} \mathrm{C}$ tetrachlorobiphenyl displays a $\mathrm{S}_{1} / \mathrm{S}_{2}$ onset overlapping approximately $6 \mathrm{~nm}$ with the solar spectrum (Figures 7.4 and 7.5). This accounts for the relatively low temperature requirement for solar enhancement of thermal destruction. The net results is a significant increase in the rate of solar radiation absorption, $\mathrm{k}_{a b}\left(\mathrm{~s}^{-1}\right)$, (see Table 7.1) as temperatures increase. For a first-order chemical reaction, the rate of destruction is exponentially dependent upon the rate of light absorption. Tetrachlorobiphenyl exhibits a near two-fold increase in $\mathrm{k}_{\mathrm{ab}}$ in the $100^{\circ} \mathrm{C}$ temperature range investigated. Chlorobenzene, non-absorbing at room temperature, shows an eightfold increase in $\mathrm{kab}_{\mathrm{ab}}$ in the $600-700^{\circ} \mathrm{C}$ interval. Thus, temperature can be used to dramatically increase the rate of reaction.

We had initially expected a conservation of oscillator strength. In other words, the area under the spectral curves was expected to remain constant, independent of temperature. However, the three samples investigated exhibited increasing integrated absorption intensities as temperatures increased. A thermally enhanced vibronic coupling mechanism may be playing a role. Here, a ground to final electronic transition $(f \leftarrow g$ ) may gain in intensity when either state becomes distorted by a suitable vibration. According to the mechanism[3], the vibrationally distorted state acquires character from a third strongly absorbing state $i$. Adopting a simplistic physical approach, we may think of the now vibronically coupled states, depicted in Figure 7.7, as $f^{\prime}=f+\alpha$ and $g^{\prime}=g+\beta i$. In benzene and chlorobenzene it is the intense $S_{3}\left(\sim 55000 \mathrm{~cm}^{-1}\right)$ which is believed to lend character to the $S_{1}$ and to a lesser extent the $S_{0}$, when either the $S_{1}$ or $S_{0}$ is distorted principally through vibrational mode $n 6$ for benzene and $v_{6 b}$ for chlorobenzene[2]. 


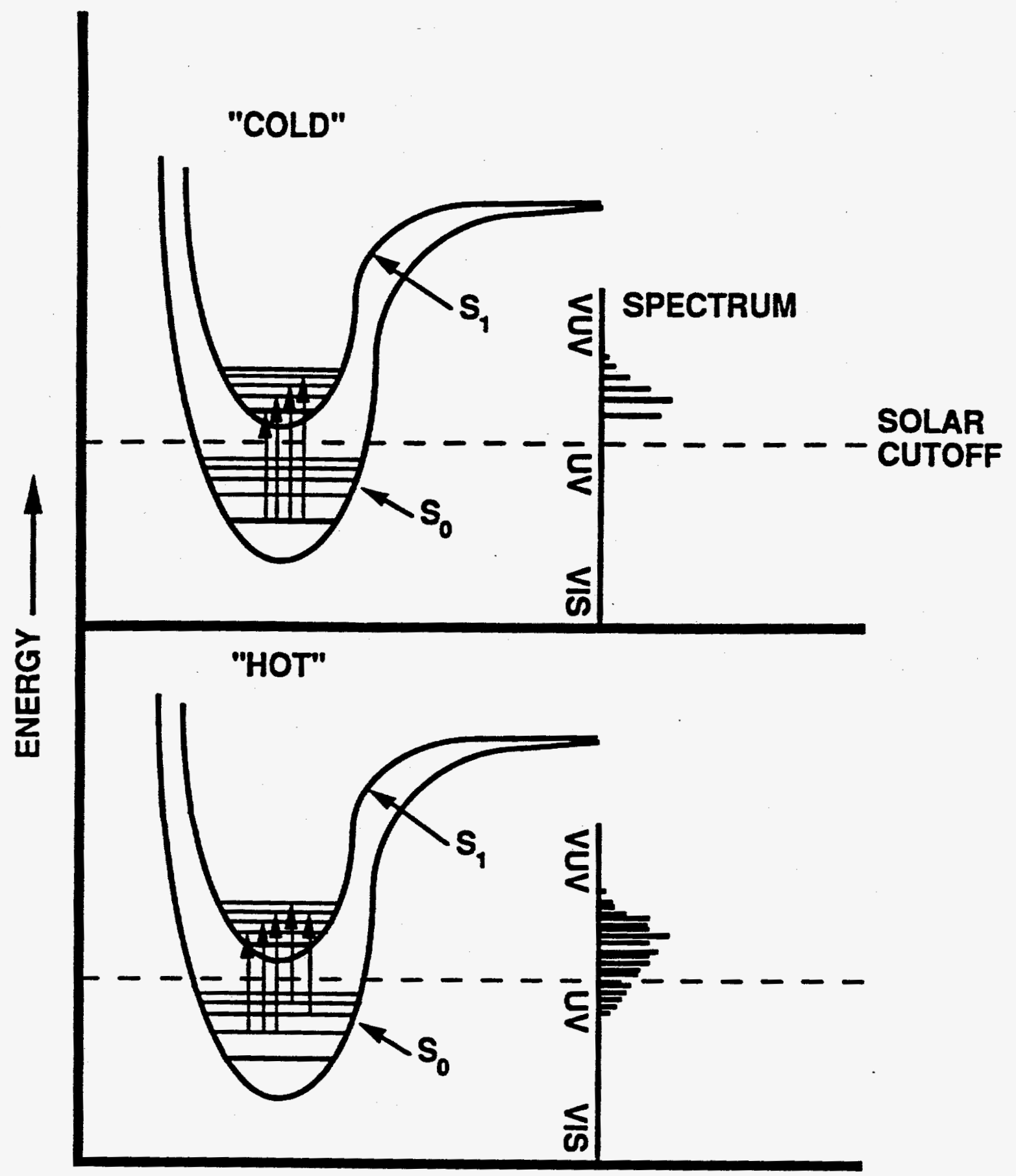

Figure 7.4. High temperature absorption spectroscopy. Ground state thermal excitation allows for a reduction of energy required to access electronically excited states. 


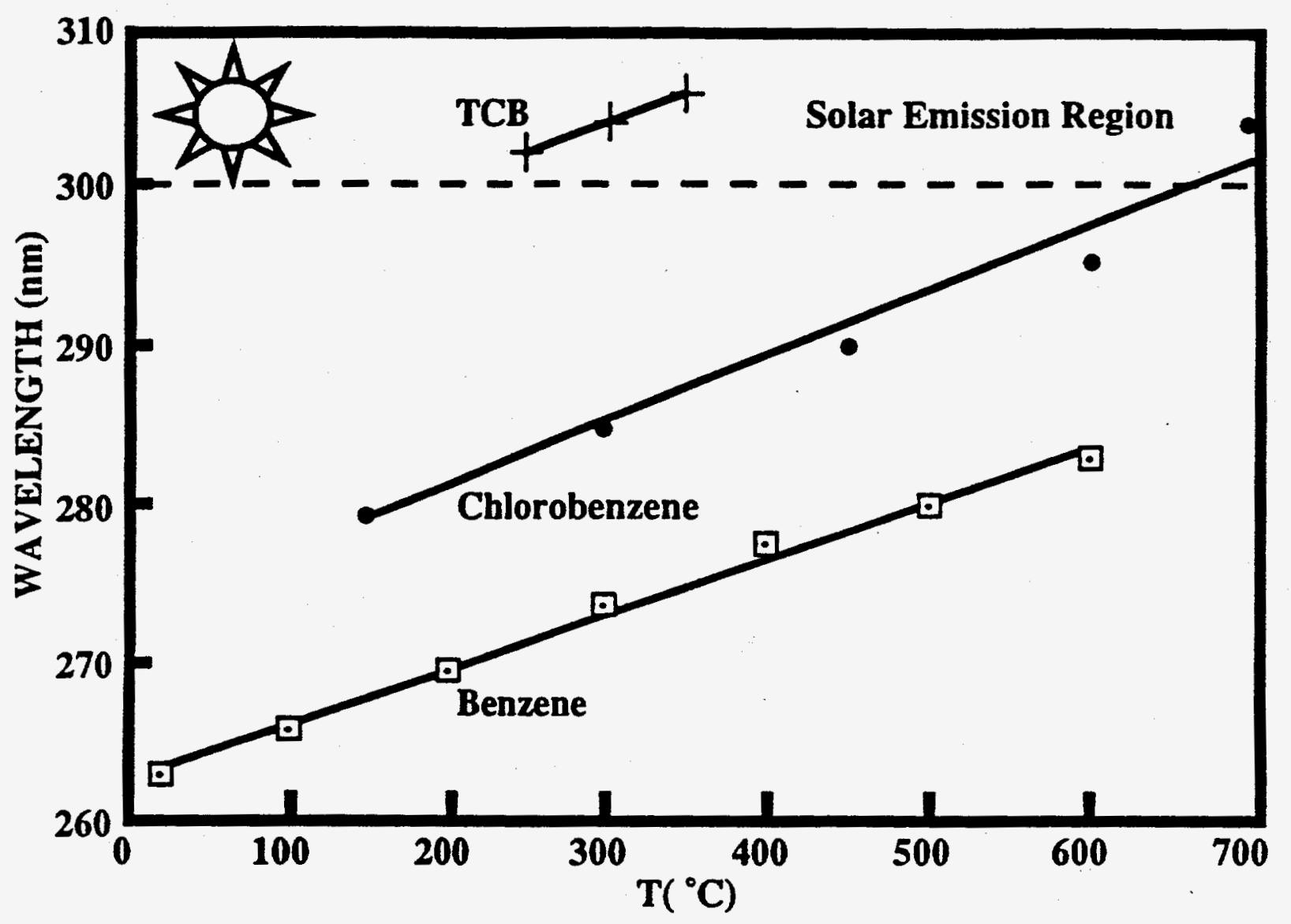

Figure 7.5. Thermally induced red shifts of the electronically excited singlet states of benzene, chlorobenzene, and tetrachlorobiphenyl. 


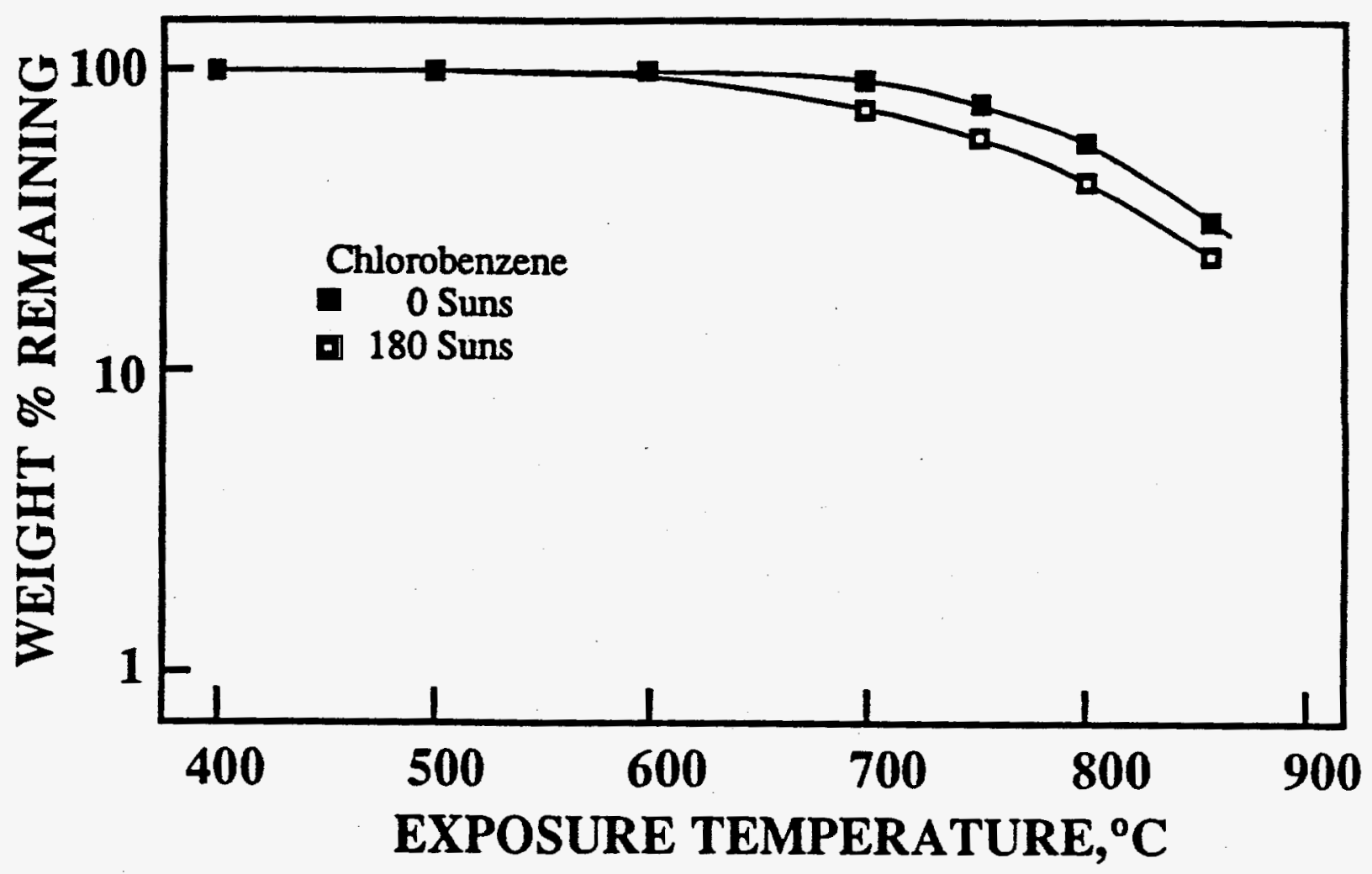

Figure 7.6. Thermal and (xenon lamp simulated) concentrated solar-assisted pyrolysis of chlorobenzene. $[\phi \mathrm{Cl}]=\left(10^{-5}\right) \mathrm{M}$, retention time $=10.0 \mathrm{~s}$, in a helium carrier. 


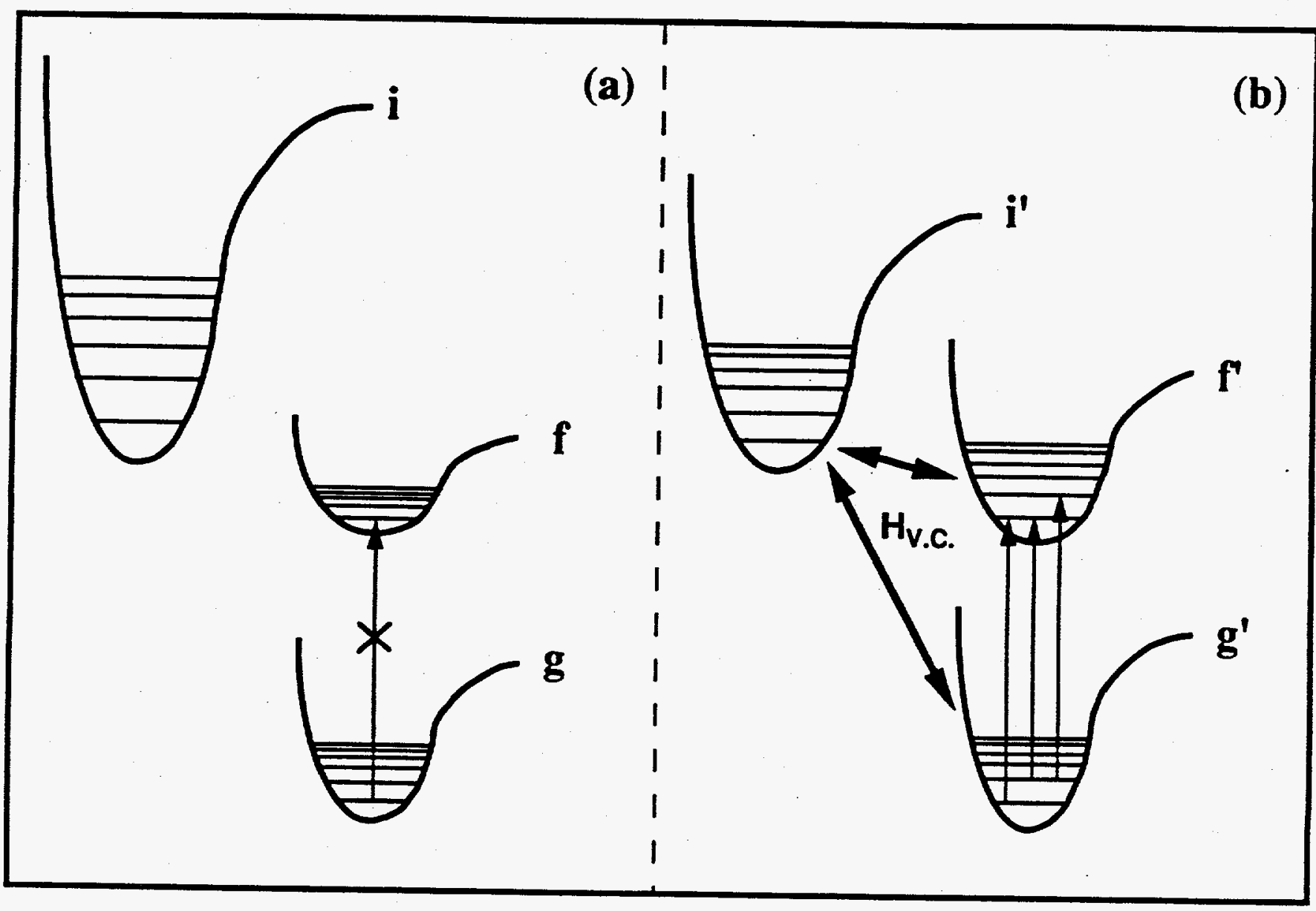

Figure 7.7. State $\mid i>$ lends character to states $\mid s$ and $\mid g>$ via a vibronic perturbation, $H_{v . c .}, f^{\prime}=f+\alpha i$, and $g^{\prime}=g+\beta i$. 


\section{TABLE 7.1}

Benzene $S_{1} \leftarrow S_{0}$, Chlorobenzene $S_{1} \leftarrow S_{0}$, and 3,3', 4,4'-Tetrachlorobiphenyl $S_{2} / S_{1} \leftarrow S_{0}$ Temperature Dependent Parameters

\begin{tabular}{|c|c|c|c|c|c|}
\hline Molecule & Temp ('C) & $\begin{array}{l}\text { Approx. } \\
\lambda_{\text {onset }}(\mathrm{nm})^{(\mathrm{a})}\end{array}$ & $\mathrm{f}_{S_{1} \leftarrow S_{0}}{ }^{\text {(a) }}$ & $10^{7} \tau_{0}(s)^{(a)}$ & $10^{6} \mathrm{k}_{\mathrm{ab}}{ }^{(\mathrm{b})}$ \\
\hline \multirow[t]{7}{*}{ Benzene } & 22 & 263 & 0.0013 & 7.8 & 0 \\
\hline & 100 & 266 & 0.0016 & 6.5 & 0 \\
\hline & 200 & 270 & 0.0017 & 6.0 & 0 \\
\hline & 300 & 274 & 0.0019 & 5.4 & 0 \\
\hline & 400 & 278 & 0.0021 & 4.9 & 0 \\
\hline & 500 & 280 & 0.0025 & 4.2 & 0 \\
\hline & 600 & 283 & 0.0029 & 3.6 & 0 \\
\hline Chloro- & 150 & 279 & 0.0037 & 3.2 & 0 \\
\hline \multirow[t]{4}{*}{ benzene } & 300 & 285 & 0.0050 & 2.4 & 0 \\
\hline & 450 & 290 & 0.0060 & 2.0 & 0 \\
\hline & 600 & 295 & 0.0093 & 1.3 & 0.7 \\
\hline & 700 & 304 & 0.0119 & 1.0 & 5.6 \\
\hline $3,3^{\prime}, 4,4^{\prime}-$ & 250 & 302 & 0.022 & 0.41 & 4.0 \\
\hline Tetrachloro- & 300 & 304 & 0.025 & 0.36 & 5.7 \\
\hline biphenyl & 350 & 306 & 0.029 & 0.31 & 7.2 \\
\hline
\end{tabular}

a $S_{2}$ contributions to the areas under the benzene and chlorobenzene $S_{1}$ profiles were estimated and subtracted. For TCB no such correction was made. Uncertainties are $\sim 10 \%$ except for TCB which is assigned $-25 \%$.

b $k_{a b}=2.11\left(10^{-5}\right) \int_{300 \mathrm{~m}}^{10} \varepsilon(\lambda) \lambda I_{0}(\lambda) d \lambda$ is the rate of solar radiation absorption where $\varepsilon(\lambda)$ is the temperature dependent molar extinction coefficient in $L /(\mathrm{cm}-\mathrm{mol}), \lambda$ is the excitation wavelength $(\mathrm{nm})$, and $\mathrm{I}_{\mathrm{o}}$ is the the intensity of incident solar radiation in $\mathrm{W} \mathrm{cm}^{-2} \mathrm{~nm}^{-1}$. 
Mathematically, the phenomenon of vibronic coupling, based on the Born-Oppenheimer principle [3] is approached via Herzberg-Teller theory[4,5]. The Herzberg-Teller vibronically induced transition

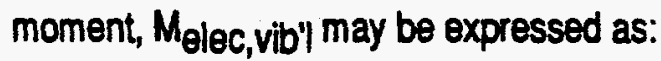

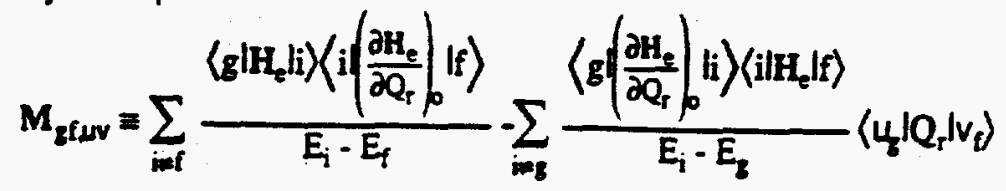

where $Q_{r}$ is the normal coordinate of vibrational mode $r\left(v_{r}\right),|g\rangle$ and $|f\rangle$ are the ground and final electronic

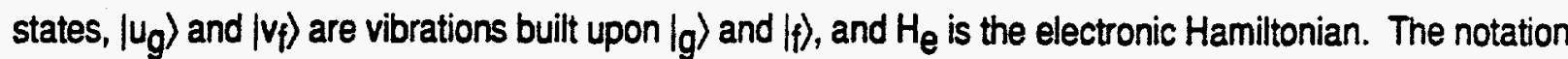
" 0 " denotes expansion over the equilibrium geometry of the molecule. The E's represent the electronic state energies. The first set of terms in the brackets may be interpreted as follows. The final electronic state $\mid f)$ in the $f \leftarrow g$ transition can be vibrationally mixed in normal coordinate $Q_{r}$ with another electronic state $|i\rangle$, the vibronic coupling state, to an extent proportional to $\left\langle i\left|\partial \partial Q_{r}\right| \hat{f}\right.$. The $f \leftarrow g$ transition may borrow intensity from the $\mathrm{i} \leftarrow \mathrm{g}$ electronic transition if the matrix element $\left\langle\mathrm{g}\left|\mathrm{H}_{\mathrm{e}}\right| \mathrm{i}\right\rangle$ is non-zero. The second set of terms in the brackets of equation 7.2 may be interpreted as vibronic coupling of the initial state $|g\rangle$ with the vibronic coupling state li) via vibration in mode $Q_{p}$. The degree of the effect is directly proportional to the inverse of the square of the energy denominator resulting often in $|f\rangle \leftrightarrow|i\rangle$ coupling favored over $|g\rangle \leftrightarrow|i\rangle$ by approximately an order of magnitude.

Unlike Franck-Condon amplitude, $\left\langle u_{g} \mid v_{f}\right\rangle$, which vanishes unless the change in vibrational quanta on going from the initial to the final state is even, $\left\langle u_{g}\left|Q_{r}\right| v_{f}\right\rangle$, the overlap integral, vanishes unless $\Delta v$ is odd and the vibration has the same force constant and equilibrium location in both $|g\rangle$ and $|f\rangle$. In the nondisplaced harmonic oscillator approximation, as $\mathrm{ug}_{\mathrm{g}}$ increases (i.e., for hotbands) these integrals become more favorable[6] perhaps accounting for some of the observed gains in oscillator strength. This is treated explicitly in equation 7.3 in terms of diatomic harmonic wave functions[6].

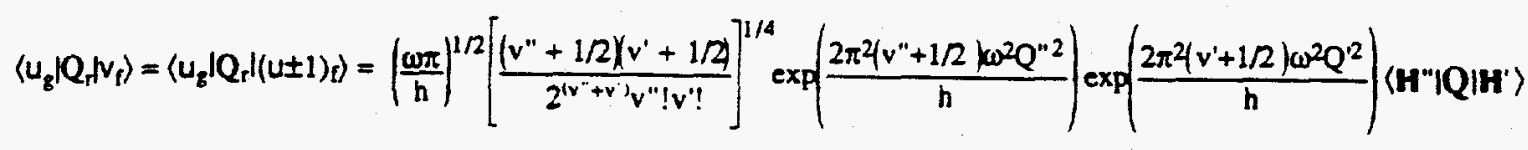

Equation 7.3 is derived from the relations given in Chapter 3 and Appendix III of Reference 6. Here, 9 and $f$ denote the ground and excited states, respectively, $v$ is the vibrational quantum number, $\mathrm{H}$ is the appropriate Hermite polynomial, $\omega$ is the frequency of the ground state vibration, and $Q$ is defined in Appendix III of Reference 6.

We are now equipped to calculate the matrix elements of equation 7.3. Results are shown in Table 7.2 where as an example we use an $\omega$ of $608 \mathrm{~cm}^{-1}$, the frequency of principle vibronic coupling mode, $v_{6}{ }_{6}$, in benzene[2]. These results are not quantitative in that we are employing a harmonic diatomic approximation to an enharmonic polyatomic problem, i.e., the vibration is assumed to have the same force 
constant and equilibrium location in both $|g\rangle$ and $|f\rangle$. The qualitative trend, however, is clear. The squares of matrix elements of the type, $\left\langle u_{g} \mid Q_{r}(u+1)_{f}\right\rangle$, show a near 14500-fold intensity growth over four quanta. Boltzman statistics heavily mitigate these numerics to the point where at $700^{\circ} \mathrm{C}$ we calculate a peak intensity growth of 430 -fold over three quanta.

Vibronic coupling may be expected to play a much greater role in polyatomic than in diatomic spectroscopic processes. Diatomic molecules have only one axial vibrational mode that can little change electronic symmetry. We look toward molecular chlorine as a simple test. Figure 7.8 shows chlorine absorption spectra taken at room temperature and at $300^{\circ} \mathrm{C}$. The near superimposibility (outside of a slight red-shift) of these spectra seems to lend support to these ideas.

\subsection{EXCITED ELECTRONIC STATES}

The mechanistic nature of high temperature photochemical kinetics and dynamics is an area of active research. Requisite to these studies is a knowledge of the excited state radiative or natural lifetimes, $\tau_{0}$. These may be calculated by relating molar extinction coefficients to radiative lifetimes via oscillator strengths. Strictly speaking, the Strickler-Berg equation[] should be used. However, knowledge of the fluorescence spectrum is required for its application. Assuming a fluorescence profile would result in an improvement in the sophistication but probably not the accuracy of our procedure. We are initiating hightemperature emission studies which in the future will allow us to remedy this deficiency. Meanwhile, we employ a crude approximation for integrated oscillator strength $[8,9]$ and from that calculate lifetimes $[10]$.

$$
\begin{aligned}
& f_{f_{g}}=\frac{2.303 m_{e} c^{2} F}{\pi N e^{2}} \int_{\omega_{g}}^{a r} \varepsilon d \omega \\
& \tau_{0}=\frac{m_{e} c^{2}}{8 \pi^{2} \omega_{f_{g}}^{2} e^{2} f_{f_{g}}}
\end{aligned}
$$

Here, $f_{f g}$ is the oscillator strength (i.e., absorption intensity) of the final $\leftarrow$ ground state transition, to is the natural lifetime in seconds, $\omega$ is in $\mathrm{cm}^{-1}$, and $\mathrm{F}$ is a number relating refractive indices which may be assumed to be $1[8-10]$. The molar extinction coefficients, $\varepsilon$, can be obtained from our absorption data and Beer's Law. Results are given in Table 7.1. It is evident from equations 7.4 and 7.5 that $f_{f g}$ and $\tau_{0}$ are inversely related such that as absorption intensity increases, excited singlet state lifetimes diminish. This, in spite of the fact that we observe increases in destruction efficiencies at elevated temperatures. This leads us to hypothesize that the rate of decomposition is accelerated due to thermal activation of $S_{1}$, or more likely, the longer-lived $T_{1}$. 


\section{TABLE 72}

Explicit Expectation Values for a Diatomic Harmonic Oscillator

\begin{tabular}{|c|c|c|c|}
\hline \multirow[b]{2}{*}{ Matrix Element } & \multirow[b]{2}{*}{ Value $^{2}$} & \multicolumn{2}{|c|}{$\left(B^{2} \times \text { Value }\right)^{2}$} \\
\hline & & $\left(22^{\circ} \mathrm{C}\right)$ & $\left(700^{\circ} \mathrm{C}\right)$ \\
\hline$\left\langle 4\left|Q_{r}\right| 3\right\rangle$ & 0.147 & $7.25\left(10^{-12}\right)$ & $1.10^{\left(10^{-4}\right)}$ \\
\hline$\left\langle 1\left|Q_{r}\right| 0\right\rangle$ & 0.398 & $1.05\left(10^{-03}\right)$ & $6.59\left(10^{-2}\right)$ \\
\hline$\left\langle 3\left|Q_{r}\right| 2\right\rangle$ & 1.053 & $1.96\left(10^{-08}\right)$ & $4.77\left(10^{-3}\right)$ \\
\hline$\left\langle 2\left|Q_{r}\right| 1\right\rangle$ & 2.332 & $1.64\left(10^{-05}\right)$ & $6.39\left(10^{-2}\right)$ \\
\hline$\left\langle 1\left|Q_{T}\right| 2\right\rangle$ & 109.265 & $2.90\left(10^{-01}\right)$ & 18.1 \\
\hline$\left\langle 2\left|Q_{r}\right| 3\right\rangle$ & 1038.966 & $7.30\left(10^{-03}\right)$ & 28.5 \\
\hline$\left\langle 3\left|Q_{r}\right| 4\right\rangle$ & 5762.324 & $.1 .07\left(10^{-04}\right)$ & 26.1 \\
\hline
\end{tabular}

a Boltzman factor, $\beta=\exp [-608 / 0.6951 / \mathrm{T}(\mathrm{K})]$. 

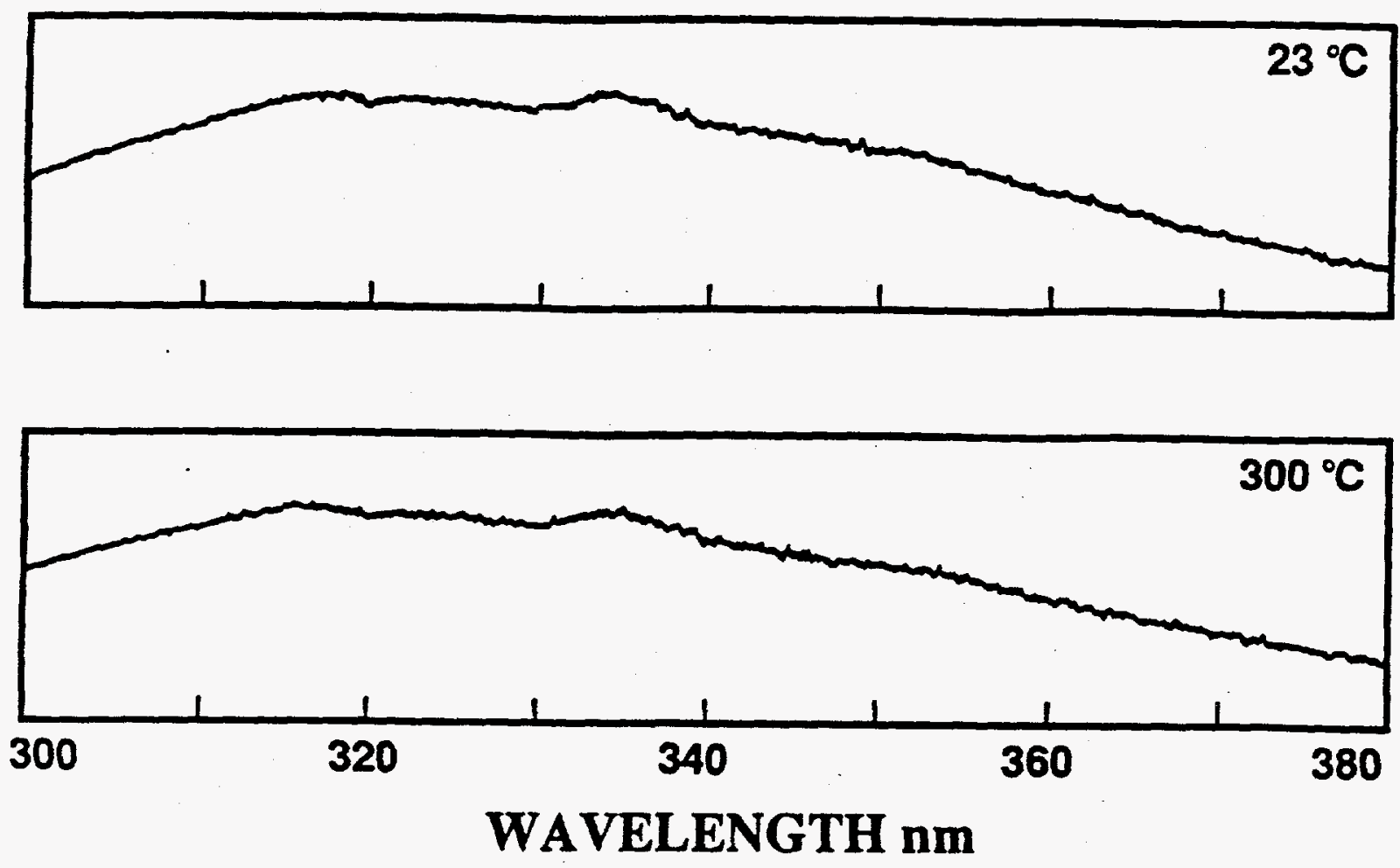

Figure 7.8. Ambient temperature $\left(-20^{\circ} \mathrm{C}\right)$ and $300^{\circ} \mathrm{C}$ absorption spectra of molecular chiorine. Note that the integrated intensity remains constant. 
As part of work co-funded by the US-EPA, we have also begun our first high-temperature dispersed emission enabling experiments. Results may not only remedy the shortcomings of applying equations 7.3 and 7.4 , but may also be of value in answering a plethora of open questions. Among them:

Are excited states in quasi-thermal (i.e., Boltzman) equilibria? A "steady-state" population of excited state thermally-populated vibronic levels may facilitate singlet-triplet intersystem crossing. Thermal population of $S_{1}$ vibronic levels may manifest itself as a blue-shift in the fluorescence spectra, a decrease in fluorescence coupled with an increase in phosphorescence intensity, or a fall-off in both fluorescence and phosphorescence intensities due to radiationless coupling out of $S_{1}$ excited vibronic states with highly excited $S_{0}$ bath states. We hope to answer this with temperature-dependent emission intensity and dispersed emission experiments.

Are emission lifetimes temperature-dependent? According to equation 7.4 the excited state lifetime is inversely dependent on oscillator strength. We have clearly observed that oscillator strength has a positive temperature dependency in benzene, chlorobenzene, and tetrachlorobiphenyl. Does the fluorescence spectrum behave commensurately? We are planning fluorescence lifetime experiments by variably delaying the boxcar gate in laser excitation fluorescence experiments. Emission lifetime studies will allow us to directly determine the rate of radiationless decay from $S_{1}$ and $T_{1}$ by comparing the measured lifetimes with the radiative lifetimes determined by absorption studies.

What roles are played by torsional modes? Do these low-lying modes facilitate intersystem crossing or radiationless processes with increasing temperature?

What roles are played by $\pi$-electron perturbations? Redistribution of the $\pi$-electron cloud in benzene derivative changes spectroscopic selection rules allowing access to electronic states and vibronic coupling routes forbidden in benzene.

Toluene and chlorobenzene respectively offer the perturbations described by the above two questions.

We have recently completed construction of a high temperature emission spectrophotometer (HTES), a schematic of which is shown in Figure 7.9. Gas and liquid samples are introduced with syringe pumps, and solids using special probes. The emission cell consists of a quartz cone surrounded by a small tube heater, which, in turn, lies in an optically accessible insulated aluminum box. 


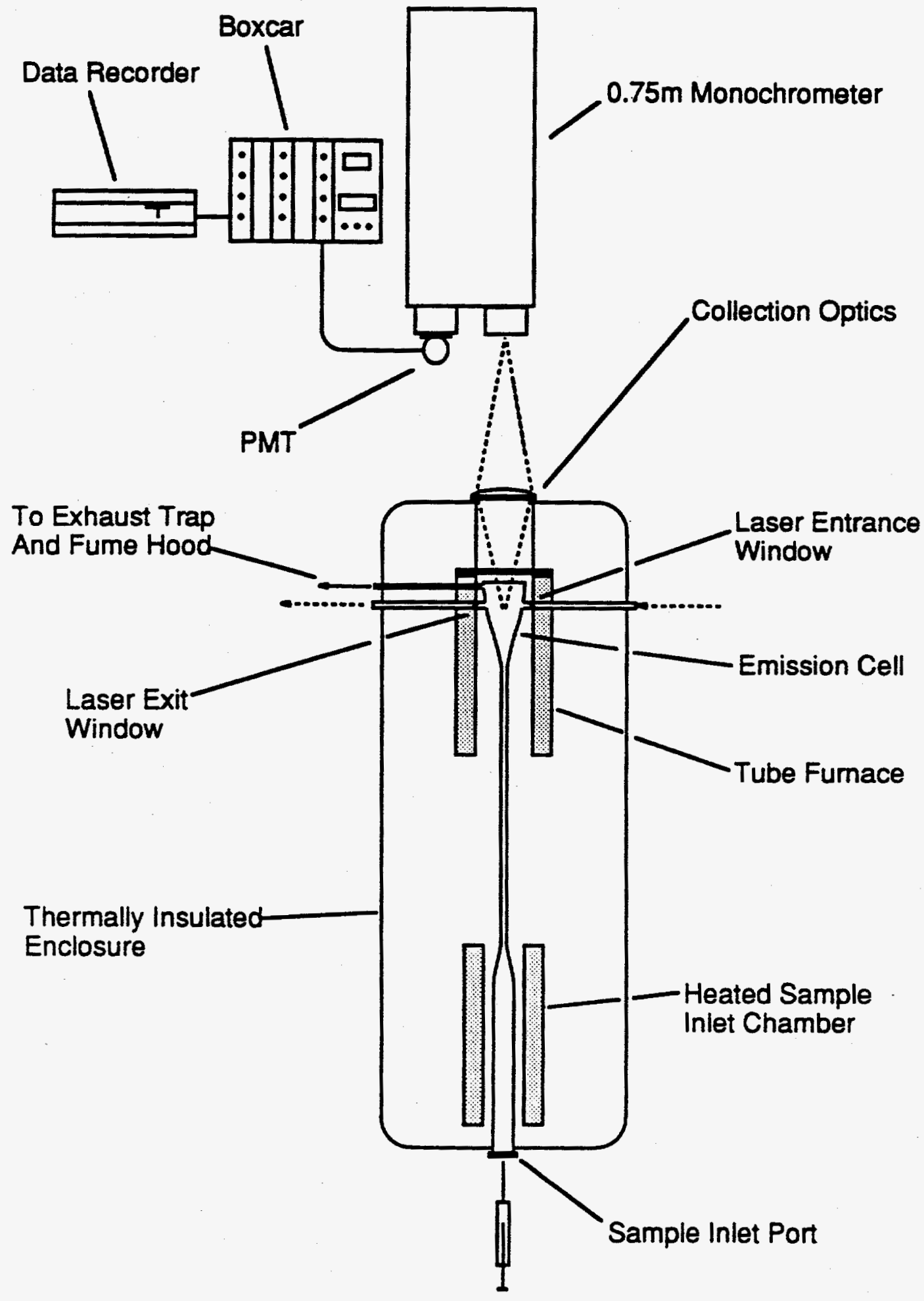

Figure 7.9. Diagram of the High Temperature Emission Spectrometer (HTES). 
Our first results, toluene, $150^{\circ} \mathrm{C}$, are shown in Figure 7.10 . The cell was illuminated with the 532 nm second harmonic of a Nd:YAG laser (Continuum Model 682-20). The UV emission resulting from the two-photon excitation was dispersed with a $0.75 \mathrm{~m}$ monochrometer (Spex Model 1702) onto a photomultiplier tube (Hamamatsu R212). The resultant fluorescence signal was gated and averaged with a boxcar (SRS Model SR-250) and displayed on an analog stripchart recorder. Toluene flowed through the cell mixed in helium. Although the cross section of two-photon transitions is much smaller than their onephoton counterparts we chose this configuration in order to make use of the favorable signal to noise ratio resulting from the large wavelength displacement between the $532 \mathrm{~nm}$ excitation and UV emission.

We observe in Figure 7.10 that the fluorescence intensity peak occurs at $288 \mathrm{~nm}$ or approximately $2760 \mathrm{~cm}^{-1}$ from the ground state origin assuming that the fluorescence originates from the zero-point of the $\mathrm{S}_{1}$. We also note that very little signal is observed at the $266.77 \mathrm{~nm} 0-0$ wavelength.

We are currently improving our optical collection system. This will allow us to narrow the slits to a point where we can vibrationally resolve our spectra. We will then continue our studies at increasing temperatures while carefully controlling residence times and concentrations.

\subsection{REFERENCES}

1. E. B. Wilson, Jr., Phys. Rey., 45, 706, 1934.

2. R. Hulstrum, R. Bird, and C. Riordan, Solar Cells, 15 (1985) 365.

3. U. Brand, H. Hippler, L. Londemann, and J. Troe, J. Phvs. Chem., 94 (1990) 6305-6316 and references therein.

4. I. Özkan and L. Goodman, Chem. Rev., 79 (1979) 275.

5. L. D. Ziegler and B. S. Hudson, Excited States, edited by E. C. Lim, Academic, N. Y., 1982, Vol. 5.

6. E. B. Wilson, Jr., J. C. Decius and P. C. Cross, Molecular Vibrations, Dover, N. Y., 1955, Appendix.

3. G. Herzberg, Spectra of Diatomic Molecules, Van Nostrand, N. Y., pp. 76-78, 1950.

7. S. J. Strictler and Robert A. Berg, J.Chem. Phys., 37, 814, 1962.

8. F. Perrin, J., Phys, Radium, 7, 390, 1926.

9. G. N. Lewis and M. Kasha, J.Am. Chem. Sec., 67, 994, 1945.

10. S. P. McGlynn, T. Azumi, and M. Kinoshita, Molecular Spectroscopy of the Triplet State, PrenticeHall, Inc., New Jersey, 1969. 


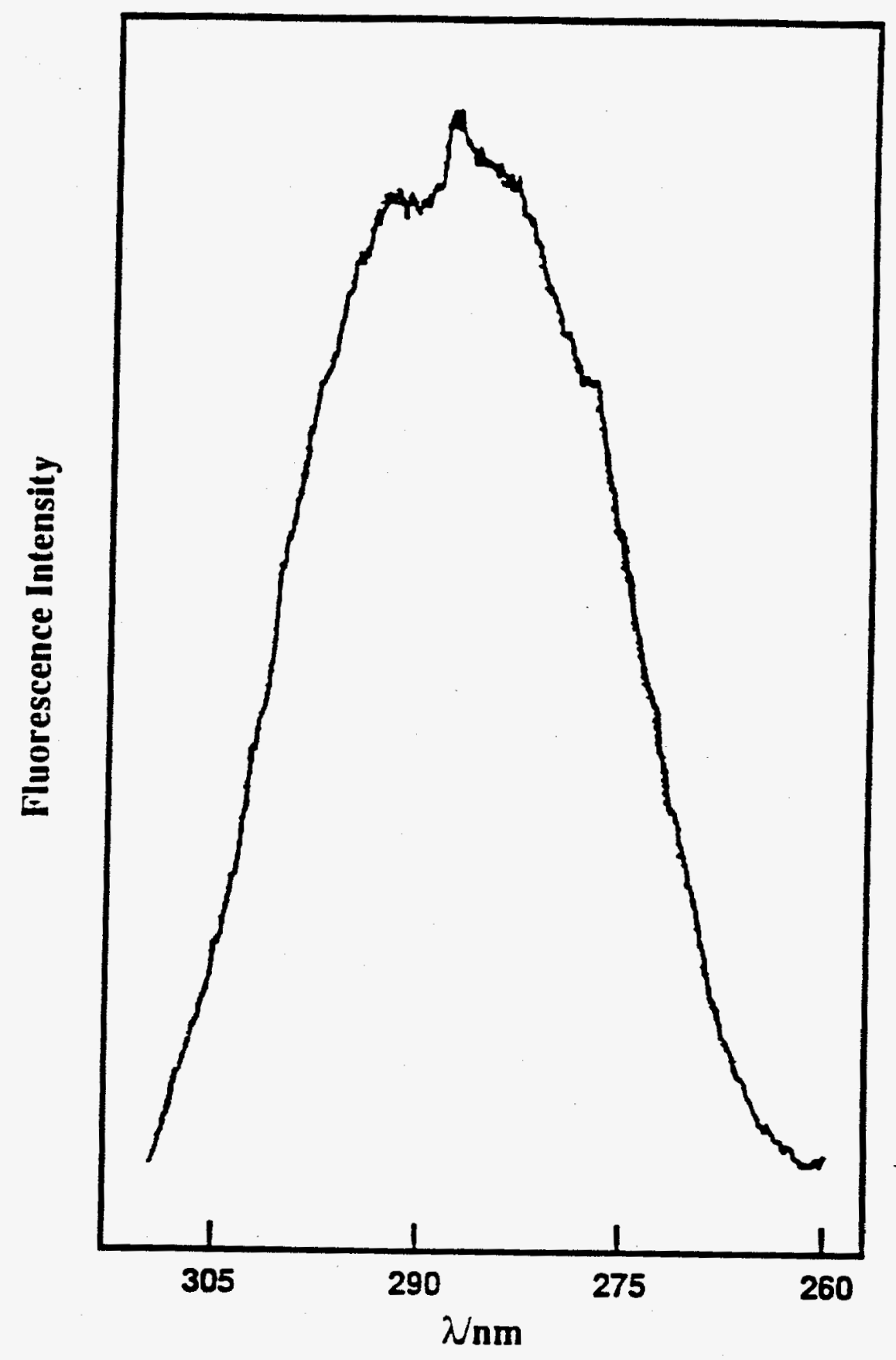

Figure 7.10. The $S_{1} \rightarrow S_{0}$ dispersed fluorescence spectrum of toluene at $150^{\circ} \mathrm{C}$. 


\section{SECTION 8.0 \\ ENGINEERING SCALE-UP}

The development of a demonstration scale solar detoxifier is currently underway as part of a TriAgency Initiative Department of Energy/Environmental Protection Agency/U.S. Army Toxic and Hazardous Materials Agency, (DOE/EPAUSTHAMA) as with any new process, many technological hurdles must be overcome.

In order to support this effort, we have performed three studies:

1. Field demonstration of destruction of 1,2,3,4-tetrachlorodibenzo- $p$-dioxin (TCDD) (jointly conducted with NREL under the direction of Dr. Greg Glatzmaier of NREL),

2. Initial evaluation of the effects of an intense radiative field on droplets,

3. Conceptual design and sizing of a solar detoxifier for remediation of contaminated soils.

\subsection{FIELD DEMONSTRATION OF DESTRUCTION OF TCDD}

Based on the initially promising laboratory results and at the urging of DoE, in 1988 a field demonstration of the solar detoxification of 1,2,3,4-TCDD. These tests were initiated by Dr. Gerry Nix of NREL and performed under the leadership of Dr. Greg Glatzmaier of NREL. UDRI was responsible for the design and fabrication of the sample feed system, effluent sampling system, and performed the analysis of the effluent samples. We also contributed to the design of the reactor and test procedure and assisted in the initial field test series. All subsequent data and data interpretation was performed exclusively by NREL personnel [1]. In this section, we provide a brief description of the test and the results of our own analysis of the data which we plan to co-publish with NREL [2].

The field tests were performed using concentrated solar energy provided by a large solar furnace located on the White Sands Missile Test Range near Las Cruces, New Mexico. This facility was ideally suited for these tests as it could deliver very high solar concentrations (up to 3,000 "suns" and $30 \mathrm{~kW}$ total power) and its optical system used front-faced, aluminized mirrors which almost completely reflect the solar energy's UV component (cf. Figure 8.1) 


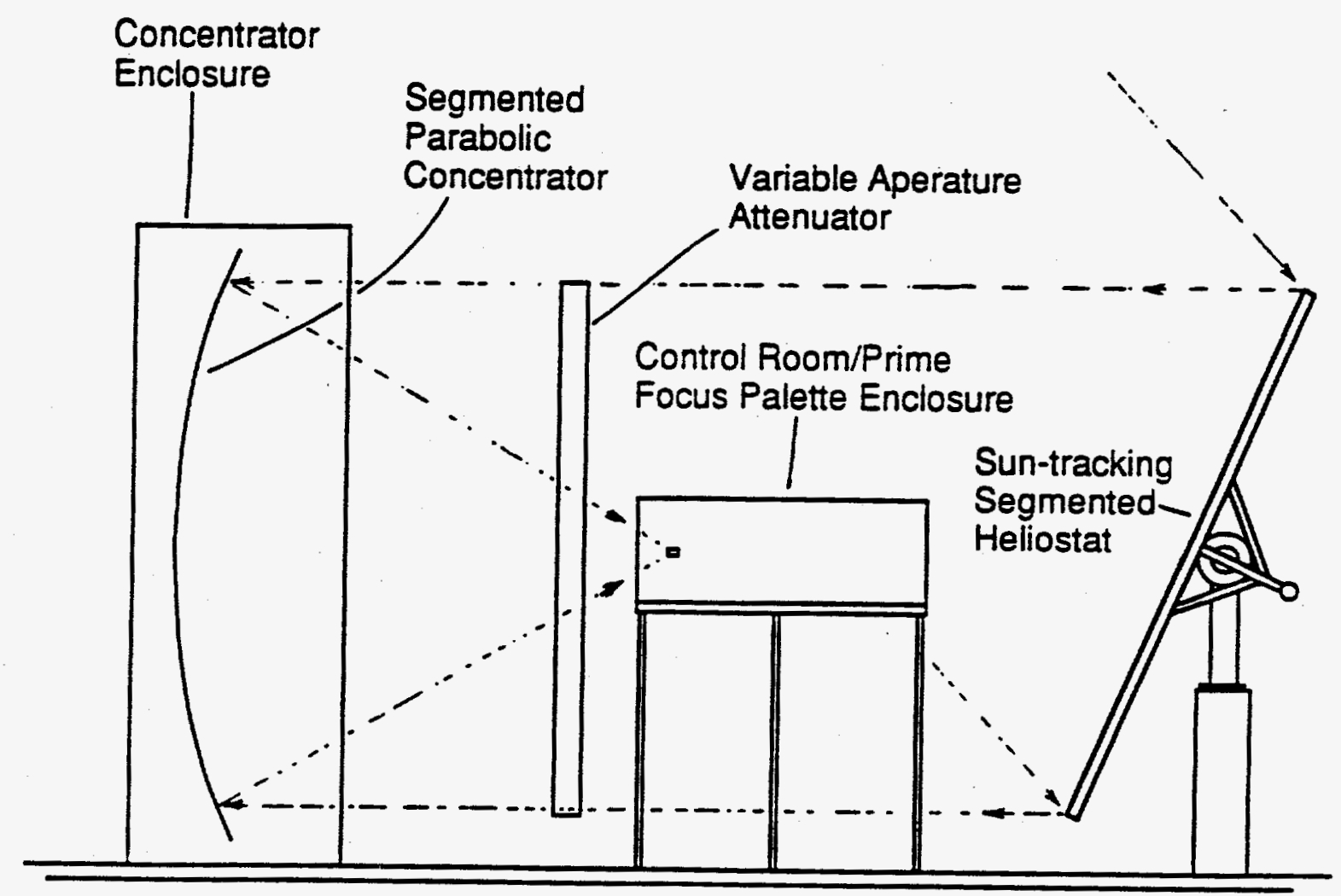

Figure 8.1. General schematic of the White Sands Solar Furnace which was used for the field tests reported here. 


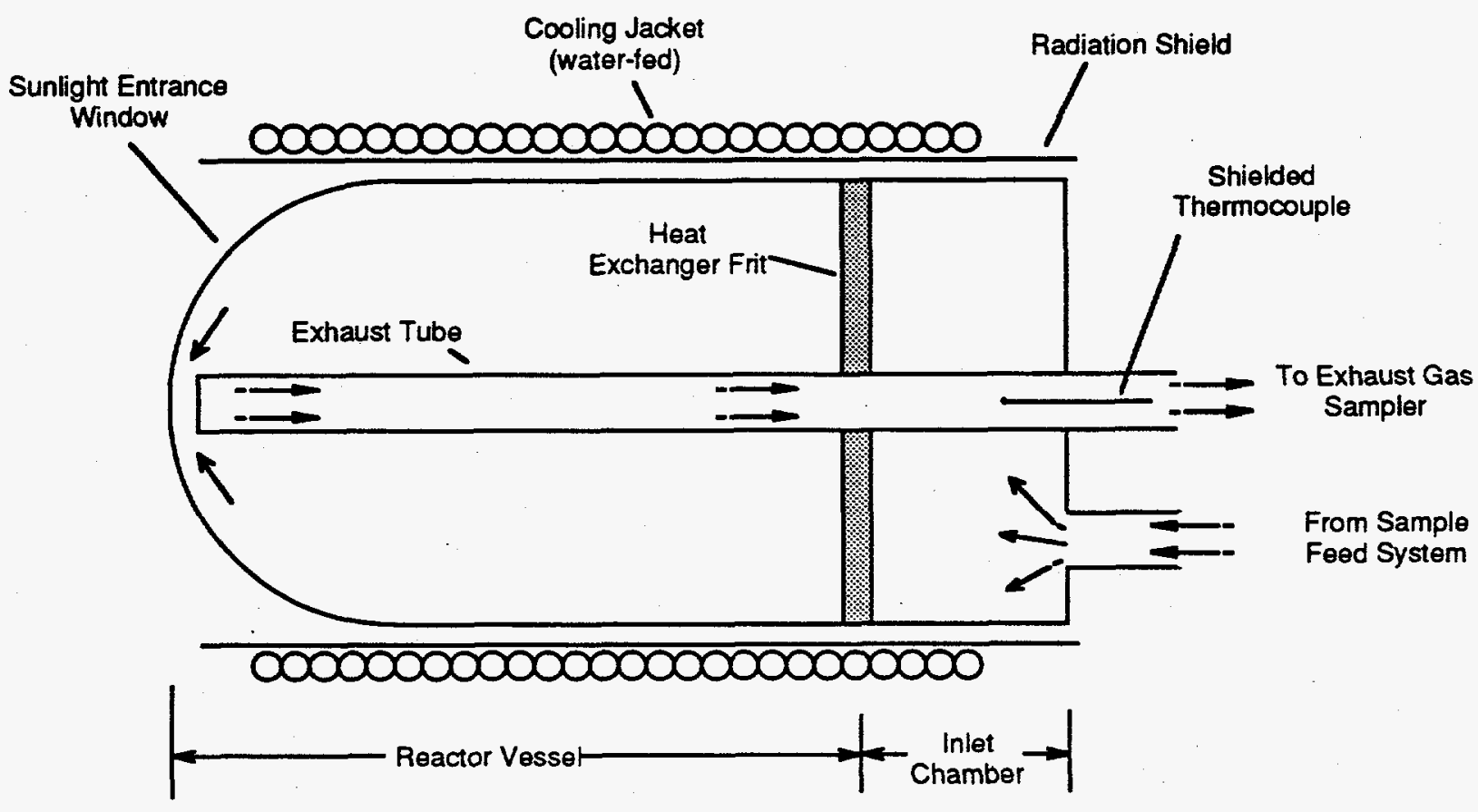

Figure 8.2. Detailed schematic of the bench-scale reactor which was designed by NREL and installed in the White Sands Solar Furnace. 


\subsubsection{EXPERIMENTAL}

The flow reactor system, shown in Figure 8.2 included a sample introduction chamber, reactor vessel, and an exhaust sampling and treatment system. The sample inlet accepted small glass cartridges that were loaded with 10 milligrams of TCDD. On heating to $300^{\circ} \mathrm{C}$, the sample sublimed and was transported through the reactor by the flowing reaction atmosphere (dry air).

The reactor was designed such that the sample laden carrier gas entered a dispersion chamber at the rear of the reactor vessel and then passed through a porous alumina disk which forms the rear wall of the actual reactor volume. This disk illuminated with concentrated sunlight and serves as an energy conversion/heat exchanger element. As the sample passes through the disk it was heated to the disk's steady state temperature of $700-1000^{\circ} \mathrm{C}$ (measured with a radiation shielded thermocouple placed in the reactor's exhaust line) which in turn is controlled by the intensity of the concentrated sunlight.

On passing through the disk, the sample enters the front of the reactor where it is exposed to the incoming concentrated sunlight, then exits the vessel via a transport line that extends from the back of the reactor to just inside the entrance window. This configuration insures that all of the sample passes through the illuminated portion of the reactor and eliminates bypassing which would limit the reactor's performance. The exhaust then passes through a glass " $U$ " tube immersed in a water ice bath which condenses the high molecular weight material (i.e., TCDD) surviving the exposure. This trap is then removed, sealed, and returned to an analytical laboratory for analysis.

The time allowed to pass to insure complete sublimation of the TCDD and pass it through the reactor was $15 \mathrm{~min}$. The transfer lines to and from the reactor were heat traced to maintain a minimum temperature of $250^{\circ} \mathrm{C}$ which was sufficient to prevent condensation of the TCDD.

The contents of each trap was extracted with toluene and analyzed for TCDD using a programmed temperature capillary column gas chromatograph (Hewlett Packard Model 5890) fitted with a scanning mass spectrometer for solute detection (Hewlett Packard Model 5970). These data were quantified using traps in which known amounts of TCDD had been deposited.

As part of the shake-down tests, as well as $Q A / Q C$ reporting during the various field trials, the temperature of the reactor's exhaust was recorded as a function of the total radiant flux measured with a calorimeter operated by the WSSF staff. These data were then used to assign mean solar flux values to the reactor data from the measured exhaust temperature. 


\section{TABLE 8.1}

Summary Of The WSSF Field Data For 1,2,3,4-Tetrachlorodibenzo-p-dioxin Exposed For 5 Seconds In Air

$\begin{array}{cccc}\text { Temp(C) } & \text { I01 } & \mathrm{fr} \text { (Thermal)2 } & \mathrm{fr} \text { (Solar) } \\ 750 & 54.5 & 2.24 & .002 \\ 750 & 54.5 & .171 & <.00004 \\ 750 & 54.5 & .472 & .000047 \\ 760 & 56.2 & .13 & .00058 \\ 760 & 56.2 & 7.49 & .00057 \\ 840 & 72.3 & .008 & - \\ 860 & 76.9 & .045 & .00019 \\ 870 & 79.4 & .0115 & .0168 \\ 870 & 79.4 & -. .- & .0498 \\ 910 & 89.9 & . .- & .046 \\ 950 & 120.0 & .0003 & - \\ 960 & 105.0 & .0014 & .0001 \\ 960 & 105.0 & <.00004 & <.00004 \\ 980 & 112.0 & --. & <.00004 \\ 980 & 112.0 & -.- & <.00004\end{array}$

1Radiant solar flux (W/cm2) estimated from the calibration equation;

Flux $=I_{0}=52.1 \exp (0.003137) \quad r=0.9815$

where $T$ is temperature (C).

2Fraction remaining expressed as weight percent. 


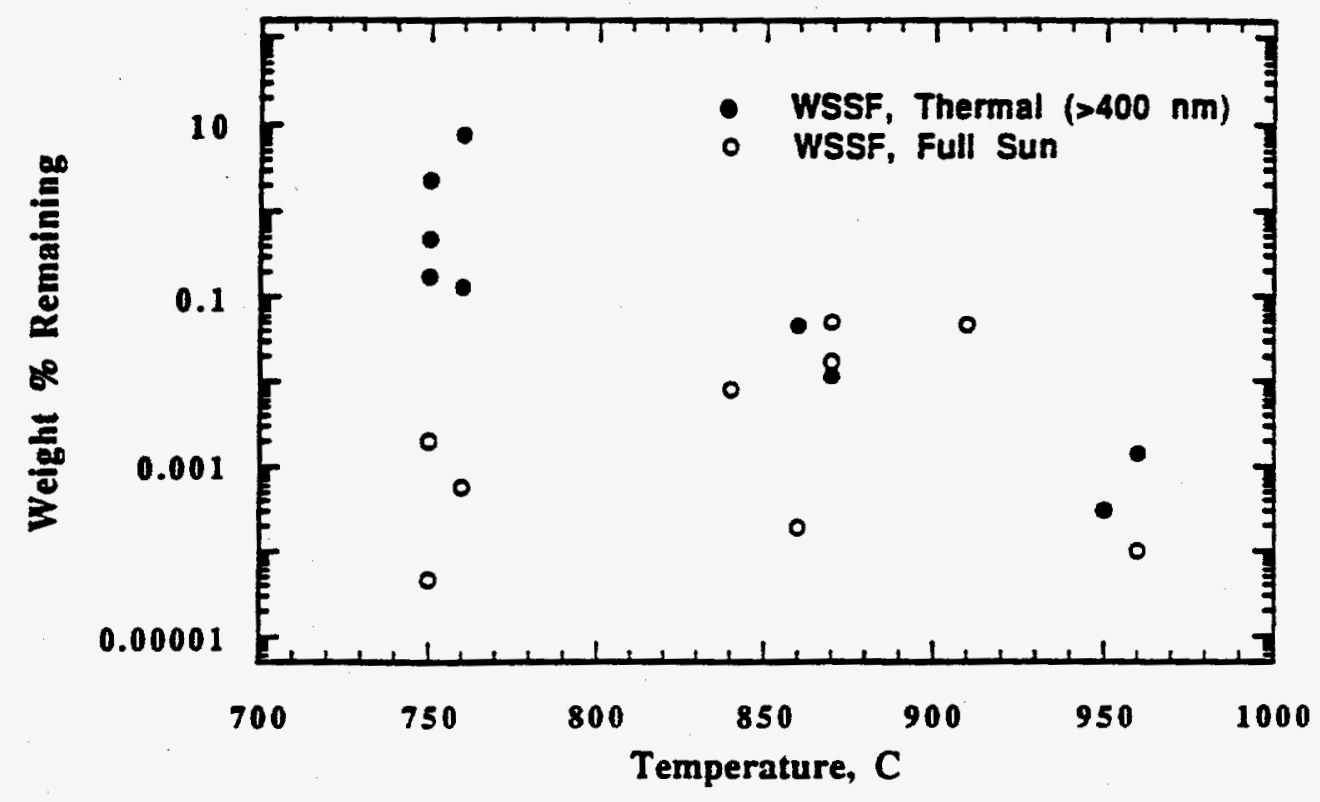

Figure 8.3. Summary of the field data for 1,2,3,4-TCDD including thermal (near-UV below $400 \mathrm{~nm}$ attenuated), solar (tull solar spectrum).

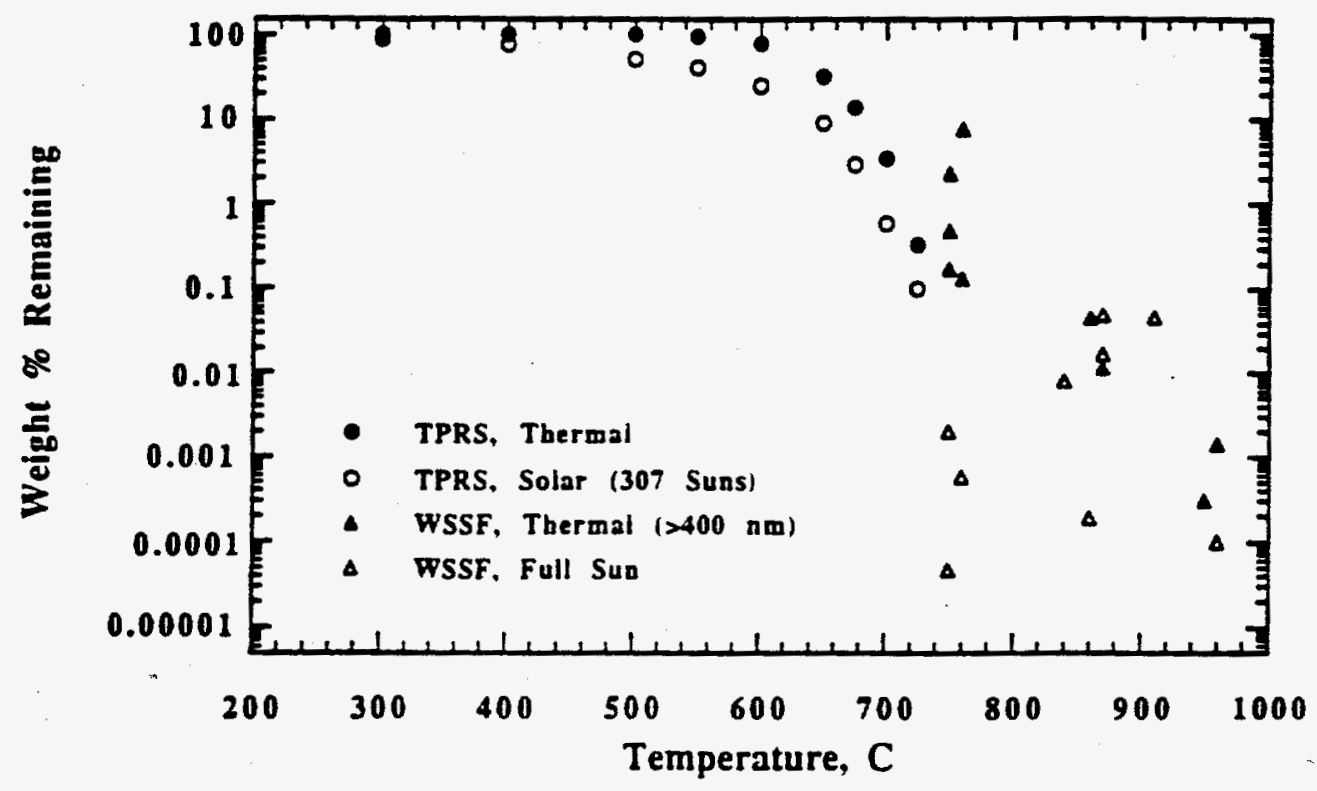

Figure 8.4. Summary of the laboratory and field data for 1,2,3,4-TCDD with a mean exposure time of $5 s$ in air. 


\subsubsection{RESULTS}

Initial shake-down tests were performed in June 1988, with additional system trials in July 1988. This led to a series of successful tests in September and October 1988 and February 1989. The data from these tests that are directly comparable to laboratory experiments (cf. Table 8.1 and Figure 8.3) (i.e., a mean residence time of 5 seconds using dry air as the reaction atmosphere) are summarized in Table 8.1 and Figure 8.4. The data described as "Full Sun" were obtained with the full solar spectrum delivered by the solar furnace (wavelengths from $\sim 300-4,000 \mathrm{~nm}$ ). The data described as "Thermal" were taken with a polymer film placed in the converging solar beam which removed wavelengths below about $400 \mathrm{~nm}$. In the latter case, the wavelengths that would induce solar photochemical reaction have been removed without significantly altering the heat content being delivered to the reactor. This allowed the reactor to be operated in an essentially thermal mode analogous to the thermal mode used in the laboratory.

\subsubsection{DISCUSSION}

The sum of all the data, laboratory and field, are summarized in Figures 8.5 and 8.6. At first glance, there appears to be such scatter in the field data that a comparison with the laboratory data is not possible. However, on closer examination several observations can be made.

Considering only the thermal data from the field tests, we find that the extent of scatter in the data is relatively small compared to the full-spectrum solar data. Furthermore, since the TPRS reactor behaves as a nearly plug flow system [1], the field data illustrates the type of behavior that would be expected from a reactor with a broad residence time distribution[2].

The schematic of the field reactor (cf. Figure 8.2) shows that the reactants (TCDD and air) enter the reactor vessel through a porous ceramic frit. This gives an initial flat velocity distribution across the diameter of the reactor of about $2.5 \mathrm{~cm} / \mathrm{s}$. As the gasses flow through the reactor they would be expected to begin to develop the characteristics of laminar flow (i.e., zero velocity at the wall increasing to a maximum near the centerline). However, the vessel is so short with respect to its diameter that this is unlikely to occur to a great extent. However, the gasses leave the reactor through a narrow exhaust line resulting in a mean exit velocity of $250 \mathrm{~cm} / \mathrm{s}$. Clearly, there is a considerable distribution of velocities within the vessel. Furthermore, the Schmidt number for gasses is typically near unity, and at the high temperatures used here, they are likely to be less than unity[2], suggesting that the diffusive component of flow is at least as important as the convective flow. Given these factors, a broad residence time distribution would be expected from this reactor. It can be shown that this broad distribution can account for the differences between the thermal data obtained from the laboratory and field reactors. 


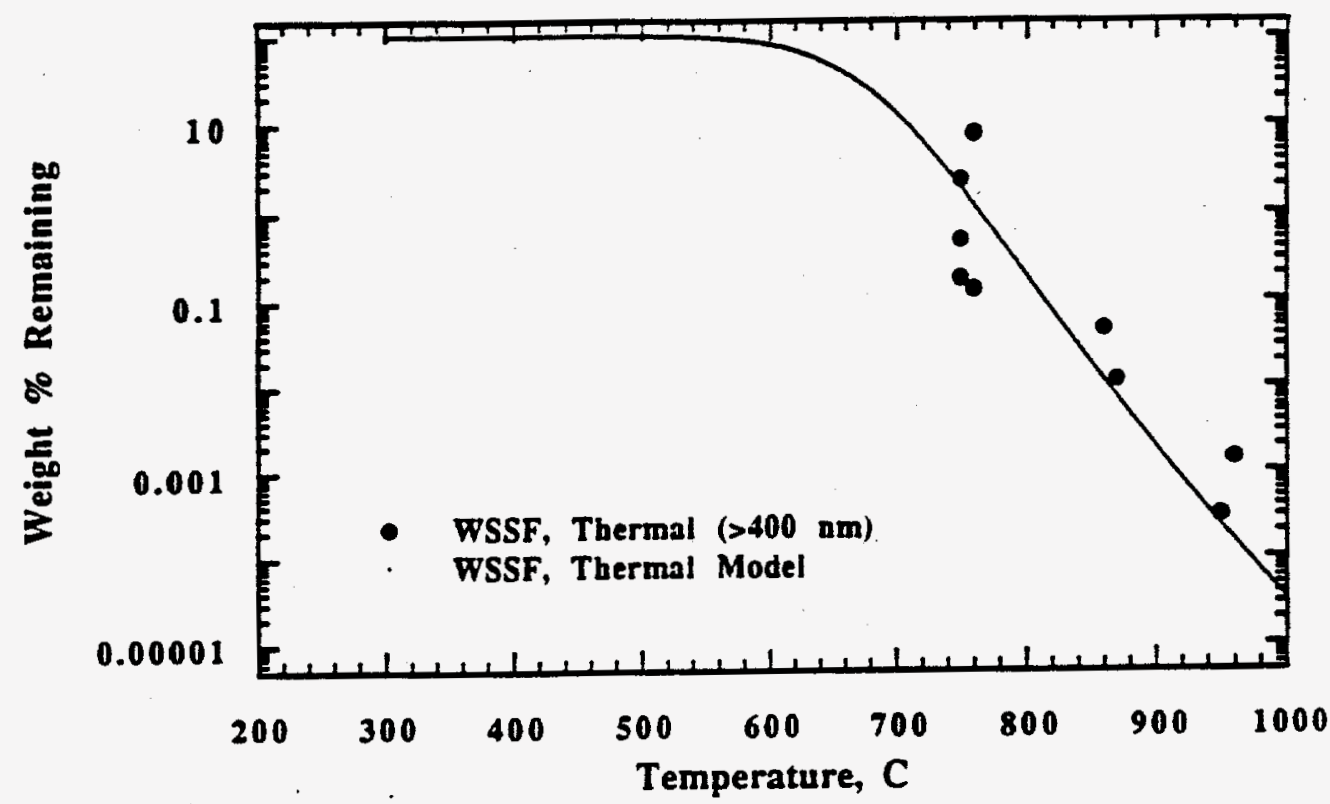

Figure 8.5. Thermal performance of the bench scaie reactor for the oxidation of $1,2,3,4-T C D D$ with a mean exposure time of $5 \mathrm{~s}$ as predicted by a compartment model of the reactor, and measured at the WSSF with wavelengths $<400 \mathrm{~nm}$ removed from the solar spectrum.

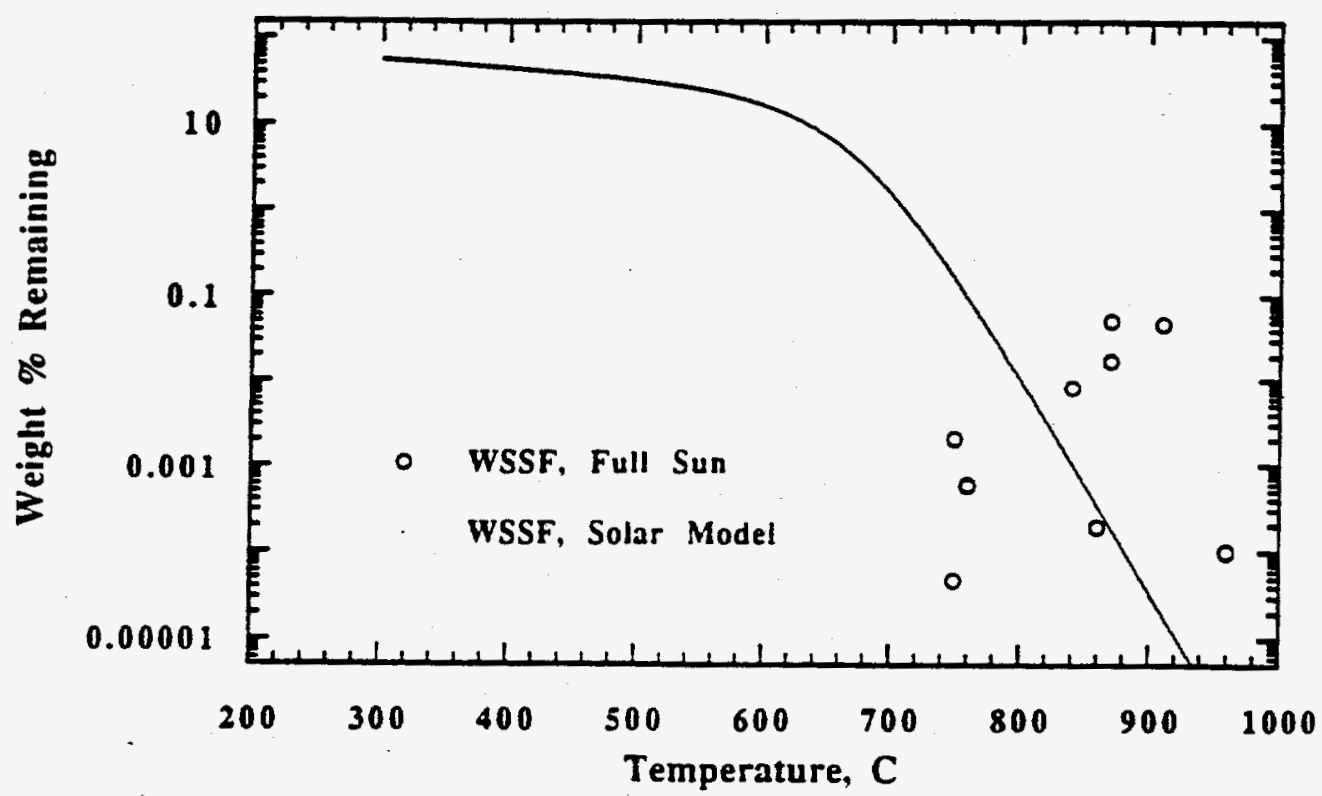

Figure 8.6. Solar periormance of the bench scale reactor for the oxidation of 1,2,3,4-TCDD with a mean exposure time of $5 \mathrm{~s}$ as predicted by a comparment model of the reactor, and measured at the WSSF using the entire ambient solar spectrum. 
Chemical reactors are often modeled using one of two approaches. These include the axial dispersion model (a diffusion process superimposed on a plug flow process) and the tanks in series model (a series of perfectly mixed vessels of equal size)[2]. These models are usually considered equivalent, and the choice of which to use is based on individual circumstances. The axial dispersion model is particularly useful, as reactor performance can be estimated from the system geometry and physical properties. Unfortunately, the field reactor's geometry (very short with respect to diameter) makes its use suspect. The tanks in series model is more empirical, but it is better suited to systems with broad residence time distributions as will be shown to be the case here.

For the first-order reactions the tanks in series model describes the reactant conversion as,

$$
f_{p}=1 /(1+k t / N)^{N}
$$

where $f_{r}$ is the fraction remaining, $N$ is the number of tanks, $k$ is the reaction rate constant $\left(\mathrm{s}^{-1}\right)$, and $t$ is the mean exposure $e_{r}$ and $t$ are known from the field tests, and $k$ can be calculated from the laboratory data.

Assuming that TCDD decomposed via first-order processes, the laboratory data can be used to estimate the Arrhenius kinetic parameters. Using the data in Table 8.1, we find $A=1.0 \times 10^{9} \mathrm{~s}^{-1}$ and $E_{a} \approx$ $41,000 \mathrm{cal} \mathrm{mol}^{-1}$. These values agree well with those found for other typical hazardous organic wastes [3].

Applying the tanks in series model (eqn. 8.1), to thermal data from the field tests shows that the reactor may be accurately described by 2.5 tanks in series. This suggests a compartment model consisting of two completely stirred tanks of equal volume in series with a third tank whose volume is half that of the first tank. Analytically, this may be described as:

$$
f_{r}=1 /\left(2\left(1+k t_{1}\right)\left(1+k t_{2}\right)\right)
$$

where $t_{1}$ is the mean residence time in each of the first two tanks ( 2.0 seconds) and $t_{2}$ is the mean residence time in the last tank ( 1.0 second). As Figure 8.5 shows, this model accurately describes the field reactor's thermal performance.

Using a mean enhancement ratio of 4.1 at $30.7 \mathrm{~W} / \mathrm{cm}^{2}$, the modeled thermal performance of the field reactor and the calibrated temperature flux data for the reactor, the solar performance of the reactor was estimated. These results, along with the actual field data, are given in Figure 8.6. This figure shows that the solar reactor performance varied from far less than expected to far better. This is in contrast to the thermal 


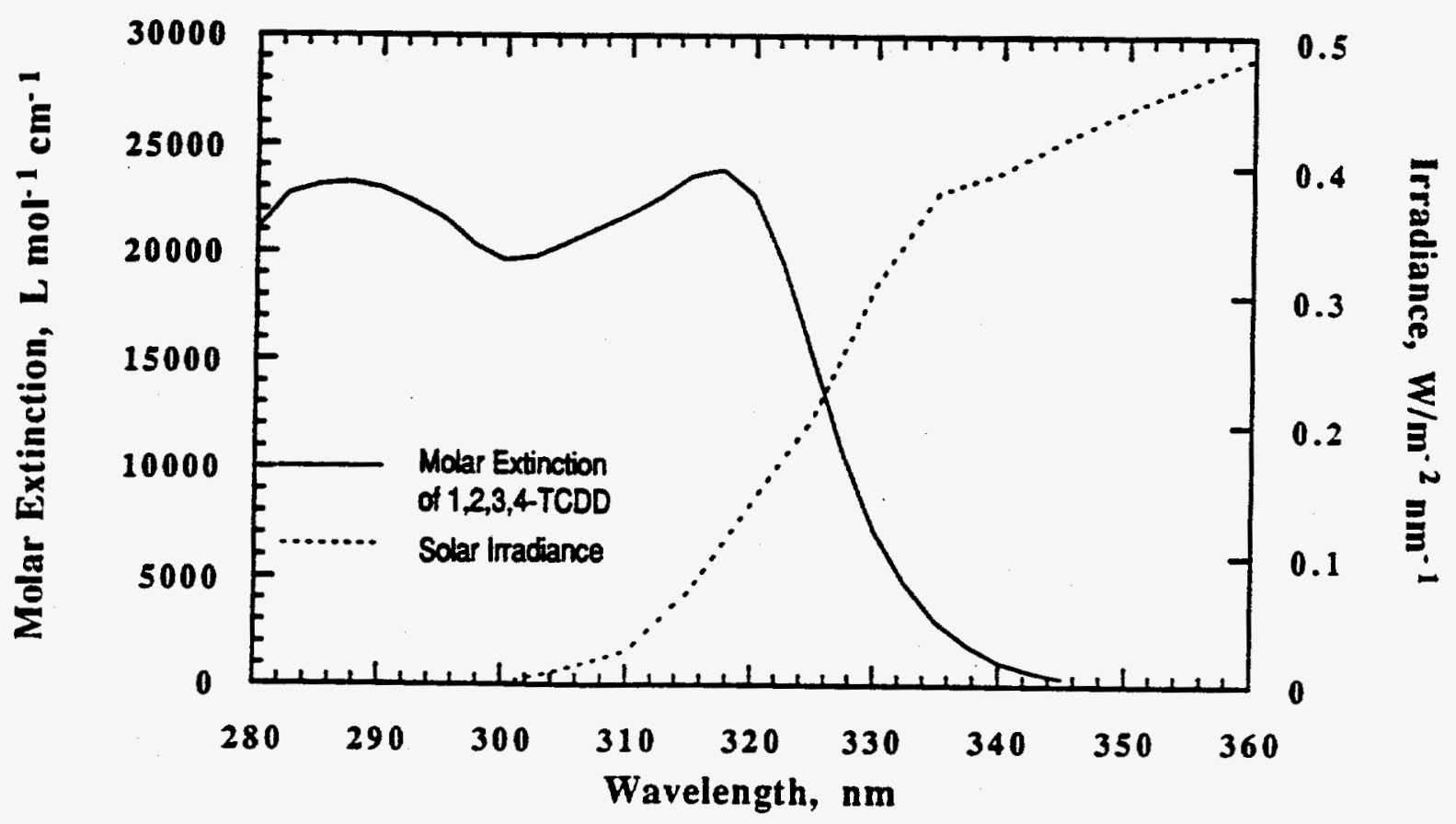

Figure 8.7. Absorption spectrum of 1,2,3,4-TCDD @ 23으 $\mathrm{C}$ in benzene and an AM 1.5 solar irradiance spectrum. 
data which shows that the performance of the reactor is fairly stable with respect to temperature and mean residence time. This suggests that source of the instability of the reactor operating in a full solar mode is related to the photolytic portion of the reaction process.

The relative molar extinction spectrum for $T C D D$ (obtained at $230^{\circ} \mathrm{C}$ in benzene solution) and the emission spectrum for an air mass 1.5 (AM1) solar spectrum is shown in Figure 8.7. This figure shows that the TCDD is a fairly strong absorber of solar photons over a narrow band from the onset of the solar spectrum $(\sim 300 \mathrm{~nm})$ to the upper limit of the TCDD absorption $(\sim 345 \mathrm{~nm})$. Furthermore, recent studies have shown that the absorption spectrum of the TCDD is expected to become more intense and extend further into the solar spectrum as the temperature is increased [4].

Unfortunately, the near UV portion of the terrestrial solar spectrum has not been extensively studied and the solar spectrum was not monitored in the course of the field tests described here. However, a recent study confirms that the near UV solar spectrum is strongly effected by air mass (the relative path length of the sunlight through the atmosphere)[5]. Furthermore, reported values for the intensity of solar radiation becomes increasingly uncertain $(>50 \%)$ at wavelengths near $300 \mathrm{~nm}$. The large scatter in the solar data relative to the thermal data suggests that the region of the solar spectrum being used to drive the solar thermal/photolytic reactions in TCDD is quite variable. Specifically, it was observed in the field data that the performance of the field reactor varied from the baseline thermal performance (due to the near-UV being naturally attenuated) to far better than expected (exceptionally clear skies) based on data from the laboratory system whose solar simulator is between these two extremes.

These tests suggest that thermal data obtained from the laboratory using the TPRS can be scaled to larger, bench scale, solar reactors if the differences in residence time distributions are taken into account. In this case, the field reactor is likely to have a broad residence time distribution equivalent to 2.5 tanks in series. This broad distribution significantly limits the ability of the reactor to destroy the TCDD to high levels $(>99.9999 \%)$ though it can do so at very high temperatures. Given the importance of this parameter it is clear that it should be taken into account of future solar reactors and carefully considered when examining the results from these systems.

The solar data is not as easily scaled due to the uncertainty in the near UV portion of the solar spectrum. However, both the laboratory and field data illustrate that solar energy has the potential to destroy organic wastes far more efficiently than thermal processing alone. This is clearly shown in the data taken in the field at temperatures around $750^{\circ} \mathrm{C}$ where a mean enhancement on the order of 1000 is observed, and in the data taken at temperatures above $900^{\circ} \mathrm{C}$ where several data points are below the detection limit of $99.99994 \%$ DRE. However, the relatively large amount of scatter in the solar data versus thermal data suggests that 
detailed characterization and monitoring of the near-UV portion (wavelengths less than $400 \mathrm{~nm}$ ) of the solar irradiance spectrum during field tests is needed to accurately interpret the results of these tests and to make it possible to extrapolate data obtained from laboratory reactors using simulated sunlight.

\subsection{DROPLETS IN AN INTENSE RADIATIVE FIELD}

Some conceptual designs for a solar detoxifier utilize spray atomization as the method for introducing waste to the reaction. Consideration of the effects of an intense radiative field on spray droplets suggest there be three primary effects. These are:

1. Decreased droplet vaporization time due to radiative heating,

2. Decreased droplet ignition time due to thermal/photolytic initiation of combustion, and

3. Micro-explosion and secondary atomization due to focused radiation on droplet interior

We have conducted preliminary calculations to determine the magnitude of each effect.

\subsubsection{RADIATIVE HEATING}

Theory and experiment [ 6 and refs. therein] indicate that the following expression governs the vaporization and burning rate of spherical, symmetrical, single droplets (1-D analysis):

$$
d^{2}=d_{0}^{2}-B t
$$

where $d_{0}$ is the original drop diameter and $d$ is the drop diameter after the time $t$. A typical gasification profile for a droplet is shown in Figure 8.8. The linear region of this plot is indicative of steady burning. Deviation of linearity for very short times is indicative of droplet ignition while deviation from linearity for very long times is indicative of droplet extinction.

It can be shown that vaporization times $\left(\tau_{v}\right)$ and burning times $\left(\tau_{b}\right)$ can be defined by the following expressions:

$$
\tau_{v}=\left(8 k d_{0}{ }^{2} / C_{p}\right) p_{l} \ln \left[1+\left\{C_{p, g}\left(T_{\infty}-T_{b}\right) / H\right]\right.
$$

and,

$$
\tau_{b}=\left(8 \mathrm{kd} d_{0}^{2} / C_{p}\right) \rho_{1} \ln \left[1+\left\{C_{p, g}\left(T_{\infty}-T_{b}\right)+Y_{o} Q_{d} / \sigma\right) / H\right]
$$




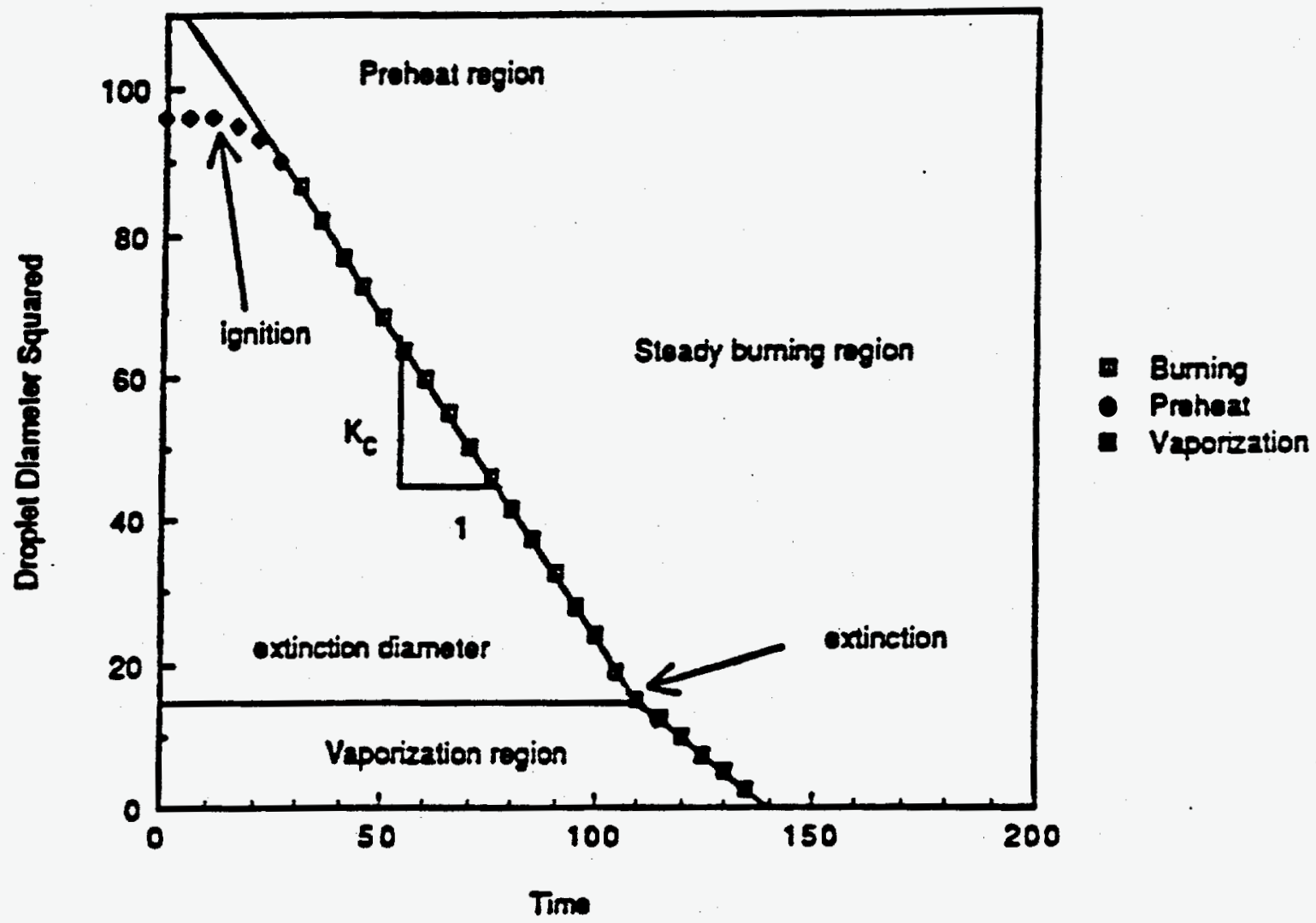

Figure 8.8. A typical gasification profile for a droplet. 
where: $k$ is thermal diffusivity $\left(\mathrm{cm}^{2} \mathrm{~s}^{-1}\right), T_{\infty}$ is temperature of the surrounding atmosphere ( $\left.{ }^{\circ} \mathrm{K}\right), \mathrm{C}_{\mathrm{p}, \mathrm{g}}$ is the droplet vapor heat capacity (cal $\left.g^{-1}{ }^{\circ} \mathrm{K}-1\right), T_{b}$ is the droplet boiling temperature $\left({ }^{\circ} \mathrm{K}\right), \rho_{1}$ is the liquid droplet density $\left(\mathrm{g} \mathrm{cm}^{-3}\right), Y_{0}$ is the oxygen mass fraction, $H$ is the effective latent heat of vaporization (i.e., energy needed for droplet heating plus latent heat of vaporization), and $Q_{c} / \rho$ is the heat of combustion per unit mass of fuel reacted divided by stoichiometric oxidizer-to-fuel ratio.

Consideration of the effect of collimated radiation falling on one hemisphere of the droplet results in the following simplified energy balance expression:

$$
\pi r^{2} H=\dot{m} q,
$$

where $q_{r}$ is the input radiant energy per unit area. The $\pi r^{2}$ term is different from that of Williams $[7$ because we have only a cross-sectional area while in the case of droplet burning, the radiant energy source intercepts the entire droplet surface. Thus,

$$
\dot{m}=\frac{-d\left(4 / 3 \pi r^{3} p_{1}\right)}{d t}=-4 \pi r^{2} \frac{d r}{d t}
$$

Substitution of 8.7 into 8.6 followed by integration yields:

$$
\Delta r=\frac{4 H p_{1} r}{q_{r}}=\frac{8 d_{o} \rho_{1}}{q_{r}} H
$$

The result of this derivation indicate that if radiant transfer becomes a dominant source of energy input to the droplet, then the $\mathrm{d}_{2}$ law becomes a simple $d$ law for the case where the Planck-mean absorption length of the gas is large compared with the droplet diameter $[7]$.

Tables 8.2 and 8.3 present droplet vaporization calculations using equations $8.4,8.5$, and 8.8 for thermal, oxidative, and radiative environments, respectively. Calculations were conducted for fuel oil and a model hazardous compound, $\mathrm{CH}_{2} \mathrm{Cl}_{2}$. The boundary conditions for each calculations are given in the bottom of each table. For both cases, the effect of a quadratic versus a linear $d_{0}$ dependence are clearly evident. For very small droplet diameters, a dominant radiation field results in a prolonged burning time compared to both thermal and oxidative environment. However, as droplet sizes increase burning times in a radiation field increase only linearly. Although some key assumptions had to be made, e.g., the fraction of the energy of the solar spectrum that was absorbed (viz., the wavelength dependent absorption cross-section), the calculation suggests that at $>500$ um radiative vaporization dominates thermal vaporization competes with vaporization in a burning droplet. Substitution of $\mathrm{CH}_{2} \mathrm{Cl}_{2}$ for fuel oil resulted in slightly reduced burning times. However, the 


\section{TABLE 8.2}

Droplet Vaporization Times for Fuel Oil in Air @ 300 Suns

\begin{tabular}{cccc}
$d_{0}(\mathrm{um})$ & thermal & $\begin{array}{c}\text { Jv }(\mathrm{s}) \\
\text { burning }\end{array}$ & solar \\
\hline 10 & 0.00917 & 0.0002 & 0.0428 \\
100 & 0.917 & 0.0402 & 0.428 \\
500 & 22.9 & 1.01 & 2.14 \\
1000 & 91.7 & 4.02 & 4.29 \\
& & \\
$T_{\infty}=300^{\circ} \mathrm{C}$ & & $Y_{0}=0.21$ \\
$T_{b}=270^{\circ} \mathrm{C}$ & $Q_{V}=55 \mathrm{cal} / \mathrm{g}$ & $\sigma=0.315$ \\
$T_{r}=20^{\circ} \mathrm{C}$ & $Q_{C}=9880 \mathrm{cal} / \mathrm{g}$ & $\rho=0.96$ \\
$C_{D}=0.4 \mathrm{cal} / \mathrm{g}$-deg & $Q_{r}=7.2 \mathrm{cal}^{2} \mathrm{~cm}^{2}-\mathrm{s}$ & $\mathrm{k}=0.1 \mathrm{~cm}^{2} / \mathrm{s}$
\end{tabular}

TABLE 8.3

Droplet Vaporization Times for Methylene Chloride in Air @ 300 Suns

\begin{tabular}{cccc}
$d_{0}$ & thermal & $\begin{array}{c}\text { Ju(s) } \\
\text { burning }\end{array}$ & solar \\
\hline 10 & 0.00064 & 0.00017 & 0.0352 \\
100 & 0.064 & 0.017 & 0.352 \\
500 & 1.595 & 0.419 & 1.76 \\
1000 & 6.378 & 1.678 & 3.52 \\
& & & \\
$T_{\infty}=300^{\circ} \mathrm{C}$ & & $Y_{D}=0.21$ \\
$T_{b}=40^{\circ} \mathrm{C}$ & $Q_{V}=89 \mathrm{cal} / \mathrm{g}$ & $\sigma=0.47$ \\
$T_{r}=20^{\circ} \mathrm{C}$ & $Q_{C}=1700 \mathrm{cal} / \mathrm{g}$ & $\rho=1.33 \mathrm{~g} / \mathrm{cm}^{3}$ \\
$C_{D}=0.3 \mathrm{cal} / \mathrm{g}$-deg & $Q_{r}=7.2 \mathrm{cal} / \mathrm{cm}^{2}-\mathrm{s}$ & $\mathrm{k}=0.24 \mathrm{~cm}^{2} / \mathrm{s}$
\end{tabular}


effect of droplet diameter on the relative burning times was similar to that observed for the fuel oil case, i.e., a radiation dominated environment reduced burning times for larger droplets.

These calculations were for a conservative value of $Q_{r}$ of $7.2 \mathrm{cal} / \mathrm{cm}^{2} \mathrm{~s}$ corresponding to 300 suns. Since $\tau_{v}$ (solar) decreases linearly with $Q_{r}$, it is clear that radiative effects become relatively more important for even smaller diameter droplets when higher solar intensities are used.

\subsubsection{PHOTO-INDUCED IGNITION}

We have previously shown evidence for photo-initiated radical chain reactions when the system temperature is elevated above about $500^{\circ} \mathrm{C}$. If the organic concentration is sufficiently high, ignition and sustained combustion may occur. One manifestation and direct measure of the effect of solar induced ignition is a reduction in the ignition delay time for the droplet, td. $t_{d}$ for droplets has been shown to empirically obey the expression:

$$
\tau_{d}=A\left[O_{2}\right]^{n} \exp E / R T
$$

Since solar induced ignition effects have been experimentally studied, we can speculate that addition of solar radiation may effect $\tau_{d}$ through an increase in $A$, decrease in $E$, or a decrease in $n$.

\subsubsection{DROPLET LENSING}

Since the indices of refraction of droplets are different then that of air they are expected to act as lenses for the incident radiation. Depending upon the index of refraction and the size of the droplet, the incident radiation may be weakly or moderately strongly focused at a point interior to the droplet.

Using Snell's law of refraction we have calculated ray traces for the case of $500 \mathrm{~mm}$ diameter droplets with indices of refraction, $n_{D}$ of 1.4 and 1.9. An no of 1.4 is typical for that of No. 5 fuel oil. As one can see from the diagrams in Figure 8.9, it is possible to focus the radiation at the droplets interior. This focusing would expect to result in uneven heating and droplet micro-exploring. The final result is solar induced secondary atomization which should result in increased destruction efficiency of the waste.

The results depicted in Figure 8.9, are based on many simplifying assumptions. These include:

- the droplet remains spherical after heating, 


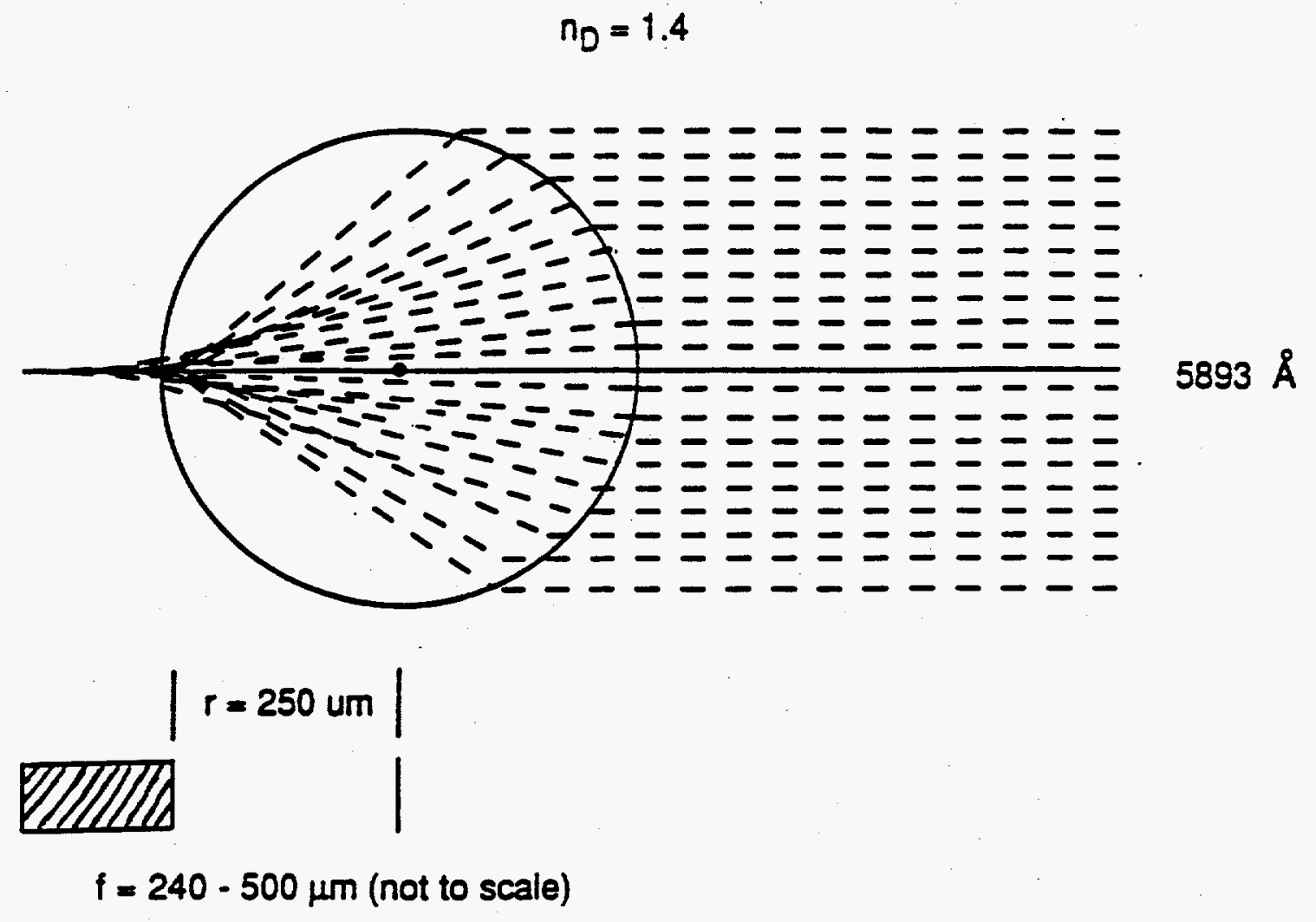

$$
n_{D}=1.9
$$
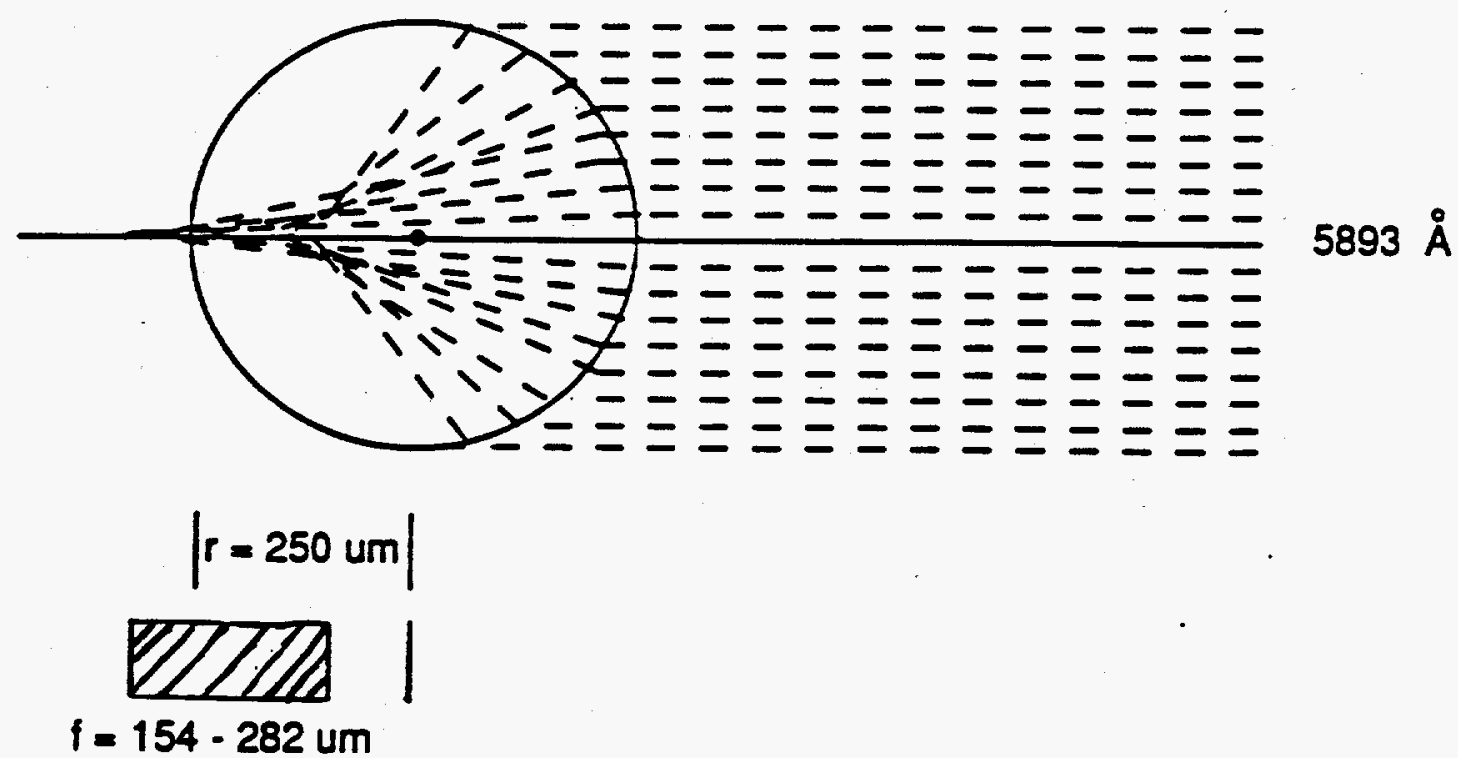

Figure 8.9. Calculated ray traces for a $500 \mu \mathrm{m}$ diameter droplets with indices of refraction, nD of 1.4 and 1.9. Note, an nD of 1.4 is typical for that of No. 5 tuel oil. 
- it does not absorb radiation,

- it is not distorted by convective waves,

- the incident radiation is monochromatic with $\lambda=589 \mathrm{~nm}$

(i.e., the tabulated values for $\mathrm{nD}_{\mathrm{D}}$ are for this wavelength)

Although our calculations are very crude, it does demonstrate how physical phenomena may effect the performance of a solar reactor. These effects are essentially unexplored and it is for this reason that we have proposed to conduct drop tube studies in droplets in an intense radiative field.

\subsection{REFERENCES}

1. Glatzmaier, G. C., Nix, R. G., and M. S. Mehos, "Solar Destruction of Hazardous Waste," J. Environ. Sci. Health, A25(5), 571-581 (1990).

2. J. L. Graham, B. Dellinger, and G. C. Glatzmaier, "The Destruction of 1,2,3,4-Tetrachlorodibenzo-p-dioxin Using Simulated and Natural Concentrated Solar Energy," submitted, Hazardous Waste and Hazardous Materials, October 1992.

3. J. L. Graham, "Determination of the Residence Time Distribution of a Laboratory Scale Flow Reactor," Graduate Thesis, School of Engineering, University of Dayton, August 1992.

4. O. Levenspiel, the Chemical Reactor Omnibook, OSU Bookstores, Inc., Corvallis, OR, 1989.

5. J. M. Berman, J. L. Graham, B. Dellinger, "High-Temperature Ultraviolet Absorption Characteristics of Three Environmentally Sensitive Compounds," accepted, Jeurnal of Photochemistry and Photobiology, 1992.

6. R. Hulstrom, R. Bird, and C. Riordan, "Spectral Solar Irradiance Data Sets for Selected Terrestrial Conditions," Solar Cells, Vol. 15, pp. 365-391, 1985.

7. C. K. Law, Proq. Energy Combust. Sci., 8: 171-202 (1982).

8. F. A. Williams, Combustion Theory, 2nd Ed. (Addison-Wesley, Reading, MA, 1990).

?. B. Dellinger, et al., "Determination of the Thermal Stability of Selected Hazardous Organic Compounds," Hazardous Waste, Vol. 1, No. 2, pp. 137-157, 1984. 


\section{SECTION 9.0 \\ DESTRUCTION OF NON-ABSORBING COMPOUNDS}

Reviewing the absorption spectra available in the literature for the types of compounds which may be present in a hazardous waste stream there are considerable numbers of compounds that will only weakly absorb solar radiation, or may not absorb it at all, even at high temperatures. If the solar detoxification process is to be broadly applied, methods must be developed to address non-absorbing compounds or accept sizable limitations on the eventual application of the technology. In Section 6 we discussed how non-absorbing compounds may be efficiently destroyed if they are in the presence of an adequate supply of other compounds that do absorb solar radiation. However, in these experiments the exact nature of the beneficial circumstances are poorly defined and elucidation of the significant mechanisms are difficult to determine. In this Section, two specific approaches to the solar enhance destruction of non-absorbing compounds are explored.

We first consider homogeneous systems (Section 9.1) in which a gas-phase initiator would be added to the feed stream to a solar reactor. We also consider a novel heterogeneous initiation process (Section 9.2) in which a photocatalytic surface would be added to a solar reactor that would generate reactive radicals within the reactor vessel itself. This concept is not a conventional photocatalytic approach in which the waste is destroyed on the surface of the catalyst. Instead, we use the catalyst to generate reactive species which are then transported into the bulk fluid where they act as homogeneous, gas-phase initiators.

\subsection{HOMOGENEOUS INITIATION}

The concept of homogeneous initiation is based on the addition of a strongly absorbing, gas-phase "sensitizer" species to an otherwise non-absorbing sample. This sensitizer may initiate waste destruction by transferring excitation energy, usually by a process called triplet-triplet energy transfer (i.e., energy transfer from an excited triplet state of one molecule directly to the excited triplet sate of another), or via electron transfer to the target compound. The presumption is that these processes convert the target waste molecules from its stable ground state into a more reactive excited state from which it can decompose. The sensitizer may also initiate waste destruction by itself decomposing and the reactive radicals, or atoms, that are formed react in turn with the more stable waste molecules.

We have performed two experimental studies of the energy transfer case, and two of the radical initiator case. Both of these studies were for relatively simple binary mixtures with one component serving 
as the initiator, and the other the target waste compound. Each of these are discussed in the following paragraphs. Data for the mixtures are found in Appendix II.

\subsubsection{ENERGY TRANSFER SENSITIZATION.}

Two binary donor/acceptor (sensitizer/target) pairs were studied; acetone/benzene, and benzophenone/naphthalene. Both acetone and benzophenone absorb in the solar spectrum. Furthermore, both of these donor's excited state transitions are to $n \pi^{*}$ singlet states which, in accord with the theory of spin-orbit coupling, promote rapid intersystem crossing to long-lived $\pi \pi^{*}$ triplet states. The two experimental pairs were selected because of the match in triplet energies of the donors with their respective acceptors which is requisite for efficient triplet-triplet energy transfer (cf. Figures 9.1 and 9.2).

Data for the destruction of benzene in the presence of acetone is summarized in Figure 9.3. As these data illustrate, no enhancement in the destruction of benzene was observed at temperatures up to $800^{\circ} \mathrm{C}$. Data was not obtained at temperatures above $800^{\circ} \mathrm{C}$ as the acetone itself was significantly decomposed by this temperature, so the donor compound was essentially dropping out of the mixture. Interestingly, a small thermal enhancement in the destruction of benzene was observed due to the thermal destruction of the acetone.

Data for the destruction of naphthalene in the presence of benzophenone is shown in Figure 9.4. This data shows a significant solar enhancement. However, benzophenone itself decomposes at a lower temperature in the presence of solar radiation (cf. Figure 9.5). In fact, benzophenone itself thermal/photolytically decomposes before naphthalene. Thus, the mechanism of enhancement of naphthalene destruction is more likely radical attack from thermal/photolytic destruction of benzophenone than energy transfer.

Consideration of the theory of energy transfer suggests that energy transfer sensitization may be of little value at high temperatures. Reversibility of is known to be a problem. In fact, to facilitate irreversible energy transfer, the donor energies are usually selected to be of a higher value than the acceptor such that the higher density of states in the acceptor favors the forward transfer over the reverse. At elevated temperatures, higher energy (and higher density) states of the donor are accessible. This means that energy transfer may be more reversible at these elevated temperatures, and thus less efficient.

In any case, the decomposition of possible donor species tends to limit the applicability of this approach. In general, large, complex molecules make good sensitizers. These same molecules tend to thermally and thermal/photolytically decompose at lower temperatures than the acceptor. 


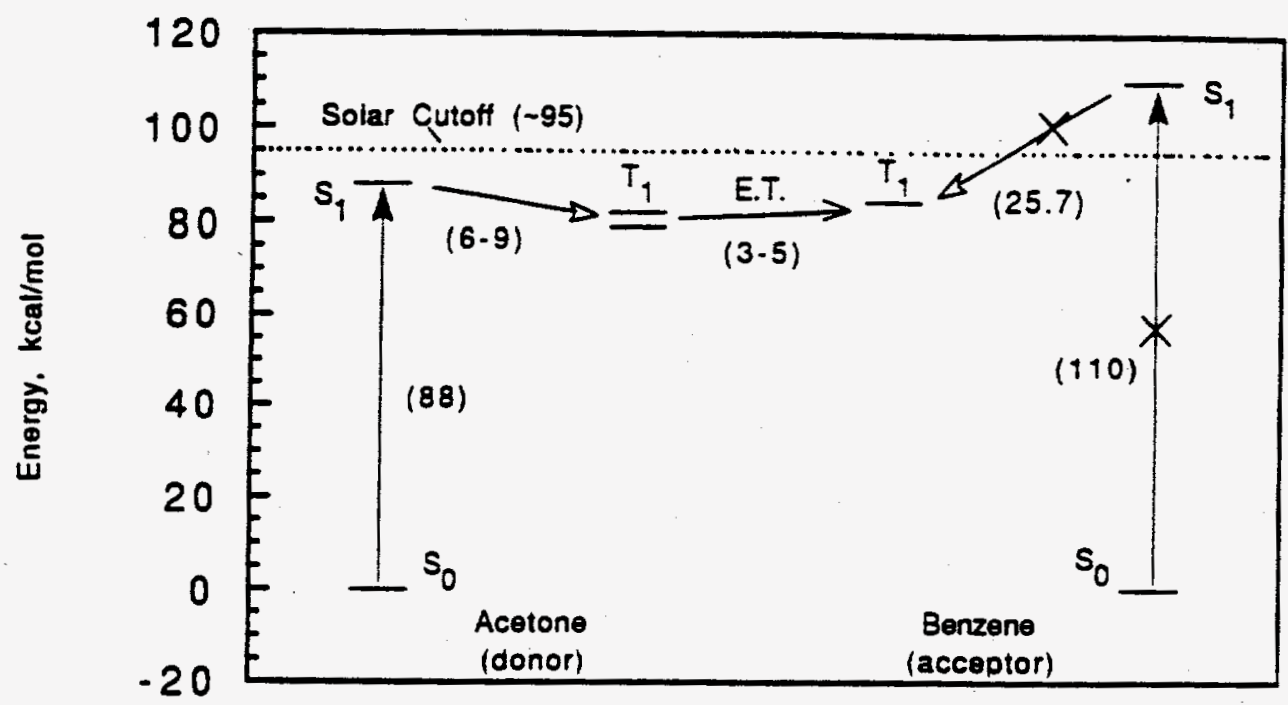

Figure 9.1. Electronic state energy diagram for acetone and benzene illustrating to slightly endothermic triplet-triplet energy transfer.

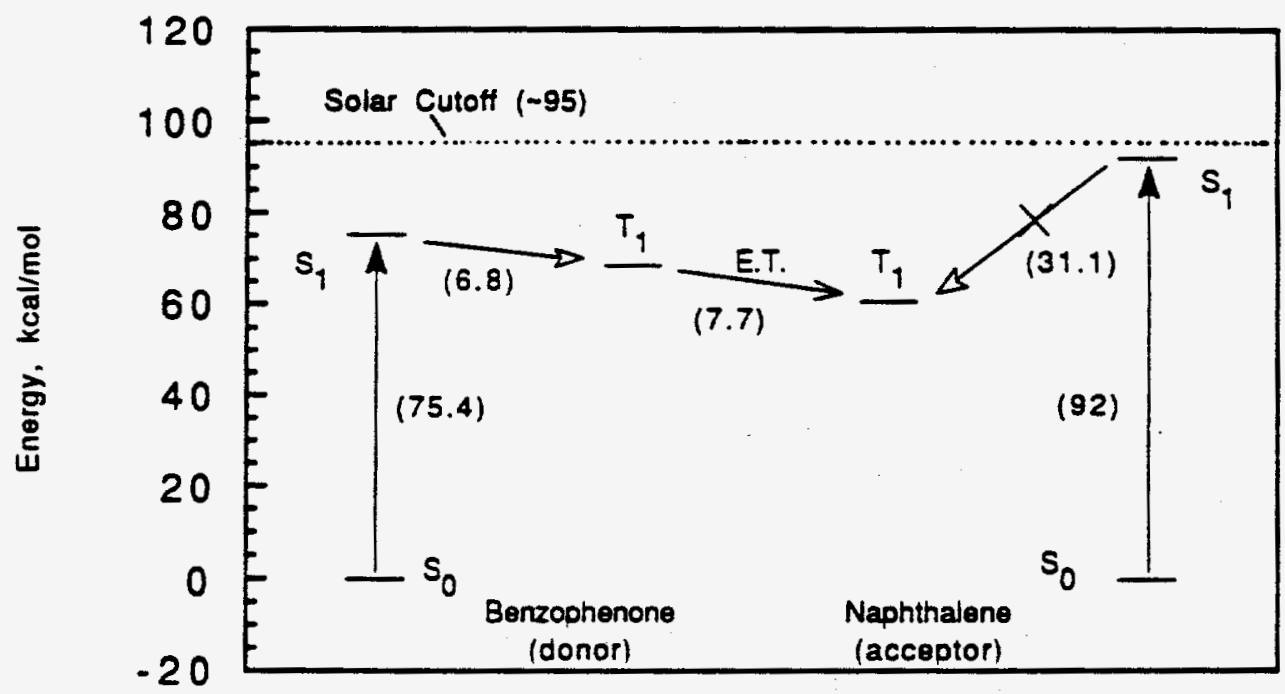

Figure 9.2. Electronic state energy diagram for benzophenone and naphthalene illustrating exothermic triplet-triplet energy tanster. 


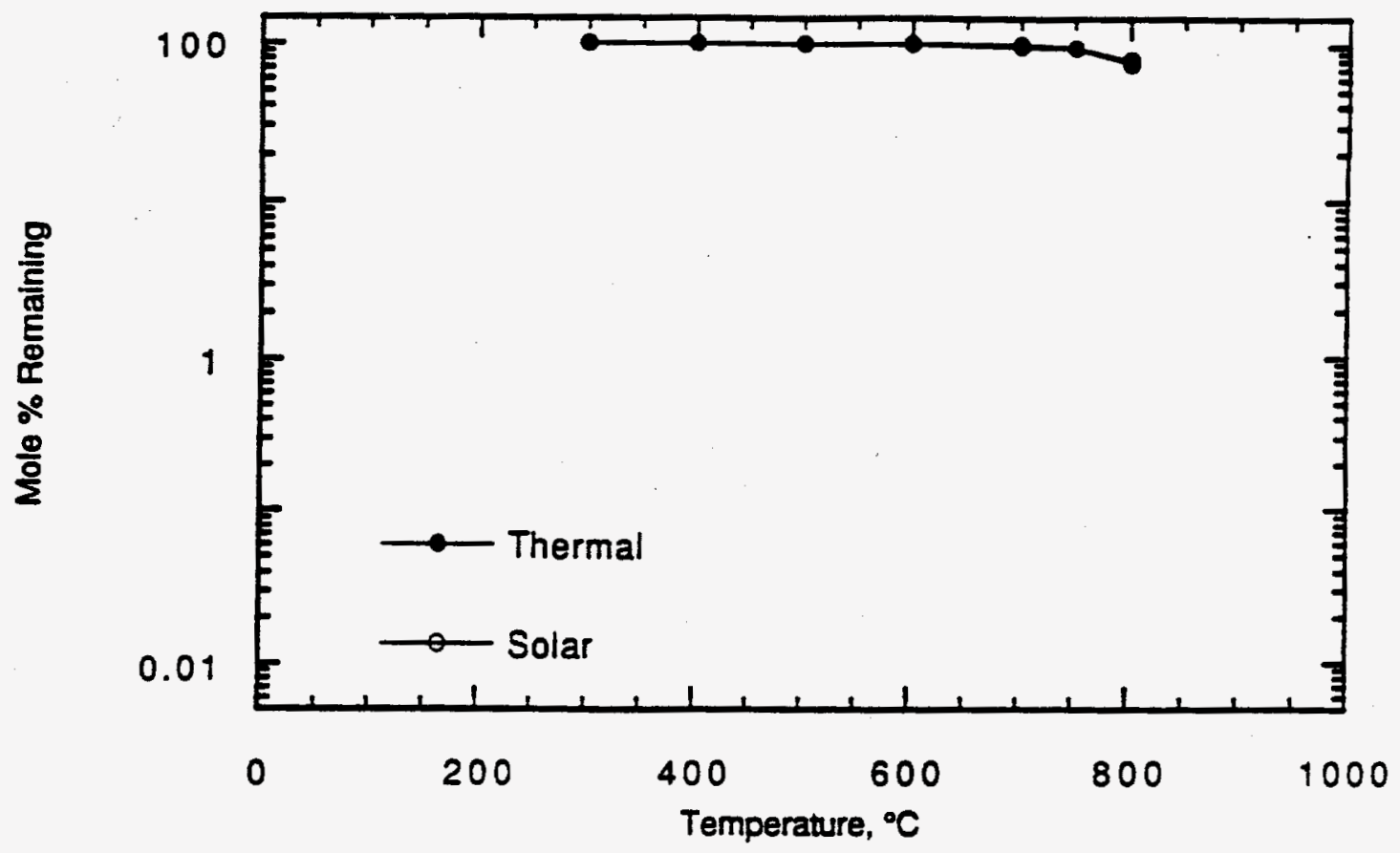

Figure 9.3. TPRS data for benzene $\left(-10^{-5} \mathrm{M}\right)$ in the presence of acetone $\left(-10^{-5} \mathrm{M}\right)$ exposed to 0 and 307 AM 1.0 suns (simulated) for $5 s$ in helium.

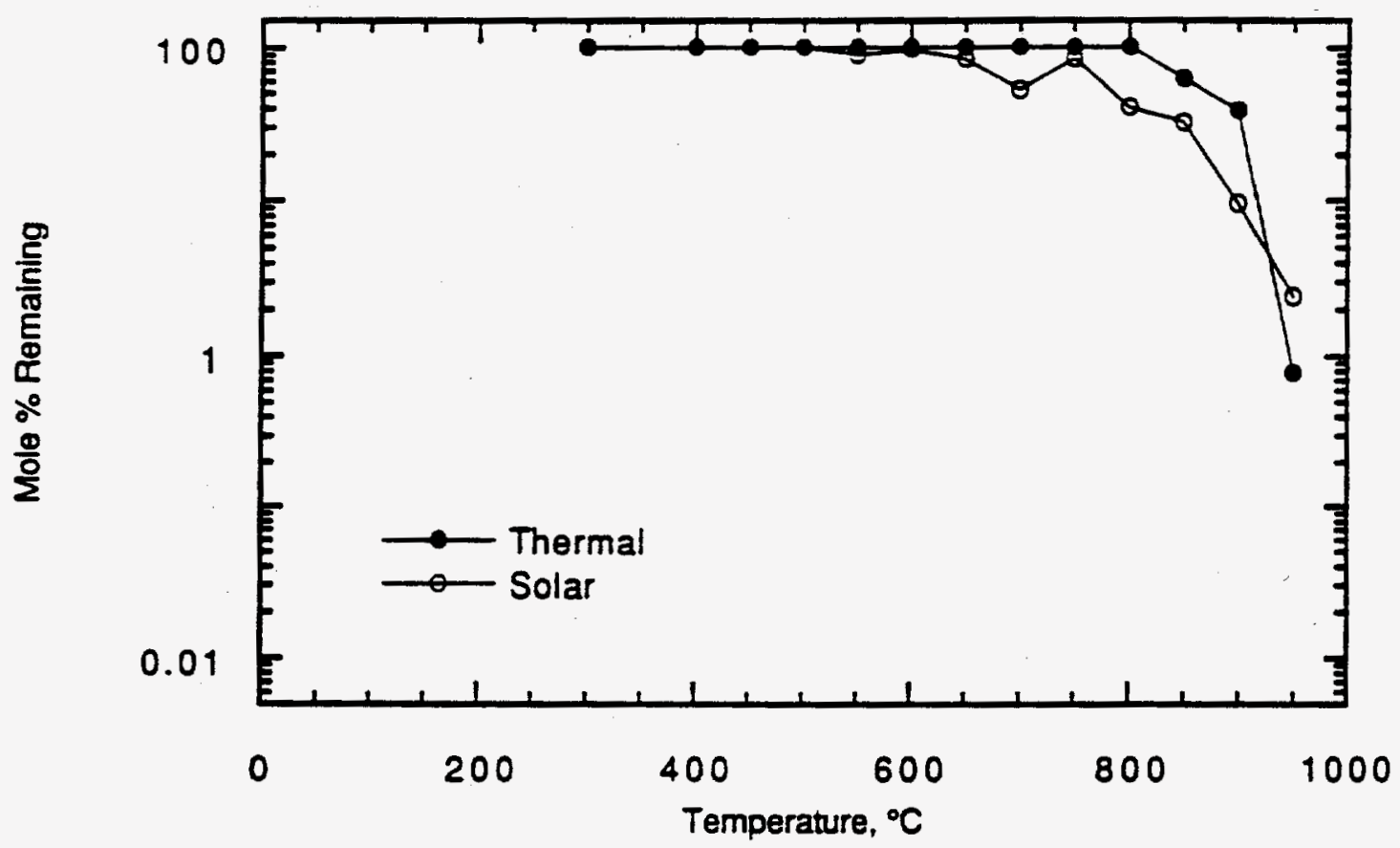

Figure 9.4. TPRS data for naphthalene $\left(\sim 10^{-5} \mathrm{M}\right)$ in the presence of benzophenone $\left(\sim 10^{-5} \mathrm{M}\right)$ exposed to 0 and 307 AM 1.0 suns (simulated) for 5 s in helium. 


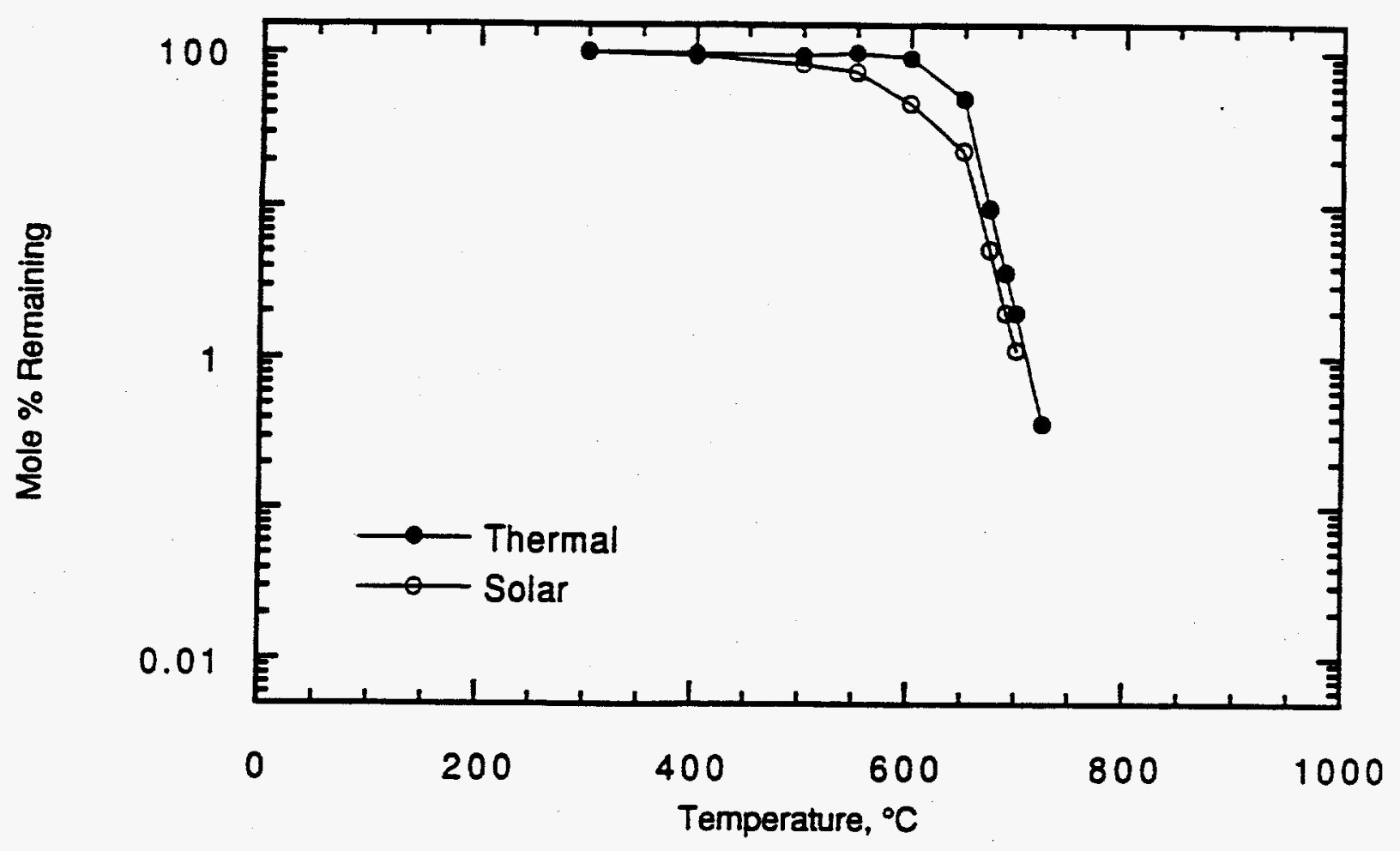

Figure 9.5. TPRS data for benzophenone $\left(-10^{-5} \mathrm{M}\right)$ exposed to 0 and 307 AM 1.0 suns (simulated) for $5 s$ in air. 


\subsubsection{PHOTO-GENERATED RADICAL INITIATION}

The effects of photo-generated radicals were studied with two binary systems; molecular chlorine with methylene chloride, and hydrogen peroxide with trichloroethylene. Molecular chlorine $\left(\mathrm{Cl}_{2}\right)$ was selected as a photo-initiator because it is well documented that $\mathrm{Cl}_{2}$ decomposes with unit quantum yield when irradiated with wavelengths within the solar spectrum. Hydrogen peroxide $\left(\mathrm{H}_{2} \mathrm{O}_{2}\right)$ was a more questionable candidate in that it may also dissociate when irradiated with wavelengths within the solar spectrum, but its absorption at wavelengths $>300 \mathrm{~nm}$ is exceedingly weak.

It was initially suggested that $\mathrm{Cl}_{2}$ would be a good photo-initiator because of the reactivity of $\mathrm{Cl}$. Although $\mathrm{Cl} \bullet$ is not as strong an oxidizer as $\bullet \mathrm{OH}$, it does track $\bullet \mathrm{OH}$ in its reactivity. Specifically, Cl• can abstract $\mathrm{H}$ to form $\mathrm{HCl}$ much as $\bullet \mathrm{OH}$ abstracts $\mathrm{H}$ to form $\mathrm{H}_{2} \mathrm{O}$.

The tests with chlorine/methylene chloride were conducted using a premixed sample with the constituents in a 3:1 volume-to-volume (chlorine:methylene chloride) ratio. This mixture was exposed to 0 and 307 AM 1.0 suns for $10 \mathrm{~s}$ in both dry helium (pyrolysis) and dry air (oxidation). As a baseline case, samples of methylene chloride by itself were exposed under the same conditions.

The tests with hydrogen peroxide/trichloroethylene were conducted using a premixed sample with the constituents in a 10:1 volume-to-volume (hydrogen peroxide:trichloroethylene) ratio. This mixture was exposed to 0 and 200 AM 1.0 suns for 10 seconds in dry air (oxidation). As a baseline case, samples of trichlororthylene by itself were exposed under the same conditions.

The TPRS data for the chlorine/methylene chloride are shown in Figure 9.6. Although these data demonstrate a very significant thermal/photolytic enhancement at temperatures up to $600^{\circ} \mathrm{C}(\mathrm{R}(307)$ of approximately 20 ), neither the thermal nor thermal/photolytic destruction exceeds $99 \%$ until the temperature reaches $500^{\circ} \mathrm{C}$, at which point the two curves coalesce. The unusual shape of the thermal decomposition curve (i.e., a two-step decomposition profile) is thought to represent an initial thermal dissociation of molecular chlorine to some steady-state value, which results in attack and destruction of methylene chloride, followed by additional, purely thermal destruction of methylene chloride (independent of $\mathrm{Cl}_{2}$ ) at temperatures above $600^{\circ} \mathrm{C}$. The convergence of the thermal/photolytic profiles at $600^{\circ} \mathrm{C}$ lend support to the hypothesis that $\mathrm{Cl} \cdot$ is at a steady-state concentration in both the thermal and photolytic cases. 


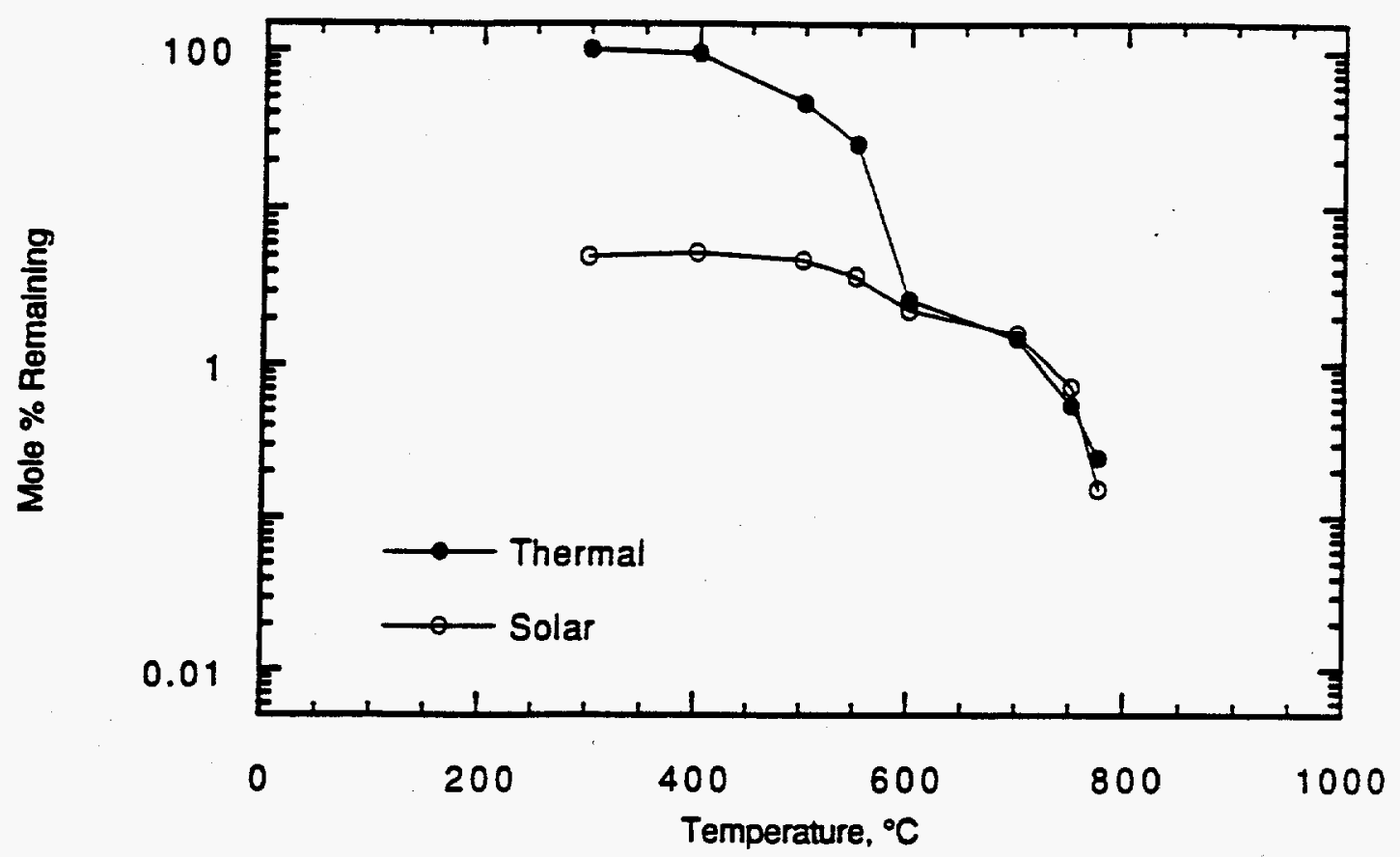

Figure 9.6. TPRS data for the methylene chloride $\left(-10^{-5} \mathrm{M}\right)$ component of a chlorine/methylene chloride mixture (3:1, v:v) exposed to 0 and 307 AM 1.0 suns (simulated) for 10s in helium.

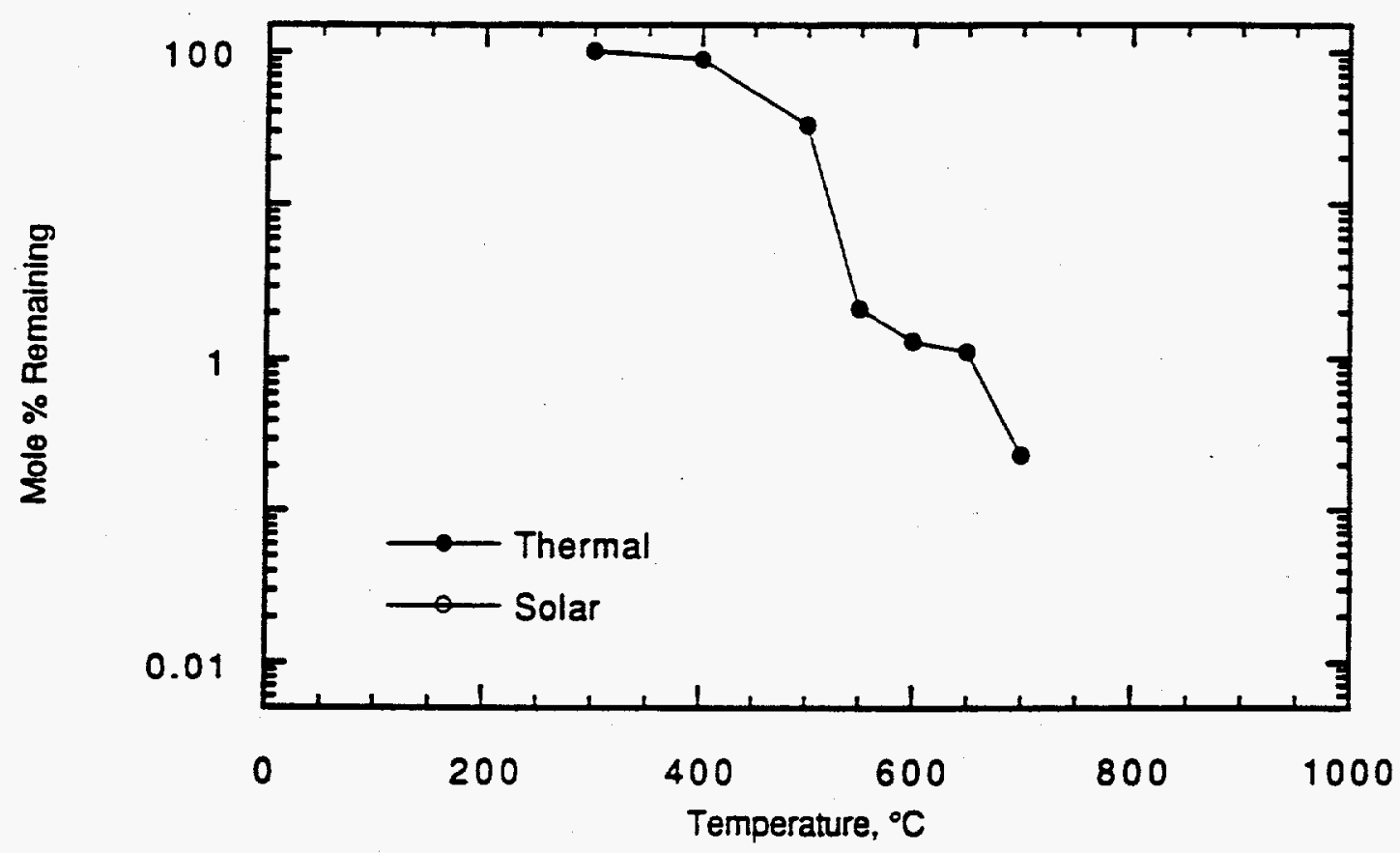

Figure 9.7. TPRS data for the methylene chioride component of a chlorine/methylene chloride mixture (3:1, v.v) exposed to 0 and 307 AM 1.0 suns (simulated) for 10 in air. Note that the fraction remainino under the simulated solar conditions is below the detection limit of $0.01 \%$ at all temoeratures. 


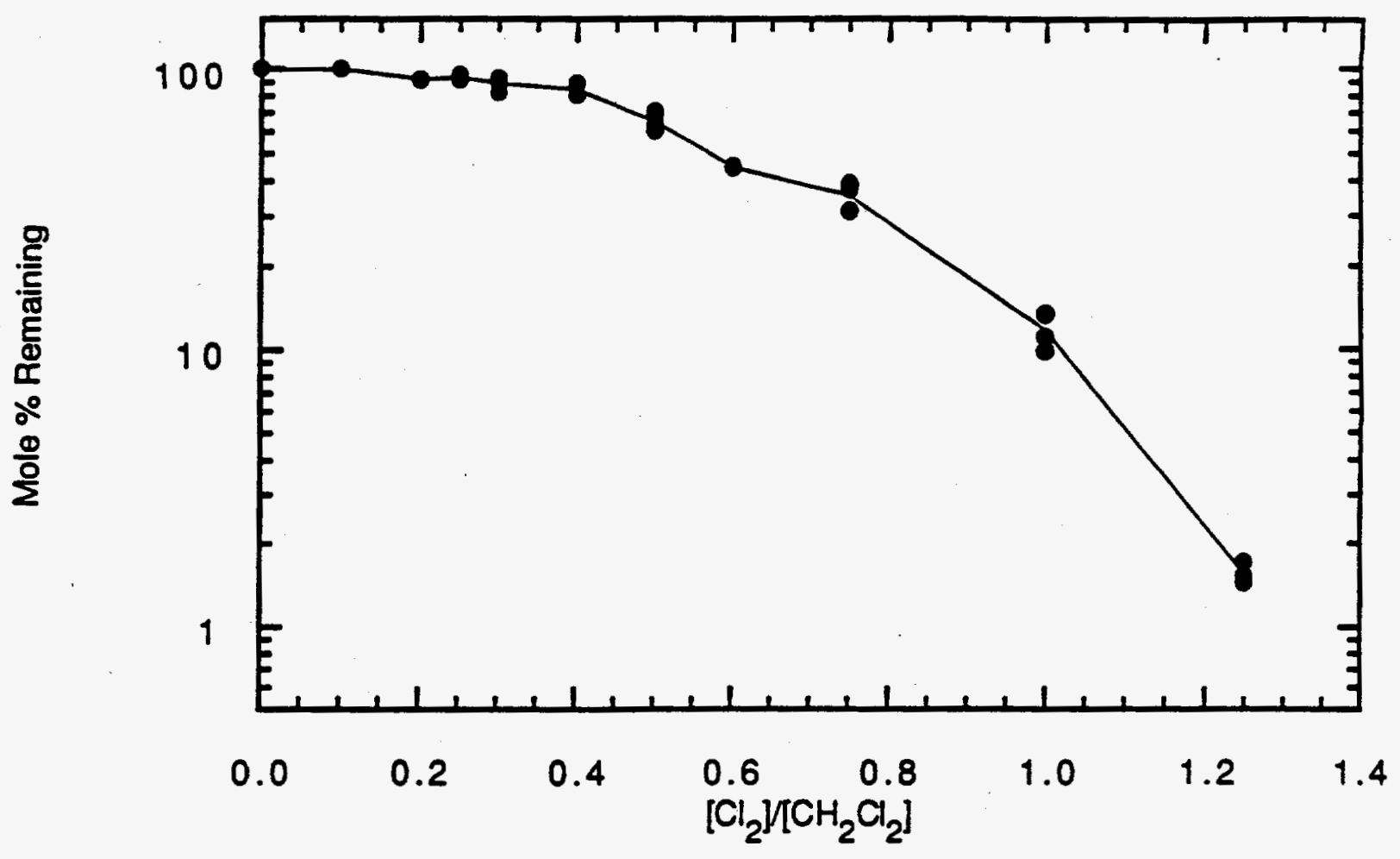

Figure 9.8. TPRS data for the methylene chloride component of a chlorine/methylene chloride mixture with the initial molar ratio of molecular chlorine to methylene chloride $\left(-10^{-5} \mathrm{M}\right)$ varying from 0 to 1.25 exposed to $307 \mathrm{AM} 1.0$ suns (simulated) and $400^{\circ} \mathrm{C}$ for $10 \mathrm{~s}$ exposure in air. 
Since the pyrolysis data for the chlorine/methylene chloride mixture showed a strong ability to initiate destruction of the target molecule, but a limited ability to achieve very high levels of conversion at low temperatures, the experiments were repeated in an oxidative environment. The results of these tests are summarized in Figure 9.7. These data demonstrate that under this condition, the methylene chloride showed thermal behavior similar to that observed under pyrolysis, but the thermal/photolytic process destroyed the sample to below the detection limit (about $0.01 \%$ ) at all temperatures studied.

Additional data was obtained on the effect of the initial ratio of molecular chlorine to methylene chloride to explore the effect of varying the donor/acceptor ratio. Figure 9.8 shows the fraction of methylene chloride remaining from a 10 s exposure in air at $400^{\circ} \mathrm{C}$ to $307 \mathrm{AM} 1.0$ suns with the initial molar ratio of molecular chlorine to methylene chloride varying from 0 to 1.25. As this Figure shows, the extent of methylene chloride conversion steadily increases throughout the concentration range.

This data still awaits a full kinetic and mechanistic analysis. We hypothesize that the addition of oxygen accelerates the destruction of initially formed radical intermediates as show below;

$$
\begin{aligned}
& \mathrm{Cl}_{2}+\mathrm{hv} \rightarrow 2 \mathrm{Cl} \cdot \\
& \cdot \mathrm{Cl}+\mathrm{CH}_{2} \mathrm{Cl}_{2} \rightarrow \cdot \mathrm{CHCl}_{2}+\mathrm{HCl} \\
& \cdot \mathrm{CHCl}_{2}+\mathrm{O}_{2} \rightarrow \mathrm{COCl}_{2}+\cdot \mathrm{OH} \\
& \mathrm{CH}_{2} \mathrm{Cl}_{2}+\cdot \mathrm{OH} \rightarrow \cdot \mathrm{CHCl}_{2}+\mathrm{H}_{2} \mathrm{O} \\
& \mathrm{COCl}_{2}+\mathrm{hv} \rightarrow \cdot \mathrm{COCl}+\cdot \mathrm{Cl} \\
& \cdot \mathrm{COCl} \rightarrow \mathrm{CO}+\cdot \mathrm{Cl}
\end{aligned}
$$

In this scheme methylene chloride is converted to $\bullet \mathrm{CHCl}_{2}$ radical by $\bullet \mathrm{H}$ abstraction by $\bullet \mathrm{Cl}$. In the presence of oxygen, $\bullet \mathrm{CHCl}_{2}$ can undergo a variety of pyrolysis reactions to produce an array of chlorinated products. Gas chromatographic analysis showed this to be the case as the chromatograms from the TPRS experiments showed a variety of products. However, when oxygen is added to the system, it can react with the $\bullet \mathrm{CHCl}_{2}$ radical and convert it into phosgene $\left(\mathrm{COCl}_{2}\right)$ and hydroxyl radical $(\bullet \mathrm{OH})$. This may even occur in an elementary reaction through a three-center transition state. The reactive $\bullet \mathrm{OH}$ radical may now attack methylene chloride in a chain process. In addition, phosgene may photodissociate to $\bullet \mathrm{COCl}, \mathrm{CO}$, and $\bullet \mathrm{Cl}$ with $\bullet \mathrm{Cl}$ attacking the methylene chloride in a chain reaction. The net effect of the addition of oxygen is an acceleration of the photo-assisted oxidation.

The TPRS data for pure trichloroethylene are shown in Figure 9.9, and data for trichloroethylene exposed in the presence of hydrogen peroxide is shown in Figure 9.10. Comparing these data demonstrates that the trichloroethylene was less stable in the presence of hydrogen peroxide, though data 


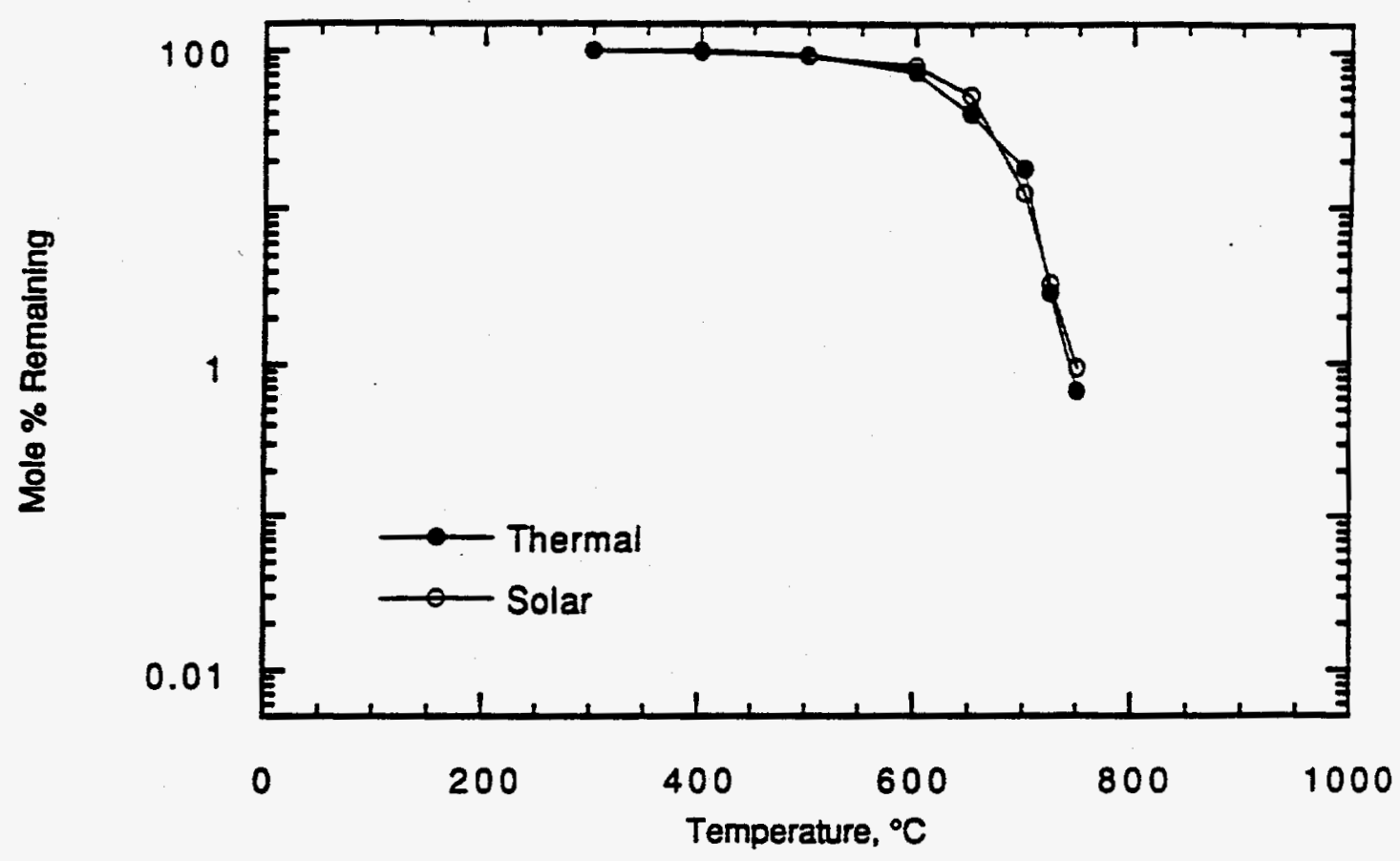

Figure 9.9. TPRS data for trichloroethylene $\left(-10^{-5} \mathrm{M}\right)$ exposed to 0 and 307 AM 1.0 suns (simulated) for $10 \mathrm{~s}$ in air.

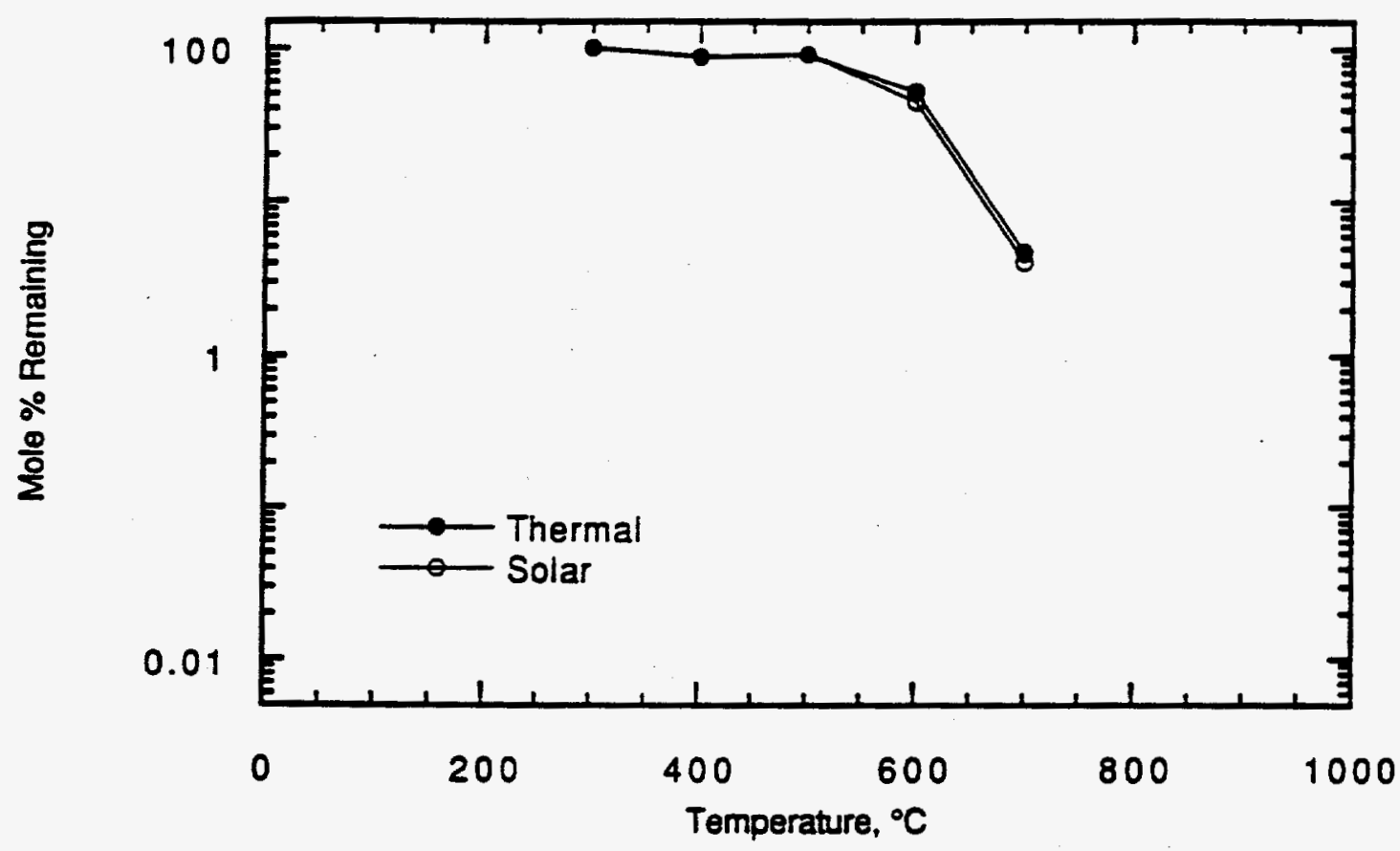

Figure 9.10. TPRS data for the trichloroethylene $\left(-10^{-5} \mathrm{M}\right)$ component of a hydrogen peroxide/trichioroethylene mixture $(10: 1, v v v)$ exposed to 0 and 307 AM 1.0 suns (simulated) for 10 s in air. 
in Figure 9.10 shows no significant effect of the simulated solar exposure. This suggests that the hydrogen peroxide is either thermally decomposing to form reactive hydroxyl radicals, or that it participates in the destruction of the trichloroethylene after decomposition of this compound begins. In any event, it is clear that homogeneous photo-decomposition of the hydrogen peroxide is not occurring to a significant extent.

The tests described above suggest that conventional photochemical sensitization may have limited usefulness as a means of enhancing the destruction of wastes which do not absorb solar energy. However, the use of reactive radical precursors may be a very promising approach if an appropriate, general purpose precursor can be identified.

\subsection{HETEROGENEOUS INITIATION}

Homogeneous photo-initiated radical-molecule, non-chain and radical-molecule, chain reactions have been experimentally shown to be modestly effective in destroying non-absorbing compounds. However, some mixture compositions have appeared to be resistant to destruction via secondary reactions. Limited, elementary reaction kinetic modeling suggests that $\mathrm{Cl}$, the principle photo-generated radical in chlorinated hydrocarbon systems is only a moderately effective oxidizer. Under certain conditions it appears to inhibit waste destruction by scavenging $\mathrm{H}$ and thereby preventing the chain branching reaction;

$$
\mathrm{H}+\mathrm{O}_{2} \rightarrow \mathrm{OH}+\mathrm{O}
$$

in the classic manner of flame inhibition[1]. This has the effect of replacing reactive $\mathrm{OH}$ by less reactive $\mathrm{Cl}$. Furthermore, as shown in the previous section, other homogeneous initiation schemes for non-absorbers, such as energy transfer sensitization, have also only been moderately effective and may be cost prohibitive due to consumption of the sensitizer.

For these reasons, there has been considerable attention placed on the application of heterogeneous photocatalysis $[2,3]$. Some photocatalysts have the advantages that they absorb strongly in the solar spectrum. Thus, if the catalyst is an efficient oxidizer, then the overall system efficiency will be high. The photo-electron transfer catalyst, titanium dioxide, $\left(\mathrm{TiO}_{2}\right)$, has been actively investigated by NREL researchers[3]. It has been shown to be effective as a gas-phase catalyst for destruction of 1,1,1trichloroethane and trichloroethylene. However, significant concerns remain about the practicability of this approach or, for that matter, any approach that relies on flowing a complex waste mixture through a catalyst bed. Components of the mixture may poison the catalyst thus reducing its actively, caustic substances may spaul the catalyst, or particulate matter may simply clog the pores. 
For these reasons, we have begun a preliminary evaluation of a process in which reactive radicals are generated on a heterogeneous, photo-catalyst and introduced into the waste stream that does not flow through the catalyst. A generalized diagram of this concept, referred to as Solar Catalytically Activated Destruction (SOl-CAD) is depicted in Figure 9.11.

In the most generalized concept of Sol-CAD, a stable precursor species is flowed through a porous catalyst. Photo-generation of the reactive species occurs at, or very near, the catalyst surface. The temperature of the catalyst and/or pre-heat zone may be increased (by solar-radiative heating) to enhance photo-catalytic radical generation. The waste is introduced at the surface of the catalyst where rapid, intense mixing is induced. This initiates the waste destruction. After initiation and consumption of the reactive species, the decomposition of the waste is completed in a down-stream completion zone. Other configurations, such as placing the catalyst on the walls, can be readily envisioned; however, the basic concepts of Sol-CAD are the same.

Three critical issues were initially identified that could control the success of the Sol-CAD concept. These are:

1. Efficiency of generation of reactive species.

2. Effectiveness of the reactive species in inducing waste destruction once they are mixed with the waste.

3. Ability to rapidly mix these species with the waste before they are otherwise quenched.

Each of these issues has been preliminarily addressed.

\subsubsection{GENERATION OF REACTIVE SPECIES}

Several precursor/reactive species systems have been identified for study. These include:

$$
\begin{aligned}
& \mathrm{O}_{2}\left({ }^{3} \Sigma^{-} \mathrm{g}\right) \rightarrow \mathrm{O}_{2}\left({ }^{1} \Delta^{+} \mathrm{g} \text { or }{ }^{1} \Sigma^{+} \mathrm{g}\right) \\
& \mathrm{H}_{2} \mathrm{O}_{2} \rightarrow 2 \cdot \mathrm{OH} \\
& \mathrm{H}_{2} \mathrm{O} \rightarrow \mathrm{H} \bullet+\bullet \mathrm{OH} \\
& \mathrm{R} \cdot \mathrm{Cl} \rightarrow \mathrm{Cl} \bullet+\mathrm{R} \bullet
\end{aligned}
$$

In addition we are considering a system for photo-catalytic ozone formation, possibly through a singlet oxygen intermediary. 


\section{Photocatalyric Radical Unitiation Reactor}

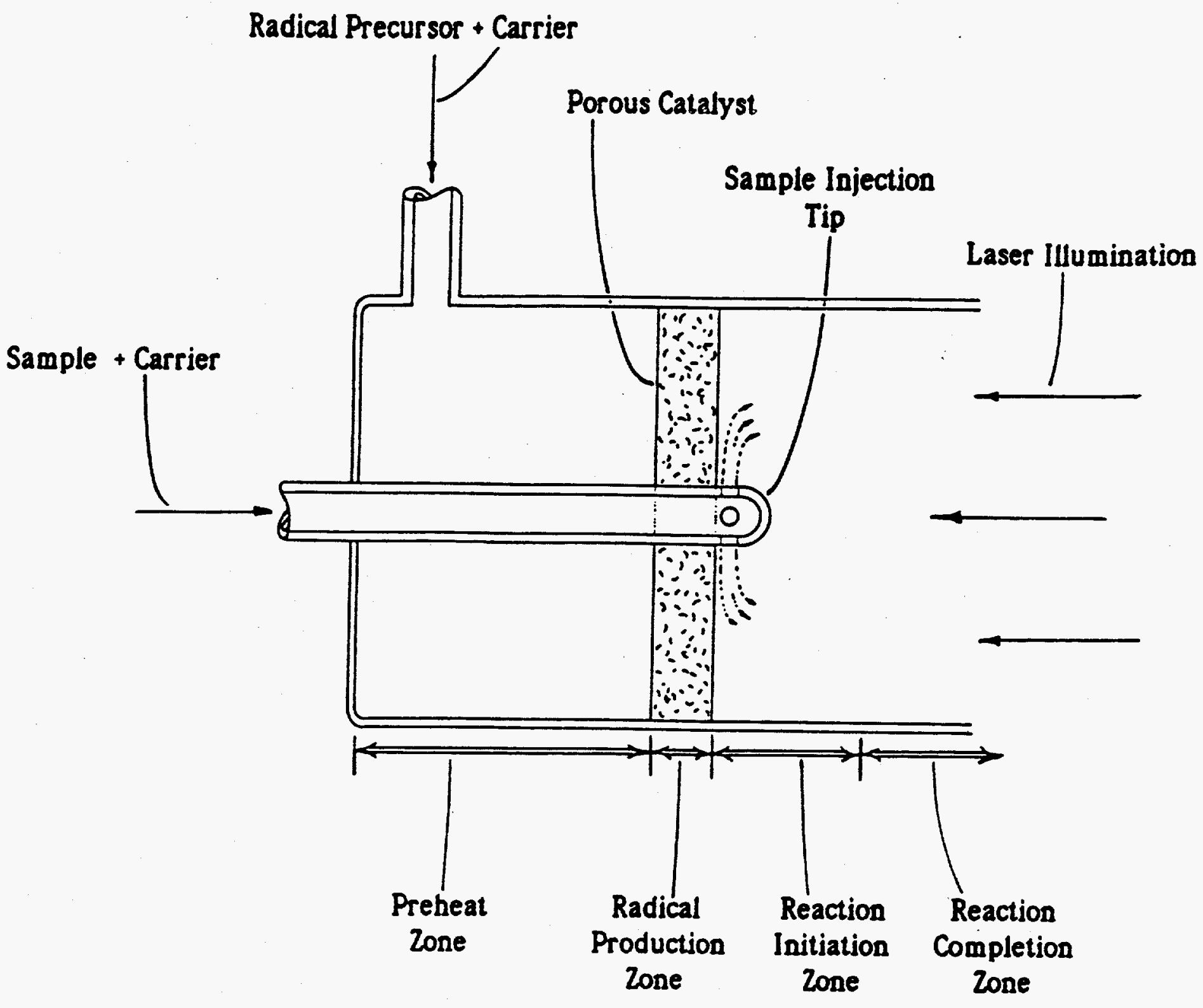

Figure 9.11. Generalized diagram of a reactor using the Solar Catalytically Activated Destruction (SolCAD) process. 
Several known photo-catalysts have also been identified for study. These include $\mathrm{TiO}_{2}$ and metal oxides such as $\mathrm{V}_{2} \mathrm{O}_{5}, \mathrm{MOO}_{2}, \mathrm{WO}_{3}$, and $\mathrm{CrO}_{3}$. Electron transfer is the suspected mechanism of photocatalysis for each $[2,3,4,5]$.

\subsubsection{PRELIMINARY STUDIES OF OH GENERATION OVER $\mathrm{TIO}_{2}$}

Because of the wide use of $\mathrm{TiO}_{2}$ by researchers involved in treatment of contaminated water as well as the apparent effectiveness of $\mathrm{TiO}_{2}$ in such systems, it was selected for initial study. $\mathrm{OH}$ generation was also selected as the reactive species for generation because of its reactivity and expected effectiveness in initiating waste destruction. In addition we have considerable experience in generating and detecting $\mathrm{OH}$ and the major experimental equipment was already available in our laboratory. An existing experimental system was adapted for these initial Sol-CAD radical generation studies $[6]$.

A diagram of the modified pump/probe experimental apparatus is depicted in Figure 9.12. A more detailed sketch of the test cell is depicted in Figure 9.13. As configured for the $\mathrm{OH}$ studies, $351 \mathrm{~nm}$ radiation from a XeF excimer laser was used to illuminate the $\mathrm{TiO}_{2}$ catalyst which was supported on a 10 $\mathrm{nm}$ OD, $6.4 \mathrm{~mm}$ thick, 60 pore/in, $92 \%$ alumina support whose face was centered on the view volume of the photo-multiplier tube (PMT) (cf. Figure 9.13). The catalyst was Degussa P-25 which is $80 \%$ anatase and $20 \%$ rutile. The $351 \mathrm{~nm}$ radiation is within the absorption band of the Degussa $\mathrm{TiO}_{2}$ but at only slightly higher energy than its work function.

The unfocused $\mathrm{OH}$ probe laser beam was positioned $1 \mathrm{~mm}$ from the surface of the catalyst. The beam was the $282.8 \mathrm{~nm}$ output of a frequency-doubled Nd:YAG pumped dye laser which selectively excites the $x(2 \pi, v=0, J=5 / 2) \rightarrow A\left({ }^{2} \Sigma^{+}, v=1, J=5 / 2\right)$ transition in $\mathrm{OH}$. Broad-band $\mathrm{OH}$ fluorescence was observed at $309 \mathrm{~nm}$ which corresponds to the 0.0 vibronic transition in emission. The combined resolution of the laser excitation and fluorescence makes this technique highly selective under our experimental conditions.

$\mathrm{OH}$ fluorescence was observed at right angles to the excimer pump and dye laser probe beam with a thermoelectrically cooled photomultiplier. To further aid in $\mathrm{OH}$ discrimination, the time decay of the fluorescence signal was observed from $0-40 \mathrm{~ms}$ by varying the delay between the pump and probe lasers. Fluorescences decay signals were signal-averaged over $500-1500$ pump/probe laser shots. The entire experiment was controlled and the data acquired and reduced using a desk top computer system.

The $\mathrm{OH}$ precursors, either $\mathrm{H}_{2} \mathrm{O}$ or $\mathrm{H}_{2} \mathrm{O}_{2}$, were flowed through the catalyst in a helium carrier. The precursor mixtures were prepared by flowing the helium through a room temperature flask containing either 


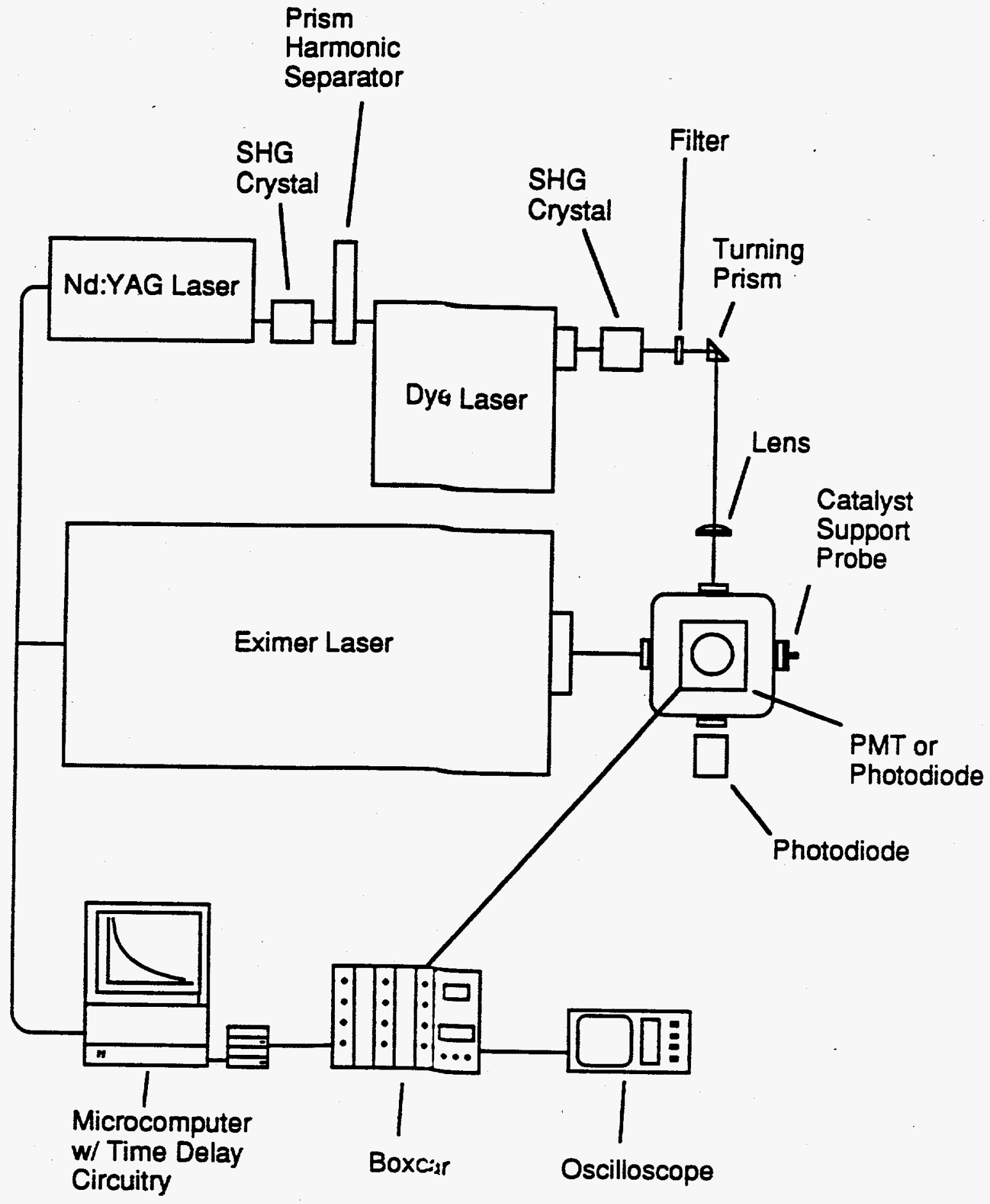

Figure 9.12. A diagram of a pump/probe experimental apparatus modified to study the Sol-CAD process. 


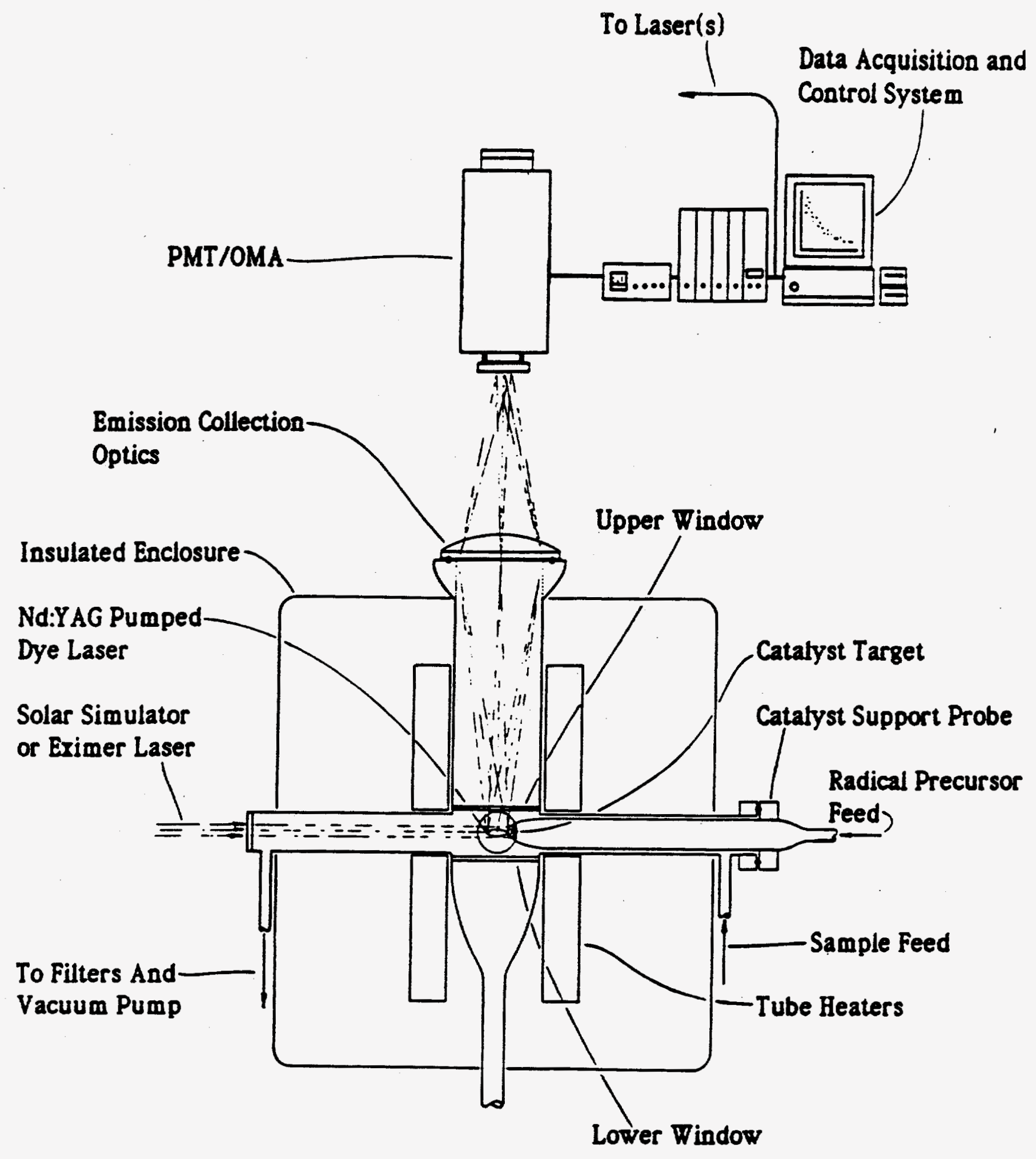

Figure 9.13. A detailed sketch of the Sol-CAD test cell assembly. 
$\mathrm{H}_{2} \mathrm{O}$ or $30 \% \mathrm{H}_{2} \mathrm{O}_{2}$ in $\mathrm{H}_{2} \mathrm{O}$. Thus, the precursor concentration in the test cell was determined by the saturation vapor pressure of the respective solutions at room temperature with correction for expansion at temperature in the cell. The cell temperature could be controlled from $300^{\circ} \mathrm{K}$ up to $1300^{\circ} \mathrm{K}$. Simple heat transfer calculations indicate that the precursor gas flow, cell flow, and catalyst support were all equilibrated at the temperature measured by a retractable thermocouple at the center of the PMT view volume.

Results of initial studies for $\mathrm{H}_{2} \mathrm{O}_{2}$ are shown in Figure 9.14 along with, for comparison, the typical results of a control experiment. Only $300^{\circ} \mathrm{K}$ experiments have been completed. When $\mathrm{H}_{2} \mathrm{O}_{2}$ is present, there is clear evidence of an $\mathrm{OH}$ signal with a lifetime of about $30 \mathrm{~ms}$. This lifetime is consistent with other $\mathrm{OH}$ studies in which $\mathrm{OH}$ was generated by more conventional means. However, a weak $\mathrm{OH}$ signal was also observed when the alumina substrate $\left(\mathrm{no} \mathrm{TiO}_{2}\right)$ was irradiated in the presence of $\mathrm{H}_{2} \mathrm{O}_{2}$. This suggests the possibility of either alumina photo-catalyzed $\mathrm{OH}$ generation or thermal dissociation due to radiative heating of the catalyst.

To determine if thermal effects were operative and to test whether $\mathrm{H}_{2} \mathrm{O}$ could be an effective initiator, similar photo-catalytic decomposition tests were conducted with $\mathrm{H}_{2} \mathrm{O}$ (cf. Figure 9.15). Water is clearly not susceptible to purely thermal decomposition at temperatures that may be achieved in this system. A weak, but clearly observable signal, was detected at $300^{\circ} \mathrm{K}$ with the signal strength appearing to increase with increasing temperature up to the maximum temperature tested at $383^{\circ} \mathrm{K}$.

This work is clearly preliminary in nature but does provide evidence of photocatalytic bond splitting.

\subsubsection{GENERATION AND DETECTION OF OTHER REACTIVE SPECIES}

Measurement of the efficiency of generation of reactive species clearly requires appropriate detection schemes. Detection is a critical aspect of demonstration of the Sol-CAD concept. The detection technique for $\mathrm{OH}$, viz. laser excitation at $283 \mathrm{~nm}$ and fluorescence detection at $309 \mathrm{~nm}$, is a well documented technique used in many laboratories and used extensively in our laboratory for the last 6 years[6]. A literature search revealed several potential detection techniques for the other reactive species under consideration for study. The detection techniques for these species that appear to be most adaptable to Sol-CAD studies in our laboratories are briefly described below. 

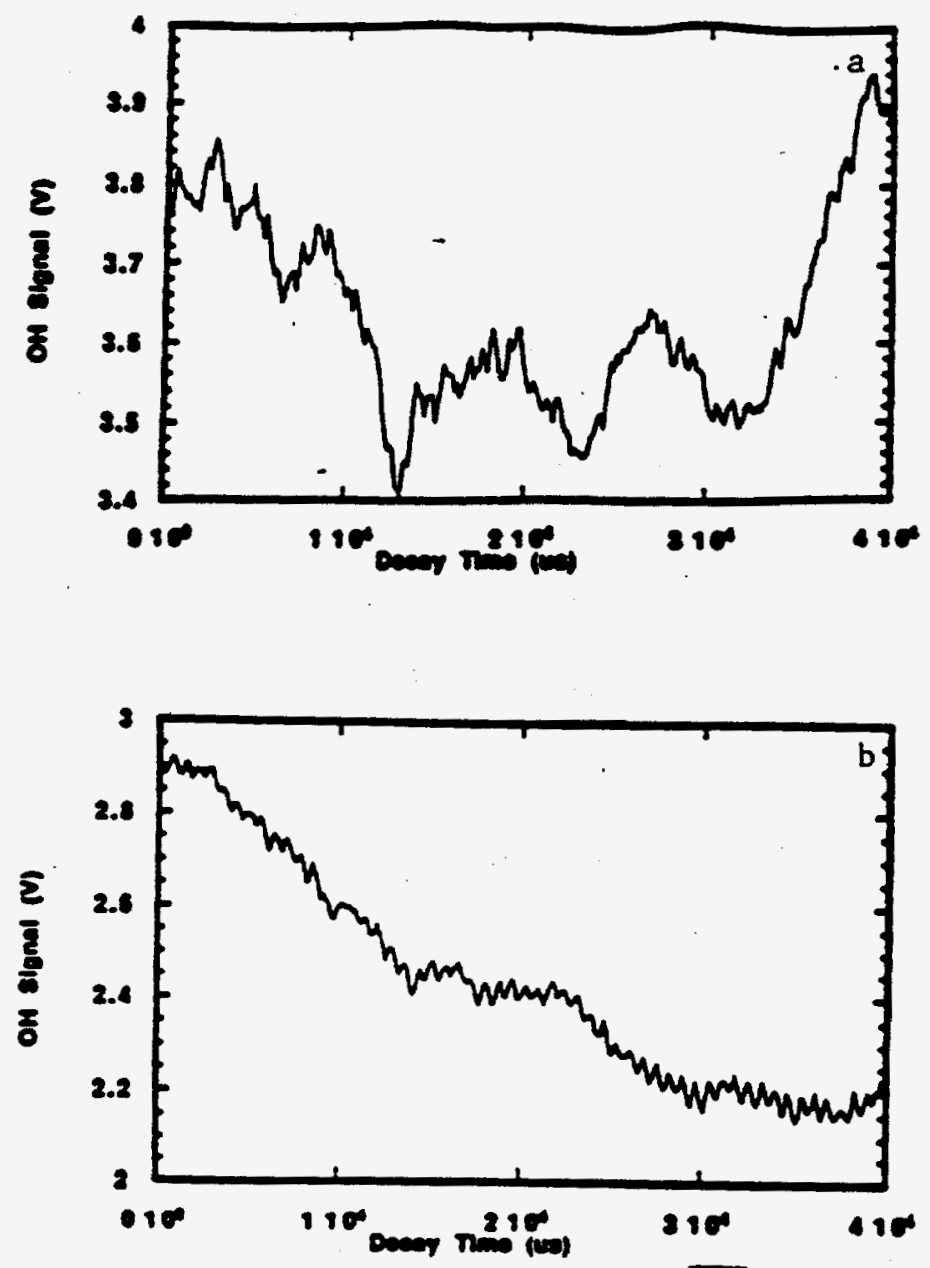

Figure 9.14. a) Typical baseline $\mathrm{OH}$ signal at $300^{\circ} \mathrm{K}$. Baseline tests consisted of no precursor, no pump laser, no probe laser, or probe laser turned off-resonance. b) Time dependent gas-phase $\mathrm{OH}$ signal from $\mathrm{H}_{2} \mathrm{O}_{2}$ over a $\mathrm{TiO}_{2}$ catalyst at $300^{\circ} \mathrm{K}$ irradiated with $351 \mathrm{~nm}$ excimer laser radiation. 

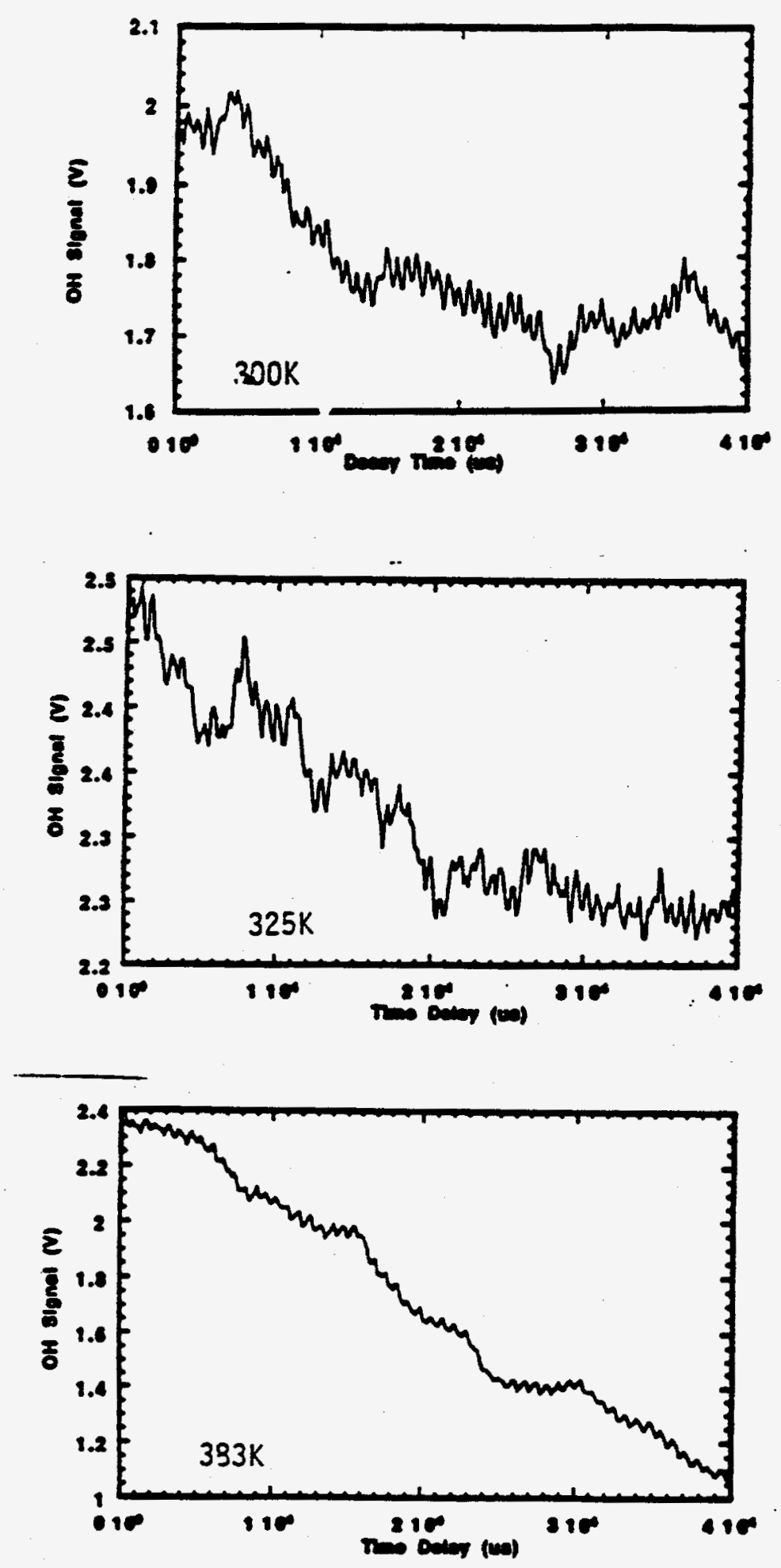

Figure 9.15. $\mathrm{OH}$ signal decays as a function of cell temperature for $\mathrm{H}_{2} \mathrm{O}$ over a $\mathrm{TiO}_{2}$ catalyst irradiated with $351 \mathrm{~nm}$ excimer radiation. 


\subsubsection{1 $\mathrm{O}_{2}\left({ }^{1} \Delta^{+} g\right)$}

Formation of $\mathrm{O}_{2}\left({ }^{1} \Delta^{+} \mathrm{g}\right)$ by a number of techniques is followed by slow, chemilumeniscent phosphorescence to the ${ }^{3} \Sigma^{+} \mathrm{g}$ ground state at $1.27 \mathrm{~mm}[7]$. This infra-red emission, where lifetimes may be several seconds under our experimental conditions, can be difficult to detect without highly sensitive detectors[8]. However, a liquid nitrogen cooled germanium photodiode gas been successfully used by many groups [8]. This detector would simply be substituted for the photomultiplier shown in Figure 9.12 to detect ${ }^{1} \Delta^{+} g$ phosphorescence as the singlet oxygen exits the catalyst.

\subsection{Cl•}

Chlorine atoms have been studied by several researchers using a variety of techniques. Coherent Anti-stokes Raman Spectroscopy (CARS) is a highly selective technique and the necessary equipment already exists in our laboratories $[9]$. In this technique, the sample is excited by two lasers with frequencies $w_{0}$ and $w_{1}$. Coherent emission is generated which is blue-shifted (anti-stokes) from $w_{0}$ and $w_{1}$ at frequency $w_{3}$ which is equal to 2 times $w_{0}$ minus $w_{1}$. The energy difference between $w_{0}$ and $w_{1}$ must be equal to that of two states, in this case the $881 \mathrm{~cm}^{-1},{ }^{2} \mathrm{P}_{3 / 2} \leftarrow 2 \mathrm{P}_{5 / 2}$ transition. Strong CARS signals have been reported in the literature with excitation at a $\lambda_{0}$ of $575 \mathrm{~nm}$ and $a \lambda_{1}$ of $605.6 \mathrm{~nm}$ which result in CARS emission at $547 \mathrm{~nm}$. These excitations wavelengths are readily achieved using our existing Nd:YAG pumped dye lasers and the emissions in the wavelength range are easily detected using conventional photomultiplier tubes. The gaseous effluent from the catalyst will be probed with co-linear aligned lasers and the co-linear $547 \mathrm{~nm}$ emission detected using a photomultiplier tube (RCA Model IP-28).

\subsection{O० and $\mathrm{H} \bullet$}

Detection schemes for other reactive species of interest including $\mathrm{O}$ and $\mathrm{H}$, are also reported in the literature. Oxygen atoms may be detected by two photon excitation to the $3 \mathrm{PO}, 1,2$ states with $226 \mathrm{~nm}$ radiation followed by detection of emission from the $5_{\Sigma_{2}}$ state at $777 \mathrm{~nm}$ [10]. Hydrogen atoms may be detected by $2+1(n=1 \rightarrow n=2+n=2 \rightarrow n=4)$ excitation at $243 \mathrm{~nm}$ and $486 \mathrm{~nm}$ using the doubled and undoubled output of a Nd:YAG (frequency tripled) pumped dye laser followed by fluorescence detection at $486 \mathrm{~nm}(n=4 \rightarrow n=2)$ [11]. Both of these techniques require acquisition of a frequency tripler for our Nd:YAG laser to be practicable in our laboratories.

In addition to $\mathrm{TiO}_{2}$, other semi-conductor and metallic catalysts have been suggested for use in various solar processes. These include, but are not limited to, $\mathrm{V}_{2} \mathrm{O}_{5}, \mathrm{MoO}_{3}, \mathrm{WO}_{3}, \mathrm{CrO}_{3}$, and $\mathrm{Pt}[5]$. These can be coated on alumina, silica or other supports and their efficiency for reactive species generation to be 
studied using the optical techniques previously discussed. The mechanism of activity of these catalysts is more fully discussed in Section 9.4 .

\subsubsection{EFFECTIVENESS OF REACTIVE SPECIES}

The effectiveness of $\mathrm{H}$ and $\mathrm{OH}$ generated from the photo-catalytic splitting of water has been addressed by detailed reaction kinetic modeling of the destruction of monochlorobenzene[12-14]. In this model, benzene was introduced at a mole fraction of 0.103 at a fuel air stoichiometry, $\phi 1 / a$, of 1.0 . Water was included in the reactant system at an initial mole fraction of 0.089 . The catalyst efficiency in splitting the water was parametrically varied from 0 to $5.0 \%$. The resulting $\mathrm{H}$ and $\mathrm{OH}$ were, in this model, instantaneously and completely mixed with the chlorobenzene and air. A 150 step chlorobenzene oxidation model was adapted from the combustion literature [12-14].

The results of the model calculation are displayed in Figure 9.16 for three reactor temperatures, 873,923 , and $973^{\circ} \mathrm{K}$. The effect of catalyst efficiency and bulk gas temperature are both dramatic. Up to $3 \%$ catalyst efficiency, a modest 2 to 5 fold increase in chlorobenzene destruction is observed, the destruction efficiency increasing slowly with increasing temperature and catalyst efficiency. At $873^{\circ} \mathrm{K}$, the destruction of chlorobenzene is increased such that the fraction of chlorobenzene remaining undestroyed is reduced to $10^{-4}$ as the catalyst efficiency is increased from 4.0 to $4.5 \%$ before reaching a steady-state value at higher catalyst efficiencies. For a bulk gas temperature of $923^{\circ} \mathrm{K}$, the catalytic effect becomes prominent at an efficiency of $>3.2 \%$ and increases chlorobenzene destruction until the fraction remaining is equal to $10^{-8}$ at a catalyst efficiency of $4.5 \%$. At $973^{\circ} \mathrm{K}$, the catalytic effect is dramatic at a catalyst efficiency of $3.5 \%$.

Sensitivity analysis calculations (cf. Table 9.1) showed, as expected based on its normalized sensitivity coefficient, that abstraction of $\mathrm{H}$ by $\mathrm{OH}$ is the dominant reaction for destruction of chlorobenzene. These analyses also showed that $\mathrm{OH}$ directly formed from the photo-catalytic splitting of water is not the only contributing source of $\mathrm{OH}$. $\mathrm{OH}$ formed from reaction of $\mathrm{H}$ and $\mathrm{O}_{2}$ is also a contributor to chlorobenzene destruction. Thus, photo-catalytic production of $\mathrm{H}$ atoms may not be significantly responsible for direct chlorobenzene destruction, however, they may contribute via chain branching to form $\mathrm{OH}$.

Time dependent calculations of $\mathrm{OH}$ and $\mathrm{H}$ mole fraction are shown in Figure 9.17. The peak $\mathrm{OH}$ concentration is reached at $\sim 10 \mathrm{~ms}$. However, its concentration remains at $\sim 25 \%$ of its peak value out to times $>0.1$ ms because of the $\mathrm{H}+\mathrm{O}_{2}$ chain branching reaction to form additional $\mathrm{OH}$. 


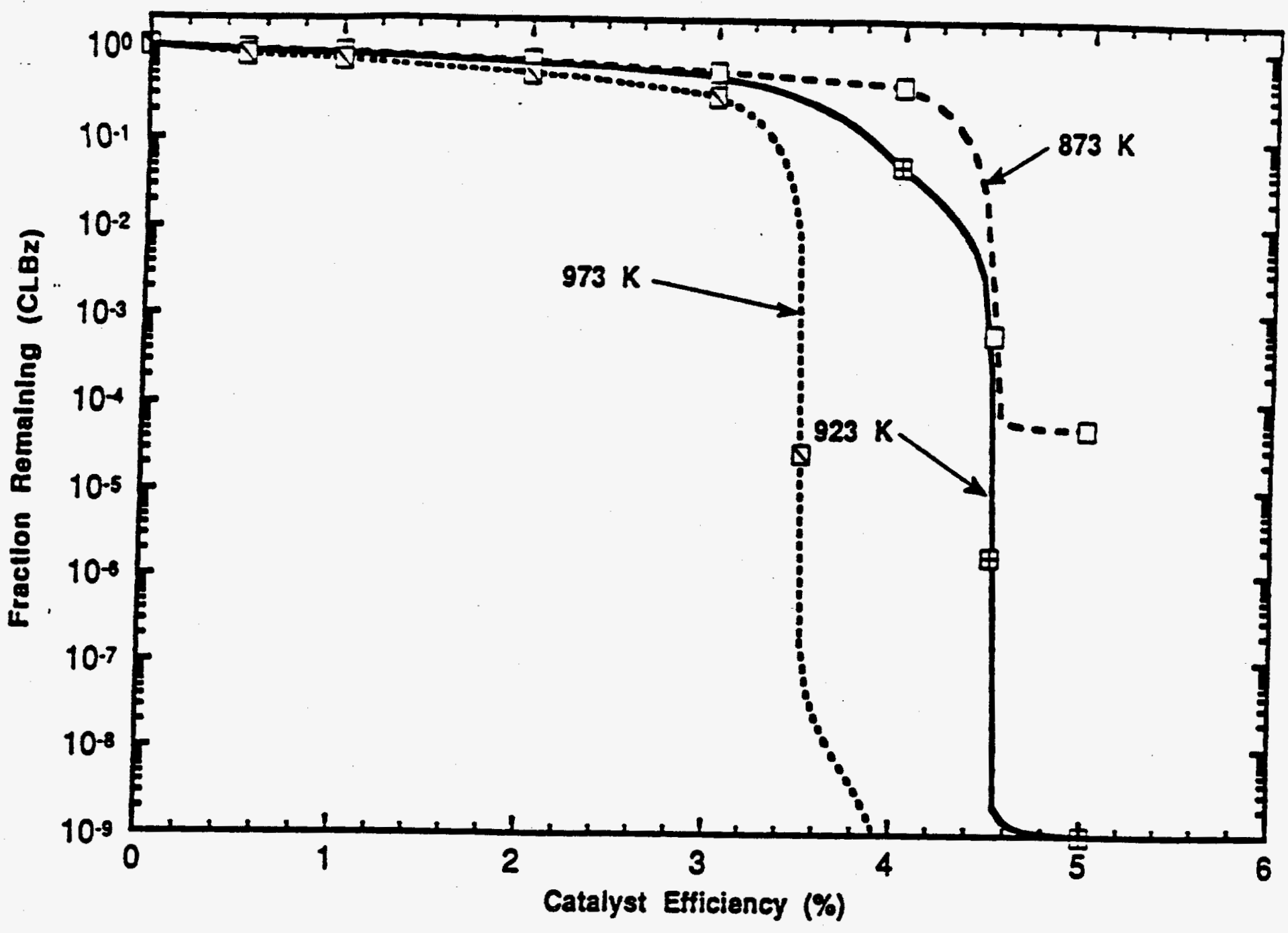

Figure 9.16. Monochlorobenzene destruction as a function of water vapor splitting catalyst efficiency. mole fraction of monochlorobenzene $=0.1028, \phi_{t / 2}=1.0$, mole fraction of water $=0.089$. 


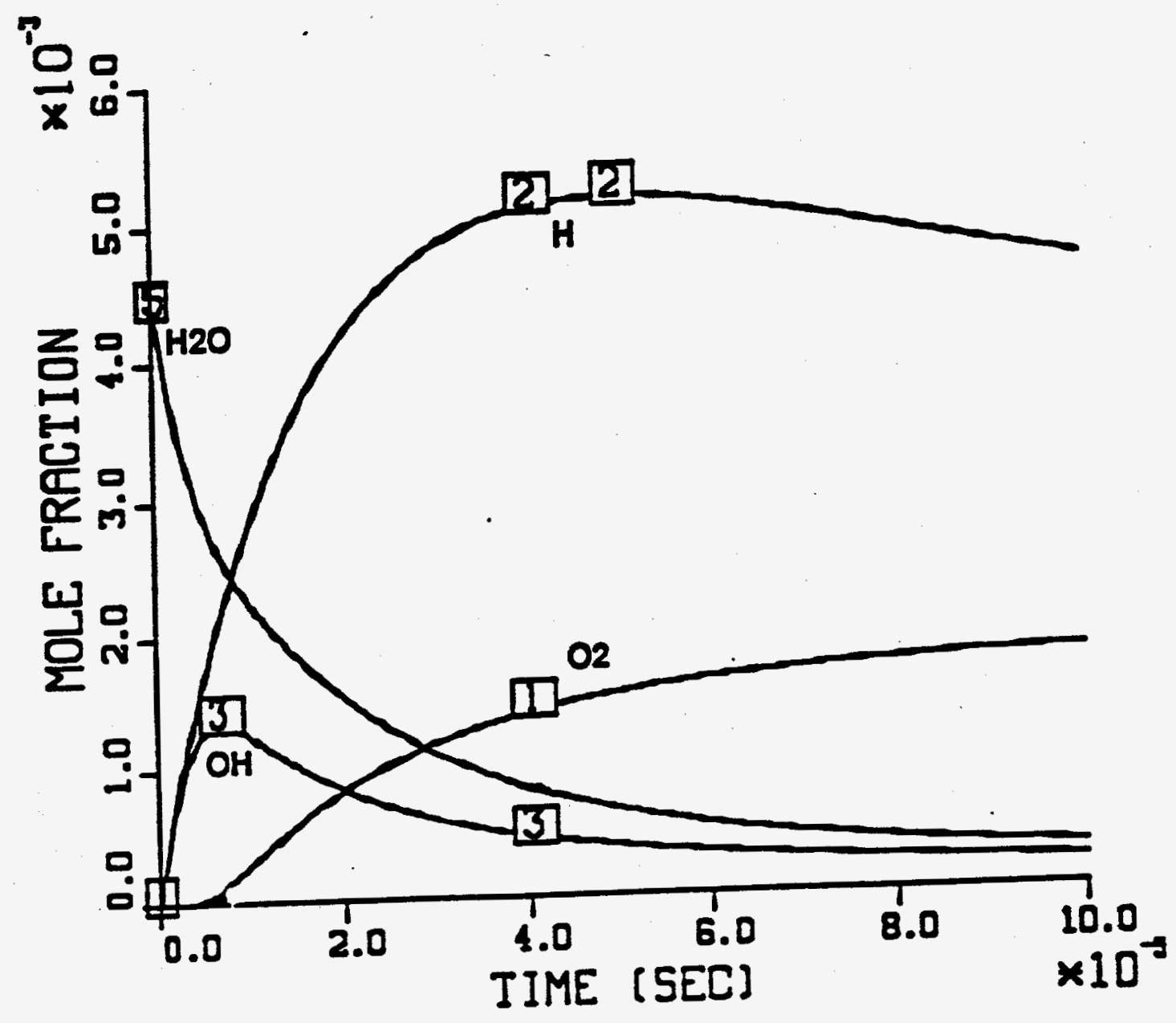

Figure 9.17. $\mathrm{H}$ and $\mathrm{OH}$ temporal profiles calculated from the rapid, irreversible, solar-catalyzed dissociation of $\mathrm{H}_{2} \mathrm{O}$ in the presence of chlorobenzene and air in $973^{\circ} \mathrm{K}$. 


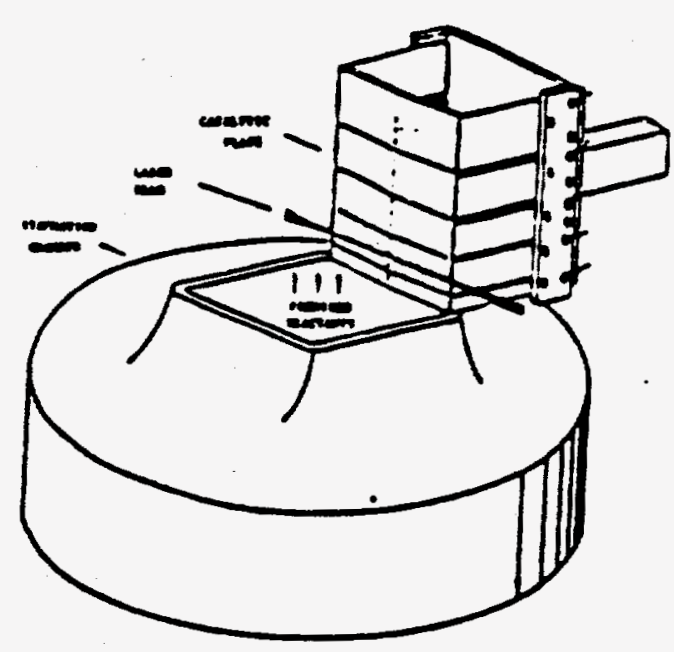

Combustion boundary byer experimental

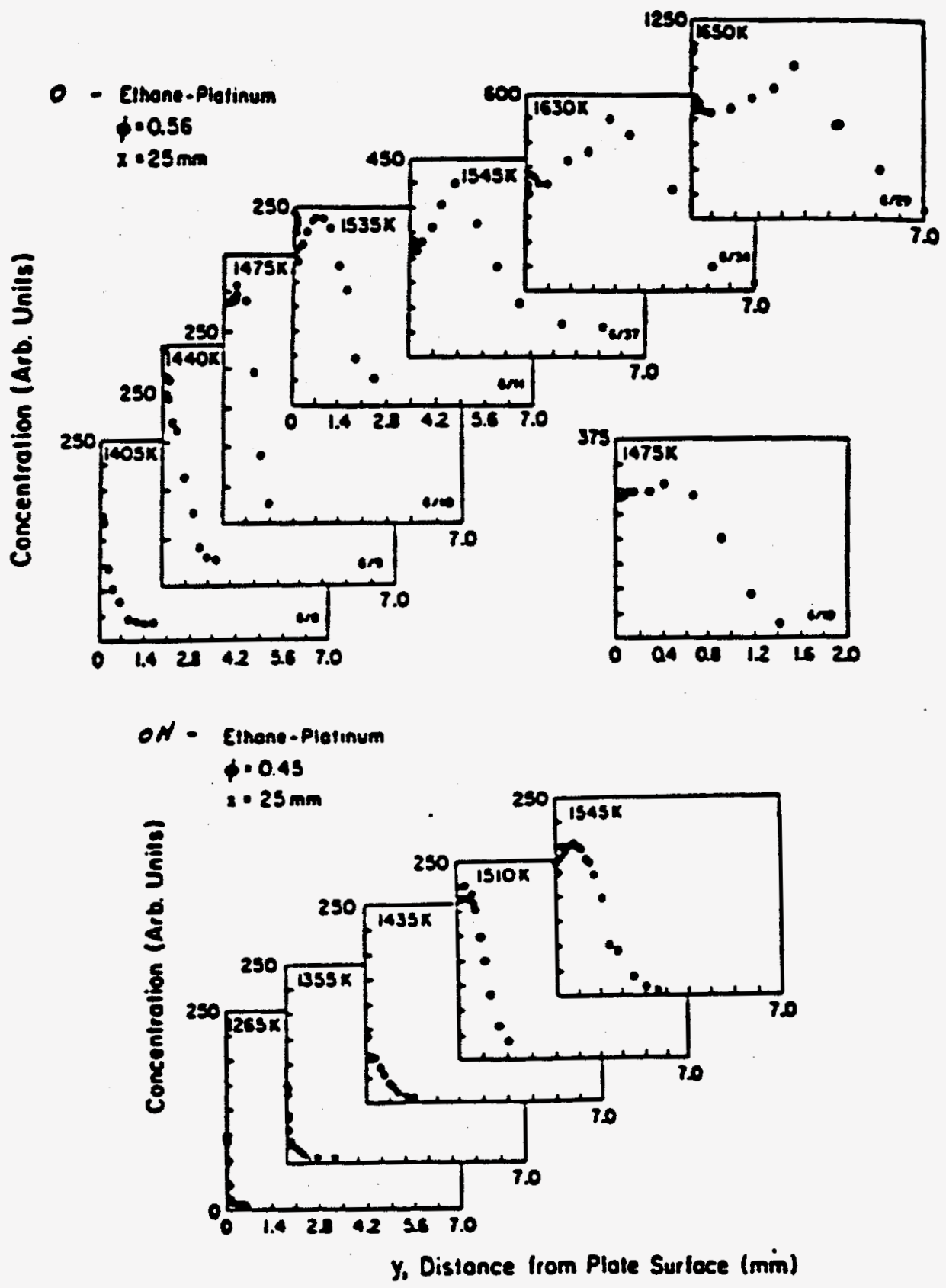

Figure 9.18. Catalytically stabilized combustion generation of gas phase $\mathrm{H}$ and $\mathrm{OH}$. 


\section{TABLE 9.1}

MONOCHLOROBENZENE SENSTTIVITY ANALYSIS CALCULATIONS

$$
(\mathrm{T}=973 \mathrm{~K}, \mathrm{t}=1.0 \mu \mathrm{s})
$$

Reaction

$$
\mathrm{C}_{6} \mathrm{H}_{5} \mathrm{Cl}+\mathrm{OH} \leftrightarrow \mathrm{C}_{6} \mathrm{H}_{4} \mathrm{Cl}+\mathrm{H}_{2} \mathrm{O}
$$

$$
\mathrm{C}_{6} \mathrm{H}_{5} \mathrm{Cl}+\mathrm{H} \leftrightarrow \mathrm{C}_{6} \mathrm{H}_{6}+\mathrm{Cl}
$$

$\mathrm{C}_{6} \mathrm{H}_{5} \mathrm{Cl}+\mathrm{H} \leftrightarrow \mathrm{C}_{6} \mathrm{H}_{4} \mathrm{Cl}+\mathrm{H}_{2}$

$\mathrm{C}_{6} \mathrm{H}_{5} \mathrm{Cl}+\mathrm{H} \leftrightarrow \mathrm{C}_{6} \mathrm{H}_{5}+\mathrm{HCl}$

$$
\mathrm{H}+\mathrm{O}_{2} \leftrightarrow \mathrm{OH}+\mathrm{O}
$$

Normalized Sensitivity Coefficient

$-2.63 \times 10^{-3}$

$-4.56 \times 10^{-4}$

$-8.01 \times 10^{-4}$

$-7.30 \times 10^{-4}$

$-1.52 \times 10^{-4}$ 
This calculation further illustrates the importance of the branching reaction. It also suggests that mixing times for the catalyst effluent gas and the waste gas flow on the order of $0.1 \mathrm{~ms}$ will be effective in affecting waste destruction.

Limited additional calculations were conducted for splitting of $\mathrm{H}_{2} \mathrm{O}_{2}$. At low catalyst efficiencies, chlorobenzene destruction was $<50 \%$ primarily due to loss of $\mathrm{OH}$ by the competing reaction;

$$
\mathrm{H}_{2} \mathrm{O}_{2}+\mathrm{OH} \rightarrow \mathrm{H}_{2} \mathrm{O}+\mathrm{HO}_{2}
$$

with $\mathrm{HO}_{2}$ being a much less reactive species. Furthermore, there was no contribution to secondary $\mathrm{OH}$ production because $\mathrm{H}_{2} \mathrm{O}_{2}$ was assumed only to dissociate into $2 \mathrm{OH}$, with no $\mathrm{H}$ production. This assumption may be invalid for photo-catalytic dissociation and only experimental studies will clarify this issue.

\subsubsection{MIXING THE REACTIVE SPECIES AND BULK WASTE}

The mixing aspect has only been indirectly addressed by considering some literature studies that may be applicable. The kinetic studies summarized in the previous section indicate that mixing times on the order of $0.1 \mathrm{~ms}$ will be effective. Research on catalytically stabilized combustion may also be relevant 15 ]. In these studies a catalytic (non-photolytic) boundary layer is established along a flow of premixed reactants (ct. Figure 9.18). In a series of studies, the ignition and combustion of several volatile organic compounds (methylene chloride, trichloroethylene, methane ethane, methyl ethyl ketone, toluene, and ethyl benzene) was stabilized and otherwise improved. These studies show that although $\mathrm{OH}$ and $\mathrm{O}$ concentrations peak at $<1 \mathrm{~mm}$ from the surface, they are effective in stabilizing the combustion of ethane (cf. Figure 9.18).

Variations on the Longwell jet-stirred and torroidal jet-stirred reactor should result in surface mixing in less than $0.1 \mathrm{~ms}[14]$. Induction of chain propagating and branching reactions can then propagate the oxidation throughout the remaining bulk of the waste to be destroyed. This propagation rate is a complex function of waste composition, equivalence ratio radical concentration and type, temperature, and mixing. It is probably best addressed by direct measurement of destruction of a waste in a laboratory Sol-CAD reactor system similar to that depicted in Figure 9.11. 


\subsubsection{MECHANISTIC ASPECTS OF PHOTO-CATALYTIC GENERATION OF REACTIVE SPECIES}

Generation of reactive species by irradiating catalysts with solar radiation may be considered to fall under the general topic area of photochemistry in the adsorbed state. This topic is not particularly well understood, especially for electron transfer reactions, as are proposed to occur in the case of $\mathrm{TiO}_{2}$. Furthermore, most research in this area has addressed photo-catalytic electron transfer in aqueous solutions. However, relatively recent reports of electron transfer to species absorbed from vapors has spurred considerable interest in gas-solid photochemistry[3,17,18].

With the exception of generation of singlet oxygen, all of the Sol-CAD concepts involve photodissociation of an adsorbed species. Singlet oxygen generation requires some type of energy transfer from the surface to the adsorbed ground state oxygen[8]. For either case, the generation of reactive species via gas-solid photo-catalysis requires the following steps:

i. Adsorption of the precursor on the surface of the catalyst;

ii. Excitation of the catalytic substrate by incident radiation (in special cases the molecule can be directly excited);

iii. Electron or energy transfer from the substrate to the absorbate;

iv. Excitation of the adsorbate from its ground electronic state to an excited ionic or neutral, valence state;

v. In the case of photo-dissociation, bond rupture to form radicals, ions, or radical-ions;

vi. Desorption of the newly formed reactive species.

A generalized discussion of each step, as it pertains to Sol-CAD follows. Specific reference is made to the potential thermal enhancement of the rate of each step.

\subsubsection{ADSORPTION OF THE PRECURSOR}

The rate of adsorption can be modeled using several available models; however, the Langmuir model is usually most applicable and widely used. Strong chemisorption is probably required for any 
useful process. Chemisorption requires activation and thus the rate can usually be described by an Arrhenius expression[4]. This suggests that an elevation in the catalyst temperature can increase the rate of chemisorption. Of course, an increase in catalyst temperature can also shitt the gas-surface equilibrium away from chemisorption. However, initial increases in temperature are expected to increase the number of chemisorbed species.

In addition, photo-excitation of catalysts has been reported to increase the number of adsorptive sites[4]. For example, in $\mathrm{V}_{2} \mathrm{O}_{5}$, excitation is presumed to involve an $\pi^{*} \leftarrow n$ transition from the 0 to the double bond in the $v^{+5}=0^{-2}$ moiety to produce $v^{+4}-0$. These charged sites can strongly adsorb polar species. Hydrogen bonding in species such as water and alcohols may be sufficiently strong to result in chemical reaction to form $\mathrm{V}+4 \cdot \mathrm{OH}+\mathrm{OH}-(\mathrm{R}-)$.

\section{ii. Photo-excitation.}

Excitation at wavelengths $<390 \mathrm{~nm}$, which corresponds to the band-gap of $3.2 \mathrm{eV}$, is required to excite the Degussa, $\mathrm{P} 25$, Anatase crystalline form of $\mathrm{TiO}_{2}[2]$. This wavelength is longer than the onset of absorption of most molecules of interest and thus catalyst excitation is the dominant mechanism of photoexcitation for most gas-solid system. However, the absorption of radiation by molecular species can be red-shifted by interaction with the surface. This can be particularly strong for hydrogen bonded species in which the hydrogen bond is strongest in the excited state of the gas-surface complex $[19,20]$. The phenomenon is schematically displayed in Figure 9.19 .

In this figure, the electronic energies of the ground and excited state hydrogen bonded complex are displayed as a function of internuclear distance between the absorbed molecule, $\mathrm{XH}$, and the surface, 5. At large internuclear distances, the energy required for excitation corresponds to that of the free molecule, hv (molec). The adsorbed molecule, however, requires lower energy radiation, hv (complex) for excitation, the energy required for excitation being reduced from that of the free molecule by the differences in excited and ground state binding energy for the molecule to the surface. This shift may be as much as $5000 \mathrm{~cm}^{-1}$ to the red for molecules with strong excited state, hydrogen bonds. This shift may be sufficient for some molecules to directly absorb solar radiation. An example could be the absorption of alcohols or water to $\mathrm{V}_{2} \mathrm{O}_{5}$, in which the excited state of the $\mathrm{V}_{2} \mathrm{O}_{5}$ is strongly charged and can result in a strong hydrogen bond.

Thermal excitation of ground state vibrational states will also red shift the absorption spectrum of the molecule or complex by $\mathrm{kT}[21]$. This corresponds to an approximately $500 \mathrm{~cm}^{-1}$ red-shift in the onset 


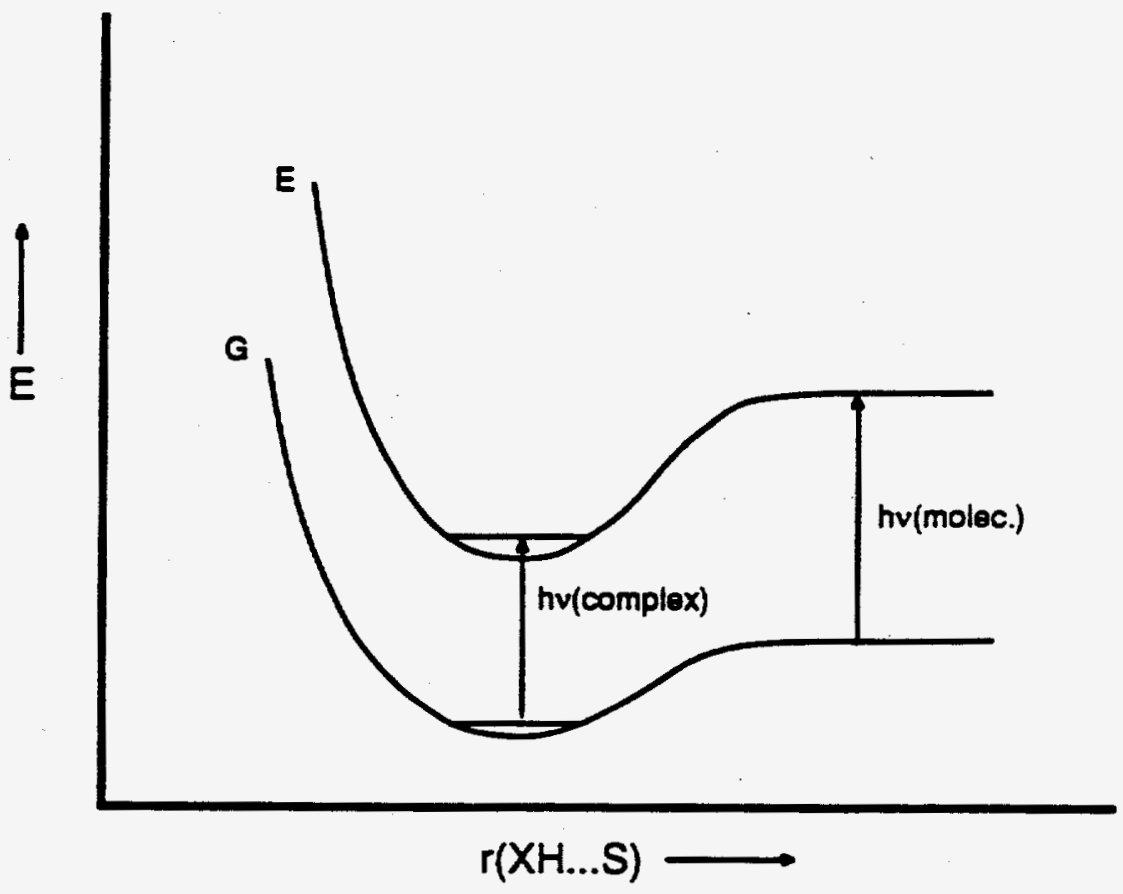

Figure 9.19. Energy required for excitation of a molecule more strongly bound in the excited electronic state, $E$, than the ground state, $G$. Note that hu (molec) $>$ hv (complex). 


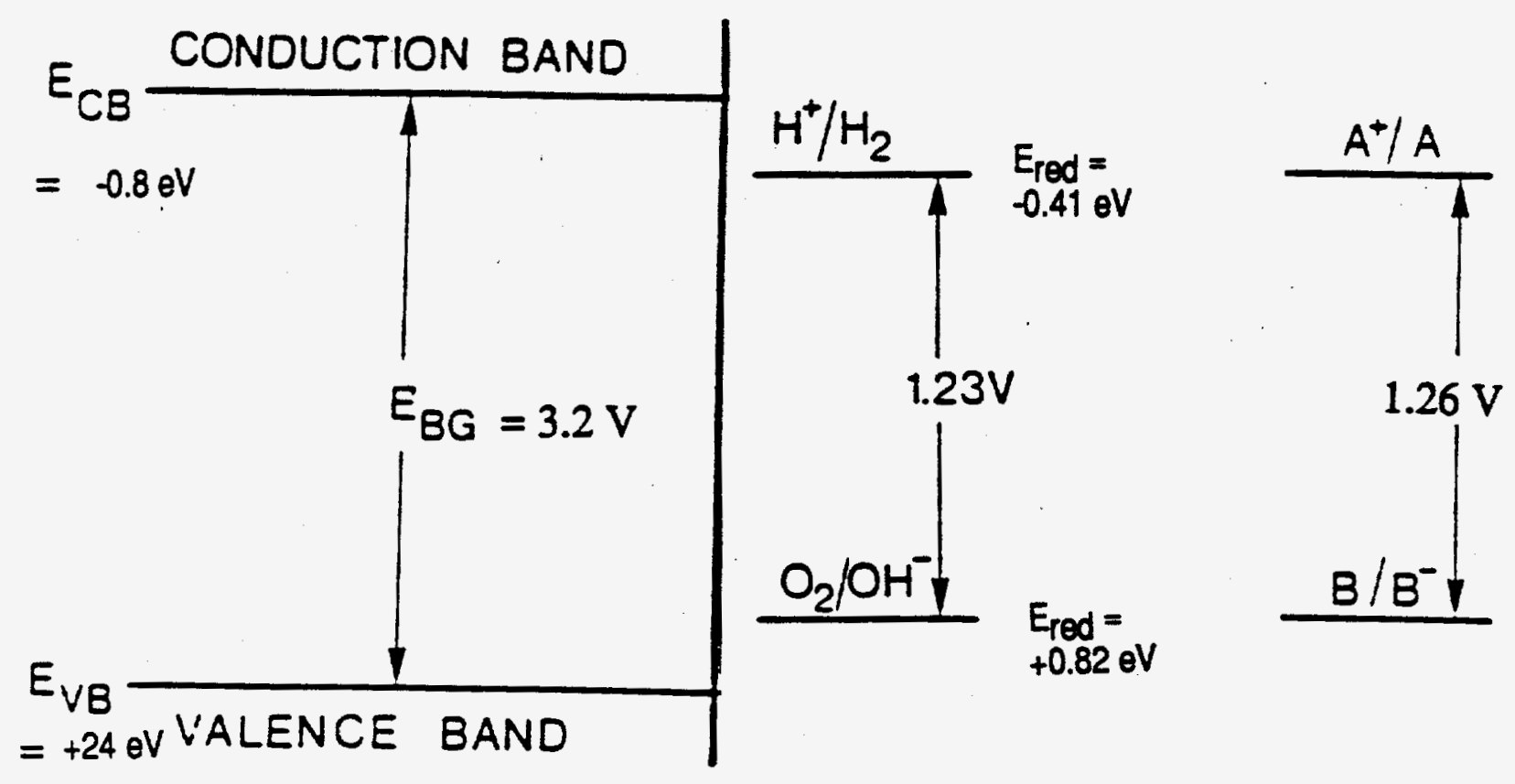

$\mathrm{TiO}_{2}$

$$
2 \mathrm{H}_{2} \mathrm{O}=2 \mathrm{H}_{2}+\mathrm{O}_{2} \quad \mathrm{CH}_{3} \mathrm{Cl}+\mathrm{H}_{2} \mathrm{O}=\mathrm{CO}+\mathrm{HCl}+2 \mathrm{H}_{2}
$$

Figure 9.20. Electron (hole) and overall energetics of the splitting of $\mathrm{H}_{2} \mathrm{O}$ to form $\mathrm{H}_{2}$ and $\mathrm{O}_{2}$ and the steam reforming of $\mathrm{CH}_{3} \mathrm{Cl}$. 
of adsorption at $700^{\circ} \mathrm{K}$. Increases in absorption intensity have also been observed as the temperature of the system is raised due to thermally induced vibronic coupling[21].

\section{iii, iv. Electron Transfer to the Adsorbant and Photoreaction.}

The initial step in all photo-catalytic electron transfer reactions is promotion of valence band electrons to the conduction band. In many semi-conductors this is a process that occurs in the visible or near UV. Transition metal oxides such as $\mathrm{TiO}_{2}$ are common electron transfer catalysts

In $\mathrm{TiO}_{2}$ the photo-reaction may be symbolized:

$$
\mathrm{TiO}_{2}+h v(\mathrm{Eg} \geq 3.2 \mathrm{eV}) \rightarrow h^{+} v b+e^{-} \mathrm{cb} \text { (exciton) }
$$

The electrons promoted to the conduction band are balanced by an equal number of resulting "holes" in the valence band. Depending upon the reduction potential for the given reaction, an adsorbed species may accept an electron from the conduction band to reduce the adsorbate or the adsorbed species may donate an electron to the valence hole resulting in an oxidized adsorbate[2]. Since electronhole generation is reversible, it is necessary to have an electron acceptor available to prevent electron-hole recombination. Since the mobility of electrons and holes increases with temperature, effective quenchers are needed if the catalyst is used at elevated temperatures[24]. Thus, electron transfer to the adsorbate and the rate of photoreaction, which limits the reversibility of the process, can usually not be decoupled.

Some commonly proposed hole reactions include[2,22,23]:

$$
\begin{aligned}
& h^{+} v b+\mathrm{H}_{2} \mathrm{O} \text { (ads) } \rightarrow \mathrm{H}_{2} \mathrm{O}^{+} \text {(ads) } \rightarrow \bullet \mathrm{OH}^{+} \mathrm{H}^{+} \\
& h^{+} v b+\mathrm{OH} \text {-(surf) } \rightarrow \bullet O H \\
& \text { also, } \\
& 2 \bullet \mathrm{OH} \rightarrow 2 \mathrm{H}_{2} \mathrm{O}_{2}
\end{aligned}
$$

Electron reactions include[1,22,23].

$$
\begin{aligned}
& e_{\mathrm{cb}}^{-}+\mathrm{O}_{2} \rightarrow \mathrm{O}_{2} \bullet \\
& \text { also, } \\
& 2 \mathrm{O}_{2} \bullet+2 \mathrm{H}_{2} \mathrm{O} \rightarrow 2 \cdot \mathrm{OH}+2 \mathrm{OH}^{-}+\mathrm{O}_{2} \\
& \mathrm{H}_{2} \mathrm{O}_{2}+e^{-} \mathrm{cb} \rightarrow \bullet \mathrm{OH}+\mathrm{OH}- \\
& \text { or, } \\
& \mathrm{H}_{2} \mathrm{O}_{2}+\mathrm{O} 2 \cdot \cdot \rightarrow \cdot \mathrm{OH}+\mathrm{OH}-+\mathrm{O}_{2}
\end{aligned}
$$


These reactions occur even though the energy of the input radiation is less than the bond dissociation energy of a free-water molecule (i.e., $\mathrm{D}_{\mathrm{O}}(\mathrm{HO}-\mathrm{H})=5.2 \mathrm{eV} / 240 \mathrm{~nm}$ )). This might initially appear to violate conservation of energy. However, the presumed overall reaction of conversion of $\mathrm{H}_{2} \mathrm{O}$ to $\mathrm{H}_{2}$ and $\mathrm{O}_{2}$ is endothermic by only $1.23 \mathrm{eV}$, a process for which $3.2 \mathrm{eV}$ is more than energetically sufficient. The additional energetics requirement, typically invoked in heterogeneous electron transfer is that the energy of the conduction band is above that of the reduction half cell potential of the redox pairs and the energy of the valence band is below that of the oxidation half cell potential of the other component of the redox pair. This results in electron transfer from the conduction band of the semi-conductor being exothermic and electron transfer to the valence band/completing the cycle) also being exothermic. An example of this is shown in Figure 9.20 for the splitting of $\mathrm{H}_{2} \mathrm{O}$ into $\mathrm{H}_{2}$ and $\mathrm{O}_{2}$.

However, a not-so-frequently acknowledged requirement is that each intermediate reaction step must also be the exothermic or nearly thermoneutral) in order to be fast enough to be feasible. For example, the splitting of $\mathrm{H}_{2} \mathrm{O}$ into $\mathrm{OH}+\mathrm{H}+$ by hole transfer would appear to be endothermic unless the energies of the products are lowered by the adsorption on the surface. Subsequent, exothermic reactions are then required to complete the overall reaction. It is in this manner that overall reactions that seemingly require an initially energetically improbable step actually occur.

Since it is already known that the initial electron and hole transfer reactions occur in the water system, it would appear that the breaking of the $\mathrm{HO}-\mathrm{H}$ bond can be accomplished over $\mathrm{TiO}_{2}$ with solar radiation if an additional reactant is available to make the overall reaction exothermic. If the overall reaction is an oxidation (or at least a reforming reaction), the overall energetic requirements appear to be readily met.

For other reactant systems in which the half cell reactions and/or reduction potentials are not known, the overall energetic requirements may be met but the electron (hole) transfer may or may not occur. Such a case is also shown in Figure 5.20 for the steam reforming of methyl chloride $\left(\mathrm{CH}_{3} \mathrm{Cl}\right)$.

In addition to the above reactions commonly quoted in aqueous photo-electron transfer studies, several gas-surface photo-dissociation reactions have been observed [25]. $\mathrm{Mo}(\mathrm{CO})_{6}$ was dissociated to yield gas phase $\mathrm{CO}$ on $\mathrm{K}$ doped $\mathrm{Ni}(\mathrm{III})$ surfaces. It was proposed that the doping lowered the work function of the substrate such that electrons of sufficient energy were generated to dissociate $\mathrm{Mo}(\mathrm{CO})_{6}$ with wavelengths from the $U V$ to at least $800 \mathrm{~nm}$. 
$\mathrm{CH}_{3} \mathrm{Cl}$ was dissociated on $\mathrm{GaAs}(100)$ using both 248 and $193 \mathrm{~nm}$ radiation. The adsorbed $\mathrm{CH}_{3} \mathrm{Cl}$ was thought to capture a photoelectron from the substrate to generate $\mathrm{CH}_{3} \mathrm{Cl}$ - (ads) which generated $\mathrm{Cl}$ (ads) and free $\mathrm{CH}_{3} \bullet$. When $\mathrm{CH}_{3} \mathrm{Cl}$ was adsorbed on $\mathrm{Ni}(\mathrm{III})$, Cl• was observed.

$\mathrm{HCl}$ photodissociation has been observed on $\mathrm{Ag}(111)$ and $\mathrm{K}$ surfaces. Dissociation was observed with an irradiation wavelength of $351 \mathrm{~nm}$ which is $0.9 \mathrm{eV}$ less than $\mathrm{D}_{0}(\mathrm{H}-\mathrm{Cl})=4.4 \mathrm{eV}$. This was attributed to formation of the $\mathrm{HCl}$ - via electron transfer which could dissociate to the more stable products $\mathrm{H} \bullet$ and $\mathrm{Cl}$. Gas phase hydrogen atoms were observed following irradiation at 248,308 , and $350 \mathrm{~nm}$.

Studies of $\mathrm{CH}_{3} \mathrm{Cl}, \mathrm{CH}_{3} \mathrm{Br}, \mathrm{CH}_{3} \mathrm{I}$, and $\mathrm{C}_{2} \mathrm{H}_{5} \mathrm{Cl}$ on $\mathrm{Pt}$ and $\mathrm{Ag}$ (III) all indicated that photo-dissociation occurred at wavelengths longer than the onset of gas-phase absorption. In some cases, wavelengths less than the work functions of the substrate were effective in initiating photo-dissociation, suggesting a mechanism of electron tunneling.

A different type of photo-catalytic reaction has been observed for $\mathrm{TiO}_{2}$ treated with carbon tetrachloride $\left(\mathrm{CCl}_{4}\right)[23]$. In this system, gas-phase $\mathrm{O}_{2}\left({ }^{1} \Delta_{g}\right)$ was evolved from the treated catalyst when irradiated with the output of medium pressure mercury arc lamp. The formation of singlet oxygen was attributed to reaction of surface hypochlorite anions $\left(\mathrm{ClO}^{-}\right)$with photo-generated $\mathrm{H}_{2} \mathrm{O}_{2}$.

$$
\text { ClO-(surf) }+\mathrm{H}_{2} \mathrm{O}_{2} \rightarrow \mathrm{H}_{2} \mathrm{O}+\mathrm{Cl} \text {-(surf) }+\mathrm{O}_{2}\left({ }^{1} \Delta_{\mathrm{g}}\right)
$$

The proposed reaction is the same as that commonly used to generate singlet oxygen in aqueous solutions[8]. These observations raise the possibility of generating long-lived reactive $\mathrm{O}_{2}\left({ }^{1} \Delta^{+} g\right)$ via heterogeneous photocatalysis. Although the rate and mechanism of reaction of $\mathrm{O}_{2}\left({ }^{1} \Delta^{+} \mathrm{g}\right)$ has not been explored at high temperature, it is known to be very reactive towards unsaturated organic species at room temperature, and this reactivity is expected to increase with increasing temperature.

\section{vi. Desorption of Photo-Products.}

The effect of catalyst temperature may be very important in this step of the Sol-CAD process. The precursor species will generally be polar and readily absorbed by the catalytic substrate. However, the reactive species that are generated are generally neutral atoms or small radicals that are not expected to be strongly adsorbed. Thus, raising the catalyst temperature may be very effective in desorbing the reactive species while not significantly affecting the adsorption of the precursor. 


\subsection{REFERENCES}

1. C. K. Westbrook, Nineteenth Symposium (International) on Combustion/The Combustion Institute, po. 127-141, 1982.

2. Photoinduced Electron Transfer, M. A. Fox and M. Chanon, ed., Elsevier, Amsterdam, 1988.

3. Proceedings of the First International Conference on Photocatalytic Treatment of Water and Air, November 8-13, 1992, London, Ontario, Canada.

4. M. Campbell, Catalysis at Surfaces, Chapman and Hall, N.Y., 1988.

5. W. E. Wentworth, M. Hamada, and C. F. Batten, "Photoassisted Hydrocarbon Reforming and Cracking Reactions," in Solar Thermal Technology - Research Develooment and Application, Proceedings of the 4th International Symposium, B. P. Gupta and W. H. Traugott, ed., Hemisphere, N.Y., 1990.

6. J. H. Kasner, P. H. Taylor, and B. Dellinger, L.Phys. Chem, 94, pp. 3250-3253, 1990.

7. W. C. Eisenberg and M. DeSilva, Tetrahedran Letters, 31, No. 41, pp. 5857-5860, 1990.

8. D. R. Kearns, Chem. Revs, Vol. 71, No. 4, pp. 395-427, 1971.

9. W. Lixin, J. Kang, N. Huang, and X. Jiren, Chemistry Phys. Letters, Z, No. 6, pp. 204267, 1990.

10. A. W. Mizialek and M. A. DeWilde, Optics Letters, 2, No. 9, pp. 390-392, 1984.

11. J. E. M. Goldsmith and N. M. Lamendeau, Optics Letters, 15, No. 10, pp. 576-578, 1990.

12. E. R. Ritter, J. W. Bozzelli, and A. M. Dean, J. Phys. Chem. 93, 1990.

13. J. H. Kiefer, et. al., J. Phys. Chem 89, pp. 2013-2019, 1985.

14. D. Bittker, Combust. Sci. and Technol, 79 pp. 49-72, 1991.

15. L. D. Pfefferle, et.al. Combustion and Flame, 76, pp. 325-338, 1989.

16. R. B. Barat, Characterization of the Mixing/Chemistry Interaction in a Torroidal Jet Stirred Combustion, Ph.D. Thesis, Dept. of Chemical Engineering, MIT, Cambridge MA. 1990.

17. L. A. Dibble and G. B. Raupp, Catalysis Letters, 4, pp. 345-354, 1990.

18. L. A. Dibble and G. B. Raupp, Environ. Sci. Technol, 26, pp. 492-495, 1992.

19. B. Dellinger, Intermolecular Perturbation of Molecular Potentials, Ph.D. Thesis, Florida State University, 1976.

20. R. S. Mullikin and W.B. Person, Molecular Comolexes. Wiley Interscience, NY, 1969.

21. J. M. Berman, J. L. Graham, and B. Dellinger, accepted, L. Photochem. and Photobiol., 1992.

22. M. Anpo, T. Shina, Y. Kubokawa, Chemistry Letters, pp. 1799-1802, 1985.

23. G. Manuera, A. Navio, and V. Rives - Arnau, J. Chem. Soc. Faraday Trans. 1, I7, pp. 2747-2749, 1981.

24. M. D. Archer and J. R. Bolton. J. Phys. Chem., 94, pp. 8028-8036, 1990.

25. J. C. Polanyi and H. Rieley, "Photochemistry in the Adsorbed Stated", in Advances in Gas Phase Photochemistry and Kinetics. Dynamics of Gas Surface Interactions, C.T. Rittmer and M. N. R. Ashford, ed., pp. 330-363, The Royal Society of Chemistry, Litchwartt, England, 1991. 


\section{SECTION 10.0 CONCLUSIONS}

The results of research to date prove that there is a solar beneficial effect on the destruction of hazardous organic wastes. Much progress has been made on determining the magnitude of the effect, development of a physical model and development of an empirical kinetic model. However, our understanding of the underlying, fundamental processes and, as a result, the generality of the solar beneficial effects is inadequate. For example, it is not clear how solar detoxification will perform on complex waste mixtures, what the capacity of a full-scale system will be, or what the cost will be. Because our fundamental understanding is still weak, we probably cannot design an optimized process. As a result, we run the danger of not being able to develop a cost-competitive system with our current knowledge.

To aid in planning of future research, we have consolidated the information gained in seven years of research. The following technical conclusions represent our attempt to define what we really know about solar detoxification as well as what we suspect based on currently available data.

1. Most compounds which absorb solar radiation exhibit a photo-enhanced rate of destruction over that achievable by purely thermal exposure. Conversely, it is apparent that elevated temperature result in a thermal enhancement of the rate of photolytic reactions. The vast majority of the compounds studied have been substituted aromatics (e.g., chlorine, bromine, iodine, nitro, carbonyl substitutional). These have all shown photo enhanced destruction at moderate radiation intensities (i.e., 57-307 suns). Unsubstituted aromatics species have not exhibited a direct photo-enhancement.

2. Four mechanisms have been identified that to varying degrees have been established as contributing to this solar enhancement. These are:

- increased absorption of solar radiation due to a red-shift and increase in absorption intensity,

- thermal activation of the photo-initiation step,

- photo thermally induced or catalyzed chain reactions at high temperature,

- light absorption and catalysis by intermediates.

Of these, only the increased absorption of radiation has been conclusively shown. Although a satisfactory theory to explain both the red-shift and increase in absorption intensity has been developed, the generality and magnitude of both effects have not been completely established. 
There is strong evidence of thermal/photolytically induced or catalyzed chain reactions at hightemperatures. However, thermal activation of the photo-initiation step and photocatalysis by intermediate can only be inferred from current kinetic experiments that have not been designed to isolate their contributions. Precise measurement of the incident broad band light intensity in a high-temperature, gasphase environment has been a major draw-back to accurately determining photochemical quantum yields.

3. A simple pseudo-first-order kinetic model that includes ground and excited state reactions accounts for the photo-enhancement in the limited number of cases in which it was studied. Considerable data has been collected that shows that the extent of photo-enhancement for pure compounds is exponentially dependent on incident light intensity in the UV $(300-400 \mathrm{~nm})$. This indicates that enhancements in full-scale systems ( $I_{0}=1000-2000$ suns) will be far greater than those observed in laboratory systems $\left(I_{0}=57-308\right.$ suns $)$.

4. PICs that absorb solar radiation have tended to show a greater photo-enhancement than the parent species. Even non-absorbing PICs tend to show a solar enhancement. This latter observation is preliminarily attributed to attack of PICs by photo formed reactive radicals and photo-dissociation of molecular and radical intermediates that absorb solar radiation.

5. Research on the more obvious photo-initiators (i.e., $\mathrm{Cl}_{2}, \mathrm{H}_{2} \mathrm{O}_{2}$, acetone, benzophenane) has shown that they are of limited utility because they tend to thermally decompose at low temperatures. This limits their effectiveness as high-temperature, energy transfer sensitizers or radical photo-initiators.

6. Research on photo-enhancement of complex mixtures has revealed that the situation is complex. The destruction of non-absorbing compounds in a mixture containing some absorbing, photoactive materials is observed to be photo-enhanced under some conditions. However, it is usually observed over a limited temperature ranges just higher in temperature than the onset of thermal destruction. This suggests that there may be a relatively narrow operating range where solar enhanced waste destruction is optimized.

7. Consideration of photo effects on droplet combustion suggest that there are a number of non gas-phase processes that may result in solar enhanced waste destruction. For droplets, these include:

- Radiative heating and an increased rate of droplet vaporization for larger droplets,

- Photo-enhanced droplet ignition,

- "Lensing" of radiation by droplets of high refractive index that can result in internal focusing, micro-explosions, and secondary atomization of sprays. 
8. A conceptual design and scaling of a solar detoxifier for soil remediation has been competed. This exercise demonstrated that intermediate trapping and storage of the material desorbed from the soil is necessary to have a reasonably efficient process. This process also demonstrated that a better understanding of the parameters that effect solar detoxification is needed before it will be possible to design and construct a reliable system. 


\section{SECTION 11.0 RECOMMENDATIONS}

The concept of solar detoxification has been demonstrated on certain wastes and the thermal enhancement of photolytic processes has been shown to be a reality. Photo kinetic studies have been very useful in advancing research to this point. However, we would appear to be at a branching point for future research.

On one hand, there is pressure to bring this technology to a reality. As such, larger scale demonstration projects are being conducted. To this end, research must be conducted to support these studies. However, we also run the risk that these demonstrations may fail because we do not have sufficient knowledge to design an efficient system and overcome the inevitable, problems that arise. Consequently, we must try to obtain as much data as possible on the anticipated performance of the fullscale system prior to conducting the difficult and expensive field tests.

Some photo-thermal kinetic studies will be needed to clarify some key issues and support field testing. Some bench scale testing will also be needed to address other key issues related to scale-up. However, these studies will be difficult to perform in a useful manner because a lack of overall understanding of key design issues. Thus, it is recommended that studies of these types be limited and highly focused.

Instead, it is recommended that the observations and preliminary findings from the early kinetic studies be verified and further explored with fundamental studies. Key issues for these fundamental studies include the temperature dependence of the initial photochemical step, the effect of temperature on absorption intensity, the role of photo-induced chain reactions, and identification of methods for destruction of non- or weakly-absorbing compounds. We thus propose the following specific recommendations:

1. Spectroscopic or time resolved photochemical studies of thermal effects on primany photochemical processes. These experiments should specifically address the issue of thermal activation of excited electronic states and its effect on photochemical reactions. The studies will probably require the use of high-temperature gas phase reactor systems in conjunction with pulsed laser systems to achieve the necessary time resolution. They should address the effect of temperature on populations and rates of decay of excited singlet and triplet states and the rate of product formation. These can be determined by measurements of the temperature-dependence of fluorescence and phosphorescence quantum yields, singlet and triplet lifetimes, and product formation. Time-resolved product radical formation may be especially helpful for determining the thermal effects on the rate of initial product formation. Use of high- 
temperature, supersonic jet spectroscopic techniques may make it possible to conduct high-resolution vibronic studies without rotational congestion that may otherwise obscure key information.

2. Additional high-temperature absorption studies should be conducted on homologous series of compounds to better discern the detailed mechanism of thermally induced vibronic coupling. These studies will help to generalize and extrapolate from the existing data base to other untested compounds.

3. Additional exploratory research is needed on methods for destroying non-absorbing compounds. The Sol-CAD technique, discussed in this report is one promising technique. However, other techniques that combine the effect of temperature and photolysis may be envisioned and should be at least preliminarily explored.

4. High-temperature photochemical kinetic studies should be conducted on a limited basis to address key issues. Simple binary mixtures studies to address photo-induced initiation of radical chain reactions that destroy non-absorbing materials may be the most timely. These experimental studies should be conducted in combination with kinetic modeling to obtain a deeper understanding of any observed effects.

5. To ensure the highest probability of success for the planned Tri-Agency, solar detoxification project, bench and laboratory treatability testing should be conducted. These should include desorbability and destructability of the contaminant to be treated. It is strongly recommended that actual samples of contaminated soils should be subjected to laboratory solar detoxification testing. This data should be used to develop a thermal/photolytic kinetic destruction model that can be used to support design of a field-scale reactor. 


\section{SECTION 12.0}

\section{BIBLIOGRAPHY}

Listed below is a summary of publications, reports and presentations on solar detoxification and related topics authored and co-authored by The University Of Dayton Research Institute's Environmental Science And Engineering Group from 1985-1993.

J. L. Graham, J. M. Berman, and B. Dellinger, "The High Temperature Thermal/Photolytic Oxidation of Monochlorobenzene," Journal Photochemistry \& Photobiology, A: Chem. 71, p. 65, 1993.

B. Dellinger, J. L. Graham, J. M. Berman, and Z. Jiang, "Photocatalytically Enhanced Gas Phase Photolysis and Oxidation of Chlorinated Hydrocarbons," presentation at the 1993 Joint Eastern and Central States Meeting of the Combustion Institute, XVIth International Conference on Photochemistry, Ottawa, Ontario, Canada, April 1993.

J. L. Graham, J. M. Berman, and B. Dellinger, "The High Temperature Thermal/Photolytic Oxidation of Monochlorobenzene," Journal of Photochemistry and Photobiology, A:Chem., 71, p. 65, 1993.

J. L. Graham, B. Dellinger, and G. Glatzmaier, "The Destruction of Polychlorinated Dibenzodioxin and Biphenyl Wastes Using Concentrated Solar Energy," presentation at the 1992 American Institute of Chemical Engineers Annual Meeting, Miami Beach, FL, November 1992.

J. L. Graham, B. Dellinger, and G. C. Glatzmaier, "The Destruction of 1,2,3,4-

Tetrachlorodibenzo-p-dioxin Using Simulated and Natural Concentrated Solar Energy," submitted, Hazardous Waste and Hazardous Materials, October 1992.

J. M. Berman, J. L. Graham, and B. Dellinger, "High-Temperature Ultraviolet Absorption Characteristics of Three Environmentally Sensitive Compounds," Journal Photochemistry \& Photobiology, A: Chem. 68, p. 353, 1992.

B. Dellinger, J. L. Graham, and J. M. Berman, "Destruction of Organic Wastes Using Concentrated Solar Radiation," presentation at the 18th Annual RREL Research Symposium, Cincinnati, OH, April, 1992.

B. Dellinger, J. L. Graham, J. M. Berman, and D. Klosterman, "High-Temperature Photochemistry Induced by Concentrated Solar Radiation," in Potential Applications of 
Concentrated Solar Energy, proceedings of NRC Workshop, National Academy Press, Washington, D. C., p. 38, 1991.

J. L. Graham, "Using Concentrated Solar Energy to Destroy Hazardous Organic Wastes," presentation at the Indiana State Legislature Study Committee on Alternative Methods of Disposal and Destruction of Hazardous Waste, Indianapolis, IN, October 1991.

J. L. Graham, B. Dellinger, D. Klosterman, G. Glatzmaier, and G. Nix, "Disposal of Toxic Wastes Using Concentrated Solar Radiation," In Emerging Technologies in Hazardous Waste Management II, American Chemical Society, Washington, DC, Chapter 6, p. 83, April 1991.

B. Dellinger, "The High Temperature Thermal and Photochemical Kinetics of Hazardous Waste Destruction," presentation at the Rensselaer Polytechnic Institute, Rensselaer, NY, February 1991.

J. L. Graham and B. Dellinger, "The High Temperature Photolytic Destruction of 3,3'4,4'Tetrachloro-biphenyl Using Simulated Solar Radiation," proceedings of the Fall Technical Meeting of the Eastern States Section - Combustion Institute, Orlando, FL, December 1990.

B. Dellinger, J. Graham, J. M. Berman, and D. Klosterman, "High Temperature Photochemistry Induced by Concentrated Solar Radiation," proceedings of the National Research Council Workshop on Potential Applications of Concentrated Solar Photons, November 1990.

G. C. Glatzmaier, J. L. Graham, and B. Dellinger, "Comparison of Laboratory and Field Experiments for the Destruction of Tetrachlorodibenzo-p-dioxin Using Concentrated Solar Energy", proceedings of the 25th Intersociety Energy Conversion Engineering Conference, Reno, NV, August 1990.

J. L. Graham and B. Dellinger, "Disposal of Toxic Wastes Using Concentrated Solar Radiation," proceedings of the I \& EC Div. Symposium, Atlantic City, NJ, June 1990.

J. L. Graham, B. Dellinger, and D. Klosterman, "An Overview of a Laboratory Investigation of Hazardous Organic Wastes," proceedings of the 12th Annual Joint Canada - U.S. Incineration Workshop, University of Dayton, Dayton, OH, April 1990.

J. L. Graham, "Destroying Hazardous Organic Wastes Using Concentrated Sunlight," presentation at the Dayton Chapter of American Institute of Chemical Engineers, Dayton, OH, September 1989.

J. L. Graham, "Solar Detoxification of Hazardous Organic Wastes," presentation at the National Energy Program Manager's Meeting, Indianapolis, IN, September 1989. 
J. L. Graham, "Incineration of Hazardous Organic Wastes Using Solar Energy," presentation at the Technology Transfer Sub-committee of Indiana State Chamber of Commerce, Indianapolis, IN, September 1989.

B. Dellinger and J. L. Graham, "The High-Temperature Photochemistry of 3,3', 4,4'Tetrachlorobiphenyl," proceedings of the Gordon Conference on Organic Photochemistry, Andover, NH, July 1989.

J. L. Graham and B. Dellinger, "Laboratory Study of the Solar Incineration of Hazardous Organic Wastes," proceedings of the AIChE Summer National Meeting, Paper 74f, Denver, CO, August 1988.

J. L. Graham and B. Dellinger, "The Role of the Triplet State in the Solar Thermal/Photolytic Destruction of Halocarbons," proceedings of the 7th International Conference on Photochemical Conversion and Storage of Solar Energy, Paper B-22, Chicago, IL, July 1988.

B. Dellinger and J. L. Graham, "Very High Temperature Photolysis of Toxic Halocarbons," proceedings of the XII IUPAC Symposium on Photochemistry, Paper PA-4, Bologna, Italy, July 1988.

J. L. Graham and B. Dellinger, "Solar Detoxification of Hazardous Organic Wastes," proceedings of the 4th International Symposium on Research, Development and Applications of Solar Thermal Technology, Sante Fe, NM, June 1988.

J. L. Graham and B. Dellinger, "Solar Thermal/Photolytic Incineration of Hazardous Organic Compounds," proceedings of the Annual SERI Symposium, Golden, CO, February 1987.

J. L. Graham and B. Dellinger, "Solar Thermal/Photolytic Destruction of Hazardous Organic Wastes," Energy, 12, No. 3/4, p. 303, 1987.

J. L. Graham and B. Dellinger, "A Laboratory Evaluation of the Solar Incinerability of Hazardous Organic Wastes," proceedings of the Solar and Thermal Research Program Annual Conference, SENS/CP-251-2680, DE85002938, Lakewood, CO, April 1985. 


\section{APPENDIX $\mid$}

\section{Thermal/Photolytic Degradation of Pure Compounds}

This Appendix summarizes all of the data obtained during this project on the thermal and thermal/photolytic decomposition of pure compounds. The data are listed alphabetically by compound with sub-ordering by reaction atmosphere (oxidation-pyrolysis), and exposure time (ascending sort). Unless otherwise noted, temperature is given in degrees Centigrade and fraction remaining is in mole percent normalized by the original amount of sample fed to the reactor. By definition, enhancement ratio is unitless. 


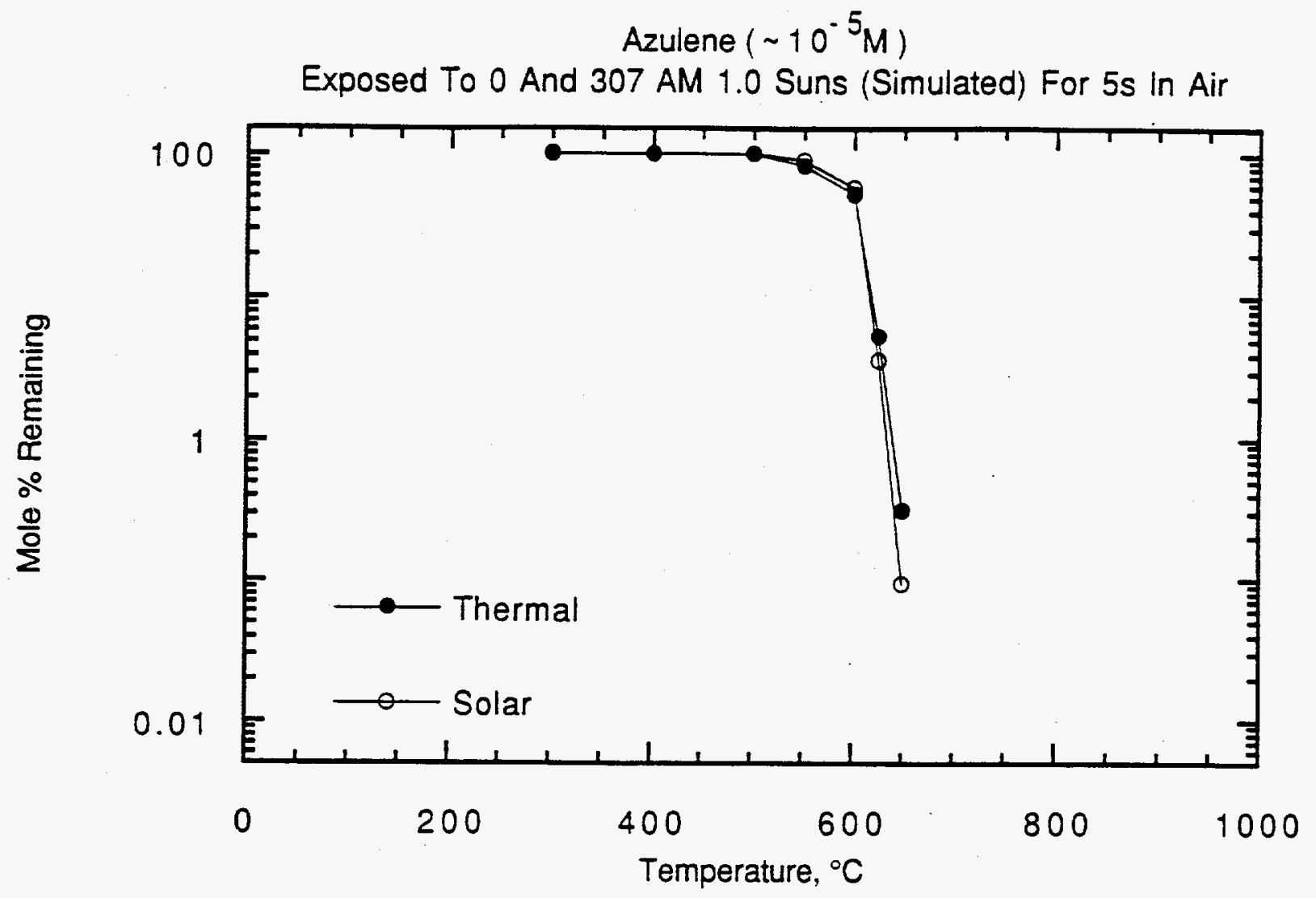

\begin{tabular}{|c|c|c|c|}
\hline Temp. & $f(0)$ & $f(10)$ & $R(10)$ \\
\hline & & & \\
300 & 100.0 & 100.0 & 1.00 \\
400 & 100.0 & 100.0 & 1.00 \\
500 & 100.0 & 100.0 & 1.00 \\
550 & 81.6 & 90.0 & 0.907 \\
600 & 51.7 & 57.3 & 0.902 \\
625 & 5.41 & 3.66 & 1.48 \\
650 & 0.310 & 0.0940 & 3.31 \\
\hline
\end{tabular}




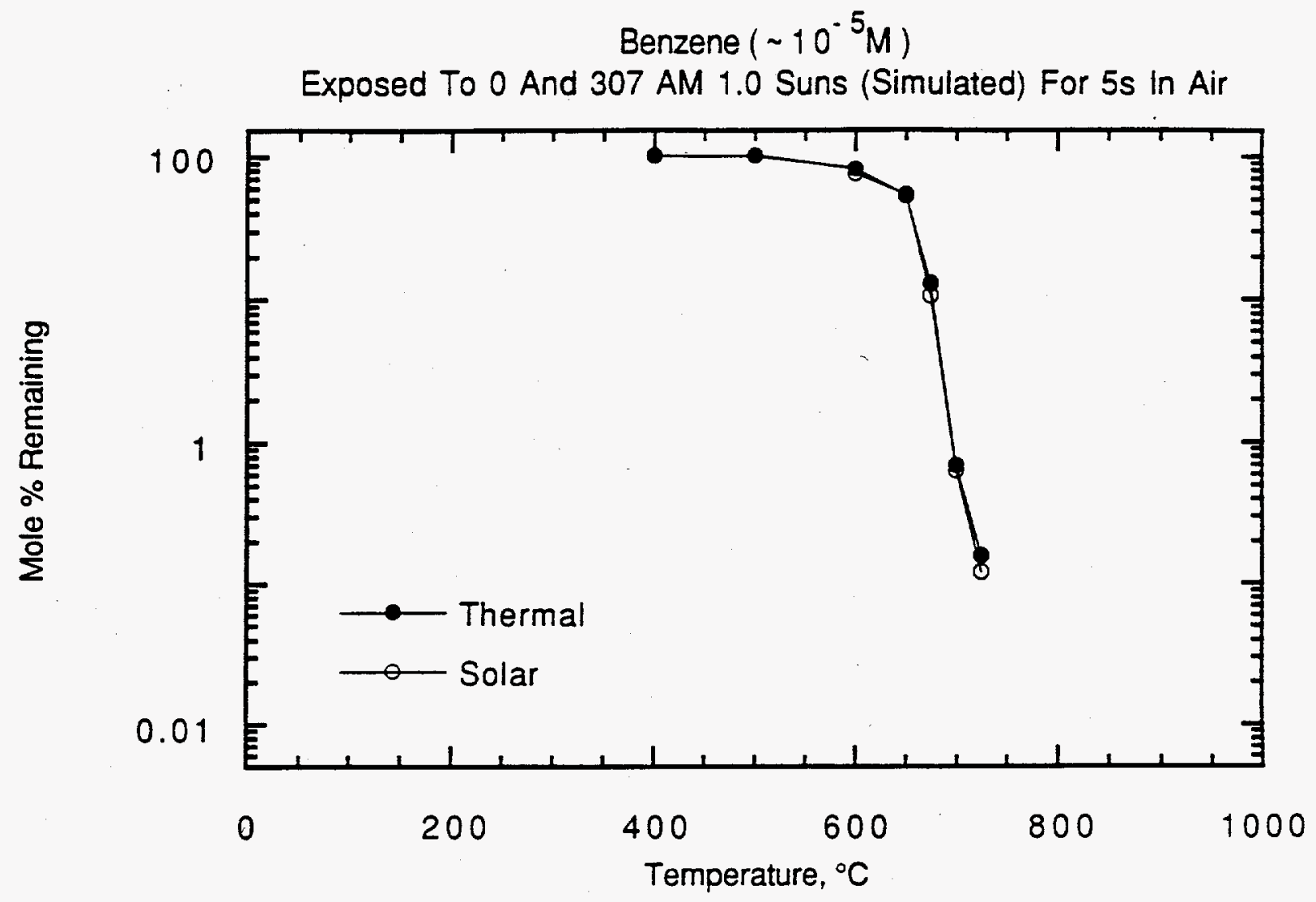

\begin{tabular}{|c|c|c|c|}
\hline Temp. & $f r(0)$ & $f f(10)$ & $R(10)$ \\
\hline 400 & 100.0 & $\ldots$ & \\
500 & 100.0 & $\ldots$ & $\cdots$ \\
600 & 80.0 & $\ldots$ & $\ldots$ \\
650 & 52.500 & 52.800 & $\ldots .994$ \\
675 & 12.900 & 10.700 & 1.206 \\
700 & 0.691 & 0.633 & 1.092 \\
725 & 0.157 & 0.120 & 1.308 \\
\hline
\end{tabular}


Benzo\{e\}pyrene $\left(\sim 10^{-5} \mathrm{M}\right)$

Exposed To 0 And 307 AM 1.0 Suns (Simulated) For $5 s$ in Air

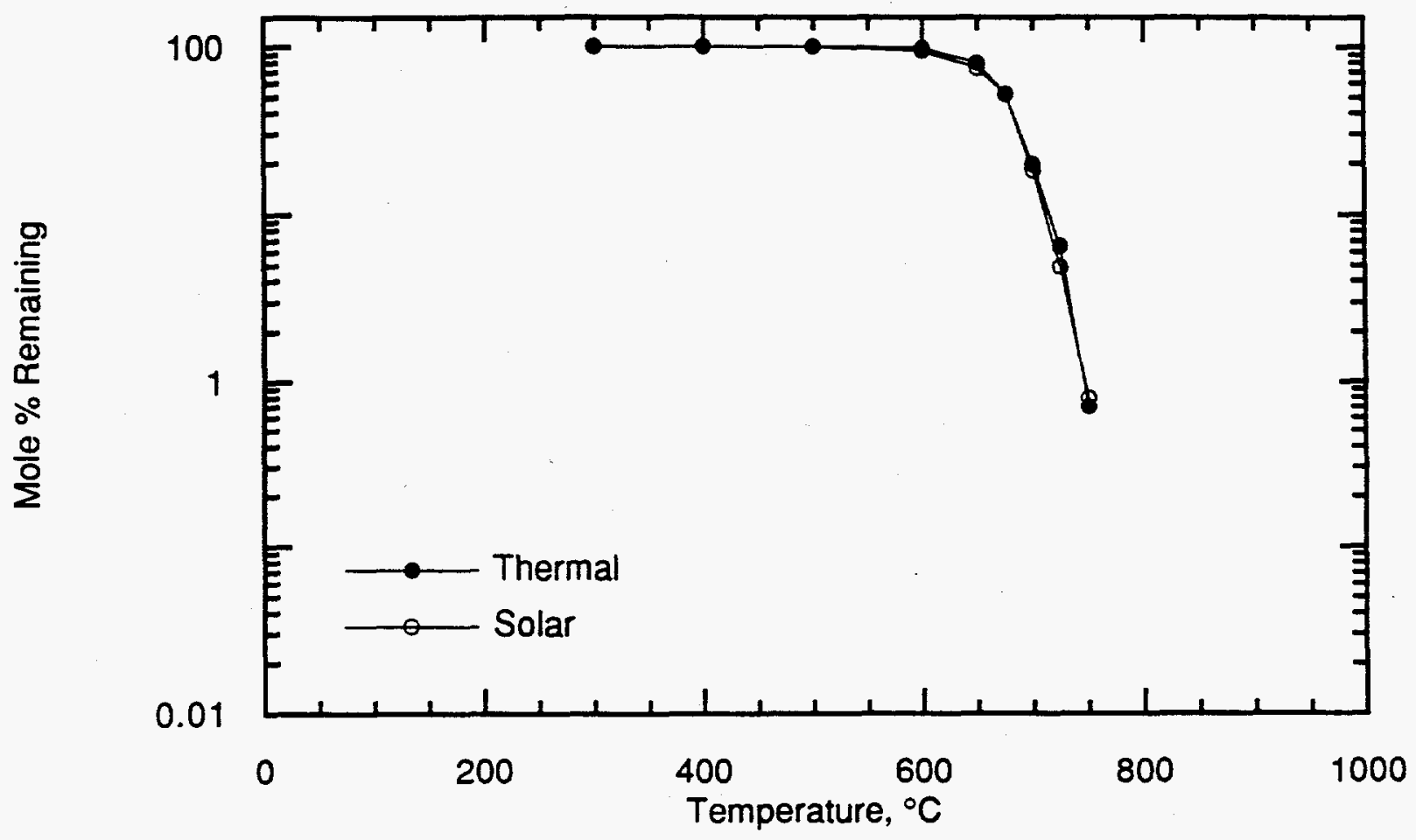

\begin{tabular}{|r|r|r|r|r|}
\hline & Temp. & $f r(0)$ & $f r(10)$ & $f(10)$ \\
\hline 0 & 300.00 & 100.00 & 100.00 & 1.0000 \\
\hline 1 & 400.00 & 100.00 & 100.00 & 1.0000 \\
\hline 2 & 500.00 & 100.00 & 100.00 & 1.0000 \\
\hline 3 & 600.00 & 98.600 & 94.400 & 1.0445 \\
\hline 4 & 650.00 & 79.800 & 74.200 & 1.0755 \\
\hline 5 & 675.00 & 52.000 & 51.800 & 1.0039 \\
\hline 6 & 700.00 & 19.800 & 18.400 & 1.0761 \\
\hline 7 & 725.00 & 6.5300 & 4.8700 & 1.3409 \\
\hline 8 & 750.00 & 0.69900 & 0.78800 & 0.88706 \\
\hline
\end{tabular}




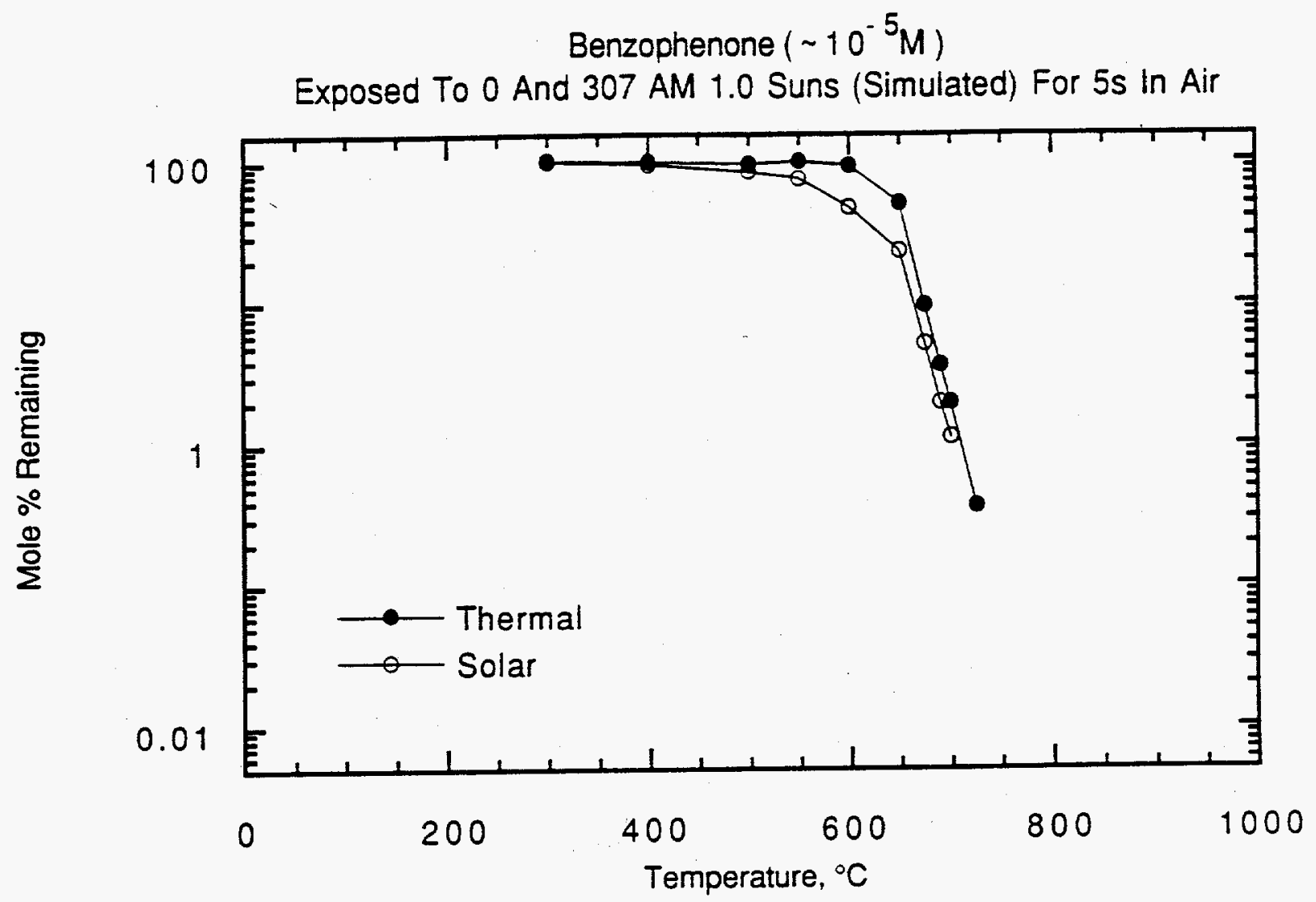

\begin{tabular}{|c|c|c|c|}
\hline Temp. & $f r(0)$ & $f(10)$ & $R($ lo $)$ \\
\hline 300 & 100.0 & 99.1 & 1.01 \\
400 & 98.1 & 93.6 & 1.05 \\
500 & 94.9 & 83.5 & 1.14 \\
550 & 98.6 & 74.0 & 1.33 \\
600 & 92.0 & 46.5 & 1.98 \\
650 & 49.7 & 22.9 & 2.17 \\
675 & 9.55 & 5.19 & 1.84 \\
690 & 3.67 & 1.96 & 1.87 \\
700 & 1.96 & 1.13 & 1.74 \\
725 & 0.363 & $\ldots$ & $\cdots$ \\
\hline
\end{tabular}




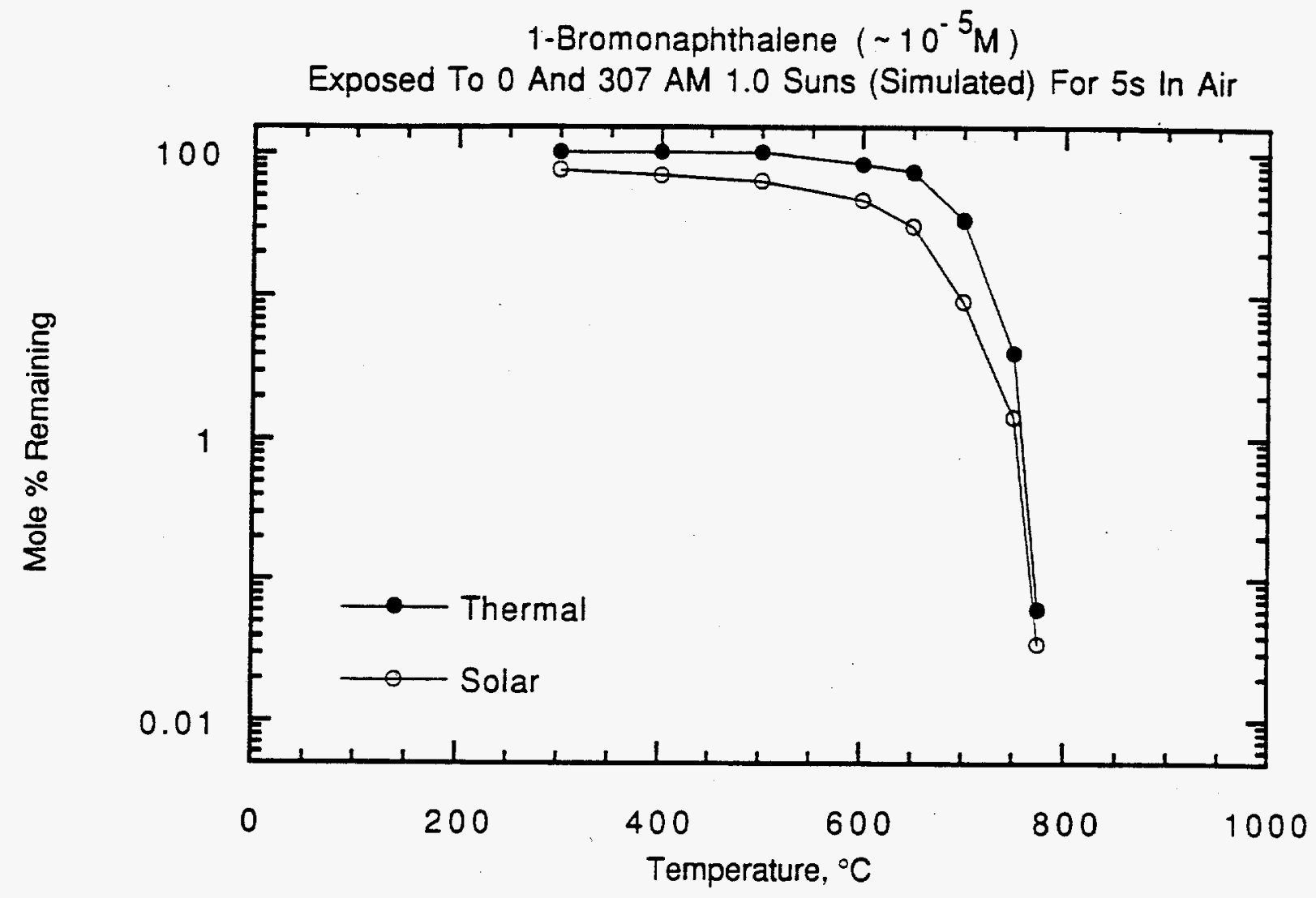

\begin{tabular}{|c|c|c|c|}
\hline Temp. & fr (0) & fr (lo) & $R(10)$ \\
\hline 300 & 100.0 & 74.6 & \\
400 & 100.0 & 68.4 & 1.34 \\
500 & 100.0 & 62.2 & 1.46 \\
600 & 82.4 & 45.8 & 1.61 \\
650 & 72.5 & 30.4 & 1.80 \\
700 & 33.5 & 9.13 & 2.39 \\
750 & 4.04 & 1.43 & 3.67 \\
775 & 0.061 & 0.035 & 2.83 \\
\hline
\end{tabular}




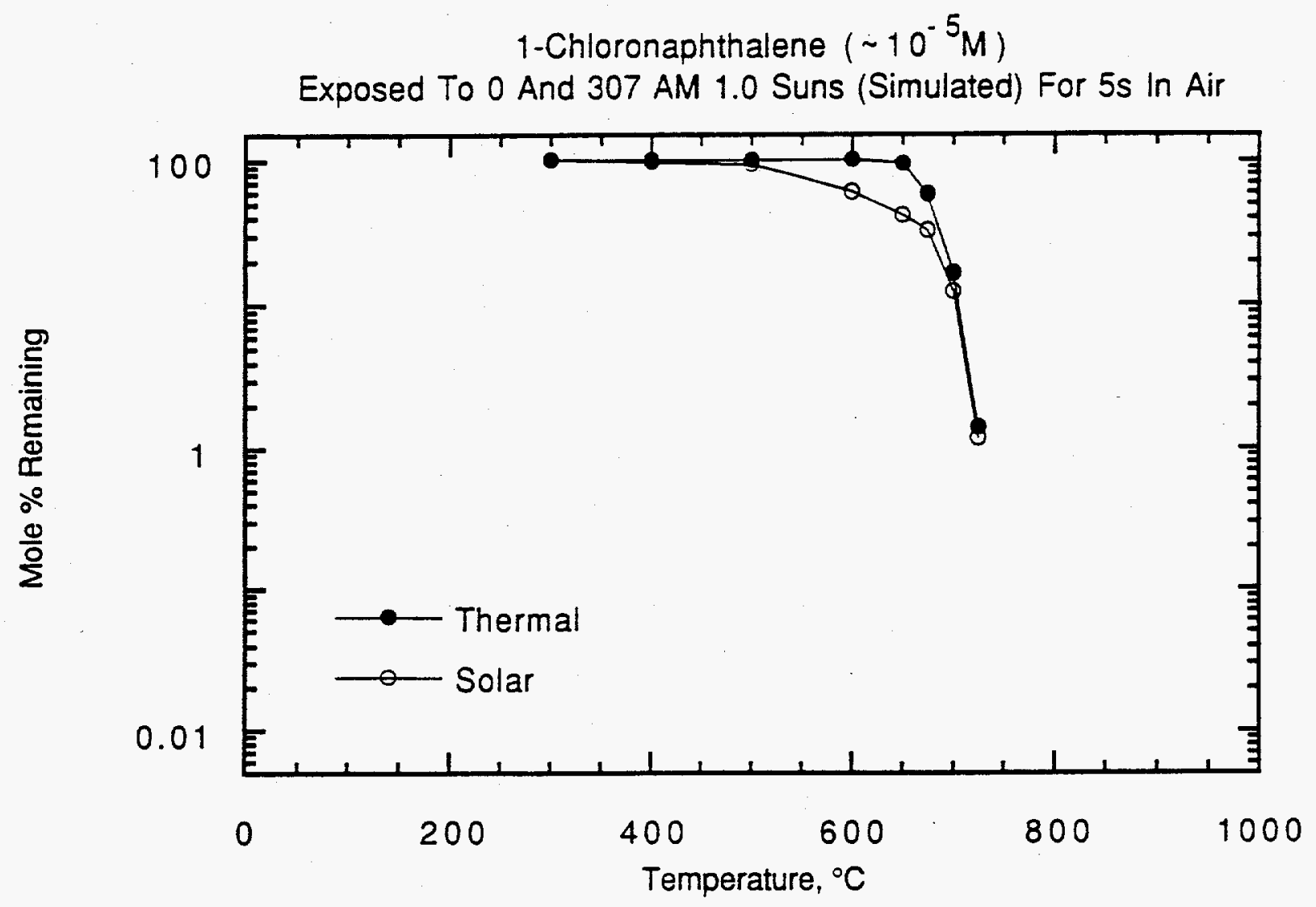

\begin{tabular}{|c|c|c|c|}
\hline Temp. & $f(0)$ & $f r(10)$ & $R(10)$ \\
\hline 300 & 100.0 & 100.0 & 1.00 \\
400 & 100.0 & 96.5 & 1.04 \\
500 & 100.0 & 93.2 & 1.07 \\
600 & 100.0 & 59.6 & 1.68 \\
650 & 93.5 & 41.0 & 2.28 \\
675 & 57.0 & 32.0 & 1.78 \\
700 & 16.4 & 12.2 & 1.34 \\
725 & 1.41 & 1.18 & 1.20 \\
\hline
\end{tabular}




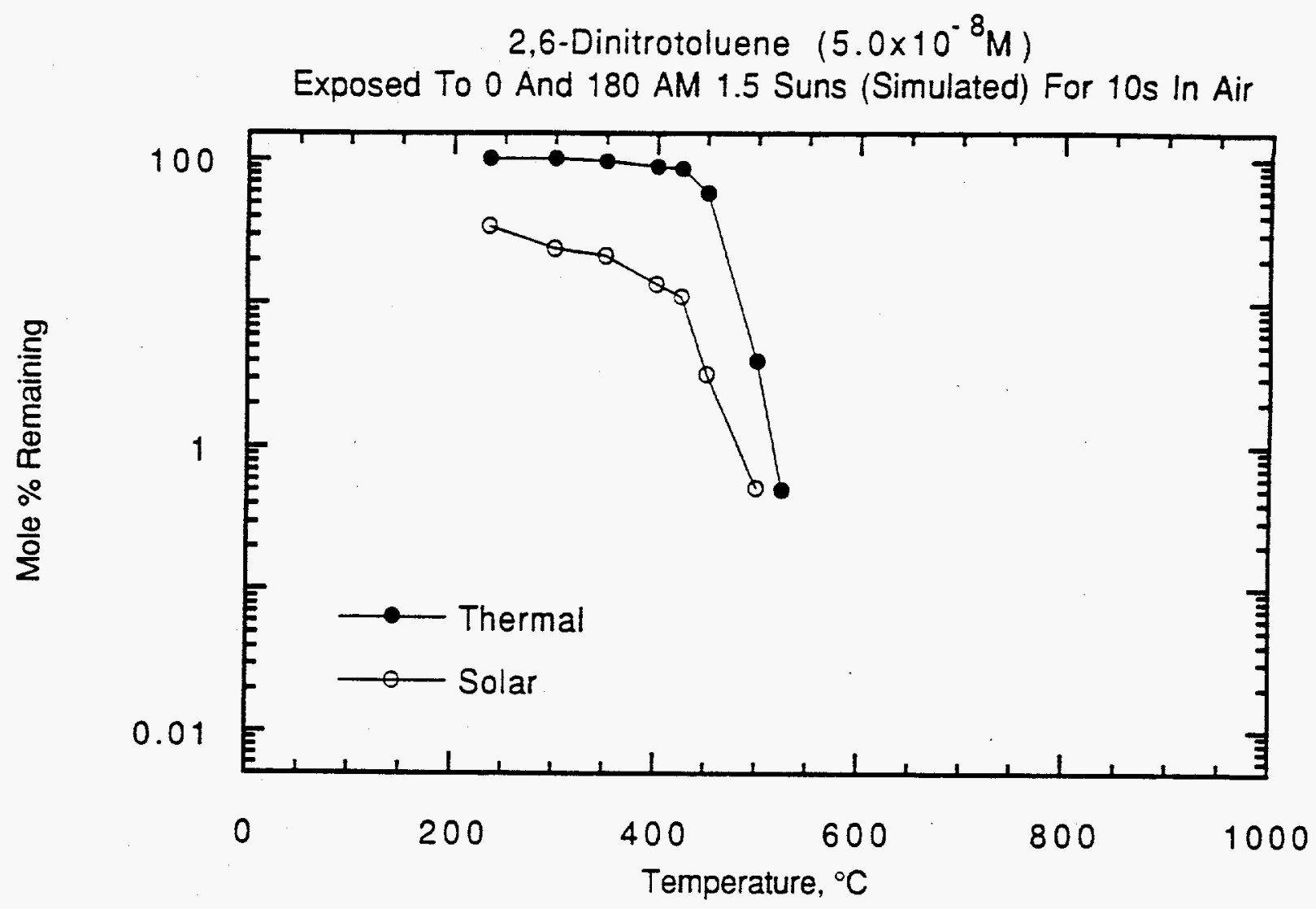

\begin{tabular}{|c|c|c|c|}
\hline Temp. & fr (0) & fr (lo) & R (lo) \\
\hline & & & \\
235 & 100.0 & 33.7 & 2.97 \\
300 & 100.0 & 23.7 & 4.22 \\
350 & 95.4 & 21.1 & 4.52 \\
400 & 87.3 & 13.3 & 6.56 \\
425 & 84.7 & 11.0 & 7.70 \\
450 & 57.5 & 3.20 & 17.97 \\
500 & 3.94 & 0.515 & 7.65 \\
525 & 0.495 & - & $\ldots$ \\
550 & $\ldots$ & $\ldots$ & $\ldots$ \\
\hline
\end{tabular}




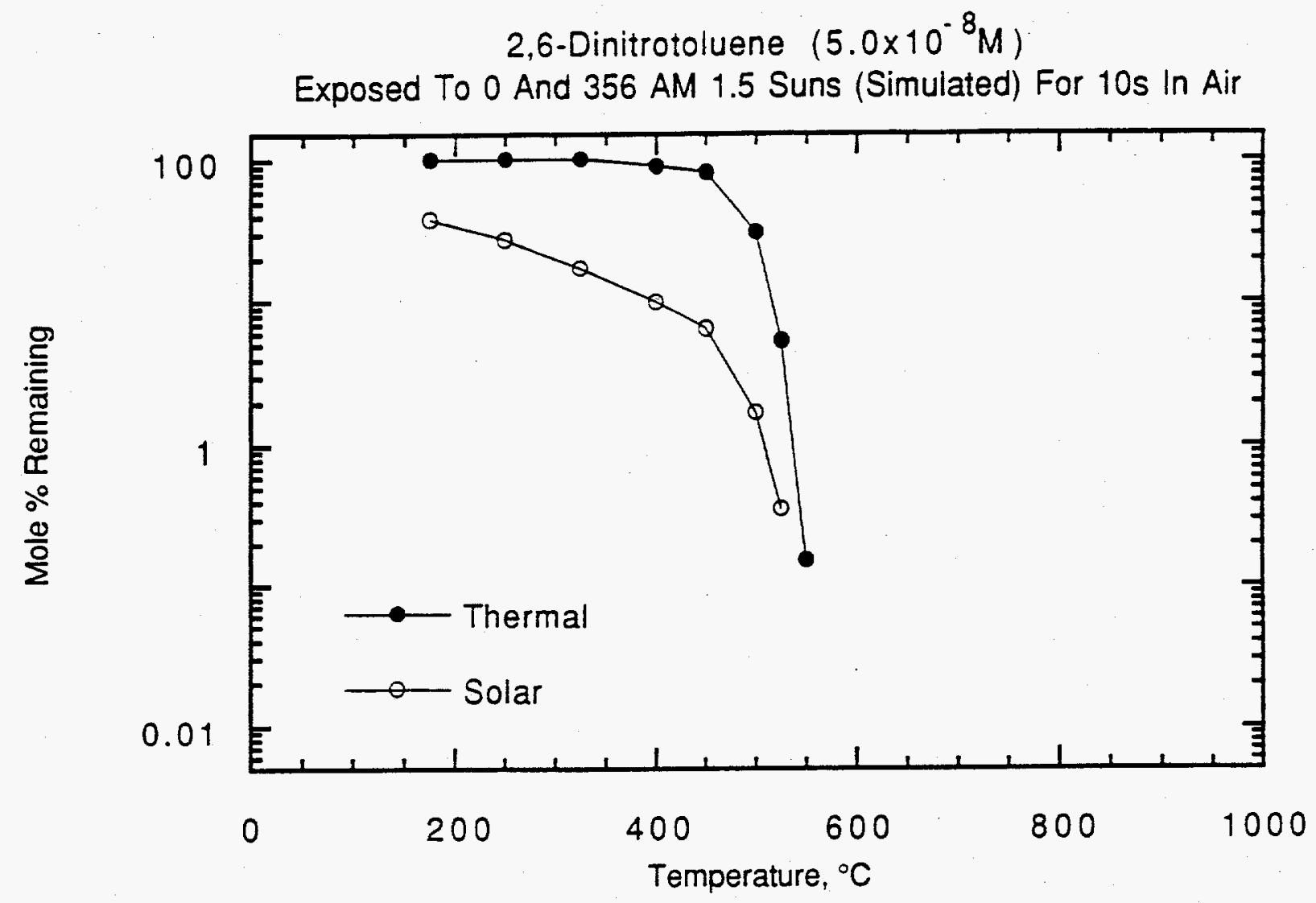

\begin{tabular}{|c|c|c|c|}
\hline Temp. & $f r(0)$ & $f r(10)$ & $R(10)$ \\
\hline & & & \\
175 & 100.0 & 37.9 & 2.64 \\
250 & 100.0 & 27.0 & 3.70 \\
325 & 100.0 & 17.0 & 5.88 \\
400 & 89.7 & 9.90 & 9.06 \\
450 & 80.9 & 6.48 & 12.5 \\
500 & 30.5 & 1.71 & 17.8 \\
525 & 5.32 & 0.355 & 15.0 \\
550 & 0.152 &.-- & $\cdots$ \\
575 & $\ldots$ & -- &.-- \\
\hline
\end{tabular}




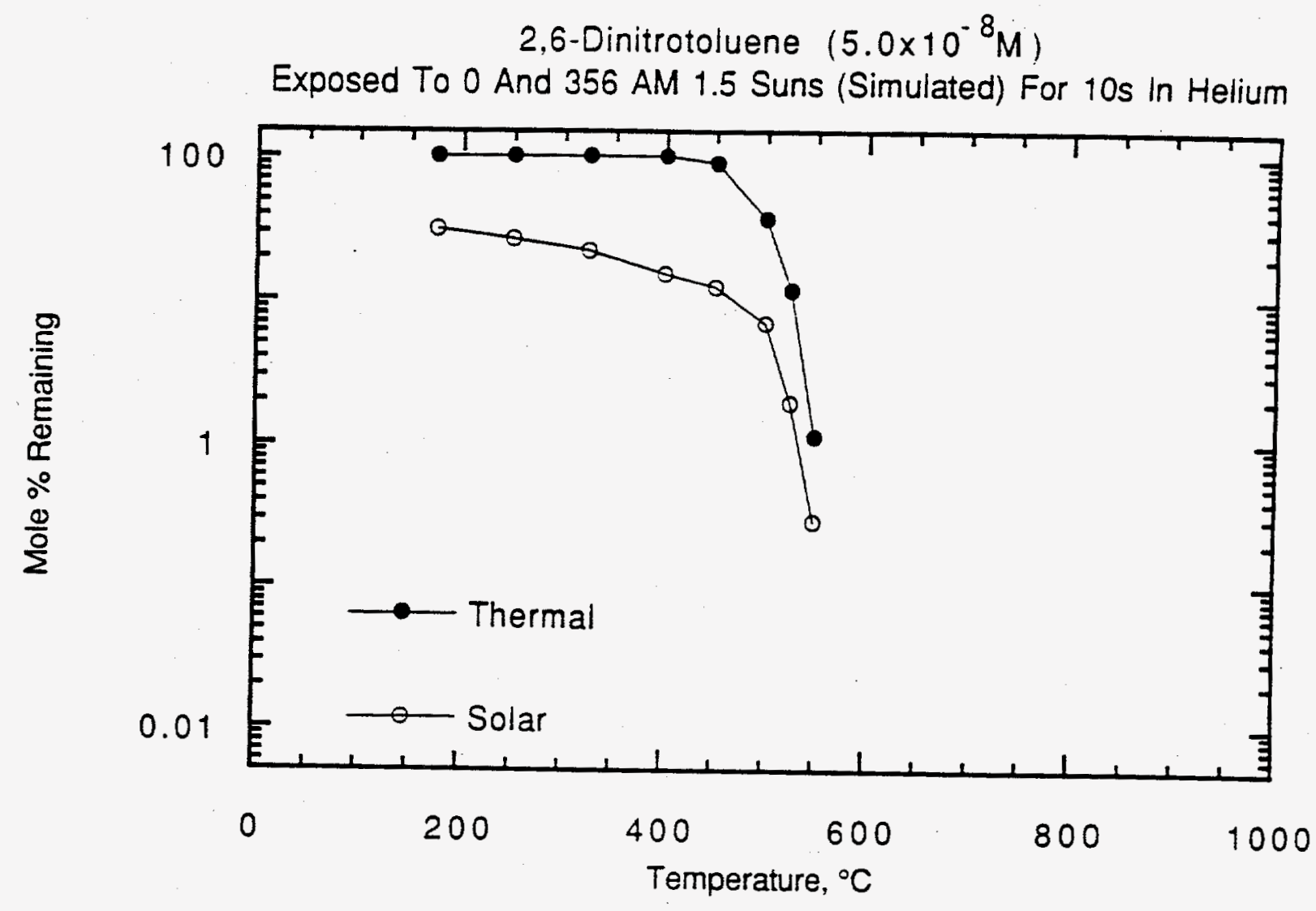

\begin{tabular}{|c|c|c|c|}
\hline Temp. & $f r(0)$ & $f r(10)$ & $R(10)$ \\
\hline 175 & 100.0 & 31.0 & 3.27 \\
250 & 100.0 & 26.4 & 3.79 \\
325 & 100.0 & 22.2 & 4.51 \\
400 & 100.0 & 15.1 & 6.62 \\
450 & 89.6 & 12.2 & 7.34 \\
500 & 36.7 & 6.88 & 5.33 \\
525 & 11.7 & 1.92 & 6.09 \\
550 & 1.12 & 0.286 & 3.92 \\
575 & $\ldots$ & $\ldots$ & $\cdots$ \\
\hline
\end{tabular}


Hexachlorobenzene $\left(\sim 10^{-5} \mathrm{M}\right)$

Exposed To 0 And 307 AM 1.0 Suns (Simulated) For $5 s$ In Air

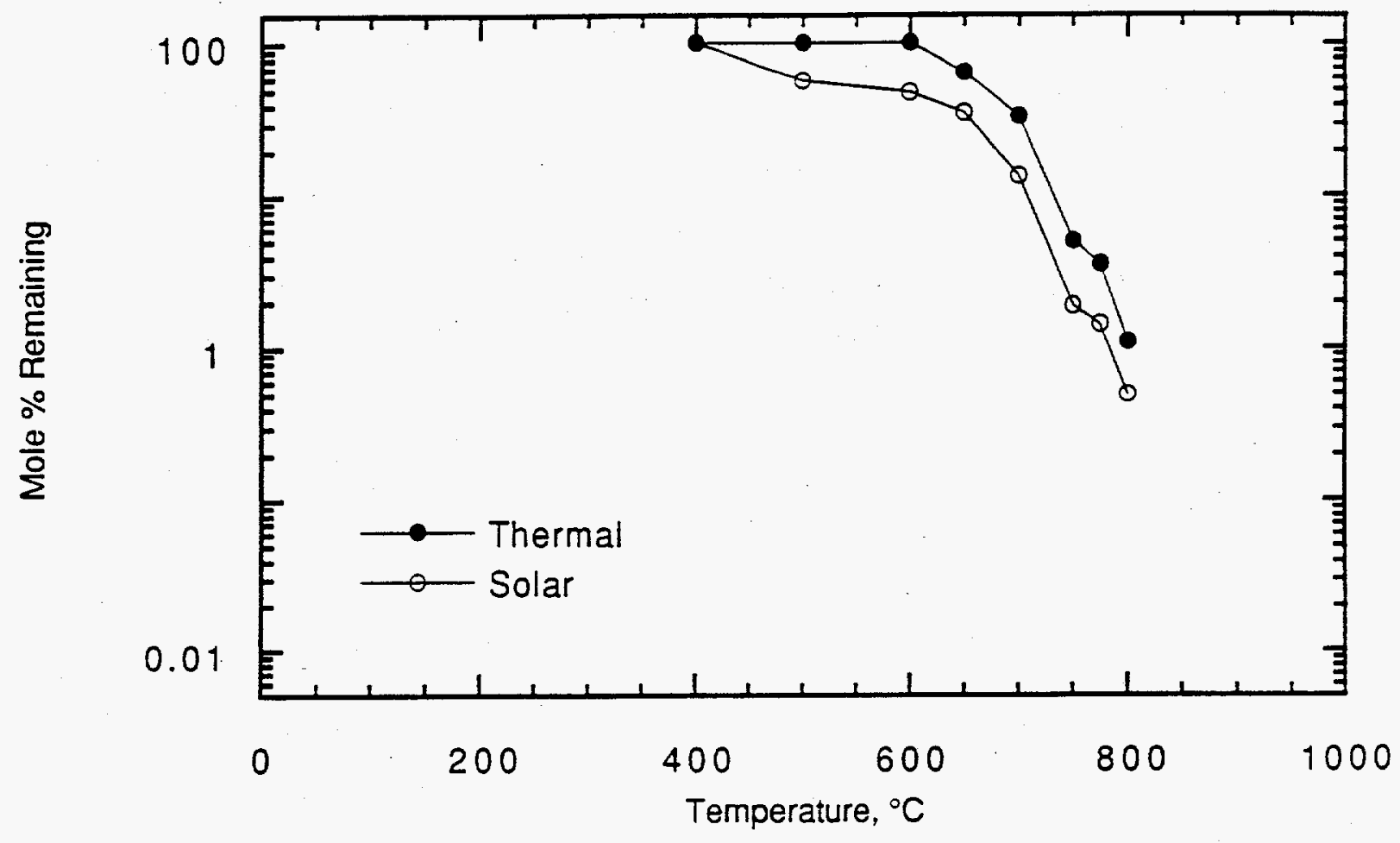

\begin{tabular}{|c|c|c|c|}
\hline Temp. & fr (0) & $f f(10)$ & $\mathrm{R}(10)$ \\
\hline & & & \\
400 & 100.0 & 100.0 & 1.00 \\
500 & 100.0 & 57.1 & 1.75 \\
600 & 100.0 & 47.9 & 2.09 \\
650 & 64.4 & 35.5 & 1.81 \\
700 & 33.6 & 13.5 & 2.49 \\
750 & 5.00 & 1.88 & 2.66 \\
775 & 3.51 & 1.42 & 2.47 \\
800 & 1.09 & 0.490 & 2.22 \\
\hline
\end{tabular}




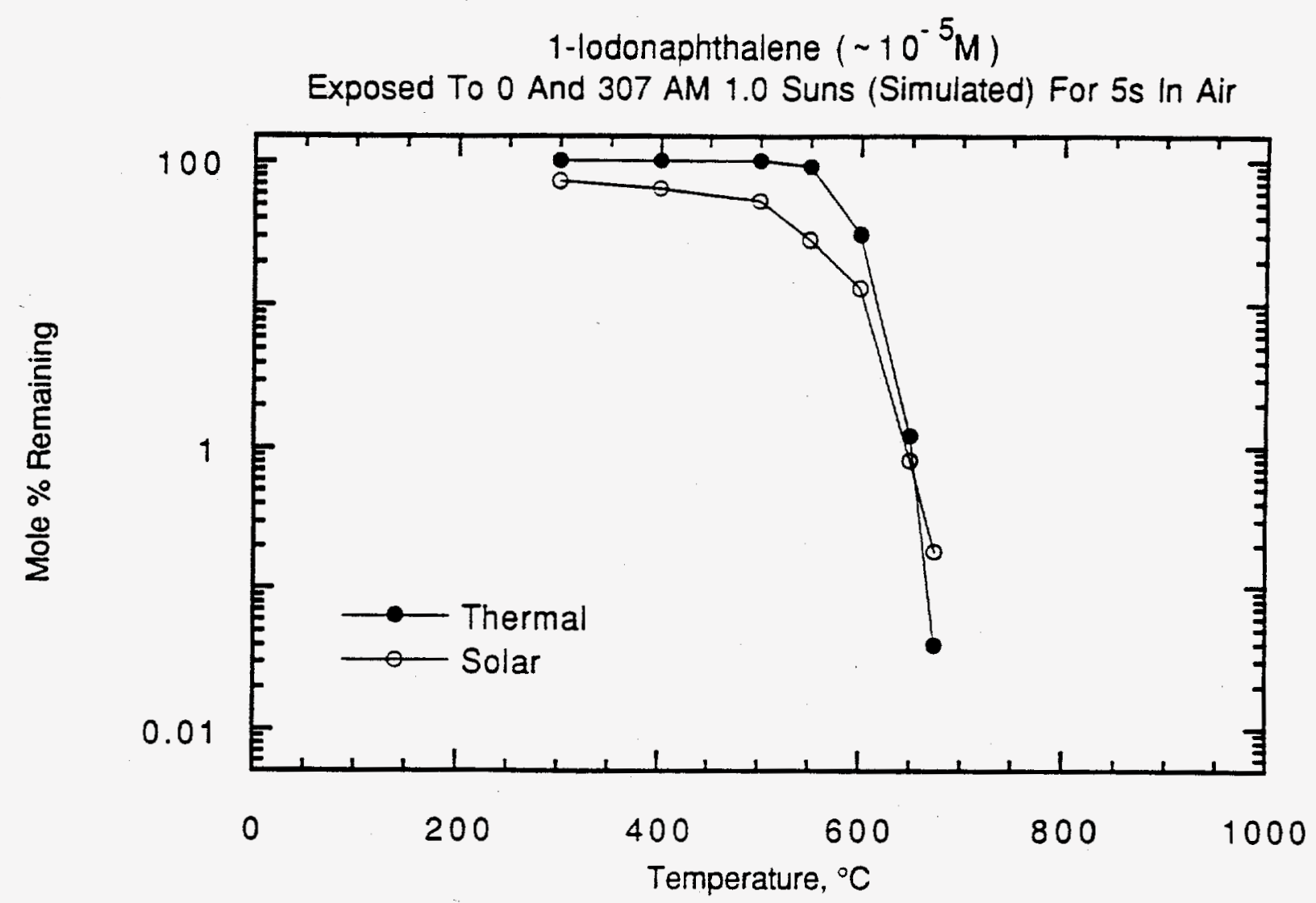

\begin{tabular}{|c|c|c|c|}
\hline Temp. & fr $(0)$ & fr $(10)$ & $R(10)$ \\
\hline & & & \\
300 & 100.0 & 71.6 & 1.40 \\
400 & 100.0 & 63.6 & 1.57 \\
500 & 100.0 & 51.7 & 1.93 \\
550 & 90.3 & 27.5 & 3.28 \\
600 & 30.20 & 13.0 & 2.32 \\
650 & 1.21 & 0.805 & 1.50 \\
675 & 0.0390 & 0.181 & 0.217 \\
\hline
\end{tabular}


Methylene Chloride $\left(\sim 10^{-5} \mathrm{M}\right)$

Exposed To 0 And 307 AM 1.0 Suns (Simulated) For $10 \mathrm{~s}$ In Helium

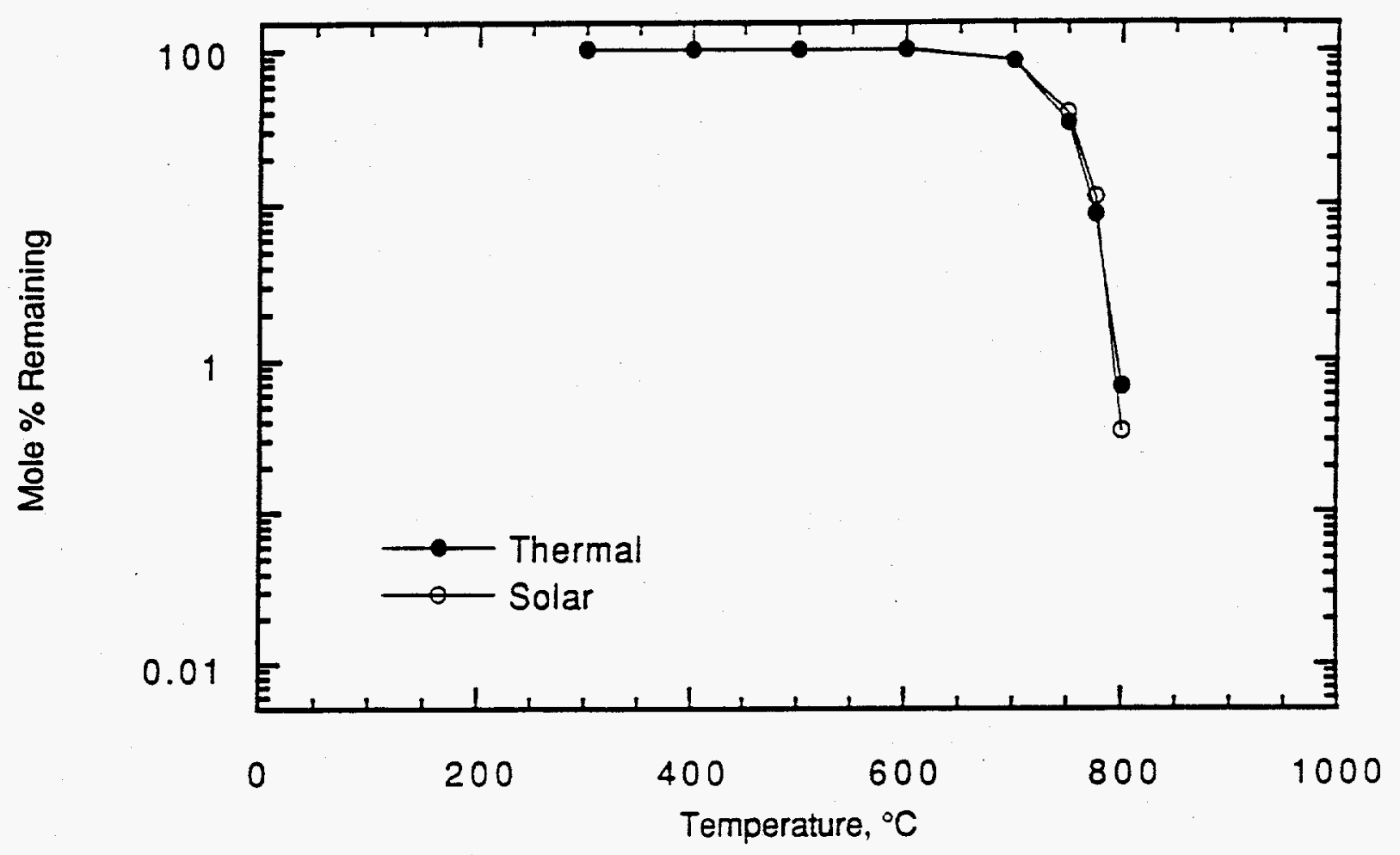

\begin{tabular}{|c|c|c|c|}
\hline Temp. & $f r(0)$ & $f r(10)$ & $R(10)$ \\
\hline & & & \\
300 & 100.0 & 100.0 & 1.00 \\
400 & 100.0 & 100.0 & 1.00 \\
500 & 100.0 & 100.0 & 1.00 \\
600 & 100.0 & 100.0 & 1.00 \\
700 & 83.6 & 83.7 & 0.999 \\
750 & 33.0 & 38.4 & 0.859 \\
775 & 8.57 & 11.0 & 0.779 \\
800 & 0.662 & 0.341 & 1.94 \\
\hline
\end{tabular}




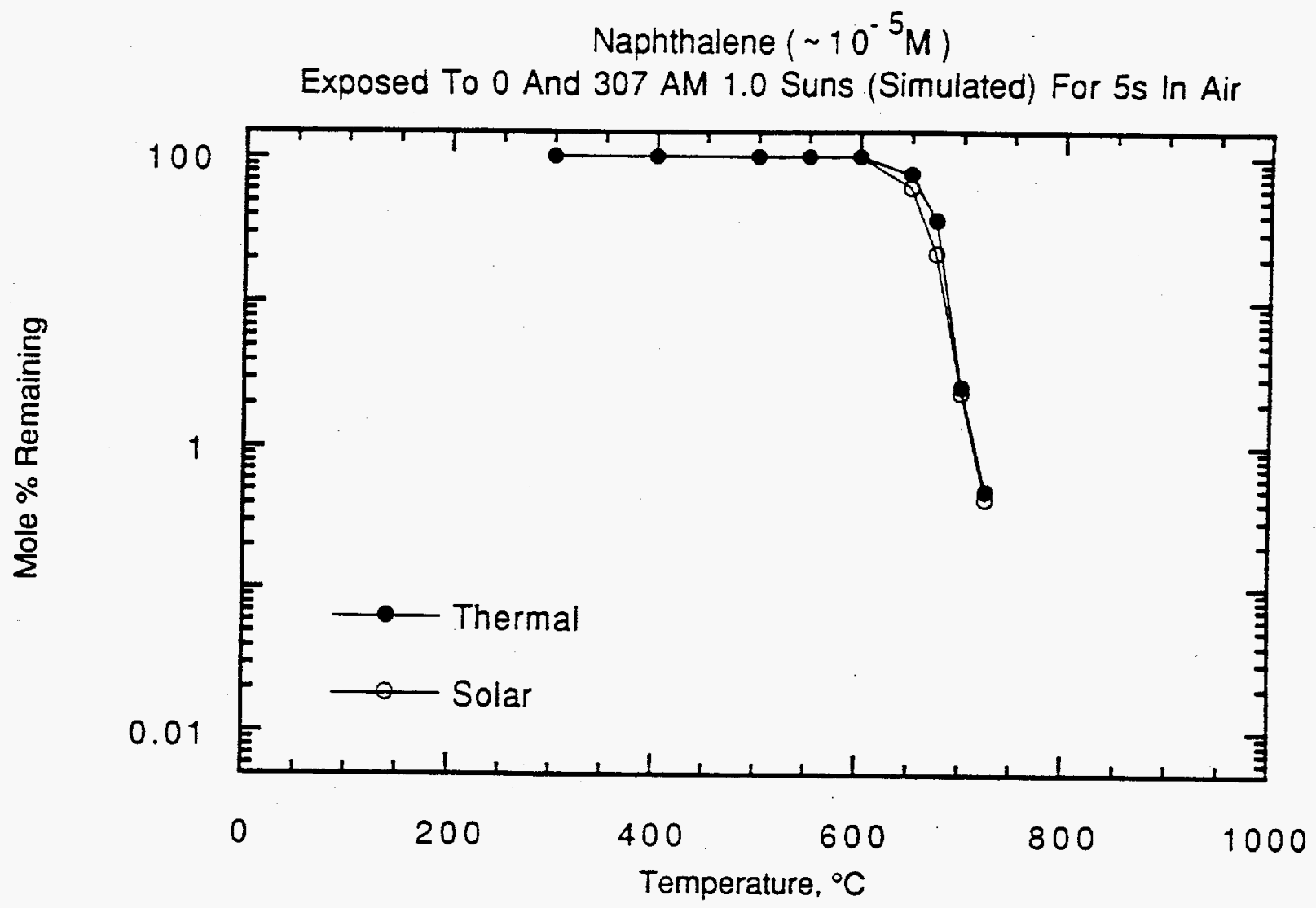

\begin{tabular}{|c|c|c|c|}
\hline Temp. & $f r(0)$ & $f(10)$ & $\mathrm{R}(10)$ \\
\hline 300 & 100.0 & 100.0 & 1.00 \\
400 & 100.0 & 100.0 & 1.00 \\
500 & 100.0 & 100.0 & 1.00 \\
550 & 100.0 & 100.0 & 1.00 \\
600 & 100.0 & 100.0 & 1.00 \\
650 & 76.3 & 60.9 & 1.25 \\
675 & 36.6 & 21.3 & 1.72 \\
700 & 2.61 & 2.36 & 1.11 \\
725 & 0.486 & 0.426 & 1.14 \\
\hline
\end{tabular}


1-Nitronaphthalene $\left(\sim 10^{-5} \mathrm{M}\right)$

Exposed To 0 And 307 AM 1.0 Suns (Simulated) For 5s In Air

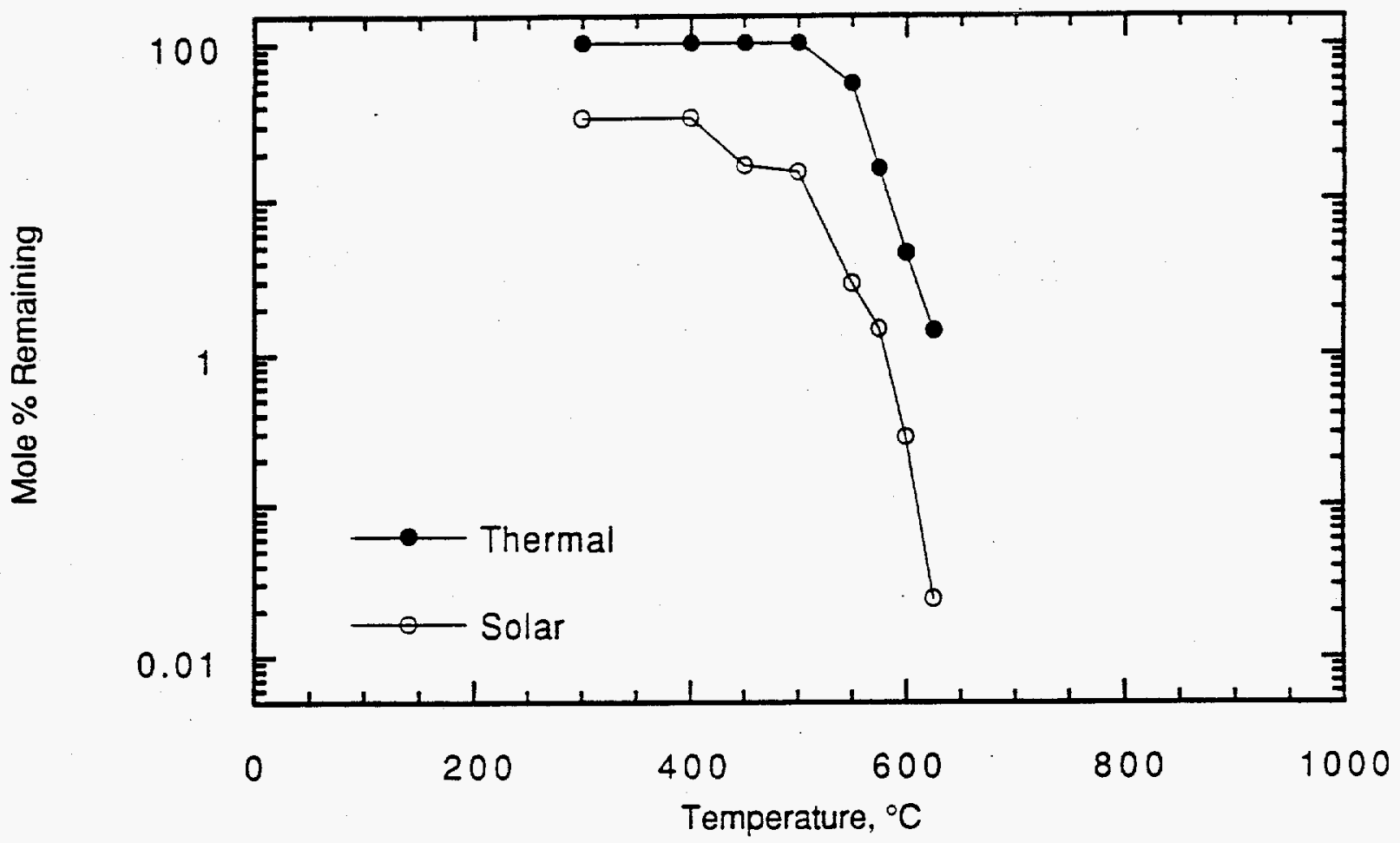

\begin{tabular}{|c|c|c|c|}
\hline Temp. & fr (0) & fr (10) & $R(10)$ \\
\hline & & & \\
300 & 100.0 & 33.4 & 2.99 \\
400 & 100.0 & 44.5 & 2.25 \\
450 & 100.0 & 16.6 & 6.02 \\
500 & 100.0 & 14.9 & 6.71 \\
550 & 55.4 & 2.89 & 19.2 \\
575 & 15.8 & 1.46 & 17.3 \\
600 & 4.51 & 0.281 & 16.1 \\
625 & 1.42 & 0.0240 & 58.4 \\
\hline
\end{tabular}


1-Nitronaphthalene $\left(\sim 10^{-5} \mathrm{M}\right)$

Exposed To 0 And 307 AM 1.0 Suns (Simulated) For 5 I In Helium

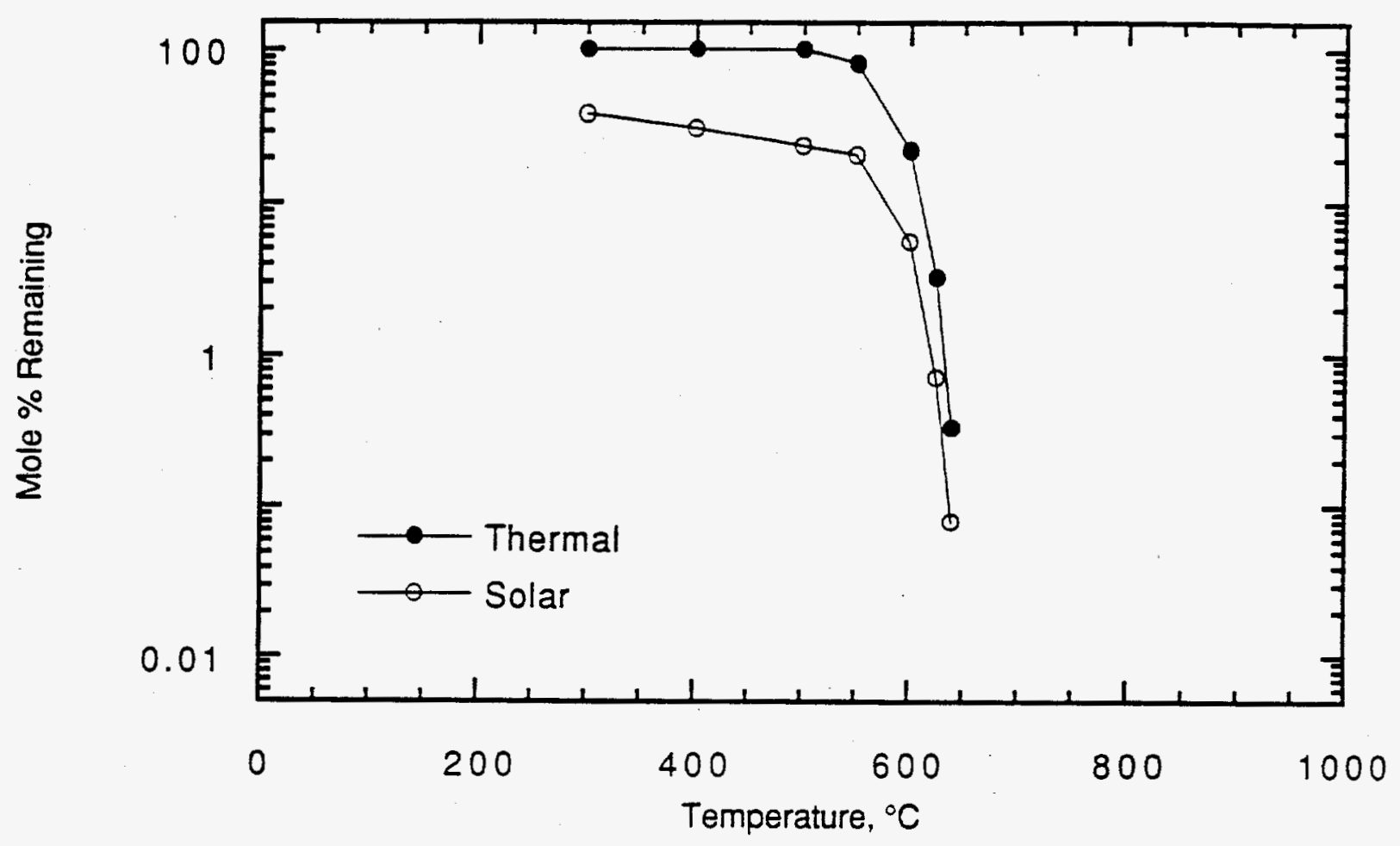

\begin{tabular}{|c|c|c|c|}
\hline Temp. & $f f(0)$ & $f f(10)$ & $\mathrm{A}(10)$ \\
\hline & & & \\
300 & 100.0 & 38.5 & 2.60 \\
400 & 100.0 & 31.0 & 3.23 \\
500 & 100.0 & 23.8 & 4.20 \\
550 & 80.8 & 21.0 & 3.85 \\
600 & 22.3 & 5.60 & 3.98 \\
625 & 3.22 & 0.700 & 4.60 \\
640 & 0.328 & 0.079 & 4.14 \\
\hline
\end{tabular}




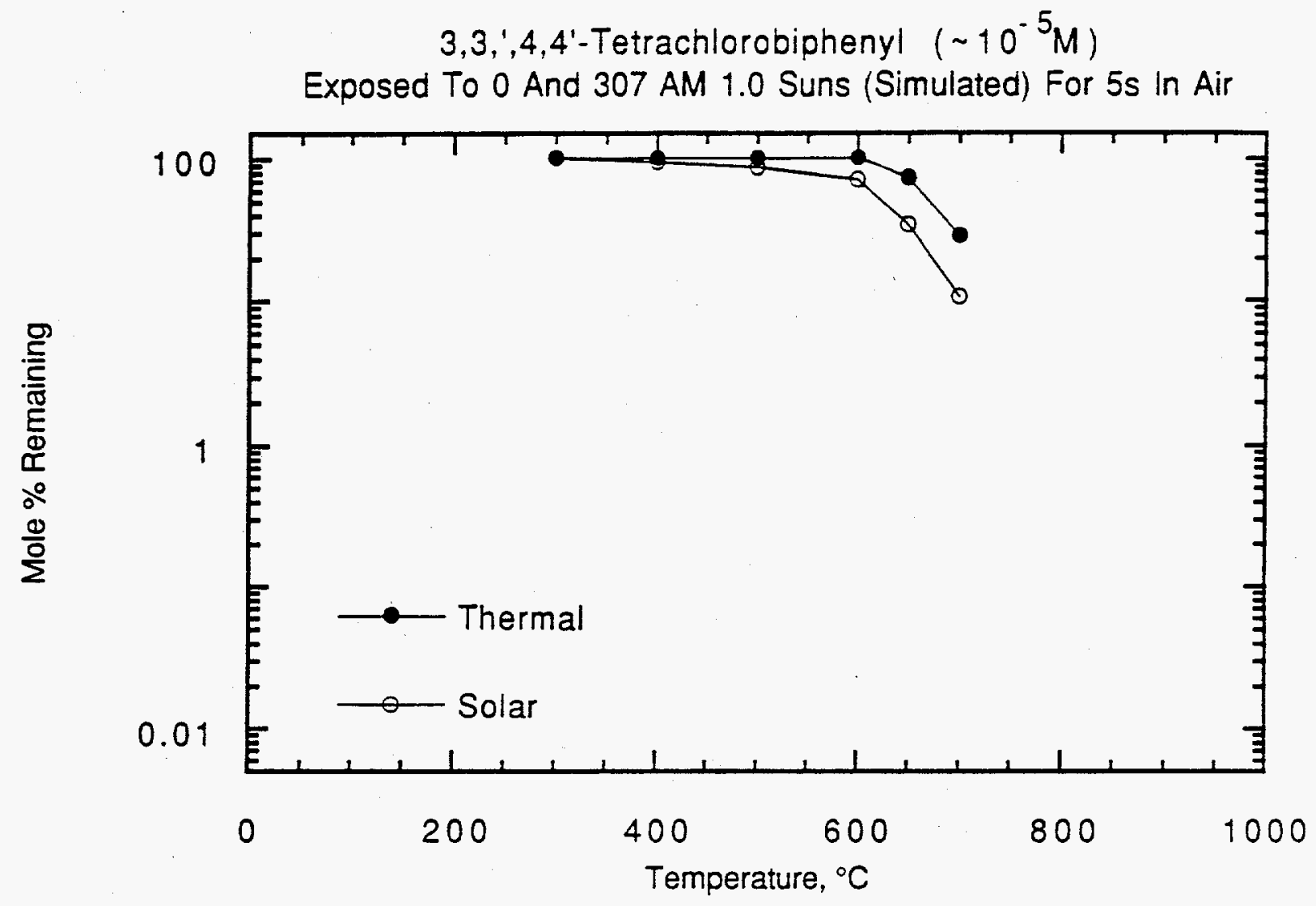

\begin{tabular}{|c|c|c|c|}
\hline Temp. & $f r(0)$ & $f r(10)$ & $R(10)$ \\
\hline & & & \\
300 & 100.0 & 100.0 & 1.00 \\
400 & 100.0 & 93.2 & 1.07 \\
500 & 100.0 & 86.4 & 1.16 \\
600 & 100.0 & 70.7 & 1.41 \\
650 & 72.8 & 34.0 & 2.14 \\
700 & 28.4 & 10.6 & 2.68 \\
\hline
\end{tabular}




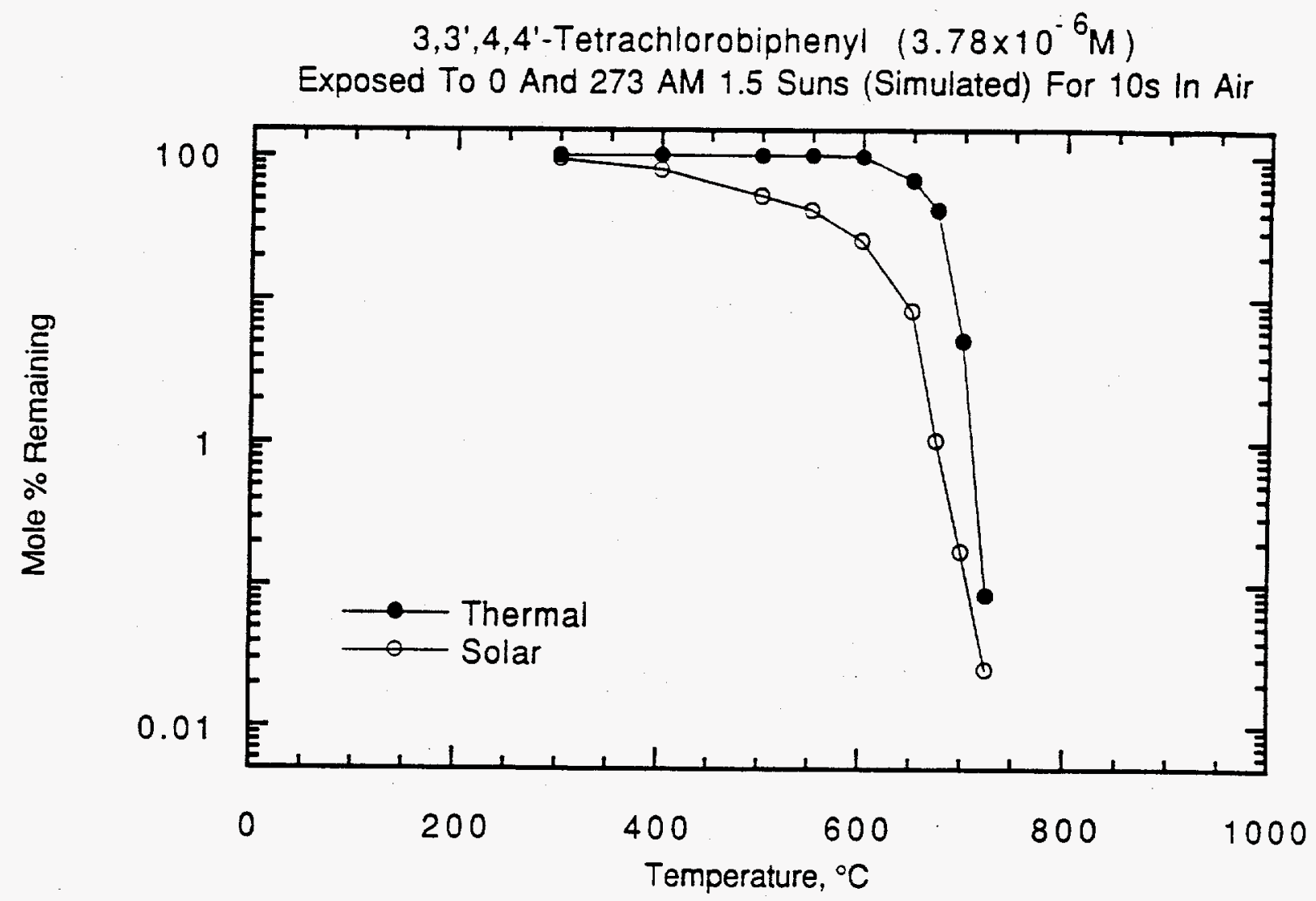

\begin{tabular}{|c|c|c|c|}
\hline Temp. & $f r(0)$ & $f r(10)$ & $R(10)$ \\
\hline & & & \\
400 & 100.0 & 94.3 & 1.06 \\
500 & 100.0 & 79.5 & 1.26 \\
550 & 100.0 & 52.3 & 1.91 \\
600 & 100.0 & 42.1 & 2.38 \\
650 & 98.1 & 25.6 & 3.83 \\
675 & 66.9 & 8.28 & 8.08 \\
700 & 42.1 & 1.03 & 40.87 \\
725 & 5.11 & 0.171 & 29.88 \\
\hline
\end{tabular}


3,3',4,4'-Tetrachlorobiphenyl $\left(3.78 \times 10^{-6} \mathrm{M}\right)$ And Its PICs

Exposed To 273 AM 1.5 Suns (Simulated) For $10 \mathrm{~s}$ In Air

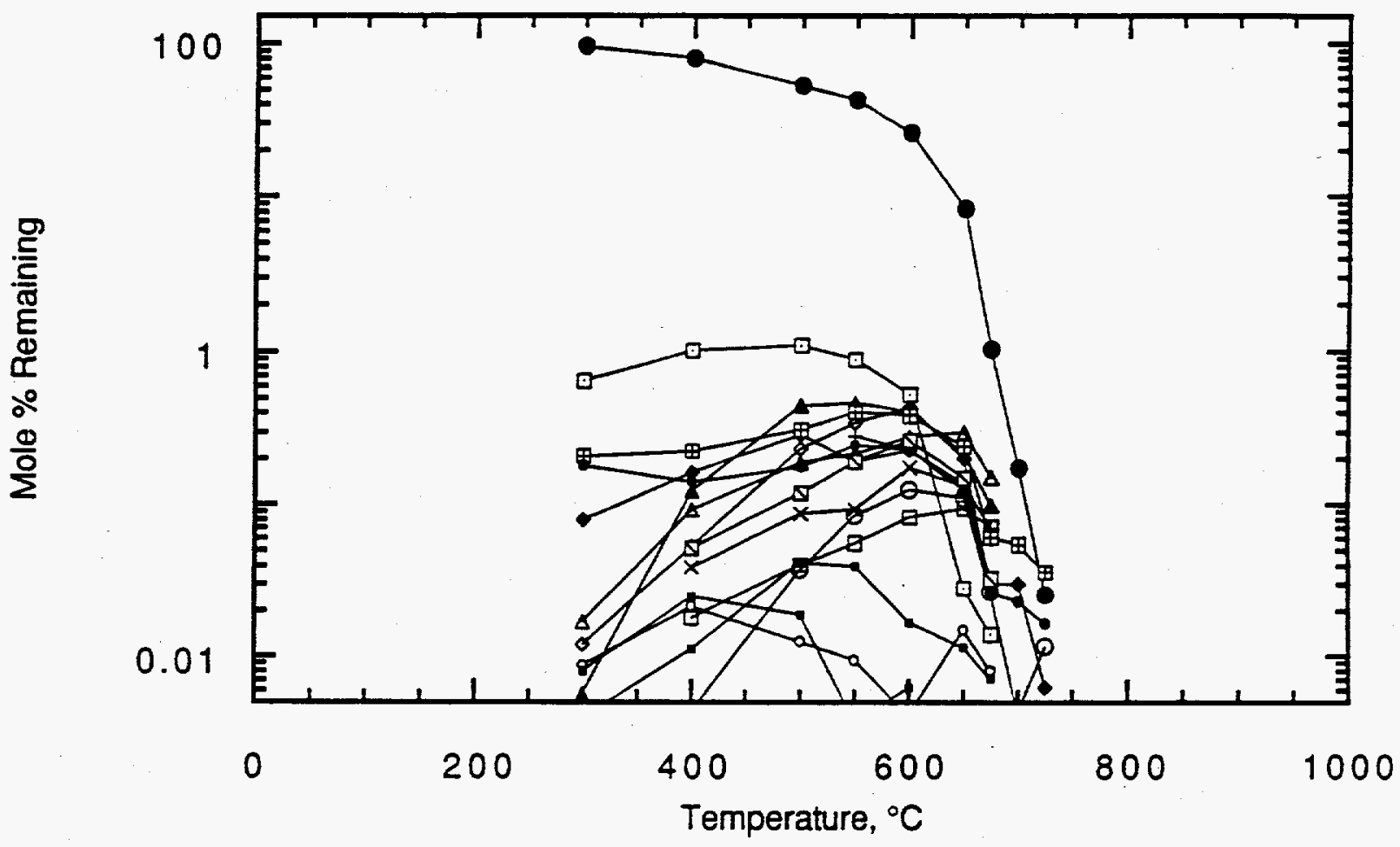

\begin{tabular}{|c|c|c|c|c|c|c|c|c|c|c|}
\hline & \multirow[b]{2}{*}{ Name } & \multicolumn{9}{|c|}{ Temberaterefe CI } \\
\hline & & 300 & 100 & 500 & 550 & $6 \infty$ & 650 & 575 & 700 & 725 \\
\hline - & $\operatorname{tc} \theta^{2}$ & 34 & 795 & 523 & 421 & 256 & 828 & 103 & 171 & 0,0253 \\
\hline$\circ$ & 1.2. Dientoroconsuner ${ }^{2}$ & - & $\cos 35$ & $207:$ & $\infty$ & 124 & .110 & .0265 & $\infty$ & - \\
\hline 口 & 1.24 Thetionobortemon ${ }^{2}$ & - & 0180 & 2010 & 557 & $\infty 23$ & $\infty$ & 0708 & - & - \\
\hline 0 & Dientorestremp ${ }^{3}$ & 0121 & 0534 & 233 & 34 & 429 & .199 & 0201 & - & $\rightarrow$ \\
\hline$\Delta$ & Dichtorroemenzotum 4 & $0 \div 70$ & $\infty 000$ & 188 & 294 & 278 & 296 & .150 & - & - \\
\hline - & 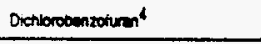 & - & - & - & - & - & - & - & - & - \\
\hline - & 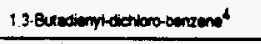 & orgt & 169 & 289 & 199 & 229 & 123 & $\infty 0301$ & $\rightarrow$ & - \\
\hline$\Delta$ & 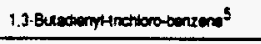 & $\cos 64$ & 124 & 47 & 464 & 402 & .217 &.$\infty 80$ & - & - \\
\hline$\times$ & 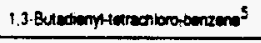 & - & $\infty 080$ & 0672 & 0932 & 174 & .132 & $\infty 28$ & - & - \\
\hline 1 & Inictioronedunemeno 6 & $\cdots$ & - & - & $2 \mathrm{B2} 2$ & 227 & .136 & $\infty 80$ & - & - \\
\hline - & 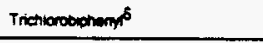 & 209 & 225 & 0300 & 40 & 384 & 241 & $\infty$ & - & - \\
\hline - & Incribordionomy ${ }^{6}$ & 181 & 141 & 176 & 245 & 228 & .133 & 0262 & - & - \\
\hline $\mathbf{\Delta}$ & 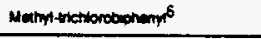 & - & 0520 & 119 & 191 & 264 & 149 & 0323 & - & $-\cdot$ \\
\hline $\mathbf{\square}$ & 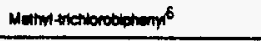 & 548 & 102 & 110 & 893 & 326 & .0280 & 0140 & - & - \\
\hline - & 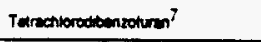 & $\infty$ & 0111 & an: & $\infty 9$ & 0167 & 0114 & $\infty 712$ & - & $\ldots$ \\
\hline - & Terractionotioneoturen ${ }^{7}$ & 00872 & 0213 & 0124 & 00996 & $\infty$ & 014 & $\infty 0002$ & - & $\ldots$ \\
\hline$=$ & Tereschlorodidonzoturen ${ }^{i}$ & $\infty 0802$ & 0248. & 0187 & $\operatorname{cosin}$ & $\infty 062$ & - & $\ldots$ & $\cdots$ & $\cdots$ \\
\hline & Carton Monoxion ${ }^{2}$ & & & & & 515 & & & & \\
\hline & Carton Droxido? & & & & & 917 & & & & \\
\hline & Totel Curbon Recovernod & & & & & 127 & & & & \\
\hline
\end{tabular}




\section{3, $3^{\prime}, 4,4^{\prime}$-Tetrachlorobiphenyl $\left(3.78 \times 10^{-6} \mathrm{M}\right)$ And Its PICs Exposed For $10 \mathrm{~s}$ In Air}

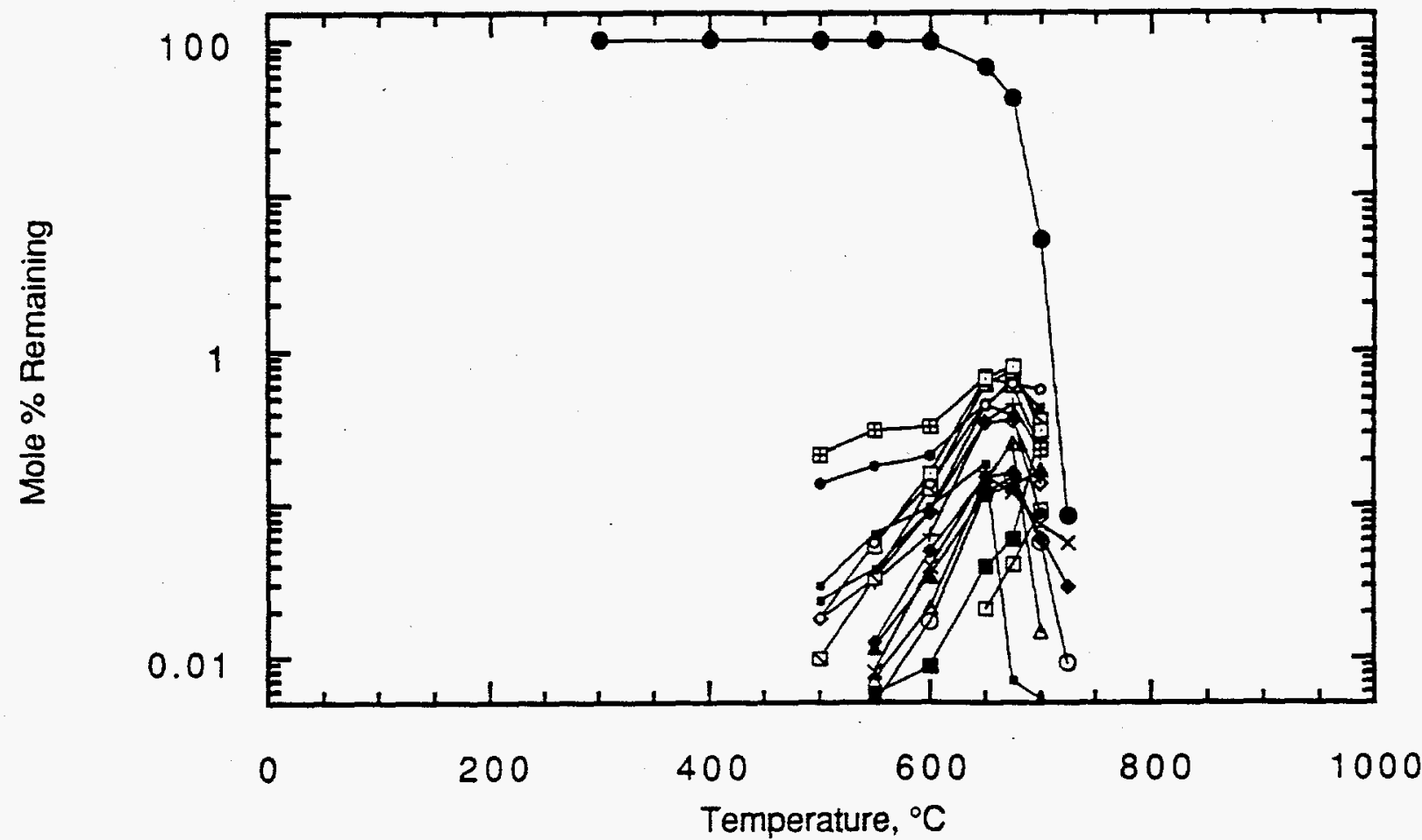

\begin{tabular}{|c|c|c|c|c|c|c|c|c|}
\hline & & \multicolumn{7}{|c|}{ Temperature $\left({ }^{\circ} \mathrm{C}\right)$} \\
\hline & Name & 500 & 550 & 600 & 550 & 675 & 700 & 725 \\
\hline - & $\mathrm{TCB}^{2}$ & 100 & 100 & 98.1 & 66.9 & 42.1 & 5.11 & 0847 \\
\hline 0 & 1,2-Dichibrobenzene 2 & & .00490 & .0172 & .120 & .153 & .0575 & .00910 \\
\hline$\square$ & 1,2,4-Trichiorobenzene 2 & & & & .0210 & .0410 & .0931 & \\
\hline 0 & Dichibrostyrene 3 & .0179 & .0331 & .0906 & .348 & .355 & .140 & \\
\hline$\Delta$ & Dichiorobenzoturan ${ }^{4}$ & & .00711 & .0244 & .144 & .256 & .0149 & \\
\hline ש & Dichlorobenzoturan ${ }^{4}$ & & .00565 & .0087 & .0399 & .0603 & .301 & - \\
\hline$\bullet$ & 1,3-8utadienyl-dichloro-benzene ${ }^{4}$ & & .0125 & .0501 & .1542 & .163 & .0614 & .0294 \\
\hline$\Delta$ & 1,3-8utadienyl-tichioro-benzenes 5 & & 0116 & .0345 & .120 & .133 & .171 & \\
\hline$x$ & 1,3-Butadienyl-retrachioro-benzene 5 & & .00799 & .0397 & .156 & .122 & .0748 & .0571 \\
\hline 1 & Trichloronaphtralene ${ }^{6}$ & & .0312 & .0646 & .354 & .453 & .220 & \\
\hline $\mathbf{E}$ & Trichlorobiphenyif & .215 & .0313 & .330 & .678 & .600 & .232 & \\
\hline - & Trichiorobionenyf & .139 & .182 & .212 & .459 & .381 & .0883 & \\
\hline $\boldsymbol{\theta}$ & Methyl-tichlorobioneny $\hat{\hat{\theta}}$ & .00983 & 0331 & .129 & .616 & .742 & .362 & \\
\hline ロ & Methyl-trichibrobionenyfo & & .0539 & .162 & .668 & .785 & .307 & \\
\hline - & Tetractiorodibenzohuran ${ }^{7}$ & .0236 & 0378 & .0919 & .444 & .644 & .432 & \\
\hline - & Tetrachlorodibenzoturan" & .0181 & .0566 & .138 & .454 & .613 & .564 & \\
\hline - & Tetrachiorodibenzoturan ${ }^{7}$ & .0294 & .0648 & .0972 & .188 & .00703 & .00530 & \\
\hline & Carbon Monoxioe ${ }^{2}$ & & & & & 475 & & \\
\hline & Carbon Dioxide 2 & & & & & 104 & & \\
\hline & Total Carbon Recovered & & & & & 136 & & \\
\hline
\end{tabular}

${ }^{1}$ Mole \% remaining reterenced to $\mathrm{TCB}$ at

4 Quantfied as 2.6. Dichiorobenzene.

$300^{\circ} \mathrm{C}$ and 0 suns (thermal).

¿Quantitied by anabycal sandaro.

50uantified as 1.2.4-Trichtorobenzene.

3Quartified as 26-Dichiorostyrene.

Quantied as 3.4'4-Trchibrobipheny.

7 Ouantied as 2.3.7.8-Tevactiorodibenzokuran. 


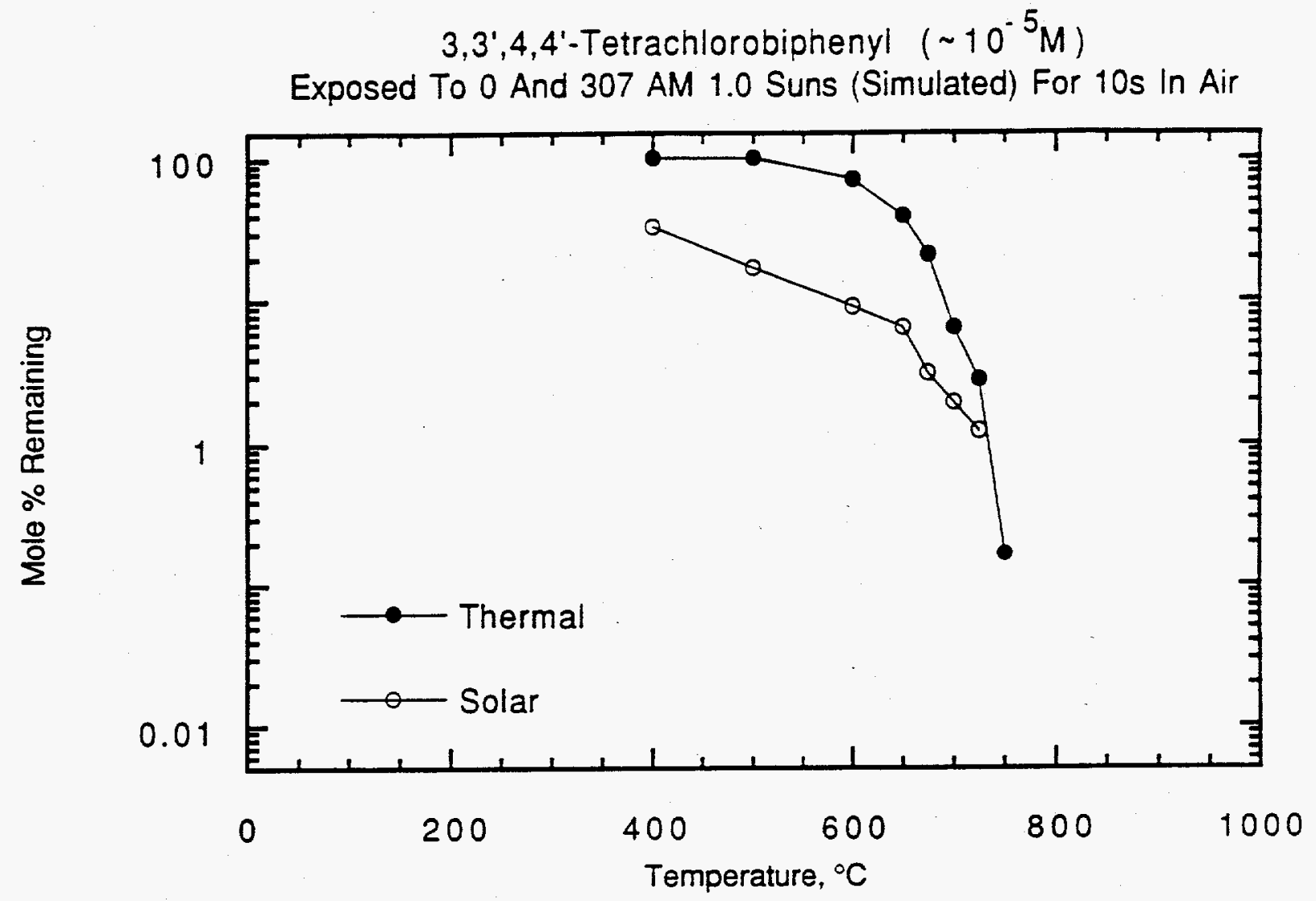

\begin{tabular}{|c|c|c|c|}
\hline Temp. & $\operatorname{fr}(0)$ & $f r(10)$ & $R(10)$ \\
\hline & & & \\
400 & 100.0 & 32.8 & 3.05 \\
500 & 100.0 & 16.9 & 5.92 \\
600 & 70.8 & 9.03 & 7.84 \\
650 & 38.6 & 6.48 & 5.96 \\
675 & 20.7 & 3.12 & 6.63 \\
700 & 6.48 & 1.94 & 3.34 \\
725 & 2.82 & 1.23 & 2.29 \\
750 & 0.165 & --- & $\cdots$ \\
\hline
\end{tabular}




\section{3,3',4,4'-Tetrachlorobiphenyl $\left(\sim 10^{-5} \mathrm{M}\right)$}

Exposed To 0 And 307 AM 1.0 Suns (Simulated) For $10 \mathrm{~s}$ in Helium

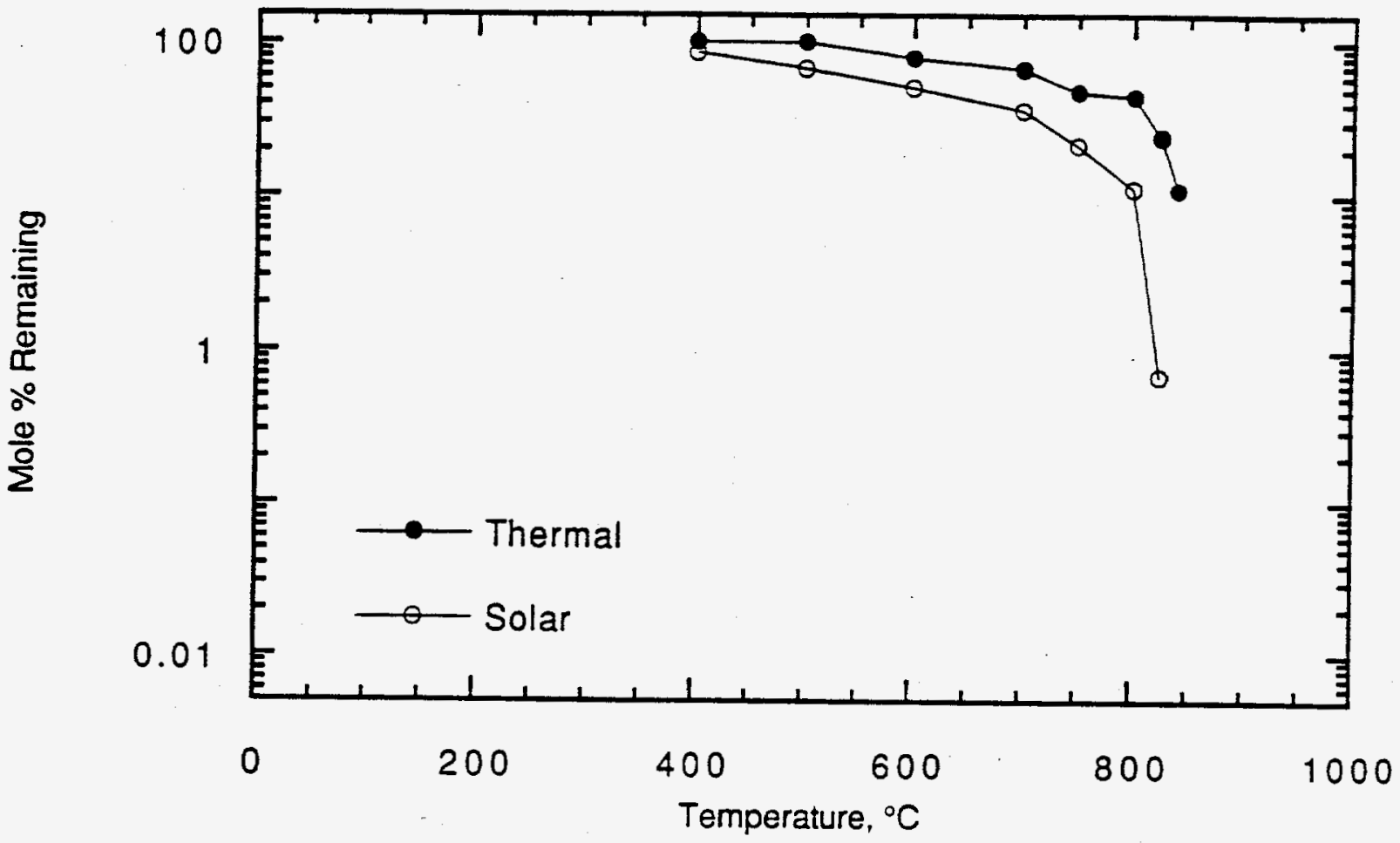

\begin{tabular}{|c|c|c|c|}
\hline Temp. & $f($ (0) & $f(10)$ & $R(10)$ \\
\hline & & & \\
400 & 100.0 & 84.4 & 1.19 \\
500 & 100.0 & 66.9 & 1.50 \\
600 & 78.6 & 50.5 & 1.56 \\
700 & 66.4 & 35.8 & 1.86 \\
750 & 47.7 & 21.5 & 2.22 \\
800 & 44.3 & 11.2 & 3.96 \\
825 & 24.2 & 0.682 & 35.48 \\
840 & 11.0 & $\ldots-$ & $\ldots$ \\
875 & $\ldots$ & $\ldots$ & $\ldots$ \\
\hline
\end{tabular}




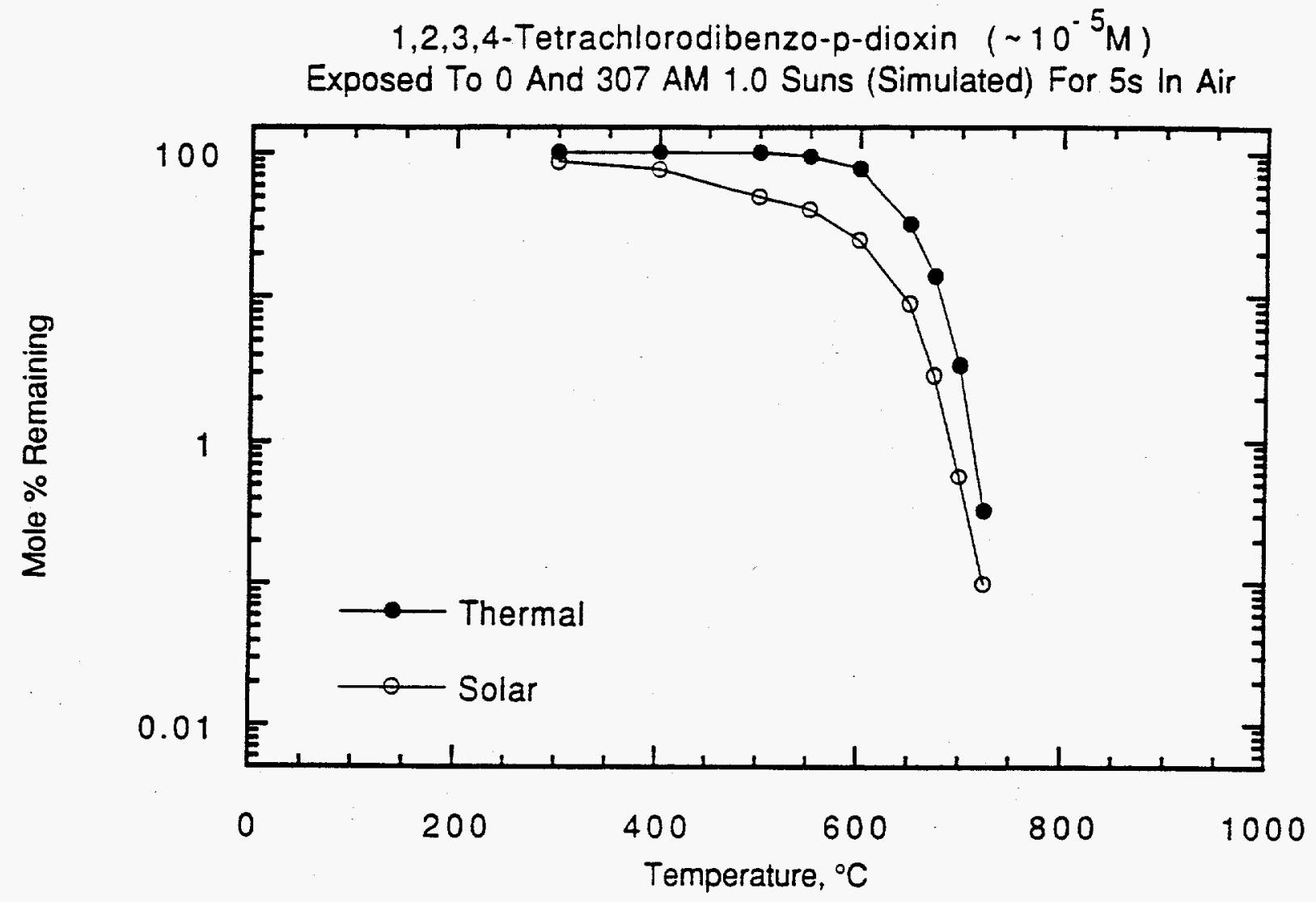

\begin{tabular}{|c|c|c|c|}
\hline Temp. & $f r(0)$ & $f r(10)$ & $R(10)$ \\
\hline & & & \\
300 & 100.0 & 86.0 & 1.16 \\
400 & 100.0 & 75.5 & 1.33 \\
500 & 100.0 & 49.6 & 2.02 \\
550 & 93.8 & 40.0 & 2.35 \\
600 & 77.0 & 24.4 & 3.16 \\
650 & 31.4 & 8.86 & 3.54 \\
675 & 13.7 & 2.85 & 4.81 \\
700 & 3.35 & 0.567 & 5.91 \\
725 & 0.325 & 0.099 & 3.28 \\
\hline
\end{tabular}




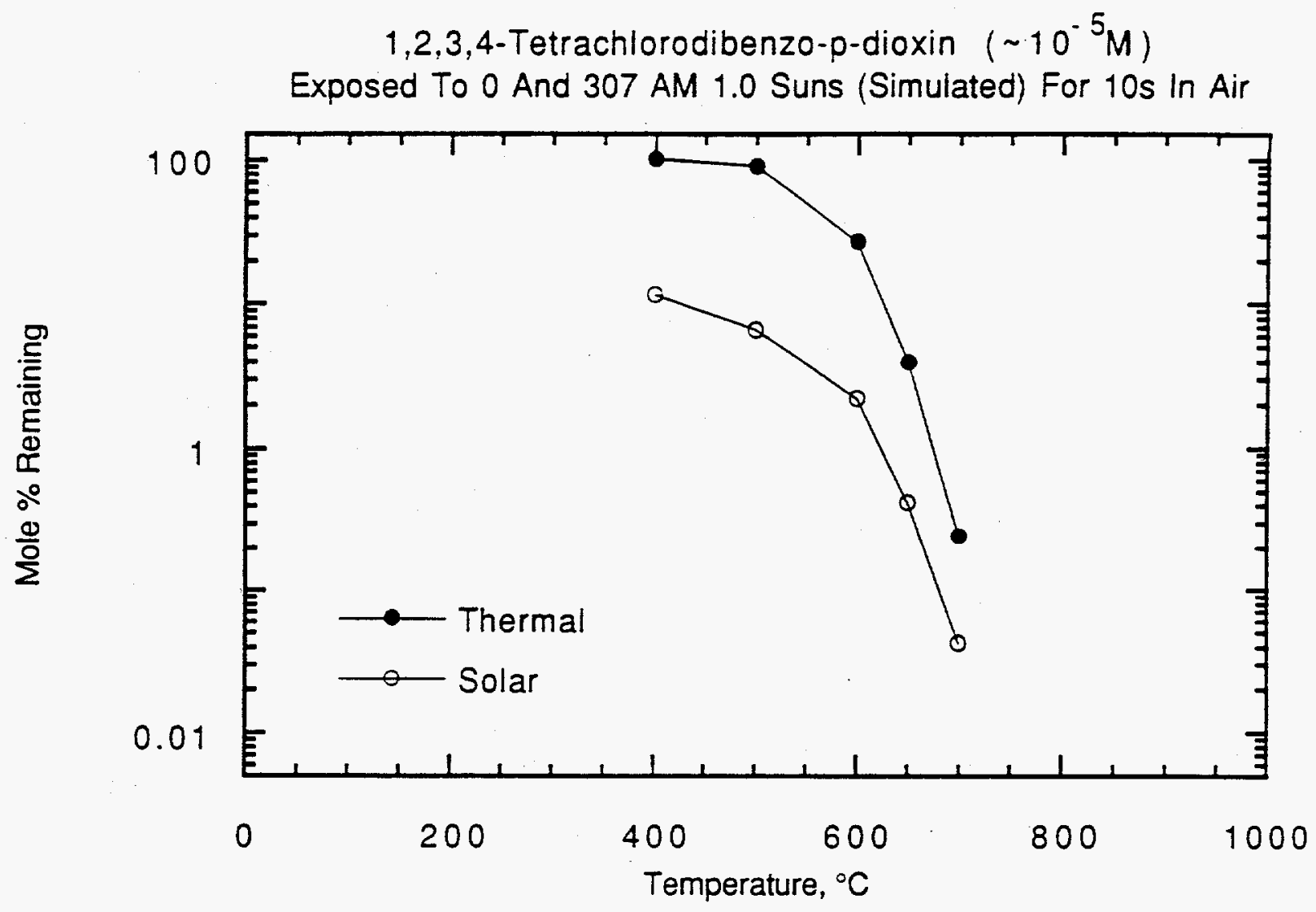

\begin{tabular}{|c|c|c|c|}
\hline Temp. & fr $(0)$ & $f r(10)$ & $R(10)$ \\
\hline & & & \\
400 & 100.0 & 11.5 & 8.70 \\
500 & 89.3 & 6.63 & 13.47 \\
600 & 26.6 & 2.19 & 12.15 \\
650 & 3.92 & 0.415 & 9.45 \\
700 & 0.242 & 0.0430 & 5.69 \\
\hline
\end{tabular}




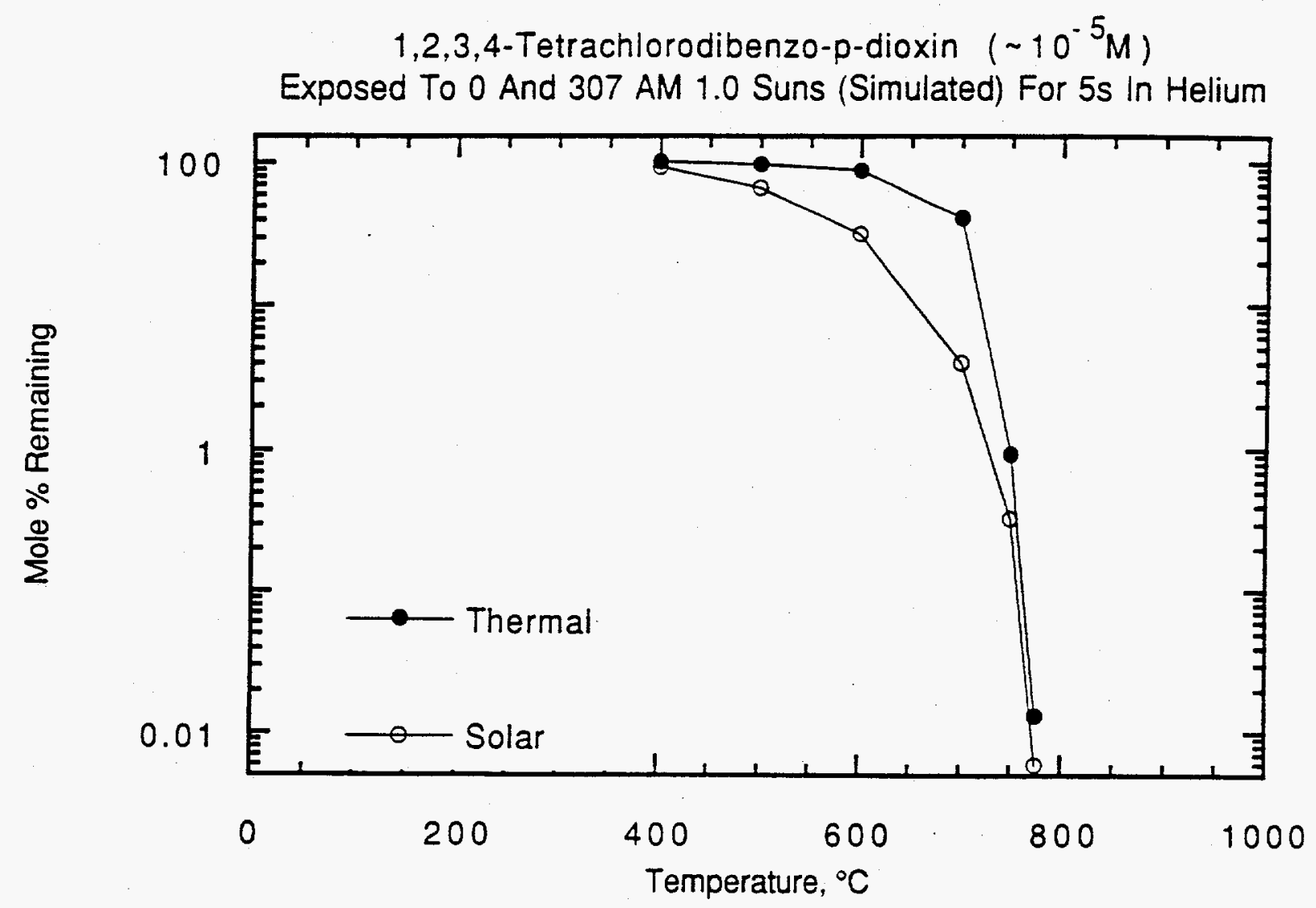

\begin{tabular}{|c|c|c|c|}
\hline Temp. & fr $(0)$ & $f r(10)$ & $R(10)$ \\
\hline & & & \\
400 & 100.0 & 91.6 & 1.09 \\
500 & 95.6 & 66.0 & 1.45 \\
600 & 86.5 & 31.5 & 2.75 \\
700 & 40.7 & 4.00 & 10.18 \\
750 & 0.928 & 0.330 & 2.81 \\
775 & 0.013 & 0.006 & 2.21 \\
\hline
\end{tabular}


1,2,3,4-Tetrachlorodibenzo-p-dioxin $\left(\sim 10^{-5} \mathrm{M}\right)$

Exposed To 0 And 307 AM 1.0 Suns (Simulated) For $10 \mathrm{~s}$ in Helium

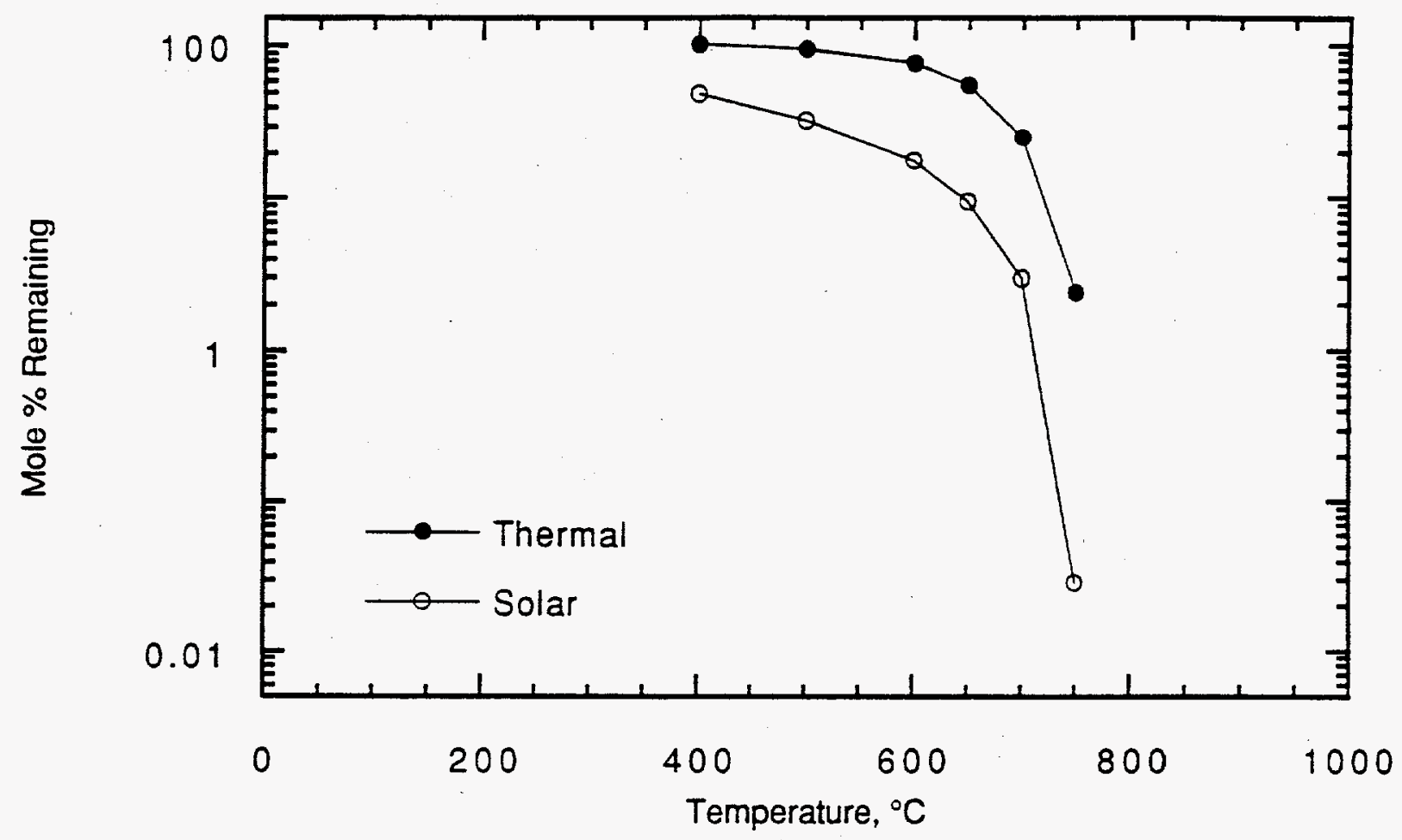

\begin{tabular}{|c|c|c|c|}
\hline Temp. & fr (0) & fr (lo) & $R(10)$ \\
\hline & & & \\
400 & 100.0 & 48.3 & 2.07 \\
500 & 94.1 & 32.4 & 2.90 \\
600 & 75.9 & 17.6 & 4.31 \\
650 & 54.5 & 9.44 & 5.77 \\
700 & 24.9 & 2.96 & 8.41 \\
750 & 2.39 & 0.029 & 83.28 \\
\hline
\end{tabular}




\section{Trichloroethyiene $\left(\sim 10^{-5} \mathrm{M}\right)$}

Exposed To 0 And 307 AM 1.0 Suns (Simulated) For $10 \mathrm{~s} \ln$ Air

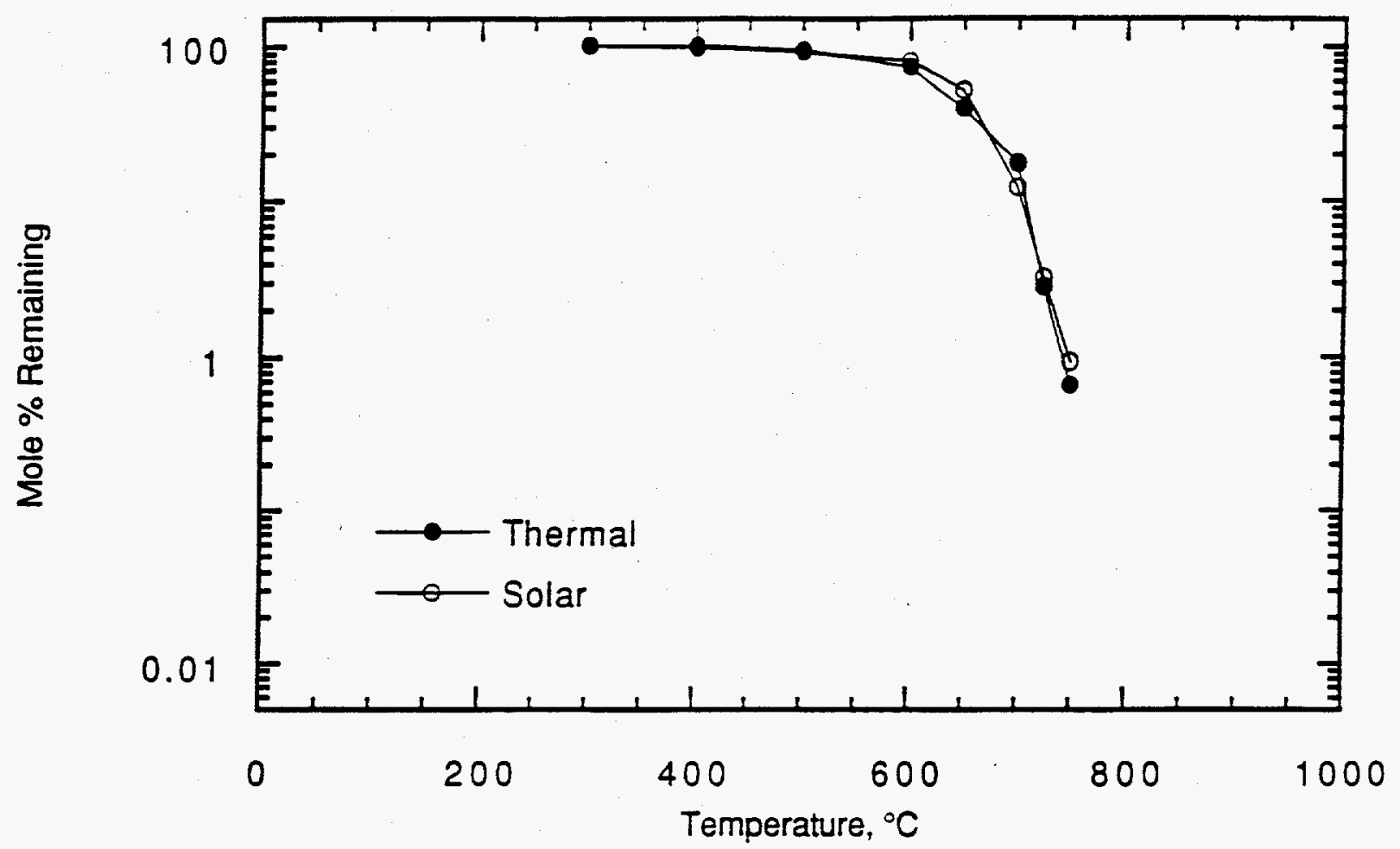

\begin{tabular}{|c|c|c|c|}
\hline Temp. & fr (0) & fr (10) & R (10) \\
\hline & & & \\
300 & 100.0 & $\ldots$. & $\ldots$ \\
400 & 100.0 & 97.1 & 1.03 \\
500 & 93.8 & 91.0 & 1.03 \\
600 & 72.1 & 79.5 & 0.907 \\
650 & 38.9 & 51.1 & 0.761 \\
700 & 17.5 & 12.3 & 1.42 \\
725 & 2.81 & 3.23 & 0.87 \\
750 & 0.658 & 0.932 & 0.706 \\
\hline
\end{tabular}


Xanthone $\left(\sim 10^{-5} \mathrm{M}\right)$

Exposed To 0 And 307 AM 1.0 Suns (Simulated) For $5 s$ In Air

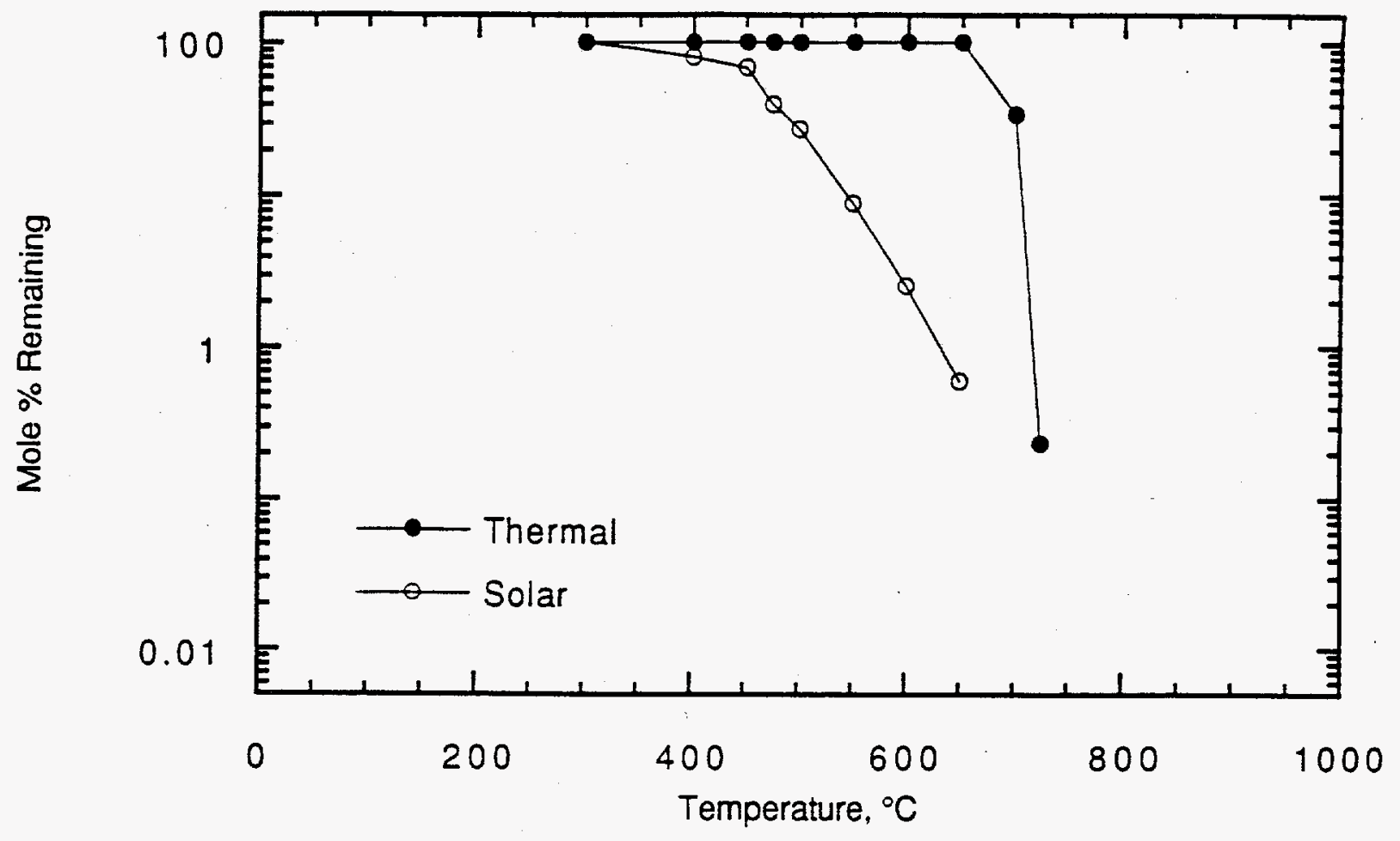

\begin{tabular}{|c|c|c|c|}
\hline Temp. & $f r(0)$ & $f r(10)$ & $R(10)$ \\
\hline 300 & 100.0 & 100.0 & 1.00 \\
400 & 100.0 & 80.3 & 1.25 \\
450 & 100.0 & 68.1 & 1.47 \\
475 & 100.0 & 39.5 & 2.53 \\
500 & 100.0 & 27.3 & 3.66 \\
550 & 100.0 & 8.93 & 11.2 \\
600 & 100.0 & 2.55 & 39.2 \\
650 & 100.0 & 0.596 & 168.0 \\
700 & 33.8 &.-- &.-- \\
725 & 0.234 &.-- & $-\cdots$ \\
\hline
\end{tabular}




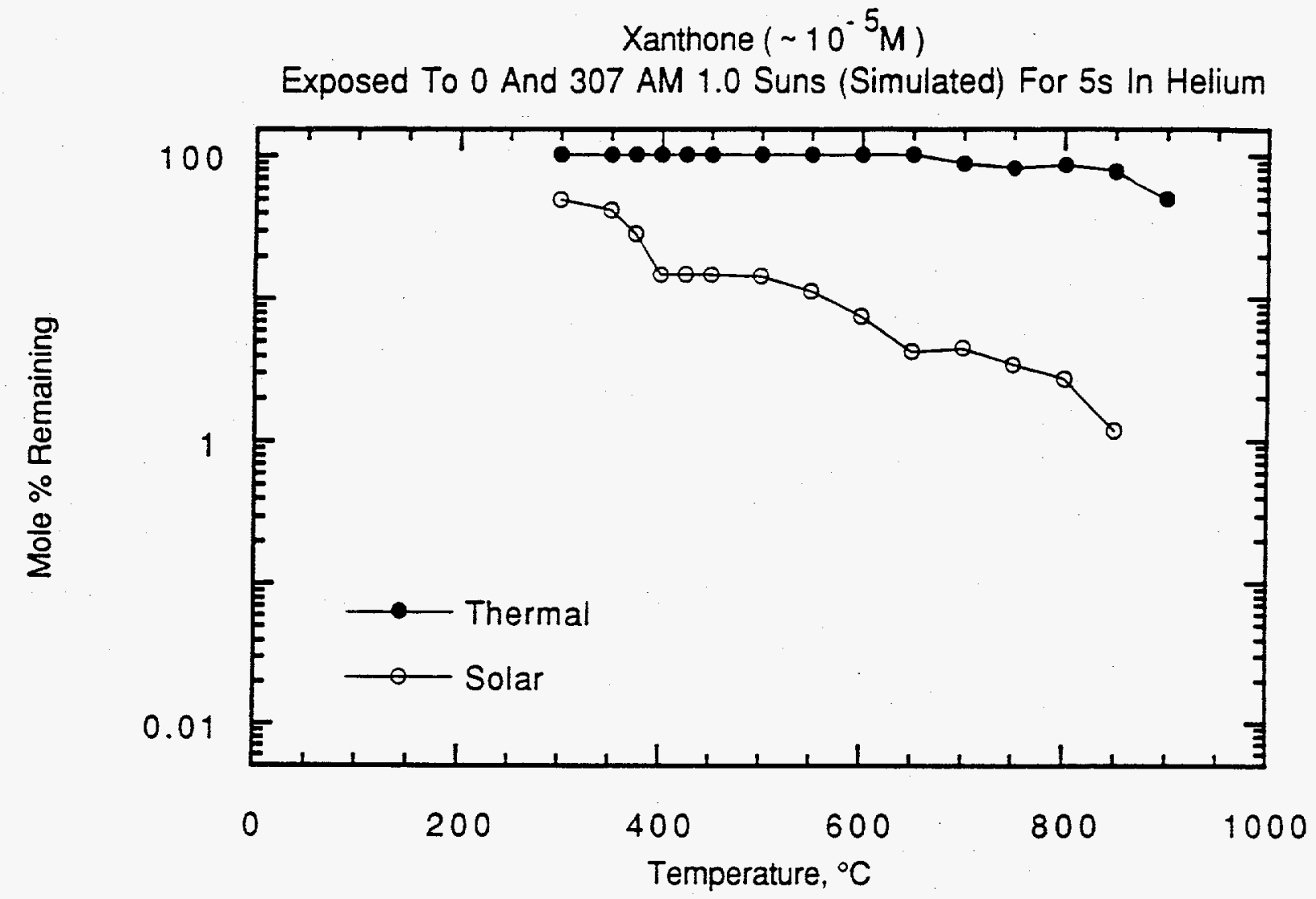

\begin{tabular}{|c|c|c|c|}
\hline Temo. & fr $(0)$ & $f r(10)$ & $R(10)$ \\
\hline 300 & 100.0 & 49.1 & \\
350 & 100.0 & 41.6 & 2.04 \\
375 & 100.0 & 28.4 & 2.40 \\
400 & 100.0 & 14.7 & 3.52 \\
425 & 100.0 & 14.8 & 6.80 \\
450 & 100.0 & 14.7 & 6.76 \\
500 & 100.0 & 14.4 & 6.80 \\
550 & 100.0 & 11.3 & 6.94 \\
600 & 100.0 & 7.48 & 8.85 \\
650 & 100.0 & 4.21 & 13.4 \\
700 & 86.9 & 4.44 & 23.8 \\
750 & 80.5 & 3.38 & 19.6 \\
800 & 85.0 & 2.66 & 23.8 \\
850 & 77.0 & 1.17 & 32.0 \\
900 & 49.3 & $\ldots$ & 65.8 \\
\hline
\end{tabular}




\section{APPENDIX II \\ Thermal/Photolytic Degradation of Binary Mixtures}

This Appendix summarizes all of the data obtained during this project on a series of binary mixtures used to evaluate the potential effectiveness of triplet-triplet evergy transfer and photoiniated radical attack as a means of destroying compounds which do not absorb near UV radiation from solar energy. Recall that for the samples for the triplet-triplet energy transfer tests consisted of acetone/benzene (donor/acceptor) and benzophenone/naphthalene. Furthermore, the photoinitiated radical studies consisted of chlorine/methylene chloride and hydrogen peroxide/trichloroethylene.

Unless otherwise noted, temperature is given in degrees Centigrade and fraction remaining is in mole percent normalized by the original amount of sample fed to the reactor. By definition, enhancement ratio is unitless. 
Acetone $\left(\sim 10^{-5} \mathrm{M}\right)$ In The Presence Of Benzene $\left(\sim 10^{-5} \mathrm{M}\right)$

Exposed To 0 And 307 AM 1.0 Suns (Simulated) For 5 s In Helium

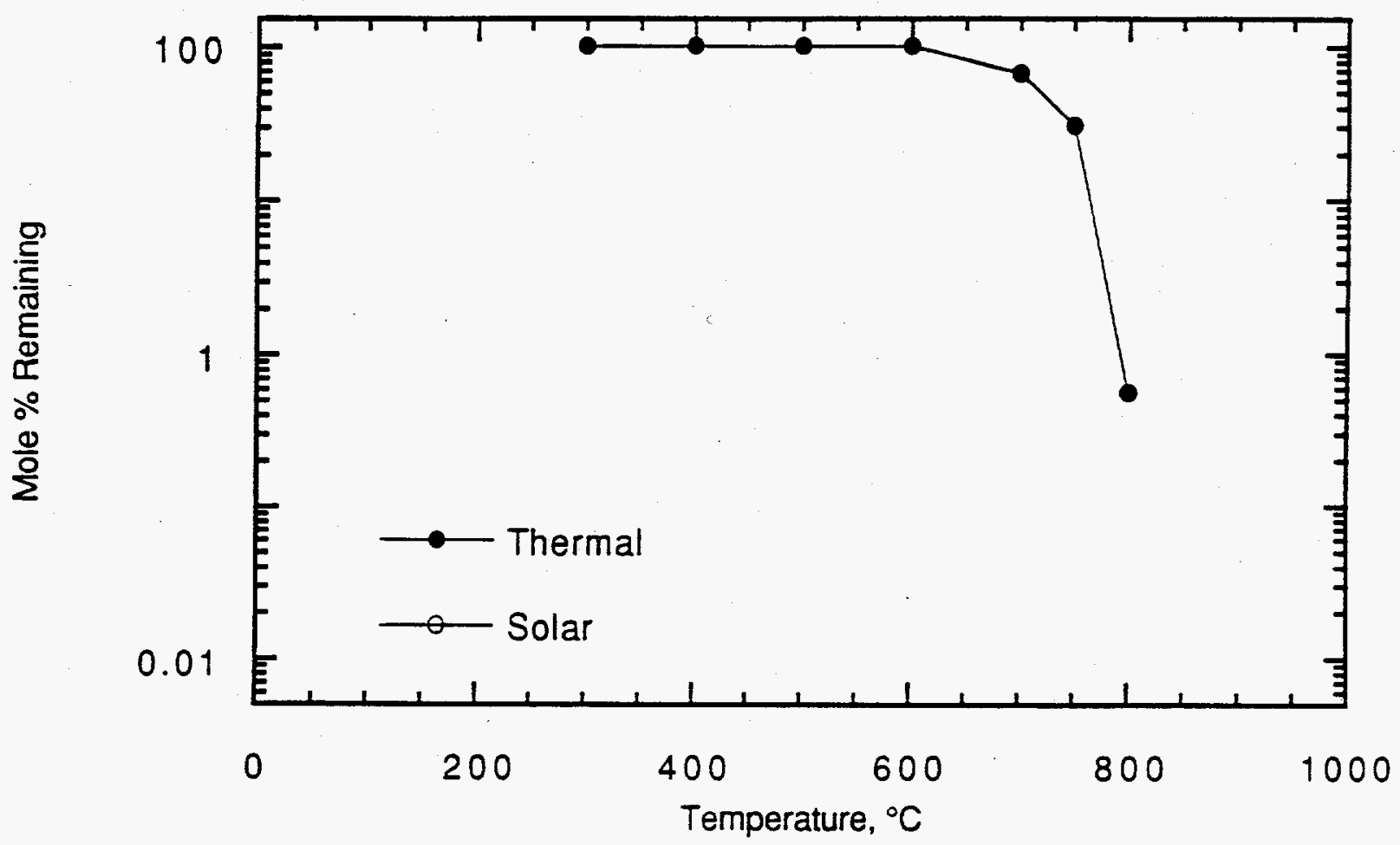

\begin{tabular}{|c|c|c|c|}
\hline Temp. & $\operatorname{fr}(0)$ & $\operatorname{fr}(10)$ & $\mathrm{R}(10)$ \\
\hline & & & \\
\hline 300 & 100 & 100 & 1.00 \\
\hline 400 & 100 & 100 & 1.00 \\
\hline 500 & 100 & 100 & 1.00 \\
\hline 600 & 100 & 100 & 1.00 \\
\hline 700 & 65.5 & 66.6 & 0.983 \\
\hline 750 & 30.1 & 30.5 & 0.987 \\
\hline 800 & 0.564 & 0.558 & 1.01 \\
\hline
\end{tabular}




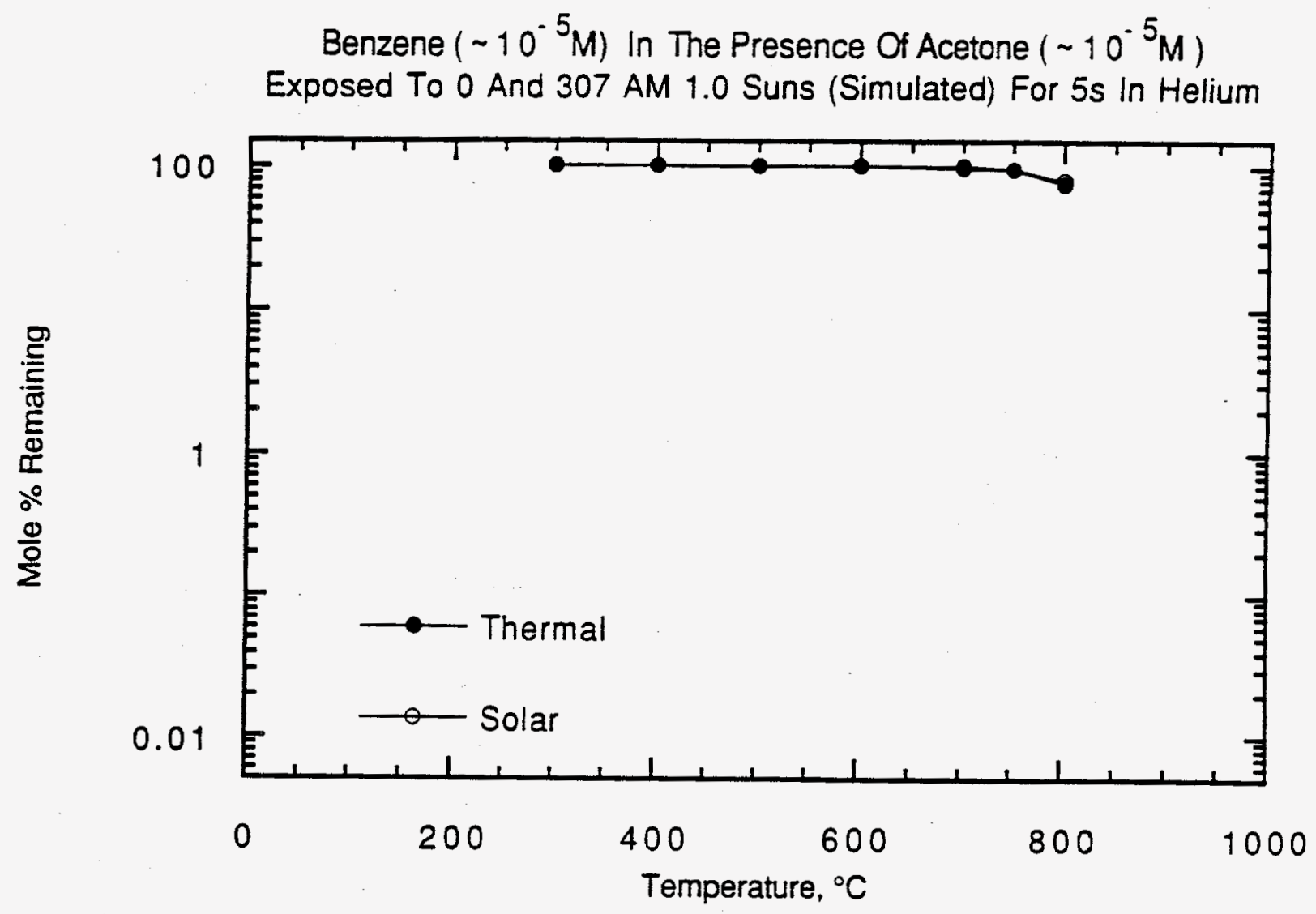

\begin{tabular}{|c|c|c|c|}
\hline Temp. & $\operatorname{fr}(0)$ & $f r(10)$ & $R(10)$ \\
\hline & & & \\
\hline 300 & 100 & 100 & 1.00 \\
\hline 400 & 100 & 100 & 1.00 \\
\hline 500 & 100 & 100 & 1.00 \\
\hline 600 & 100 & 100 & 1.00 \\
\hline 700 & 100 & 96.4 & 1.04 \\
\hline 750 & 94.9 & 94.6 & 1.00 \\
\hline 800 & 74.7 & 78.8 & 0.948 \\
\hline
\end{tabular}


Benzophenone $\left(\sim 10^{-5} \mathrm{M}\right)$ In The Presence Of Naphthalene $\left(\sim 10^{-5} \mathrm{M}\right)$

Exposed To 0 And 307 AM 1.0 Suns (Simulated) For $5 s$ In Helium

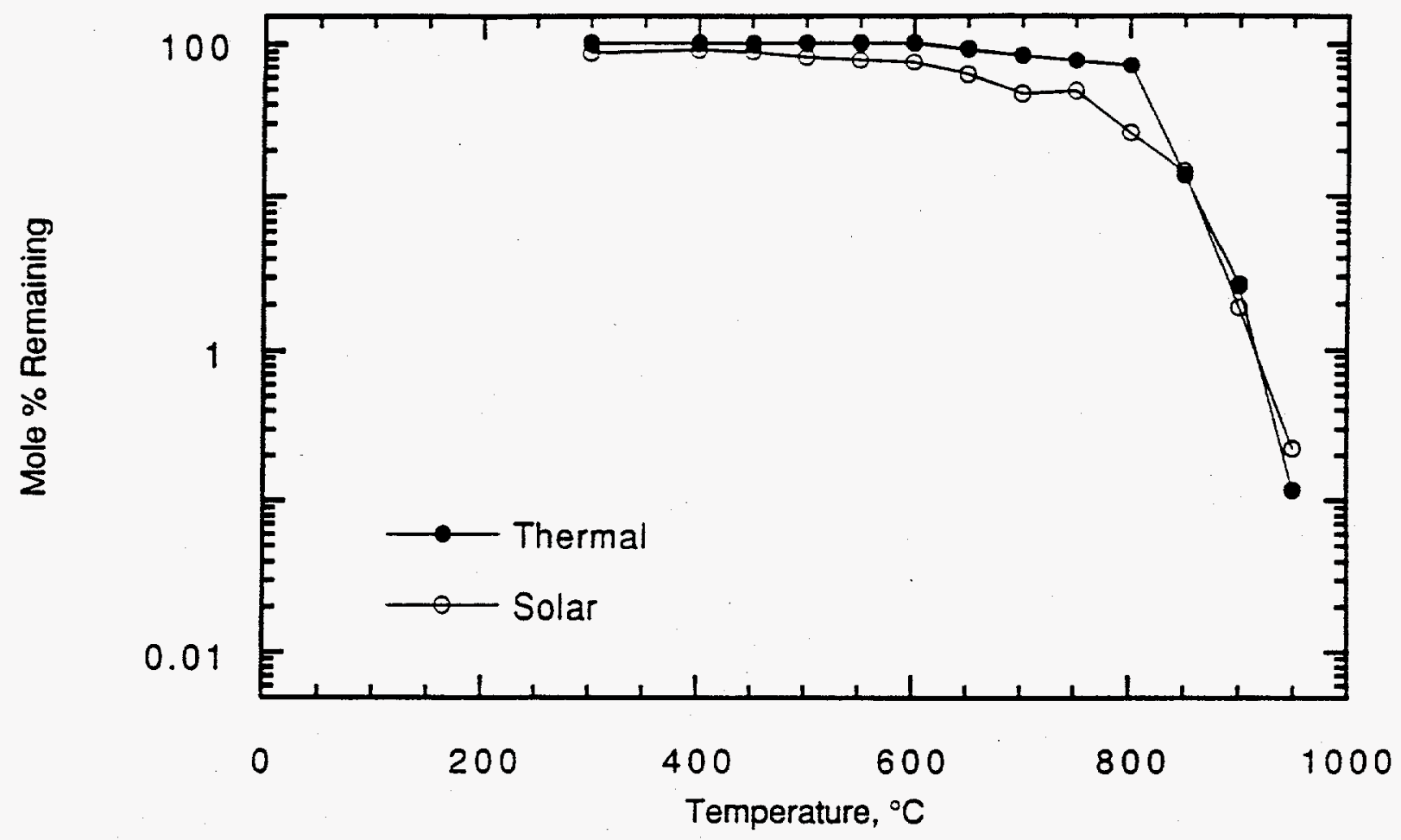

\begin{tabular}{|c|c|c|c|}
\hline Temp. & $\operatorname{fr}(0)$ & $\operatorname{fr}(10)$ & $\mathrm{R}(10)$ \\
\hline & & & \\
\hline 300 & 100 & 86.7 & 1.15 \\
\hline 400 & 100 & 90.5 & 1.11 \\
\hline 450 & 100 & 88.0 & 1.14 \\
\hline 500 & 100 & 81.0 & 1.24 \\
\hline 550 & 100 & 77.3 & 1.29 \\
\hline 600 & 100 & 75.2 & 1.33 \\
\hline 650 & & 61.9 & \\
\hline 700 & 83.1 & 46.2 & 1.80 \\
\hline 750 & & 48.4 & \\
\hline 800 & 71.0 & 25.7 & 2.76 \\
\hline 850 & & 14.4 & \\
\hline 900 & 2.65 & 1.88 & 1.41 \\
\hline 950 & 0.116 & 0.219 & 0.530 \\
\hline
\end{tabular}


Naphthalene $\left(\sim 10^{-5} \mathrm{M}\right)$ In The Presence Of Benzophenone $\left(\sim 10^{-5} \mathrm{M}\right)$ Exposed To 0 And 307 AM 1.0 Suns (Simulated) For $5 s$ in Helium

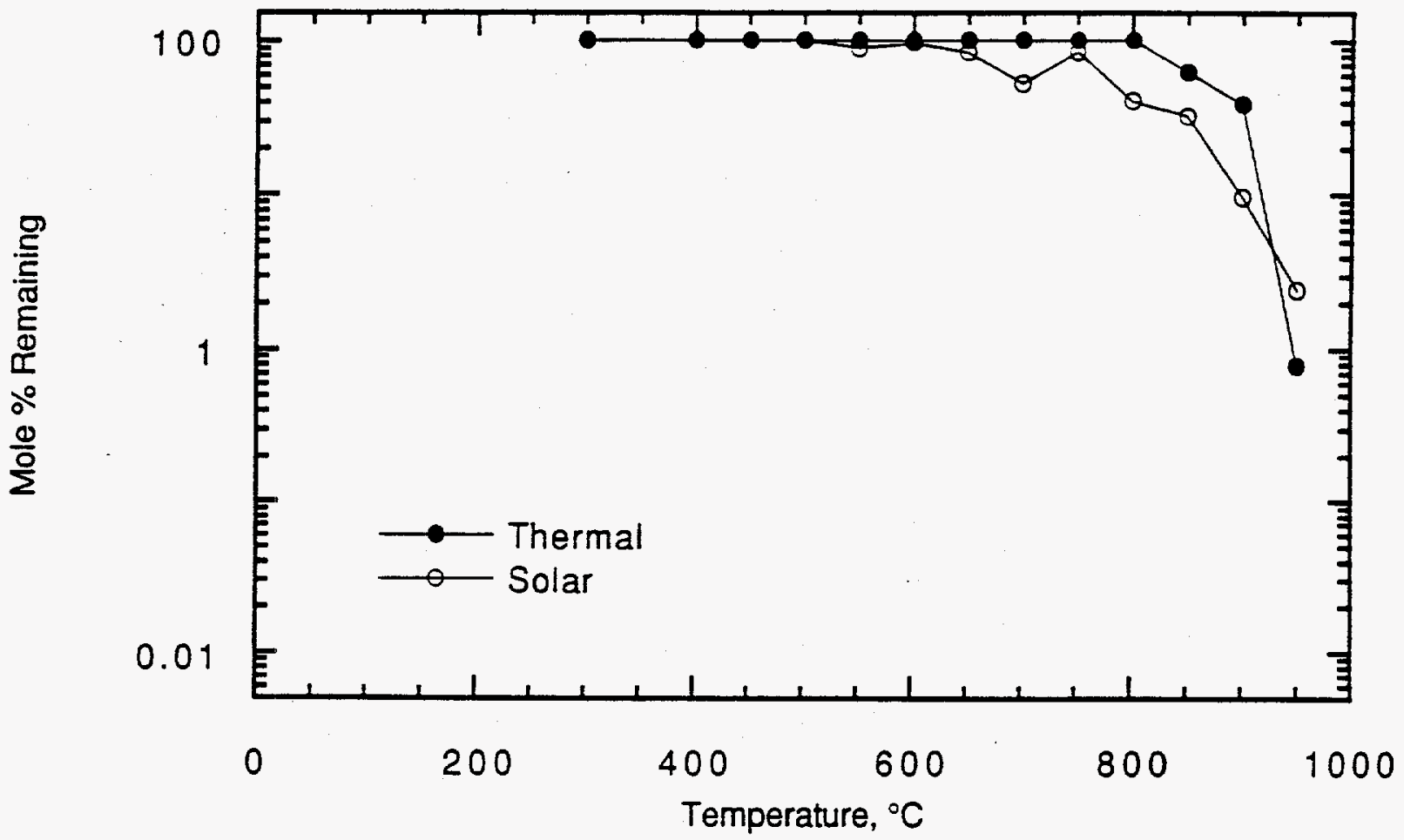

\begin{tabular}{|c|c|c|c|}
\hline Temp. & $\operatorname{fr}(0)$ & $\operatorname{fr}(10)$ & $\mathbf{R}(10)$ \\
\hline & & & \\
\hline 300 & 100 & 100 & 1.00 \\
\hline 400 & 100 & 100 & 1.00 \\
\hline 450 & 100 & 100 & 1.00 \\
\hline 500 & 100 & 100 & 1.00 \\
\hline 550 & 100 & 89.0 & 1.12 \\
\hline 600 & 100 & 96.3 & 1.04 \\
\hline 650 & 100 & 83.4 & 1.20 \\
\hline 700 & 100 & 52.3 & 1.91 \\
\hline 750 & 100 & 84.0 & 1.19 \\
\hline 800 & 100 & 40.3 & 2.48 \\
\hline 850 & & 32.2 & \\
\hline 900 & 38.4 & 9.55 & 4.02 \\
\hline 950 & 0.778 & 2.41 & 0.323 \\
\hline
\end{tabular}


Methylene Chloride $\left(\sim 10^{-5} \mathrm{M}\right)$

Exposed To 0 And 307 AM 1.0 Suns (Simulated) For 10s in Helium

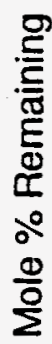

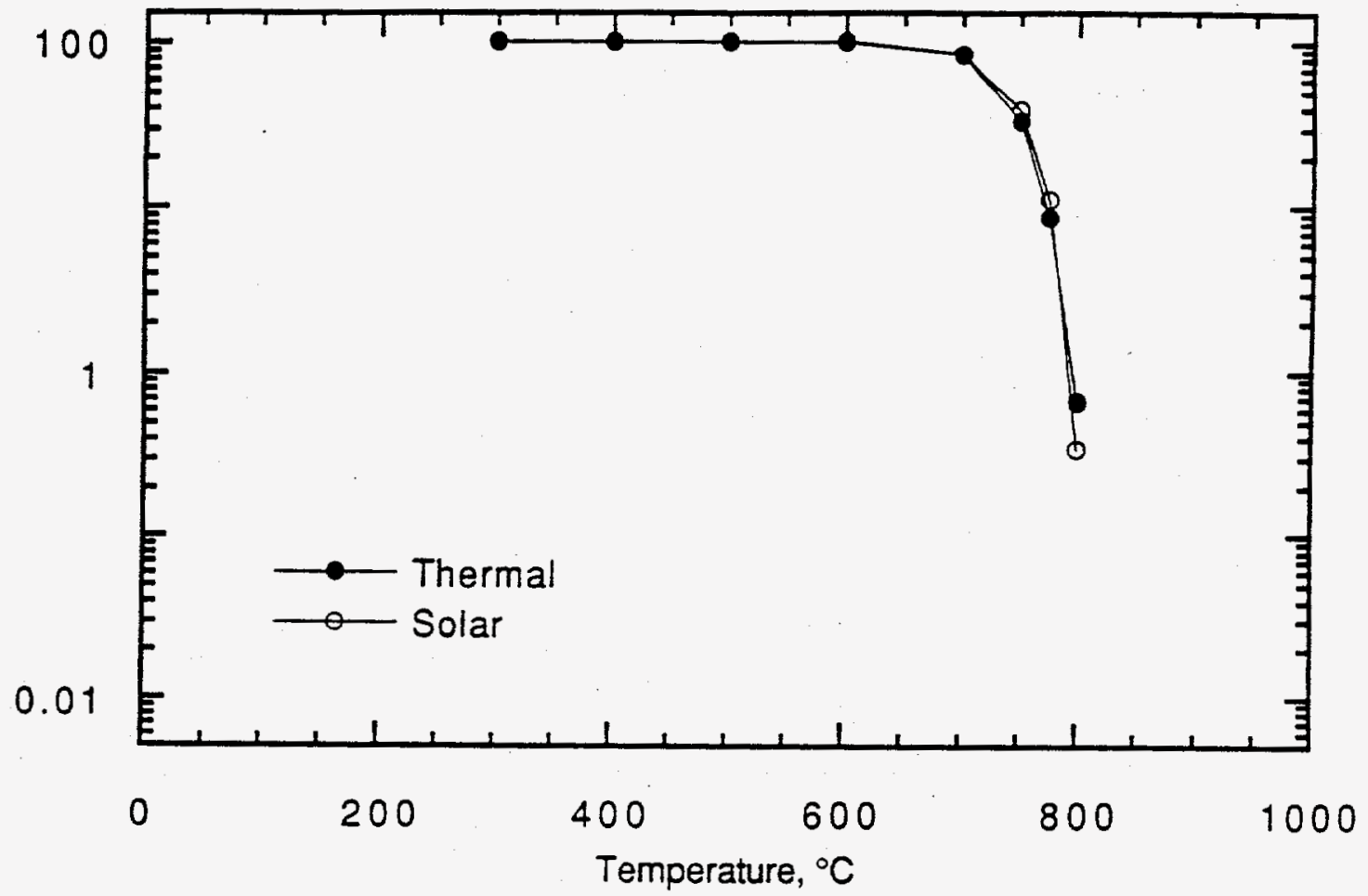

\begin{tabular}{|c|c|c|c|}
\hline Temp. & $f r(0)$ & $\operatorname{fr}(10)$ & $R(10)$ \\
\hline 300 & & & \\
\hline 400 & 100 & 100 & 1.00 \\
\hline 500 & 100 & 100 & 1.00 \\
\hline 600 & 100 & 100 & 1.00 \\
\hline 700 & 100 & 100 & 1.00 \\
\hline 750 & 83.6 & 83.7 & 0.999 \\
\hline 775 & 33.0 & 38.4 & 0.859 \\
\hline 800 & 8.57 & 11.0 & 0.779 \\
\hline
\end{tabular}


Methylene Chloride $\left(\sim 10^{-5} \mathrm{M}\right)$ In The Presence Of Chlorine $\left(\sim 3 \times 10^{-5} \mathrm{M}\right)$ Exposed To 0 And 307 AM 1.0 Suns (Simulated) For 10s in Helium

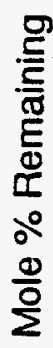

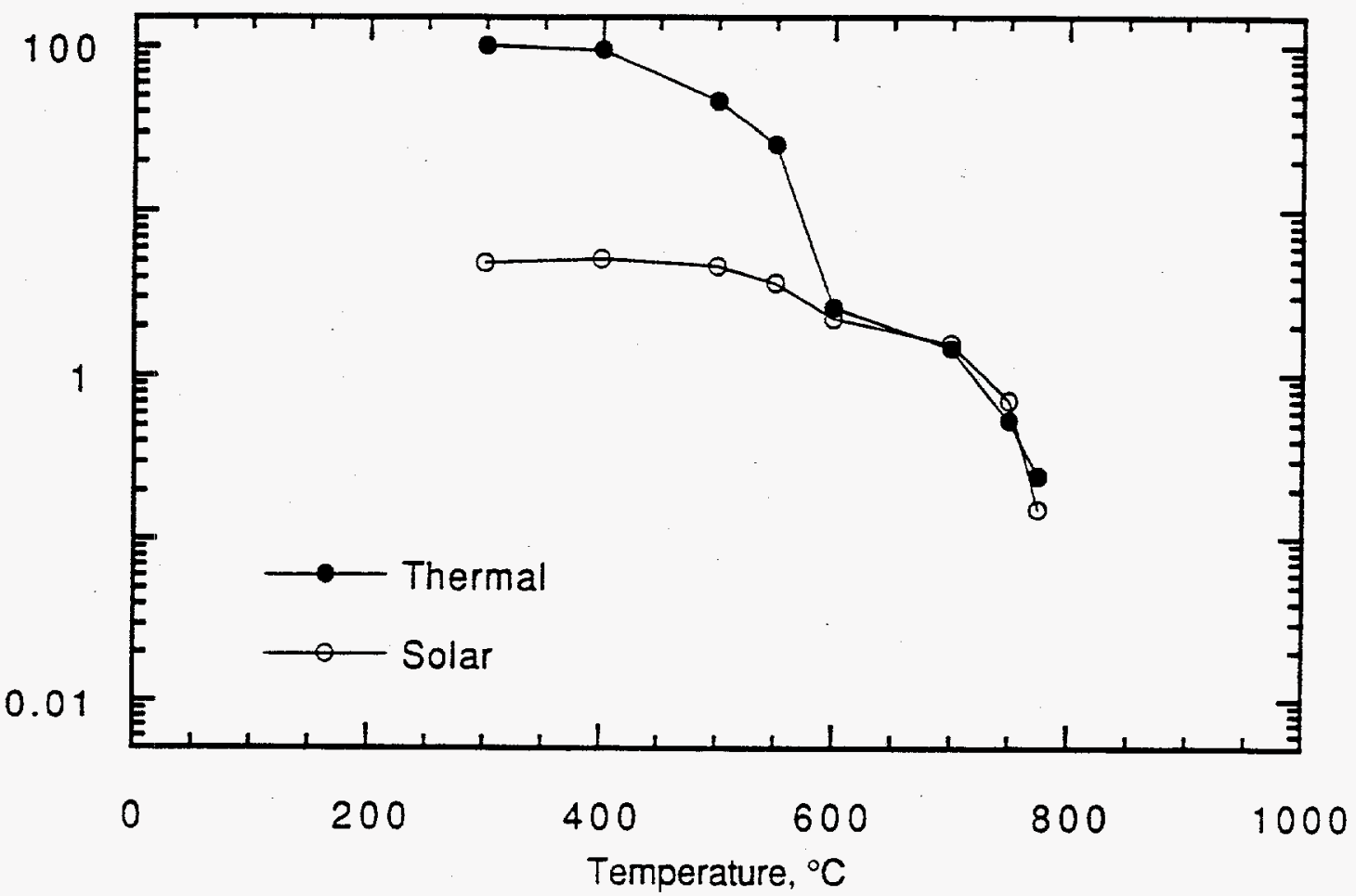

\begin{tabular}{|c|c|c|c|}
\hline Temp. & $\operatorname{fr}(0)$ & $\operatorname{fr}(10)$ & $\mathrm{R}(10)$ \\
\hline & & & \\
\hline 300 & 100 & 4.91 & 20.4 \\
\hline 400 & 94.5 & 5.16 & 18.3 \\
\hline 500 & 45.6 & 4.62 & 9.87 \\
\hline 550 & 25.0 & 3.63 & 6.88 \\
\hline 600 & 2.57 & 2.20 & 1.17 \\
\hline 700 & 1.45 & 1.55 & 0.935 \\
\hline 750 & 0.524 & 0.694 & 0.755 \\
\hline 775 & 0.240 & 0.150 & 1.60 \\
\hline
\end{tabular}


Methylene Chloride $\left(\sim 10^{-5} \mathrm{M}\right)$ In The Presence of Chlorine $\left(\sim 3 \times 10^{-5} \mathrm{M}\right)$ Exposed To 0 And 307 AM 1.0 Suns (Simulated) For $10 \mathrm{~s}$ In Air

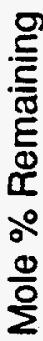

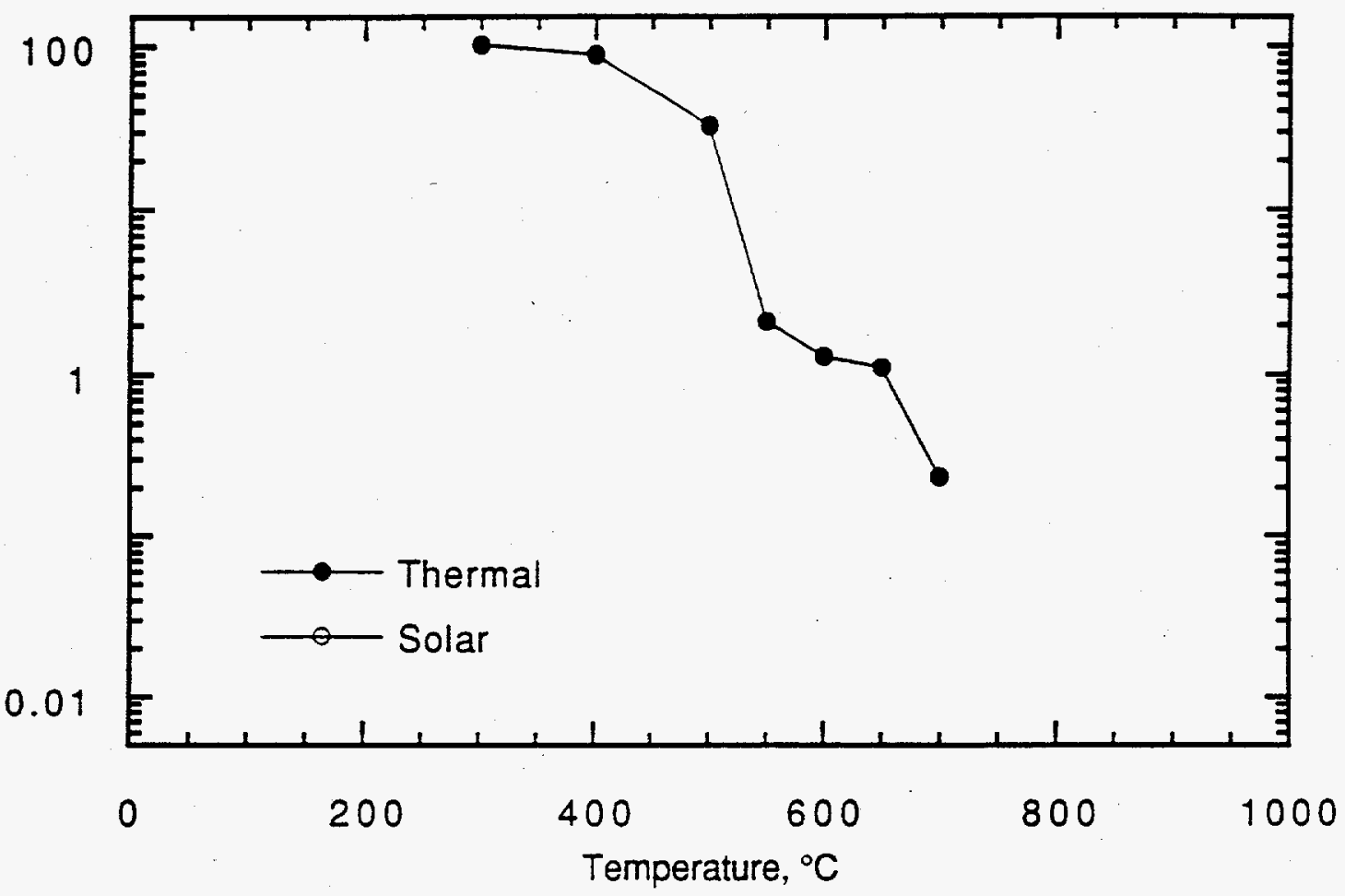

\begin{tabular}{|c|c|c|c|}
\hline Temp. & $\operatorname{fr}(0)$ & $\operatorname{fr}(10)$ & $R(10)$ \\
\hline & & & \\
\hline 300 & 100 & bdl & \\
\hline 400 & 87.8 & bdl & \\
\hline 500 & 32.3 & bdl & \\
\hline 550 & 2.11 & bdl & \\
\hline 600 & 1.29 & bdl & \\
\hline 650 & 1.10 & bdl & \\
\hline 700 & 0.230 & bdl & \\
\hline
\end{tabular}

bdl $=$ below detection limit $(\sim 0.01 \%$ 
Methylene Chloride $\left(\sim 10^{-5} \mathrm{M}\right)$ With Chlorine

Exposed To 307 AM 1.0 Suns (Simulated) At $400^{\circ} \mathrm{C}$ For $10 \mathrm{~s}$ In Air

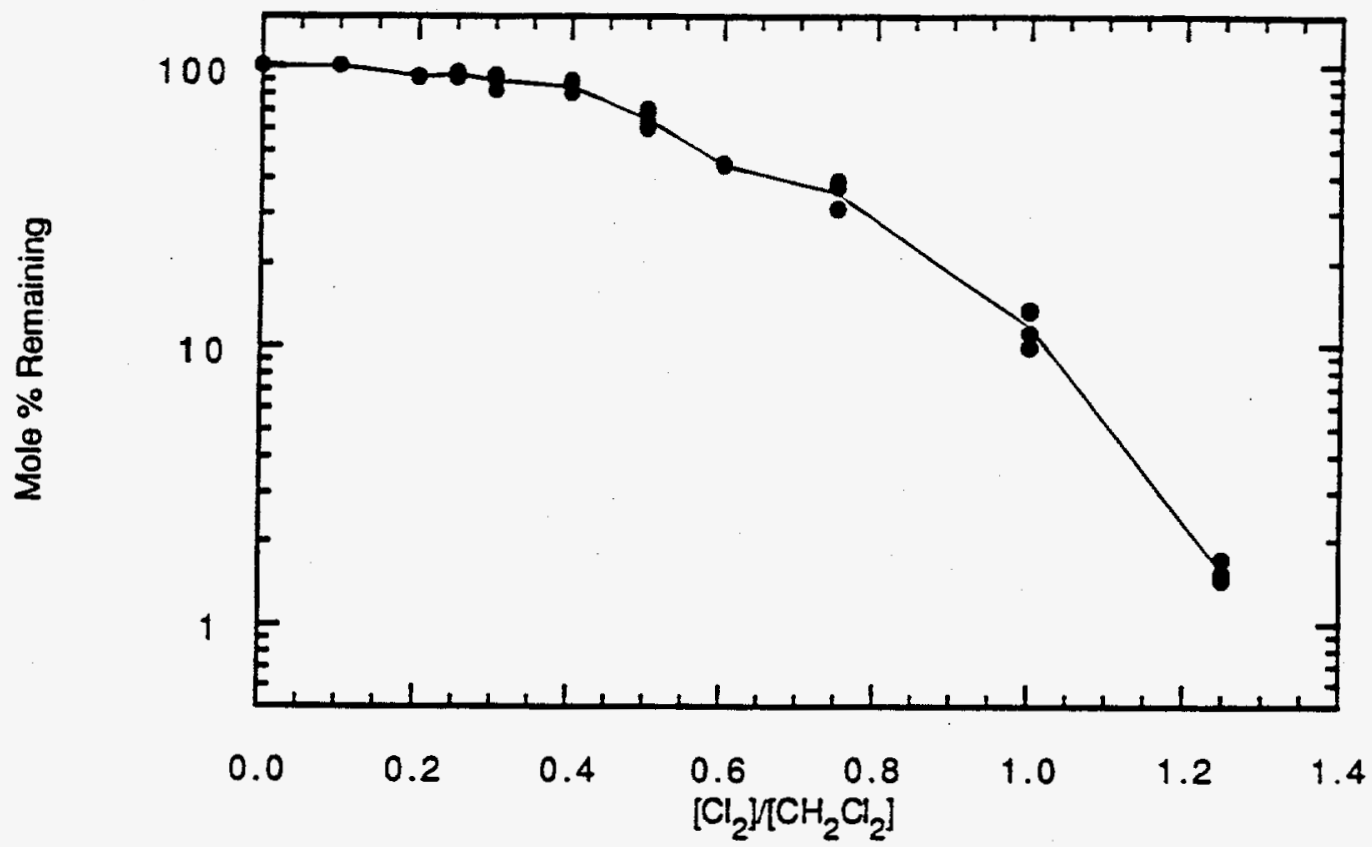

\begin{tabular}{|c|c|c|}
\hline$\left.\left[\mathrm{Cl}_{2}\right] \mathrm{CH}_{2} \mathrm{Cl}\right]$ & frro) & $R(10)$ \\
\hline 0 & 100 & 1.00 \\
\hline 0 & 100 & 1.00 \\
\hline 0.100 & 100 & 1.00 \\
\hline 0.100 & 100 & 1.00 \\
\hline 0.200 & 91.4 & 1.09 \\
\hline 0.250 & 95.0 & 1.05 \\
\hline 0.250 & 93.6 & 1.07 \\
\hline 0.250 & 91.6 & 1.09 \\
\hline 0.300 & 82.7 & 1.21 \\
\hline 0.300 & 89.3 & 1.12 \\
\hline 0.300 & 91.8 & 1.09 \\
\hline 0.300 & 92.2 & 1.09 \\
\hline 0.400 & 80.3 & 1.25 \\
\hline 0.400 & 88.2 & 1.13 \\
\hline 0.500 & 63.0 & 1.59 \\
\hline 0.500 & 70.1 & 1.43 \\
\hline 0.500 & 68.4 & 1.46 \\
\hline 0.500 & 63.5 & 1.58 \\
\hline 0.500 & 60.3 & 1.66 \\
\hline 0.600 & 44.6 & 2.24 \\
\hline 0.750 & 39.0 & 2.56 \\
\hline 0.750 & 37.3 & 2.68 \\
\hline 0.750 & 31.3 & 3.20 \\
\hline 1.000 & 13.5 & 7.41 \\
\hline 1.000 & 13.4 & 7.46 \\
\hline 1.000 & 11.1 & 9.01 \\
\hline 1.000 & 9.90 & 10.1 \\
\hline 1250 & 1.71 & 58.5 \\
\hline 1250 & 1.54 & 64.9 \\
\hline 1250 & 2101.45 & 69.0 \\
\hline
\end{tabular}


Trichloroethylene $\left(\sim 10^{-5} \mathrm{M}\right)$

Exposed To 0 And 307 AM 1.0 Suns (Simulated) For 10s In Air

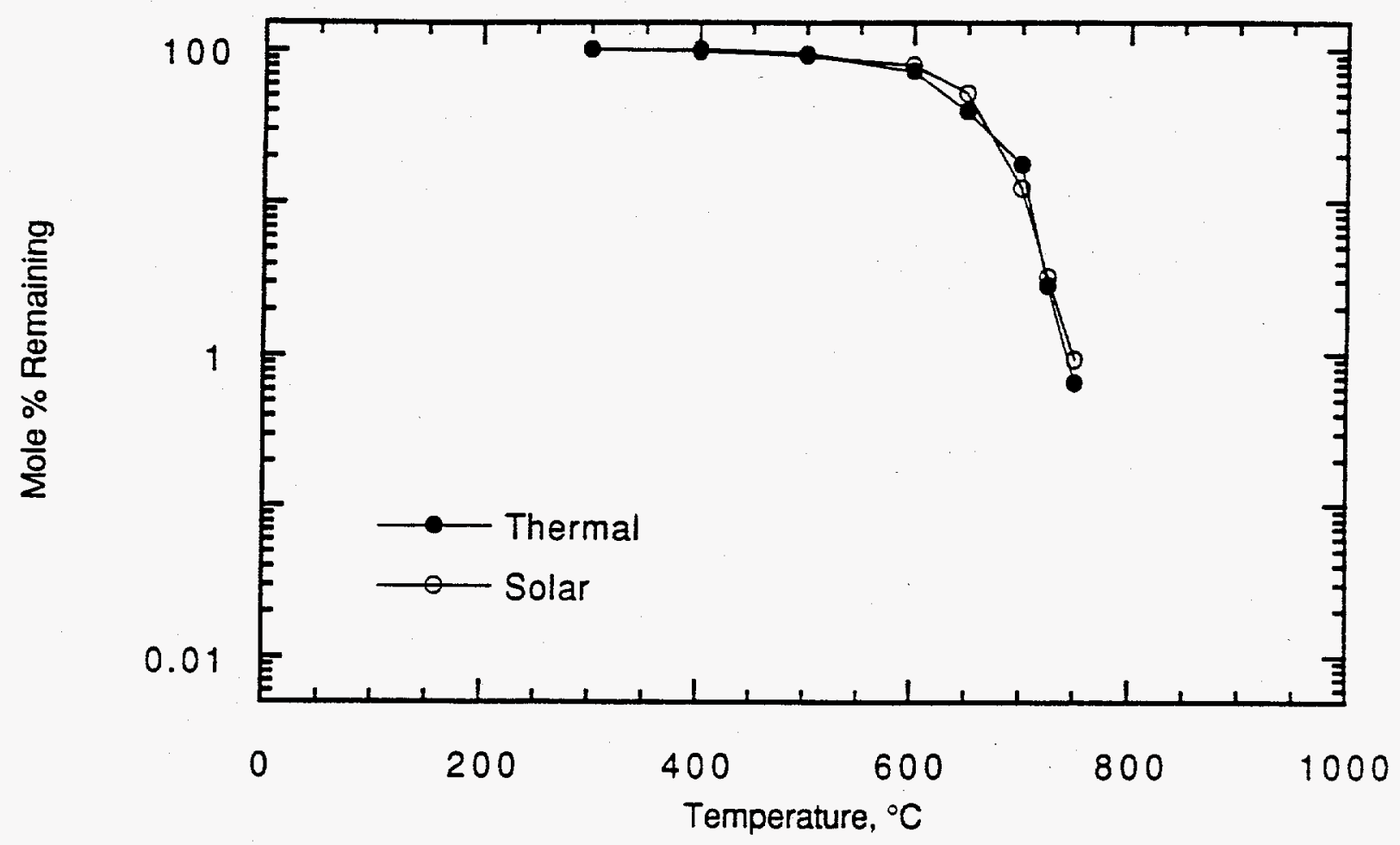

\begin{tabular}{|c|c|c|c|c|}
\hline Temp. & $\operatorname{fr}(0)$ & $\operatorname{fr}(10)$ & $\operatorname{R}(10)$ & TCE \\
\hline & & & & \\
\hline 300 & 100 & & & \\
\hline 400 & 100 & 97.100 & 1.030 & 307 \\
\hline 500 & 93.800 & 91.000 & 1.031 & 1 \\
\hline 600 & 72.100 & 79.500 & .907 & 10 \\
\hline 650 & 38.900 & 51.100 & .761 & air \\
\hline 700 & 17.500 & 12.300 & 1.423 & \\
\hline 725 & 2.810 & 3.230 & .870 & \\
\hline 750 & .658 & .932 & .706 & \\
\hline
\end{tabular}


Trichloroethylene $\left(\sim 10^{-5} \mathrm{M}\right)$ In The Presence Of Hydrogen Peroxide $\left(\sim 10^{-4} \mathrm{M}\right)$ Exposed To 0 And 307 AM 1.0 Suns (Simulated) For 10s In Air

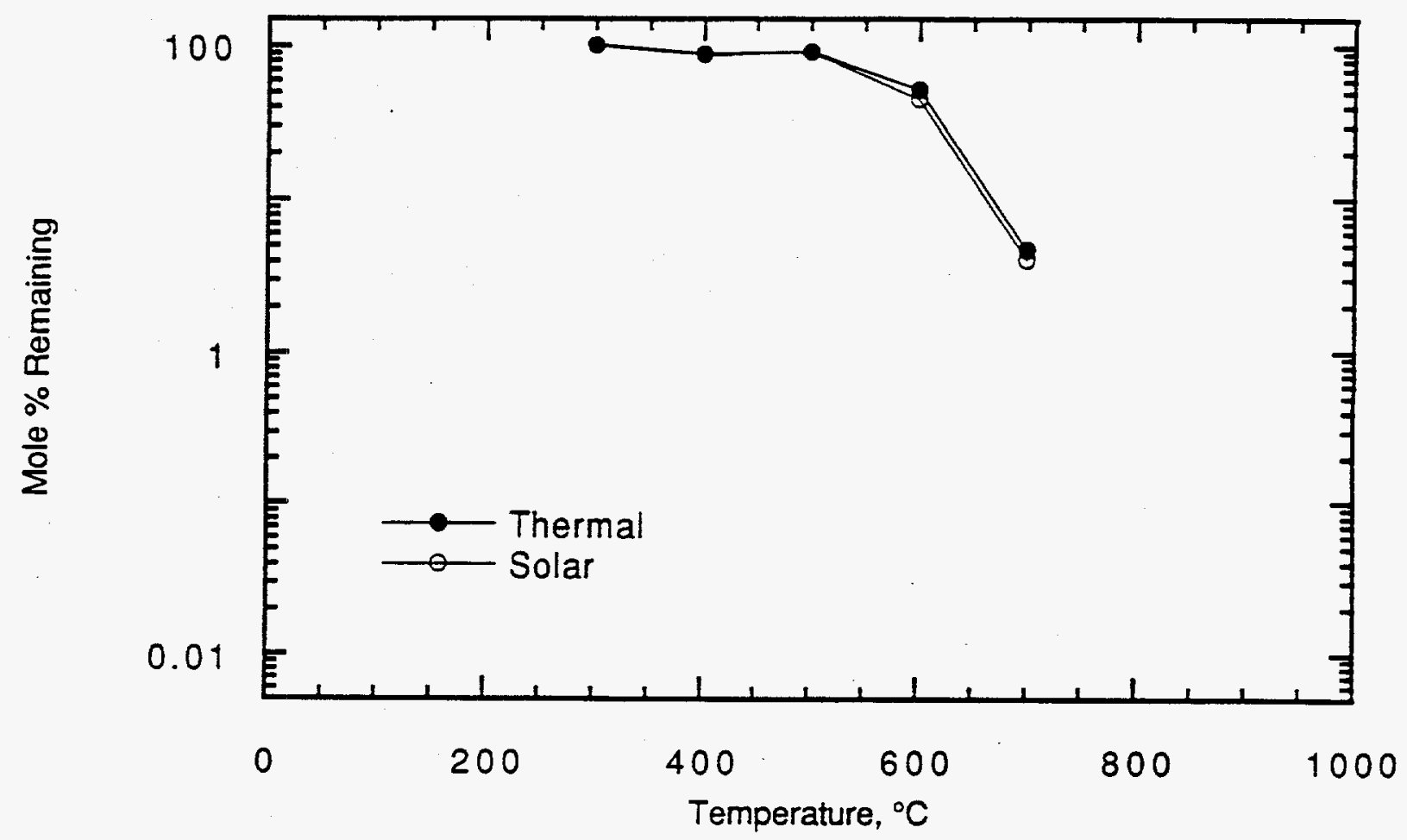

\begin{tabular}{|c|c|c|c|}
\hline Temp. & fr(0) & fr $(10)$ & $R(10)$ \\
\hline & & & \\
\hline 300 & 100 & & \\
\hline 400 & 86.7 & 87.9 & 0.986 \\
\hline 500 & 90.8 & 90.9 & 0.999 \\
\hline 600 & 51.2 & 44.5 & 1.15 \\
\hline 700 & 4.67 & 4.04 & 1.16 \\
\hline 750 & bdl & bdl & \\
\hline
\end{tabular}

bdl $=$ below detection limit $(\sim 0.01 \%)$ 


\section{APPENDIX III \\ Thermal/Photolytic Degradation of Multi-Component Mixtures}

This Appendix summarizes all of the data obtained during this project on complex mixtures which were either actual waste streams, or waste mixtures blended in the laboratory. The first is an 8-component mixture composed of selected compounds which absorb near-UV solar radiation to various degrees. The second is a series of three variants of a spiked fuel oil. Fuel Oil-I was a waste stream which was being burned in a commercial incinerator, while variant-la was additionally spiked with 1-bromonaphthalene and variant-lb was spiked with 1-nitronaphthalene. The last mixture, referred to as spiked Fuel Oil-II, was a mixture blended by Midwest Research Institute for use in trial with a pilot scale reactor.

Unless otherwise noted, temperature is given in degrees Centigrade and fraction remaining is in mole percent normalized by the original amount of sample fed to the reactor. By definition, enhancement ratio is unitless. Entries marked as "bdl" are below the system detection limit of $\sim 0.01 \%$. 
Parent Compounds And PICs Of An 8-Component Mixture

Exposed To 0 And 192 AM 1.0 Suns (Simulated) For $10 \mathrm{~s}$ in Air

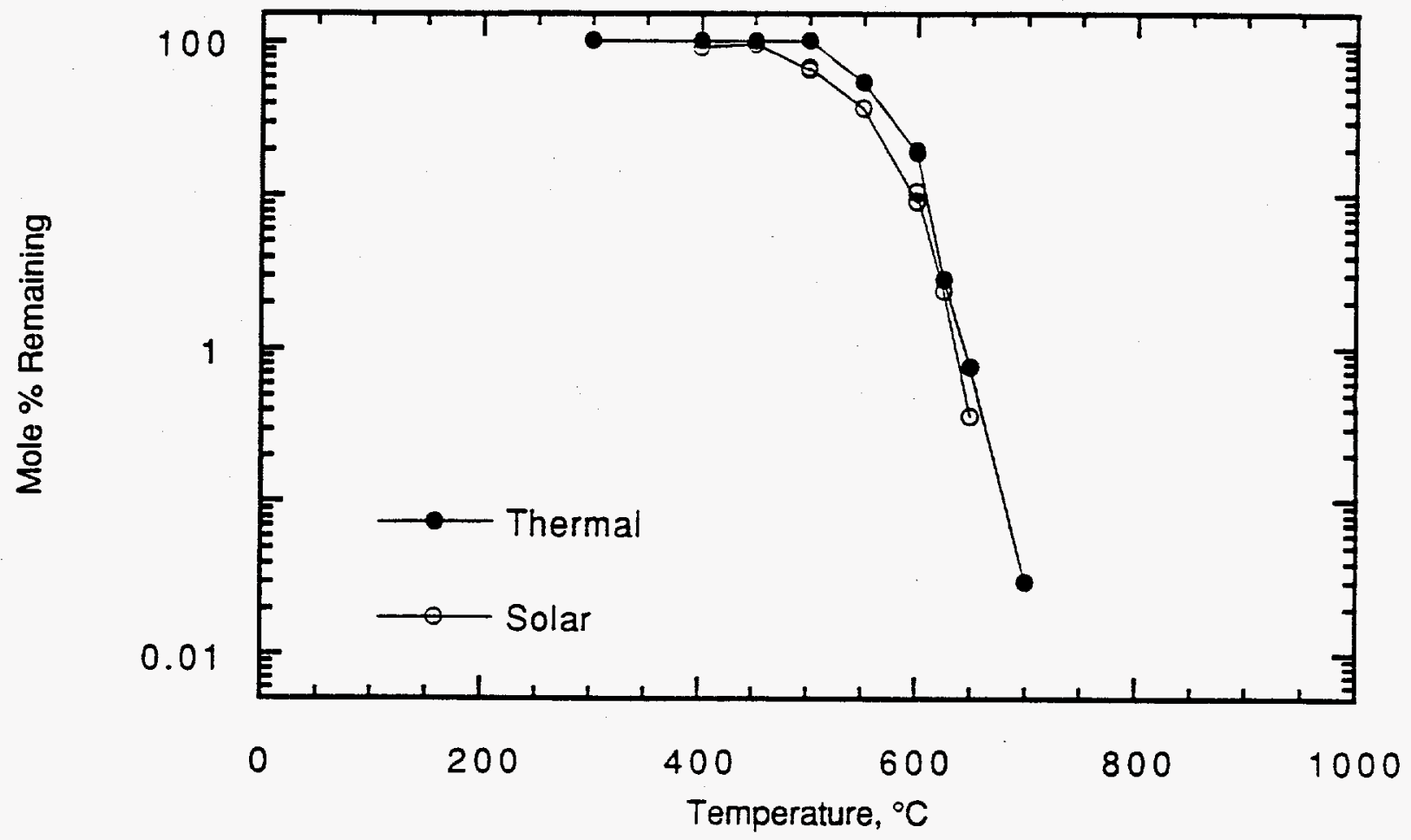

\begin{tabular}{|c|c|c|c|}
\hline Temp. & $\operatorname{fr}(0)$ & $\operatorname{fr}(10)$ & $\mathbf{R}(10)$ \\
\hline & & & \\
\hline 300 & 100 & & \\
\hline 400 & 100 & 90.8 & 1.10 \\
\hline 450 & 100 & 94.7 & 1.06 \\
\hline 500 & 100 & 64.9 & 1.54 \\
\hline 500 & 100 & 67.4 & 1.48 \\
\hline 550 & 53.7 & 36.4 & 1.48 \\
\hline 600 & 18.7 & 9.08 & 2.06 \\
\hline 600 & 19.4 & 9.12 & 2.123 \\
\hline 600 & & 10.4 & \\
\hline 625 & 2.81 & 2.37 & 1.19 \\
\hline 650 & 0.750 & 0.360 & 2.08 \\
\hline 700 & 0.0300 & & \\
\hline
\end{tabular}


Parent Compounds Of An 8-Component Mixture

Exposed To 0 And 192 AM 1.0 Suns (Simulated) For $10 \mathrm{~s}$ In Air

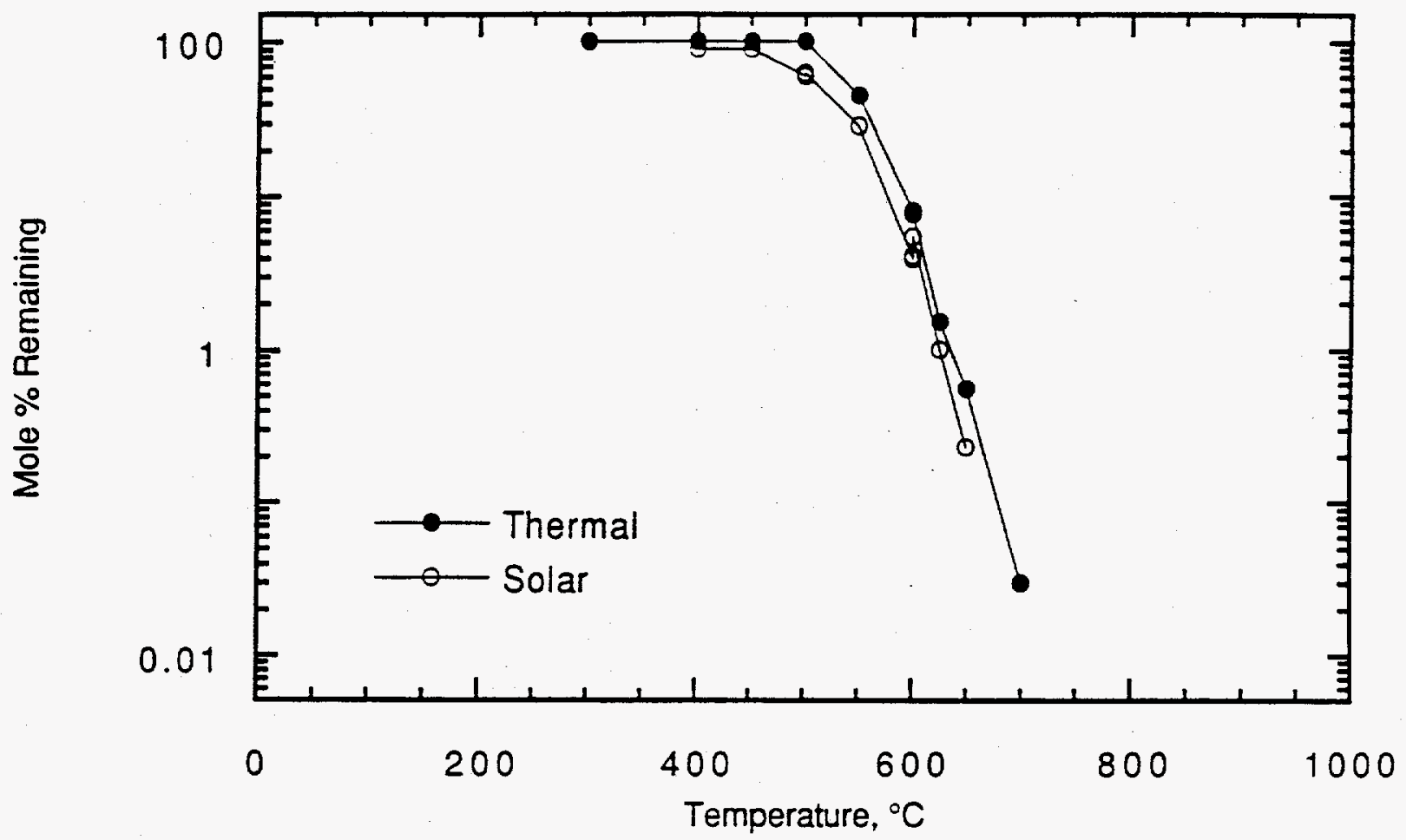

\begin{tabular}{|c|c|c|c|}
\hline Temp. & $\operatorname{fr}(0)$ & $\operatorname{fr}(10)$ & $\mathrm{R}(10)$ \\
\hline & & & \\
\hline 300 & 100 & & \\
\hline 400 & 100 & 89.2 & 1.12 \\
\hline 450 & 100 & 89.9 & 1.11 \\
\hline 500 & 100 & 59.6 & 1.68 \\
\hline 500 & 100 & 62.7 & 1.60 \\
\hline 550 & 44.7 & 28.6 & 1.56 \\
\hline 600 & 8.08 & 3.96 & 20.4 \\
\hline 600 & 7.66 & 4.15 & 1.85 \\
\hline 600 & & 5.45 & \\
\hline 625 & 1.52 & 1.01 & 1.51 \\
\hline 650 & 0.560 & 0.230 & 2.44 \\
\hline 700 & 0.030 & bdl & \\
\hline
\end{tabular}


Total PICs From An 8-Component Mixture

Exposed To 0 And 192 AM 1.0 Suns (Simulated) For $10 \mathrm{~s}$ In Air

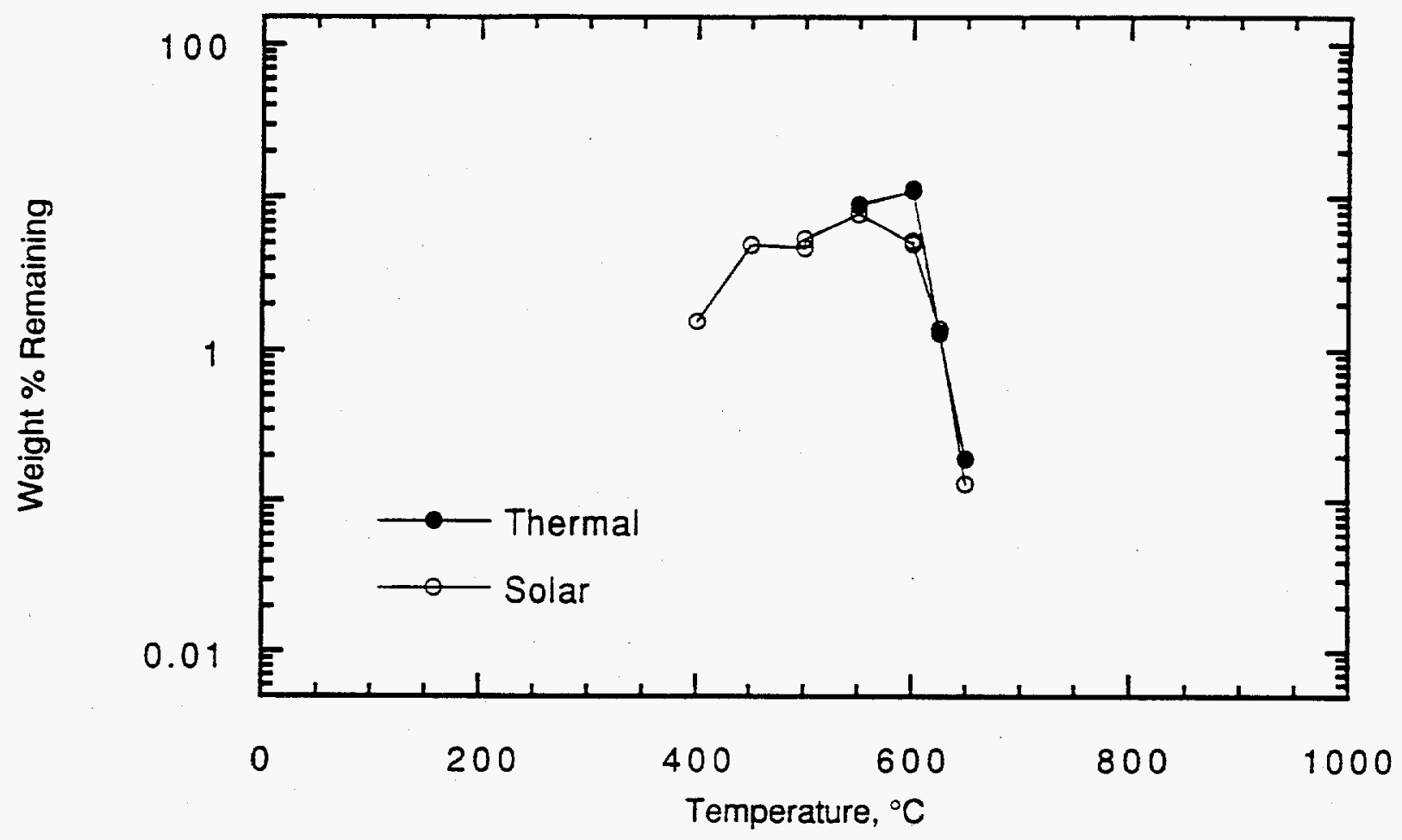

\begin{tabular}{|c|c|c|c|}
\hline Temp. & $\operatorname{fr}(0)$ & $\operatorname{fr}(10)$ & $\mathbf{R}(\mathbf{l 0})$ \\
\hline & & & \\
\hline 300 & bdl & bdl & \\
\hline 400 & bdl & 1.53 & \\
\hline 450 & bdl & 4.88 & \\
\hline 500 & bdl & 5.34 & \\
\hline 550 & 8.96 & 7.80 & \\
\hline 600 & 11.0 & 4.93 & \\
\hline 600 & 11.3 & 5.16 & \\
\hline 600 & & 4.95 & \\
\hline 625 & 1.29 & 1.37 & \\
\hline 650 & 0.190 & 0.130 & \\
\hline 700 & bdl & bdl & \\
\hline
\end{tabular}


Aniline $\left(\sim 2 \times 10^{-6} \mathrm{M}\right)$ Component of An 8-Component Mixture Exposed To 0 And 192 AM 1.0 Suns (Simulated) For 10s In Air

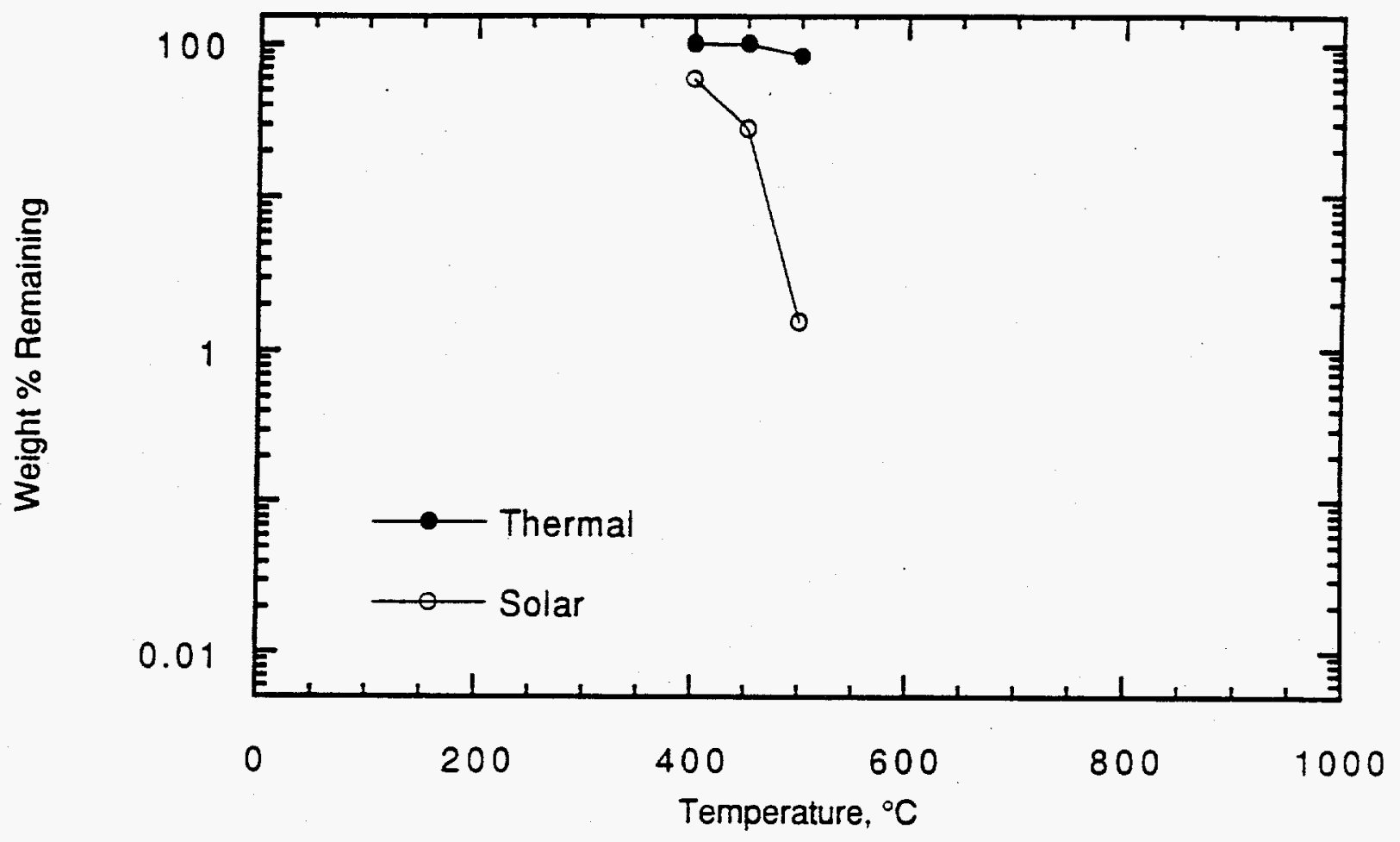

\begin{tabular}{|c|c|c|c|}
\hline Temp. & $\operatorname{fr}(0)$ & $\operatorname{fr}(10)$ & $R(10)$ \\
\hline & & & \\
\hline 400 & 100 & 58.3 & 1.72 \\
\hline 450 & 100 & 27.6 & 3.62 \\
\hline 500 & 82.9 & 1.54 & 53.8 \\
\hline 550 & bdl & bdl & \\
\hline 600 & bdl & bdl & \\
\hline 600 & bdl & bdl & \\
\hline 625 & bdl & bdl & \\
\hline 650 & bdl & bdl & \\
\hline 700 & bdl & bdl & \\
\hline
\end{tabular}


Methylene Chloride $\left(\sim 2 \times 10^{-6} \mathrm{M}\right)$ Component of An 8-Component Mixture Exposed To 0 And 192 AM 1.0 Suns (Simulated) For 10s in Air

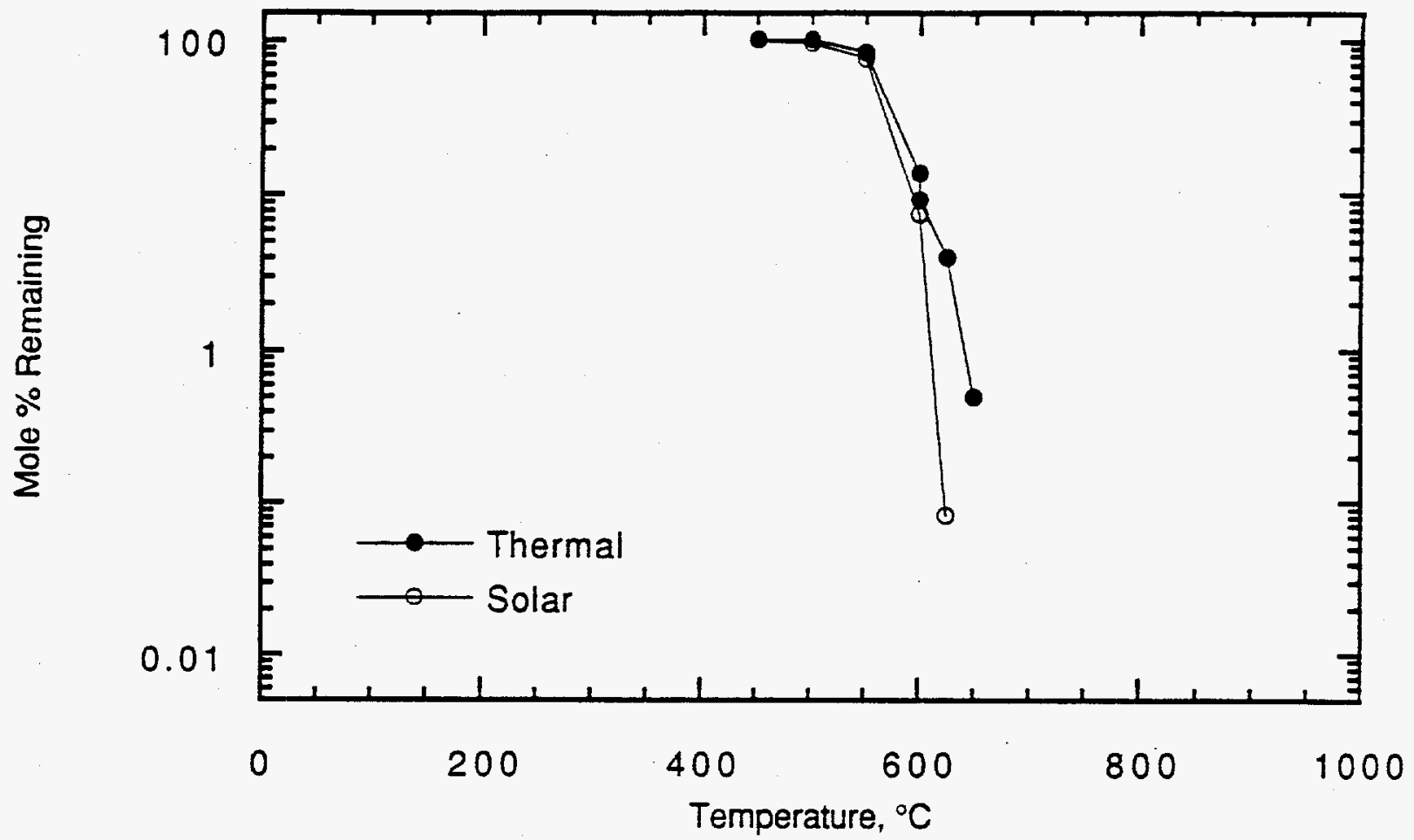

\begin{tabular}{|c|c|c|c|}
\hline Temp. & $\operatorname{fr}(0)$ & $\mathrm{fr}(10)$ & $\mathrm{R}(10)$ \\
\hline & & & \\
\hline 450 & 100 & 99.7 & 1.00 \\
\hline 500 & 100 & 94.8 & 1.06 \\
\hline 550 & 81.9 & 74.8 & 1.10 \\
\hline 600 & 13.7 & 7.50 & 1.83 \\
\hline 600 & 9.18 & 7.49 & 1.23 \\
\hline 625 & 3.93 & 0.0820 & 47.9 \\
\hline 650 & 0.493 & bdl & \\
\hline 700 & bdl & bdl & \\
\hline
\end{tabular}


Naphthalene $\left(\sim 10^{-7} \mathrm{M}\right)$ Component of An 8-Component Mixture Exposed To 0 And 192 AM 1.0 Suns (Simulated) For $10 \mathrm{~s}$ In Air

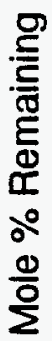

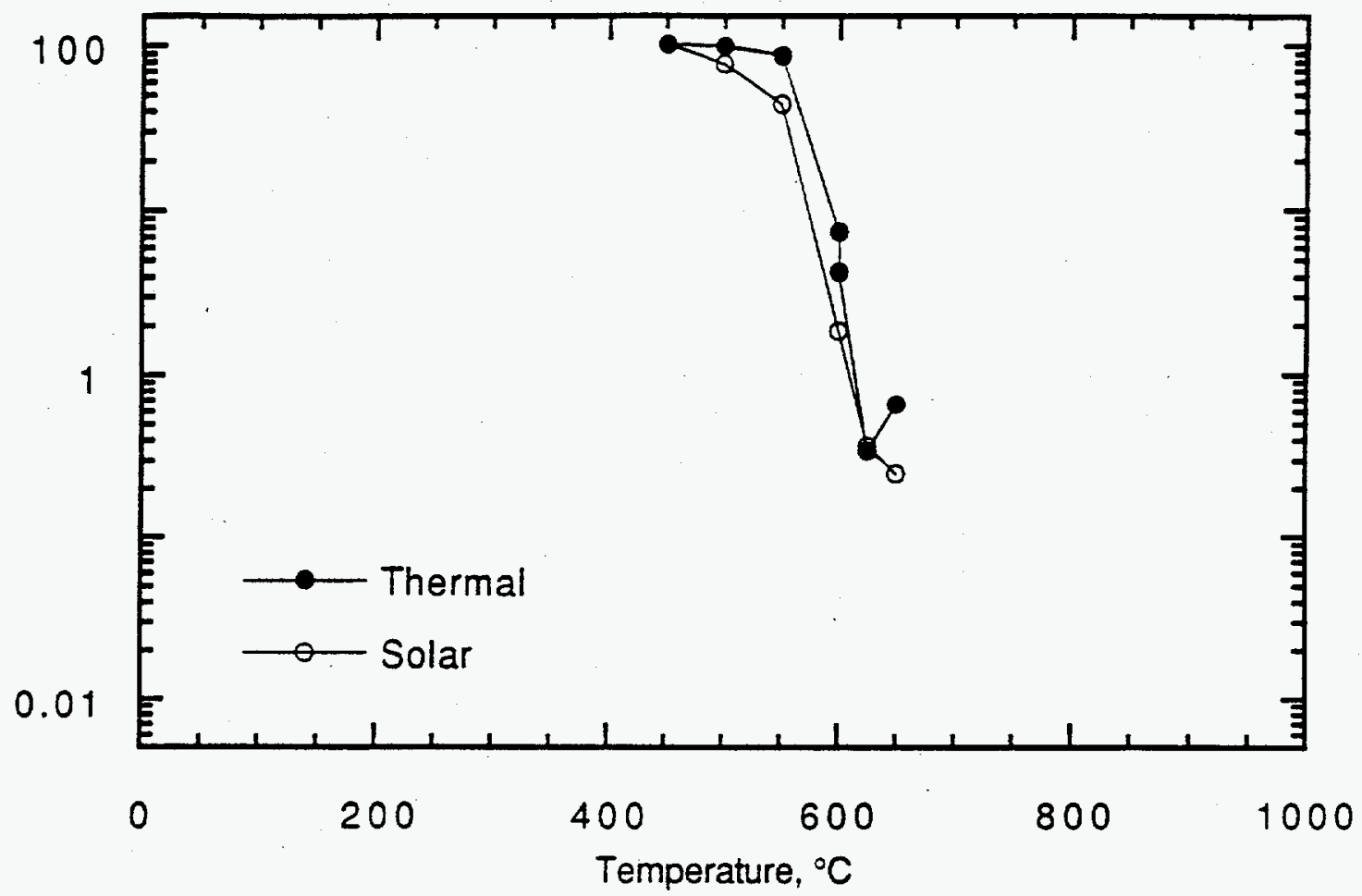

\begin{tabular}{|c|c|c|c|}
\hline Temp. & $\operatorname{fr}(0)$ & $\operatorname{fr}(10)$ & $\mathbf{R}(10)$ \\
\hline & & & \\
\hline 450 & 100 & 100 & 1.00 \\
\hline 500 & 98.6 & 77.0 & 1.28 \\
\hline 550 & 85.8 & 43.1 & 1.99 \\
\hline 600 & 7.35 & 1.84 & 4.00 \\
\hline 600 & 4.21 & 1.84 & 2.29 \\
\hline 625 & 0.340 & 0.363 & 0.937 \\
\hline 650 & 0.654 & 0.247 & 2.65 \\
\hline 700 & bdl & bdl & \\
\hline
\end{tabular}




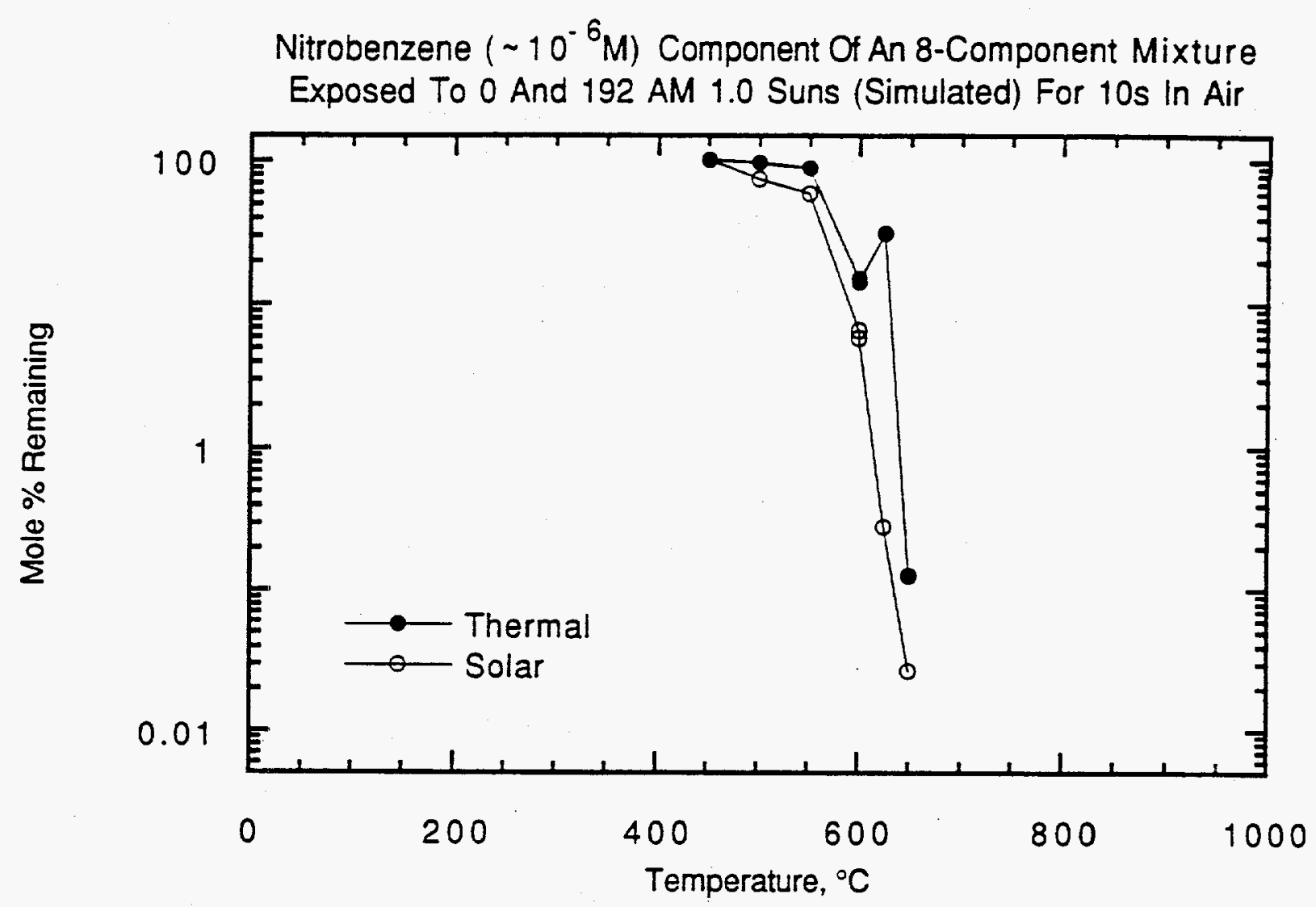

\begin{tabular}{|c|c|c|c|}
\hline Temp. & fr(0) & fr(lo) & R(lo) \\
\hline & & & \\
\hline 450 & 100 & 100 & 1.00 \\
\hline 500 & 96.2 & 74.3 & 1.30 \\
\hline 550 & 88.1 & 58.4 & 1.51 \\
\hline 600 & 15.0 & 6.62 & 2.27 \\
\hline 600 & 14.3 & 5.85 & 2.44 \\
\hline 625 & 31.0 & 0.278 & 112 \\
\hline 650 & 0.126 & 0.0270 & 4.72 \\
\hline 700 & bdl & bdl & \\
\hline
\end{tabular}


1-Nitronaphthalene $\left(\sim 10^{-7} \mathrm{M}\right)$ Component of An 8-Component Mixture Exposed To 0 And 192 AM 1.0 Suns (Simulated) For 10s In Air

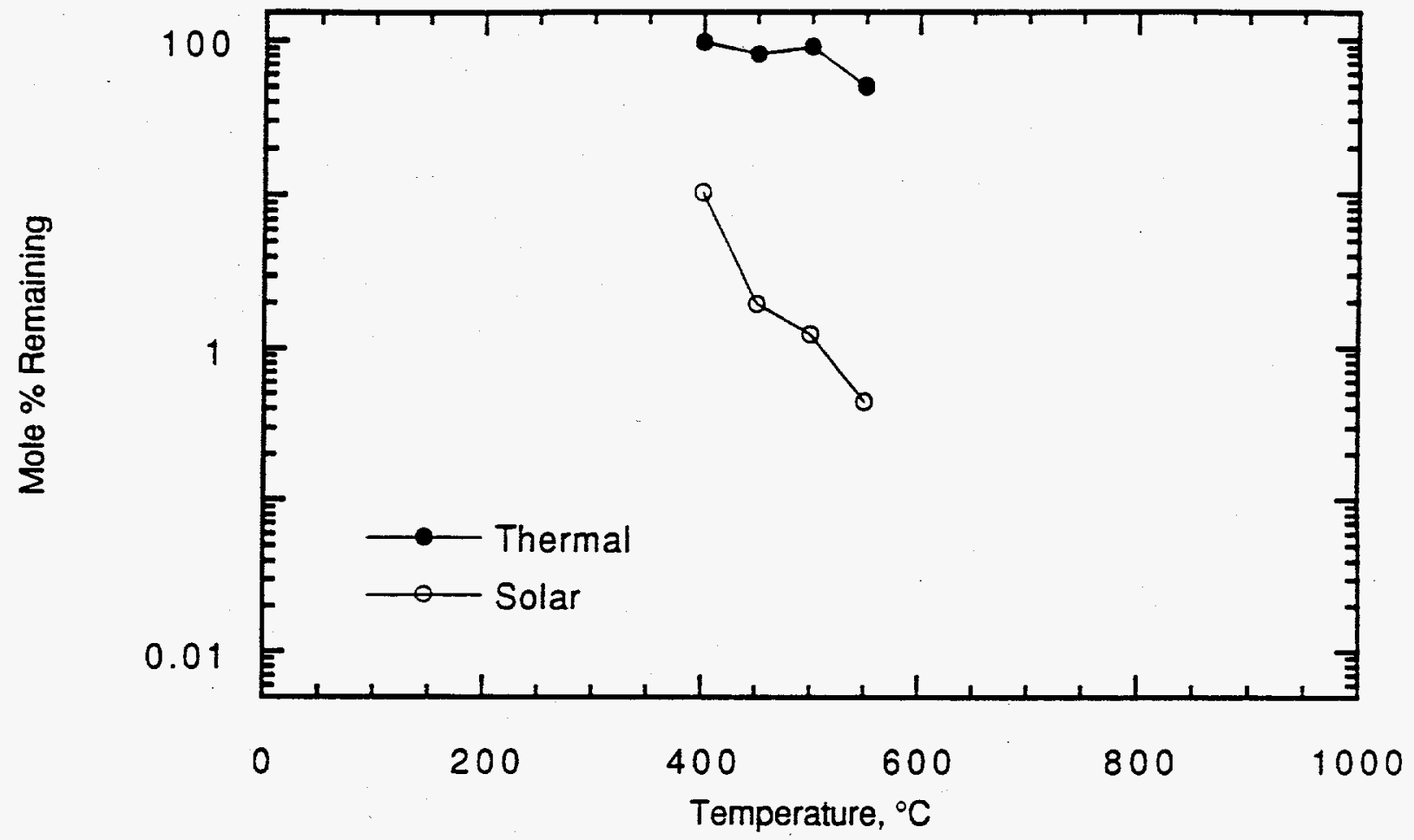

\begin{tabular}{|c|c|c|c|}
\hline Temp. & $\operatorname{fr}(0)$ & $\operatorname{fr}(10)$ & $\mathbf{R}(10)$ \\
\hline & & & \\
\hline 400 & 96.0 & 10.3 & 9.32 \\
\hline 450 & 79.8 & 1.95 & 40.9 \\
\hline 500 & 89.4 & 1.24 & 72.1 \\
\hline 550 & 49.2 & 0.441 & 112 \\
\hline 600 & bdl & bdl & \\
\hline 600 & bdl & bdl & \\
\hline 625 & bdl & bdl & \\
\hline 650 & bdl & bdl & \\
\hline 700 & bdl & bdl & \\
\hline
\end{tabular}




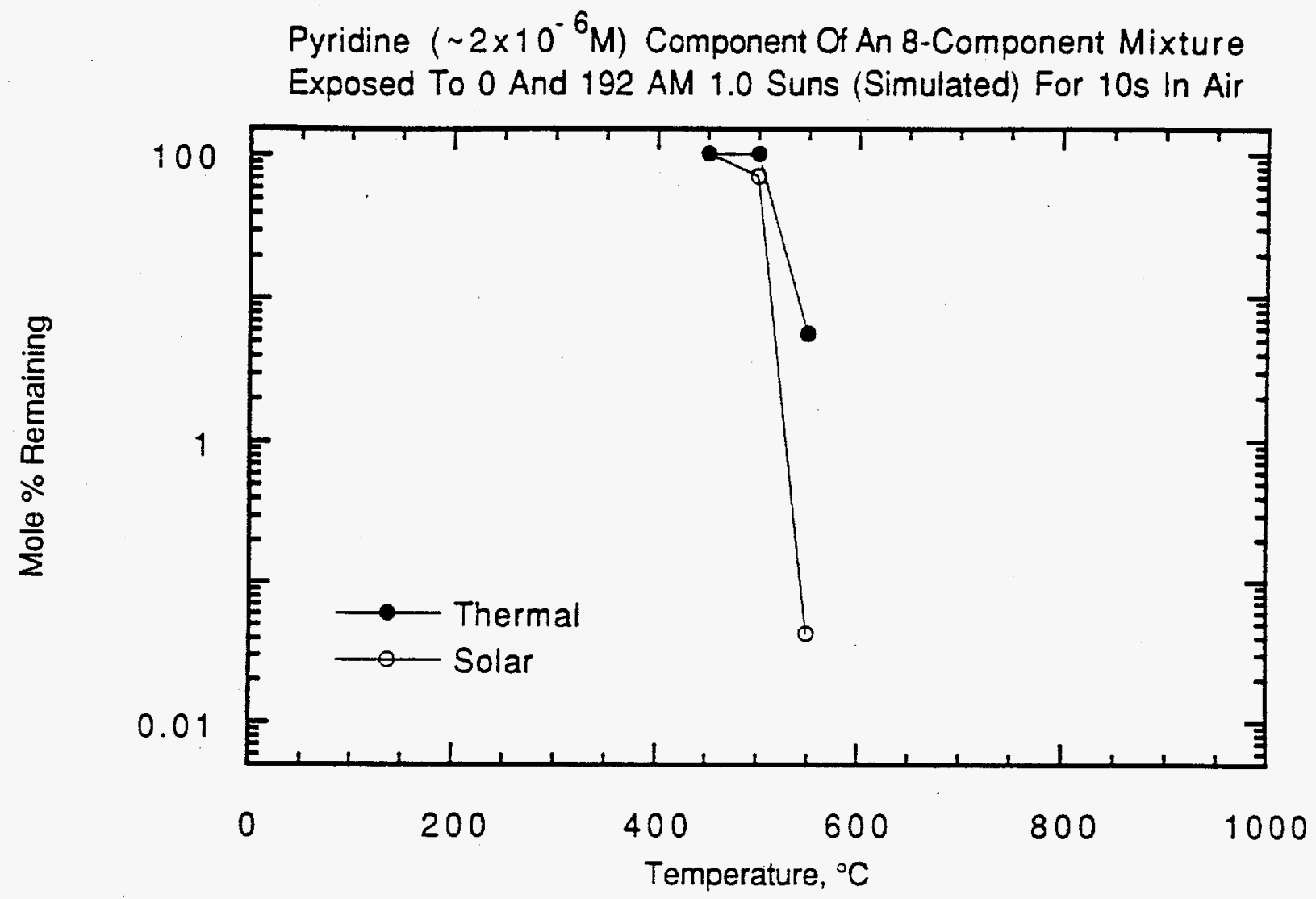

\begin{tabular}{|c|c|c|c|}
\hline Temp. & fr(0) & fr(lo) & $R(10)$ \\
\hline & & & \\
\hline 450 & 100 & 100 & 1.00 \\
\hline 500 & 100 & 69.9 & 1.43 \\
\hline 550 & 5.56 & 0.0430 & 129 \\
\hline 600 & bdl & bdl & \\
\hline 600 & bdl & bdl & \\
\hline 625 & bdl & bdl & \\
\hline 650 & bdl & bdl & \\
\hline 700 & bdl & bdl & \\
\hline
\end{tabular}


Trichloroethylene $\left(2 \times 10^{-6} \mathrm{M}\right)$ Component of An 8-Component Mixture Exposed To 0 And 192 AM 1.0 Suns (Simulated) For 10 s In Air

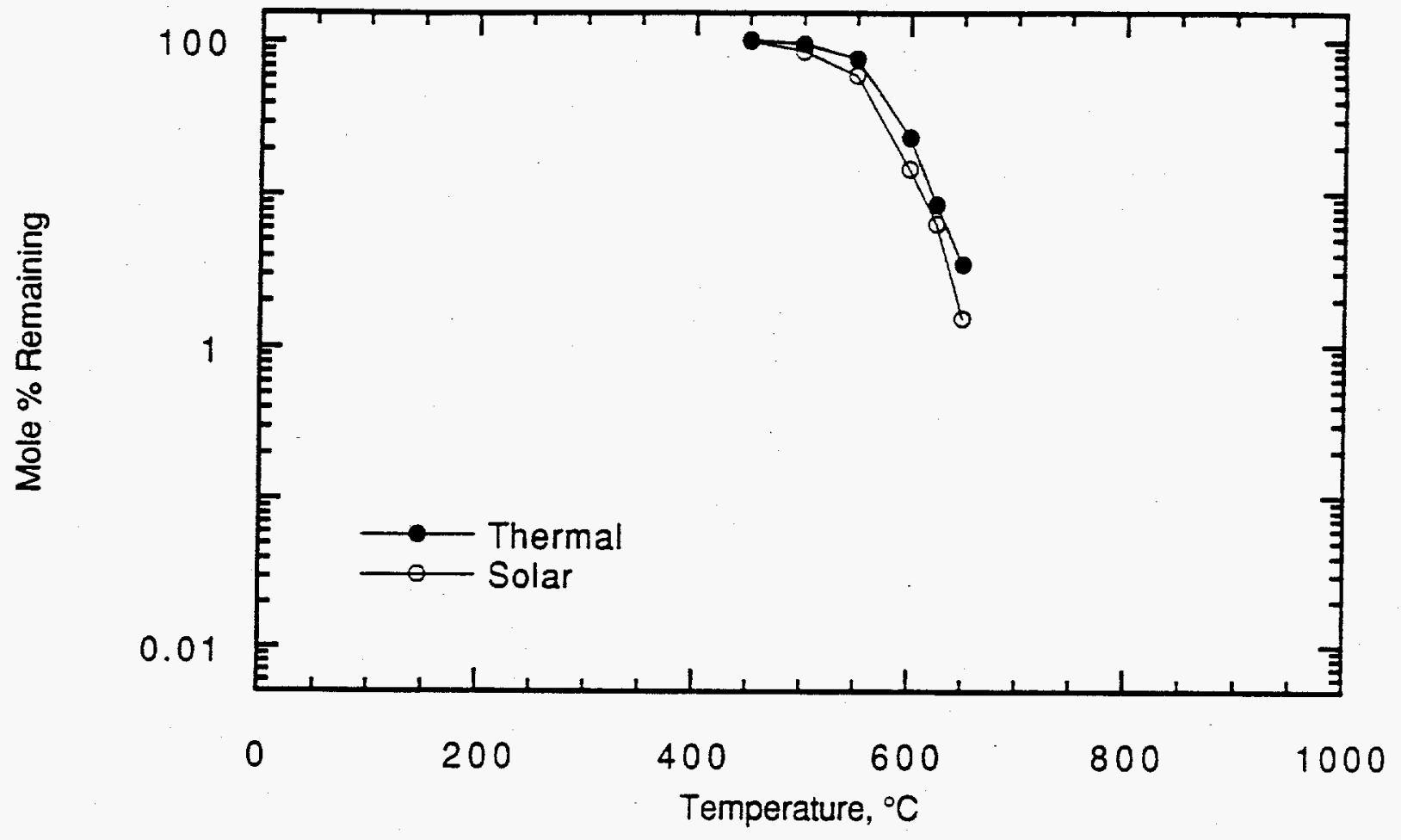

\begin{tabular}{|c|c|c|c|}
\hline Temp. & $\operatorname{fr}(0)$ & $\operatorname{fr}(10)$ & $\mathrm{R}(10)$ \\
\hline & & & \\
\hline 450 & 100 & 99.5 & 1.01 \\
\hline 500 & 94.9 & 84.7 & 1.12 \\
\hline 550 & 75.8 & 59.5 & 1.27 \\
\hline 600 & 23.5 & 14.4 & 1.63 \\
\hline 600 & 23.1 & 14.4 & 1.60 \\
\hline 625 & 8.48 & 6.34 & 1.34 \\
\hline 650 & 3.44 & 1.53 & 2.25 \\
\hline 700 & bdl & bdl & \\
\hline
\end{tabular}


Parent Compounds And PICs Of A Contaminated Fuel Oil-I ( 1000ppm) Exposed To 0 And 307 AM 1.0 Suns (Simulated) For 5 s in Air

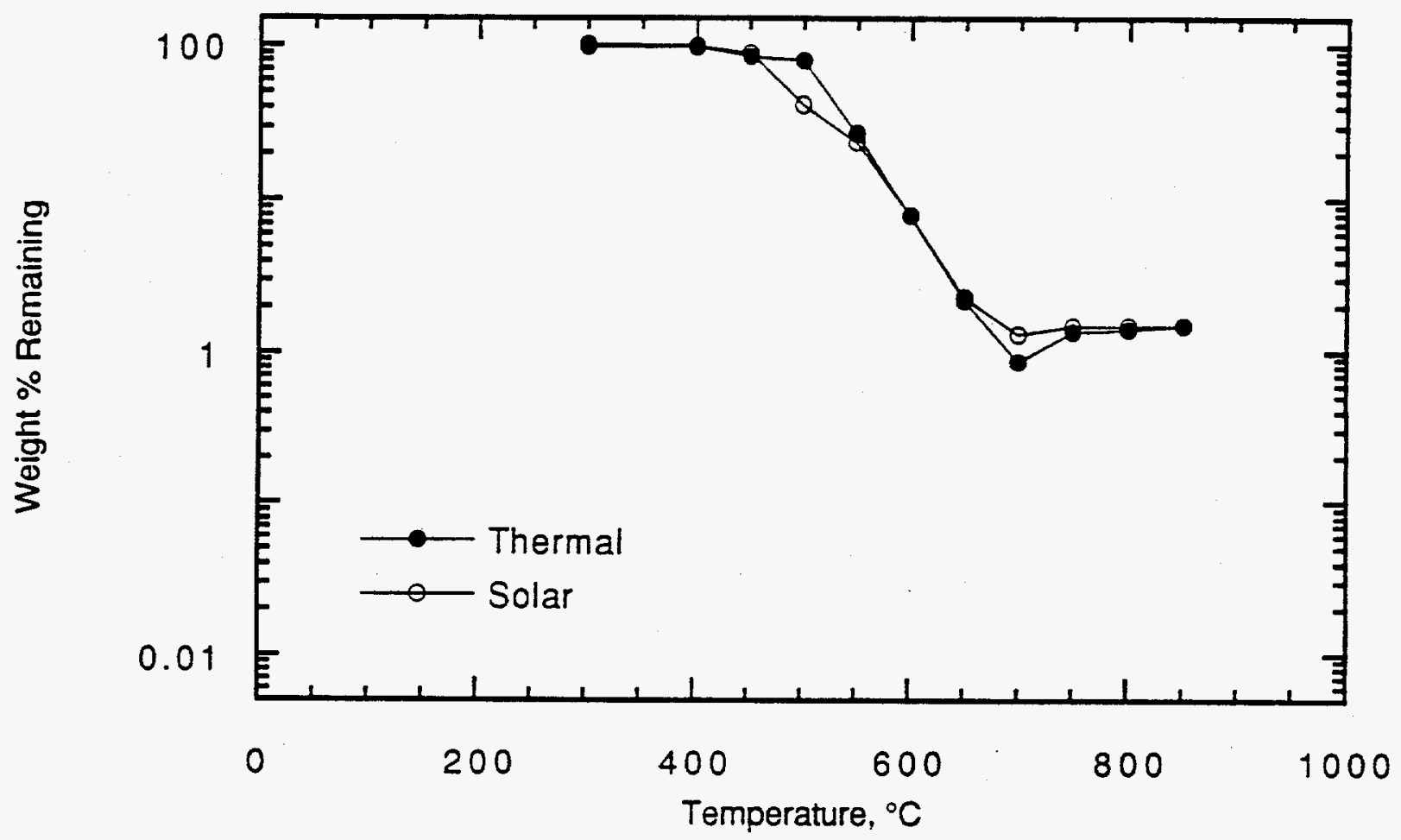

\begin{tabular}{|c|c|c|c|}
\hline Temp. & $\operatorname{fr}(0)$ & $\mathrm{fr}(10)$ & $\mathrm{A}(10)$ \\
\hline & & & \\
\hline 300 & 100 & 96.6 & 1.04 \\
\hline 400 & 97.7 & 95.6 & 1.02 \\
\hline 450 & 83.2 & 88.1 & 0.945 \\
\hline 500 & 78.5 & 40.8 & 1.92 \\
\hline 550 & 26.6 & 23.4 & 1.14 \\
\hline 600 & 7.78 & 7.83 & 0.994 \\
\hline 650 & 2.18 & 2.29 & 0.952 \\
\hline 700 & 0.863 & 1.30 & 0.664 \\
\hline 750 & 1.34 & 1.47 & 0.912 \\
\hline 800 & 1.39 & 1.47 & 0.946 \\
\hline 850 & 1.47 & 1.48 & 0.993 \\
\hline
\end{tabular}


Carbontetrachloride ( $100 \mathrm{ppm})$ And Trichloroethylene ( 100ppm) Components Of A Contaminated Fuel Oil-1 Exposed To 0 And 307 AM 1.0 Suns (Simulated) For $5 \mathrm{~s}$ In Air

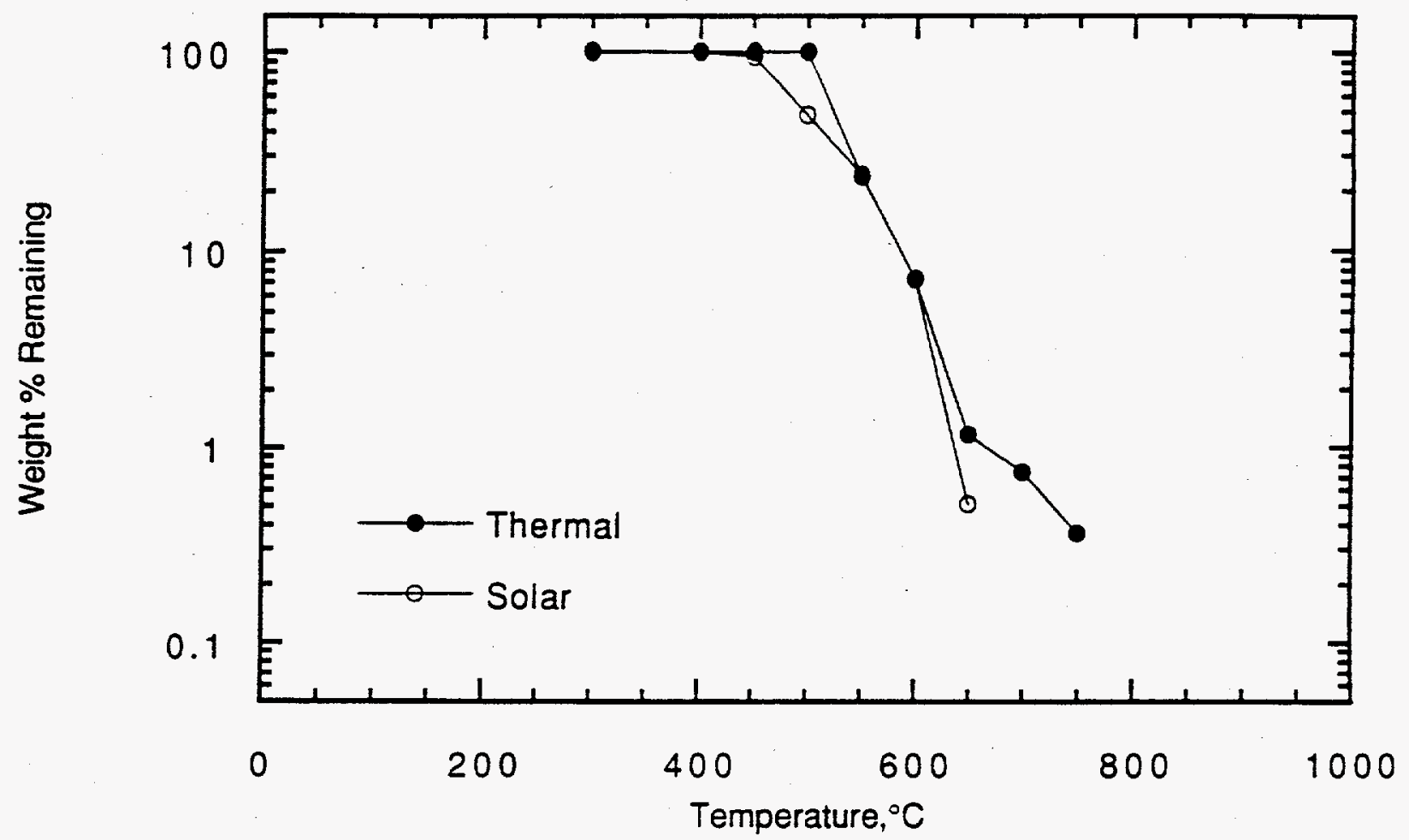

\begin{tabular}{|c|c|c|c|}
\hline Temp. & $\operatorname{fr}(0)$ & $\operatorname{fr}(10)$ & $\mathbf{R}(10)$ \\
\hline & & & \\
\hline 300 & 100 & 100 & 1.00 \\
\hline 400 & 100 & 100 & 1.00 \\
\hline 450 & 100 & 94.8 & 1.05 \\
\hline 500 & 99.5 & 47.8 & 2.08 \\
\hline 550 & 23.5 & 23.9 & 0.983 \\
\hline 600 & 7.27 & 7.22 & 1.01 \\
\hline 650 & 1.16 & 0.509 & 2.28 \\
\hline 700 & 0.741 & bdl & \\
\hline 750 & 0.361 & bdl & \\
\hline
\end{tabular}


Chloronitrobenzene ( $300 \mathrm{ppm})$ Component Of A Contaminated Fuel Oil-1 Exposed To 0 And 307 AM 1.0 Suns (Simulated) For $5 \mathrm{~s}$ in Air

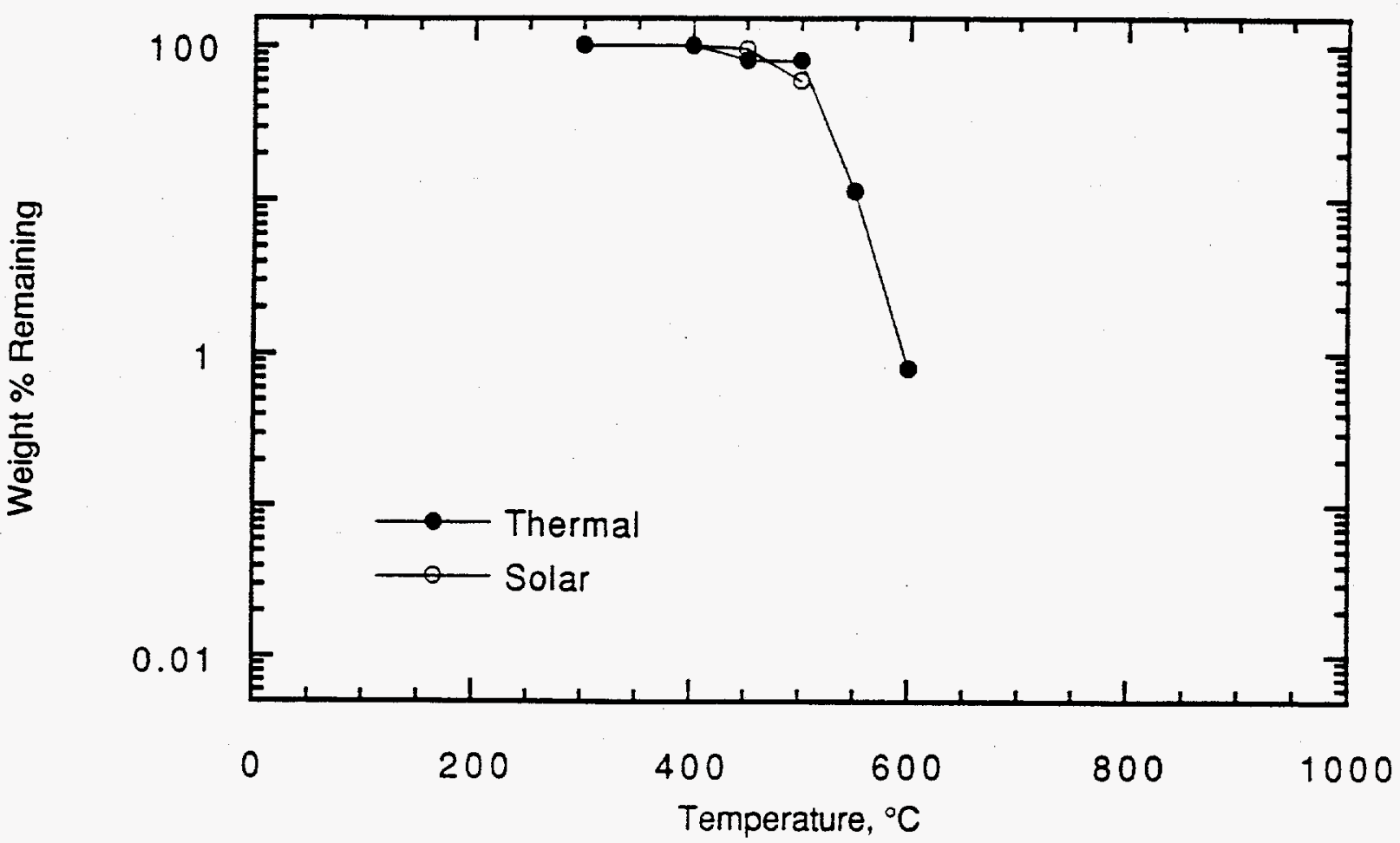

\begin{tabular}{|c|c|c|c|}
\hline Temp. & $\operatorname{fr}(0)$ & $\operatorname{fr}(10)$ & $\mathbf{R}(10)$ \\
\hline & & & \\
\hline 300 & 100 & 100 & 1.00 \\
\hline 400 & 100 & 99.0 & 1.01 \\
\hline 450 & 80.3 & 95.1 & .844 \\
\hline 500 & 80.0 & 58.9 & 1.36 \\
\hline 550 & 11.4 & bdl & \\
\hline 600 & 0.793 & bdl & \\
\hline
\end{tabular}


Dichlorobenzene ( $100 \mathrm{ppm})$ Component Of A Contaminated Fuel Oill-1 Exposed To 0 And 307 AM 1.0 Suns (Simulated) For 5s In Air

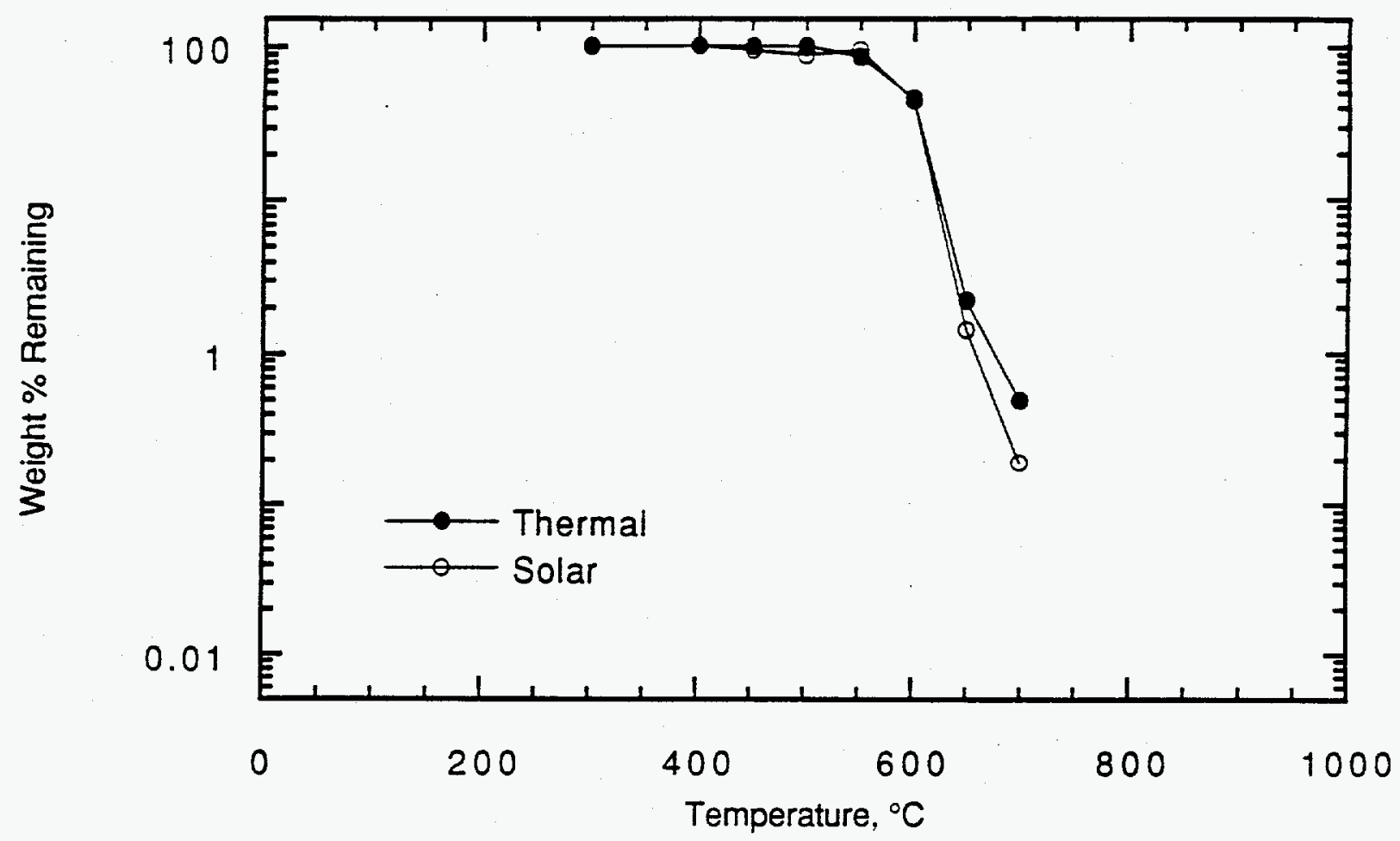

\begin{tabular}{|c|c|c|c|}
\hline Temp. & $\operatorname{fr}(0)$ & $\mathrm{fr}(10)$ & $\mathrm{R}(10)$ \\
\hline & & & \\
\hline 300 & 100 & 100 & 1.00 \\
\hline 400 & 100 & 100 & 1.00 \\
\hline 450 & 100 & 94.4 & 1.06 \\
\hline 500 & 100 & 87.6 & 1.14 \\
\hline 550 & 85.2 & 94.0 & 0.906 \\
\hline 600 & 45.2 & 43.7 & 1.03 \\
\hline 650 & 2.21 & 1.43 & 1.55 \\
\hline 700 & 0.494 & 0.191 & 2.59 \\
\hline
\end{tabular}


Parent Compounds and PICs Of A Contaminated Fuel Oil-la ( -1000ppm) Exposed To 0 And 307 AM 1.0 Suns (Simulated) For $5 s$ in Air

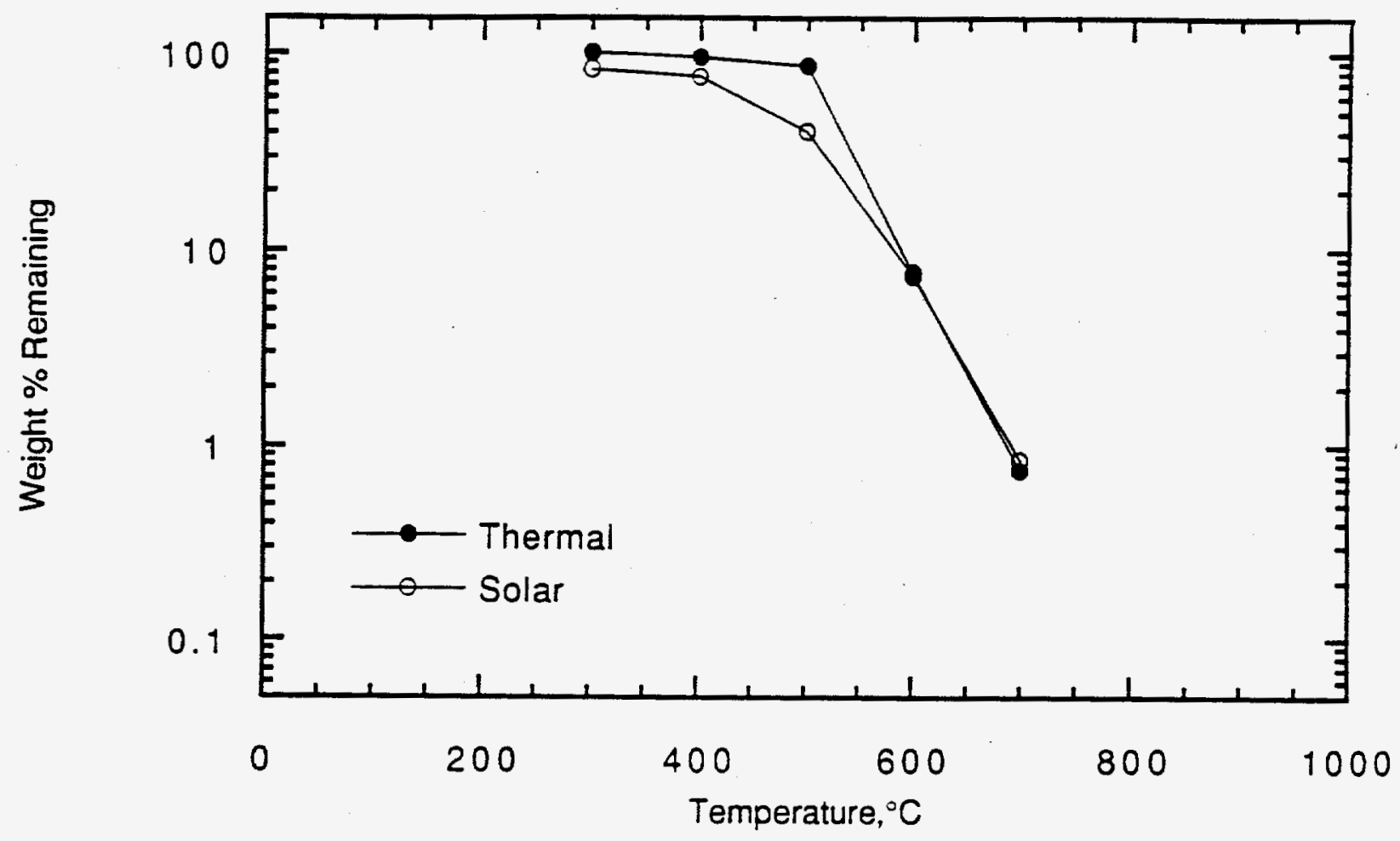

\begin{tabular}{|c|c|c|c|}
\hline Temp. & fr(0) & fr(10) & $\mathrm{R}(10)$ \\
\hline & & & \\
\hline 300 & 100 & 82.6 & 1.21 \\
\hline 400 & 94.7 & 75.4 & 1.26 \\
\hline 500 & 86.1 & 40.0 & 2.15 \\
\hline 600 & 7.69 & 7.39 & 1.04 \\
\hline 700 & 0.751 & 0.846 & 0.999 \\
\hline 800 & bdl & bdl & \\
\hline
\end{tabular}


Carbon Tetrachloride ( 100ppm) And Trichloroethylene ( 100ppm) Components Of A Contaminated Fuel Oil-la Exposed To 0 And 307 AM 1.0 Suns (Simulated) For $5 s$ in Air

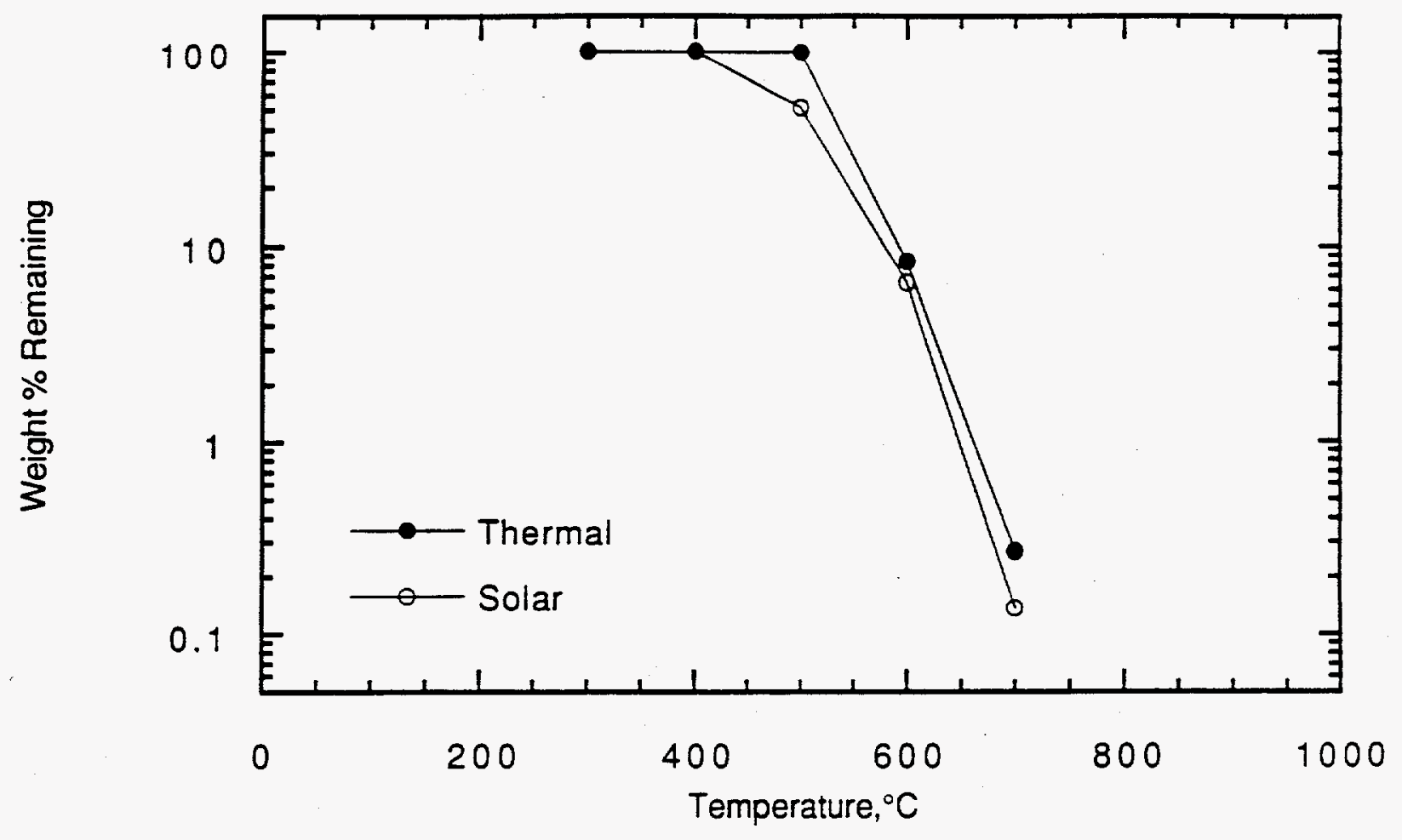

\begin{tabular}{|c|c|c|c|}
\hline Temp. & $\operatorname{fr}(0)$ & $\operatorname{fr}(10)$ & $\mathrm{R}(10)$ \\
\hline & & & \\
\hline 300 & 100 & 100 & 1.00 \\
\hline 400 & 100 & 100 & 1.00 \\
\hline 500 & 98.8 & 51.3 & 1.93 \\
\hline 600 & 8.32 & 6.48 & 1.28 \\
\hline 700 & 0.268 & 0.136 & 1.97 \\
\hline 800 & bdl & bdl & \\
\hline
\end{tabular}


Chloronitrobenzene ( 300ppm) Component Of A Contaminated Fuel Oil-la Exposed To 0 And 307 AM 1.0 Suns (Simulated) For 5 s in Air

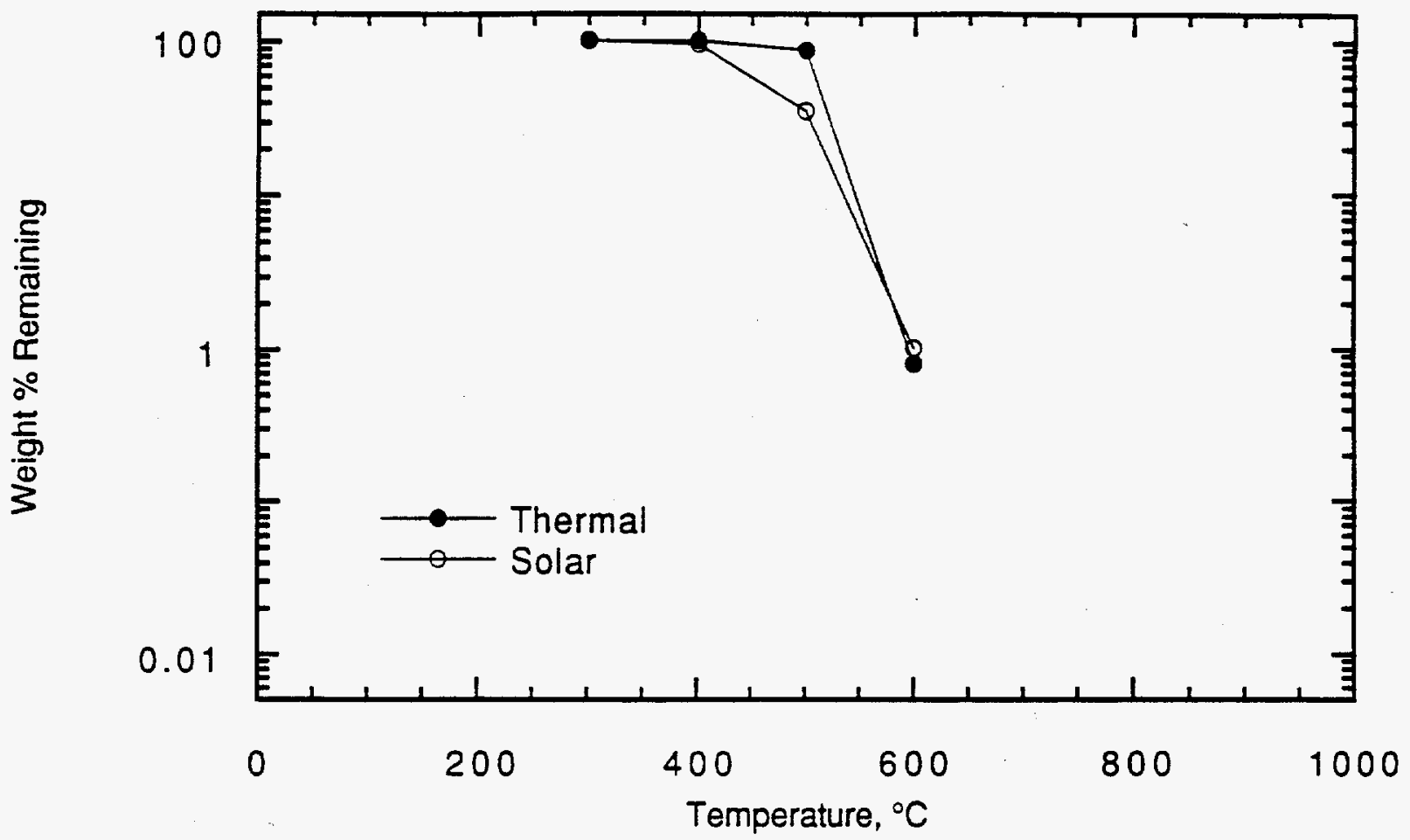

\begin{tabular}{|c|c|c|c|}
\hline Temp. & $\operatorname{tr}(0)$ & $\operatorname{tr}(10)$ & $\mathbf{R}(10)$ \\
\hline & & & \\
\hline 300 & 100 & 100 & 1.00 \\
\hline 400 & 100 & 94.8 & 1.00 \\
\hline 500 & 86.7 & 34.9 & 2.48 \\
\hline 600 & 0.800 & 1.01 & 0.792 \\
\hline 700 & bdl & bdl & \\
\hline 800 & bdl & bdl & \\
\hline
\end{tabular}


Dichlorobenzene ( 100ppm) Component Of A Contaminated Fuel Oil-la Exposed To 0 And 307 AM 1.0 Suns (Simulated) For $5 s$ In Air

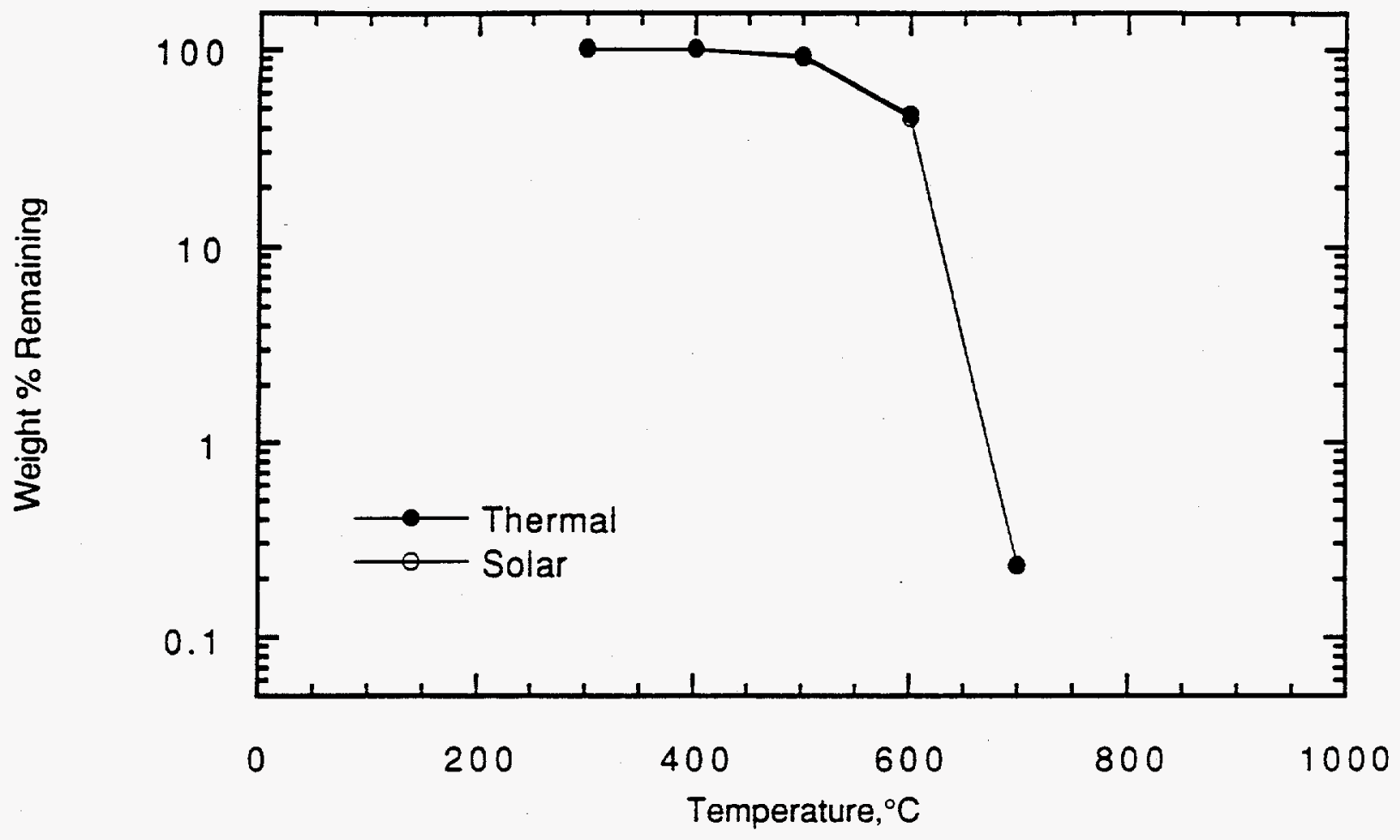

\begin{tabular}{|c|c|c|c|}
\hline Temp. & $\operatorname{fr}(0)$ & $\operatorname{fr}(10)$ & $R(10)$ \\
\hline & & & \\
\hline 300 & 100 & 100 & 1.00 \\
\hline 400 & 100 & 100 & 1.00 \\
\hline 500 & 93.0 & 89.6 & 1.04 \\
\hline 600 & 46.3 & 44.5 & 1.04 \\
\hline 700 & 0.232 & 0.231 & 1.00 \\
\hline 800 & bdl & bdl & \\
\hline
\end{tabular}


Nitronaphthalene ( 100ppm) Component Of A Contaminated Fuel Oil-la Exposed To 0 And 307 AM 1.0 Suns (Simulated) For $5 \mathrm{~s}$ In Air

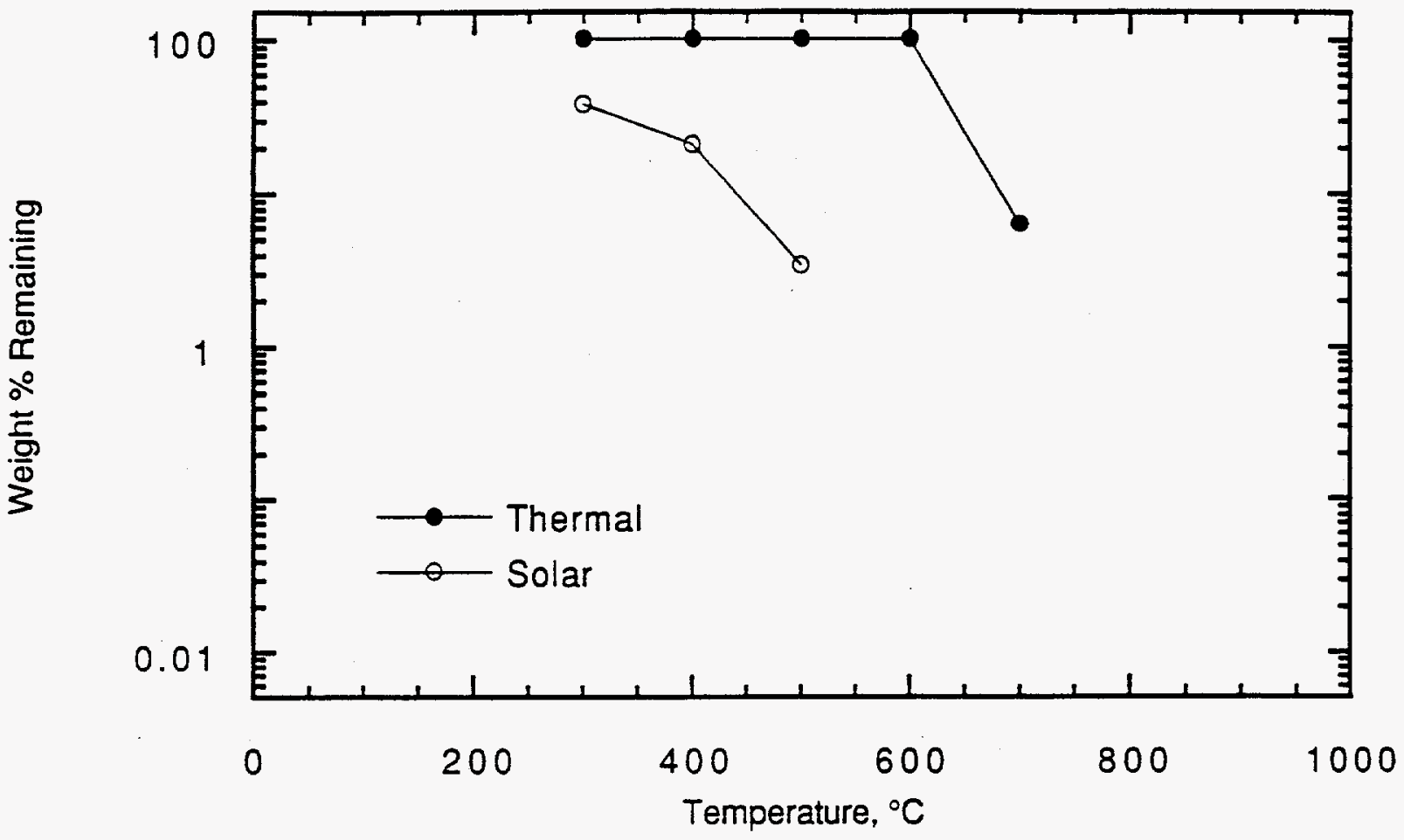

\begin{tabular}{|c|c|c|c|}
\hline Temp. & $\operatorname{fr}(0)$ & $\operatorname{fr}(10)$ & $\mathrm{R}(10)$ \\
\hline & & & \\
\hline 300 & 100 & 38.0 & 2.63 \\
\hline 400 & 100 & 20.9 & 4.78 \\
\hline 500 & 100 & 3.41 & 29.3 \\
\hline 600 & 6.29 & bdl & \\
\hline 700 & bdl & bdl & \\
\hline 800 & bdl & bdl & \\
\hline
\end{tabular}


Parent Compounds And PICs Of A Contaminated Fuel Oil-lb ( 1000ppm)

Exposed To 0 And 307 AM 1.0 Suns (Simulated) For $5 \mathrm{~s}$ in Air

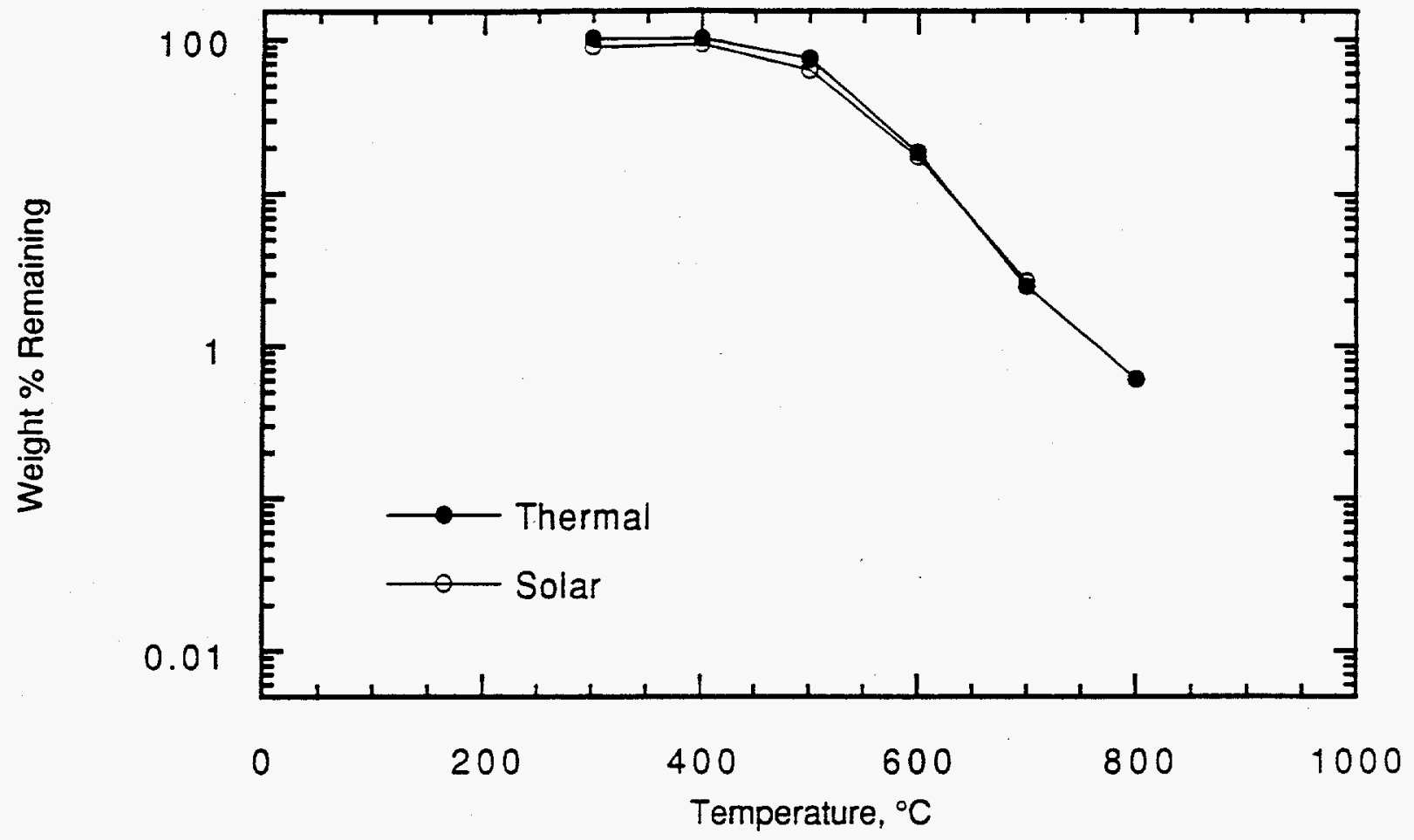

\begin{tabular}{|c|c|c|c|}
\hline Temp. & $\operatorname{fr}(0)$ & $\operatorname{fr}(10)$ & $\mathbf{R}(10)$ \\
\hline & & & \\
\hline 300 & 100 & 88.2 & 1.13 \\
\hline 400 & 100 & 91.4 & 1.09 \\
\hline 500 & 74.0 & 62.7 & 1.18 \\
\hline 600 & 18.4 & 17.3 & 1.06 \\
\hline 700 & 2.47 & 2.66 & 0.929 \\
\hline 800 & 0.604 & bdl & \\
\hline
\end{tabular}


Bromonaphthalene ( 200ppm) Component Of A Contaminated Fuel Oil-1b Exposed To 0 And 307 AM 1.0 Suns (Simulated) For 5 s In Air

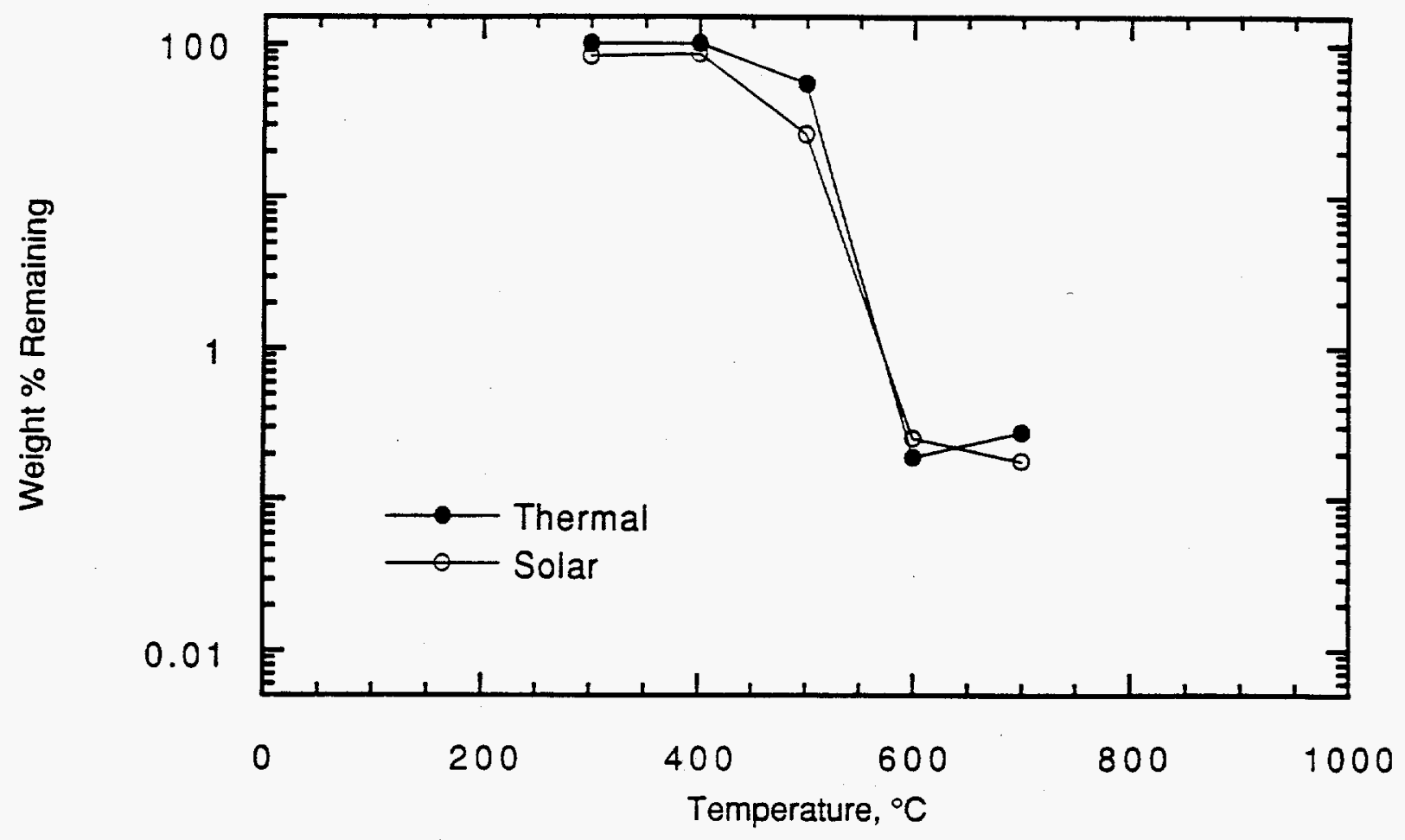

\begin{tabular}{|c|c|c|c|}
\hline Temp. & $\operatorname{fr}(0)$ & $\operatorname{fr}(10)$ & $R(10)$ \\
\hline & & & \\
\hline 300 & 100 & 82.5 & 1.21 \\
\hline 400 & 100 & 86.0 & 1.16 \\
\hline 500 & 54.9 & 35.9 & 1.53 \\
\hline 600 & 0.189 & 0.250 & 0.756 \\
\hline 700 & 0.272 & 0.177 & 1.54 \\
\hline 800 & bdl & bdl & \\
\hline
\end{tabular}


Carbontetrachloride ( 100ppm) And Trichloroethylene ( 100ppm) Components Of A Contaminated Fuel Oil-lb Exposed To 0 And 307 AM 1.0 Suns (Simulated) For $5 s$ In Air

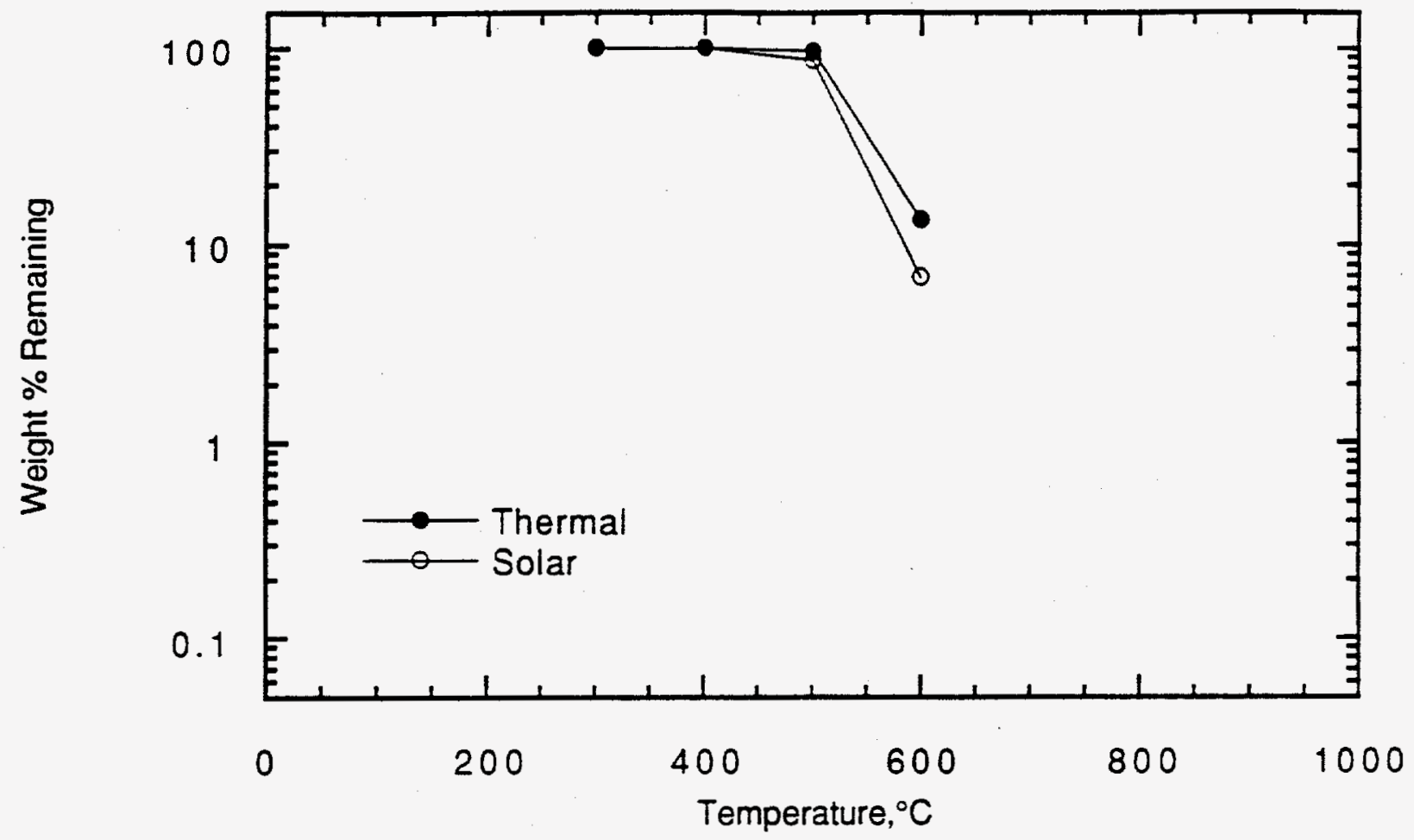

\begin{tabular}{|c|c|c|c|}
\hline Temp. & $\operatorname{tr}(0)$ & $\operatorname{fr}(10)$ & $R(10)$ \\
\hline & & & \\
\hline 300 & 100 & 100 & 1.00 \\
\hline 400 & 100 & 100 & 1.00 \\
\hline 500 & 96.1 & 86.3 & 1.11 \\
\hline 600 & 13.4 & 6.94 & 1.93 \\
\hline 700 & bdl & bdl & \\
\hline 800 & bdl & bdl & \\
\hline
\end{tabular}


Chloronitrobenzene ( 300ppm) Component Of A Contaminated Fuel Oil-1b Exposed To 0 And 307 AM 1.0 Suns (Simulated) For $5 s$ In Air

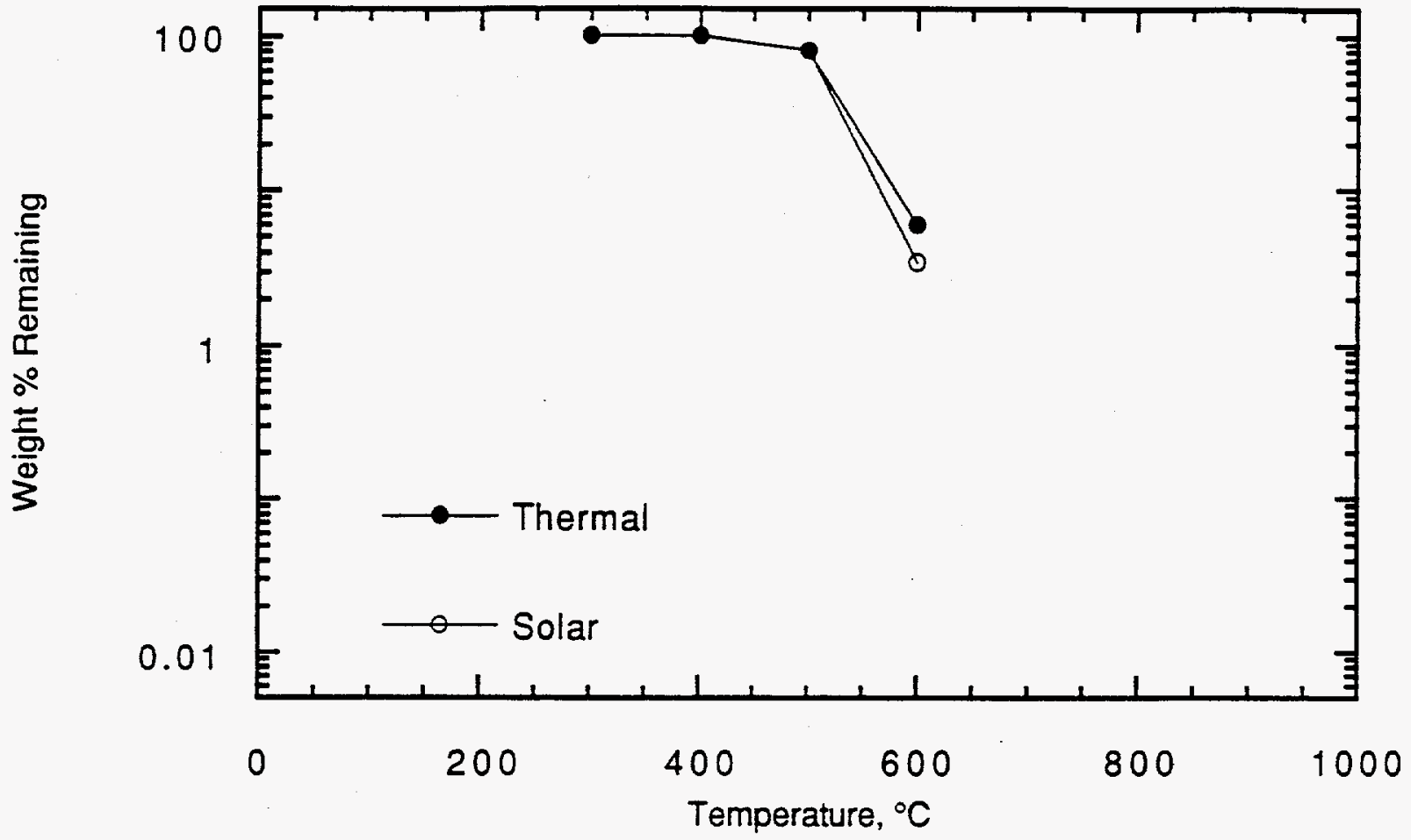

\begin{tabular}{|c|c|c|c|}
\hline Temp. & $\operatorname{fr}(0)$ & $\operatorname{fr}(10)$ & $R(10)$ \\
\hline & & & \\
\hline 300 & 100 & 100 & 1.00 \\
\hline 400 & 100 & 100 & 1.00 \\
\hline 500 & 80.6 & 79.7 & 1.01 \\
\hline 600 & 6.00 & 3.44 & 1.74 \\
\hline 700 & bdl & bdl & \\
\hline 800 & bdl & bdl & \\
\hline
\end{tabular}


Dichlorobenzene ( 100ppm) Component Of A Contaminated Fuel Oil-lb Exposed To 0 And 307 AM 1.0 Suns (Simulated) For 5s In Air

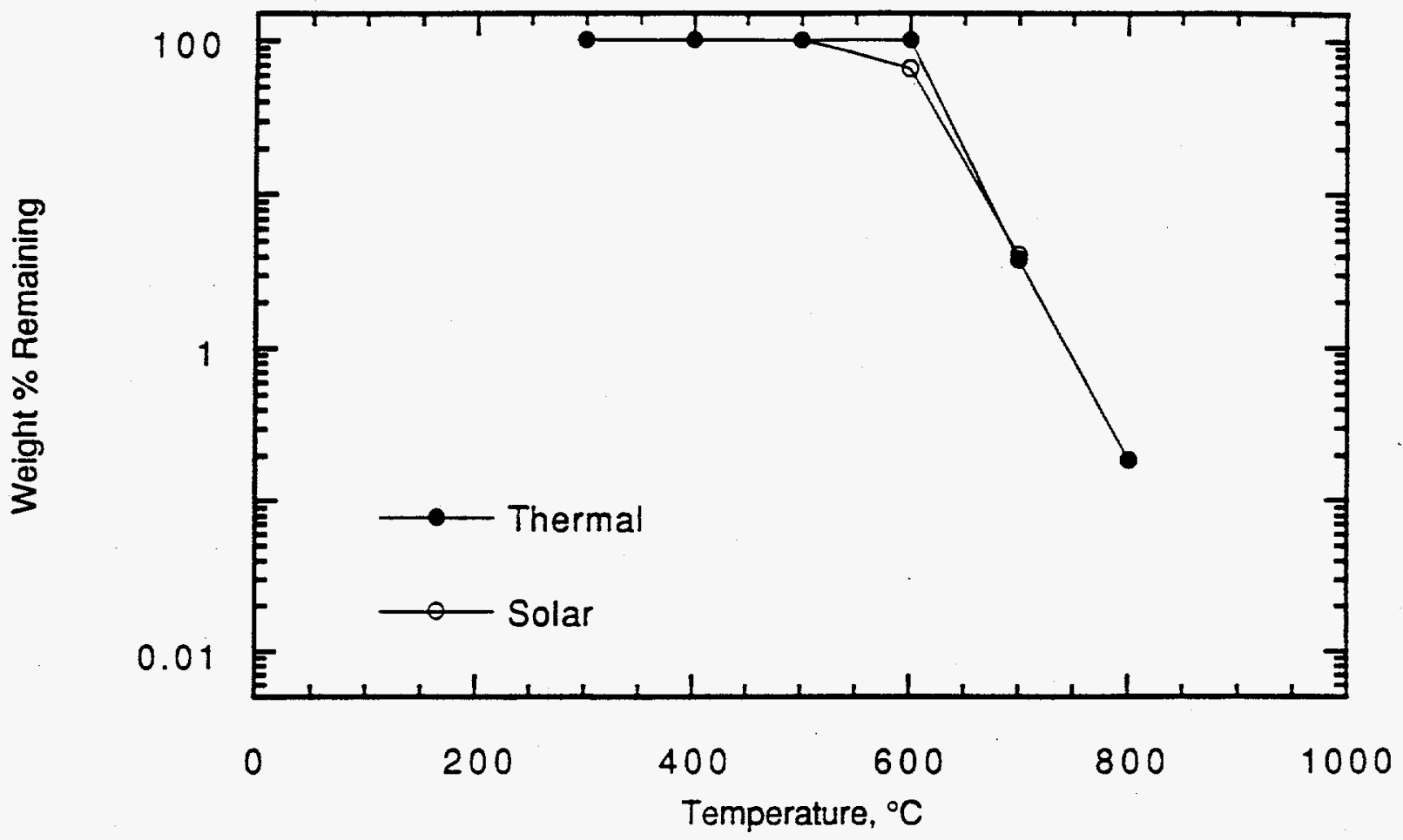

\begin{tabular}{|c|c|c|c|}
\hline Temp. & $\operatorname{tr}(0)$ & $\operatorname{tr}(10)$ & $R(10)$ \\
\hline & & & \\
\hline 300 & 100 & 100 & 1.00 \\
\hline 400 & 100 & 100 & 1.00 \\
\hline 500 & 100 & 100 & 1.00 \\
\hline 600 & 100 & 65.7 & 1.52 \\
\hline 700 & 3.77 & 4.03 & 0.935 \\
\hline 800 & 0.183 & bdl & \\
\hline
\end{tabular}


Parent Compounds And PICs ( 1000ppm) Of A Contaminated Fuel Oil-1I

Exposed To 0 And 273 AM 1.5 Suns (Simulated) For 10s In Air

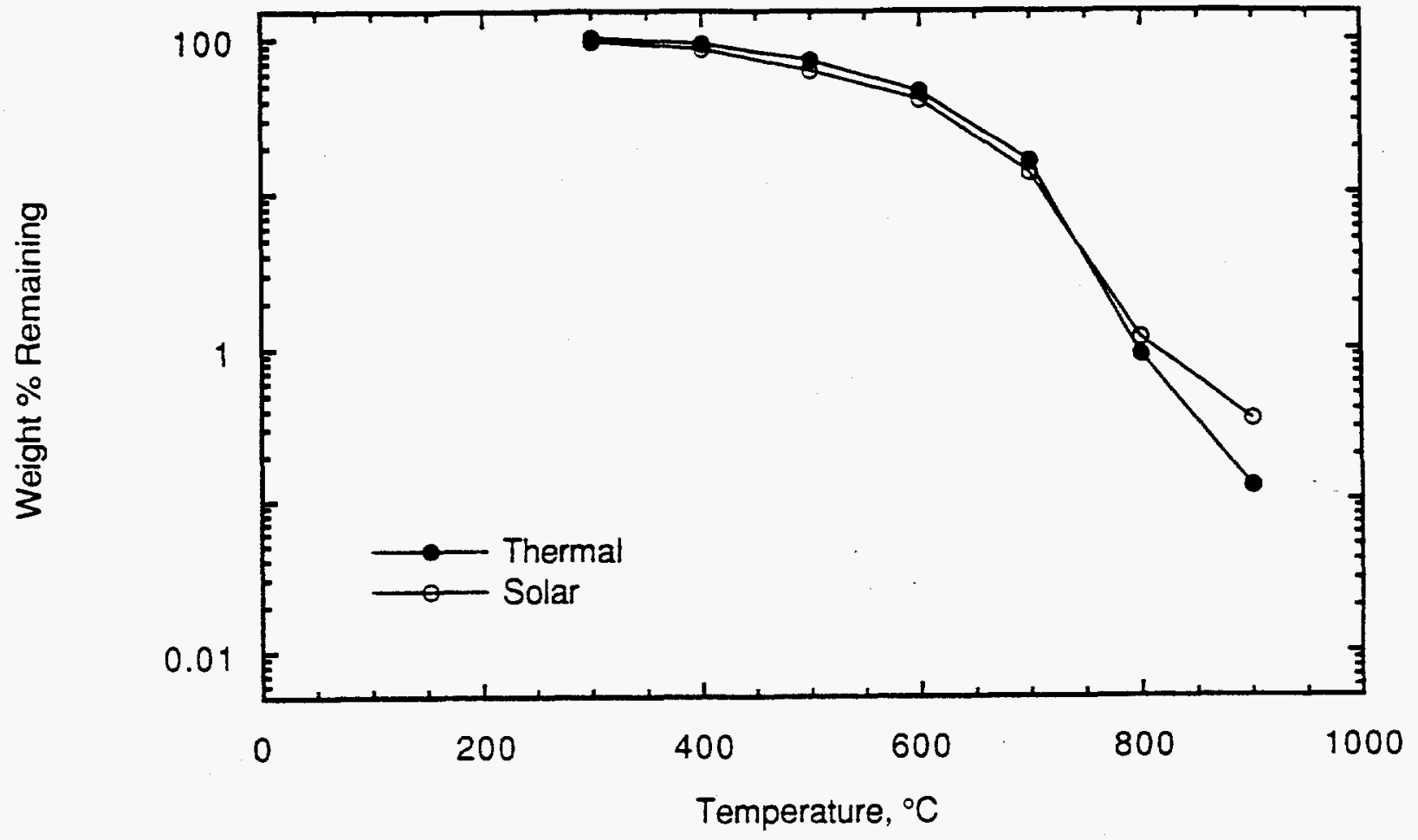

\begin{tabular}{|c|c|c|c|}
\hline Temp. & $\operatorname{fr}(0)$ & $\operatorname{fr}(10)$ & $\mathrm{R}(10)$ \\
\hline & & & \\
\hline 300 & 100 & 94.9 & 1.05 \\
\hline 400 & 91.5 & 85.2 & 1.07 \\
\hline 500 & 71.9 & 61.2 & 1.17 \\
\hline 600 & 45.0 & 40.0 & 1.13 \\
\hline 700 & 15.9 & 13.3 & 1.20 \\
\hline 800 & 0.891 & 1.15 & 0.775 \\
\hline 900 & 0.122 & 0.336 & 0.363 \\
\hline
\end{tabular}


Dichlorobenzene ( 400ppm) Component Of A Contaminated Fuel Oil- 11

Exposed To 0 And 273 AM 1.5 Suns (Simulated) For 10s In Air

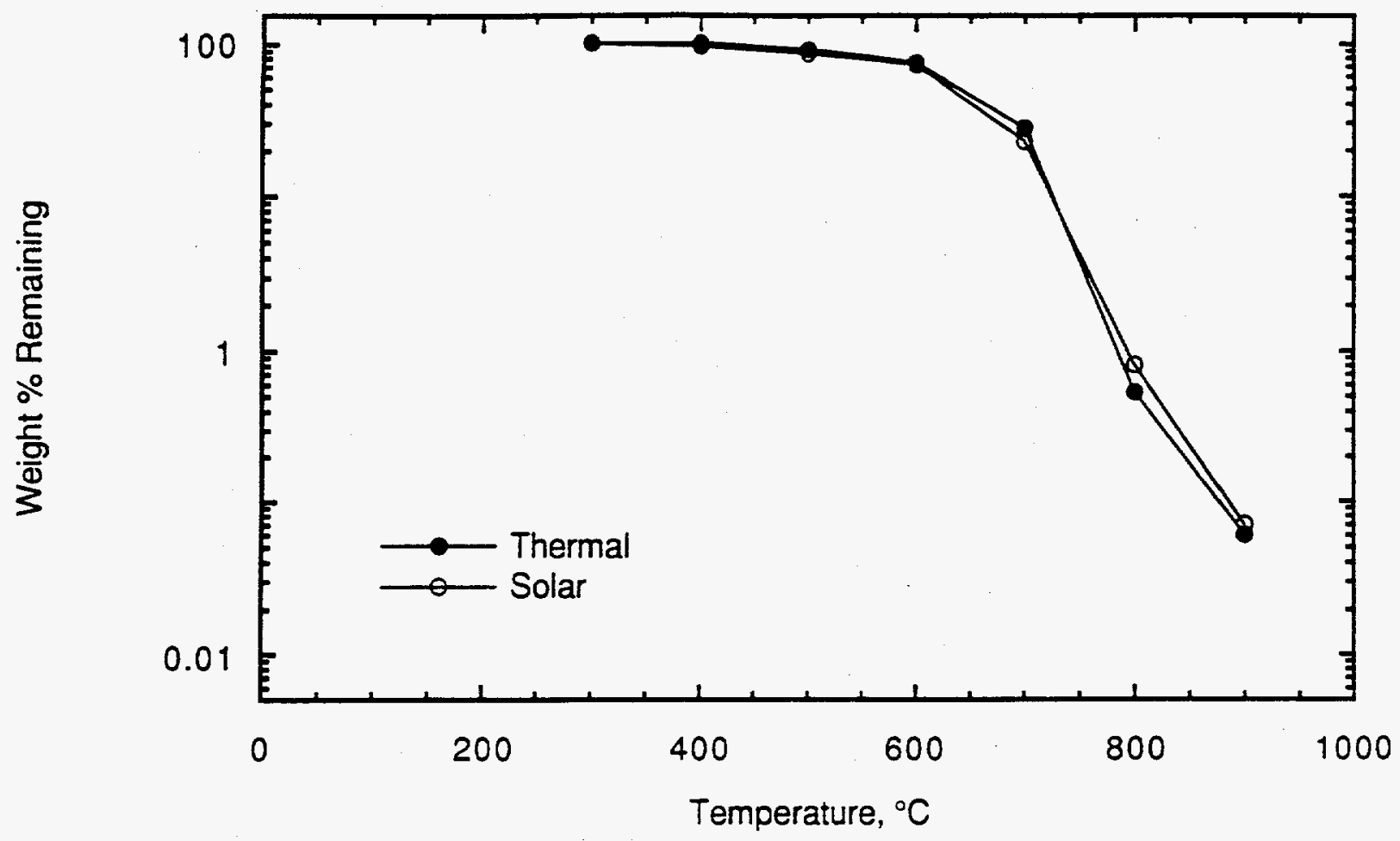

\begin{tabular}{|c|c|c|c|}
\hline Temp. & $\operatorname{fr}(0)$ & $\operatorname{fr}(10)$ & $\mathbf{R}(10)$ \\
\hline & & & \\
\hline 300 & 100 & 99.6 & 1.00 \\
\hline 400 & 100 & 94.5 & 1.06 \\
\hline 500 & 90.7 & 85.0 & 1.07 \\
\hline 600 & 73.5 & 71.1 & 1.03 \\
\hline 700 & 27.5 & 22.5 & 1.22 \\
\hline 800 & 0.527 & 0.802 & 0.657 \\
\hline 900 & 0.0563 & 0.0679 & 0.829 \\
\hline
\end{tabular}


Naphthalene ( 50ppm) Component Of A Contaminated Fuel Oil-II Exposed To 0 And 273 AM 1.5 Suns (Simulated) For 10s In Air

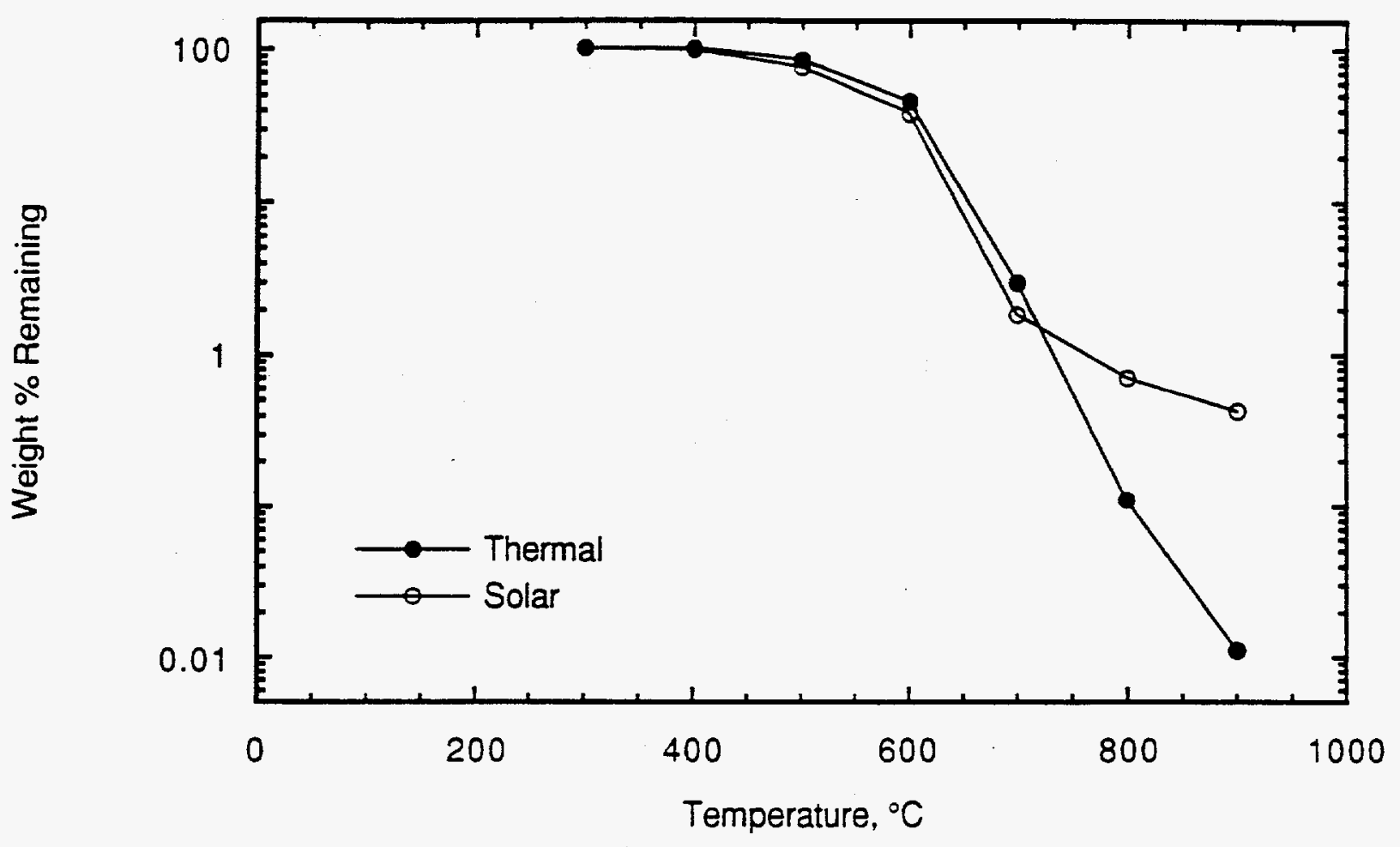

\begin{tabular}{|c|c|c|c|}
\hline Temp. & $\operatorname{fr}(0)$ & $\operatorname{fr}(10)$ & $\mathrm{R}(10)$ \\
\hline & & & \\
\hline 300 & 100 & 100 & 1.00 \\
\hline 400 & 100 & 97.2 & 1.03 \\
\hline 500 & 84.1 & 74.8 & 1.12 \\
\hline 600 & 45.2 & 37.6 & 1.20 \\
\hline 700 & 2.94 & 1.82 & 1.61 \\
\hline 800 & 0.114 & 0.688 & 0.166 \\
\hline 900 & 0.108 & 0.417 & 0.259 \\
\hline
\end{tabular}


Toluene ( $50 \mathrm{ppm})$ Component Of A Contaminated Fuel Oil-II

Exposed To 0 And 273 AM 1.5 Suns (Simulated) For $10 \mathrm{~s}$ in Air

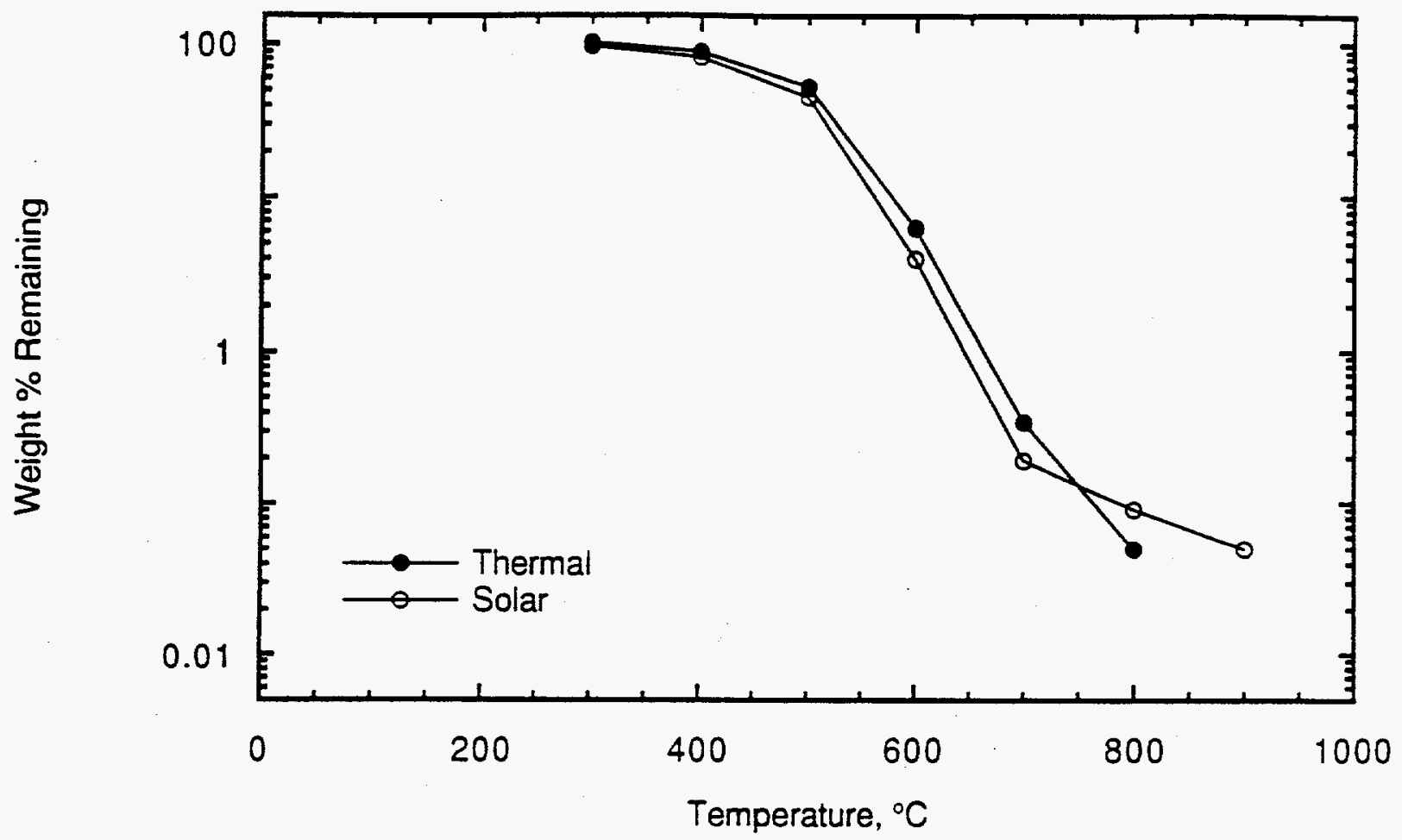

\begin{tabular}{|c|c|c|c|}
\hline Temp. & $\operatorname{fr}(0)$ & $\operatorname{fr}(10)$ & $\mathbf{R}(10)$ \\
\hline & & & \\
\hline 300 & 100 & 96.1 & 1.04 \\
\hline 400 & 87.4 & 80.7 & 1.08 \\
\hline 500 & 51.6 & 44.3 & 1.16 \\
\hline 600 & 6.30 & 3.95 & 1.60 \\
\hline 700 & 0.343 & 0.193 & 1.78 \\
\hline 800 & 0.0508 & 0.0909 & 0.559 \\
\hline 900 & bdl & 0.0453 & \\
\hline
\end{tabular}


Benzene From A Contaminated Fuel Oil-1I

Exposed To 0 And 273 AM 1.5 Suns (Simulated) For $10 \mathrm{~s}$ in Air

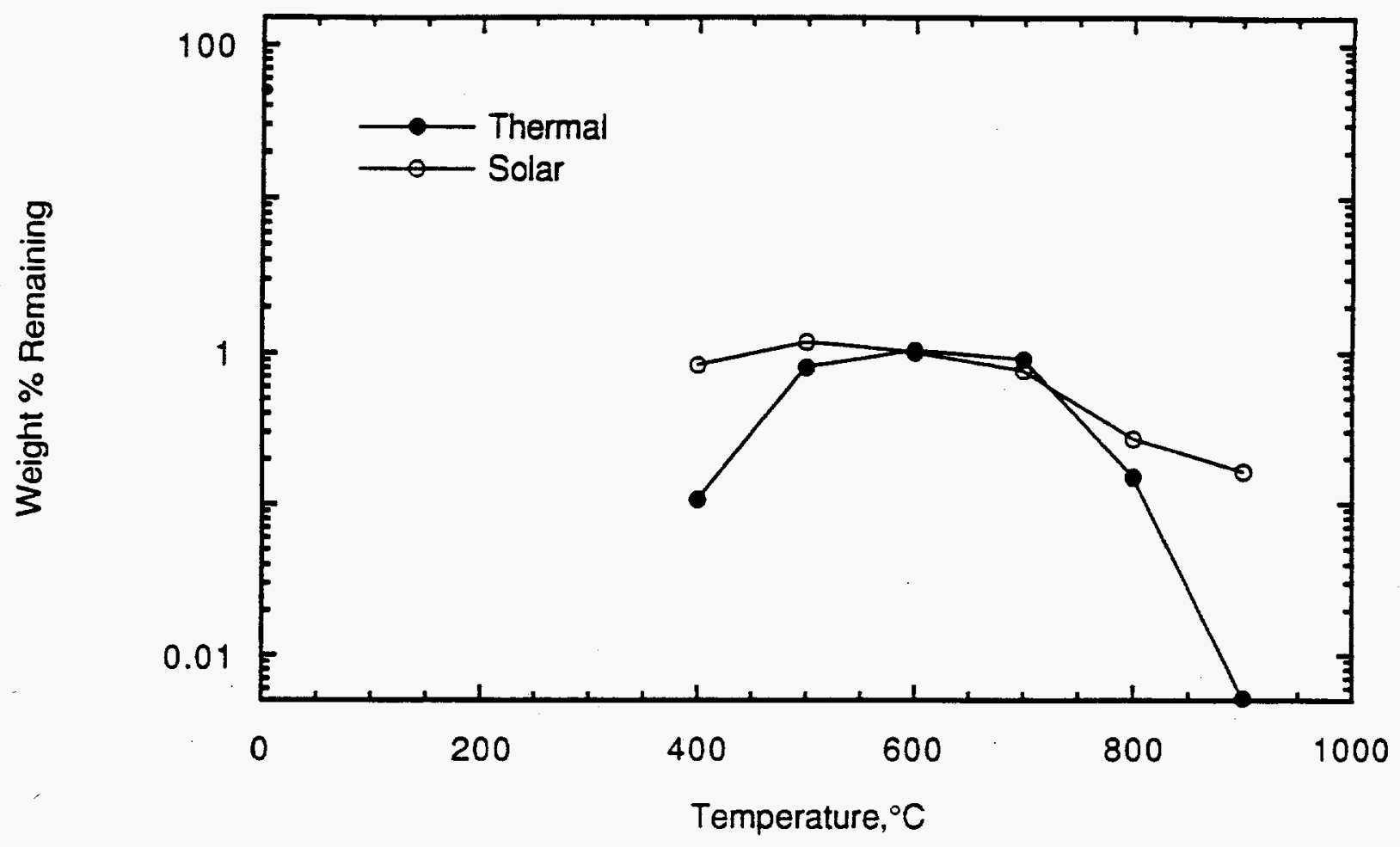

\begin{tabular}{|c|c|c|}
\hline Temp. & $\operatorname{fr}(0)$ & $\operatorname{fr}(10)$ \\
\hline & & \\
\hline 400 & .106 & .828 \\
\hline 500 & .795 & 1.16 \\
\hline 600 & 1.03 & .990 \\
\hline 700 & .894 & .745 \\
\hline 800 & .148 & .264 \\
\hline 900 & .00513 & .161 \\
\hline & & \\
\hline
\end{tabular}


Chlorobenzene From A Contaminated Fuel Oil-11

Exposed To 0 And 273 AM 1.5 Suns (Simulated) For $10 \mathrm{~s}$ in Air

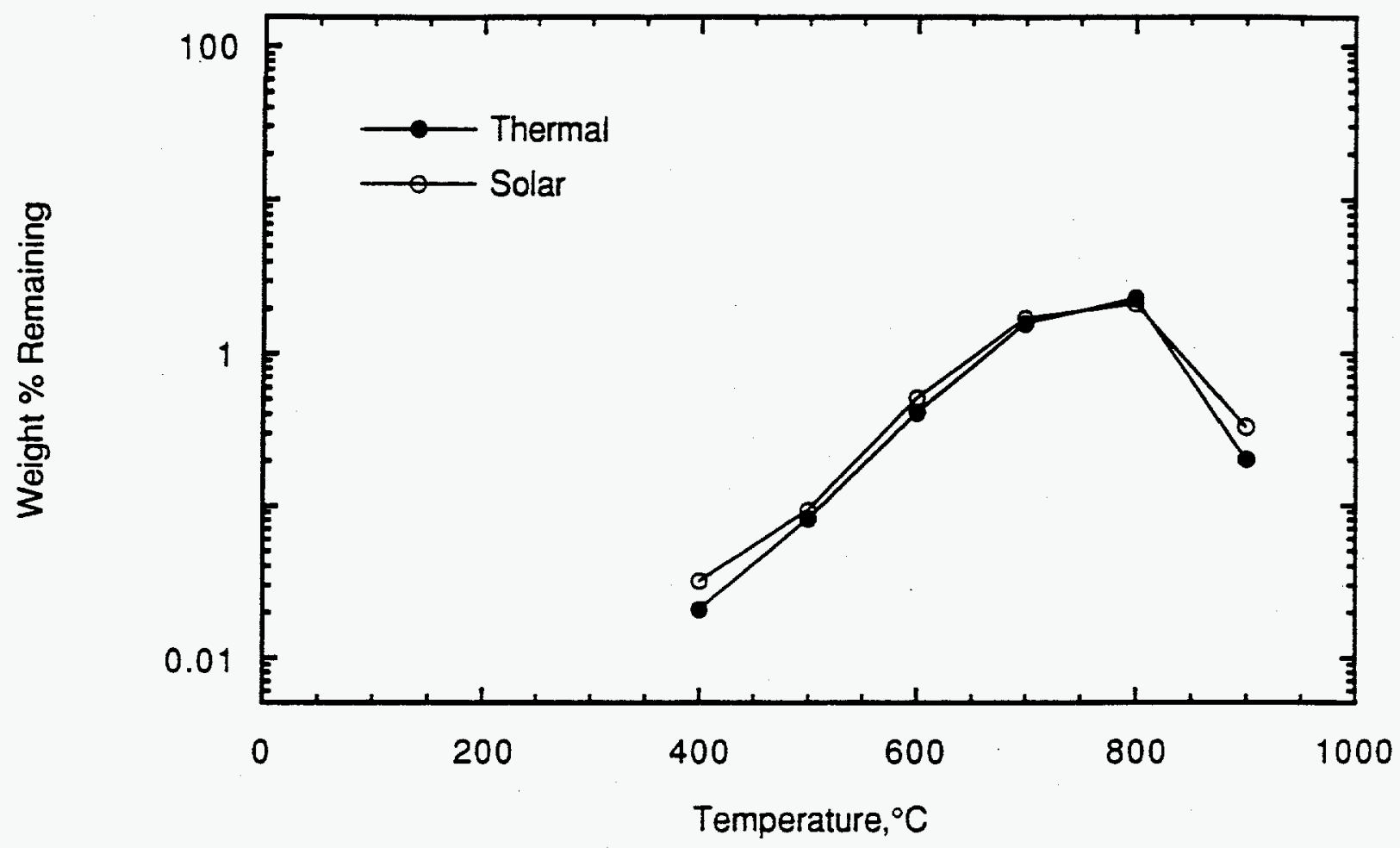

\begin{tabular}{|c|c|c|}
\hline Temp. & $\operatorname{fr}(0)$ & $\operatorname{fr}(10)$ \\
\hline & & \\
\hline 300 & .0205 & .0318 \\
\hline 400 & .0809 & .0925 \\
\hline 500 & .404 & .501 \\
\hline 600 & 1.56 & 1.69 \\
\hline 700 & 2.28 & 2.10 \\
\hline 800 & .200 & .325 \\
\hline 900 & .00348 & .0301 \\
\hline
\end{tabular}




\section{APPENDIX IV \\ Thermal/Photolytic Destruction Involving $280 \mathrm{~nm}$ Laser Excitation}

This Appendix summarizes all of the data obtained during this project using $280 \mathrm{~nm}$ pulsed laser radiation as the illumination source. Recall that at this wavelength only the first excited singlet state is assessed in most molecules, so the resulting photochemical reactions should be comparable to those induced by broad band solar radiation with wavelengths greater than $300 \mathrm{~nm}$.

Unless otherwise noted, temperature is given in degrees Centigrade and fraction remaining is in mole percent normalized by the original amount of sample fed to the reactor. By definition, enhancement ratio is unitless. 
Monochlorobenzene $\left(2.95 \times 10^{-5} \mathrm{M}\right)$

Exposed To 0 and $883 \mathrm{~mW} / \mathrm{cm}^{2}$ Of $280 \mathrm{~nm}$ Laser Radiation For $10 \mathrm{~s}$ In Air

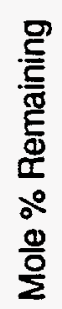

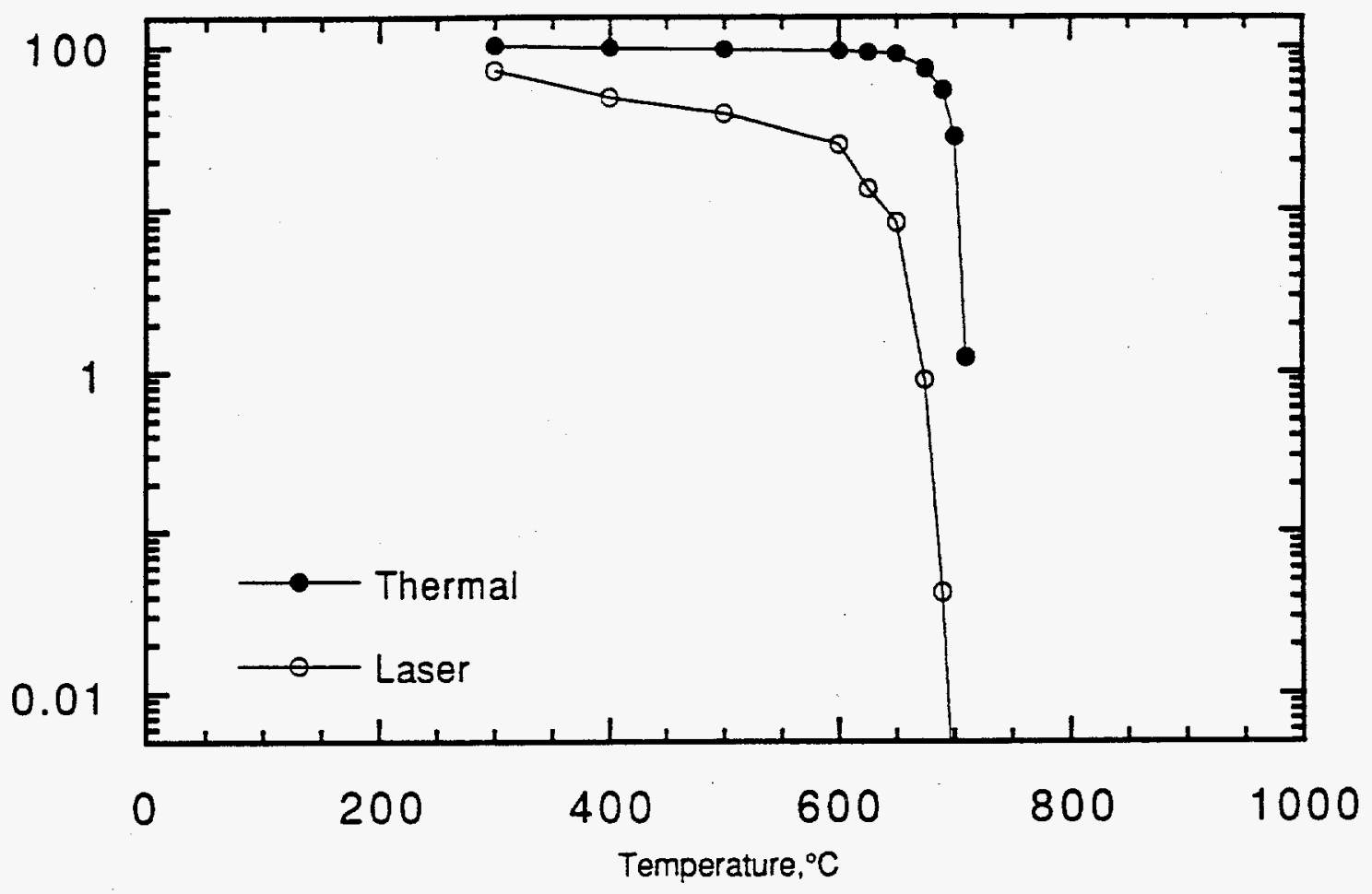

\begin{tabular}{|c|c|c|c|}
\hline Temp & fr(0) & $f r(10)$ & $\mathrm{R}(10)$ \\
\hline 300 & 100 & 69.8 & 1.43 \\
\hline 400 & 96.6 & 47.8 & 2.02 \\
\hline 500 & 94.9 & 38.5 & 2.47 \\
\hline 600 & 92.9 & 24.7 & 3.76 \\
\hline 625 & 90.7 & 13.5 & 6.72 \\
\hline 650 & 88.6 & 8.36 & 10.6 \\
\hline 675 & 71.6 & 0.895 & 80.0 \\
\hline 690 & 52.7 & 0.0420 & 1240 \\
\hline 700 & 27.6 & 0.00200 & 13700 \\
\hline 710 & 1.23 & bdl & \\
\hline
\end{tabular}


Monochlorobenzene $\left(2.95 \times 10^{-5} \mathrm{M}\right)$

Exposed To 0 and $2400 \mathrm{~mW} / \mathrm{cm}^{2}$ of $280 \mathrm{~nm}$ Laser Radiation For $10 \mathrm{~s}$ In Helium

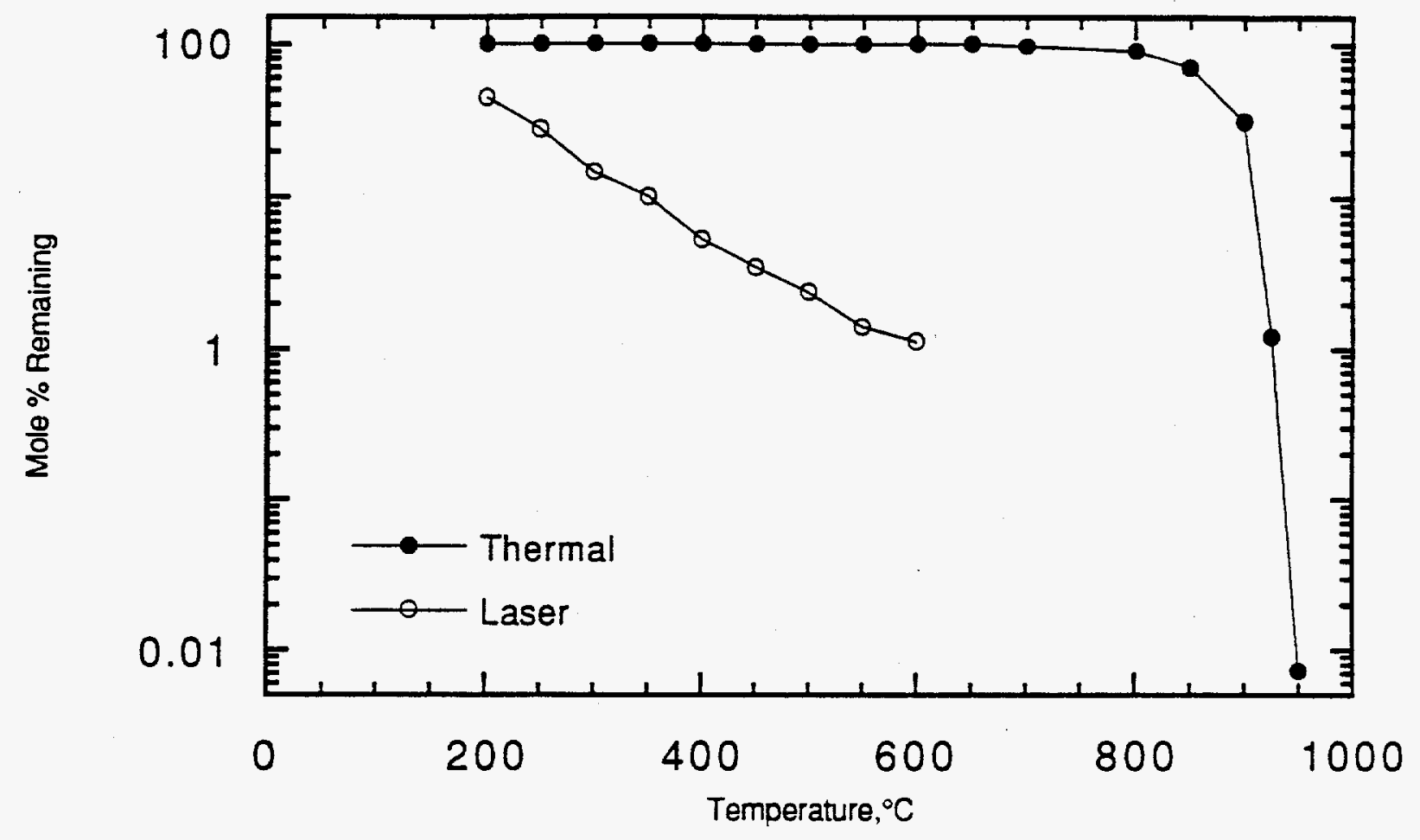

\begin{tabular}{|c|c|c|c|}
\hline Temp & $f(0)$ & $f r(10)$ & $R(10)$ \\
\hline 200 & 100 & 44.3 & 2.26 \\
\hline 250 & 100 & 27.8 & 3.60 \\
\hline 300 & 100 & 14.7 & 6.80 \\
\hline 350 & 100 & 10.2 & 9.80 \\
\hline 400 & 100 & 5.37 & 18.6 \\
\hline 450 & 100 & 3.50 & 28.6 \\
\hline 500 & 100 & 2.40 & 41.7 \\
\hline 550 & 100 & 1.41 & 70.9 \\
\hline 600 & 100 & 1.12 & 89.3 \\
\hline 650 & 100 & no data & \\
\hline 700 & 96.5 & & \\
\hline 800 & 89.1 & & \\
\hline 850 & 69.2 & & \\
\hline 900 & 31.0 & & \\
\hline 925 & 1.20 & & \\
\hline 950 & .00700 & & \\
\hline & & & \\
\hline
\end{tabular}


Monochlorobenzene $\left(2.95 \times 10^{-5} \mathrm{M}\right)$

Exposed To $280 \mathrm{~nm}$ Laser Radiation At $600^{\circ} \mathrm{C}$ For $10 \mathrm{~s}$ In Helium

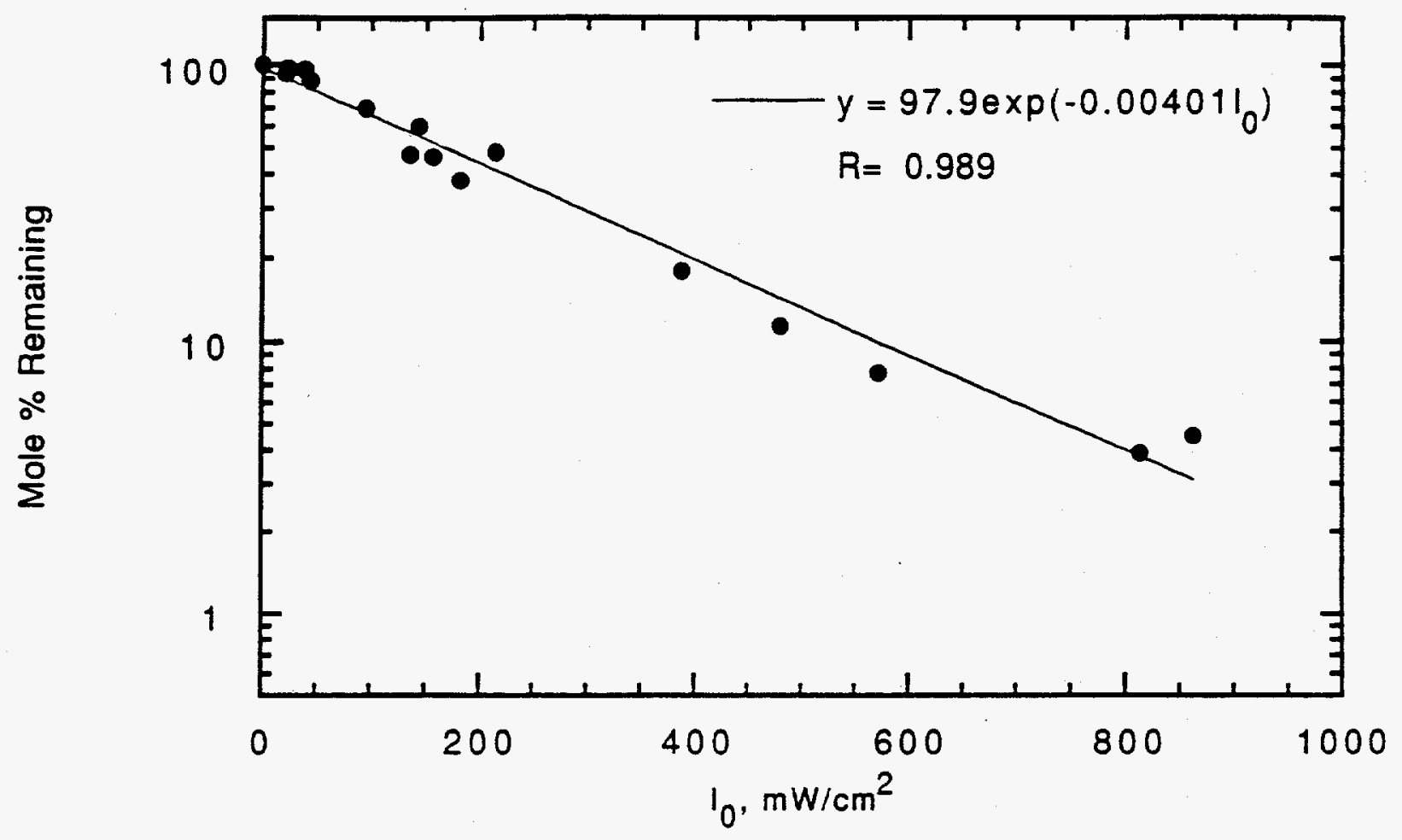

\begin{tabular}{|c|c|c|}
\hline 10 & fr(lo) & $\mathrm{R}(10)$ \\
\hline 0 & 100 & 1.00 \\
\hline 21.7 & 93.1 & 1.07 \\
\hline 23.6 & 97.5 & 1.03 \\
\hline 24.8 & 96.9 & 1.03 \\
\hline 38.9 & 96.0 & 1.04 \\
\hline 44.0 & 87.4 & 1.14 \\
\hline 44.8 & 87.6 & 1.14 \\
\hline 95.5 & 69.7 & 1.44 \\
\hline 136 & 47.1 & 2.12 \\
\hline 144 & 59.8 & 1.67 \\
\hline 157 & 46.2 & 2.17 \\
\hline 182 & 37.9 & 2.64 \\
\hline 215 & 48.0 & 2.08 \\
\hline 388 & 17.9 & 5.59 \\
\hline 480 & 11.3 & 8.85 \\
\hline 572 & 7.65 & 13.1 \\
\hline 813 & 3.87 & 25.8 \\
\hline 862 & 4.48 & 22.3 \\
\hline
\end{tabular}




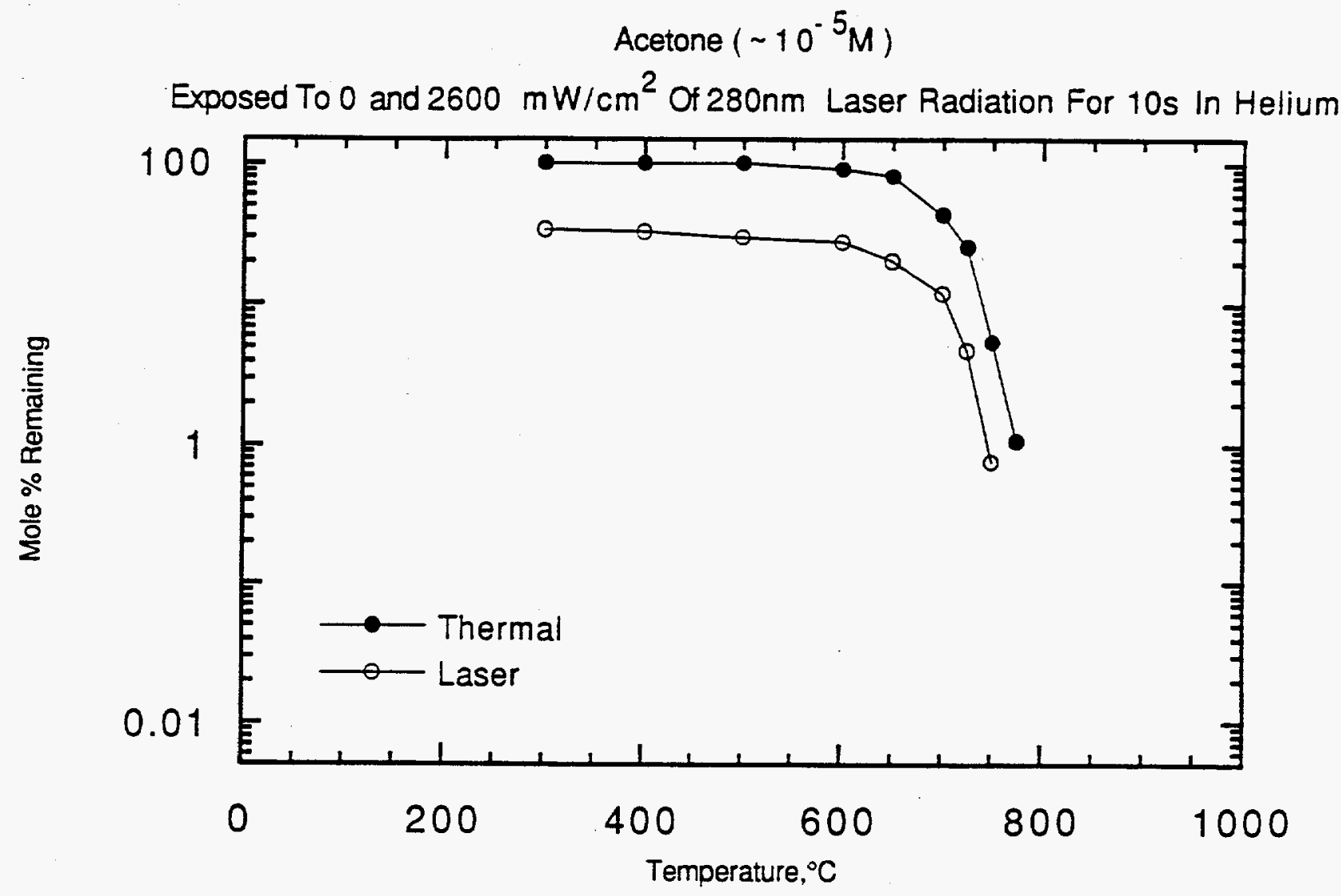

\begin{tabular}{|c|c|c|c|}
\hline Temp & fr(0) & fr $(10)$ & R(10) \\
\hline 300 & 100 & 33.5 & 2.99 \\
\hline 400 & 100 & 32.4 & 3.09 \\
\hline 500 & 100 & 29.7 & 3.37 \\
\hline 600 & 90.3 & 27.3 & 3.31 \\
\hline 650 & 80.5 & 20.2 & 3.99 \\
\hline 700 & 42.6 & 11.9 & 3.58 \\
\hline 725 & 25.2 & 4.72 & 5.34 \\
\hline 750 & 5.43 & .757 & 7.17 \\
\hline 775 & 1.06 & bd & \\
\hline
\end{tabular}


Acetone $\left(\sim 10^{-5} \mathrm{M}\right)$

Exposed To $280 \mathrm{~nm}$ Laser Radtiation At $150^{\circ} \mathrm{C}$ For $13.5 \mathrm{~s}$ In Helium

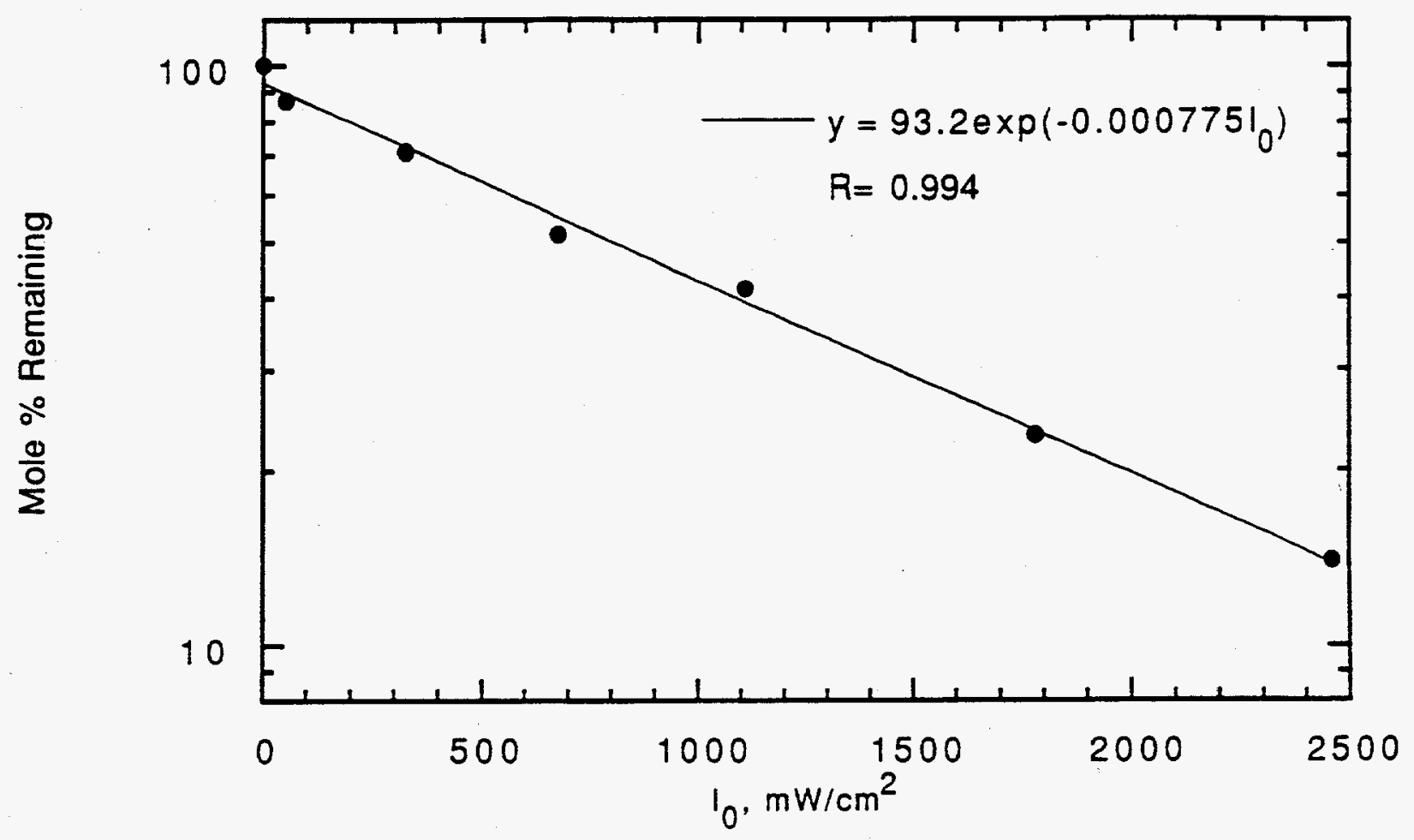

\begin{tabular}{|c|c|c|}
\hline 10 & $\operatorname{fr}(10)$ & $\mathrm{R}(10)$ \\
\hline 0 & 100 & 1.00 \\
\hline 54.0 & 86.8 & 1.15 \\
\hline 326 & 70.9 & 1.41 \\
\hline 677 & 51.6 & 1.94 \\
\hline 1110 & 41.6 & 2.40 \\
\hline 1780 & 23.0 & 4.35 \\
\hline 2460 & 14.0 & 7.14 \\
\hline
\end{tabular}


Naphthalene $\left(\sim 10^{-5} \mathrm{M}\right)$

Exposed To $280 \mathrm{~nm}$ Laser Radtiation At $300^{\circ} \mathrm{C}$ For $10 \mathrm{~s}$ In Helium

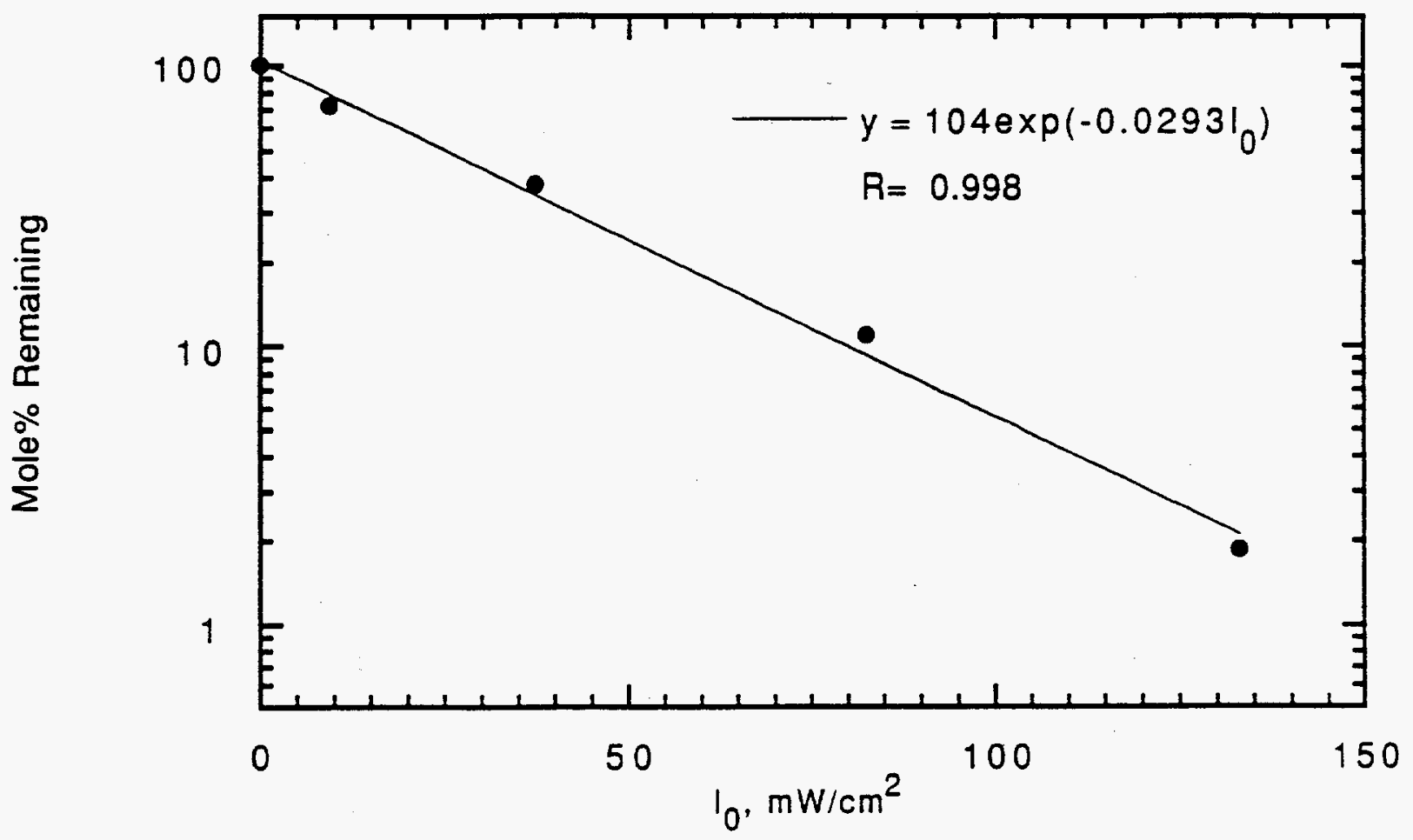

\begin{tabular}{|c|c|c|}
\hline 10 & $f r(10)$ & $\mathrm{R}(10)$ \\
\hline 0 & 100 & 1.00 \\
\hline 9.30 & 72.2 & 1.39 \\
\hline 37.2 & 38.1 & 2.63 \\
\hline 82.5 & 11.0 & 9.09 \\
\hline 133 & 1.87 & 53.5 \\
\hline 340 & bdl & \\
\hline
\end{tabular}




\section{Monochlorobenzene $\left(2.95 \times 10^{-5} \mathrm{M}\right)$ And its PICs Exposed To $883 \mathrm{~mW} / \mathrm{cm}^{2}$ For 10s in Air}

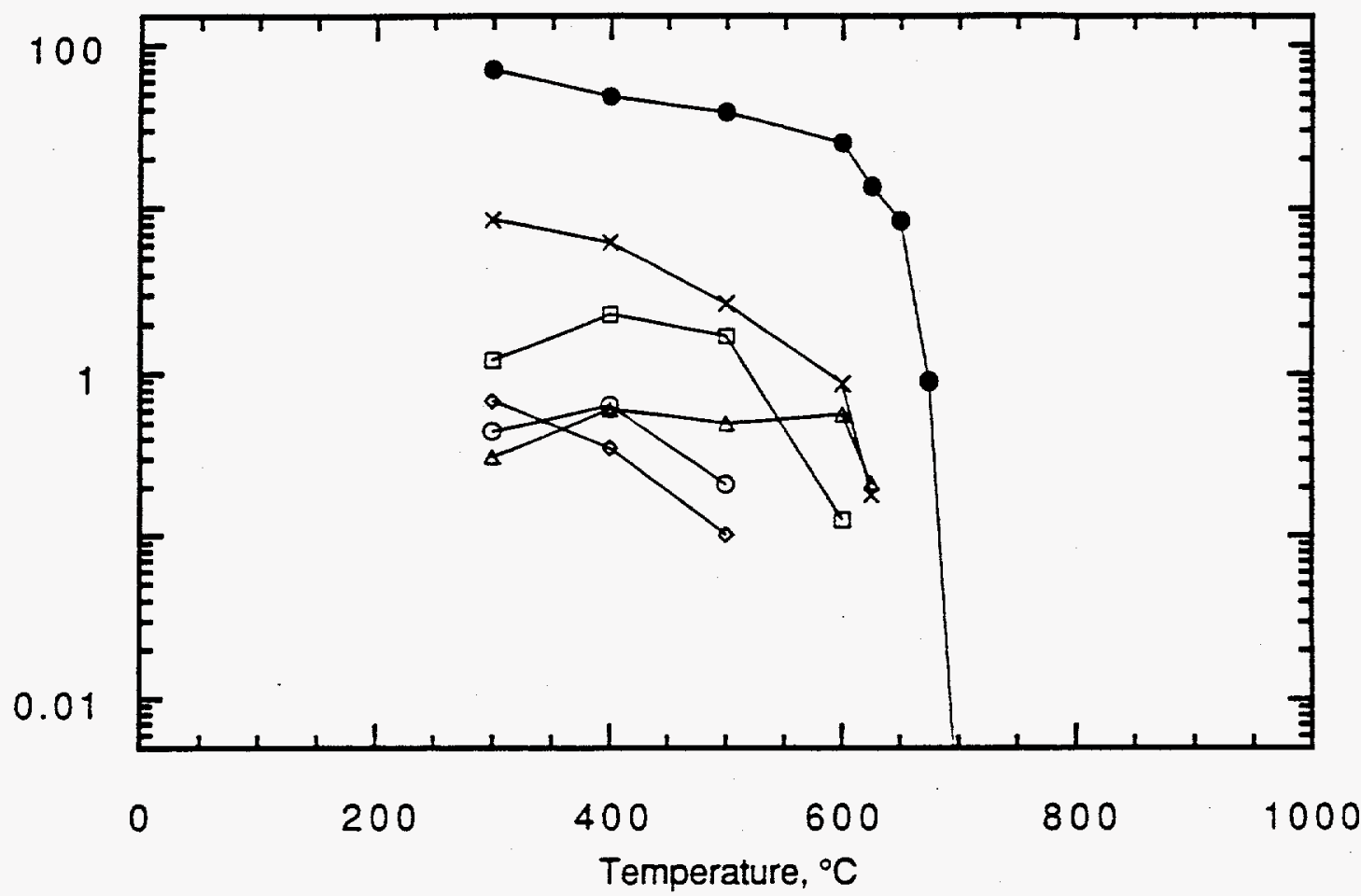

\begin{tabular}{|c|c|c|c|c|c|c|c|c|c|}
\hline & & \multicolumn{8}{|c|}{ Temperalure. ${ }^{\circ} \mathrm{C}$} \\
\hline & Viame & $3 m$ & 100 & 500 & 600 & 625 & 650 & 675 & 700 \\
\hline & & & & & & & & & \\
\hline 0 & Chlorobenzene 1 Parenty ${ }^{2}$ & 69.8 & 47.8 & 38.5 & 24.7 & 13.5 & 8.36 & 0.895 & 0.00201 \\
\hline & Carbon monoxide & 203 & 356 & $\$ 65$ & 516 & 564 & $6 ! 2$ & 565 & 518 \\
\hline & Carbon dioxide & 58.4 & 52.4 & 73.8 & 79.7 & 79.7 & 796 & 91.2 & 103 \\
\hline 0 & $2(3 \mathrm{H})$-Furnnone & 0.447 & 0.647 & 0.207 & & & & & \\
\hline$\square$ & Maleic Anhvonde & 1.22 & 2.31 & 1.71 & 0.125 & & & & \\
\hline 0 & 2-Chloropnenol- & 0.685 & 0.354 & 0.102 & & & & & \\
\hline 1 & Benzene- & 0.312 & 0.612 & 0.500 & 0.564 & 0.211 & & & \\
\hline$x$ & Phenol- & 8.60 & 6.28 & 2.68 & 0.869 & 0.178 & & & \\
\hline & Total Carbon Recovered & $124 \%$ & $126 \%$ & $133 \%$ & $126 \%$ & $121 \%$ & $124 \%$ & $110 \%$ & $104 \%$ \\
\hline
\end{tabular}

Mole \% remaining of species 1 at temperature $T=$ Moles $T(i) /$ Moles $_{3} 00(\mathrm{CIBz}) \times 100 \%$.

${ }^{2}$ Quantified by analytical standard. Two GC peaks were observed that were identified as maleic anhydride.

${ }^{3}$ Quanufied as $2 \mathrm{H}-\mathrm{Py}$ ranone.

${ }^{4}$ Sum of the cotal carbon measured in the output normalized by the total carbon input $(5.343 \mathrm{nMol})$. 
Monochiorobenzene $\left(2.95 \times 10^{-5} \mathrm{M}\right)$ and its PICs (Thermal Desruction only)
Exposed for $10 \mathrm{~s}$ in Air

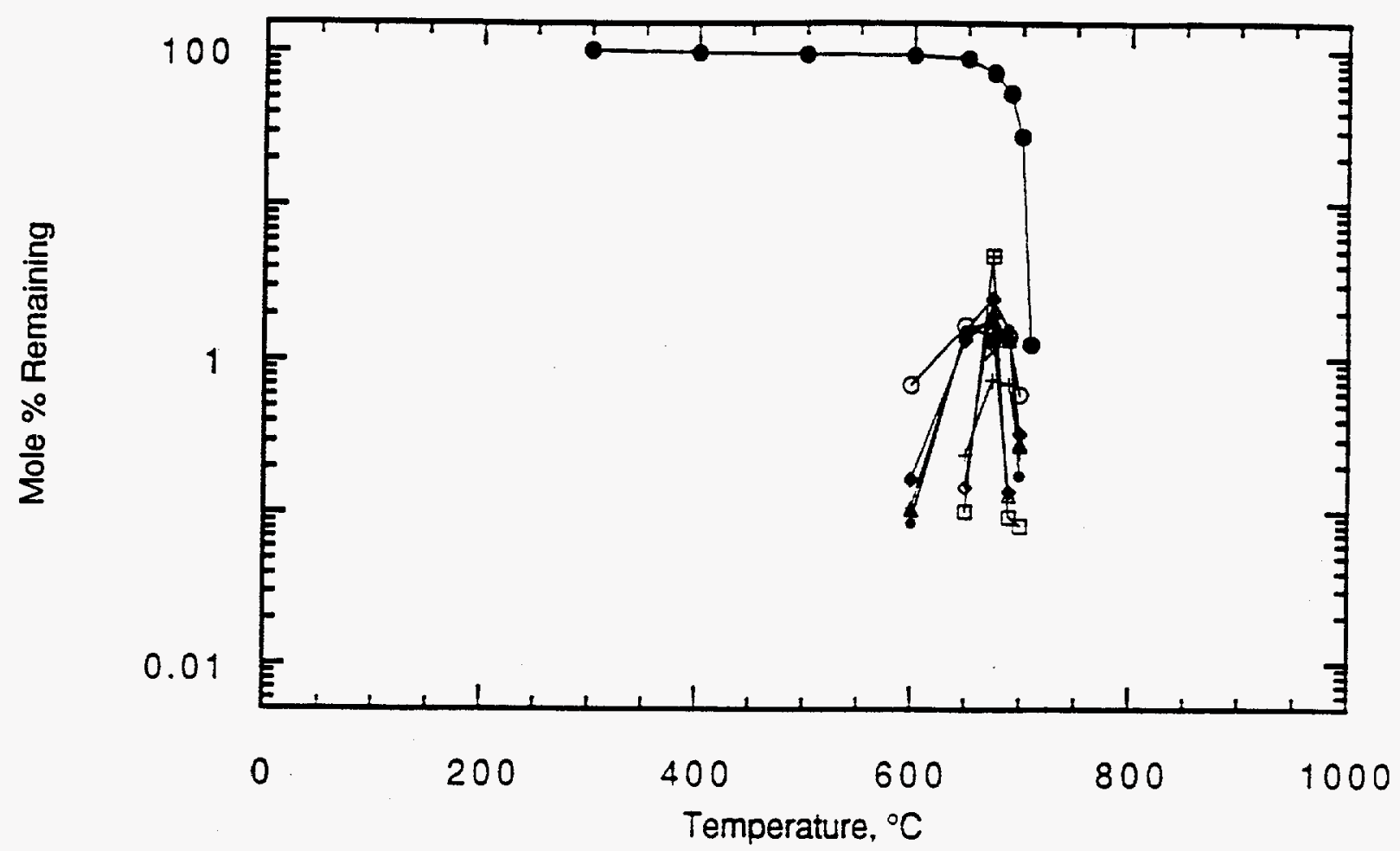

\begin{tabular}{|c|c|c|c|c|c|c|c|c|c|c|}
\hline & & \multicolumn{9}{|c|}{ Temperature. ${ }^{\circ} \mathrm{C}$} \\
\hline & Nime & in & $\pm m$ & 590 & $\tan$ & 650 & 675 & 690 & 700 & 710 \\
\hline & 1 & & $\rightarrow$ & & $T$ & & & & & \\
\hline e & Chlomber:zene (Parent) $)^{2}$ & 100 & 96.6 & 94.6 & 93.9 & 88.6 & 71.6 & 52.7 & 27.6 & 1.93 \\
\hline & Carbon $m$ Jnoxide & & & & & 45.9 & 138 & 388 & 488 & 579 \\
\hline & Carbon dis side & & & & $1=$ & 5.30 & $\overline{11.2}$ & Ii.t & 68.7 & 94.6 \\
\hline 0 & 1. Burene- 1. une & & & & 1.680 & 1.64 & 1.43 & 1.0 & 0.586 & \\
\hline D & $\begin{array}{l}\text { 1. Ethymyl 4-meunyl } \\
\text {-henzenet }\end{array}$ & & & & & 0.102 & 4.67 & 0.093 & 0.081 & \\
\hline 0 & 1 2-Methvi-11aonunalenet & & & & 1 & 0.146 & 2.45 & 0.136 & & \\
\hline 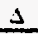 & 1.Methyi-unphunalenem & & & & 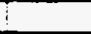 & & 1.65 & 0.130 & & \\
\hline 므 & 11.Propeny -benzonst & & & & & & 1.34 & & & \\
\hline+ & 19.-Chloropienol- & & & & 0.165 & 1.33 & 1.90 & 1.36 & 0.327 & \\
\hline 1 & 3-Chloropl enol- & & & & 0.106 & 1.49 & $\overline{1.84}$ & 1.33 & 0.284 & \\
\hline$x$ & 1 Acenapenayene- & & & & i & & 1.11 & & & \\
\hline 1 & I Benzene- & & & & 1 & 0.239 & 0.735 & 0.689 & 0.248 & $0.1+1$ \\
\hline $\bar{E}$ & Naphehalen $=-$ & & & & & & 4.67 & & & \\
\hline$\cdot$ & Thonol" & & & & 0.085 & 1.94 & 2.48 & 1.55 & 0.172 & \\
\hline & Total Carben Recovereds & $100 \%$ & $96.6 \%$ & $04.9 \%$ & 93.30 & $103 \%$ & $131 \%$ & 1319 & $122 \%$ & $113 \%$ \\
\hline
\end{tabular}

Mole \% remaning sif species $i$ at temperature $T=$ Moles $T(i) / M o l e s 2001 \mathrm{ClBz}) \times 100 \%$.

2Quanufied by analyucal standard.

${ }^{3}$ Quanutied as butadi ene.

${ }^{4}$ Quantufied as ethynyibenzene.

S Sum of the total carbon measured in the output normalized by the total carbon input ( $5.343 \mathrm{nMol}$ ). 


\section{APPENDIX V}

\section{High-Temperature Absorption Spectra}

This Appendix summarizes all of the data obtained during this project on the high-temperature, gas-phase absorption spectra. The data are listed by name alphabetically by compound. Unless otherwise noted, temperature is given in degrees Centigrade and extinction is in $L / \mathrm{mol} \mathrm{cm}$. 
Gas-phase Molar Extinction Spectra

For Benzene At 22, 200, 400, and $600^{\circ} \mathrm{C}$

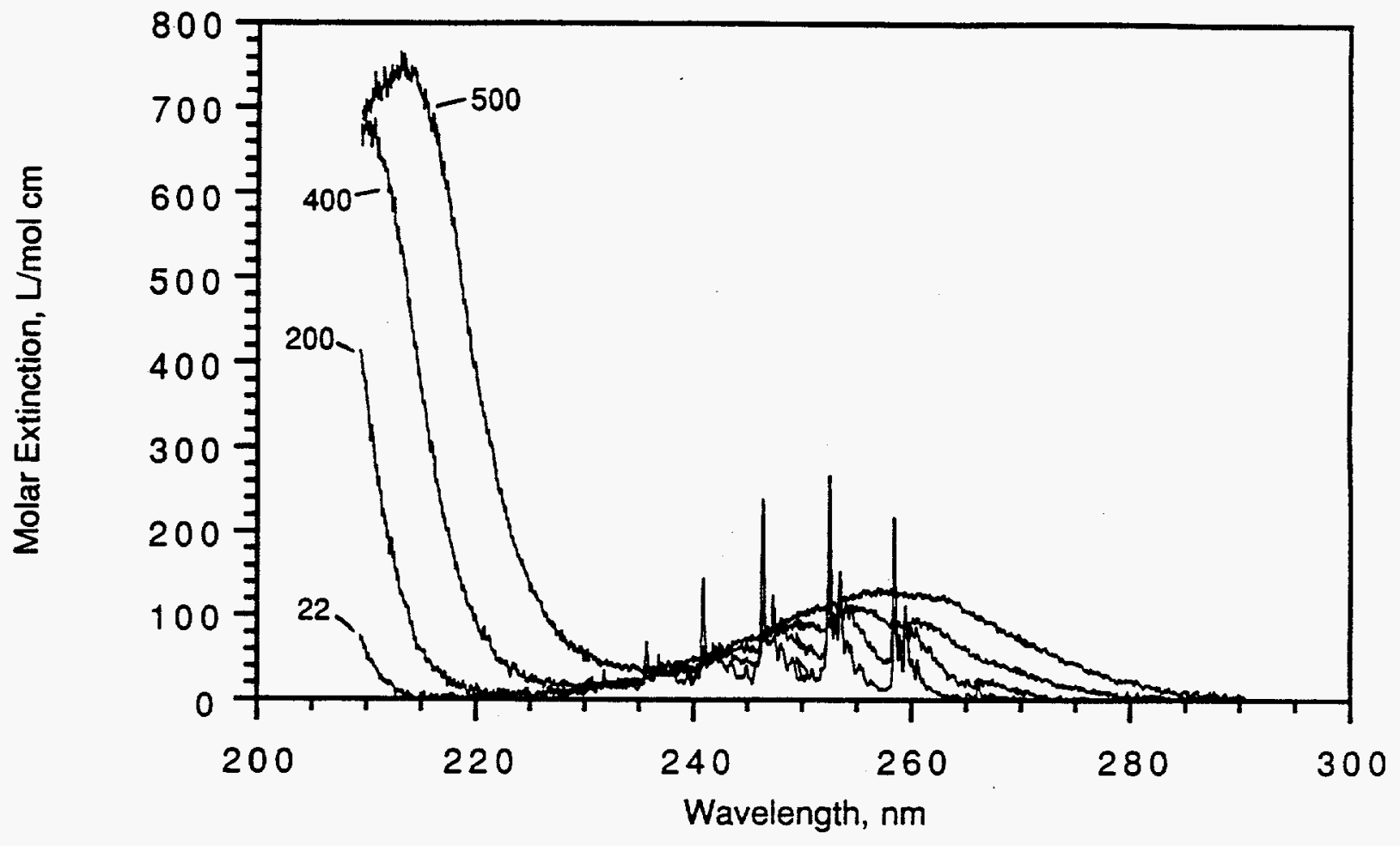


Gas-phase Molar Extinction Spectra

For Chlorine At 23 And $300^{\circ} \mathrm{C}$

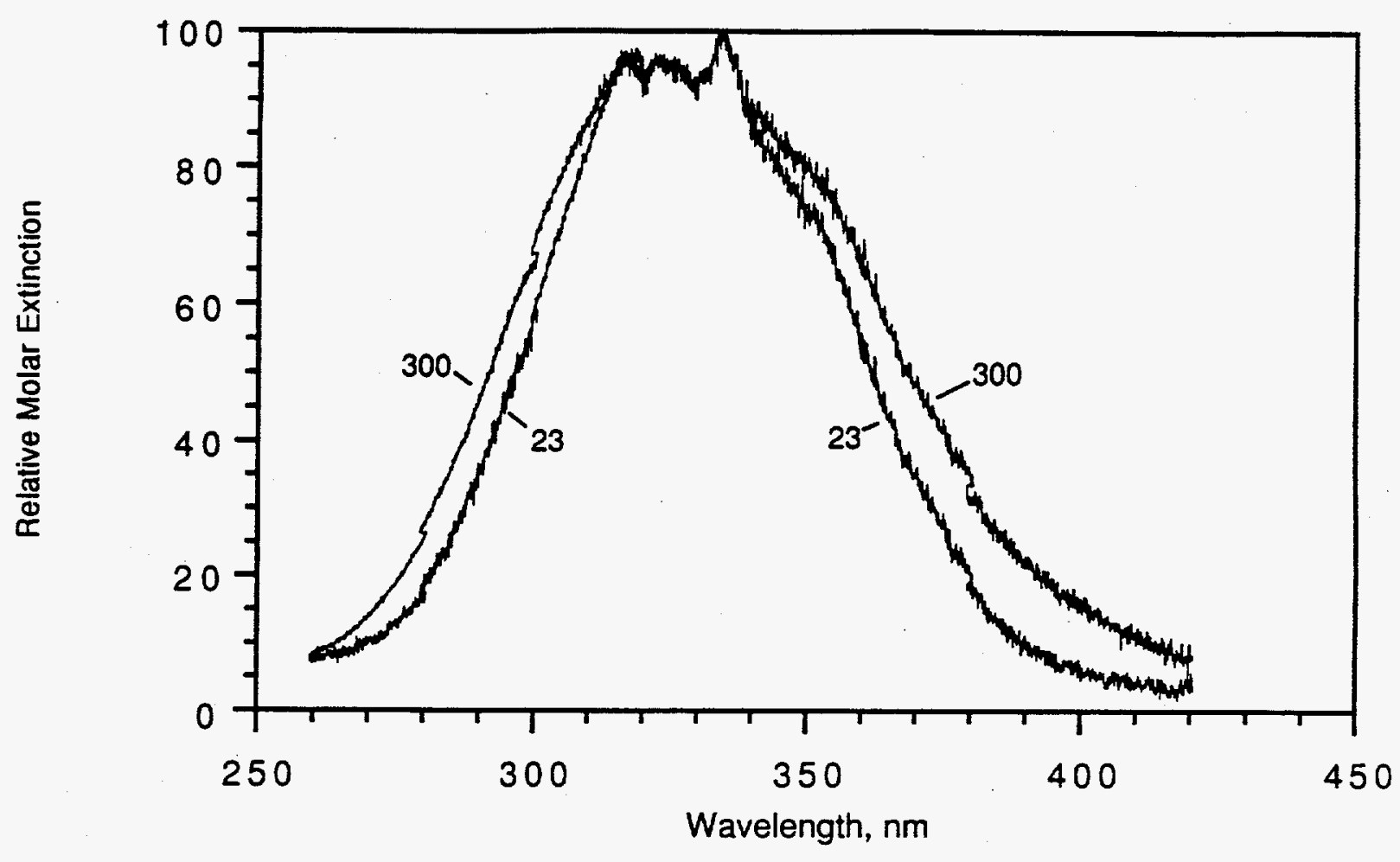


Gas-phase Molar Extinction Spectra

For 1-Chloronaphthalene At 100,300 , and $500^{\circ} \mathrm{C}$

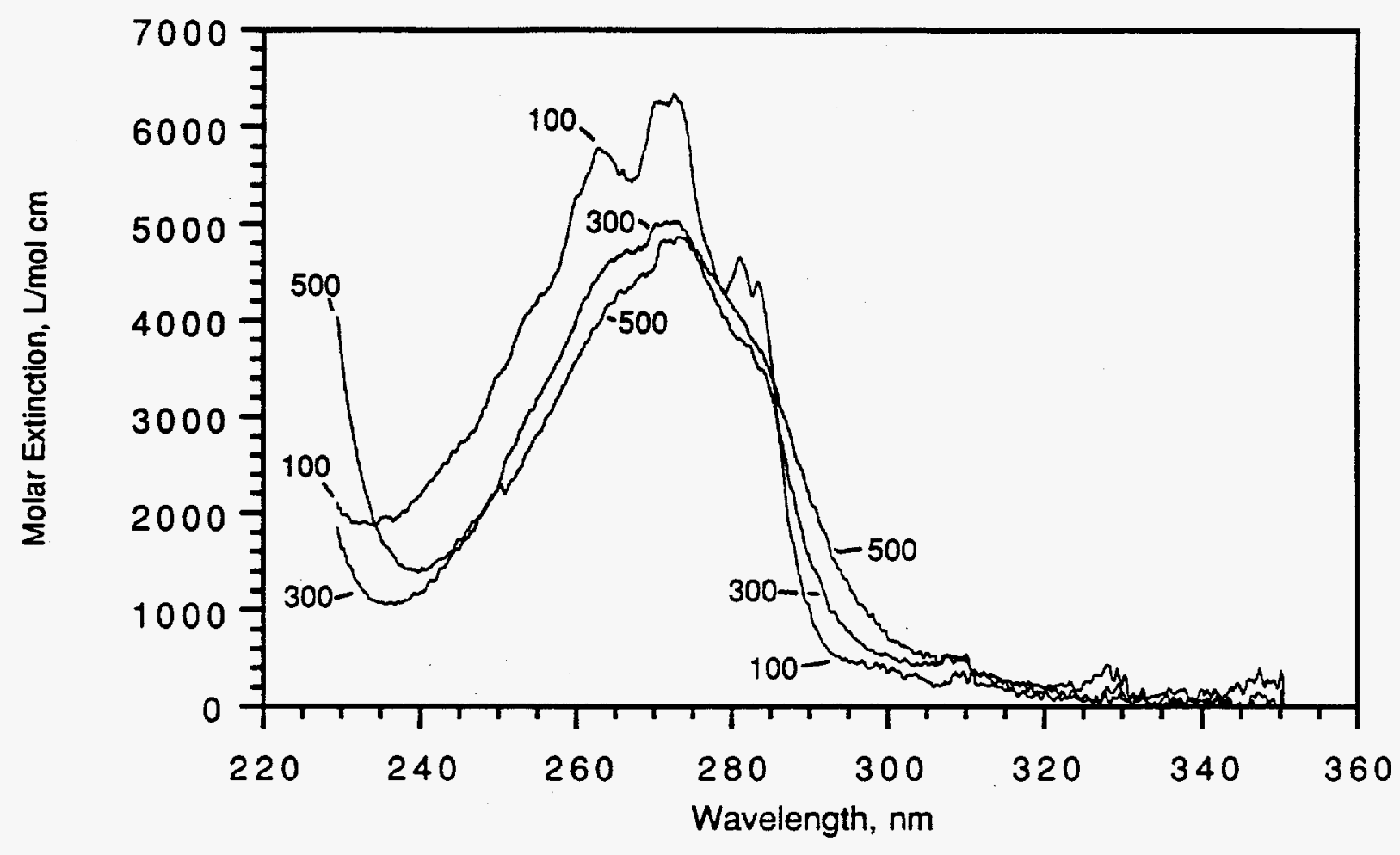


Gas-phase Molar Extinction Spectra

For 2,6-Dinitrotoluene At 200,225 , and $250^{\circ} \mathrm{C}$

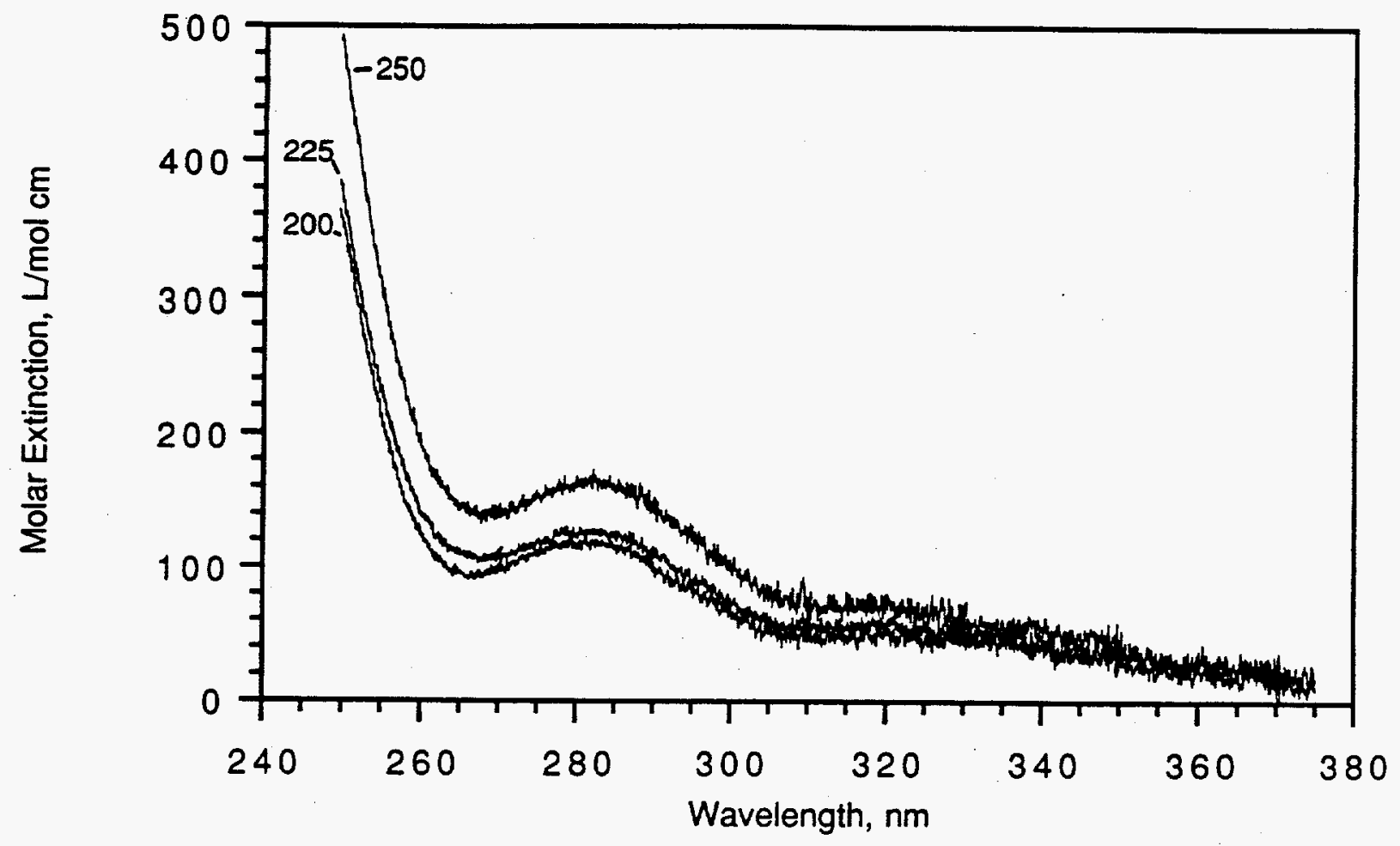


Gas-phase Molar Extinction Spectra

For Methylene Chloride At $200,400,600$, and $700^{\circ} \mathrm{C}$

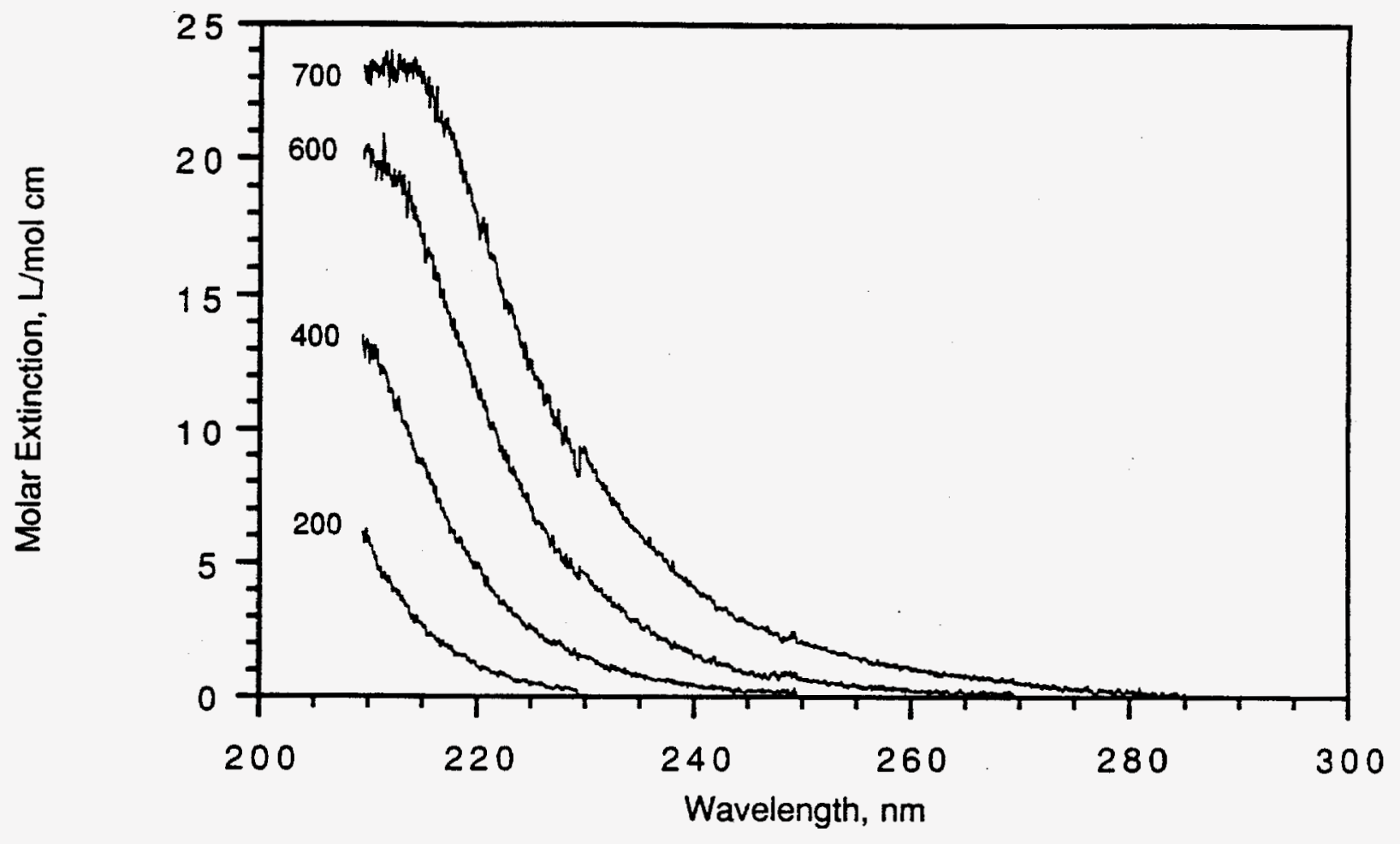


Gas-phase Molar Extinction Spectra

For Monochlorobenzene At 150,400 , and $700^{\circ} \mathrm{C}$

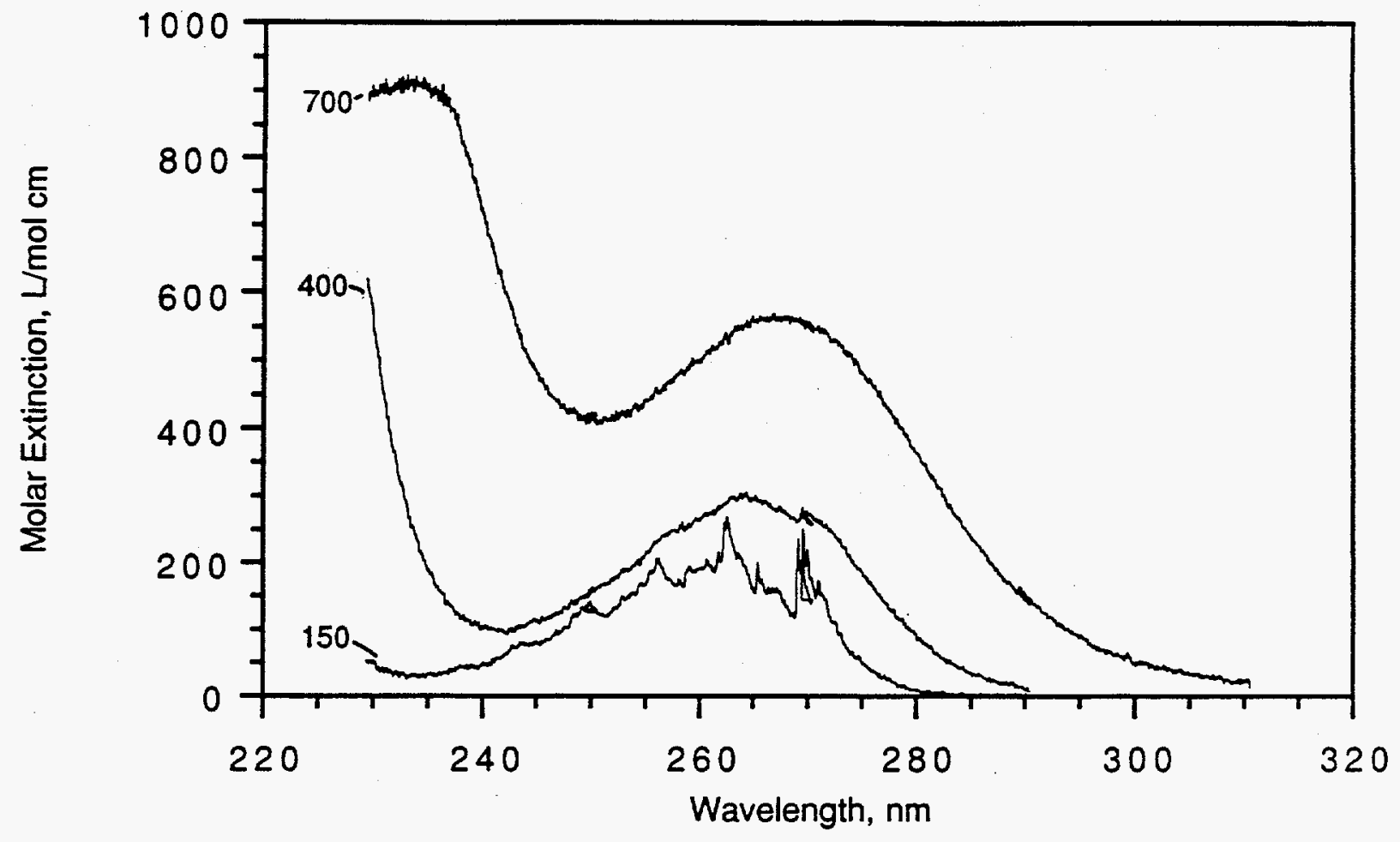


Gas-phase Molar Extinction Spectra

For $3,3^{\prime}, 4,4^{\prime}-$ Tetrachlorobiphenyl At 300,500 , and $700^{\circ} \mathrm{C}$

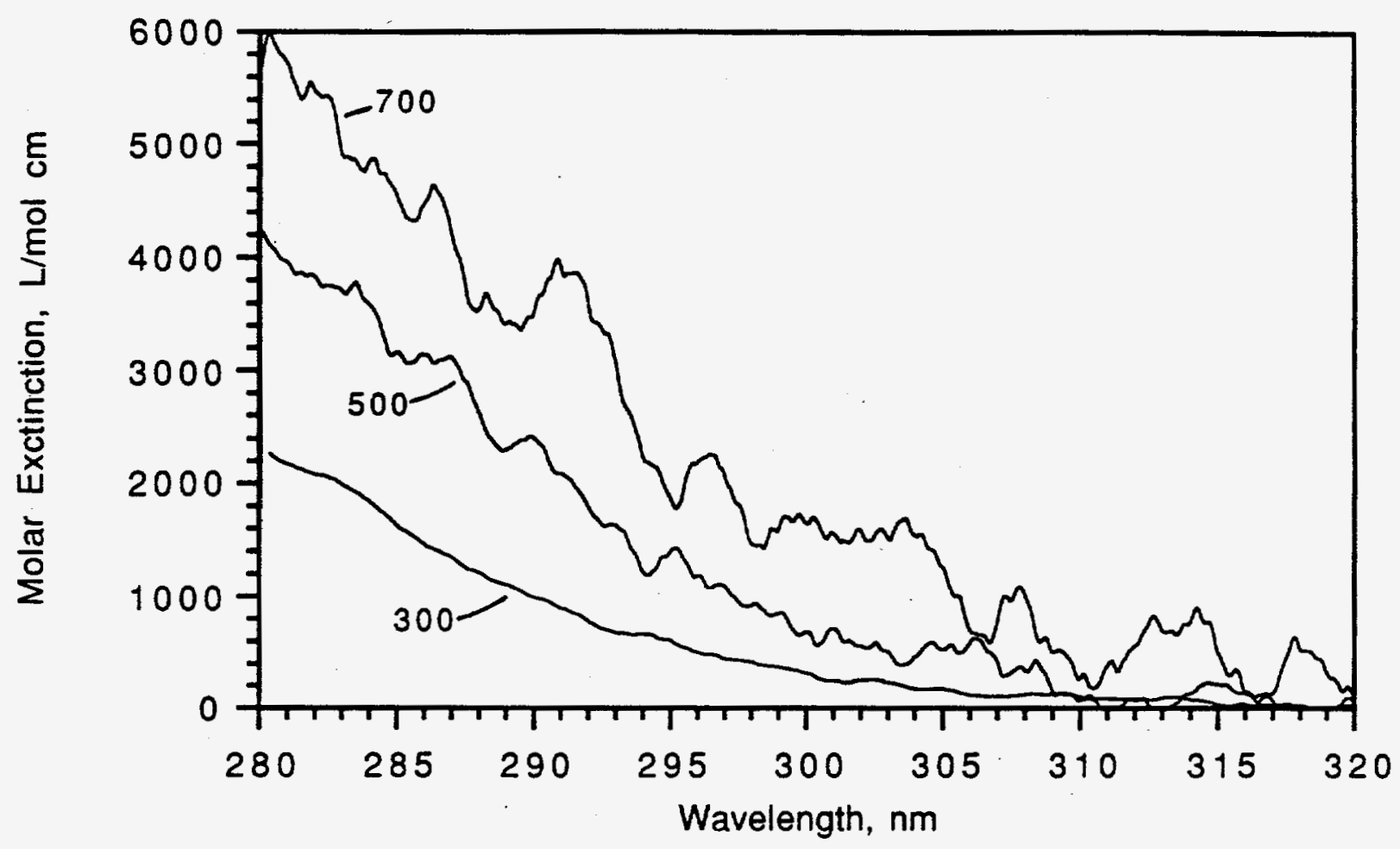




\section{APPENDIX VI}

\section{Detailed Kinetic Modeling of Solar-Enhanced Destruction of Organic Compounds and Mixtures \\ (Co-funded by US-EPA under CR-813938)}

In an effort to better understand the effects of solar-enhanced destruction of toxic organic compounds, we undertook an effort to develop detailed elementary reaction kinetic models. Our approach was to take existing, purely thermal models and modify them to include photolytic effects. Our initial work focused on modeling of chlorobenzene, a compound for which we have conducted detailed thermal and thermal/photolytic studies. We then extended the study to include modeling of solar-induced photochemical effects on a five-component mixture. The following paragraphs describe the current status of this effort.

\section{VI.1 Chlorobenzene}

Our initial modeling studies of the photo-assisted thermal destruction of hazardous organic compounds have concentrated on the model compound, chlorobenzene $\left(\mathrm{C}_{6} \mathrm{H}_{5} \mathrm{Cl}\right)$. The photodynamics and spectroscopy of this compound are illustrated in Figure Vl.1. This compound of environmental significance was chosen to relate red-shifting of the $\mathrm{S}_{1}$ state at elevated temperatures to enhanced destruction in the presence of ultraviolet radiation are $2800 \AA$ (viz., the lower wavelength limit of solar radiation at the earth's surface).

A high temperature spectrometer was used to obtain the $S_{1} \leftarrow S_{0}$ oscillator strength, natural lifetime, and molar extinction coefficient over a temperature range of 150 to $700^{\circ} \mathrm{C}$. The absorption spectrum is shown in Figure VI.2. The data indicates that red-shifting of the $S_{1}$ state occurs, as evidenced by the increase in molar extinction coefficient $(\varepsilon)$ at $2800 \AA$. This is numerically summarized in the second column of Table VI.1. This data illustrates how the extinction coefficient increases by a factor of 50 from 423 to $973 \mathrm{~K}$.

Preliminary high-temperature thermal and photo-assisted thermal decomposition data for $\mathrm{C}_{6} \mathrm{H} \mathrm{H}_{5} \mathrm{Cl}$ were obtained with the Advanced Thermal/Photolytic Reactor System. Thermal/ photolytic data were 


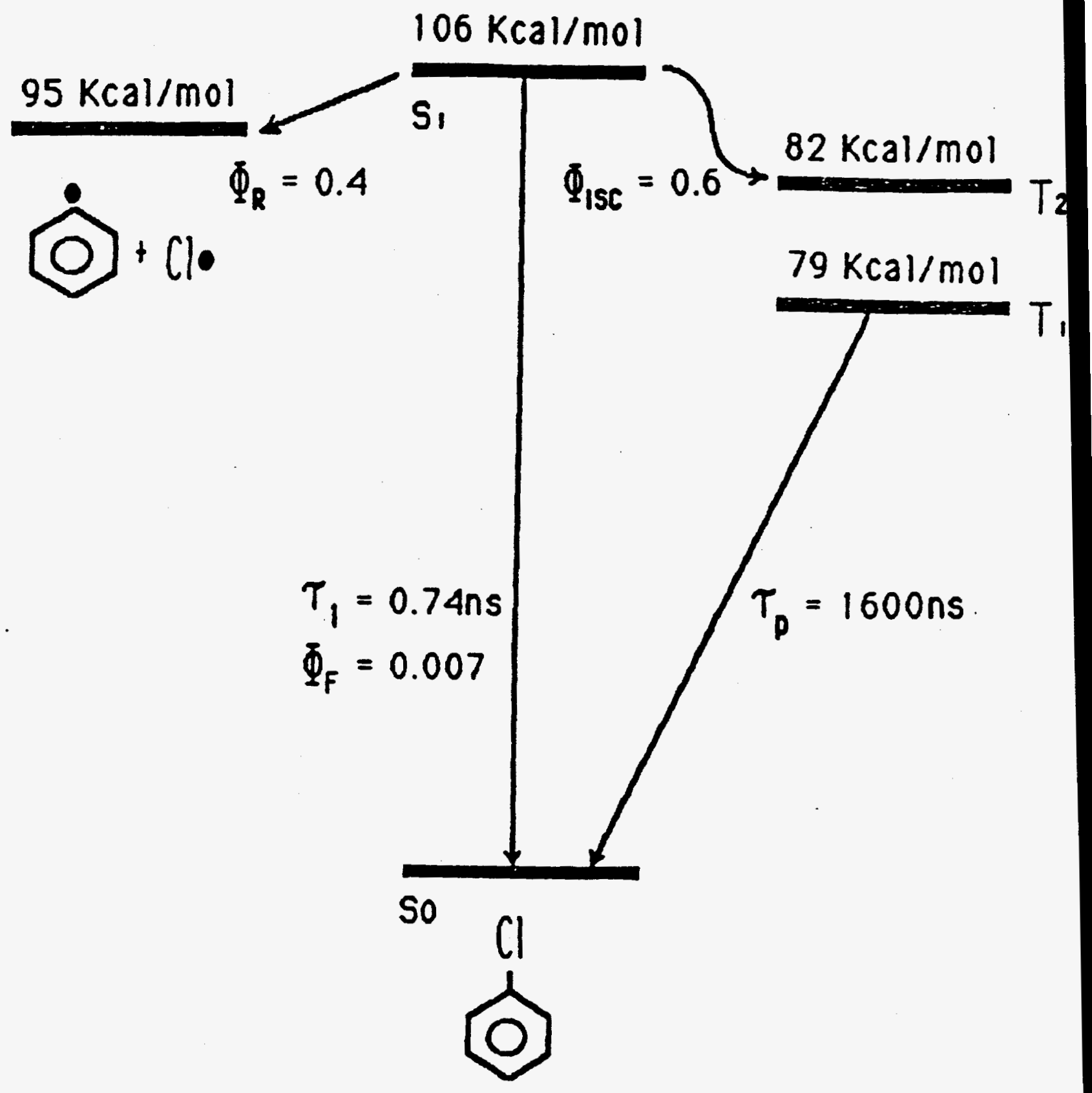

Figure VI.1. State energy/process diagram for $\mathrm{C}_{6} \mathrm{H}_{5} \mathrm{Cl}$. 

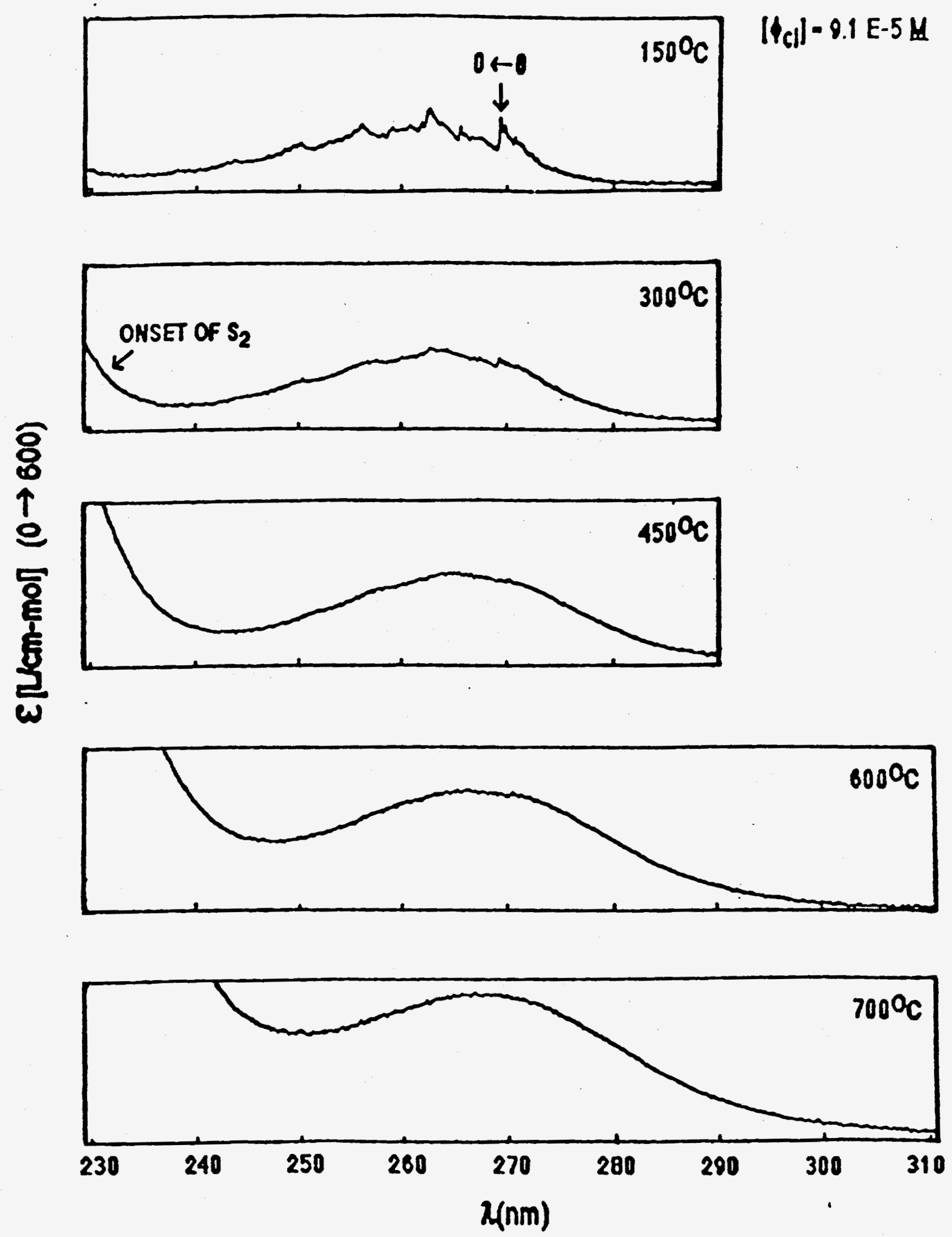

Figure VI.2. Chlorobenzene $S_{1} \leftarrow S_{0}$ absorption spectrum as a function of temperature. 
obtained with helium carrier gas using coherent, monochromatic radiation of $2800 \AA$ at initial $\mathrm{C}_{6} \mathrm{H}_{5} \mathrm{Cl}$ concentrations of $29 \mathrm{ppmv}$ and for maximum laser fluences of $25.2 \mathrm{GW} / \mathrm{cm}^{2}$. The thermal/photolytic data were obtained at a repetition rate of $20 \mathrm{~Hz}$. Two-dimensional "discs" of reactor gas were irradiated with $2800 \AA$ radiation 200 times while traversing the reactor. This number is based on the dimensions of the reactor and a mean, gas-phase, residence time of $10.0 \mathrm{~s}$.

TABLE V 1.1

DATA FOR PHOTODISSOCIATION RATE

\begin{tabular}{ccccc}
$T(\mathrm{~K})$ & $\varepsilon(\mathrm{L} \mathrm{cm}-\mathrm{mol})$ & $\lambda(\mathrm{nm})$ & $\mathrm{P} / \mathrm{A}\left(\mathrm{W} / \mathrm{cm}^{2}\right)$ & $k \operatorname{exc}(1 / \mathrm{s})$ \\
\hline 423 & 7 & 280 & $2.52 \mathrm{E}+07$ & $9.47 \mathrm{E}+02$ \\
473 & 12 & 280 & $2.52 \mathrm{E}+07$ & $1.62 \mathrm{E}+03$ \\
523 & 27 & 280 & $2.52 \mathrm{E}+07$ & $3.65 \mathrm{E}+03$ \\
573 & 43 & 280 & $2.52 \mathrm{E}+07$ & $5.82 \mathrm{E}+03$ \\
623 & 60 & 280 & $2.52 \mathrm{E}+07$ & $8.12 \mathrm{E}+03$ \\
673 & 87 & 280 & $2.52 \mathrm{E}+07$ & $1.18 \mathrm{E}+04$ \\
713 & 117 & 280 & $2.52 \mathrm{E}+07$ & $1.58 \mathrm{E}+04$ \\
773 & 144 & 280 & $2.52 \mathrm{E}+07$ & $1.95 \mathrm{E}+04$ \\
823 & 176 & 280 & $2.52 \mathrm{E}+07$ & $2.38 \mathrm{E}+04$ \\
873 & 229 & 280 & $2.52 \mathrm{E}+07$ & $3.10 \mathrm{E}+04$ \\
923 & 281 & 280 & $2.52 \mathrm{E}+07$ & $3.80 \mathrm{E}+04$ \\
973 & 357 & 280 & $2.52 \mathrm{E}+07$ & $4.83 \mathrm{E}+04$ \\
\hline
\end{tabular}

Figures VI. 3 - VI. 5 present a comparison of the thermal and thermal/photolytic data for the parent compound and three observed organic products, benzene, ethynyl benzene, and naphthalene. The data indicates an $\sim 100$-fold decrease in $\mathrm{C}_{6} \mathrm{H}_{5} \mathrm{Cl}$ fraction remaining at $600^{\circ} \mathrm{C}$ in the presence of $2800 \AA$ radiation. This corresponds to an $300^{\circ} \mathrm{C}$ shift (to lower temperatures) in $\mathrm{Tgg}$ (10). A comparison of the product data indicated an 100-fold decrease (based on approximate detection limits) in yields of benzene, ethynyl benzene, and naphthalene at $800^{\circ} \mathrm{C}$ in the presence of $2800 \AA$ radiation. Other reaction products not shown included vinyl-acetylene $\left(\mathrm{CH}_{2}=\mathrm{CHC} \equiv \mathrm{CH}\right)$ and diacetylene $(\mathrm{CH} \equiv \mathrm{CC} \equiv \mathrm{CH})$. These products also exhibited an $\sim 100$-fold decrease in yields at $800^{\circ} \mathrm{C}$ in the presence of $2800 \AA$ radiation.

Kinetic modeling of thermal experiments was accomplished with a 149-step elementary reaction mechanism shown in Table VI.2. This mechanism is based on previous studies of the thermal 


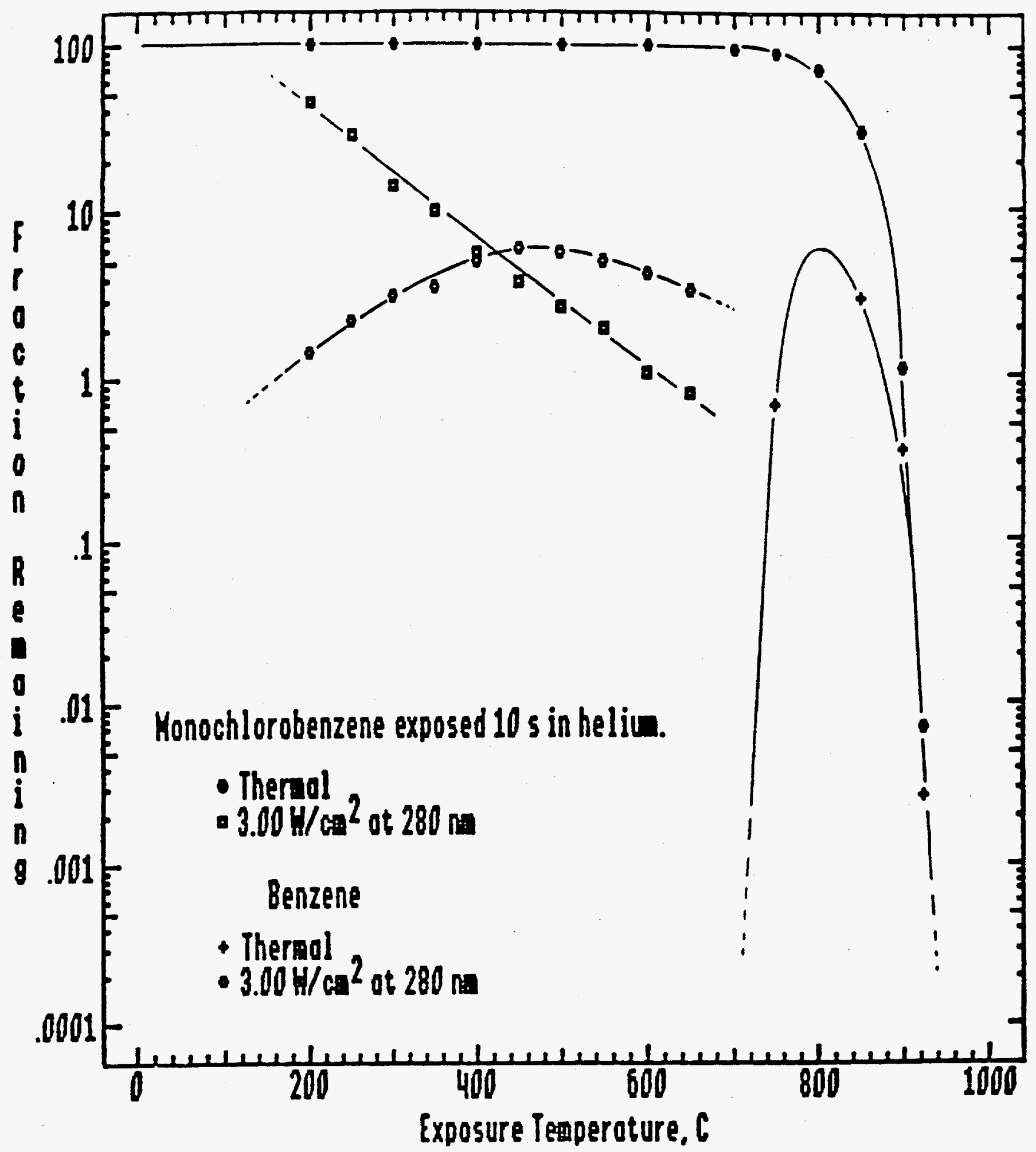

Figure VI.3. Thermal decomposition profile of $\mathrm{C}_{6} \mathrm{H}_{5} \mathrm{Cl}$ in flowing helium. Also shown is the formation/destruction profile for the major PIC $\mathrm{C}_{6} \mathrm{H}_{6}$. Filled symbols: thermal, open symbols: $3 \mathrm{~W} / \mathrm{cm}^{2}$ at $2800 \dot{A}$. 


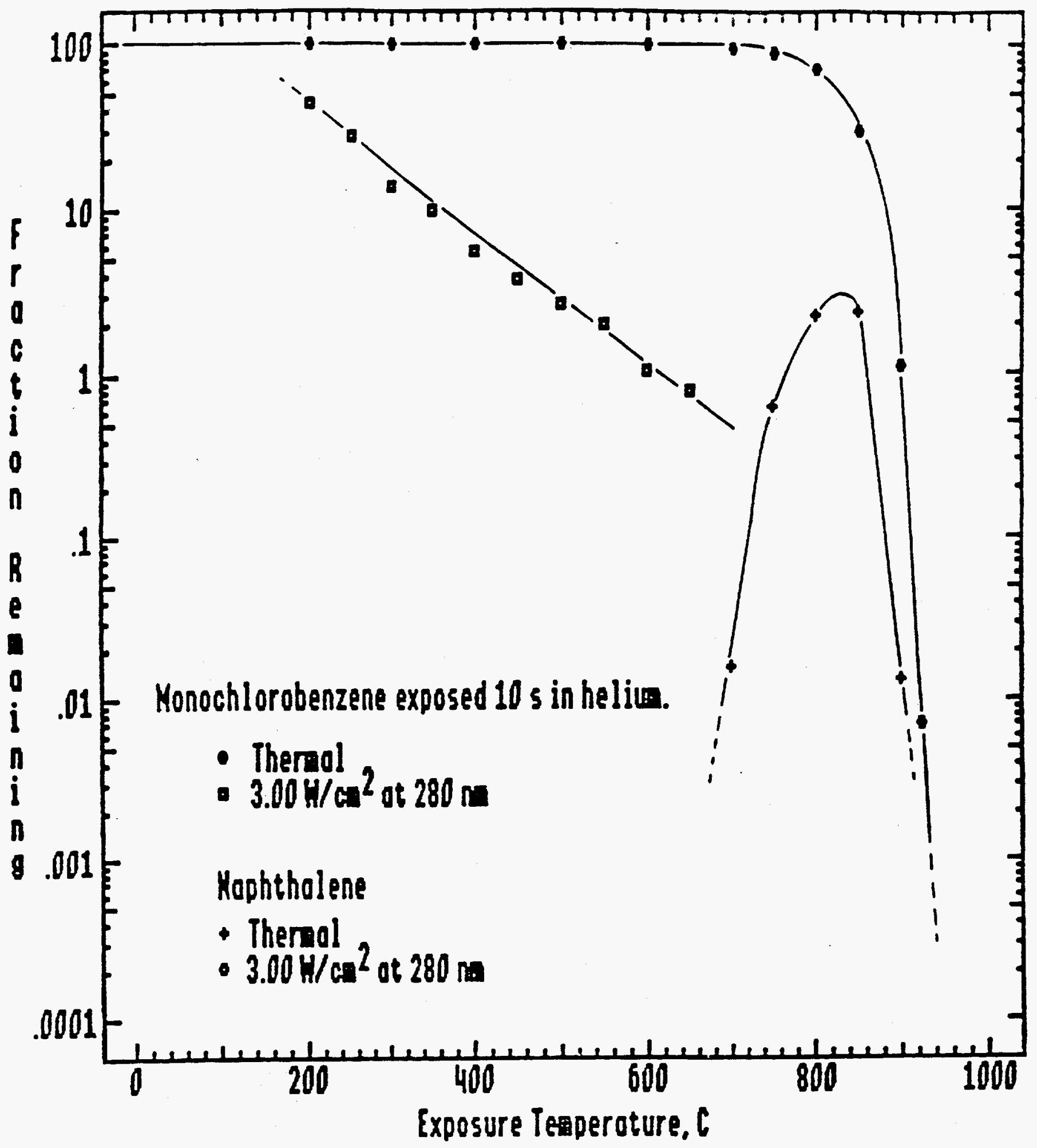

Figure VI.4. Thermal decomposition profile of $\mathrm{C}_{6} \mathrm{H}_{5} \mathrm{Cl}$ in flowing helium. Also shown is the formation/destruction profile for naphthalene. 


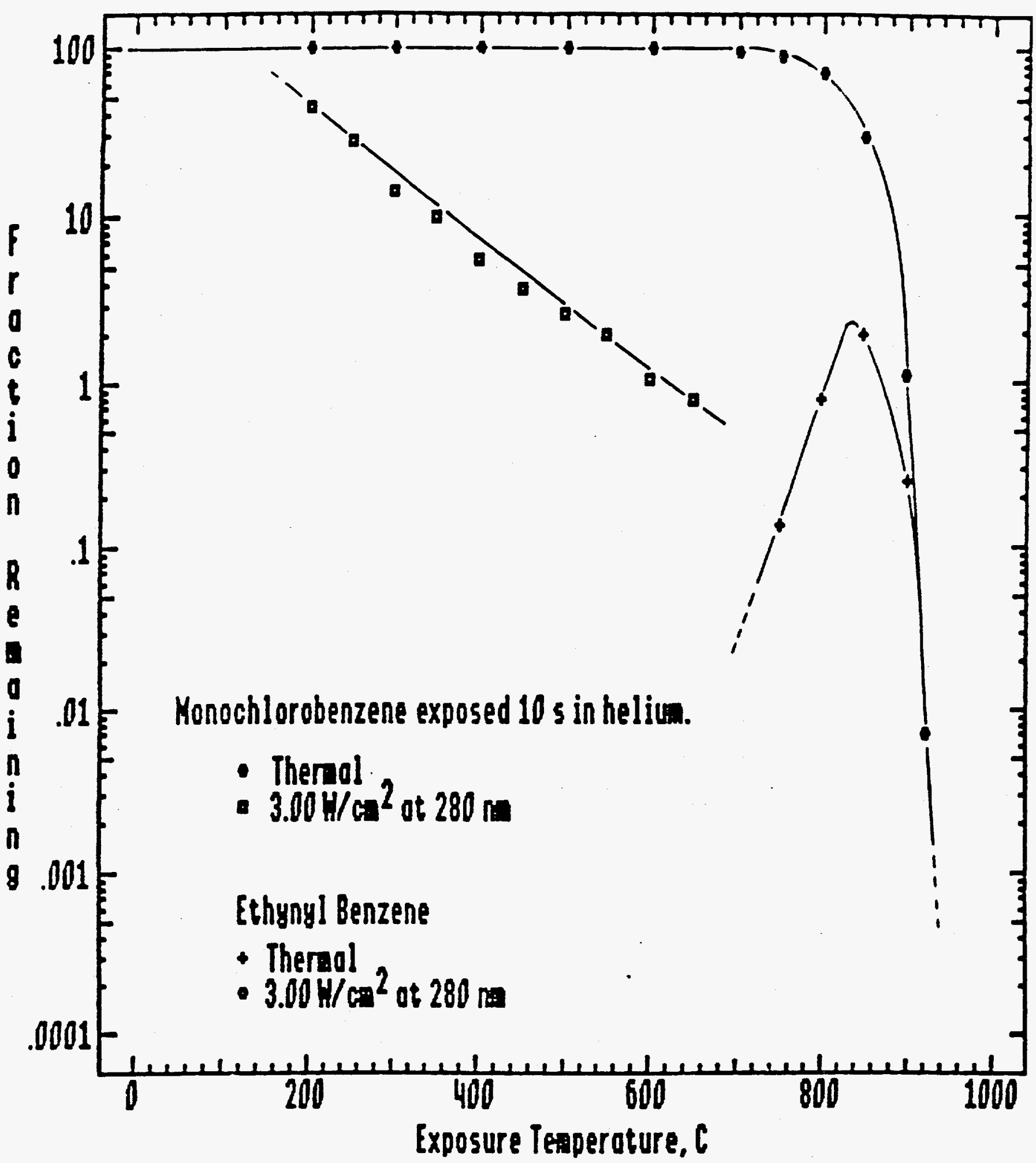

Figure VI.5. Thermal decomposition profile of $\mathrm{C}_{6} \mathrm{H}_{5} \mathrm{Cl}$ in flowing helium. Also shown is the formation/destruction profile for ethynyl benzene. 
TABLE VI.2

$\mathrm{C}_{6} \mathrm{H}_{5} \mathrm{Cl}$ PYROLYSIS OXIDATION MECHANISM

\section{REACTIONS CONSIDERED}

1. $\mathrm{C} 6 \mathrm{H} 5 \mathrm{CL}=\mathrm{C} 6 \mathrm{H} 5+\mathrm{CL}$

2. $\mathrm{C} 6 \mathrm{H} 5 \mathrm{CL}+\mathrm{CL}=\mathrm{C} 6 \mathrm{H} 4 \mathrm{CL}+\mathrm{HCL}$

3. $\mathrm{C} 6 \mathrm{H} 5 \mathrm{CL}+\mathrm{H}=\mathrm{C} 6 \mathrm{H} 6+\mathrm{CL}$

4. $\mathrm{C} 6 \mathrm{H} 5 \mathrm{CL}+\mathrm{H}=\mathrm{C} 6 \mathrm{H} 4 \mathrm{CL}+\mathrm{H} 2$

5. $\mathrm{C} 6 \mathrm{H} 5 \mathrm{CL}+\mathrm{H}=\mathrm{C} 6 \mathrm{H} 5+\mathrm{HCL}$

6. $\mathrm{C} 6 \mathrm{H} 5 \mathrm{CL}+\mathrm{OH}=\mathrm{C} 6 \mathrm{H} 4 \mathrm{CL}+\mathrm{H} 2 \mathrm{O}$

7. $\mathrm{C} 6 \mathrm{H} 5 \mathrm{CL}+\mathrm{O}=\mathrm{C} 6 \mathrm{H} 5 \mathrm{O}+\mathrm{CL}$

8. $\mathrm{C} 6 \mathrm{H} 5 \mathrm{CL}+\mathrm{O} 2=\mathrm{C} 6 \mathrm{H} 4 \mathrm{CL}+\mathrm{HO} 2$

9. $\mathrm{C} 6 \mathrm{H} 6=\mathrm{C} 6 \mathrm{H} 5+\mathrm{H}$

10. $\mathrm{C} 6 \mathrm{H} 6+\mathrm{O} 2=\mathrm{C} 6 \mathrm{H} 5+\mathrm{HO} 2$

11. $C 6 H 6+C 6 H 5=C 12 H 1 O+H$

12. $\mathrm{C} 5 \mathrm{H} 6+\mathrm{H}=\mathrm{C} 6 \mathrm{H} 5+\mathrm{H} 2$

13. $\mathrm{C} 6 \mathrm{H} 6+\mathrm{O}=\mathrm{C} 6 \mathrm{H} 5 \mathrm{O}+\mathrm{H}$

14. $\mathrm{C} 6 \mathrm{H} 6+\mathrm{OH}=\mathrm{C} 6 \mathrm{H} 5+\mathrm{H} 2 \mathrm{O}$

15. $\mathrm{C} 6 \mathrm{H} 5 \mathrm{OH}=\mathrm{C} 6 \mathrm{H} 5 \mathrm{O}+\mathrm{H}$

15. $\mathrm{C} 6 \mathrm{H} 5 \mathrm{OH}+\mathrm{CL}=\mathrm{C} 6 \mathrm{H} 4 \mathrm{OH}+\mathrm{HCL}$

17. $\mathrm{C} 6 \mathrm{H} 5 \mathrm{OH}+\mathrm{H}=\mathrm{C} 6 \mathrm{H} 6+\mathrm{OH}$

18. $\mathrm{C} 6 \mathrm{H} 5 \mathrm{OH}+\mathrm{H}=\mathrm{C} 6 \mathrm{H} 5 \mathrm{O}+\mathrm{H} 2$

19. $\mathrm{C} 6 \mathrm{H} 5 \mathrm{OH}+\mathrm{C} 5 \mathrm{HS}(\mathrm{CY})=\mathrm{C} 6 \mathrm{H} 5 \mathrm{O}+\mathrm{C} 5 \mathrm{H} 6$

20. $\mathrm{C} 6 \mathrm{H} 5 \mathrm{OH}+\mathrm{OH}=\mathrm{C} 6 \mathrm{H} 5 \mathrm{O}+\mathrm{H} 2 \mathrm{O}$

21. $\mathrm{C} 6 \mathrm{H} 4 \mathrm{OH}=\mathrm{C} 5 \mathrm{H} 4(\mathrm{CY})+\mathrm{HCO}$

22. $\mathrm{C} 6 \mathrm{H} 5 \mathrm{O}=\mathrm{C} 5 \mathrm{HS}(\mathrm{CY})+\mathrm{CO}$

23. $\mathrm{C} 5 \mathrm{H} 4(\mathrm{CY})+\mathrm{H}=\mathrm{C} 5 \mathrm{H} 5(\mathrm{CY})$

24. $\mathrm{C}$ HS $(\mathrm{CY})+\mathrm{O}=\mathrm{C} 5 \mathrm{H} 5 \mathrm{O}(\mathrm{CY})$

25. $\mathrm{C} 5 \mathrm{H} 5(\mathrm{CY})+\mathrm{OH}=\mathrm{C} 5 \mathrm{H} 4 \mathrm{OH}+\mathrm{H}$

26. $\mathrm{C} 5 \mathrm{HS}(\mathrm{CY})+\mathrm{HO} 2=\mathrm{C} 5 \mathrm{H} 5 \mathrm{O}(\mathrm{CY})+\mathrm{OH}$

27. $\mathrm{C} 5 \mathrm{H} 6+\mathrm{O}=\mathrm{C} 5 \mathrm{H} 5 \mathrm{O}(\mathrm{CY})+\mathrm{H}$

28. $\mathrm{C} 5 \mathrm{H} 4 \mathrm{OH}=\mathrm{CH} 2 \mathrm{CHCCH}+\mathrm{HCO}$

29. $\mathrm{C} 6 \mathrm{H} 5+\mathrm{O} 2=\mathrm{C} 6 \mathrm{H} 5 \mathrm{O}+\mathrm{O}$

30. $\mathrm{C} 6 \mathrm{H} 5+\mathrm{HO} 2=\mathrm{C} 6 \mathrm{H} 5 \mathrm{O}+\mathrm{OH}$

31. $\mathrm{C} 6 \mathrm{H} 5=\mathrm{C} 4 \mathrm{H3}+\mathrm{C} 2 \mathrm{H} 2$

32. $2 \mathrm{C} 6 \mathrm{H} 5=\mathrm{C} 12 \mathrm{H} 1 \mathrm{O}$

33. $\mathrm{C} 6 \mathrm{H} 5+\mathrm{OH}=\mathrm{C} 6 \mathrm{H} 5 \mathrm{OH}$

34. $\mathrm{C} 6 \mathrm{H} 5+\mathrm{O}=\mathrm{C} 6 \mathrm{H} 5 \mathrm{O}$

35. $\mathrm{C} 5 \mathrm{H} 5 \mathrm{O}(\mathrm{CY})=\mathrm{CH} 2 \mathrm{CHCCH} 2+\mathrm{CO}$

36. $\mathrm{C} 6 \mathrm{H} 4 \mathrm{CL}=\mathrm{C} 4 \mathrm{H} 3+\mathrm{C} 2 \mathrm{HCL}$

37. $\mathrm{C} 5 \mathrm{H} 4 \mathrm{CL}+\mathrm{OH}=\mathrm{C} 6 \mathrm{H} 4 \mathrm{CLOH}$

38. $\mathrm{C} 6 \mathrm{H} 4 \mathrm{CL}+\mathrm{HO} 2=\mathrm{C} 6 \mathrm{H} 4 \mathrm{CLO}+\mathrm{OH}$

39. $\mathrm{C} 6 \mathrm{H} 4 \mathrm{CL}+\mathrm{O}=\mathrm{C} 6 \mathrm{H} 4 \mathrm{CLO}$

40. $\mathrm{C} 6 \mathrm{H} 4 \mathrm{CLOH}=\mathrm{C} 6 \mathrm{H} 4 \mathrm{CLO}+\mathrm{H}$

41. $\mathrm{C} 6 \mathrm{H} 4 \mathrm{CLOH}+\mathrm{H}=\mathrm{C} 6 \mathrm{H} 5 \mathrm{CL}+\mathrm{OH}$

42. $\mathrm{C} 6 \mathrm{H} 4 \mathrm{CLOH}+\mathrm{H}=\mathrm{C} 6 \mathrm{H} 4 \mathrm{OH}+\mathrm{HCL}$

43. $\mathrm{C} 6 \mathrm{H} 4 \mathrm{CLOH}+\mathrm{OH}=\mathrm{C} 6 \mathrm{H} 3 \mathrm{CLOH}+\mathrm{H} 2 \mathrm{O}$

44. $\mathrm{CH} 2 \mathrm{CHCCH} 2=\mathrm{C} 2 \mathrm{H} 3+\mathrm{C} 2 \mathrm{H} 2$

45. $\mathrm{C} 4 \mathrm{H} 3+\mathrm{M}=\mathrm{C} 4 \mathrm{H} 2+\mathrm{H}+\mathrm{M}$

46. $\mathrm{C} 4 \mathrm{H} 2+\mathrm{O}=\mathrm{HCCO}+\mathrm{C} 2 \mathrm{H}$

47. $\mathrm{C} 4 \mathrm{H} 2+\mathrm{O}=\mathrm{CO}+\mathrm{C} 3 \mathrm{H} 2$

48. $\mathrm{C} 4 \mathrm{H} 2+\mathrm{OH}=\mathrm{HCO}+\mathrm{C}_{3} \mathrm{H}_{2}$

49. $\mathrm{C} 2 \mathrm{H} 4+\mathrm{M}=\mathrm{C} 2 \mathrm{H} 2+\mathrm{H} 2+\mathrm{M}$

50. $\mathrm{C} 2 \mathrm{H} 4+\mathrm{OH}=\mathrm{C} 2 \mathrm{H} 3+\mathrm{H} 2 \mathrm{O}$

51. $\mathrm{C} 2 \mathrm{H} 4+\mathrm{OH}=\mathrm{CH} 3+\mathrm{CH} 2 \mathrm{O}$

52. $\mathrm{C} 2 \mathrm{H} 4+\mathrm{O}=\mathrm{CH} 3+\mathrm{HCO}$

53. $\mathrm{C} 2 \mathrm{H} 4+\mathrm{O}=\mathrm{CH} 2 \mathrm{O}+\mathrm{CH} 2$

54. $\mathrm{C} 2 \mathrm{H} 3+\mathrm{M}=\mathrm{C} 2 \mathrm{H} 2+\mathrm{H}+\mathrm{M}$

55. $\mathrm{C} 2 \mathrm{H3}+\mathrm{O} 2=\mathrm{CH} 2 \mathrm{O}+\mathrm{HCO}$

56. $\mathrm{C} 2 \mathrm{H} 3+\mathrm{H}=\mathrm{C} 2 \mathrm{H} 2+\mathrm{H}_{2}$

57. $\mathrm{C} 2 \mathrm{H} 3+\mathrm{OH}=\mathrm{C} 2 \mathrm{H} 2+\mathrm{H} 2 \mathrm{O}$

58. $\mathrm{C} 2 \mathrm{H}_{3}+\mathrm{CH} 2=\mathrm{C} 2 \mathrm{H}_{2}+\mathrm{CH} 3$

59. $\mathrm{C} 2 \mathrm{H} 3+\mathrm{C} 2 \mathrm{H}=2 \mathrm{C} 2 \mathrm{H} 2$

6O. $\mathrm{C} 2 \mathrm{H}_{3}+\mathrm{O}=\mathrm{CH} 2 \mathrm{CO}+\mathrm{H}$
$(k=A T * b \underset{b}{\exp (-E / R T))}$

3. $00 E+15$

$2.50 E+13$

2. $20 E+13$

1. $00 \mathrm{E}+14$

2. $50 E+13$

$2.10 E+13$

2. $50 E+13$

$5.00 E+13$

5. $00 \mathrm{E}+15$

6. $30 E+13$

4. $00 E+11$

$3.00 E+12$

2. $80 \mathrm{E}+13$

2. $10 E+13$

3. $00 \mathrm{E}+15$

$1.00 E+13$

2. $20 E+13$

1. $20 \mathrm{E}+14$

$4.00 E+14$

3. $00 E+13$

2. $50 E+11$

2. $50 E+11$

1. $00 \mathrm{E}+12$

5. $00 E+13$

1. $00 E+13$

2. $00 E+13$

1. $00 \mathrm{E}+11$

1. $00 E+15$

2. $10 E+12$

5. $00 \mathrm{E}+13$

1. $60 \mathrm{E}+15$

2. $00 E+14$

1. $00 E+14$

1. $00 E+13$

3. $00 E+16$

1. $00 E+14$

1. $00 E+14$

1. $00 E+13$

1. $00 \mathrm{E}+13$

$4.00 E+15$

2. $20 E+13$

1. $00 \mathrm{E}+14$

$1.00 \mathrm{E}+13$

1. $40 E+13$

1. $00 \mathrm{E}+16$

1. $00 E+13$

1. $20 E+12$

3. $00 E+13$

9. $30 E+16$

2. $00 E+13$

2. $00 E+12$

3. $30 \mathrm{E}+12$

2. $50 \mathrm{E}+13$

7. $90 E+14$

4. $00 \mathrm{E}+12$

6. $00 E+12$

5. $00 E+12$

3. $00 E+13$

3. $00 E+13$
0.0

0.0

0.0

0.0

0.0

0.0

0.0

0.0

0.0

0.0

0.0

0.0

0.0

0.0

0.0

0.0

0.0

0.0

0.0

0.0

0.0

0.0

0.0

0.0

0.0

0.0

0.0

0.0

0.0

0.0

0.0

0.0

0.0

0.0

0.0

0.0

0.0

0.0

0.0

0.0

0.0

0.0

0.0

0.0

0.0

0.0

0.0

0.0

0.0

0.0

0.0

0.0

0.0

0.0

0.0

0.0

0.0

0.0

0.0

0.0
95500.0 3000.0

9004.0

10000.0

7500.0

4580.0

5000.0

50000.0

108000.0

60000.0

4000.0

8100.0

4910.0

4580.0

88000.0

6000.0

7929.0

12400.0

25200.0

0.0

40000.0

43900.0

0.0

0.0

0.0

0.0

0.0

0.0

7470.0

1000.0

82000.0

0.0

0.0

0.0

15000.0

58000.0

0.0

1000.0

0.0

80000.0

8000.0

8000.0

5000.0

32900.0

60000.0

0.0

0.0

0.0

77200.0

5955.0

960.0

1130.0

5000.0

31500.0

$-250.0$

0.0

0.0

0.0

0.0

0.0 
TABLE VI.2

$\mathrm{C}_{6} \mathrm{H}_{5} \mathrm{Cl}$ PYROLYSIS OXIDATION MECHANISM (CONTINUED)

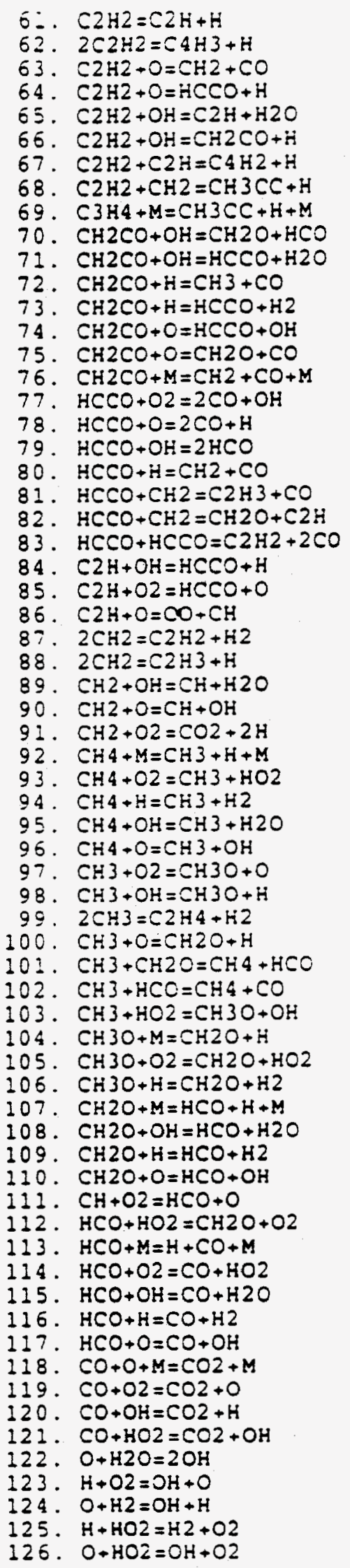

\begin{tabular}{|c|c|c|}
\hline 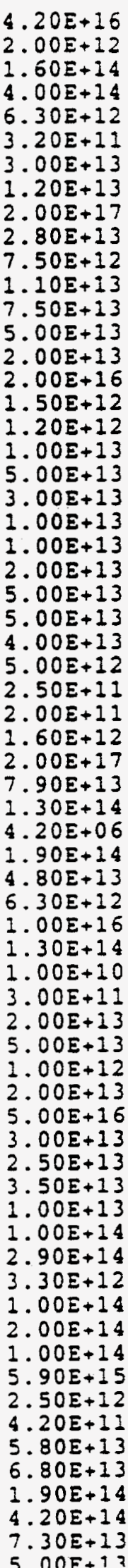 & $\begin{array}{l}0.0 \\
0.0 \\
0.0 \\
0.0 \\
0.0 \\
0.0 \\
0.0 \\
0.0 \\
0.0 \\
0.0 \\
0.0 \\
0.0 \\
0.0 \\
0.0 \\
0.0 \\
0.0 \\
0.0 \\
0.0 \\
0.0 \\
0.0 \\
0.0 \\
0.0 \\
0.0 \\
0.0 \\
0.0 \\
0.0 \\
0.0 \\
0.0 \\
0.7 \\
0.7 \\
0.0 \\
0.0 \\
0.0 \\
0.0 \\
2.0 \\
0.0 \\
0.0 \\
0.0 \\
0.0 \\
0.0 \\
0.5 \\
0.5 \\
0.0 \\
0.0 \\
0.0 \\
0.0 \\
0.0 \\
0.0 \\
0.0 \\
0.0 \\
0.0 \\
0.0 \\
0.0 \\
0.0 \\
0.0 \\
0.0 \\
0.0 \\
0.0 \\
0.0 \\
0.0 \\
0.0 \\
0.0 \\
0.0 \\
0.0 \\
0.0 \\
010\end{array}$ & 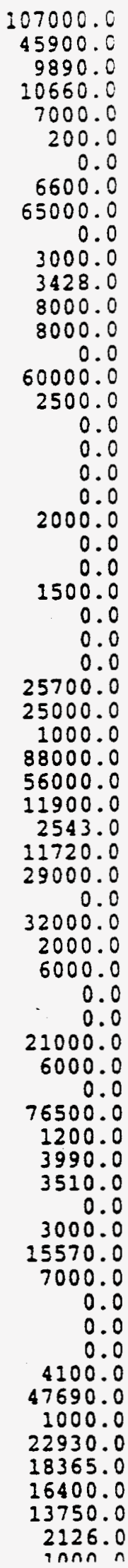 \\
\hline
\end{tabular}


TABLE VI.2

\section{$\mathrm{C}_{6} \mathrm{H}_{5} \mathrm{Cl}$ PYROL YSIS OXIDATION MECHANISM (CONTINUED)}

127. $\mathrm{HO} 2+\mathrm{OH}=\mathrm{H} 2 \mathrm{O}+\mathrm{O} 2$

128. $\mathrm{H}+\mathrm{HO} 2=2 \mathrm{OH}$

129. $\mathrm{H} 2+\mathrm{HO} 2=\mathrm{H} 2 \mathrm{O} 2+\mathrm{H}$

$130 . \mathrm{OH}+\mathrm{H} 2 \mathrm{O} 2=\mathrm{H} 2 \mathrm{O}+\mathrm{HO} 2$

131. $2 \mathrm{HO} 2=\mathrm{H} 2 \mathrm{O} 2+\mathrm{O} 2$

132. $\mathrm{H}+\mathrm{H} 2 \mathrm{O} 2=\mathrm{OH}+\mathrm{H} 2 \mathrm{O}$

133. $\mathrm{H} 2 \mathrm{O} 2+\mathrm{M}=2 \mathrm{OH}+\mathrm{M}$

134. $\mathrm{H} 2+\mathrm{OH}=\mathrm{H} 2 \mathrm{O}+\mathrm{H}$

135. $\mathrm{H}+\mathrm{O} 2+\mathrm{M}=\mathrm{HO} 2+\mathrm{M}$

136. $\mathrm{H} 2 \mathrm{O}+\mathrm{M}=\mathrm{H}+\mathrm{OH}+\mathrm{M}$

$137 . \mathrm{H}+\mathrm{O}+\mathrm{M}=\mathrm{OH}+\mathrm{M}$

138. $H 2+M=2 H+M$

139. $O 2+M=2 O+M$

140. $2 C L+M=C L 2+M$

141. $\mathrm{HCL}+\mathrm{M}=\mathrm{H}+\mathrm{CL}+\mathrm{M}$

142. $\mathrm{HCL}+\mathrm{H}=\mathrm{H} 2+\mathrm{CL}$

143. $\mathrm{H}+\mathrm{CL} 2=\mathrm{HCL}+\mathrm{CL}$

$144 . \mathrm{O}+\mathrm{HCL}=\mathrm{OH}+\mathrm{CL}$

145. $\mathrm{OH}+\mathrm{HCL}=\mathrm{CL}+\mathrm{H} 2 \mathrm{O}$

146. $\mathrm{O}+\mathrm{CL} 2=\mathrm{CLO}+\mathrm{CL}$

$147 . \mathrm{O}+\mathrm{CLO}=\mathrm{CL}+\mathrm{O} 2$

148. $\mathrm{CL}+\mathrm{HO} 2=\mathrm{HCL}+\mathrm{O} 2$

149. $\mathrm{CL}+\mathrm{HO} 2=\mathrm{OH}+\mathrm{CLO}$
8. $00 E+12$

1. $30 E+14$

$7.90 E+13$

$6.10 E+12$

1. $80 E+12$

7. $80 E+11$

1. $40 \mathrm{E}+17$

4. $70 E+13$

1. $50 E+15$

1. $30 E+15$

7. $10 E+18$

2. $20 E+14$

1. $80 E+18$

$7.90 E+14$

2. $80 E+13$

$7.90 E+12$

8. $50 E+12$

3. $20 E+13$

2. $20 E+12$

1. $30 E+13$

5. $60 E+13$

7. $90 \mathrm{E}+12$

$6.30 \mathrm{E}+13$
0.0

0.0

0.0

0.0

0.0

0.0

0.0

0.0

0.0

0.0

$-1.0$

0.0

$-1.0$

0.0

0.0

0.0

0.0

0.0

0.0

0.0

0.0

0.0

0.0
0.0

1070.0

25000.0

1430.0

0.0

0.0

45510.0

6098.0

$-1000.0$ 105140.0 0.0 96000.0 118000.0 0.0 81800.0 3400.0 1200.0 6700.0 1000.0 2800.0 400.0 0.0 1700.0

NOTE: A units mole-cm-sec-K, E units cal/mole 
decomposition of $\mathrm{C}_{6} \mathrm{H}_{5} \mathrm{Cl} / \mathrm{H}_{2}$ mixtures by Ritter and Bozzelli [1], shock-tube studies of benzene pyrolysis by Kiefer et al. [2], and a detailed mechanism for benzene oxidation proposed by Bittker [3]. Elementary reaction steps incorporating oxygen and oxygen-containing radicals were included in the mechanism to account for the significant initial concentrations of oxygen $(-10 \mathrm{ppmv})$ present in these experiments. Arrhenius rate parameters for hydrocarbon molecular growth reactions were estimated by analogy with previous studies by Frenklach [4]. $\mathrm{HCl} / \mathrm{Cl}_{2}$ and $\mathrm{CO} / \mathrm{H}_{2} / \mathrm{O}_{2}$ reaction sets are well established and were taken without modification from literature compilations. Reverse rates (not shown) were calculated from thermochemistry and principles of detailed balancing.

The photo-assisted thermal experiments were modeled with the same mechanism and the inclusion of the following additional photochemical, or excited state, reaction:

$$
\mathrm{C}_{6} \mathrm{H}_{5} \mathrm{Cl}+\mathrm{hv}_{2800} \stackrel{\mathrm{k}_{\text {exf }}}{\longrightarrow} \mathrm{C}_{6} \mathrm{H}_{5}+\mathrm{Cl} \text {. }
$$

First-order rate parameters for this reaction were obtained from the following equation which can be derived starting with the Beer-Lambert relation and assuming weak absorption of callimated radiation:

$$
k_{e x c}=1.92 \times 10^{-5} \varepsilon \lambda(P / A) \Phi
$$

where $\varepsilon=$ molar extinction coefficient $(\mathrm{L} / \mathrm{cm} / \mathrm{mol}), \lambda=$ excitation wavelength $(\mathrm{nm}), P / A=$ laser fluence $\left(W / \mathrm{cm}^{2}\right)$, and $\Phi=$ quantum yield for the reaction. Values for $\varepsilon$, and $P / A$ used to derive $k_{e x c}$ are given in Table VI.1. In the calculation of $k_{\text {exc }}(1 / s)$ as a function of temperature, $\Phi$ was assumed to be constant with a magnitude of unity. Values of $k_{\text {exc }}$ are presented in the last column of Table VI.1. The excitation rate constant was fit to the following three-parameter relation for inclusion in the model:

$$
k=A T^{n} \exp (-E a / R T)
$$

Figures VI. 6 and VI.7 present a comparison of model predictions with experimental results for the thermal destruction of $\mathrm{C}_{6} \mathrm{H} 5 \mathrm{Cl}$. Model predictions for parent destruction are in good agreement with experiment. Sensitivity analyses indicate that the activation energy of $\mathrm{C}-\mathrm{Cl}$ bond rupture from $\mathrm{C}_{6} \mathrm{H}_{5} \mathrm{Cl}$ exhibits a significant influence on the predictive capability of the model. Model predictions for five of the observed products (benzene $\left(\mathrm{C}_{6} \mathrm{H}_{6}\right)$, ethynyl benzene $\left(\mathrm{C}_{8} \mathrm{H}_{6}\right)$, naphthalene $\left(\mathrm{C}_{10} \mathrm{H}_{8}\right)$, vinyl acetylene $\left(\mathrm{CH}_{2}=\mathrm{CHC} \equiv \mathrm{CH}\right)$, and diacetylene $\left.(\mathrm{CH} \equiv \mathrm{CC} \equiv \mathrm{CH})\right)$, indicate large discrepancies with experiment. Model predictions of $\mathrm{C}_{6} \mathrm{H}_{5}$ and $\mathrm{C}_{10} \mathrm{H}_{8}$ are within a factor of 10 of maximum experimental yields. However, 


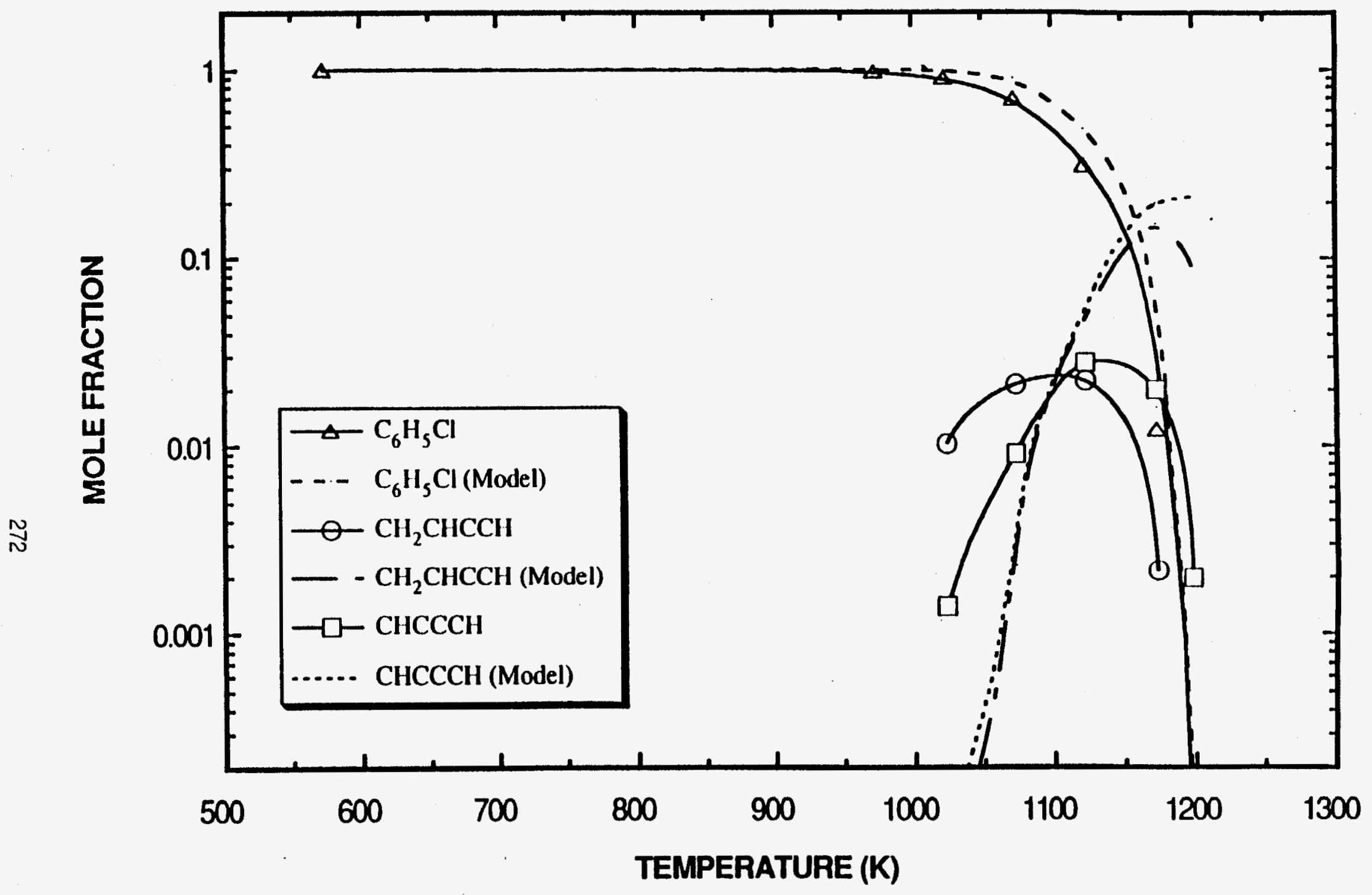

Figure VI.6. Comparison of model predictions vs experiment for the thermal decomposition of $\mathrm{C}_{6} \mathrm{H}_{5} \mathrm{Cl}$ in flowing helium. Formation/destruction profiles for $\mathrm{CH}_{2} \mathrm{CH} \mathrm{CCH}$ and $\mathrm{CHCCCH}$ are also shown. 


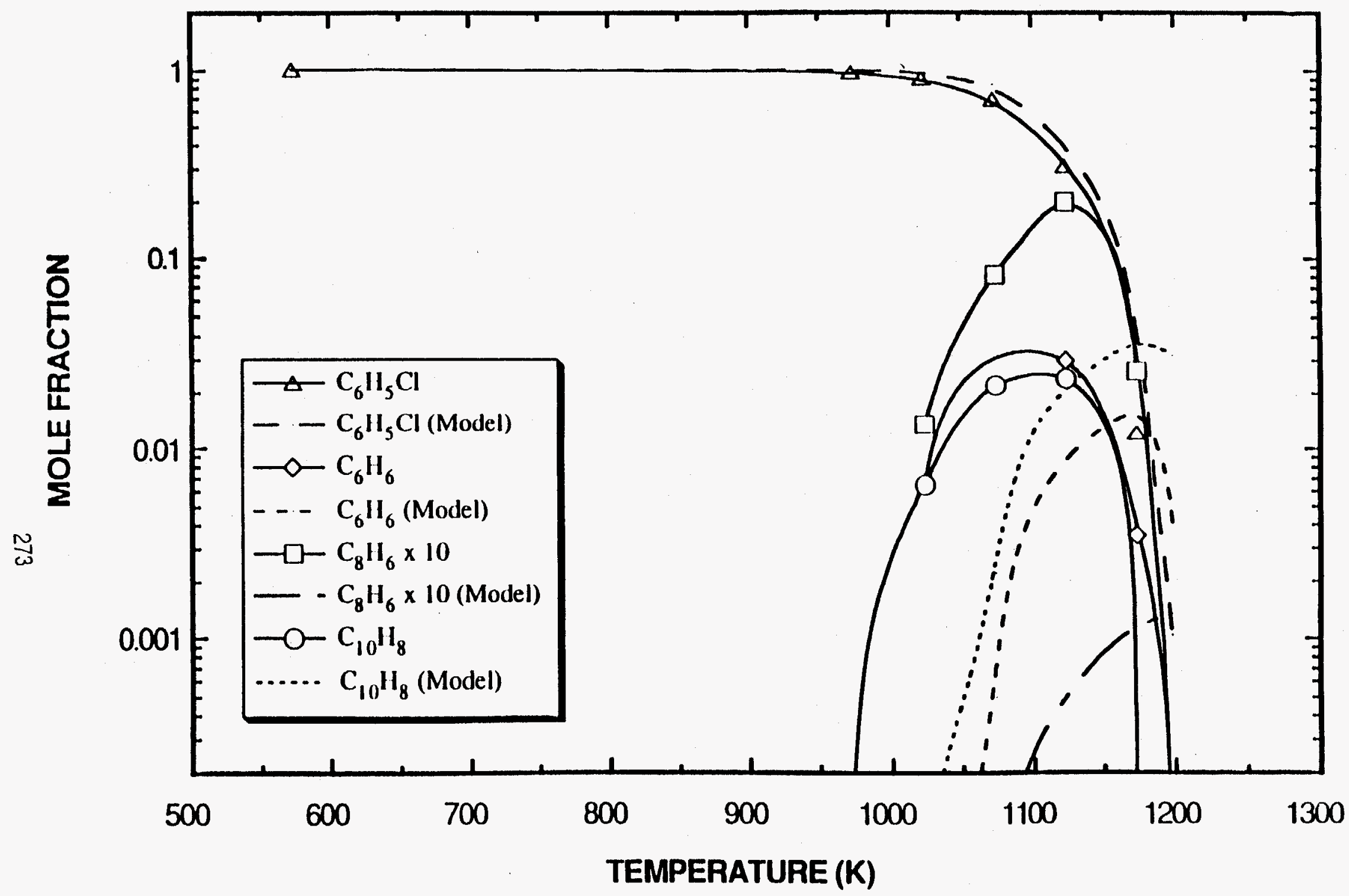

Figure VI.7 Comparison of model predictions vs experiment for the thermal decomposition of $\mathrm{C}_{6} \mathrm{H}_{5} \mathrm{Cl}$ in flowing helium. Formation/destruction profiles for $\mathrm{C}_{6} \mathrm{H}_{6}, \mathrm{C}_{8} \mathrm{H}_{6}$, and $\mathrm{C}_{10} \mathrm{H}_{8}$ are also shown. 
the model predicts initial formation of these compounds at $\sim 100^{\circ} \mathrm{C}$ higher temperatures. Model predictions of $\mathrm{CH}_{2}=\mathrm{CHC} \equiv \mathrm{CH}$ and $\mathrm{CH} \equiv \mathrm{CCH} \equiv \mathrm{CH}$ are also shifted to higher temperatures and yields substantially overshoot the experimental data. This suggests that rates for reaction channels leading to the consumption of these compounds may be under-estimated.

The modeling of the photo-enhancement is only in its initial stages. The pulsed nature of the light source required a "pulsed" photo-model in which there were short "light" reaction periods of $10 \mathrm{~ns}$ followed by long "dark" reaction periods of $50 \mathrm{~ms}$. The modeling was executed by initiating the photo model, stopping the photo-model, entering the results into the thermal model, initiating the thermal model, stopping the thermal model, and repeating this procedure until the $10.0 \mathrm{~s}$ reaction time was achieved. This approach proved to be too time consuming for the computer and the modeling effort was suspended until more computer time could be made available for this relatively costly procedure or a new procedure could be developed.

Initial model predictions of $\mathrm{C}_{6} \mathrm{H}_{5} \mathrm{Cl}$ destruction in the presence of $2800 \AA$ radiation are in qualitative agreement with experiment. Model predictions based on 20 laser pulses at a temperature of $700^{\circ} \mathrm{C}$ indicated a fraction remaining of less than 0.001 . This result is consistent with below detection limit yields determined from experimental measurements.

\section{VI.2 A Five Component Mixture}

The objective of this study was to combine validated chemical kinetic models of hydrocarbon fuels and hazardous waste compounds and simulate the solar-induced photo/thermal oxidation of a mixture of these compounds at elevated temperatures. Simulations were conducted using chlorobenzene $\left(\mathrm{C}_{6} \mathrm{H}_{5} \mathrm{Cl}\right)$ and simulated sunlight as a photo-catalyst. The hazardous waste mixture also contained the following compounds: methane $\left(\mathrm{CH}_{4}\right)$, methylene chloride $\left(\mathrm{CH}_{2} \mathrm{Cl}_{2}\right)$, benzene $\left(\mathrm{C}_{6} \mathrm{H}_{6}\right)$, and trichloroethene $\left(\mathrm{C}_{2} \mathrm{HCl}_{3}\right)$. These components were selected because thermal models already existed and they were representative of the classes of compounds in typical waste streams. 
A kinetic model for the oxidation of $\mathrm{CH}_{4}$ and $\mathrm{CH}_{2} \mathrm{Cl}_{2}$ was obtained from $\mathrm{Ho}$ and Bozzelli [5]. The kinetic models for the oxidation of $\mathrm{C}_{6} \mathrm{H}_{6}$ and $\mathrm{C}_{2} \mathrm{HCl}_{3}$ were obtained from Bittker [3] and $\mathrm{Chang}$ and Senkan [6], respectively. The solar photo-absorption rate constant for $\mathrm{C}_{6} \mathrm{H}_{5} \mathrm{Cl}$ was obtained from photo absorption studies recently published from our laboratories [7]. A listing of the mechanism is given in Table VI.3 along with rate coefficients for the forward reactions. Rates for the reverse reaction were obtained by detailed balancing. The mechanism was quite large and consisted of 405 reactions and 106 species. Numerous reactions were subsequently added to the model to improve its accuracy. These reactions resulted in interaction of stable species or radicals from the degradation of the individual starting materials, e.g.,

$$
\mathrm{C}_{6} \mathrm{H}_{5}(\mathrm{cy})+\mathrm{C}_{2} \mathrm{HCl}_{3}=\mathrm{C}_{2} \mathrm{HCl}_{2}+\mathrm{C}_{6} \mathrm{H}_{5} \mathrm{Cl} \mathrm{k}_{16}
$$

The CHEMKIN-II/SENKIN homogeneous kinetics code was used to conduct the simulations. The program has previously been modified to run on a CRAY mainframe computer at the Ohio Super Computer Center.

Several variables were parametrically addressed during the course of running these simulations. These are: intensity of simulated sunlight, hazardous waste mixture relative concentrations, absorption cross section, and residence time. Two initial mixture compositions were chosen for these simulations. They are given in Table VI.4.

Exposure temperature was the independent parameter with simulations covering the range 573 $1273 \mathrm{~K}$. The control simulation was a series of kinetic simulations in the absence of solar radiation. Subsequent simulation in the presence of sunlight were then used determine the shift in concentration profiles for the starting materials and important intermediates.

Numerical simulations of the solar-catalyzed photo/thermal oxidation of a mixture of $\mathrm{CH}_{4}, \mathrm{CH}_{2} \mathrm{Cl}_{2}$, $\mathrm{C}_{2} \mathrm{HCl}_{3}, \mathrm{C}_{6} \mathrm{H}_{6}$ and $\mathrm{C}_{6} \mathrm{H}_{5} \mathrm{Cl}$ have been completed. The results of these simulations are summarized in Figure VI.8 - Vl.11. Open symbols denote simulations with 2,000 suns. Closed symbols denote purely thermal simulations. 
Figure VI.8a. Solar Simulation, $\phi=0.5, \mathrm{H}: \mathrm{Cl}=12, \mathrm{t}_{\mathrm{r}}=1 \mathrm{~s}$

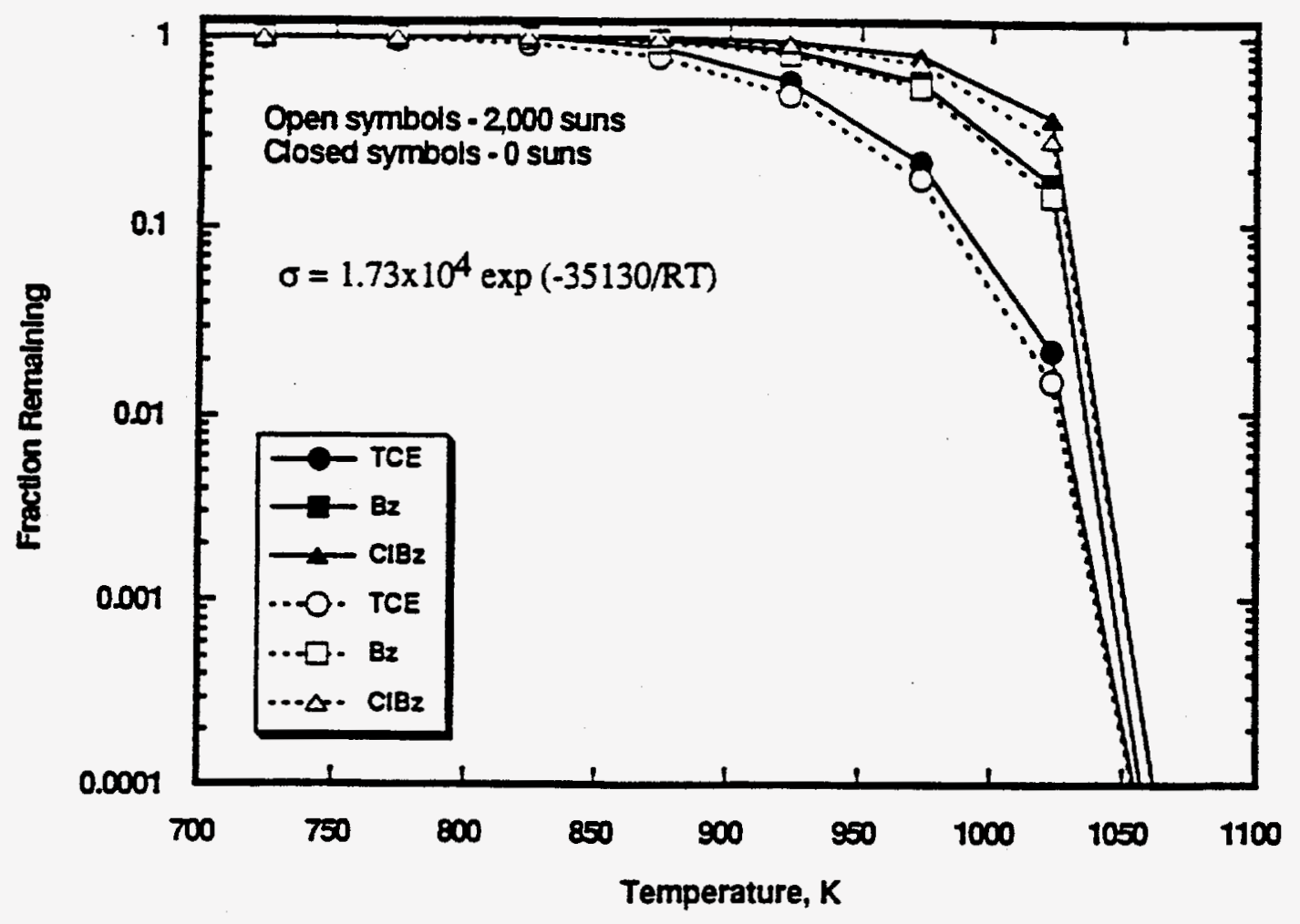

Figure VI.8b. Solar Simulation, $\phi=0.5, \mathrm{H}: \mathrm{Cl}=12, \mathrm{t}_{r}=1 \mathrm{~s}$

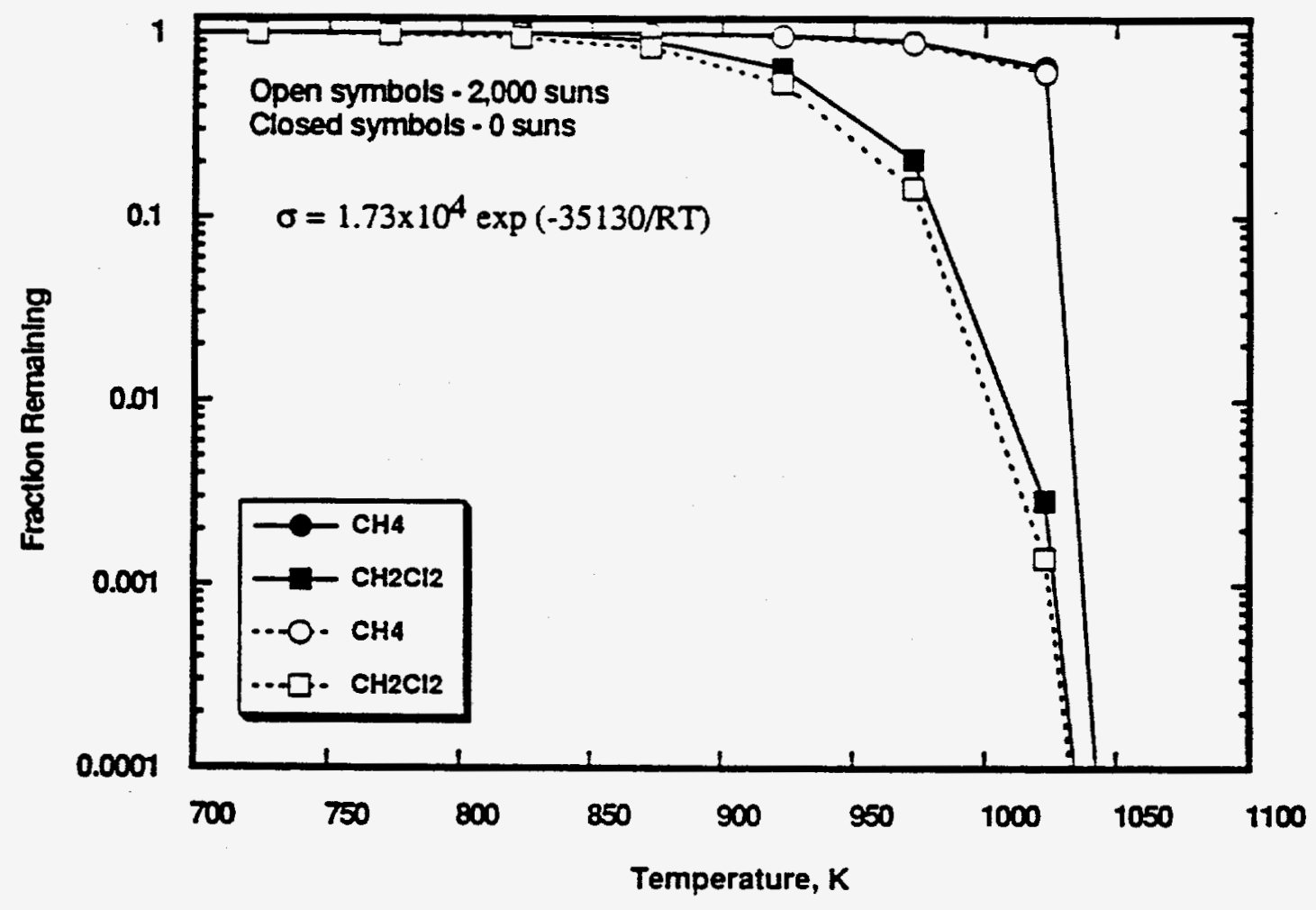


Figure VI.9a. Solar Simulation, $\phi=0.5, \mathrm{H}: \mathrm{Cl}=12, \mathrm{t}_{r}=10 \mathrm{~s}$

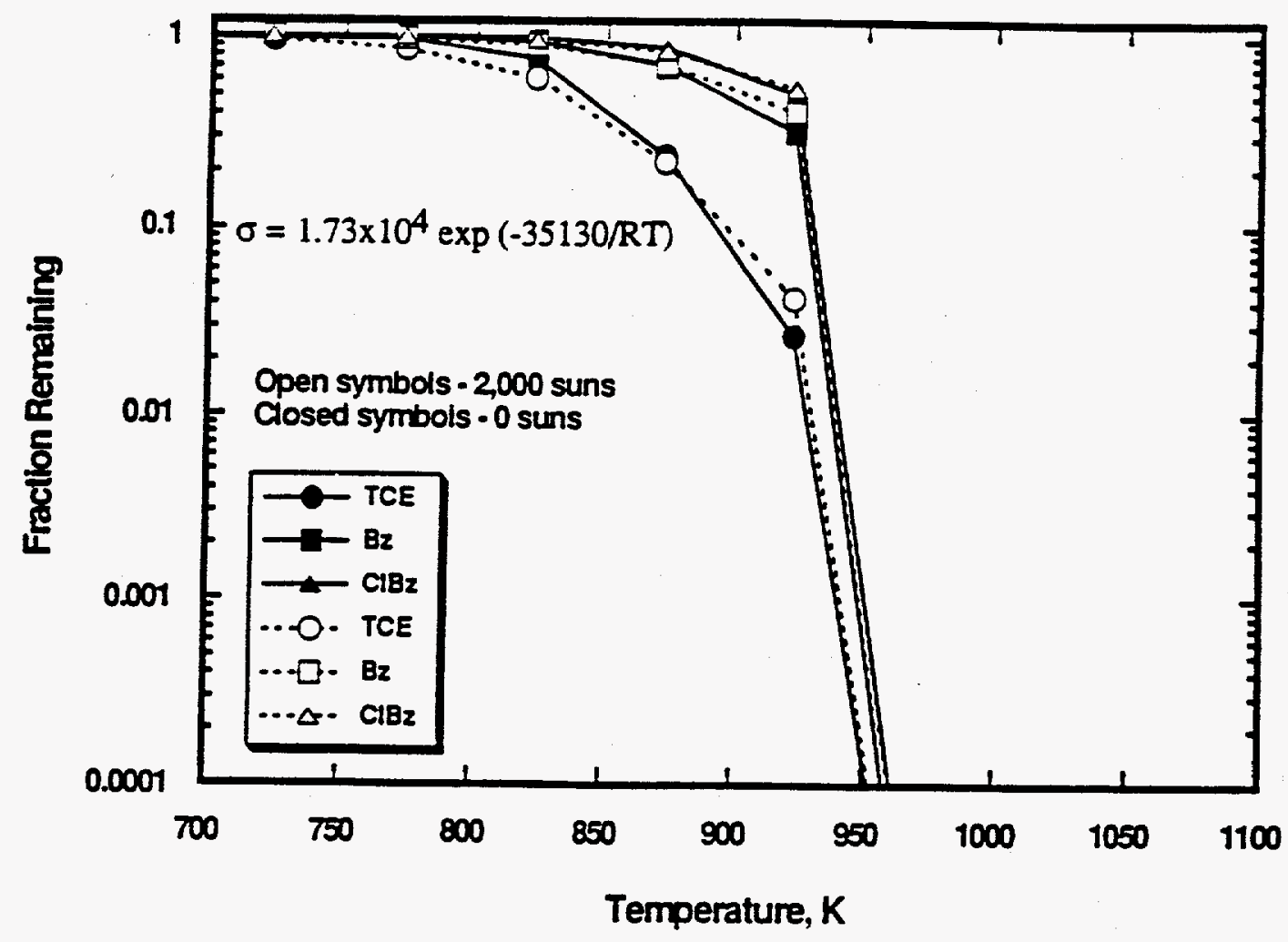

Figure VI.9b. Solar Simulation, $\phi=0.5, \mathrm{H}: \mathrm{Cl}=12, \mathrm{t}_{\mathrm{r}}=10 \mathrm{~s}$

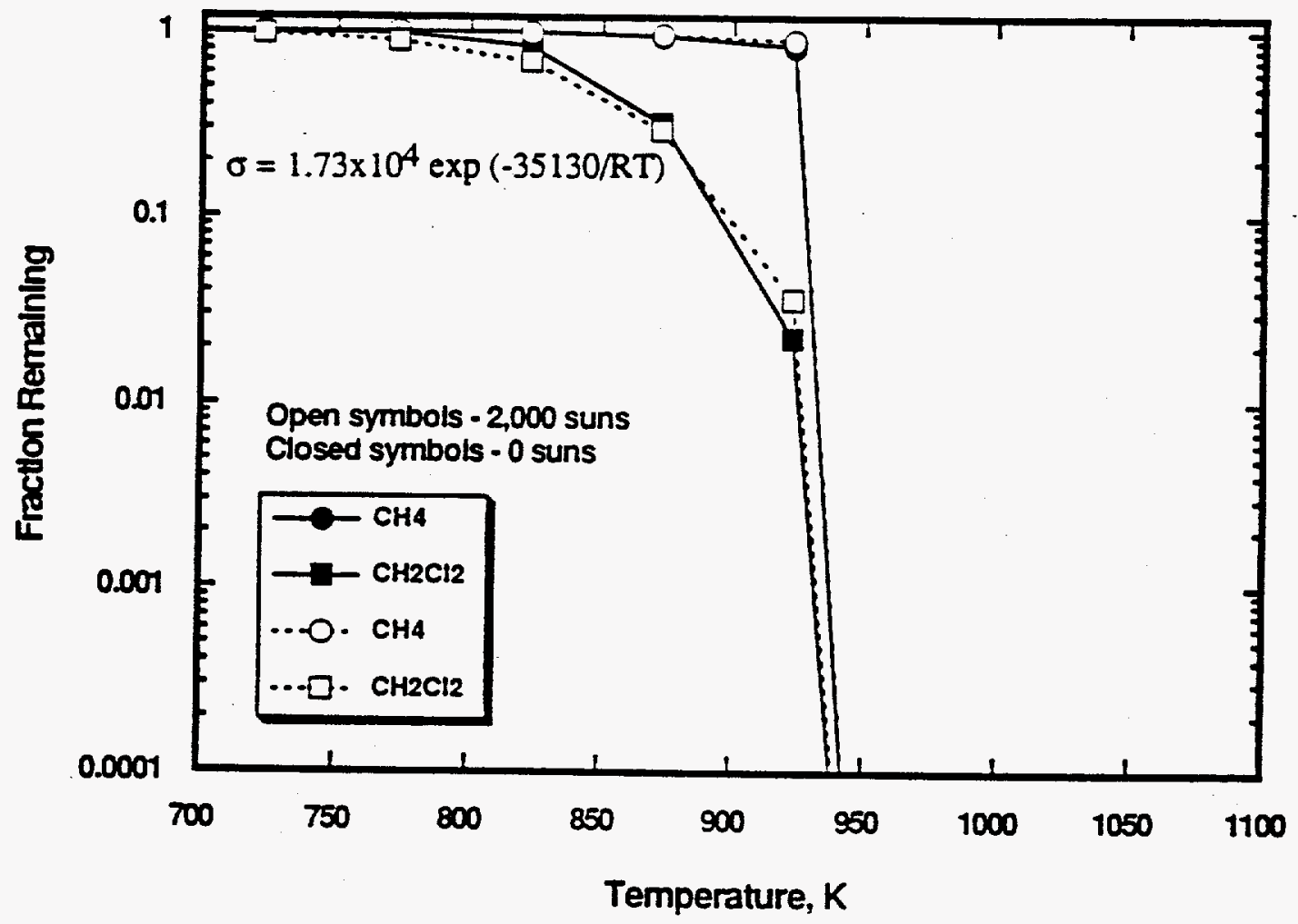


Figure VI.10a. Solar Simulation, $\phi=0.5, \mathrm{H}: \mathrm{Cl}=3, t_{r}=1 \mathrm{~s}$

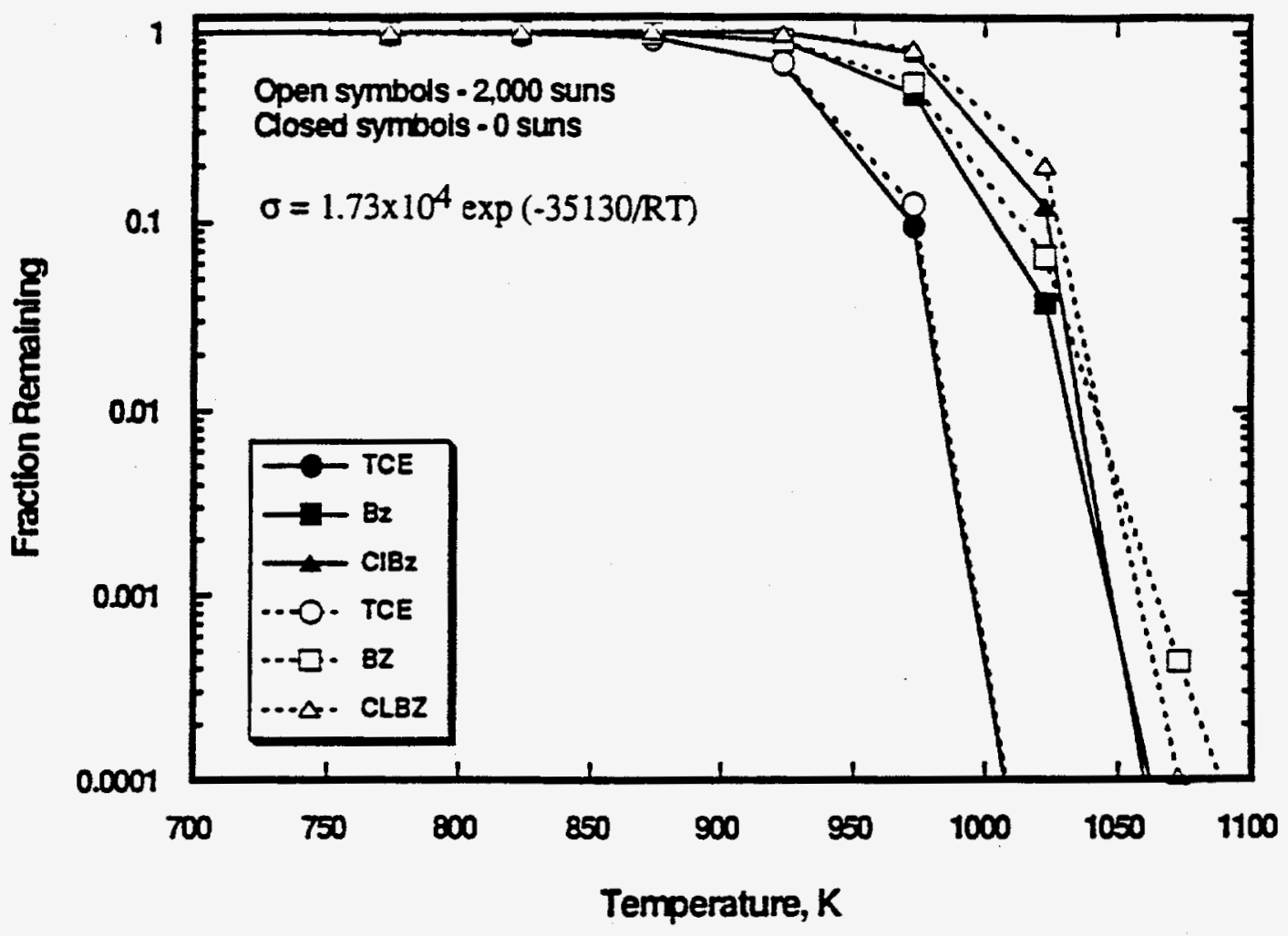

Figure VI.10b. Solar Simulation, $\phi=0.5, \mathrm{H}: \mathrm{Cl}=3, t_{r}=1 \mathrm{~s}$

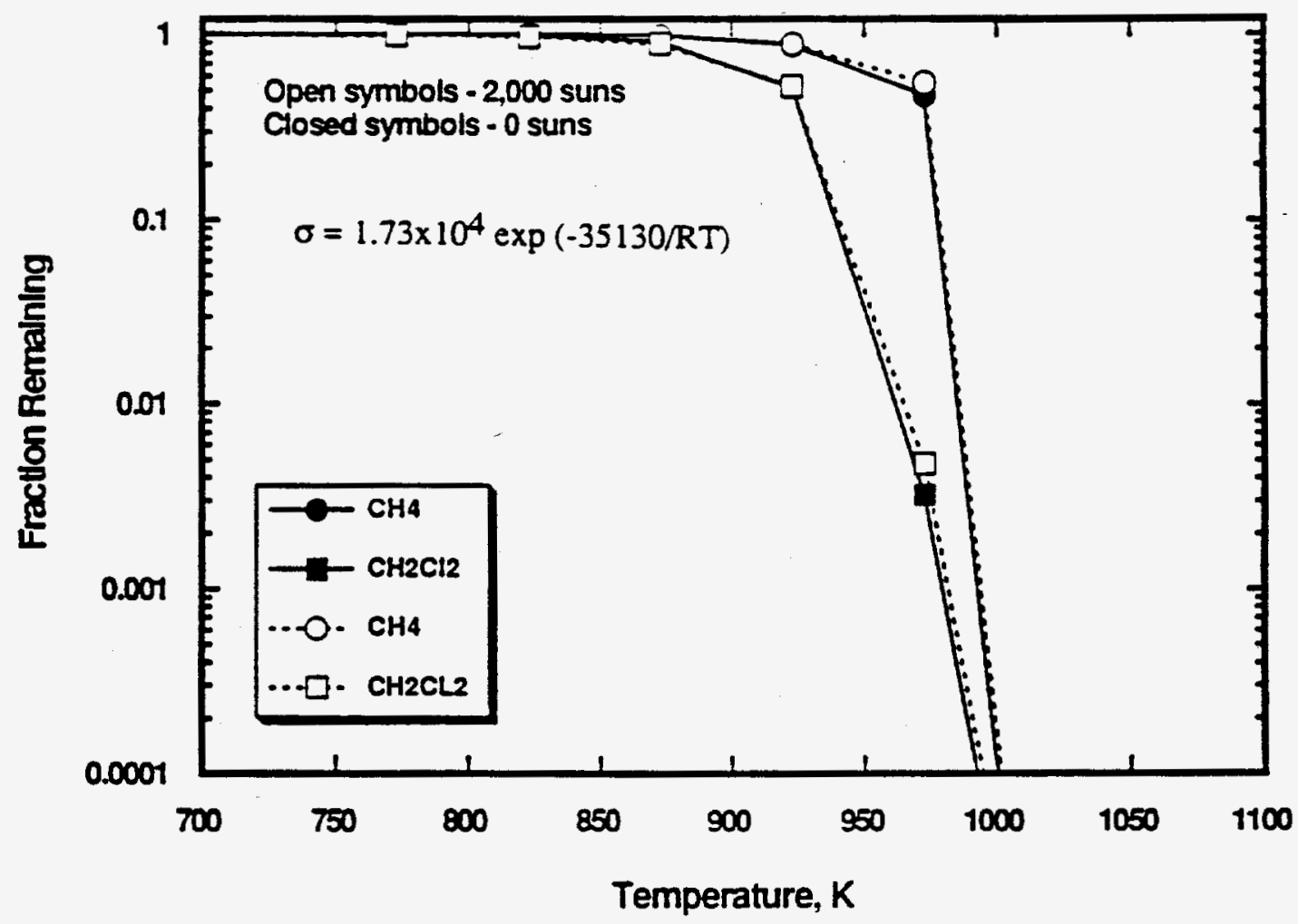


Figure VI.11a. Solar Simulation, $\phi=0.5, \mathrm{H:Cl}=3, t_{t}=10 \mathrm{~s}$

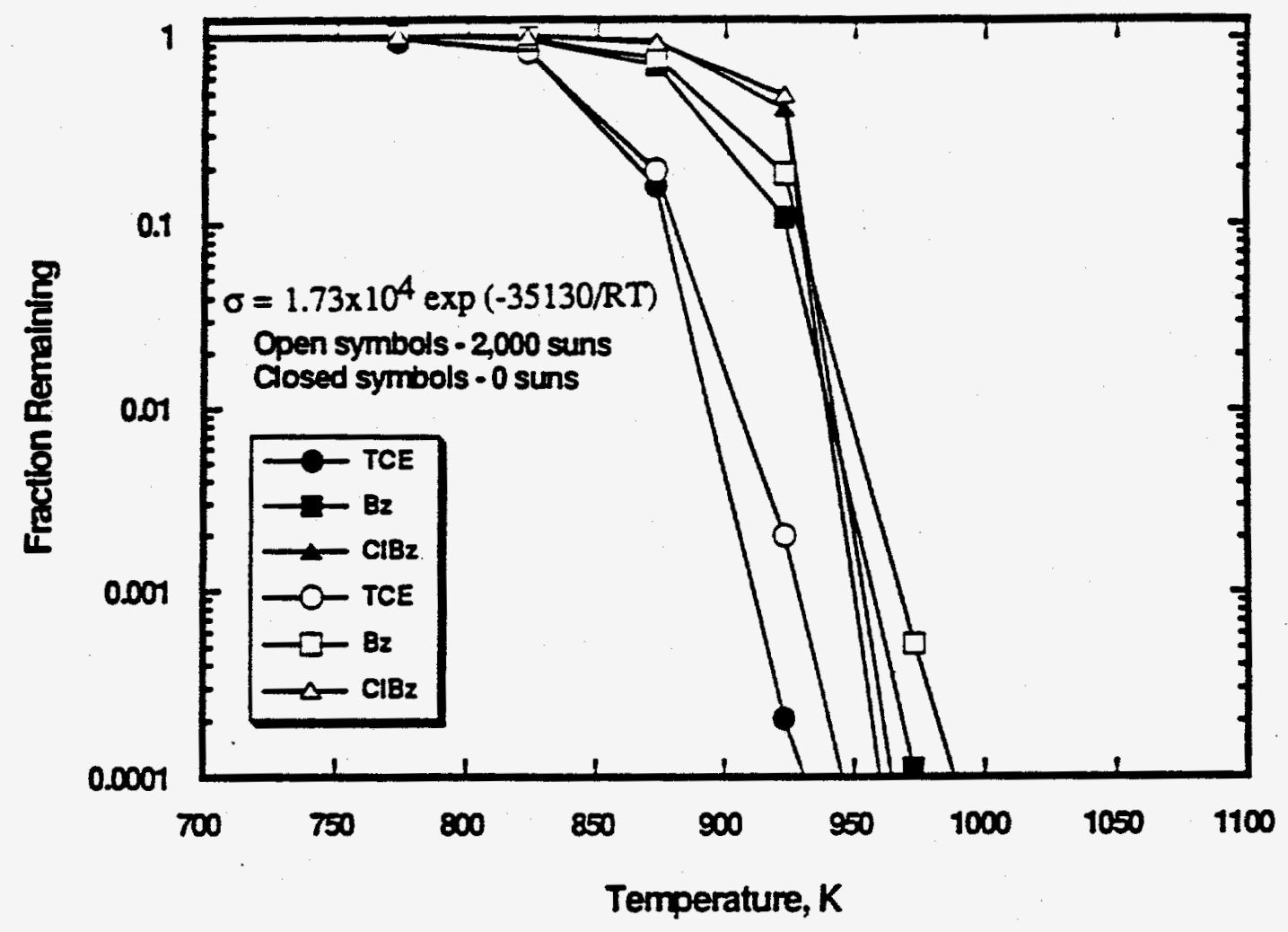

Figure VI.11b. Solar Simulation, $\phi=0.5, \mathrm{H}: \mathrm{Cl}=3, \mathrm{t}_{\mathrm{r}}=10 \mathrm{~s}$

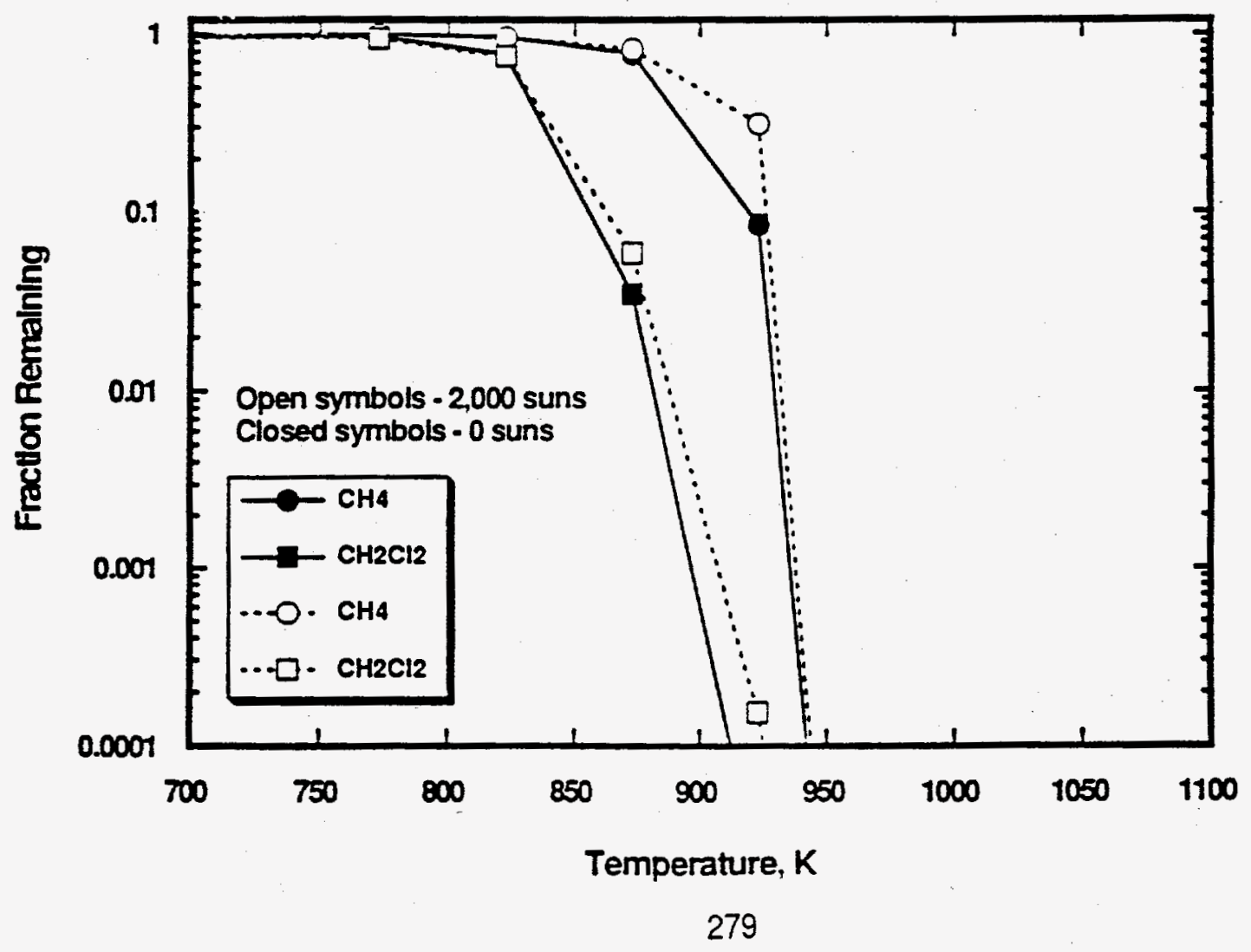




\section{PHOTO-THERMAL OXIDATION MECHANISM FOR A 5 COMPONENT MIXTURE}

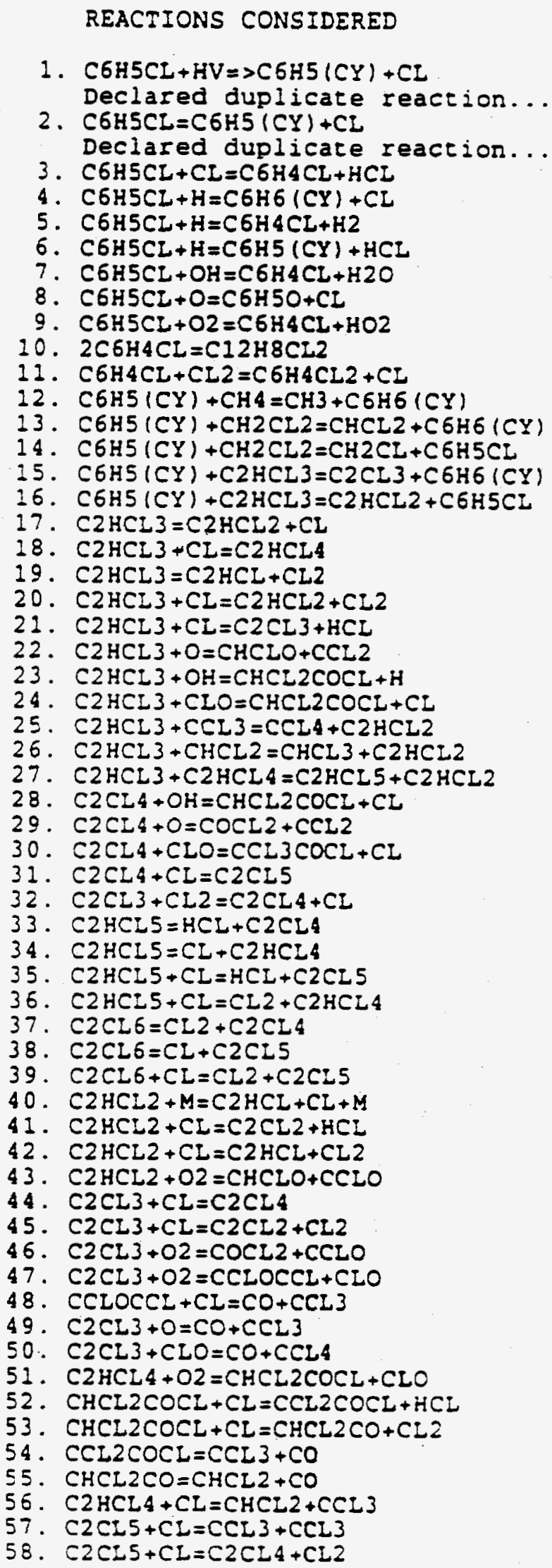

REACTIONS CONSIDERED

C6H5CL $+H V=>C 6 H 5(C Y)+C I$

C6HSCL $=$ C6H5 $(\mathrm{CY})+\mathrm{CL}$

Declared duplicate reaction...

3. $\mathrm{C} 6 \mathrm{H} 5 \mathrm{CL}+\mathrm{CL}=\mathrm{C} 6 \mathrm{H} 4 \mathrm{CL}+\mathrm{HCL}$

4. $\mathrm{C} 6 \mathrm{H} 5 \mathrm{CL}+\mathrm{H}=\mathrm{C} 6 \mathrm{H} 6(\mathrm{CY})+\mathrm{CL}$

5. $\mathrm{C} 6 \mathrm{H} 5 \mathrm{CL}+\mathrm{H}=\mathrm{C} 5 \mathrm{H} 4 \mathrm{CL}+\mathrm{H} 2$

6. $\mathrm{C} 6 \mathrm{H} 5 \mathrm{CL}+\mathrm{H}=\mathrm{C} 6 \mathrm{H} 5(\mathrm{CY})+\mathrm{HCL}$

7. $\mathrm{C} 6 \mathrm{H} 5 \mathrm{CL}+\mathrm{OH}=\mathrm{C} 6 \mathrm{H} 4 \mathrm{CL}+\mathrm{H} 2 \mathrm{O}$

8. $C 6 H 5 C L+O=C 6 H 5 O+C L$

9. $\mathrm{C} 6 \mathrm{H} 5 \mathrm{CL}+\mathrm{O} 2=\mathrm{C} 6 \mathrm{H} 4 \mathrm{CL}+\mathrm{HO} 2$

$\mathrm{CL}=\mathrm{C} 12 \mathrm{H} 8 \mathrm{CL}$

13. $\mathrm{C} 6 \mathrm{H} 5(\mathrm{CY})+\mathrm{CH} 2 \mathrm{CL} 2=\mathrm{CHCL} 2+\mathrm{C} 6 \mathrm{H} 6(\mathrm{CY})$

4. $\mathrm{C} 6 \mathrm{H} 5(\mathrm{CY})+\mathrm{CH} 2 \mathrm{CL} 2=\mathrm{CH} 2 \mathrm{CL}+\mathrm{C} 6 \mathrm{H} 5 \mathrm{CI}$

15. $\mathrm{C} 6 \mathrm{HS}(\mathrm{CY})+\mathrm{C} 2 \mathrm{HCL} 3=\mathrm{C} 2 \mathrm{CL} 3+\mathrm{C} 6 \mathrm{H} 6(\mathrm{CY})$

7. $\mathrm{C} 2 \mathrm{HCl} 3=\mathrm{C} 2 \mathrm{HC} 2 \mathrm{CL}+\mathrm{Cl}$

18. $\mathrm{C} 2 \mathrm{HCL} 3+\mathrm{CL}=\mathrm{C} 2 \mathrm{HCL}$

19. $\mathrm{C} 2 \mathrm{HCL} 3=\mathrm{C} 2 \mathrm{HCL}+\mathrm{CL} 2$

20. $\mathrm{C} 2 \mathrm{HCL} 3+\mathrm{CL}=\mathrm{C} 2 \mathrm{HCL} 2+\mathrm{CL} 2$

21. $\mathrm{C} 2 \mathrm{HCL} 3+\mathrm{CL}=\mathrm{C} 2 \mathrm{CL} 3+\mathrm{HCL}$

2. $\mathrm{C} 2 \mathrm{HCL} 3+\mathrm{O}=\mathrm{CHCLO}+\mathrm{CCL} 2$

23. $\mathrm{C} 2 \mathrm{HCL} 3+\mathrm{OH}=\mathrm{CHCL} 2 \mathrm{COCI}+\mathrm{H}$

24. $\mathrm{C} 2 \mathrm{HCL} 3+\mathrm{CLO}=\mathrm{CHCL} 2 \mathrm{COCL}+\mathrm{CL}$

25. $\mathrm{C}_{2} \mathrm{HCL} 3+\mathrm{CCL} 3=\mathrm{CCL} 4+\mathrm{C} 2 \mathrm{HCL} 2$

26. $\mathrm{C}_{2} \mathrm{HCL} 3+\mathrm{CHCL2}=\mathrm{CHCL} 3+\mathrm{C} 2 \mathrm{HCL} 2$

27. $\mathrm{C} 2 \mathrm{HCL} 3+\mathrm{C} 2 \mathrm{HCL} 4=\mathrm{C} 2 \mathrm{HCL} 5+\mathrm{C} 2 \mathrm{HCL} 2$

28. $\mathrm{C} 2 \mathrm{CL} 4+\mathrm{OH}=\mathrm{CHCL} 2 \mathrm{COCL}+\mathrm{CL}$

29. $\mathrm{C} 2 \mathrm{CL} 4+\mathrm{O}=\mathrm{COCL} 2+\mathrm{CCL} 2$

30. $C 2 C L A+C L O=C C L 3 C O C L+C L$

31. $C 2 C L 4+C L=C 2 C L 5$

32. $C 2 C L 3+C L 2=C 2 C L 4+C L$

33. $\mathrm{C} 2 \mathrm{HCL}=\mathrm{HCL}+\mathrm{C} 2 \mathrm{CLA}$

34. $C 2 \mathrm{HCLS}=\mathrm{CL}+\mathrm{C} 2 \mathrm{HCL}$

35. $\mathrm{C2} \mathrm{HCL} 5+\mathrm{CL}=\mathrm{HCL}+\mathrm{C2CL}$

36. $\mathrm{C} 2 \mathrm{HCL} 5+\mathrm{CL}=\mathrm{CL} 2+\mathrm{C} 2 \mathrm{HCL} 4$

37. $C 2 C L E=C L 2+C 2 C L 4$

38. $C 2 C L 6=C L+C 2 C L 5$

39. $C 2 C L 6+C L=C L 2+C 2 C L 5$

40. $\mathrm{C} 2 \mathrm{HCL} 2+\mathrm{M}=\mathrm{C} 2 \mathrm{HCL}+\mathrm{CL}+\mathrm{M}$

41. $\mathrm{C} 2 \mathrm{HCL2}+\mathrm{CL}=\mathrm{C} 2 \mathrm{CL} 2+\mathrm{HCL}$

42. $\mathrm{C} 2 \mathrm{HCL} 2+\mathrm{CL}=\mathrm{C} 2 \mathrm{HCL}+\mathrm{CL} 2$

43. $\mathrm{C} 2 \mathrm{HCL} 2+\mathrm{O} 2=\mathrm{CHCLO}+\mathrm{CCLO}$

44. $\mathrm{C} 2 \mathrm{CL} 3+\mathrm{CL}=\mathrm{C} 2 \mathrm{CL}$

45. $\mathrm{C} 2 \mathrm{CL} 3+\mathrm{CL}=\mathrm{C} 2 \mathrm{CL} 2+\mathrm{CL} 2$

46. $\mathrm{C} 2 \mathrm{CL} 3+\mathrm{O} 2=\mathrm{COCL} 2+\mathrm{CCLO}$

47. $\mathrm{C} 2 \mathrm{CL}_{3}+\mathrm{O} 2=\mathrm{CCLOCCL}+\mathrm{CLO}$

4. $\mathrm{CCLOCCL}+\mathrm{CI}=\mathrm{CO}+\mathrm{CCL}$

49. $\mathrm{C}_{2} \mathrm{CL}_{3}+\mathrm{O}=\mathrm{CO}+\mathrm{CCL}_{3}$

50. $\mathrm{C} 2 \mathrm{CL} 3+\mathrm{CLO}=\mathrm{CO}+\mathrm{CCL}_{4}$

51. $\mathrm{C} 2 \mathrm{HCL} 4+\mathrm{O2}=\mathrm{CHCL} 2 \mathrm{COCL}+\mathrm{CLO}$

52. $\mathrm{CHCL} 2 \mathrm{COCL}+\mathrm{CL}=\mathrm{CCL} 2 \mathrm{COCL}+\mathrm{HCL}$

53. $\mathrm{CHCL} 2 \mathrm{COCL}+\mathrm{CL}=\mathrm{CHCL} 2 \mathrm{CO}+\mathrm{CL} 2$

54. $\mathrm{CCL} 2 \mathrm{COCL}=\mathrm{CCL} 3+\mathrm{CO}$

55. $\mathrm{CHCL} 2 \mathrm{CO}=\mathrm{CHCL} 2+\mathrm{CO}$

5. $\mathrm{C} 2 \mathrm{HCL} 4+\mathrm{CL}=\mathrm{CHCL}_{2}+\mathrm{CCL}_{3}$

58. $C 2 \mathrm{CL} 5+\mathrm{CL}=\mathrm{C} 2 \mathrm{CL} 4+\mathrm{Cl} 2$ $\left\langle k=A T * b_{b} \exp (-E / R T)\right.$

$\begin{array}{lll}3.45 E+06 & 0.0 & 35136.0\end{array}$

$2.50 E+13$

$2.20 E+13$

$1.00 E+14$

$2.50 E+13$

2. $10 E+13$

$2.50 E+13$

5. $00 E+13$

$2.00 E+13$

2. $50 \mathrm{E}+12$

8. $00 E+13$

$4.00 E+13$

$4.00 E+13$

$2.00 E+14$

$6.00 E+13$

$3.02 \mathrm{E}+33$

$3.63 E+32$

$9.55 E+35$

$3.16 E+13$

$2.00 E+12$

$1.00 E+13$

3. $26 E+11$

$1.00 E+11$

3. $16 E+11$

$2.51 E+12$

$1.00 E+11$

$5.62 E+12$

$1.00 E+13$

$1.00 E+11$

$2.63 E+35$

$2.51 E+12$

1. $66 E+33$

$2.18 E+29$

$2.00 E+12$

1. $00 E+13$

1. $62 E+35$

1. $35 E+36$

$6.31 E+13$

$7.94 E+14$

$7.24 E+15$

$5.25 E+05$

$1.00 E+11$

$4.47 E+33$

$5.25 E+17$

$3.16 E+11$

$3.16 E+11$

1. $00 E+13$

1. $00 E+13$

$1.00 E+12$

$1.00 E+11$

1. $00 E+13$

1. $00 E+14$

1. $00 E+12$

3. $16 E+13$

$6.17 E+20$

$1.70 E+27$

I. $26 E+27$
0.0

0.0

0.0

0.0

0.0

0.0

0.0

0.0

0.0

0.0

0.0

0.0

0.0

0.0

$-5.9$

$-6.5$

$-7.1$

0.0

0.0

0.0

0.0

0.0

0.0

0.0

0.0

0.0

0.0

0.0

$-7.7$

0.0

$-5.8$

$-4.1$

0.0

0.0

$-6.5$

$-6.5$

0.0

0.0

$-1.0$

1.6

0.0

$-7.2$

$-1.7$

0.0

0.0

0.0

0.0

0.0

0.0

0.0

0.0

0.0

0.0

$-1.7$

$-4.0$

$-4.7$
95500.0

3000.0

9004.0

10000.0

7500.0

4580.0

5000.0

58000.0

0.0

4000.0

8000.0

8000.0

7000.0

8000.0

7000.0

88300.0

55200.0

83500.0

20000.0

5000.0

2000.0

$-900.0$

2000.0

8000.0

5000.0

8000.0

2400.0

5000.0

5000.0

5300.0

3000.0

68100.0

72000.0

3300.0

16600.0

63200.0

74400.0

18300.0

28000.0

1900.0

1100.0

5000.0

5700.0

5100.0

5000.0

5000.0

0.0

0.0

0.0

5000.0

5000.0

17600.0

5000.0

13500.0

16500.0

12100.0

8900.0 
TABLE VI.3

PHOTO-THERMAL OXIDATION MECHANISM FOR A 5 COMPONENT MIXTURE (continued)

59. $C 2 C L 5+02=C C L 3 C O C L+C L O$

60. $\mathrm{CCL} 3 \mathrm{COCL}+\mathrm{CL}=\mathrm{CCL} 3 \mathrm{CO}+\mathrm{CL} 2$

61. $\mathrm{CCL} 3 \mathrm{CO}=\mathrm{CCL} 3+\mathrm{CO}$

62. $\mathrm{C} 2 \mathrm{CL} 2+\mathrm{O}=\mathrm{CCL} 2+\mathrm{CO}$

63. $C 2 C L 2+O 2=C C L O+C C L O$

64. $C 2 C L 2+C L 2=C 2 C L 4$

65. $C 2 C L 3+M=C 2 C L 2+C L+M$

66. $\mathrm{C} 2 \mathrm{CL} 2+\mathrm{CLO}=\mathrm{CO}+\mathrm{CCL}_{3}$

67. $\mathrm{C} 2 \mathrm{CL}_{2}+\mathrm{OH}=\mathrm{CO}+\mathrm{CHCL}_{2}$

68. $\mathrm{C} 2 \mathrm{HCL}+\mathrm{CL}=\mathrm{C} 2 \mathrm{CL}+\mathrm{HCL}$

69. $\mathrm{CHCL} 3+\mathrm{O}=\mathrm{COCL} 2+\mathrm{HCL}$

70. $\mathrm{CHCL}_{3}+\mathrm{O}=\mathrm{CCL} 3+\mathrm{OH}$

71. $\mathrm{CHCL} 3+\mathrm{CL}=\mathrm{CHCL} 2+\mathrm{CL} 2$

72. $\mathrm{CHCL} 3+\mathrm{CL}=\mathrm{CCL} 3+\mathrm{HCL}$

73. $\mathrm{CCL} 4=\mathrm{CCL}_{3}+\mathrm{CL}$

74. $\mathrm{CCL} 4+\mathrm{O}=\mathrm{CCL} 3+\mathrm{CLO}$

75. $\mathrm{CCL}+\mathrm{O} 2=\mathrm{COCL} 2+\mathrm{CLO}$

76. $\operatorname{CCL} 3+O=\operatorname{COCL} 2+C L$

77. $\operatorname{CCL} 3+\operatorname{CL} 2=\operatorname{CCL} 4+C L$

78. $\mathrm{CCL} 3+\mathrm{CCL} 3=\mathrm{C} 2 \mathrm{CL} 6$

79. $\mathrm{CCL}_{3}+\mathrm{CCL}_{3}=\mathrm{C} 2 \mathrm{CL} 4+\mathrm{CL} 2$

80. CCL $3+C H C L 2=C 2$ HCL 5

81. CCL3 $+\mathrm{CHCL} 2=\mathrm{C} 2 \mathrm{CL} 4+\mathrm{HCL}$

82. $\mathrm{CHCL} 2+\mathrm{O} 2=\mathrm{CHCLO}+\mathrm{CLO}$

83. $\mathrm{CHCL} 2+\mathrm{O}=\mathrm{CHCLO}+\mathrm{CL}$

84. $\mathrm{CCL} 2+02=C L O+C C L O$

85. $\mathrm{CCL} 2+\mathrm{CL} 2=\mathrm{CCL} 3+\mathrm{CL}$

86. $\mathrm{C} 2 \mathrm{H} 6+\mathrm{CH} 3=\mathrm{C} 2 \mathrm{H} 5+\mathrm{CH} 4$

87. $\mathrm{CH} 3+\mathrm{C} 2 \mathrm{H} 5=\mathrm{CH} 4+\mathrm{C} 2 \mathrm{H} 4$

88. $\mathrm{CH} 2 \mathrm{CL} 2=\mathrm{CHCL}+\mathrm{HCL}$

89. $\mathrm{CH} 2 \mathrm{CL} 2=\mathrm{CH} 2 \mathrm{CL}+\mathrm{CL}$

90. $\mathrm{CH} 3 \mathrm{CL}=\mathrm{CH} 3+\mathrm{CL}$

91. $\mathrm{CH} 3 \mathrm{CL}=\mathrm{CH} 2 \mathrm{~S}+\mathrm{HCL}$

92. $\mathrm{CH} 2 \mathrm{CL} 2+\mathrm{H}=\mathrm{CH} 2 \mathrm{CL}+\mathrm{HCL}$

93. $\mathrm{CHCL} 2+\mathrm{H} 2=\mathrm{CH} 2 \mathrm{CL} 2+\mathrm{H}$

94. $\mathrm{CH} 2 \mathrm{CL}+\mathrm{H} 2=\mathrm{CH} 3 \mathrm{CL}+\mathrm{H}$

95. $\mathrm{CH} 2 \mathrm{CL} 2+\mathrm{CL}=\mathrm{CHCL} 2+\mathrm{HCL}$

96. $\mathrm{CH} 3 \mathrm{CL}+\mathrm{H}=\mathrm{CH} 3+\mathrm{HCL}$

97. $\mathrm{CH} 4=\mathrm{CH} 3+\mathrm{H}$

98. $\mathrm{CH} 4+\mathrm{H}=\mathrm{CH} 3+\mathrm{H}_{2}$

99. $\mathrm{CH} 4+\mathrm{CL}=\mathrm{CH} 3+\mathrm{HCL}$

100. $\mathrm{CH} 3 \mathrm{CL}+\mathrm{CL}=\mathrm{CH} 2 \mathrm{CL}+\mathrm{HCL}$

101. $\mathrm{CH} 2 \mathrm{CL} 2+\mathrm{CH} 3=\mathrm{CH} 4+\mathrm{CHCL} 2$

102. $\mathrm{CH} 2 \mathrm{CL} 2+\mathrm{CH} 3=\mathrm{CH} 3 \mathrm{CL}+\mathrm{CH} 2 \mathrm{CL}$

103. $\mathrm{CH} 3 \mathrm{CL}+\mathrm{CH} 3=\mathrm{CH} 4+\mathrm{CH} 2 \mathrm{CL}$

104. CHCL $2+\mathrm{CHCL} 2=\mathrm{C} 2 \mathrm{H} 2 \mathrm{CL} 4$

105. $\mathrm{CHCL} 2+\mathrm{CHCL} 2=\mathrm{C}_{2} \mathrm{H}_{2} \mathrm{CL}_{3}+\mathrm{CL}$

106. $\mathrm{CHCL} 2+\mathrm{CHCL} 2=\mathrm{C} 2 \mathrm{HCL} 3+\mathrm{HCL}$

107. $\mathrm{CH} 2 \mathrm{CL}+\mathrm{CH} 2 \mathrm{CL}=\mathrm{C} 2 \mathrm{H} 4 \mathrm{CL} 2$

108. $\mathrm{CH} 2 \mathrm{CL}+\mathrm{CH} 2 \mathrm{CL}=\mathrm{CH} 2 \mathrm{CLCH} 2+\mathrm{CL}$

109. $\mathrm{CH} 2 \mathrm{CL}+\mathrm{CH} 2 \mathrm{CL}=\mathrm{C} 2 \mathrm{H} 3 \mathrm{CL}+\mathrm{HCL}$

110. $\mathrm{CH} 2 \mathrm{CL}+\mathrm{CHCL} 2=\mathrm{C}_{2} \mathrm{H}_{3} \mathrm{CL} 3$

111. $\mathrm{CH} 2 \mathrm{CL}+\mathrm{CHCL} 2=\mathrm{CH} 2 \mathrm{CCL} 2+\mathrm{HCL}$

112. $\mathrm{CH} 2 \mathrm{CL}+\mathrm{CHCL} 2=\mathrm{CHCLCHCL}+\mathrm{HCL}$

113. $\mathrm{CH} 2 \mathrm{CL}+\mathrm{CH} 3=\mathrm{C} 2 \mathrm{HSCL}$

114. $\mathrm{CH} 2 \mathrm{CL}+\mathrm{CH} 3=\mathrm{C} 2 \mathrm{H} 4+\mathrm{HCL}$

115. $\mathrm{CH} 2 \mathrm{CL}+\mathrm{CH} 3=\mathrm{C} 2 \mathrm{H} 5+\mathrm{CL}$

116. $\mathrm{CHCL} 2+\mathrm{CH} 3=\mathrm{CH} 3 \mathrm{CHCL} 2$

117. $\mathrm{CHCL} 2+\mathrm{CH} 3=\mathrm{C} 2 \mathrm{H} 3 \mathrm{CL}+\mathrm{HCL}$

118. $\mathrm{CHCL} 2+\mathrm{CH} 3=\mathrm{CH} 3 \mathrm{CHCL}+\mathrm{CL}$

119. $\mathrm{CH} 2 \mathrm{CL}+\mathrm{H}=\mathrm{CH} 3 \mathrm{CL}$

120. $\mathrm{CH} 2 \mathrm{CL}+\mathrm{H}=\mathrm{CH} 2 \mathrm{~S}+\mathrm{HCL}$

121. $\mathrm{CH} 2 \mathrm{CL}+\mathrm{H}=\mathrm{CH} 3+\mathrm{CL}$

122. $\mathrm{CHCL} 2+\mathrm{H}=\mathrm{CH} 2 \mathrm{CL}_{2}$

123. $\mathrm{CHCL} 2+\mathrm{H}=\mathrm{CH} 2 \mathrm{CL}+\mathrm{CL}$

124. $\mathrm{C} 2 \mathrm{H} 3 \mathrm{CL}+\mathrm{H}=\mathrm{CH} 2 \mathrm{CLCH} 2$
1. $00 E+12$

1. $00 E+14$

3. $16 E+13$

$7.08 E+13$

1. $00 E+11$

2. $63 E+36$

$7.94 E+14$

$1.00 \mathrm{E}+11$

1. $00 E+12$

1. $00 E+13$

1. $00 \mathrm{E}+11$

$2.88 E+12$

1. $00 E+14$

$6.91 E+12$

7. $41 E+35$

2. $51 E+10$

1. $00 E+13$

$1.00 E+14$

$2.51 E+12$

1. $41 E+36$

2. $24 E+26$

1. $66 E+34$

2. $34 E+20$

1. $00 E+13$

1. $00 E+14$

1. $00 E+13$

$5.01 E+12$

2.70E-01

5. $50 E+11$

$1.44 E+37$

7. $31 E+40$

1. $26 E+37$

8. $52 E+27$

7. $00 E+13$

$4.63 \mathrm{E}+12$

3. $90 E+12$

2. $79 E+13$

$6.64 E+13$

1. $03 E+33$

$1.55 E+14$

3. $09 \varepsilon+13$

3. $16 E+13$

6. $76 E+10$

1. $40 E+11$

3. $30 E+11$

9. $08 E+45$

1. $36 E+30$

$6.72 E+35$

3. $92 E+45$

4. $67 E+29$

1. $88 E+35$

$6.41 E+33$

3. $75 E+36$

1. $22 E+37$

3. $27 E+40$

3. $50 E+28$

9. $27 E+19$

2. $28 E+41$

1. $35 E+30$

2. $74 E+25$

3. $04 E+25$

$9.48 E+04$

$5.12 E+14$

4. $81 E+26$

1. $25 E+14$

$5.01 E+23$
0.0

0.0

0.0

0.0

0.0

$-7.2$

0.0

0.0

0.0

0.0

0.0

0.0

0.0

0.0

$-6.5$

0.0

0.0

0.0

0.0

$-7.5$

$-4.4$

$-6.8$

$-2.5$

0.0

0.0

0.0

0.0

4.0

0.0

$-7.6$

$-8.0$

$-6.9$

$-5.1$

0.0

0.0

0.0

0.0

0.0

$-5.6$

0.0

0.0

0.0

0.0

0.0

0.0

$-10.6$

$-5.2$

$-7.1$

$-10.2$

$-4.9$

$-6.7$

$-10.2$

$-7.2$

$-7.2$

$-8.5$

$-4.5$

$-2.1$

$-8.7$

$-5.0$

$-3.5$

$-4.5$

1. 9

$-0.2$

$-4.8$

0.0

$-4.2$
12000.0

17600.0

8000.0

0.0

5000.0

40900.0

28000.0

0.0 0.0

3000.0

4000.0

5000.0

21000.0

3300.0

75400.0

2300.0

28000.0

0.0

6000.0

6700.0

9000.0

6000.0

6400.0

28000.0

0.0

1000.0

3000.0

8280.0

0.0

86180.0

84340.0

90540.0

109640.0

7100.0

15295.0

14059.0

2940.0

7620.0

111810.0

11000.0

3600.0

3300.0

7200.0

4900.0

9400.0

13170.0

14180.0

13210.0

13150.0

14070.0

13160.0

12910.0

13620.0

13640.0

10590.0

9180.0

10130.0

11620.0

11550.0

12810.0

3490.0

2600.0

310.0

3810.0

570.0

8470.0 
TABLE VI.3

PHOTO-THERMAL OXIDATION MECHANISM FOR A 5 COMPONENT MLXTURE (continued)

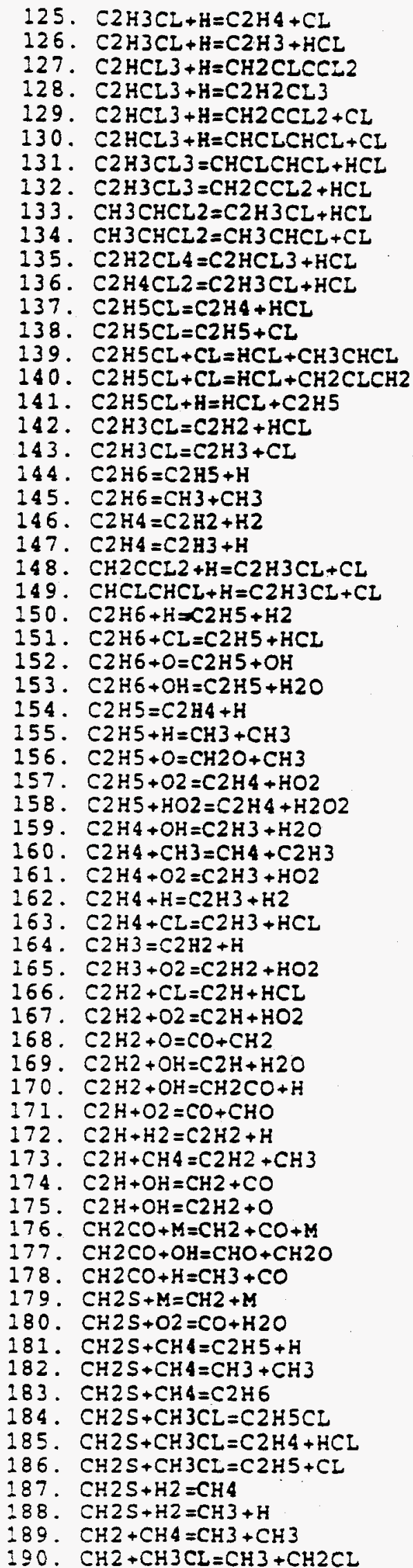

125. $\mathrm{C2H} 3 \mathrm{CL}+\mathrm{H}=\mathrm{C} 2 \mathrm{H} 4+\mathrm{CL}$

126. $\mathrm{C} 2 \mathrm{H} 3 \mathrm{CL}+\mathrm{H}=\mathrm{C} 2 \mathrm{H} 3+\mathrm{HCL}$

127. $\mathrm{C} 2 \mathrm{HCL} 3+\mathrm{H}=\mathrm{CH} 2 \mathrm{CLCCL} 2$

128. $\mathrm{C} 2 \mathrm{HCL} 3+\mathrm{H}=\mathrm{C} 2 \mathrm{H} 2 \mathrm{CL}$

129. $\mathrm{C} 2 \mathrm{HCL} 3+\mathrm{H}=\mathrm{CH} 2 \mathrm{CCL} 2+\mathrm{CL}$

130. $\mathrm{C} 2 \mathrm{HCL}+\mathrm{H}=\mathrm{CHCLCHCL}+\mathrm{CI}$

$132 . \mathrm{C} 2 \mathrm{H} 3 \mathrm{CL} 3=\mathrm{CH} 2 \mathrm{CCL} 2+\mathrm{HCL}$

133. $\mathrm{CH} 3 \mathrm{CHCL} 2=\mathrm{C} 2 \mathrm{H} 3 \mathrm{CL}+\mathrm{HCL}$

134. $\mathrm{CH} 3 \mathrm{CHCL} 2=\mathrm{CH} 3 \mathrm{CHCL}+\mathrm{CL}$

135. $\mathrm{C} 2 \mathrm{H} 2 \mathrm{CL} 4=\mathrm{C} 2 \mathrm{HCL} 3+\mathrm{HCL}$

C2 $\mathrm{H}$ C $2=\mathrm{C} 2 \mathrm{H} 3 \mathrm{CL}+\mathrm{HC}$

139. $\mathrm{C} 2 \mathrm{H} 5 \mathrm{CL}+\mathrm{CL}=\mathrm{HCL}+\mathrm{CH} 3 \mathrm{CHCL}$

140. $\mathrm{C} 2 \mathrm{H} 5 \mathrm{CL}+\mathrm{CL}=\mathrm{HCL}+\mathrm{CH} 2 \mathrm{CLCH} 2$

143. $\mathrm{C} 2 \mathrm{H} 3 \mathrm{CL}=\mathrm{C} 2 \mathrm{H3}+\mathrm{CI}$

144. $\mathrm{C} 2 \mathrm{H} 6=\mathrm{C} 2 \mathrm{H} 5+\mathrm{H}$

$\mathrm{C} 2 \mathrm{H} 6=\mathrm{CH} 3+\mathrm{CH} 3$

146. $\mathrm{C} 2 \mathrm{H} 4=\mathrm{C} 2 \mathrm{H} 2+\mathrm{H} 2$

$47 . \mathrm{C} 2 \mathrm{H} 4=\mathrm{C} 2 \mathrm{H} 3+\mathrm{H}$

150. $\mathrm{C} 2 \mathrm{H} 6+\mathrm{H}=\mathrm{C} 2 \mathrm{H} 5+\mathrm{H} 2$

151. $\mathrm{C} 2 \mathrm{H} 6+\mathrm{CL}=\mathrm{C} 2 \mathrm{H} 5+\mathrm{HCL}$

152. $\mathrm{C} 2 \mathrm{H} 6+\mathrm{O}=\mathrm{C} 2 \mathrm{H} 5+\mathrm{OH}$

53. $\mathrm{C} 2 \mathrm{H} 6+\mathrm{OH}=\mathrm{C} 2 \mathrm{H} 5+\mathrm{H} 2 \mathrm{O}$

154. $\mathrm{C} 2 \mathrm{H} 5=\mathrm{C} 2 \mathrm{H} 4+\mathrm{H}$

155. $\mathrm{C} 2 \mathrm{H} 5+\mathrm{H}=\mathrm{CH} 3+\mathrm{CH}_{3}$

156. $\mathrm{C} 2 \mathrm{H} 5+\mathrm{O}=\mathrm{CH} 2 \mathrm{O}+\mathrm{CH} 3$

157. $\mathrm{C} 2 \mathrm{H} 5+\mathrm{O} 2=\mathrm{C} 2 \mathrm{H} 4+\mathrm{HO} 2$

158. $\mathrm{C} 2 \mathrm{H} 5+\mathrm{HO} 2=\mathrm{C} 2 \mathrm{H} 4+\mathrm{H} 2 \mathrm{O} 2$

159. $\mathrm{C} 2 \mathrm{H} 4+\mathrm{OH}=\mathrm{C} 2 \mathrm{H} 3+\mathrm{H} 2 \mathrm{O}$

政 2

161. $\mathrm{C} 2 \mathrm{H} 4+\mathrm{O} 2=\mathrm{C} 2 \mathrm{H} 3+\mathrm{HO} 2$

62. $\mathrm{C} 2 \mathrm{H} 4+\mathrm{H}=\mathrm{C} 2 \mathrm{H}_{3}+\mathrm{H}_{2}$

163. $\mathrm{C} 2 \mathrm{H} 4+\mathrm{CL}=\mathrm{C} 2 \mathrm{H} 3+\mathrm{HCL}$

$164 . \mathrm{C} 2 \mathrm{H} 3=\mathrm{C} 2 \mathrm{H} 2+\mathrm{H}$

165. $\mathrm{C} 2 \mathrm{H} 3+\mathrm{O} 2=\mathrm{C} 2 \mathrm{H} 2+\mathrm{HO}_{2}$

166. $\mathrm{C} 2 \mathrm{H} 2+\mathrm{CL}=\mathrm{C} 2 \mathrm{H}+\mathrm{HCL}$

167. $\mathrm{C} 2 \mathrm{H} 2+\mathrm{O} 2=\mathrm{C} 2 \mathrm{H}+\mathrm{HO} 2$

168. $\mathrm{C} 2 \mathrm{H} 2+\mathrm{O}=\mathrm{CO}+\mathrm{CH} 2$

169. $\mathrm{C} 2 \mathrm{H} 2+\mathrm{OH}=\mathrm{C} 2 \mathrm{H}+\mathrm{H} 2 \mathrm{O}$

170. $\mathrm{C} 2 \mathrm{H} 2+\mathrm{OH}=\mathrm{CH} 2 \mathrm{CO}+\mathrm{H}$

171. $\mathrm{C} 2 \mathrm{H}+\mathrm{O} 2=\mathrm{CO}+\mathrm{CHO}$

172. $\mathrm{C} 2 \mathrm{H}+\mathrm{H} 2=\mathrm{C} 2 \mathrm{H} 2+\mathrm{H}$

173. $\mathrm{C} 2 \mathrm{H}+\mathrm{CH} 4=\mathrm{C} 2 \mathrm{H}_{2}+\mathrm{CH}_{3}$

174. $\mathrm{C} 2 \mathrm{H}+\mathrm{OH}=\mathrm{CH} 2+\mathrm{CO}$

175. $\mathrm{C} 2 \mathrm{H}+\mathrm{OH}=\mathrm{C} 2 \mathrm{H} 2+\mathrm{O}$

176. $\mathrm{CH} 2 \mathrm{CO}+\mathrm{H}=\mathrm{CH} 2+\mathrm{CO}+\mathrm{H}$

177. $\mathrm{CH} 2 \mathrm{CO}+\mathrm{OH}=\mathrm{CHO}+\mathrm{CH} 2 \mathrm{O}$

186. $\mathrm{CH} 2 \mathrm{~S}+\mathrm{CH} 3 \mathrm{CL}=\mathrm{C} 2 \mathrm{H} 5+\mathrm{CL}$

$187 . \mathrm{CH} 2 \mathrm{~S}+\mathrm{H} 2=\mathrm{CH} 4$

189. $\mathrm{CH} 2+\mathrm{CH} 4=\mathrm{CH} 3+\mathrm{CH} 3$

190. $\mathrm{CH} 2+\mathrm{CH} 3 \mathrm{CL}=\mathrm{CH} 3+\mathrm{CH} 2 \mathrm{CL}$

\begin{tabular}{|c|c|c|}
\hline 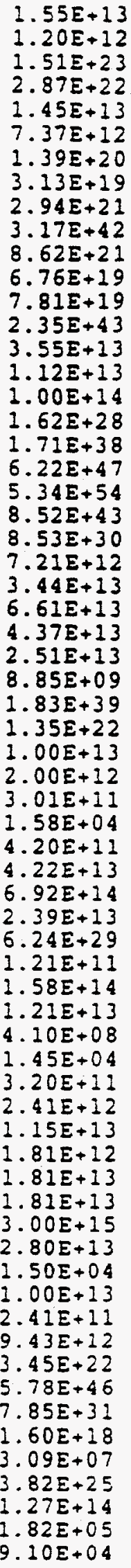 & $\begin{array}{r}0.0 \\
0.0 \\
-4.2 \\
-4.1 \\
0.0 \\
0.0 \\
-2.0 \\
-2.0 \\
-2.4 \\
-8.1 \\
-2.6 \\
-1.9 \\
-2.0 \\
-8.5 \\
0.0 \\
0.0 \\
0.0 \\
-4.3 \\
-7.1 \\
-9.8 \\
-11.1 \\
-8.3 \\
-5.9 \\
0.0 \\
0.0 \\
0.0 \\
0.0 \\
0.0 \\
-10 \\
-1.0 \\
-6.3 \\
-1.5 \\
-1.5 \\
-7.7 \\
-4.5 \\
-0.1 \\
-2.2 \\
0.0 \\
0.0 \\
0.0 \\
0.0 \\
0.0 \\
0.0 \\
0.0 \\
0.0 \\
0.0 \\
0.0 \\
0.0 \\
-5.3 \\
0.0 \\
0.0 \\
0.0 \\
0.0 \\
1.5 \\
2.7 \\
0.0 \\
0.0 \\
0.0 \\
0.0 \\
0.0\end{array}$ & 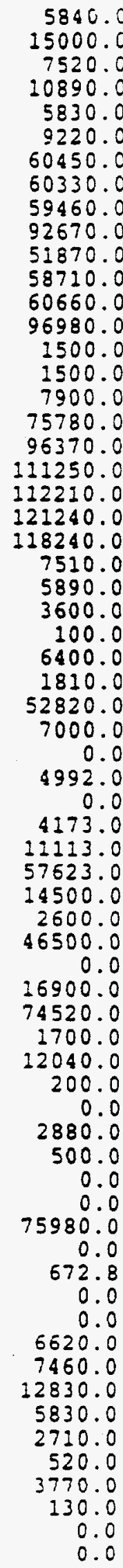 \\
\hline
\end{tabular}


TABLE VI.3

PHOTO-THERMAL OXIDATION MECHANISM FOR A 5 COMPONENT MLXTLRE (conunued)

291. $\mathrm{CH} 2+\mathrm{H} 2=\mathrm{CH} 3+\mathrm{H}$

i 92 . $\mathrm{CH} 2+\mathrm{H} 2 \mathrm{O}=\mathrm{CH} 3+\mathrm{OH}$

193. $\mathrm{CH} 4+\mathrm{O} 2=\mathrm{CH} 3+\mathrm{HO} 2$

194. $\mathrm{CH} 4+\mathrm{O}=\mathrm{CH} 3+\mathrm{OH}$

195. $\mathrm{CH} 4+\mathrm{OH}=\mathrm{CH} 3+\mathrm{H} 2 \mathrm{O}$

196. $\mathrm{CH} 4+\mathrm{HO} 2=\mathrm{CH} 3+\mathrm{H} 2 \mathrm{O} 2$

197. $\mathrm{CH} 3+\mathrm{O} 2=\mathrm{CH} 2 \mathrm{O}+\mathrm{OH}$

198. $\mathrm{CH} 3+\mathrm{O} 2=\mathrm{CH} 3 \mathrm{O}+\mathrm{O}$

199. $\mathrm{CH} 3+\mathrm{O}=\mathrm{CH} 2 \mathrm{O}+\mathrm{H}$

200. $\mathrm{CH} 3+\mathrm{OH}=\mathrm{CH} 3 \mathrm{O}+\mathrm{H}$

201. $\mathrm{CH} 3+\mathrm{HO} 2=\mathrm{CH} 3 \mathrm{O}+\mathrm{OH}$

202. $\mathrm{CH} 3+\mathrm{CL} 2=\mathrm{CH} 3 \mathrm{CL}+\mathrm{CL}$

203. $\mathrm{CH} 3 \mathrm{O}+\mathrm{O} 2=\mathrm{CH} 2 \mathrm{O}+\mathrm{HO} 2$

204. $\mathrm{CH} 3 \mathrm{O}+\mathrm{M}=\mathrm{CH} 2 \mathrm{O}+\mathrm{H}+\mathrm{M}$

205. $\mathrm{CH} 3 \mathrm{O}+\mathrm{CO}=\mathrm{CO} 2+\mathrm{CH} 3$

206. $\mathrm{CH} 3 \mathrm{O}+\mathrm{HO} 2=\mathrm{CH} 2 \mathrm{O}+\mathrm{H} 2 \mathrm{O} 2$

207. $\mathrm{CH} 3 \mathrm{O}+\mathrm{CH} 3=\mathrm{CH} 4+\mathrm{CH} 2 \mathrm{O}$

208. $\mathrm{CH} 3 \mathrm{O}+\mathrm{O}=\mathrm{OH}+\mathrm{CH} 2 \mathrm{O}$

209 . $\mathrm{CH} 3 \mathrm{O}+\mathrm{OH}=\mathrm{H} 2 \mathrm{O}+\mathrm{CH} 2 \mathrm{O}$

210. $\mathrm{CH} 3 \mathrm{O}+\mathrm{H}=\mathrm{CH} 2 \mathrm{O}+\mathrm{H} 2$

211. $\mathrm{CH} 3 \mathrm{O}+\mathrm{CH} 2=\mathrm{CH} 3+\mathrm{CH} 2 \mathrm{O}$

212. $\mathrm{CH} 3 \mathrm{O}+\mathrm{C} 2 \mathrm{H} 5=\mathrm{C} 2 \mathrm{H} 6+\mathrm{CH} 2 \mathrm{O}$

213. $\mathrm{CH} 3 \mathrm{O}+\mathrm{CLO}=\mathrm{HOCL}+\mathrm{CH} 2 \mathrm{O}$

214. $\mathrm{CH} 3 \mathrm{O}+\mathrm{CL}=\mathrm{HCL}+\mathrm{CH} 2 \mathrm{O}$

215. $\mathrm{CH} 2 \mathrm{O}+\mathrm{CLO}=\mathrm{CHO}+\mathrm{HOCL}$

216. $\mathrm{CH} 2 \mathrm{O}+\mathrm{C} 2 \mathrm{H} 5=\mathrm{CHO}+\mathrm{C} 2 \mathrm{H} 6$

217. $\mathrm{CH} 2 \mathrm{O}+\mathrm{CH} 3=\mathrm{CH} 4+\mathrm{CHO}$

218. $\mathrm{CH} 2 \mathrm{O}+\mathrm{H}=\mathrm{CHO}+\mathrm{H} 2$

219. $\mathrm{CH} 2 \mathrm{O}+\mathrm{O}=\mathrm{CHO}+\mathrm{OH}$

220. $\mathrm{CH} 2 \mathrm{O}+\mathrm{OH}=\mathrm{CHO}+\mathrm{H} 2 \mathrm{O}$

221. $\mathrm{CH} 2 \mathrm{O}+\mathrm{HO} 2=\mathrm{CHO}+\mathrm{H} 2 \mathrm{O} 2$

222. $\mathrm{CH} 2 \mathrm{O}+\mathrm{CL}=\mathrm{CHO}+\mathrm{HCL}$

223. $\mathrm{CH} 2 \mathrm{O}+\mathrm{M}=\mathrm{CHO}+\mathrm{H}+\mathrm{M}$

224. $\mathrm{CH} 2 \mathrm{O}+\mathrm{O} 2=\mathrm{CHO}+\mathrm{HO} 2$

225. $\mathrm{CHO}=\mathrm{H}+\mathrm{CO}$

226. $\mathrm{CHO}+\mathrm{H}=\mathrm{CO}+\mathrm{H} 2$

227. $\mathrm{CHO}+\mathrm{O} 2=\mathrm{CO}+\mathrm{HO} 2$

228. $\mathrm{CHO}+\mathrm{O}=\mathrm{CO}+\mathrm{OH}$

229. $\mathrm{CHO}+\mathrm{O}=\mathrm{H}+\mathrm{CO} 2$

230 . $\mathrm{CHO}+\mathrm{OH}=\mathrm{CO}+\mathrm{H} 2 \mathrm{O}$

231. $\mathrm{CO}+\mathrm{OH}=\mathrm{CO} 2+\mathrm{H}$

232. $\mathrm{CO}+\mathrm{HO} 2=\mathrm{CO} 2+\mathrm{OH}$

233. $\mathrm{CO}+\mathrm{O} 2=\mathrm{CO} 2+\mathrm{O}$

$234 . \mathrm{CO}+\mathrm{O}+\mathrm{M}=\mathrm{CO} 2+\mathrm{M}$

235. $\mathrm{H}+\mathrm{O} 2=\mathrm{O}+\mathrm{OH}$

236. $\mathrm{HO} 2+\mathrm{M}=\mathrm{H}+\mathrm{O} 2+\mathrm{M}$

$237 . \mathrm{O}+\mathrm{H} 2=\mathrm{H}+\mathrm{OH}$

$238 . \mathrm{O}+\mathrm{H} 2 \mathrm{O}=\mathrm{OH}+\mathrm{OH}$

239. $\mathrm{H}+\mathrm{H} 2 \mathrm{O}=\mathrm{H} 2+\mathrm{OH}$

240. $\mathrm{H}+\mathrm{OH}+\mathrm{M}=\mathrm{H} 2 \mathrm{O}+\mathrm{M}$

241. $O 2+M=O+O+M$

242. $\mathrm{H}+\mathrm{O}+\mathrm{M}=\mathrm{OH}+\mathrm{M}$

243. $\mathrm{H}+\mathrm{HO} 2=\mathrm{OH}+\mathrm{OH}$

$244 . \mathrm{H}+\mathrm{HO} 2=\mathrm{H} 2+\mathrm{O} 2$

245. $\mathrm{O}+\mathrm{HO} 2=\mathrm{OH}+\mathrm{O} 2$

246 . OH+HO2 $=\mathrm{H} 2 \mathrm{O}+\mathrm{O} 2$

$247 . \mathrm{OH}+\mathrm{H} 2 \mathrm{O} 2=\mathrm{HO} 2+\mathrm{H} 2 \mathrm{O}$

248. $\mathrm{O}+\mathrm{H} 2 \mathrm{O} 2=\mathrm{HO} 2+\mathrm{OH}$

249. $\mathrm{H}+\mathrm{H} 2 \mathrm{O} 2=\mathrm{H} 2+\mathrm{HO} 2$

250. $\mathrm{H}+\mathrm{H} 2 \mathrm{O} 2=\mathrm{OH}+\mathrm{H} 2 \mathrm{O}$

251. $\mathrm{O} 2+\mathrm{H} 2 \mathrm{O} 2=\mathrm{HO} 2+\mathrm{HO} 2$

252. $\mathrm{H} 2 \mathrm{O} 2+\mathrm{M}=\mathrm{OH}+\mathrm{OH}+\mathrm{M}$

253. $\mathrm{O}+\mathrm{HCL}=\mathrm{OH}+\mathrm{CL}$

254. $\mathrm{OH}+\mathrm{HCL}=\mathrm{CL}+\mathrm{H} 2 \mathrm{O}$

255. $\mathrm{H} 2+\mathrm{M}=\mathrm{H}+\mathrm{H}+\mathrm{M}$

$2 \equiv 6 . C L+C L+M=C L 2+M$
$9.64 E+07$

4. $04 E+13$

$1.02 E+09$

$1.93 E+05$

2. $00 E+13$

$3.59 E+09$

2. $88 E+15$

$7.00 E+13$

$3.87 E+12$

2. $00 \mathrm{E}+13$

2. $50 \mathrm{E}+12$

$6.62 E+10$

1. $00 \mathrm{E}+14$

1. $57 E+13$

3. $01 E+11$

$2.41 E+13$

$6.03 E+12$

$1.81 E+13$

$1.99 E+13$

$1.81 E+13$

$2.41 E+13$

$2.41 E+13$

$4.00 E+14$

$5.50 E+03$

$5.50 E+03$

$1.00 E+11$

$2.50 E+13$

$3.50 E+13$

$3.00 E+13$

1. $00 E+12$

5. $00 E+13$

$5.00 E+16$

2. $05 E+13$

2. $50 E+14$

$2.00 \mathrm{E}+14$

$5.12 E+13$

3. $01 E+13$

$3.01 E+13$

$3.01 E+13$

$4.40 E+06$

$5.80 E+13$

2. $50 E+12$

6.17E+14

1. $69 E+17$

$1.21 E+19$

$1.08 E+04$

1. $50 E+10$

4. $60 \mathrm{E}+08$

7. $50 E+23$

1. $20 \mathrm{E}+14$

2. $29 E+14$

1. $69 \mathrm{E}+14$

$6.62 E+13$

2. $00 E+13$

$2.00 E+13$

1. $75 E+12$

$9.63 E+06$

4. $82 E+13$

$2.41 E+13$

$5.42 E+13$

1. $29 E+33$

$5.24 E+12$

$2.45 E+12$

4. $57 E+19$
$3.01 E+00$

$\begin{array}{rr}0.0 & 0.0 \\ 0.0 & 0.0 \\ 0.0 & 55910.0 \\ 1.5 & 8600.0 \\ 2.4 & 2110.0 \\ 0.0 & 18000.0 \\ -0.1 & 10150.0 \\ -1.1 & 30850.0 \\ 0.0 & 0.0 \\ -0.2 & 13741.0 \\ 0.0 & 0.0 \\ 0.0 & 4000.0 \\ 0.0 & 2600.0 \\ 0.0 & 25100.0 \\ 0.0 & 11800.0 \\ 0.0 & 0.0 \\ 0.0 & 0.0 \\ 0.0 & 0.0 \\ 0.0 & 0.0 \\ 0.0 & 0.0 \\ 0.0 & 0.0 \\ 0.0 & 0.0 \\ 0.0 & 0.0 \\ 0.0 & 0.0 \\ 2.8 & 5860.0\end{array}$

5860.0

5860.0

6090.0

3990.0

3510.0

1190.0

8000.0

500.0

76200.0

38945.0

16790.0

0.0

1690.0

0.0

0.0

0.0

$-741.0$

22934.0

47800.0

3000.0

17390.0

48610.0

5920.0

17240.0

18560.0

0.0

$-2.6$

$-4.9$

2. $34 E+14$
0.0

0.0

0.0

0.0

0.0

0.0

0.0

2.0

0.0

0.0

0.0

0.0

0.0

107552.0

3900.0

870.0

2130.0

0.0

0.0

320.0

3970.0

7950.0

3970.0

39740.0

53250.0

6400.0

1100.0

$-1.4 \quad 104390.0$

0.0

$-1800.0$ 
TABLE VI.3

PHOTO-THERMAL OXIDATION MECHANISM FOR A 5 COMPONENT MIXTURE (continued)

257. $\mathrm{H}+\mathrm{CL}+\mathrm{M}=\mathrm{HCL}+\mathrm{M}$

258. $\mathrm{H}+\mathrm{HCL}=\mathrm{H} 2+\mathrm{CL}$

259. $\mathrm{CL}+\mathrm{HO} 2=\mathrm{HCL}+\mathrm{O} 2$

260. $\mathrm{CL}+\mathrm{HO}=\mathrm{CLO}+\mathrm{OH}$

261. $\mathrm{CLO}+\mathrm{CO}=\mathrm{CL}+\mathrm{CO} 2$

262. CHCLO $+\mathrm{H}=\mathrm{CHO}+\mathrm{HCL}$

263. $\mathrm{CHCLO}+\mathrm{H}=\mathrm{CH} 2 \mathrm{O}+\mathrm{CL}$

$264 . \mathrm{CH} 3+\mathrm{CLO}=\mathrm{CH} 3 \mathrm{O}+\mathrm{CL}$

255. $\mathrm{CH} 3+\mathrm{CLO}=\mathrm{CH} 2 \mathrm{O}+\mathrm{HCL}$

266. $\mathrm{CH} 2 \mathrm{CL}_{2}+\mathrm{O} 2=\mathrm{CHCL} 2+\mathrm{HO}_{2}$

267. $\mathrm{CH} 2 \mathrm{CL} 2+\mathrm{HO} 2=\mathrm{CHCL} 2+\mathrm{H} 2 \mathrm{O} 2$

268. $\mathrm{CH} 2 \mathrm{CL} 2+\mathrm{OH}=\mathrm{CHCL} 2+\mathrm{H} 2 \mathrm{O}$

269. $\mathrm{CH} 2 \mathrm{CL} 2+\mathrm{O}=\mathrm{CHCL} 2+\mathrm{OH}$

270. $\mathrm{CH} 2 \mathrm{CL}+\mathrm{O} 2=\mathrm{CH} 2 \mathrm{CLOO}$

271. $\mathrm{CH} 2 \mathrm{CL}+\mathrm{O} 2=\mathrm{CH} 2 \mathrm{O}+\mathrm{CLO}$

272 . $\mathrm{CH} 2 \mathrm{CI}+\mathrm{O} 2=\mathrm{CHCLO}+\mathrm{OH}$

273. $\mathrm{CH} 2 \mathrm{CL}+\mathrm{O}=\mathrm{CH} 2 \mathrm{CLO}$

274. $\mathrm{CH} 2 \mathrm{CL}+\mathrm{O}=\mathrm{CH} 2 \mathrm{O}+\mathrm{CL}$

275. $\mathrm{CH} 2 \mathrm{CL}+\mathrm{OH}=\mathrm{CH} 2 \mathrm{O}+\mathrm{HCL}$

276. $\mathrm{CH} 2 \mathrm{CI}+\mathrm{OH}=\mathrm{CH} 3 \mathrm{O}+\mathrm{CL}$

277. $\mathrm{CH} 2 \mathrm{CI}+\mathrm{HO} 2=\mathrm{CH} 2 \mathrm{CLO}+\mathrm{OH}$

278. $\mathrm{CH} 2 \mathrm{CLO}=\mathrm{CHCLO}+\mathrm{H}$

279. $\mathrm{CH} 2 \mathrm{CLO}=\mathrm{CH} 2 \mathrm{O}+\mathrm{CL}$

280. $\mathrm{CHCLO}=\mathrm{CHO}+\mathrm{CL}$

281. $\mathrm{CHCLO}=\mathrm{CO}+\mathrm{HCL}$

282. $\mathrm{CH} 2 \mathrm{CL}+\mathrm{OLO}=\mathrm{CH} 2 \mathrm{CLO}+\mathrm{CL}$

283. $\mathrm{CH} 2 \mathrm{CL}+\mathrm{CLO}=\mathrm{CHCLO}+\mathrm{HCL}$

284. $\mathrm{CH} 2 \mathrm{CL}+\mathrm{CH} 2 \mathrm{O}=\mathrm{CH} 3 \mathrm{CL}+\mathrm{CHO}$

285. $\mathrm{CH} 3 \mathrm{CL}+\mathrm{O} 2=\mathrm{CH} 2 \mathrm{CL}+\mathrm{HO} 2$

286. $\mathrm{CH} 3 \mathrm{CL}+\mathrm{O}=\mathrm{CH} 2 \mathrm{CL}+\mathrm{OH}$

287. $\mathrm{CH} 3 \mathrm{CL}+\mathrm{OH}=\mathrm{CH} 2 \mathrm{CL}+\mathrm{H} 2 \mathrm{O}$

288. $\mathrm{CH} 3 \mathrm{CL}+\mathrm{HO} 2=\mathrm{CH} 2 \mathrm{CL}+\mathrm{H} 2 \mathrm{O2}$

$289 . \mathrm{H} 2 \mathrm{O} 2+\mathrm{CL}=\mathrm{HCL}+\mathrm{HO} 2$

290. $\mathrm{CLO}+\mathrm{CH} 4=\mathrm{CH} 3+\mathrm{HOCL}$

291. $\mathrm{CLO}+\mathrm{CH} 3 \mathrm{CL}=\mathrm{CH} 2 \mathrm{CL}+\mathrm{HOCL}$

292. $\mathrm{CLO}+\mathrm{H2}=\mathrm{HOCL}+\mathrm{H}$

293. $\mathrm{OH}+\mathrm{HOCL}=\mathrm{H} 2 \mathrm{O}+\mathrm{CLO}$

294. $\mathrm{H}+\mathrm{HOCL}=\mathrm{HCL}+\mathrm{OH}$

295. $\mathrm{CL}+\mathrm{HOCL}=\mathrm{CL} 2+\mathrm{OH}$

296. $C L+H O C L=H C L+C L O$

$297 . \mathrm{O}+\mathrm{HOCL}=\mathrm{OH}+\mathrm{CLO}$

298. $\mathrm{HOCL}=\mathrm{CL}+\mathrm{OH}$

299. $\mathrm{HOCL}=\mathrm{H}+\mathrm{CLO}$

$300.0+C L 2=C L+C L O$

301. $\mathrm{H}+\mathrm{CLZ}=\mathrm{HCL}+\mathrm{CL}$

$302 . \mathrm{C} 2 \mathrm{H} 3+\mathrm{CL} 2=\mathrm{C} 2 \mathrm{H} 3 \mathrm{CL}+\mathrm{CL}$

303. $\mathrm{CHCLO}+\mathrm{OH}=\mathrm{CCLO}+\mathrm{H} 2 \mathrm{O}$

$304 . \mathrm{CHCLO}+\mathrm{O}=\mathrm{CCLO}+\mathrm{OH}$

305. CHCLO $+\mathrm{O} 2=\mathrm{CCLO}+\mathrm{HO} 2$

306. $\mathrm{CHCLO}+\mathrm{CI}=\mathrm{CCLO}+\mathrm{HCL}$

$307 . \mathrm{CHCLO}+\mathrm{CH} 3=\mathrm{CCLO}+\mathrm{CH} 4$

308 . $\mathrm{CHCLO}+\mathrm{CH} 3=\mathrm{CHO}+\mathrm{CH} 3 \mathrm{CL}$

309. $\mathrm{CHCLO}+\mathrm{CLO}=\mathrm{CCLO}+\mathrm{HOCL}$

310. $C C L O=C O+C L$

311. $\mathrm{CCLO}+\mathrm{OH}=\mathrm{CO}+\mathrm{HOCL}$

312. $\mathrm{CCLO}+\mathrm{O} 2=\mathrm{CO} 2+\mathrm{CLO}$

313. $\mathrm{CCLO}+\mathrm{CL}=\mathrm{CO}+\mathrm{CL} 2$

314. $\operatorname{COCL} 2+M=C C L O+C L+M$

315. $\mathrm{COCL} 2+\mathrm{OH}=\mathrm{CCLO}+\mathrm{HOCL}$

316. $\operatorname{COCL} 2+\mathrm{O}=\mathrm{CCLO}+\mathrm{CLO}$

$317 . \mathrm{COCL} 2+\mathrm{H}=\mathrm{CCLO}+\mathrm{HCL}$

318. $\mathrm{COCL} 2+\mathrm{CL}=\mathrm{CCLO}+\mathrm{CL} 2$

$319 . \mathrm{COCL} 2+\mathrm{CH} 3=\mathrm{CCLO}+\mathrm{CH} 3 \mathrm{CL}$

320. $\mathrm{CHCL} 3=\mathrm{CHCL} 2+\mathrm{CL}$

321. $\mathrm{CHCL} 3=\mathrm{CCL} 2+\mathrm{HCL}$

$322 . \operatorname{CCL} 2+02=\operatorname{COCL} 2+0$
$1.00 \varepsilon+17$

2. $30 E+13$

1. $08 E+13$

$2.47 \bar{E}+13$

$6.03 E+11$

8. $33 E+13$

$6.99 E+14$

$3.33 E+11$

$3.47 E+18$

1. $35 E+13$

$6.67 E+12$

$2.83 E+12$

$6.00 E+12$

$2.73 E+33$

1. $91 E+14$

4. $00 E+13$

1. $29 E+15$

5. $59 E+13$

1. $24 E+22$

2. $00 \mathrm{E}+12$

1. $00 E+13$

1. $83 E+27$

4. $53 E+31$

$8.86 E+29$

1. $10 E+30$

4. $15 E+12$

$4.13 E+19$

2. $00 E+11$

2. $02 E+13$

1. $70 E+13$

2. $45 E+12$

1. $00 \mathrm{E}+13$

6. $62 \mathrm{E}+12$

$6.03 E+11$

3. $03 E+11$

$6.03 E+11$

1. $81 E+12$

9. $55 E+13$

1. $81 E+12$

$7.28 E+12$

6. $03 E+12$

1. $76 E+20$

8. $12 E+14$

2. $51 E+12$

8. $59 \mathrm{E}+13$

5. $25 E+12$

7. $50 E+12$

8. $80 E+12$

4. $50 E+12$

1. $25 E+13$

$2.50 E+13$

1. $50 E+13$

1. $10 E+13$

1. $30 E+14$

3. $30 E+12$

1. $00 \mathrm{E}+13$

$4.00 E+14$

1. $20 E+16$

1. $00 E+13$

2. $.00 E+13$

5. $00 \mathrm{E}+13$

3. $20 E+14$

1. $90 E+13$

5. $70 E+12$

5. $20 \mathrm{E}+12$

5. $78 E+10$
0.0

0.0

0.0

0.0

0.0

0.0

$-0.6$

0.5

$-1.8$

0.0

0.0

0.0

0.0

$-7.5$

$-1.3$

0.0

$-2.0$

$-0.1$

$-2.7$

0.3

0.0

$-5.1$

$-6.4$

$-5.2$

$-5.2$

0.1

$-2.2$

0.0

0.0

0.0

0.0

0.0

0.0

0.0

0.0

0.0

0.0

0.0

0.0

0.0

0.0

$-3.0$

$-2.1$

0.0

0.0

0.0

0.0

0.0

0.0

0.0

0.0

0.0

0.0

0.0

0.0

0.0

0.0

0.0

0.0

0.0

0.0

0.0

0.0

0.0

0.0

0.0 c. $\hat{\circ}$

3500.0

$-338.0$

894.0

17400.0

7400.0

6360.0

30.0

2070.0

51800.0

18270.0

2090.0

5760.0

4440.0

3810.0

34000.0

1100.0

710.0

3860.0

3270.0

0.0

21170.0

22560.0

92920.0

92960.0

1110.0

2360.0

6000.0

54000.0

7300.0

2700.0

21660.0

1950.0

15000.0

10700.0

14100.0

990.0

7620.0

260.0

100.0

4370.0

56720.0

93690.0

2720.0

1170.0

$-480.0$

1200.0

3500.0

41800.0

500.0

6000.0

8800.0

500.0

8000.0

0.0

0.0

800.0

75500.0

23300.0

17000.0

6300.0

23500.0

12900.0

67700.0

51500.0

4100.0 
TABLE VI.3

PHOTO-THERMAL OXIDATION MECHANISM FOR A 5 COMPONENT MLXTURE (continued)

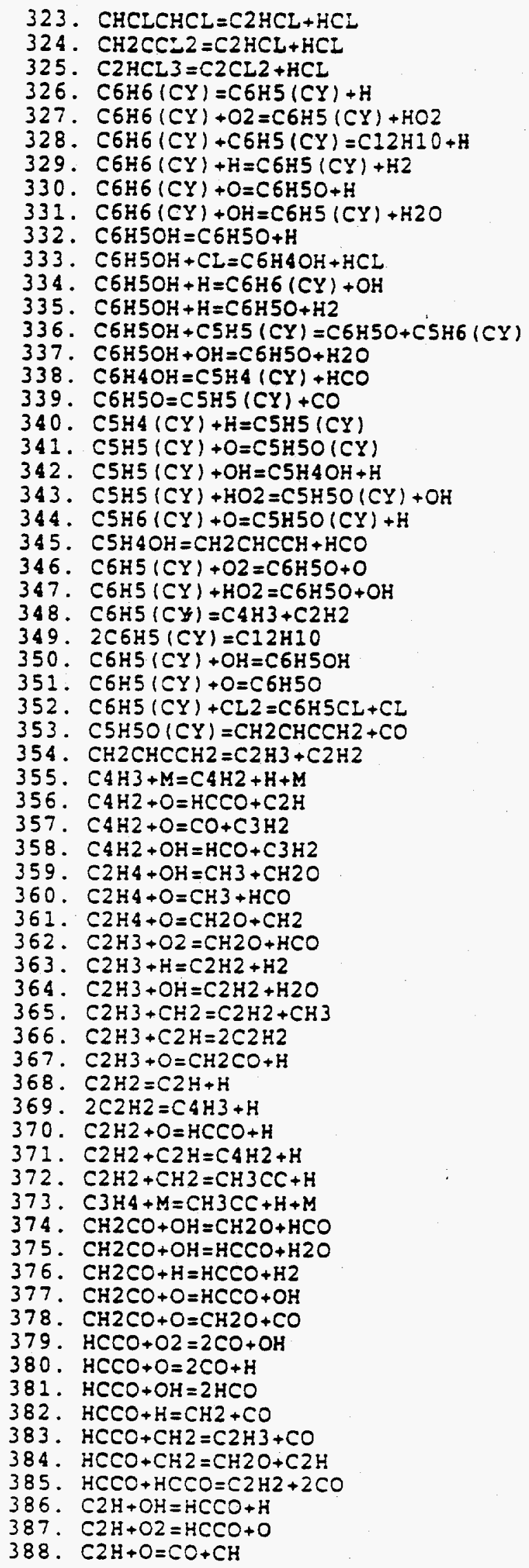

$7.26 E+13$

$1.45 E+14$

$7.26 E+13$

$5.00 E+15$

$6.30 E+13$

4. $00 E+11$

$3.00 E+12$

$2.80 E+13$

$2.10 E+13$

$3.00 E+15$

$1.00 E+13$

$2.20 E+13$

$1.20 E+14$

$4.00 E+14$

$3.00 E+13$

$2.50 E+11$

$2.50 E+11$

1. $00 E+12$

$5.00 E+13$

$1.00 E+13$

$2.00 E+13$

$1.00 \mathrm{E}+11$

1. $00 E+15$

2. $10 E+12$

$5.00 E+13$

$1.60 \mathrm{E}+15$

2. $00 E+14$

$1.00 E+14$

$1.00 E+13$

2. $50 E+12$

$3.00 E+16$

1. $40 E+13$

1. $00 E+16$

$1.00 E+13$

1. $20 E+12$

$3.00 E+13$

2. $00 E+12$

$3.30 E+12$

$2.50 E+13$

$4.00 E+12$

$6.00 E+12$

$5.00 E+12$

$3.00 E+13$

3. $00 E+13$

$3.30 E+13$

$4.20 E+16$

$2.00 E+12$

$4.00 E+14$

3. $00 E+13$

1. $20 E+13$

$2.00 E+17$

$2.80 E+13$

$7.50 E+12$

$7.50 E+13$

5. $00 \mathrm{E}+13$

$2.00 E+13$

$1.50 E+12$

1. $20 E+12$

$1.00 E+13$

$5.00 E+13$

$3.00 E+13$

$1.00 E+13$

1. $00 E+13$

2. $00 E+13$

$5.00 \mathrm{E}+13$

$5.00 E+13$
0.0

0.0

0.0

0.0

0.0

0.0

0.0

0.0

0.0

0.0

0.0

0.0

0.0

0.0

0.0

0.0

0.0

0.0

0.0

0.0

0.0

0.0

0.0

0.0

0.0

0.0

0.0

0.0

0.0

0.0

0.0

0.0

0.0

0.0

0.0

0.0

0.0

0.0

0.0

0.0

0.0

0.0

0.0

0.0

0.0

0.0

0.0

0.0

0.0

0.0

0.0

0.0

0.0

0.0

0.0

0.0

0.0

0.0

0.0

0.0

0.0

0.0

0.0

0.0

0.0

0.0
69090.0 69220.0

74440.0

108000.0

60000.0

4000 . 0

8100.0

4910.0

4580.0

88000.0

6000.0

7929.0

12400.0

25200.0

0.0

40000.0

43900.0

0.0

0.0

0.0

0.0

0.0

0.0

7470.0

1000.0

82000.0

0.0

0.0

0.0

4000.0

15000.0

32900.0

60000.0

0.0

0.0

0.0

960.0

1130.0

5000.0

$-250.0$

0.0

0.0

0.0

0.0

0.0

107000.0

45900.0

10660.0 0.0

6600.0

65000.0 0.0

3000.0

8000.0

8000.0

0.0

2500.0

0.0

0.0

0.0

0.0

2000.0

0.0

1500.0

0.0 
TABLE VI.3

PHOTO-THERMAL OXIDATION MECHANISM FOR A 5 COMPONENT MIXTURE (continued)

\author{
389. $2 \mathrm{CH} 2=\mathrm{C} 2 \mathrm{H} 2+\mathrm{H} 2$ \\ $390.2 \mathrm{CH} 2=\mathrm{C} 2 \mathrm{H} 3+\mathrm{H}$ \\ 391. $\mathrm{CH} 2+\mathrm{OH}=\mathrm{CH}+\mathrm{H} 2 \mathrm{O}$ \\ 392. $\mathrm{CH} 2+\mathrm{O}=\mathrm{CH}+\mathrm{OH}$ \\ 393. $\mathrm{CH} 2+\mathrm{O} 2=\mathrm{CO} 2+2 \mathrm{H}$ \\ 394. $2 \mathrm{CH} 3=\mathrm{C} 2 \mathrm{H} 4+\mathrm{H}_{2}$ \\ 395. $\mathrm{CH} 3+\mathrm{CH} 2 \mathrm{O}=\mathrm{CH} 4+\mathrm{HCO}$ \\ 396. $\mathrm{CH} 3+\mathrm{HCO}=\mathrm{CH} 4+\mathrm{CO}$ \\ 397. $\mathrm{CH} 2 \mathrm{O}+\mathrm{M}=\mathrm{HCO}+\mathrm{H}+\mathrm{M}$ \\ 398. $\mathrm{CH} 2 \mathrm{O}+\mathrm{OH}=\mathrm{HCO}+\mathrm{H} 2 \mathrm{O}$ \\ 399. $\mathrm{CH} 2 \mathrm{O}+\mathrm{H}=\mathrm{HCO}+\mathrm{H} 2$ \\ $40 \mathrm{O}$. $\mathrm{CH} 2 \mathrm{O}+\mathrm{O}=\mathrm{HCO}+\mathrm{OH}$ \\ 401. $\mathrm{CH}+\mathrm{O} 2=\mathrm{HCO}+\mathrm{O}$ \\ 402 . $\mathrm{HCO}+\mathrm{HO}_{2}=\mathrm{CH} 2 \mathrm{O}+\mathrm{O} 2$ \\ 403. $\mathrm{HCO}+\mathrm{M}=\mathrm{H}+\mathrm{CO}+\mathrm{M}$ \\ $404 . \mathrm{HCO}+\mathrm{O} 2=\mathrm{CO}+\mathrm{HO} 2$ \\ 405. $\mathrm{HCO}+\mathrm{OH}=\mathrm{CO}+\mathrm{H} 2 \mathrm{O}$ \\ $406 . \mathrm{HCO}+\mathrm{H}=\mathrm{CO}+\mathrm{H} 2$ \\ $407 . \mathrm{HCO}+\mathrm{O}=\mathrm{CO}+\mathrm{OH}$
}

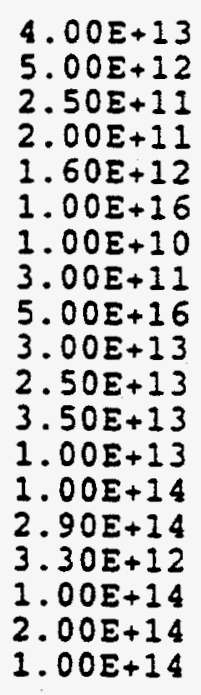

0.0

0.0

0.7

0.7

0.0

0.0

0.5

0.5

0.0

0.0

0.0

0.0

0.0

0.0

0.0

0.0

0.0

0.0

0.0

0.0

0.0

25700.0

25000.0

1000.0

32000.0

6000.0

0.0

76500.0

1200.0

3990.0

3510.0

0.0

3000.0

15570.0

7000.0

0.0

0.0

0.0

NOTE: A units mole-cm-sec-K, E units cal/mole 
Numerical simulations for high $\mathrm{H}: \mathrm{Cl}$ ratios indicated a modest solar enhancement for $\mathrm{t}_{\mathrm{f}}=1 \mathrm{~s}$ (cf., Figure VI.8). Surprisingly, $\mathrm{CH}_{2} \mathrm{Cl}_{2}$, not $\mathrm{C}_{6} \mathrm{H}_{5} \mathrm{Cl}$, showed the largest effect, followed by $\mathrm{C}_{2} \mathrm{HCl}_{3}$ and $\mathrm{C}_{6} \mathrm{H}_{5} \mathrm{Cl}$. Smaller solar enhancements were observed for $\mathrm{CH}_{4}$ and $\mathrm{C}_{6} \mathrm{H}_{6}$. An increase in $\mathrm{t}_{\mathrm{r}}$ to $10.0 \mathrm{~s}$ produced an unexpected result (cf. Figure VI.9). These simulations indicated a net reduction of the rate of decomposition of the starting materials in the presence of solar energy. This was most evident for $\mathrm{CH}_{2} \mathrm{Cl}_{2}$ $\mathrm{C}_{2} \mathrm{HCl}_{3}$, and $\mathrm{C}_{6} \mathrm{H}_{5} \mathrm{Cl}$ and occurred at temperatures above $1073 \mathrm{~K}$.

Numerical simulations for low $\mathrm{H}: \mathrm{Cl}$ ratios also showed a reduction in the ratio of decomposition for each starting material (ct. Figures VI.10 and VI.11). This effect was observed regardless of residence time but tended to increase with longer residence times. Largest effects were observed for $\mathrm{C}_{2} \mathrm{HCl}_{3}, \mathrm{CH}_{4}$, $\mathrm{C}_{6} \mathrm{H}_{6}$, and $\mathrm{CH}_{2} \mathrm{Cl}_{2}$.

\section{TABLE VI.4}

INITIAL MODELING CONDITIONS

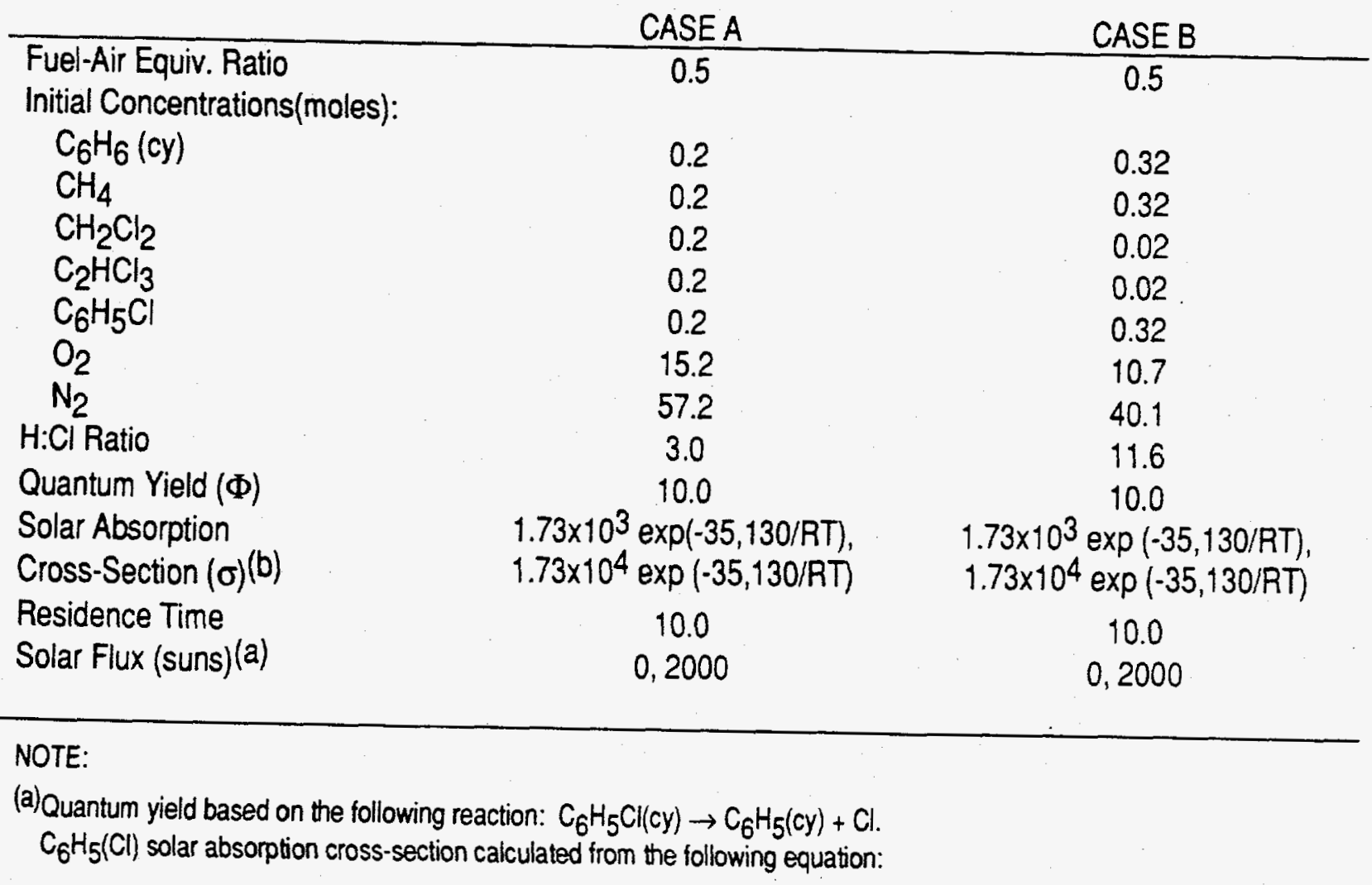




$$
\sigma=1.92 \times 10^{-5} \int_{300 \mathrm{~nm}}^{\lambda_{\text {onet }}} \varepsilon(\lambda) f(\lambda) d \lambda
$$

where: $f(\lambda)$ is the air mass 2.0 solar irradiance spectrum and $\varepsilon(\lambda)$ is the molar extinction spectrum for chlorobenzene.

(b) Values tor $\sigma$ were obtained from Reference 4. The temperature dependence of $\sigma$ was calculated assuming an Arthenius temperature dependence. $\sigma$ was varied by a factor of 10 to account for the uncertainty in the rate of absorption of solar radiation under various atmospheric conditions.

The numerical results indicate the presence of two competing kinetic phenomena. The effect of interest, i.e., photo-initiated chlorine-catalyzed destruction of non-absorbing organic compounds was observed at modest levels for low initial chlorine concentrations and relatively short residence times $\left(t_{r}=\right.$ $1 \mathrm{~s})$. The reason for the modest increase in destruction of organics was the small increase in the production of $\mathrm{Cl}$ atoms in the presence of sunlight (cf. Table VI.5). This, in turn, was due to the solarinduced dissociation of $\mathrm{C}_{6} \mathrm{H}_{5} \mathrm{Cl}$ with the relatively slow rate:

$$
\mathrm{C}_{6} \mathrm{H}_{5} \mathrm{Cl}+\mathrm{hv} \stackrel{\mathrm{k}_{1}}{\longrightarrow} \mathrm{C}_{6} \mathrm{H}_{5}+\mathrm{Cl}
$$

where:

$$
\begin{gathered}
\log k_{1}=6.54-35.5 / \Theta \\
\text { TABLE VI.5 } \\
\text { MAXIMUM CI ATOM FORMATION RATE } \\
\text { (moles } / \mathrm{cm}^{3}-\mathrm{s} \text { ) at } t=0.19 \mathrm{~s}
\end{gathered}
$$
MAXIMUM CI ATOM FORMATION RATE $\left(\mathrm{moles} / \mathrm{cm}^{3}-\mathrm{s}\right)$ at $\mathrm{t}=0.19 \mathrm{~s}$

$\frac{\text { I.K. }}{1073} \quad \frac{20.000 \text { suns }}{5.56 \times 10^{-5}} \quad \frac{\text { esuns }}{4.68 \times 10^{-5}}$

For high initial chlorine concentrations, the numerical results indicated an inhibitory effect. It is well documented that halogen atoms, e.g., $\mathrm{Br}, \mathrm{Cl}$, have the capacity to reduce the propagation rate of both premixed and diffusion flame combustion [8]. The general mechanism is believed to be chemical in nature and results in enhanced removal ratio of $\mathrm{OH}$ radicals and $\mathrm{H}$ atoms, i.e.: 


$\begin{array}{lll}\mathrm{HCl}+\mathrm{OH} & \rightarrow \mathrm{H}_{2} \mathrm{O}+\mathrm{Cl} & k_{254} \\ \mathrm{Cl}_{2}+\mathrm{H} & \rightarrow \mathrm{HCl}+\mathrm{Cl} & \mathrm{k}_{304} \\ \mathrm{HCl}+\mathrm{H} & \rightarrow \mathrm{H}_{2}+\mathrm{Cl} & k_{258} \\ \mathrm{H}+\mathrm{Cl}+\mathrm{M} & \rightarrow \mathrm{HCl}+\mathrm{M} & k_{257} \\ \mathrm{Cl}+\mathrm{OH} & \rightarrow \mathrm{HCl}+\mathrm{O} & k_{253} \\ \mathrm{O}+\mathrm{Cl} & \rightarrow \mathrm{ClO}+\mathrm{O} & k_{300}\end{array}$

These reactions indicate that chlorine inhibit oxidation processes by at least two chemical mechanisims. First, through $\mathrm{k}_{254}$ and $\mathrm{k}_{253}, \mathrm{HCl}$ and $\mathrm{Cl}$ directly compete with oxidation of organic species and $\mathrm{CO}$. Secondly, $\mathrm{Cl}_{2}, \mathrm{HCl}$, and $\mathrm{Cl}$ all remove $\mathrm{H}$ atoms from the reacting system $\left(\mathrm{k}_{257}, \mathrm{k}_{258}\right.$, and $\left.\mathrm{k}_{301}\right)$, thereby inhibiting the chain-branching reaction:

$$
\mathrm{H}+\mathrm{O}_{2} \rightarrow \mathrm{OH}+\mathrm{O} \quad \mathrm{k}_{235}
$$

Evidence for an inhibiting effect was observed through increased $\mathrm{CO}$ yields in the presence of sunlight. These results are shown in Table VI.6. Similar results were observed for $t_{r}=10.0 \mathrm{~s}$.

TABLE VI.6

CO YIELDS

\begin{tabular}{cccc}
\hline $\mathrm{T}, \mathrm{K}$ & O Suns & 2,000 Suns & $(\mathrm{CO})_{2000} /(\mathrm{CO}) 0$ \\
\hline 923 & $2.14 \mathrm{E}-3$ & $1.88 \mathrm{E}-3$ & 0.88 \\
973 & $1.05 \mathrm{E}-2$ & $7.95 \mathrm{E}-3$ & 0.76 \\
1023 & $1.60 \mathrm{E}-2$ & $1.47 \mathrm{E}-2$ & 0.92 \\
1073 & $8.00 \mathrm{E}-3$ & $9.16 \mathrm{E}-3$ & 1.15 \\
1123 & $1.65 \mathrm{E}-3$ & $2.51 \mathrm{E}-3$ & 1.52 \\
\hline
\end{tabular}

The goals of these numerical simulations were to demonstrate the most favorable conditions for chlorine-catalyzed solar destruction of a mixture of hazardous organic compounds. Only modest 
enhancements in the destruction rate were observed, and under most of the conditions simulated, a competing effect was evident. With the exception of fuel/air equivalence ratio, all of the kinetic parameters were studied over a considerable range, e.g., $\mathrm{t}_{\mathrm{\gamma}}=0.0-10.0 \mathrm{~s}, \mathrm{H}: \mathrm{Cl}$ ratios of 3.0 and 12.0 , and solar flux ranging from $0-2,000$ suns. These detailed parametric studies did not produce more favorable conditions for chlorine-catalyzed solar destruction using $\mathrm{C}_{6} \mathrm{H}_{5} \mathrm{Cl}$ as the photo-initiator.

The major implication of the modeling studies was the inefficiency of $\mathrm{Cl}$-catalyzed organics destruction under fuel-lean (excess air) conditions. Although some enhanced destruction was observed at low $\mathrm{Cl}$ concentrations, a chemical inhibition effect $(\mathrm{OH}, \mathrm{H}$, and $\mathrm{O}$ atom scavenging by $\mathrm{Cl}$ atoms) became dominant at higher initial $\mathrm{Cl}$ concentrations. These results infer that for enhanced $\mathrm{Cl}$ destruction, one needs to minimize the inhibitory effect. It thus seems logical to conduct some additional simulations under sub-stoichiometric conditions.

A key parameter to be varied in these simulations will be the oxygen concentration. It will likely be desirable to have enough oxygen present to convert radical intermediates and $\mathrm{CO}$ to $\mathrm{CO}_{2}$. On the other hand, a limiting oxygen concentration will also be desirable to suppress the $\mathrm{Cl}$ inhibition chemistry. A major goal of this continuing work will be the elucidation of the oxygen "window" where enhanced organics destruction can occur.

It would also be desirable to study other photo-initiators. A major limitation of $\mathrm{C}_{6} \mathrm{H}_{5} \mathrm{Cl}$ is its very slow photo-dissociation rate at low temperatures. More promising candidates would include more highly chorinated aromatic compounds which exhibit faster low temperature photo-dissociation rates.

\section{VI.3 References}

1. E. R. Ritter, J. W. Bozzelli, and A. M. Dean, J.Phys. Chem. 93, (1990).

2. J. H. Kiefer, et al., J. Phys. Chem., 89, 2013-2019 (1985).

3. D. Bittker, Combust. Sci. Technol., in press (1992).

4. M. Frenklach et al., Combust. Sci. Tech., 50, 79-115 (1986). 
5. W. Ho and J.W. Bozzelli, 24th Symp. (Int.) on Combustion, in press (1992).

6. W.D. Chang and S.M. Senkan, Environ. Sci. Technol. 23, 442 (1989).

7. J. Berman, J. Graham, and B. Dellinger, L L Photochem. Photobiol., in press (1992).

8. D. Bose and S.M. Senkan, Combust. Sci. Technol, 35, 187 (1983), and references therein. 


\section{APPENDIX VII \\ Thermal And Thermal/Photolytic Destruction Of Organic Compounds Volatilized From Virgin And Contaminated Soil (co-funded by the US-EPA Under CR-818614)}

One promising application for solar detoxification is the destruction of hazardous organic wastes from soils. Specifically, a variety of soil vapor extraction (SVE) techniques have been developed for the removal of volatile and semi-volatile compounds from contaminated soils $[1,2,3]$. The relative energy requirements to detoxify the dilute process streams from these processes makes conventional treatments (i.e., thermal oxidation) economically unfavorable [4]. Therefore, on-site concentration and storage via carbon absorption or liquid condensation is often employed for collecting the contaminants for later destruction. This general scheme is ideally suited for solar detoxification as it overcomes one of the largest economic challenges of utilizing concentrated solar energy caused by the intermittent nature of the energy source. Specifically, the wastes collected on-site could be destroyed in a suitably scaled solar reactor when sunlight is available. It would also be possible to operate an off-site solar detoxification facility processing wastes from a variety of sites.

One concern of the proposed process is it's ability to destroy the targeted wastes, particularly the low molecular weight species which may not absorb solar radiation. However, the organic content of the off-gas stream from SVE processes are likely to contain significant amounts of organic material from the soil's humus in addition to the waste material. We have hypothesized that these naturally occurring compounds, which are likely to include derivatives of humic acids [5,6], will serve as photosensitizers which could enhance the solar detoxification process through either energy or electron transfer reactions or radical induced chain reactions.

To examine the thermal and thermal/photolytic destruction of the volatile compounds from soil, the volatile components from an organic rich soil spiked with a synthetic waste mixture was studied using the TPRS.

\section{VII.1 Thermal Desorption Characteristics of Humus Rich Soil}

The first step in examining the detoxification of organic contaminants from soil was to select a soil matrix, and evaluate it's thermal desorption characteristics. To provide a humus rich and reasonably reproducible soil matrix for these tests, the fine particulate fraction from a commercially available source was chosen. Specifically, a stock sample of approximately 100 grams was obtained from a 1 cubic foot 
bail of Canadian Sphagnum Peat Moss (Fertilome) by sieving a portion of the bail through a 50 mesh screen, giving particles with sizes less than $300 \mathrm{~mm}$.

To measure the volatile content of the soil matrix, $10 \mathrm{mg}$ sample were tested by thermogravimetric analysis (TGA) in atmospheres of flowing dry air (oxidation) and nitrogen (pyrolysis). The data from these tests (weight \% remaining as a function of temperature) are summarized in Figures VII.1 and VII.2. The initial weight loss (from 100 to $~ 75 \%$ ) evident in both Figures suggest a water content of approximately $25 \%$ by weight. The second weight loss (from $\sim 75$ to $\sim 45 \%$ ), also evident in both Figures, suggests a volatile organic content of $\sim 30 \%$ by weight. The relatively rapid weight loss seen in Figure VII. 1 starting at $\sim 340^{\circ} \mathrm{C}$ (from $\sim 45 \%$ to $\sim 10 \%$ ) indicates ignition of the remaining sample, indicating the sample consists of $\sim 35 \%$ nonvolatile organic material. The gradual weight loss over a similar weight range (from $\sim 45 \%$ to $\sim 13 \%$ ) shown in Figure VII.2, but over a broad temperature range (from 340 to $780^{\circ} \mathrm{C}$ ), suggests that the nonvolatile organic fraction encompasses a very broad molecular weight range, and this weight loss is the result of thermal degradation (fragmentation) of these materials. Finally, these Figures show that the sample consists of $\sim 10 \%$ nonvolatile, non-combustible ash.

\section{VII.2 Thermal and Thermal/Photolytic Destruction of a Synthetic Contaminant Mixture}

To simulate the contamination at a remediation site, a complex mixture of organic compounds was constructed. This mixture included $40 \%$ (v/v) No. 2 fuel oil, $40 \%$ dichlorobenzene, $10 \%$ carbon tetrachloride, $5 \%$ toluene, and $5 \%$ naphthalene. The GC/FID chromatogram for this mixture shown in Figure VII. 3 illustrates the extreme complexity of this mixture.

Prior to testing the synthetic waste sample on the TPRS, its volatilization characteristics were evaluated via TGA, and its high temperature absorption spectra was obtained with the HTAS. The TGA data for the sample in an atmosphere of flowing dry air, shown in Figure VII.4, illustrates that the mixture contains only volatile components, and readily vaporizes at temperatures below $200^{\circ} \mathrm{C}$. The high temperature absorption spectra (shown as a molar extinction spectra by assigning the mixture a mean molecular weight of $200 \mathrm{~g} / \mathrm{g}-\mathrm{mol}$ ) is given in Figure VII.5. This Figure shows that the mixture is a relatively modest absorber of solar photons (i.e., wavelengths $>300 \mathrm{~nm}$ ).

The thermal and thermal/photolytic decomposition of the synthetic mixture was measured with the TPRS from 300 to $900^{\circ} \mathrm{C}$ in an atmosphere of flowing dry air (oxidation) with a mean exposure time of $10 \mathrm{~s}$ and radiant exposures equivalent to 0 (thermal) and 273 air mass (AM) 1.5 suns (i.e., 273 times typical terrestrial solar radiation). The data for the conversion of the sum of all the mixture components, and the individual compounds toluene, dichlorobenzene, and naphthalene, are summarized in Tables VII. 1 and 


\section{TABLE VII.1}

Summary Of TPRS Data ${ }^{1}$ For the Synthetic Waste Mixture Exposed For $10 \sin$ Air

\begin{tabular}{llllllll} 
& \multicolumn{8}{c}{ Temperature, C } \\
Compound & 300 & 400 & 500 & 600 & 700 & 800 & 900 \\
\hline Mixture & 100 & 91.5 & 71.9 & 45.0 & 15.9 & 0.891 & 0.122 \\
Toluene & 100 & 87.4 & 51.6 & 6.30 & 0.34 & 0.0500 & \\
Dichlorobenzene & 100 & 100 & 90.7 & 73.5 & 27.5 & 0.530 & 0.0600 \\
Naphthalene & 100 & 100 & 84.0 & 45.2 & 2.94 & 0.110 & 0.0110
\end{tabular}

TABLE VII.2

Summary Of TPRS Data ${ }^{1}$ For the Synthetic Waste Mixture Exposed To 273 AM 1.5 Suns For $10 s \ln$ Air

Compound

Mixture

Toluene

Dichlorobenzene

Naphthalene
Temperature, $\mathrm{C}$

\begin{tabular}{lllllll}
300 & 400 & 500 & 600 & 700 & 800 & 900 \\
\hline 94.9 & 85.2 & 61.2 & 40.0 & 13.3 & 1.15 & 0.336 \\
96.1 & 80.7 & 44.3 & 3.95 & 0.190 & 0.090 & 0.0500 \\
99.6 & 94.5 & 85.0 & 71.1 & 22.5 & 0.800 & 0.0700 \\
100 & 97.2 & 74.8 & 37.6 & 1.82 & 0.690 & 0.420
\end{tabular}




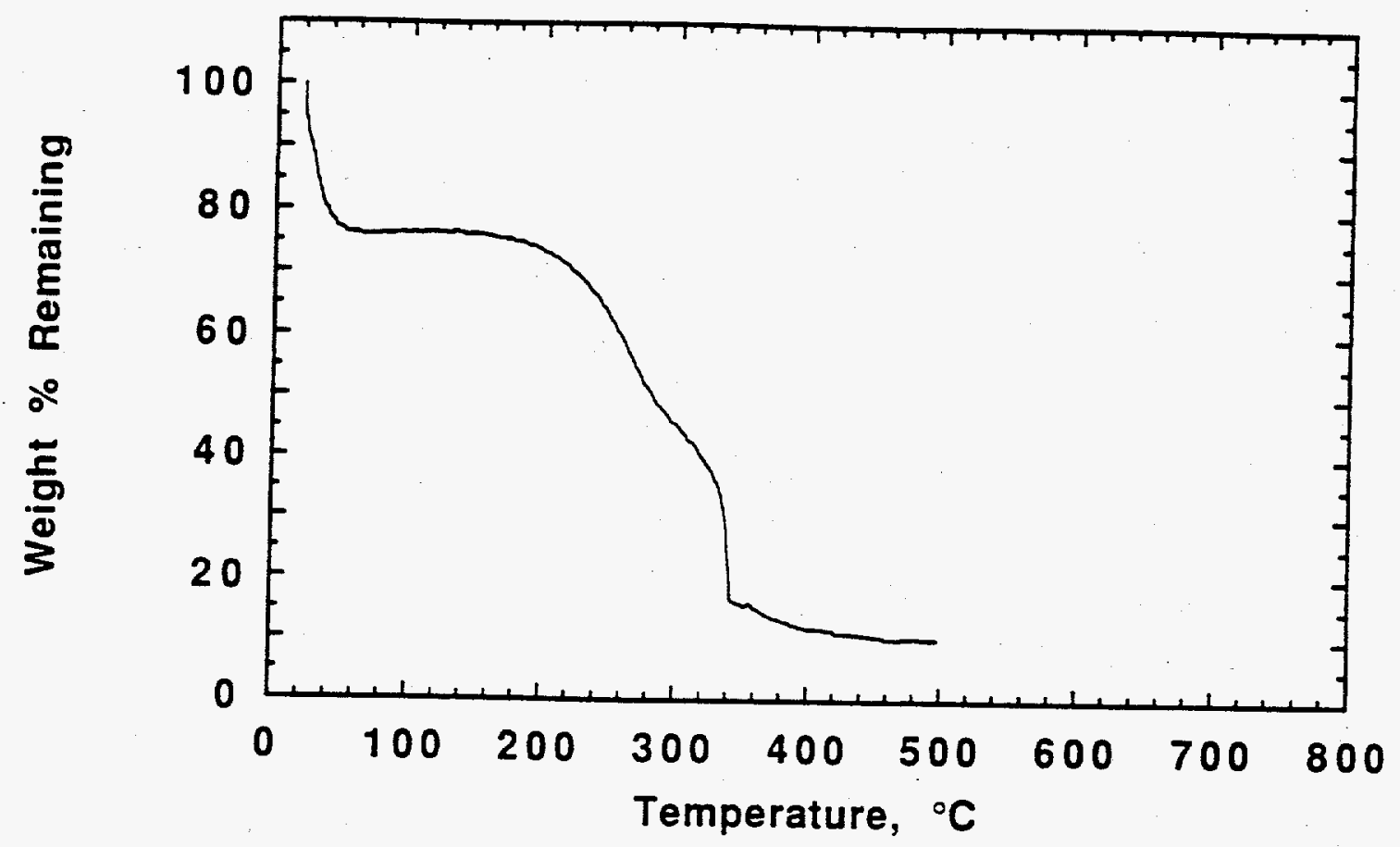

Figure VIl.1. TGA data for fine mesh ( $<300 \mathrm{~mm})$ Sphagnum Peat Moss in air.

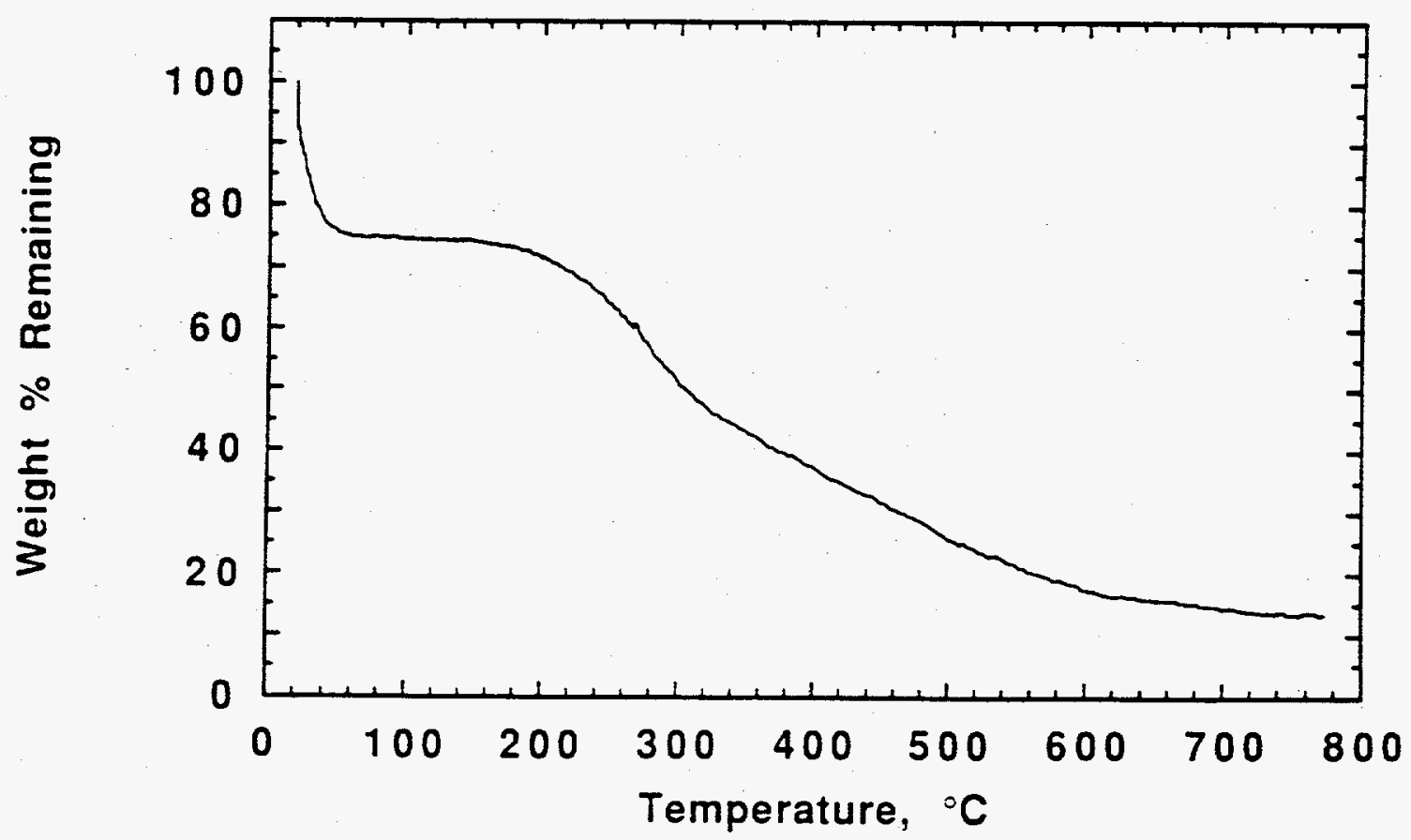

Figure VII.2. TGA data for fine mesh (<300 mm) Sphagnum Peat Moss in nitrogen. 


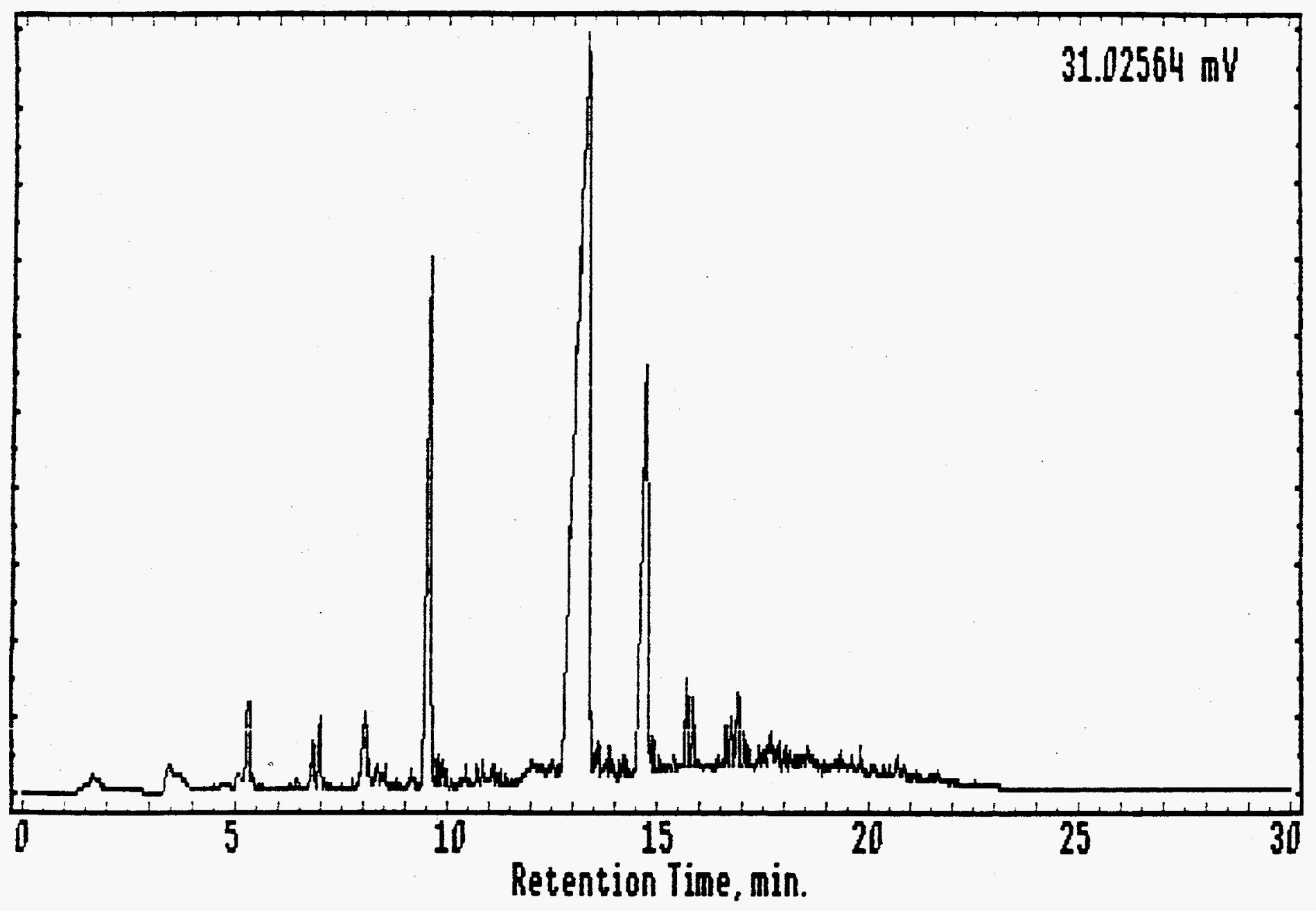

Figure VII.3. TPRS GC/FID chromatogram for the synthetic waste mixture. 


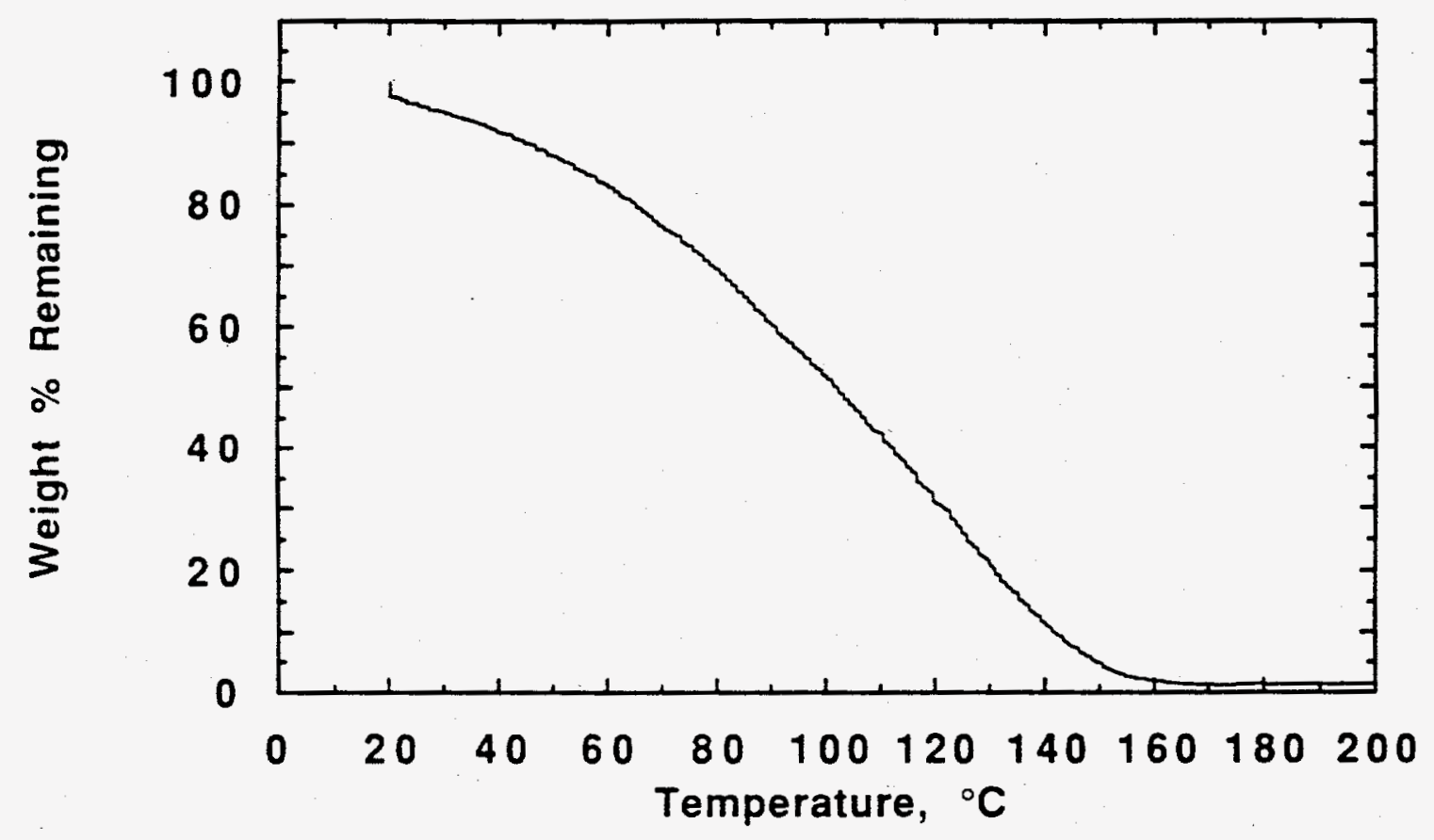

Figure VIl.4. TGA data for the synthetic waste mixture in air. 


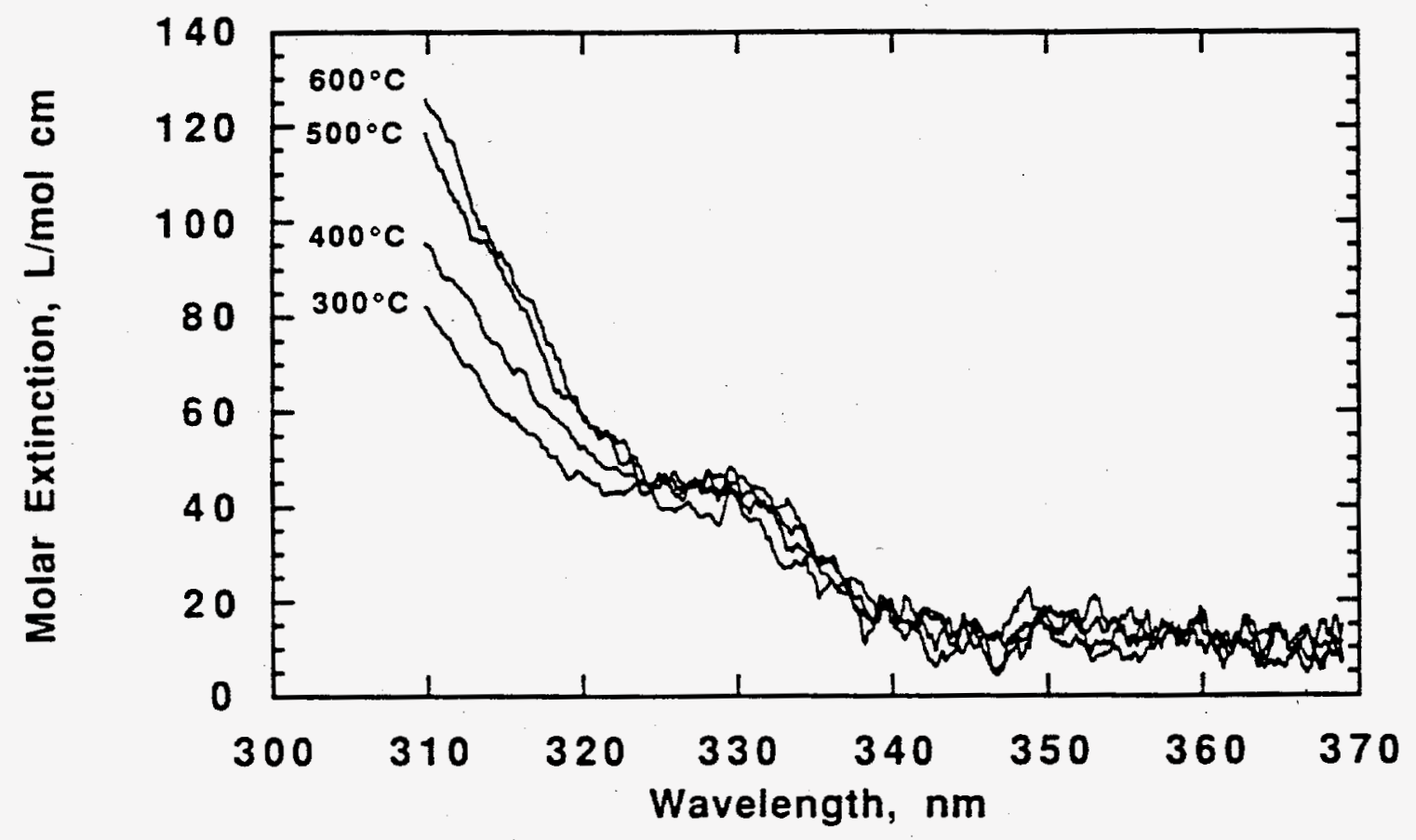

Figure VII.5. High temperature absorption spectra for the synthetic waste mixture.

Note that a mean molecular weight of $200 \mathrm{~g} / \mathrm{g}$-mol was assigned to the sample. 
VII.2, and in Figures VII.6 through VII.9, respectively. Quantitative data for carbon tetrachloride is not available as its chromatographic peak co-eluted with that of a product of incomplete conversion.

Data for the waste mixture taken as a whole (cf. Figure VII.6) shows nearly identical decomposition behavior from 300 to $800^{\circ} \mathrm{C}$. At $900^{\circ} \mathrm{C}$, the thermal exposure performed approximately two times better than the simulated solar exposure. Similar behavior is seen in the data for the toluene and naphthalene (cf. Figures VII.7 and VII.9) with comparable reactor performance from 300 to $700^{\circ} \mathrm{C}$, then the thermal exposures performing significantly better than the solar at higher temperatures. In contrast, the reactor performance on the destruction of dichlorobenzene component (cf. Figure VII.8) remained comparable from the thermal and simulated solar exposures throughout the temperature range studied.

The absence of significant response to the simulated solar exposures can be understood by examining the nature of the photon absorption by the sample. Specifically, considering the chemical composition of the sample, only the highest molecular weight components of the fuel are likely to be capable of absorbing the simulated solar radiation. The vast bulk of the aliphatic components which make up the fuel, and the contaminants which were added to it, should not absorb at wavelengths $>300 \mathrm{~nm}$ (i.e., within the solar spectrum).

The apparent reduction in bulk reactor performance at temperatures $>900^{\circ} \mathrm{C}$, and the similar reduction in performance on destroying toluene and naphthalene at temperatures $>800^{\circ} \mathrm{C}$, is unclear. Since these molecules should not absorb the simulated solar radiation, this observed effect should not be the result of a direct photochemical mechanism and an indirect photochemical process is implicated. One possibility is that the simulated sunlight interacts with reactive intermediates in such a way as to suppress species which participate in the decomposition of these components. Another possibility which should be considered is the production of these compounds as PICs. While a thorough understanding of the undoubtedly complex reaction pathways is necessary to explain the data, the fact remains that the simulated solar exposure reduced the performance of the TPRS reactor in destroying the waste mixture.

\section{VII.3 Soil Oft-Gas Destruction}

The study of the contaminant mixture showed no minimal enhancement. The next step was to study the effect of a soil matrix on the thermal and thermal/photolytic destruction of the individual contaminants. 


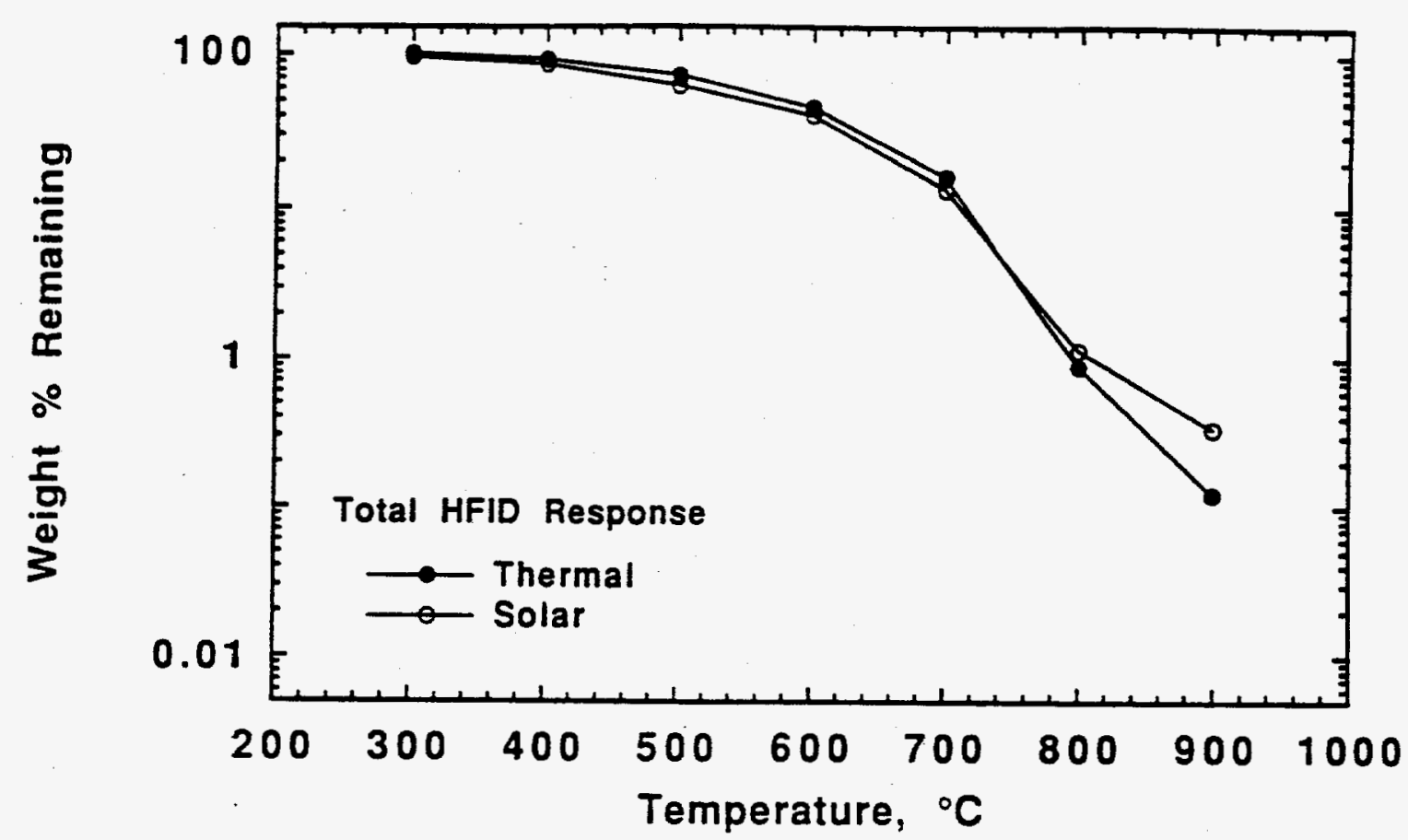

Figure VII.6. Summary of TPRS data for the synthetic waste mixture exposed to 0 and 273 AM 1.5 suns (simulated) for $10 \mathrm{~s}$ in air.

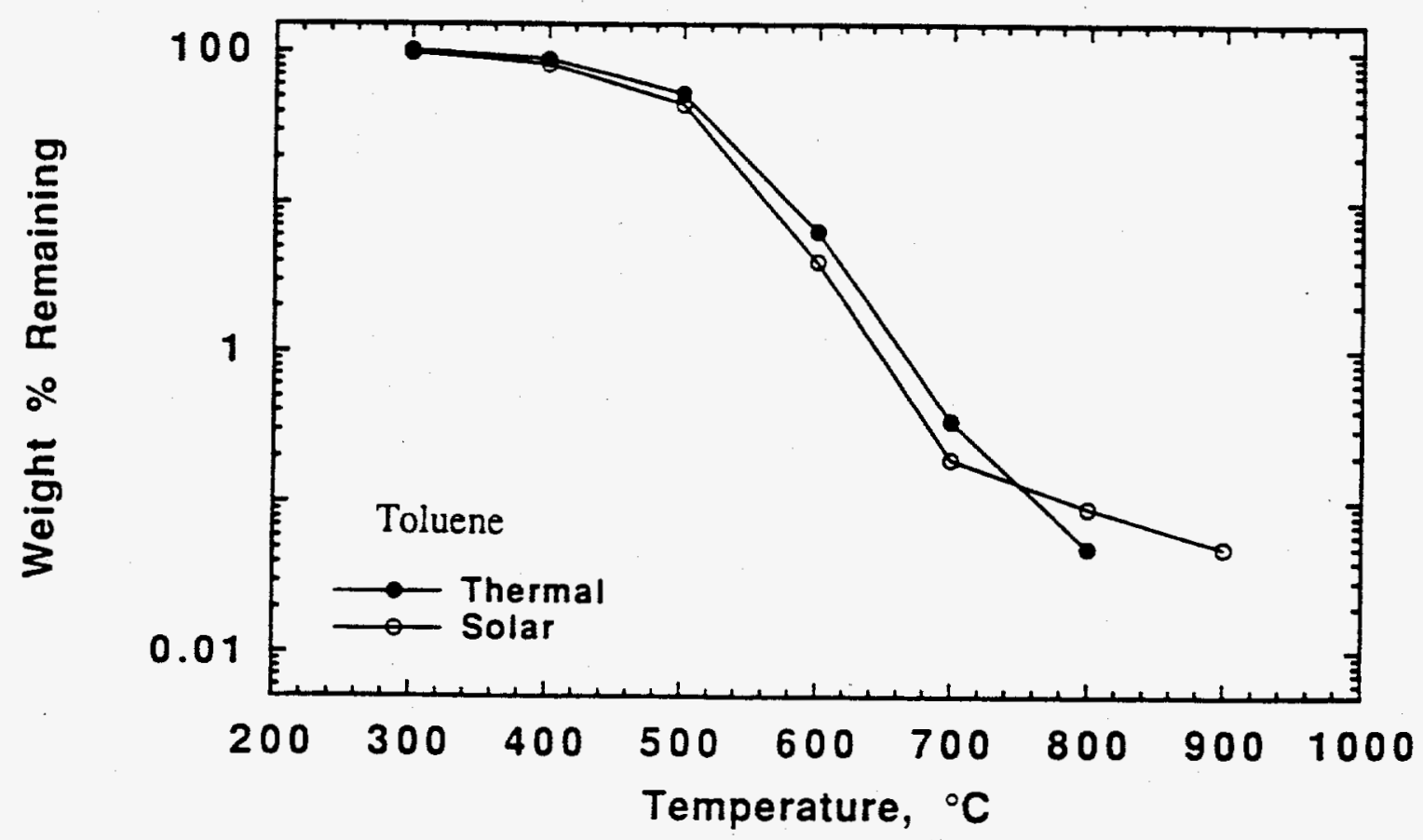

Figure VII.7. Summary of TPRS data for the toluene component of the synthetic waste mixture exposed to 0 and $273 \mathrm{AM} 1.5$ suns (simulated) for $10 \mathrm{~s}$ in air. 


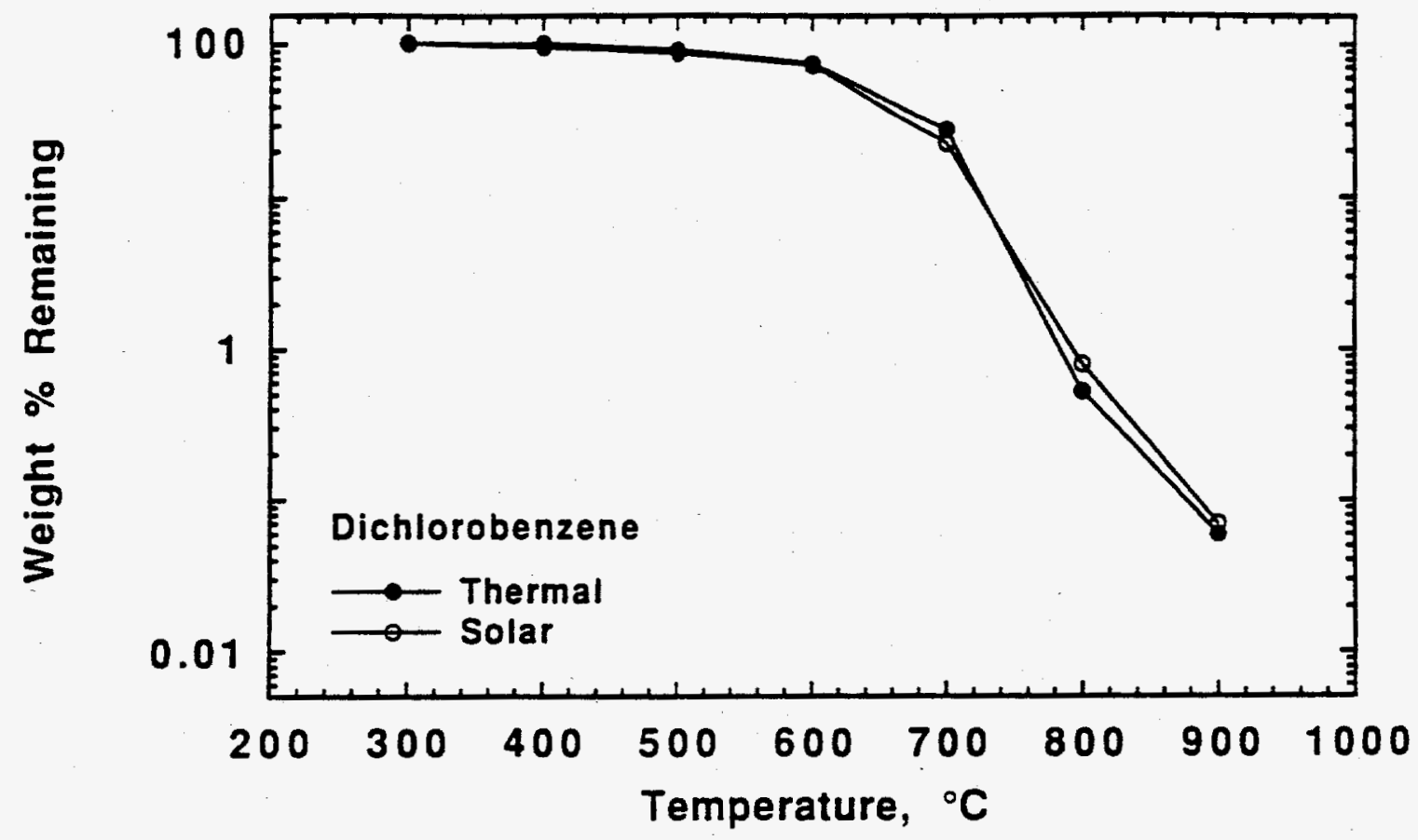

Figure VII.8. Summary of TPRS data for the dichlorobenzene component of the synthetic waste mixture exposed to 0 and $273 \mathrm{AM} 1.5$ suns (simulated) for $10 \mathrm{~s}$ in air.

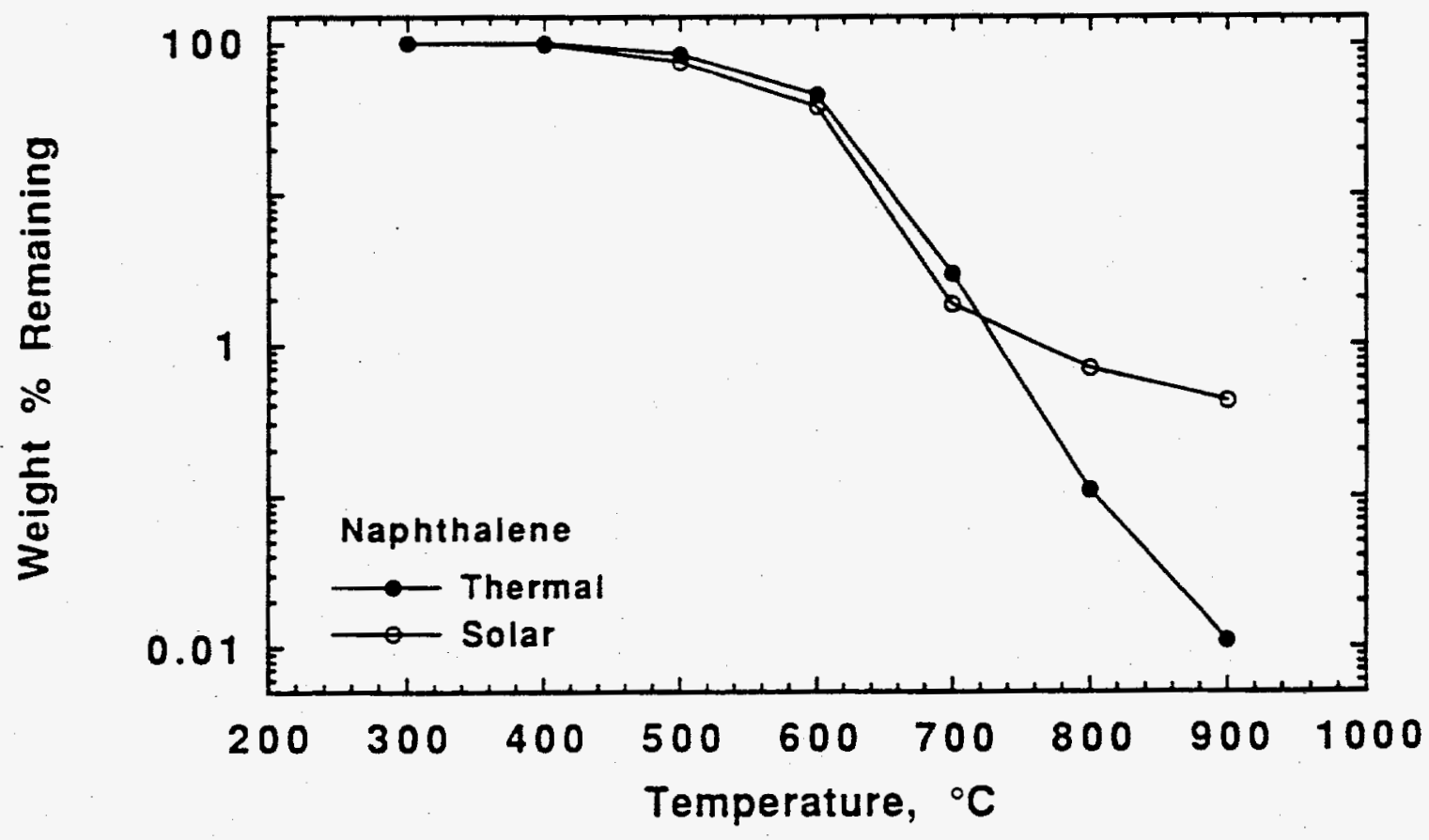

Figure VII.9. Summary of TPRS data for the naphthalene component of the synthetic waste mixture exposed to 0 and $273 \mathrm{AM} 1.5$ suns (simulated) for $10 \mathrm{~s}$ in air. 


\section{VII.3.1 Virgin Soils}

Prior to conducting a full series of tests of the destruction of the synthetic waste mixture desorbed from a humus rich soil, a small number of tests were conducted on the effect of the simulated solar exposure on the organic compounds that thermally desorb from virgin soil. For each analysis, approximately $100 \mathrm{mg}$ of the soil was gently packed into a specially fabricated miniature $(1 \times 30 \mathrm{~mm})$ test tube. The sample tube was then sealed in the TPRS's insertion chamber using a quartz carrier probe. The temperature of the inlet was maintained at $330^{\circ} \mathrm{C}$ to promote rapid evaporation of the volatile compounds from the soil. After a period of 10 minutes, the probe and sample tube were removed from the inlet, and the inlet was then resealed with a clean probe. After an additional period of 5 minutes to ensure that all of the gaseous sample had been swept through the system, the material collected on the TPRS's cryogenic trap was thermally transferred to the GC/FID system for analysis.

The procedure described above was conducted with a reactor temperature of $300^{\circ} \mathrm{C}$ with an atmosphere of flowing dry air and a mean exposure time of $10 \mathrm{~s}$ to 0 (thermal) and 273 AM 1.5 suns. The TPRS chromatograms from these exposures are shown in Figure VII.10. The upper chromatogram in Figure VII. 10 clearly shows the extreme complexity of the mixture of organic compounds evolving from the virgin soil. Furthermore, weighing the samples before and after the analysis revealed that the samples lost approximately $45 \%$ of their original mass. Considering the TGA data given in Figures VII.1 and VII.2, this suggests the material volatilizing from the neat soil includes the water vapor, and only the volatile fraction represented by the second weight loss in these figures. Therefore, the material volatilizing from each 100 $\mathrm{mg}$ of soil consists of approximately $25 \mathrm{mg}$ of water, and $20 \mathrm{mg}$ of organic material.

Upon exposure to the 273 AM 1.5 suns (cf. the lower chromatogram in Figure VII.10), the total integrated area of the volatile released from the soil was reduced by $47.6 \%$. This is a very significant result, showing that volatile organic component of humus-rich soil is highly susceptible to solar degradation. The resulting decomposition products may also provide a reactive atmosphere for destruction of hazardous contaminants in the soil.

\section{VII.3.2 Contaminated Soil}

To test the effect of the naturally occurring arganic compounds from soils on the destruction of the synthetic waste mixture described in Section 5.2, soil samples similar to those described above (i.e., 100 $\mathrm{mg}$ in miniature test tubes) where injected with $0.1 \mathrm{ml}$ (approximately $0.1 \mathrm{mg}$ ) of the mixture. To encourage efficient mixing of the volatized waste mixture with the compounds volatilizing from the soil, the waste was injected with the syringe needle pushed though the soil and onto the bottom of the sample tube. This 

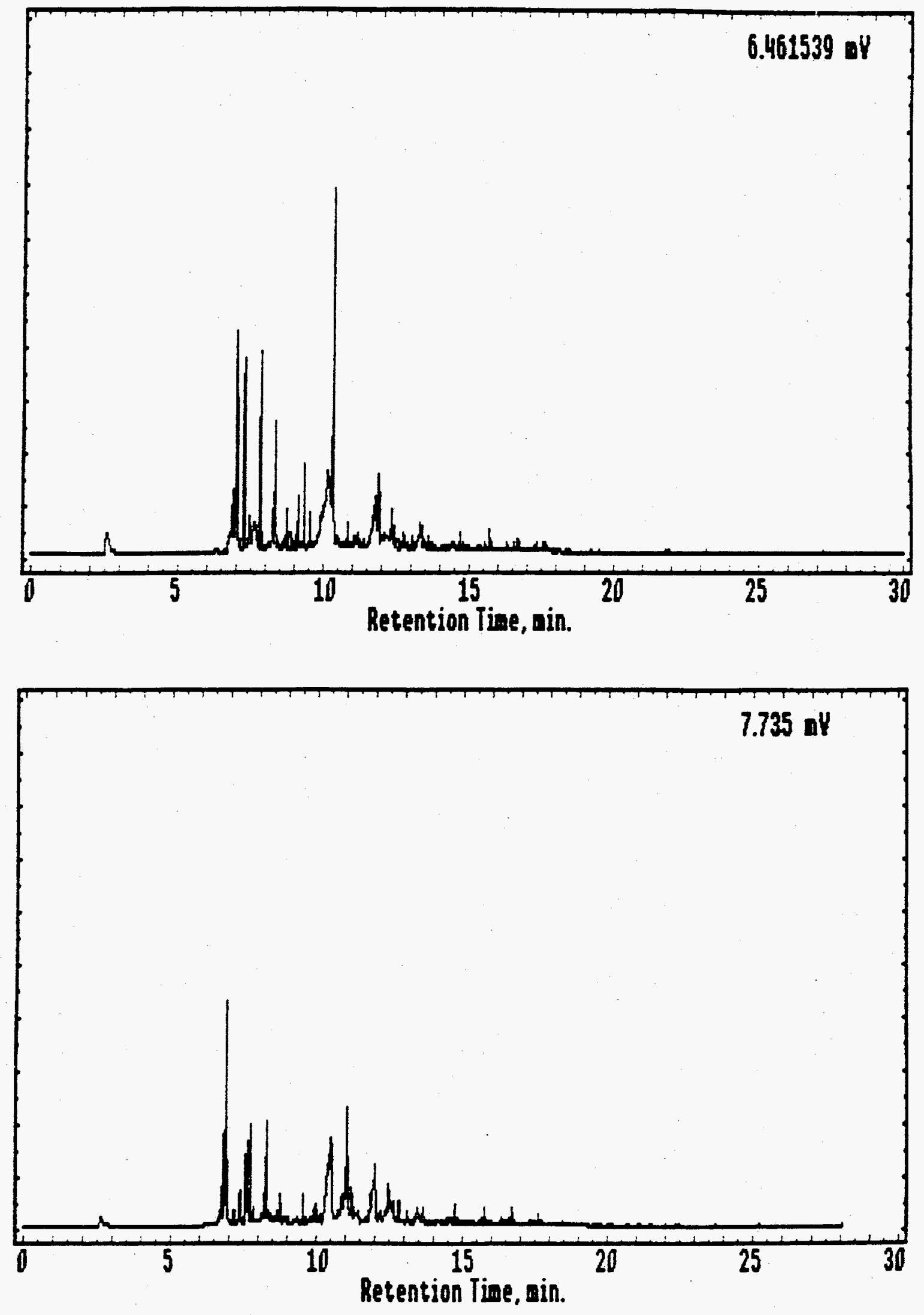

Figure VII.10. TPRS GC/FID chromatogram for the volatile organic components desorbed from fine mesh (<300 mm) Sphagnum Peat Moss exposed to 0 (top) and 273 AM 1.5 suns (bottom) and $300 \mathrm{C}$ for $10 \mathrm{~s}$ in air. 
forced the mixture vapors to pass though the soil as it volatilized from the tube. The TPRS data for the resulting mixture taken as a whole, and for the toluene, dichlorobenzene, and naphthalene components are summarized in Tables VII.3 and VII.4, and in Figures VII.11 through VII.14, respectively. To clearly show the effect of the soil components, the data are also plotted as neatsoil pairs for the thermal and solar data in Figures VII.15 through VII.22.

The data shown in Figures VII.11 through VII.14 illustrate comparable reactor performance from both thermal and solar exposures throughout the temperature range studied. This contrasts with the data reported for the neat waste where the thermal performance was significantly better than the solar at temperatures above $700-900^{\circ} \mathrm{C}$. From these results one may question the value of pursuing a solar reactor in lieu of a thermal system. In considering this, it is important to recall other factors which are not explicitly a part of this study. Specifically, a since a properly design solar reactor will derive it's heat from the conversion of the visible and infrared portion of the spectrum, the addition of a combustible fuel stock is not required, and the additional cost and pollution from the use of a fossil fuel will not be encountered. Therefore, since the data suggest that there will be at least no significant performance penalty by using concentrated sunlight as the energy source for the detoxification reactor, conducting an economic evaluation of such a system is justifiable.

The data summarized in Figure VII.15 through VII.22 show interesting results for both the thermal and solar aspects of detoxifying the process stream from SVE operations. Specifically, with the exception of the destruction of toluene shown in Figures VII.16 and VII.20, the organic material evolving from the soil was destroyed far more efficiently than from the neat waste. This clearly shows that the decomposition of the naturally occurring components from the soil has a positive impact on the overall performance of the reactor. In contrast, toluene appeared more stable when volatilized from the soil as compared to the neat waste mixture. Furthermore, it's destruction from the soil was not effected by the simulated solar radiation. This is likely to be due to the formation of toluene from the decomposition of the soil matrix.

\section{VII.4 References}

1. M. F. Kress, et al., "Field Investigation of Soil Vapor Extraction Technology," proceedings of the 1992 U.S. EPAAir \& Waste Management Association International Symposium, Cincinnati, Ohio, February, 1992.

2. D. W. Chartrand, et al., "Application of Stream Injection/Vapor Extraction Treatment Systems," proceedings of the 1992 U.S. EPAAAir \& Waste Management Association International Symposium, Cincinnati, Ohio, February, 1992. 
3. R. A. Brown, et al., "In Situ of Chlorinated Solvents in Shallow Aquifers with Air Sparging/Soil Venting (ASV Technology)," proceedings of the 1992 U.S. EPA/Air \& Waste Management Association International Symposium, Cincinnati, Ohio, February 1992.

4. G. R. Allen, et al., "Case Study: Vacuum Extraction to Remediate Contaminated Soil - Selection of Optimum Extraction and Emission Control Equipment," proceedings of the 1992 U.S. EPA/Air \& Waste Management Association International Symposium, Cincinnati, Ohio, February 1992.

5. C. E. Marshall, The Physical Chemistry and Mineralogy of Soils. Vol. I: Soil Materials, Robert E. Krieger Publishing Co., Huntington, NY, 1975.

6. M. M. Kononova, et al., Soil Organic Matter, Its Nature. its Role in Soil Formation and in Soil Fertility, 2nd English Ed., Pergamon Press, Oxford, England, 1966. 
TABLE VII.3

Summary Of TPRS Data ${ }^{1}$ For The Synthetic Waste MixtureDesorbed From Soil Exposed For $10 \mathrm{~s} \ln$ Air

Compound

Mixture

Toluene

Dichlorobenzene

Naphthalene

\begin{tabular}{llllll}
\multicolumn{7}{c}{ Temperature, C } \\
300 & 400 & 500 & 600 & 700 & 800 \\
\hline 100 & 100 & 100 & 25.8 & 5.13 & 0.0376 \\
100 & 100 & 100 & 20.7 & 1.05 & 0.124 \\
100 & 100 & 100 & 68.3 & 18.1 & 0.00834 \\
100 & 100 & 100 & 8.71 & .646 &
\end{tabular}

TABLE VII. 4

Summan Of TPRS Data ${ }^{1}$ For The Synthetic Waste Mixture Desorbed From Soil Exposed To 273 AM 1.5 Suns For $10 \mathrm{~s} \ln$ Air

Compound

Mixture

Toluene

Dichlorobenzene

Naphthalene

\begin{tabular}{llllll}
\multicolumn{7}{c}{ Temperature, C } \\
300 & 400 & 500 & 600 & 700 & 800 \\
\hline 87.8 & 81.4 & 90.0 & 21.6 & 3.84 & 0.0691 \\
100 & 94.5 & 100 & 14.9 & 1.12 & 0.157 \\
100 & 94.0 & 98.2 & 62.2 & 14.8 & 0.0187 \\
100 & 94.1 & 93.3 & 6.17 & 0.263 & 0.055
\end{tabular}

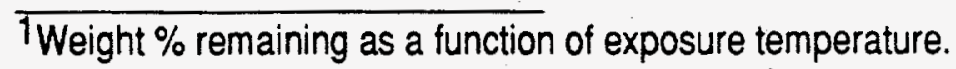




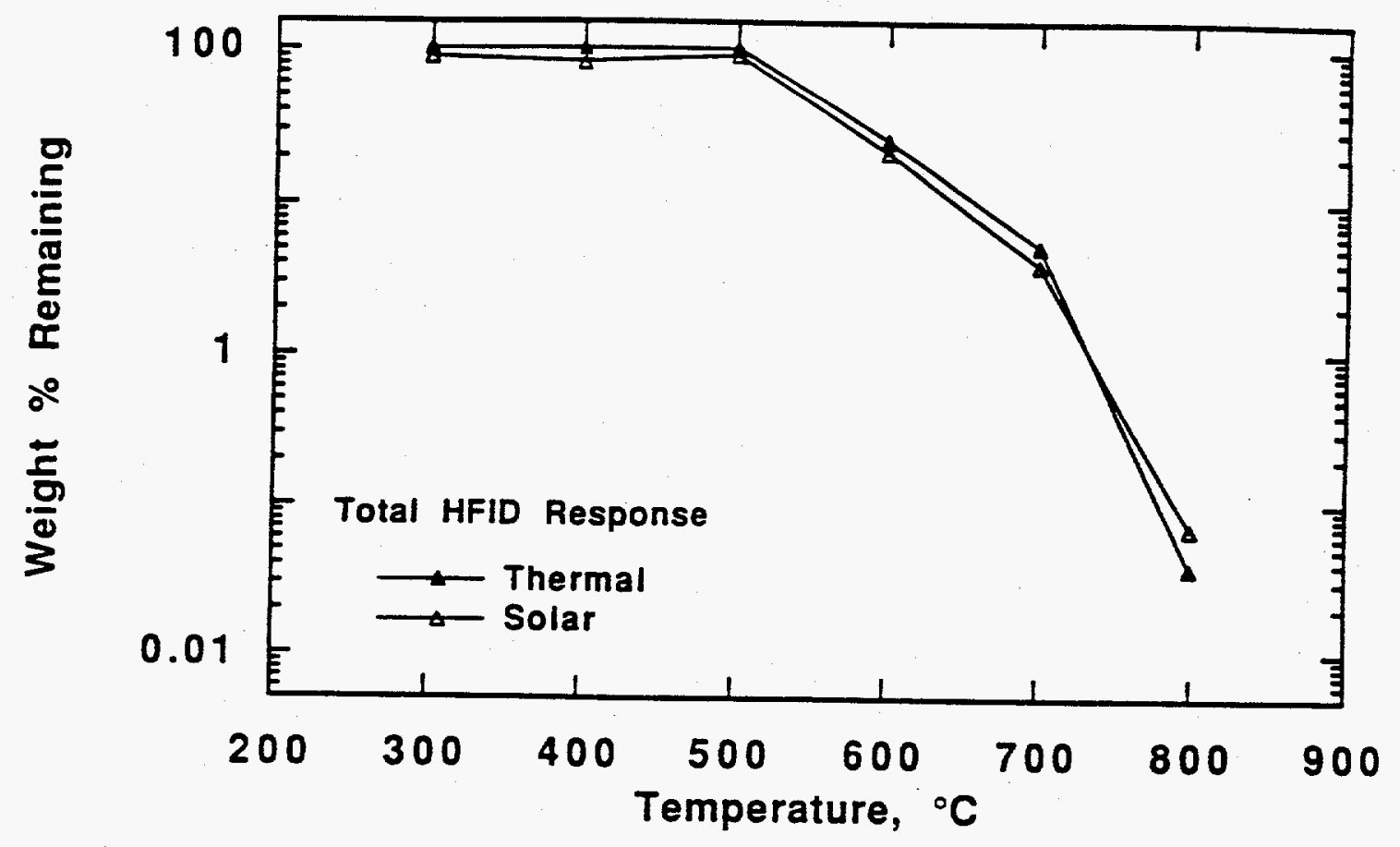

Figure VII.11. Summary of TPRS data for the synthetic waste mixture desorbed from soil exposed to 0 and $273 \mathrm{AM} 1.5$ suns (simulated) for $10 \mathrm{~s}$ in air.

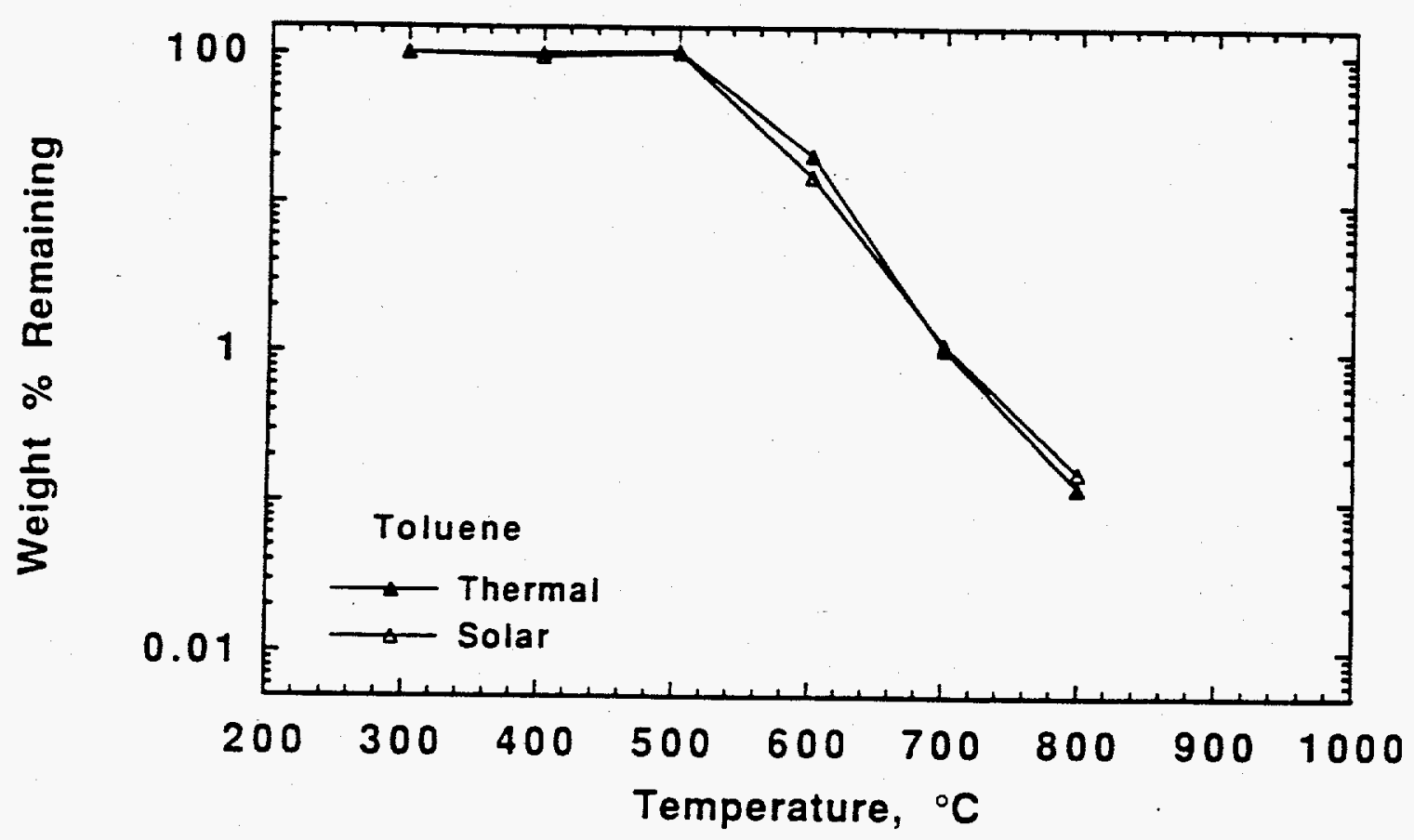

Figure VII.12. Summary of TPRS data for the toluene component of the synthetic waste mixture desorbed from soil exposed to 0 and $273 \mathrm{AM} 1.5$ suns (simulated) for $10 \mathrm{~s}$ in air. 


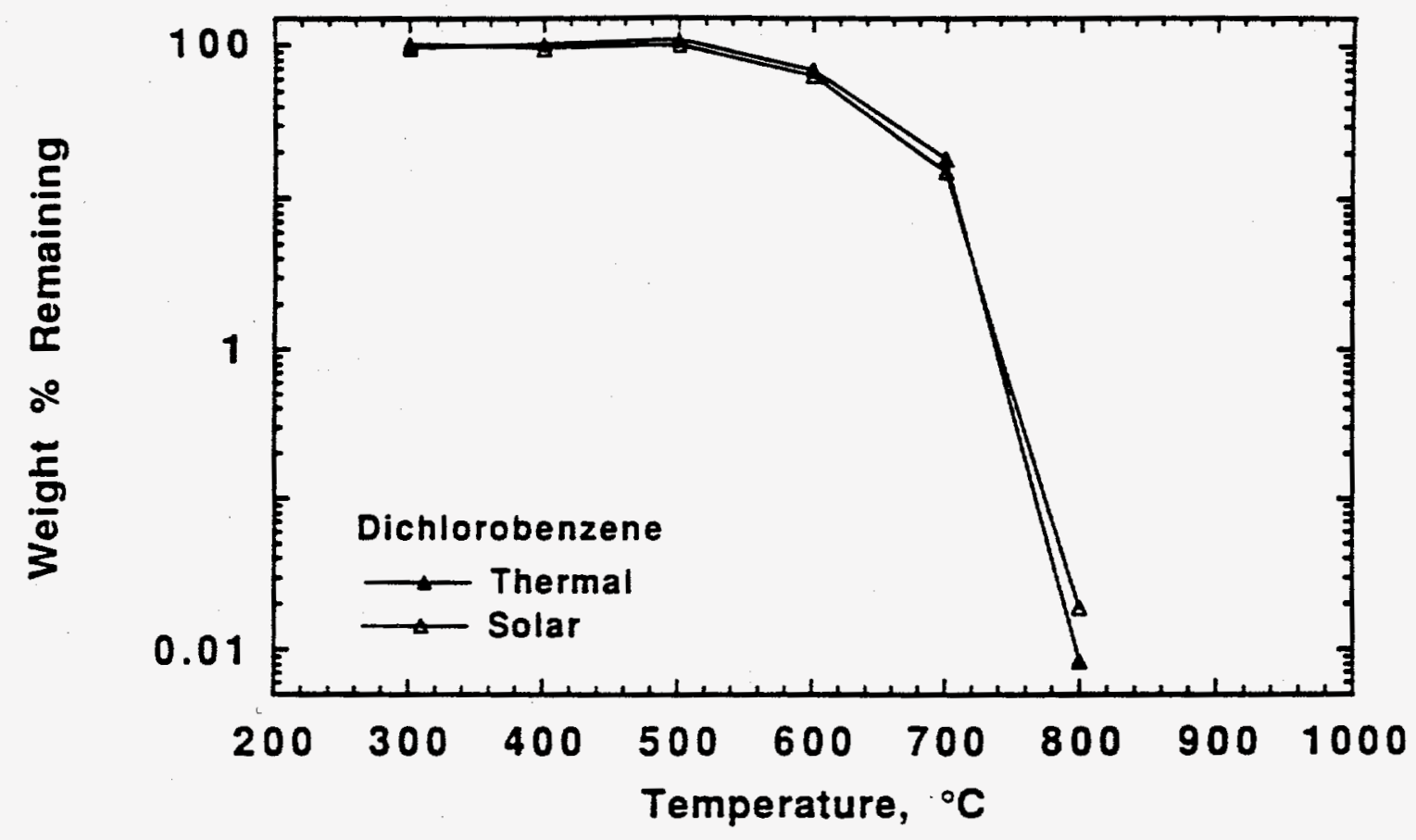

Figure VII.13. Summary of TPRS data for the dichlorobenzene component of the synthetic waste mixture desorbed from soil exposed to 0 and 273 AM 1.5 suns (simulated) for $10 \mathrm{~s}$ in air.

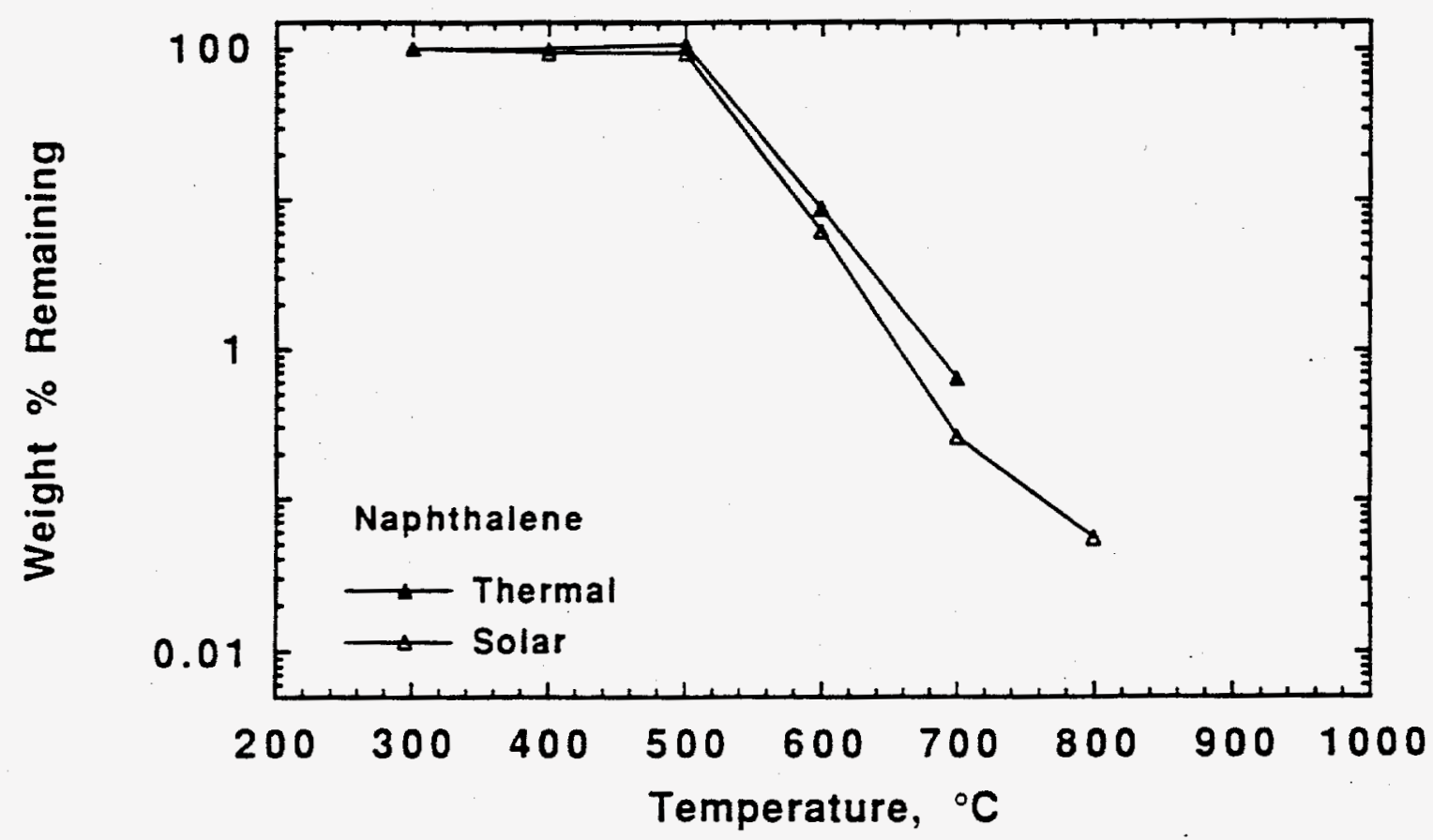

Figure VII.14. Summary of TPRS data for the naphthalene component of the synthetic waste mixture desorbed from soil exposed to 0 and 273 AM 1.5 suns (simulated) for $10 \mathrm{~s}$ in air. 


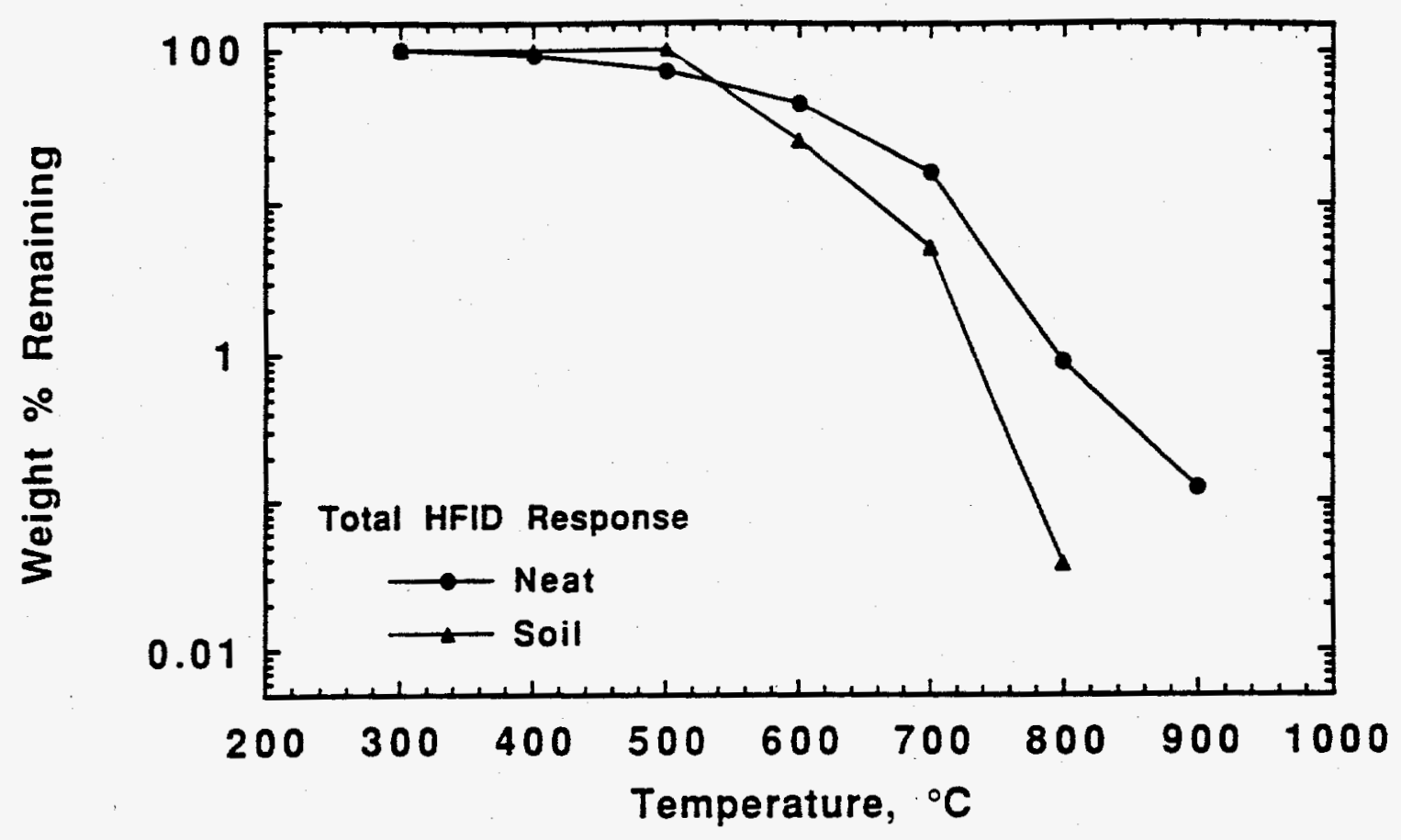

Figure VII.15. Summary of thermal TPRS data for the syntheric waste mixture desorbed from soil exposed for $10 \mathrm{~s}$ in air.

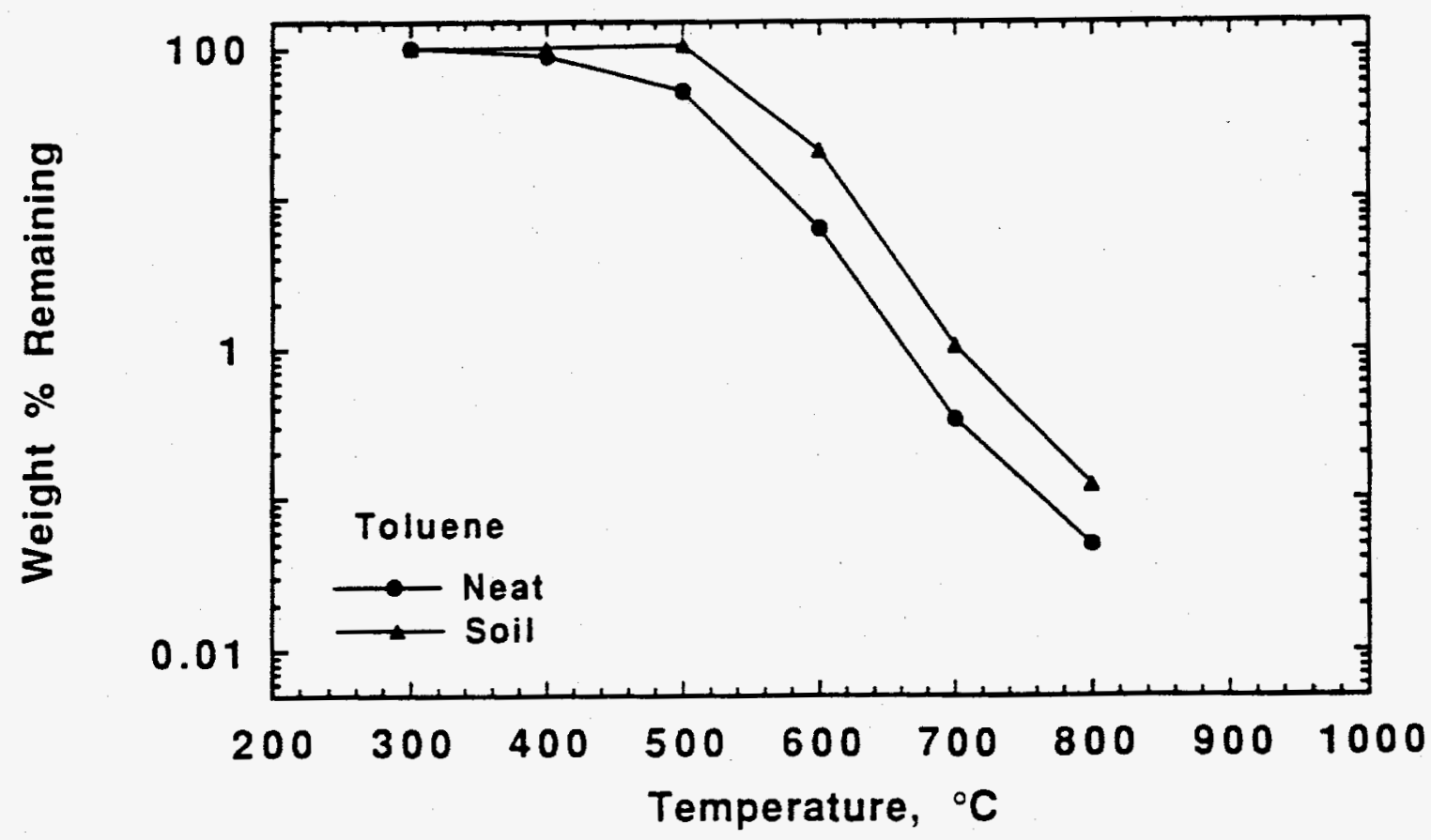

Figure VII.16. Summary of thermal TPRS data for the toluene component of the synthetic waste mixture desorbed from soil exposed for $10 \mathrm{~s}$ in air. 


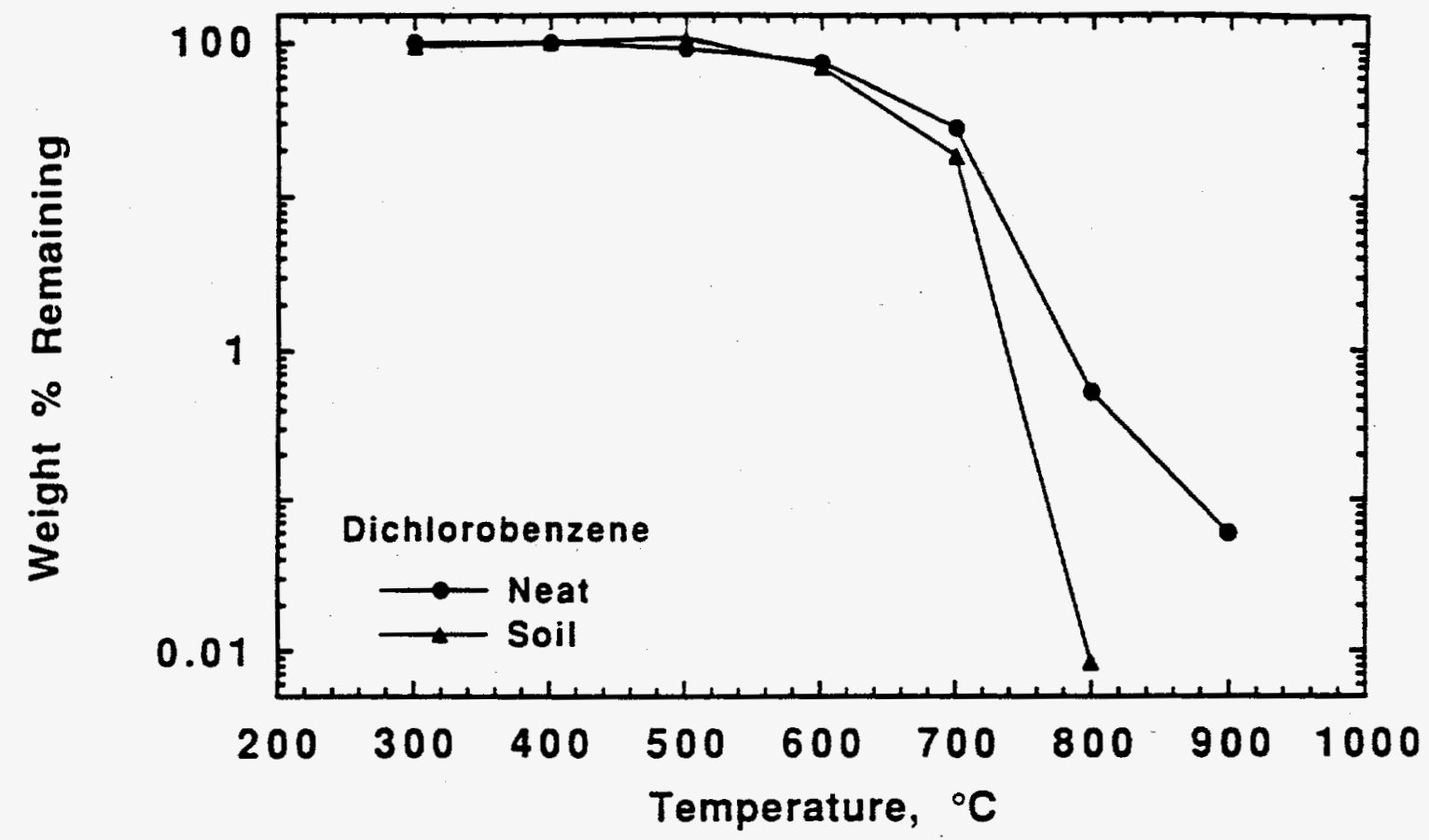

Figure VII.17. Summary of thermal TPRS data for the dichlorobenzene component of the synthetic waste mixture desorbed from soil exposed for $10 \mathrm{~s}$ in air.

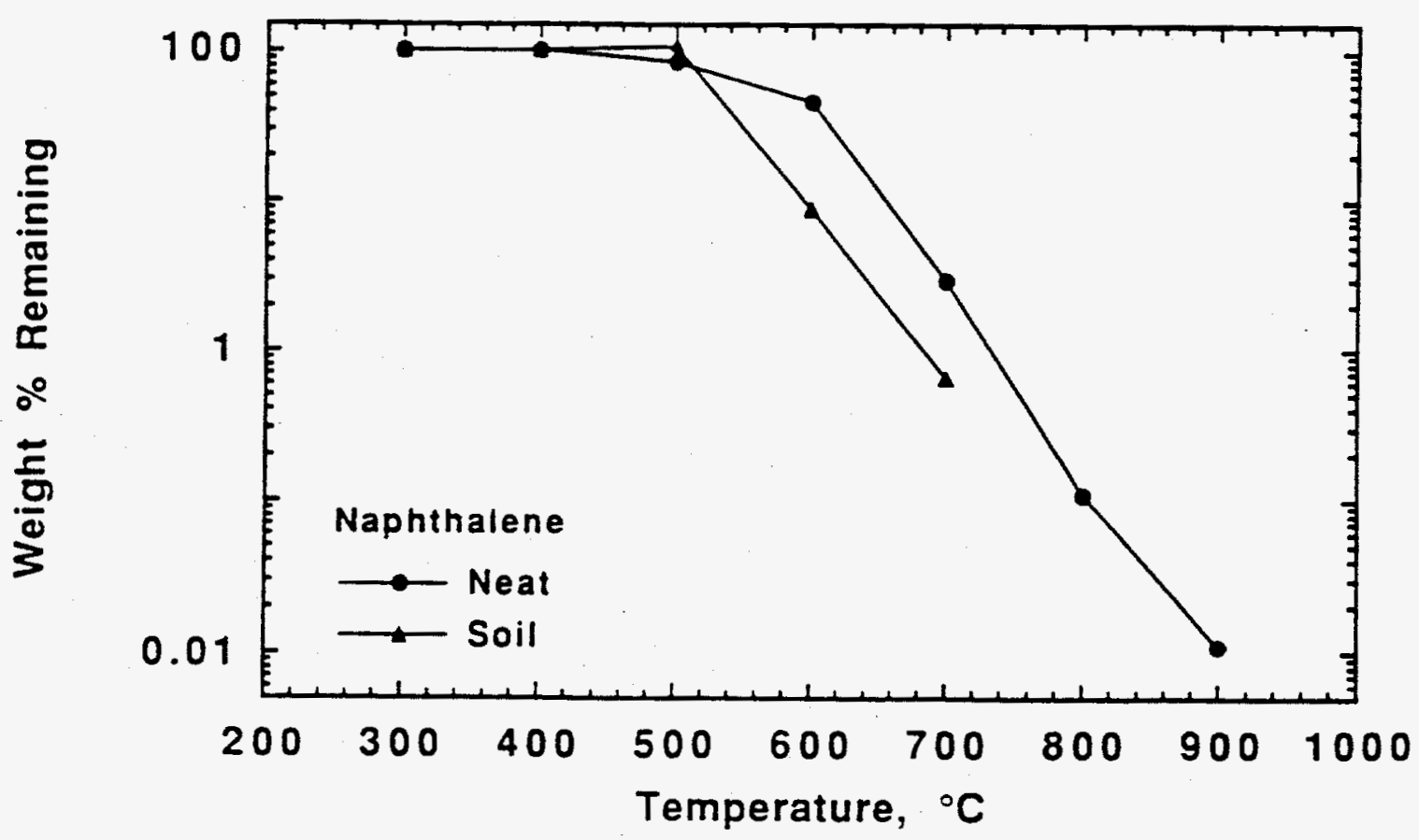

Figure VII.18. Summary of thermal TPRS data for the naphthalene component of the synthetic waste mixture desorbed from soil exposed for $10 \mathrm{~s}$ in air. 


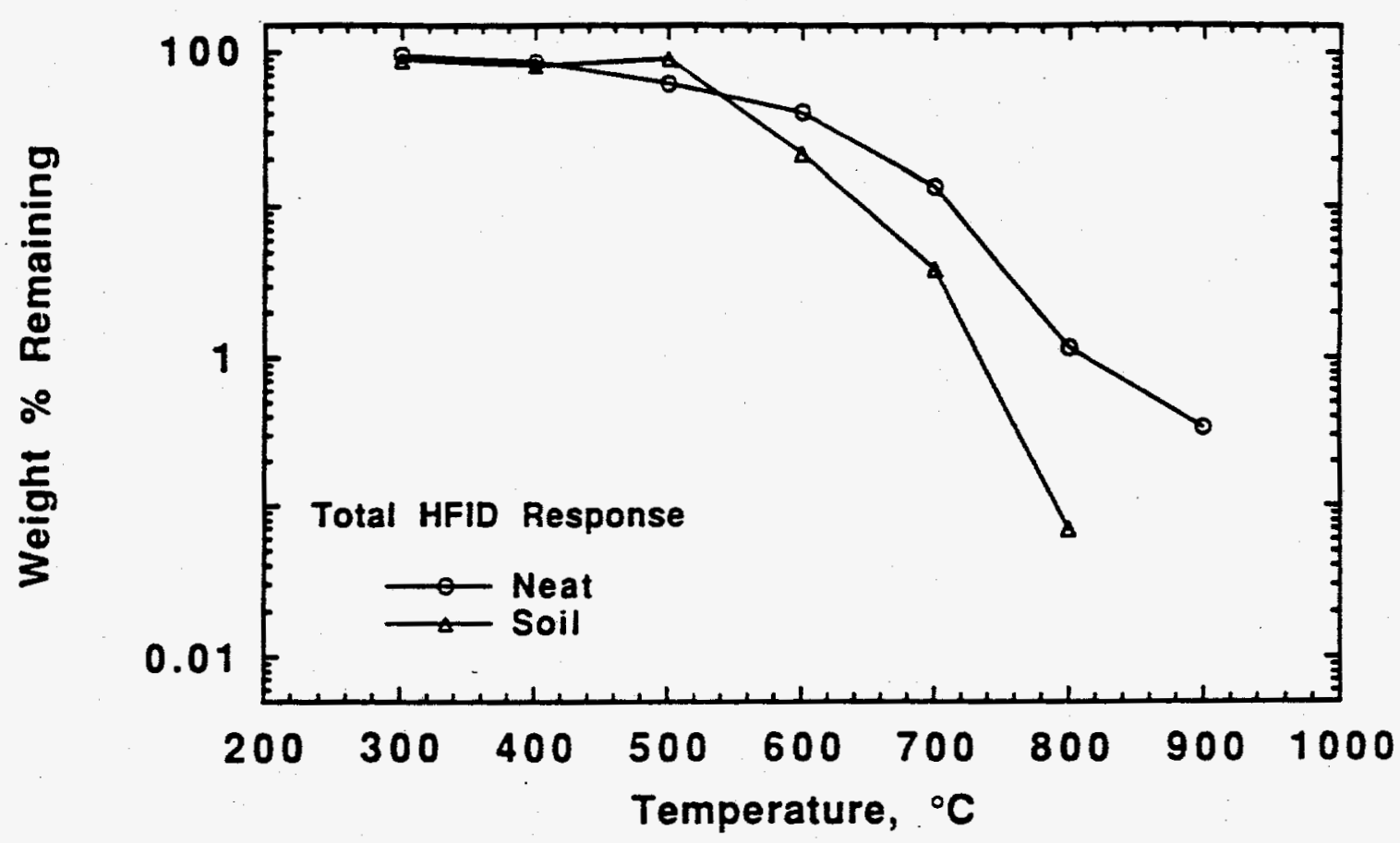

Figure VII.19. Summary of thermal TPRS data for the synthetic waste mixture desorbed from soil exposed to 273 AM 1.5 suns (simulated) for $10 \mathrm{~s}$ in air.

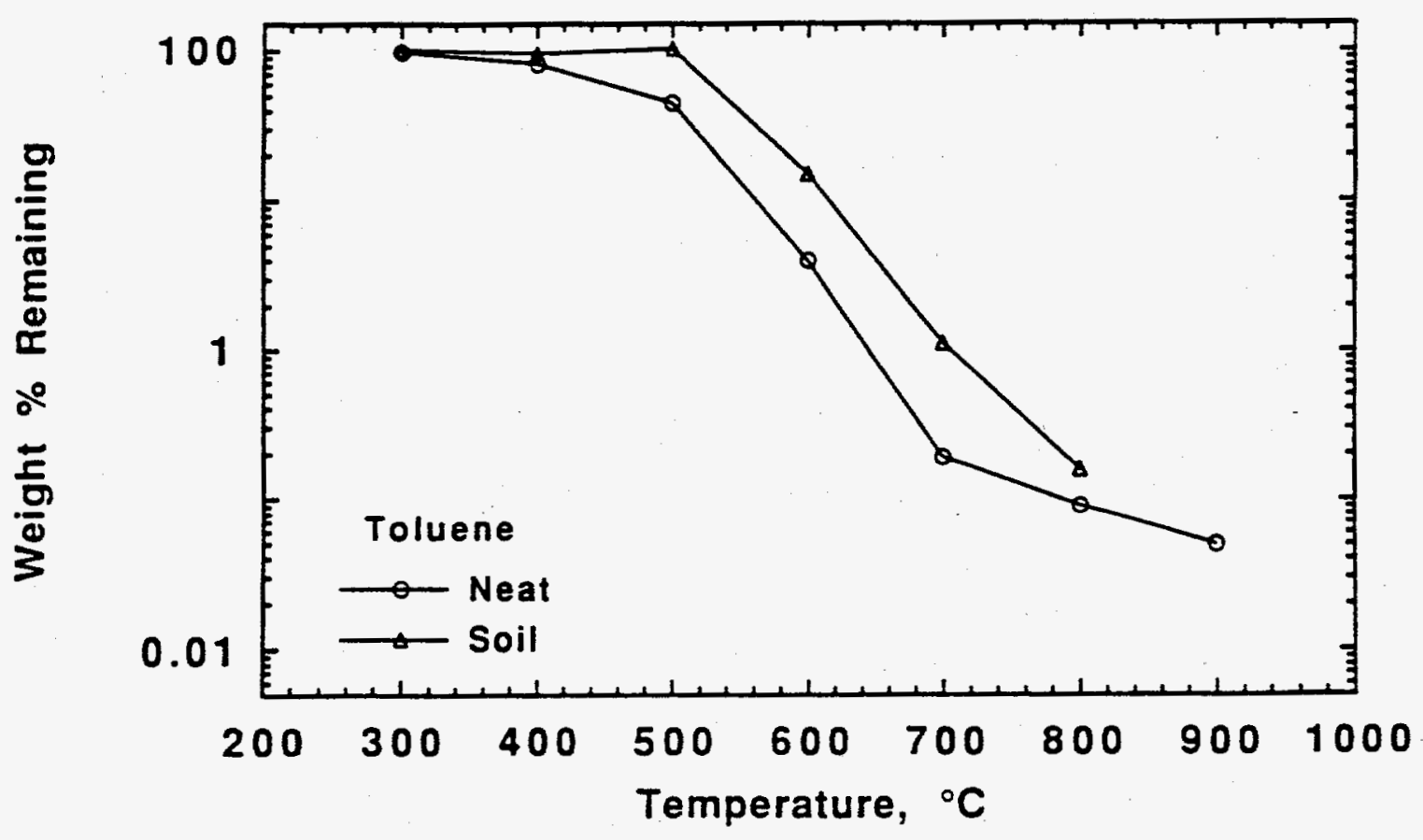

Figure VII.20. Summary of thermal TPRS data for the toluene component of the synthetic waste mixture desorbed from soil exposed to $273 \mathrm{AM} 1.5$ suns (simulated) for $10 \mathrm{~s}$ in air. 


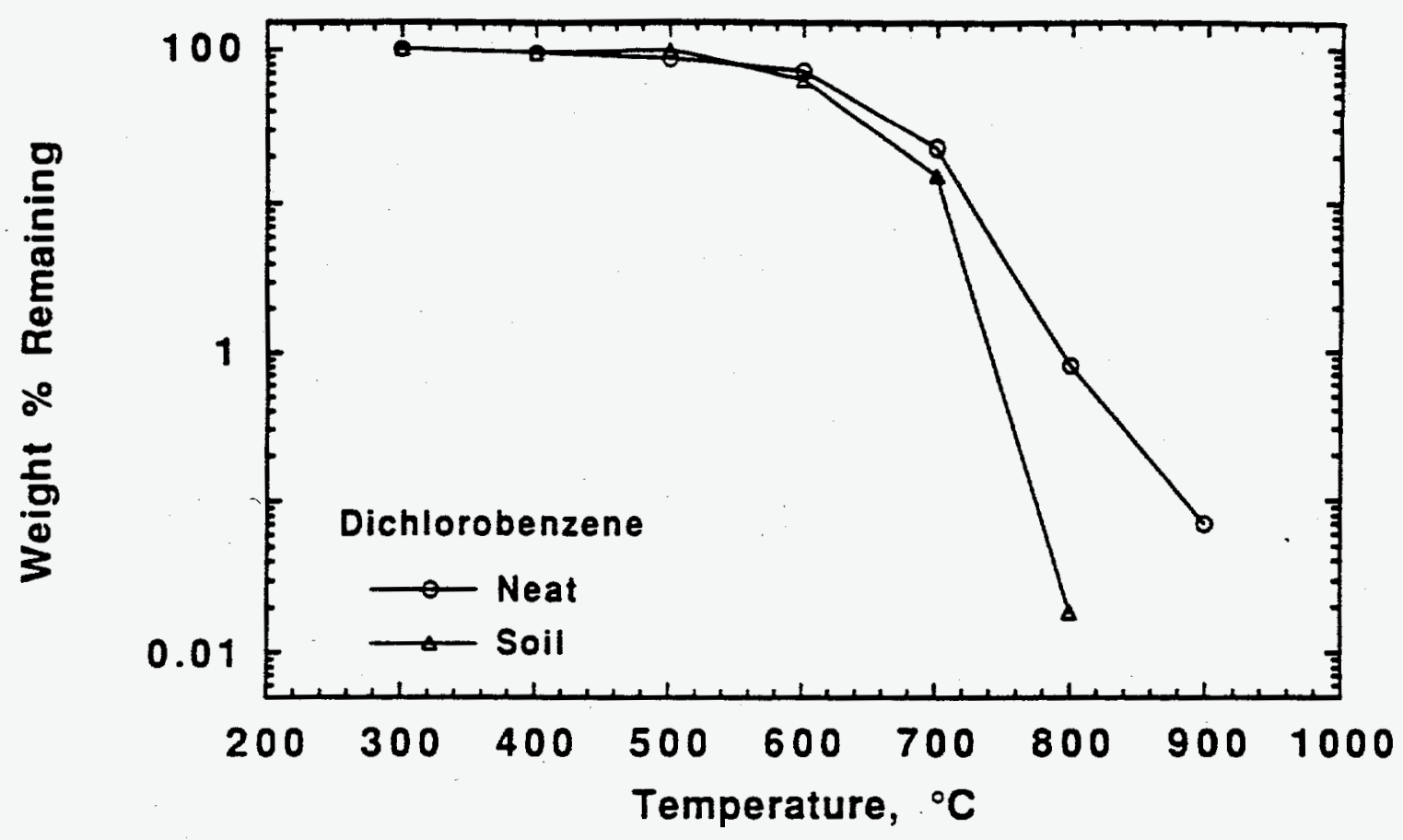

Figure VII.21. Summary of thermal TPRS data for the dichlorobenzene component of the synthetic waste mixture desorbed from soil exposed to 273 AM 1.5 suns (simulated) for $10 \mathrm{~s}$ in air.

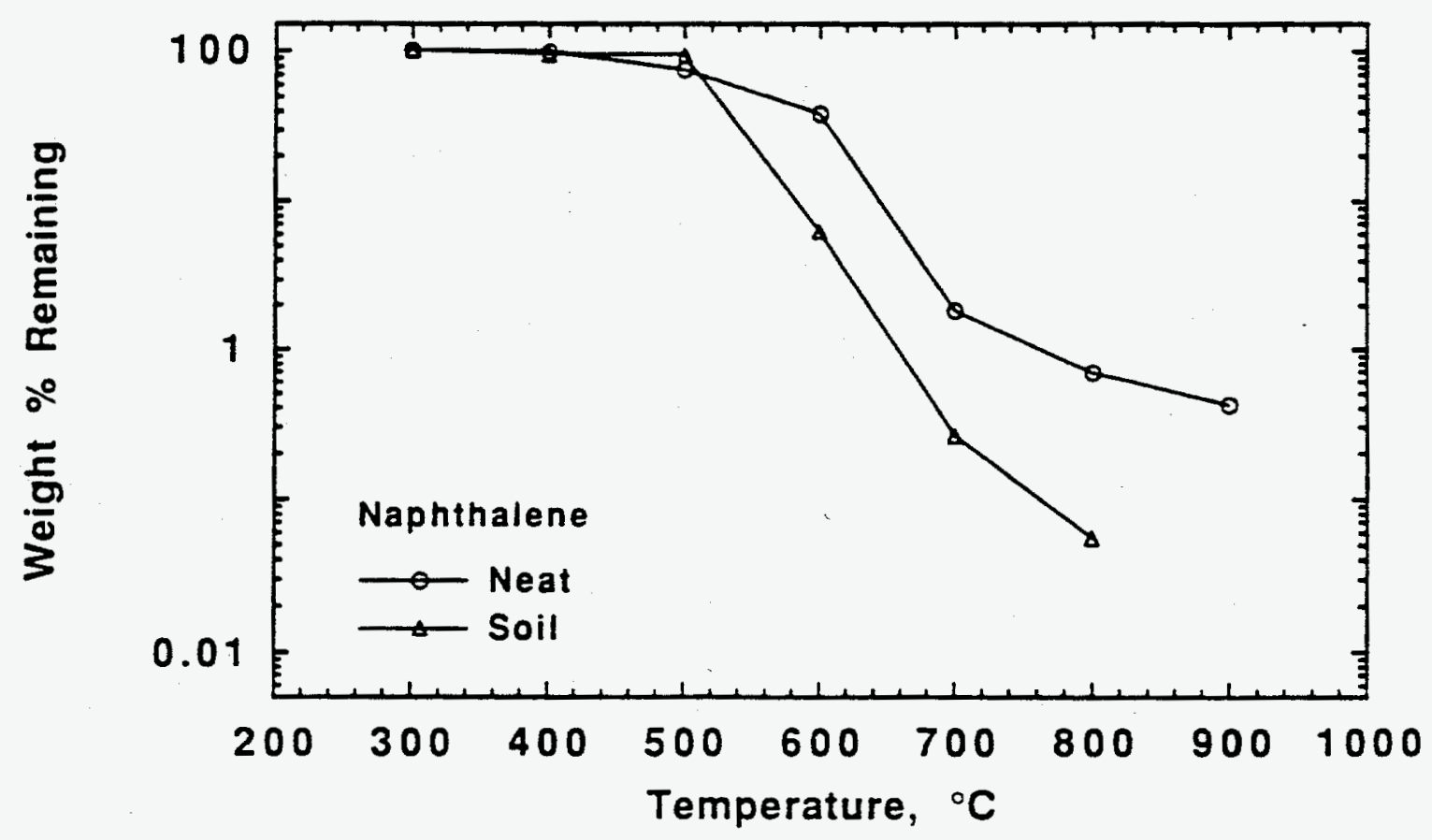

Figure VII.22. Summary of themal TPRS data for the naphthalene component of the synthetic waste mixture desorbed from soil exposed to 273 AM 1.5 suns (simulated) for $10 \mathrm{~s}$ in air. 


\begin{tabular}{|c|c|c|c|}
\hline $\begin{array}{l}\text { Document Control } \\
\text { Page }\end{array}$ & $\begin{array}{l}\text { 1. NREL Report No. } \\
\text { NREL/TP-430-6534 }\end{array}$ & $\begin{array}{l}\text { 2. NTIS Accession No. } \\
\text { DE94006912 }\end{array}$ & 3. Recipient's Accession No. \\
\hline \multirow{2}{*}{\multicolumn{3}{|c|}{$\begin{array}{l}\text { 4. Title and Subtitle } \\
\text { High Temperature Photochemical Destruction of Toxic Organic Wastes Using } \\
\text { Concentrated Solar Radiation }\end{array}$}} & $\begin{array}{l}\text { 5. Publication Date } \\
\text { May } 1994\end{array}$ \\
\hline & & & 6. \\
\hline \multicolumn{3}{|c|}{$\begin{array}{l}\text { 7. Author(s) } \\
\text { B. Dellinger; J.L. Graham; J.M. Berman; P.Taylor }\end{array}$} & $\begin{array}{l}\text { 8. Performing Organization Rept. No. } \\
\text { NREL/TP-430-6534 }\end{array}$ \\
\hline \multicolumn{3}{|c|}{ 9. Performing Organization Name and Address } & $\begin{array}{l}\text { 10. Project/Task/Work Unit No. } \\
\text { SI } 41.3020\end{array}$ \\
\hline \multicolumn{3}{|c|}{$\begin{array}{l}\text { Environmental Science and Engineering } \\
\text { University of Dayton } \\
300 \text { College Park Ave. } \\
\text { Dayton, Ohio } 45469-0132\end{array}$} & $\begin{array}{l}\text { 11. Contract (C) or Grant (G) No. } \\
\text { (C) XX-6-06082-1 } \\
\text { (G) }\end{array}$ \\
\hline \multicolumn{3}{|c|}{ 12. Sponsoring Organization Name and Address } & $\begin{array}{l}\text { 13. Type of Report \& Period Covered } \\
\text { Subcontract Report }\end{array}$ \\
\hline \multicolumn{3}{|c|}{$\begin{array}{l}\text { National Renewable Energy Laboratory } \\
1617 \text { Cole Blvd. } \\
\text { Golden, CO } 80401-3393\end{array}$} & 14. \\
\hline \multicolumn{4}{|c|}{$\begin{array}{l}\text { 15. Supplementary Note } \\
\text { NREL technical contact, Dr. Thomas Milne, (303) 231-7000, x1440 }\end{array}$} \\
\hline \multicolumn{4}{|c|}{$\begin{array}{l}\text { 16. Abstract (Limit: } 200 \text { words) } \\
\text { The report is concerned with using concentrated solar radiation to destroy hazardous waste. As opposed to using just the } \\
\text { thermal energy in solar radiation to "burn" the waste, the concept focused on the synergistic combination of thermal energy (viz. } \\
\text { infrared and visible solar radiation) and photolytic energy (viz. ultraviolet solar radiation) to induce a thermally enhanced } \\
\text { photochemical destruction of the waste. }\end{array}$} \\
\hline \multicolumn{4}{|l|}{$\begin{array}{l}\text { 17. Document Analysi } \\
\text { a. Descriptors } \\
\text { photocatalyti } \\
\text { b. Identifiers/Open- } \\
\text { c. UC Categories } \\
241\end{array}$} \\
\hline \multirow{2}{*}{\multicolumn{2}{|c|}{$\begin{array}{l}\text { 18. Availability Statement } \\
\text { National Technical Information Service } \\
\text { U.S. Department of Commerce } \\
5285 \text { Port Royal Road } \\
\text { Springfield, VA } 22161\end{array}$}} & & $\begin{array}{l}\text { 19. No. of Pages } \\
336\end{array}$ \\
\hline & & & $\begin{array}{l}\text { 20. Price } \\
\text { A13 }\end{array}$ \\
\hline
\end{tabular}

Form No. 0069E (6-30-87) 